\title{
DOE/DP/40030-1
}

KMSF-U884

\section{ANNUAL REPORT ON LASER FUSION RESEARCH}

\author{
Fiscal Year 1978
}

Editor: R. R. Johnson

WORK PERFORMED UNDER CONTRACT

ED-78-C-08-1598

KMS FUSION, INC.

3621 S. STATE RD.

ANN ARBOR, MI 48104

This book was prepared as an account of work sponsored by an agency of the United States Government. Neither the United States Government nor any agency thereof, nor any of their employees, makes any warranty, express or implied, or assumes any legal liability or responsibility for the accuracy,

completeness, or usefulness of any information, apparatus, product, or process disclosed, or
represents that its use would not infringe privately owned rights, Reference herein to any specitic

represents that its use would not infringe, privately owned rights. Reference herein to any specitic
commercial product, process, or service by trade name, trademark, manufacturer, or otherwise, does

not necessarily constitute or imply its endorsement, recommendation, or favoring by the United 


\section{DISCLAIMER}

This report was prepared as an account of work sponsored by an agency of the United States Government. Neither the United States Government nor any agency Thereof, nor any of their employees, makes any warranty, express or implied, or assumes any legal liability or responsibility for the accuracy, completeness, or usefulness of any information, apparatus, product, or process disclosed, or represents that its use would not infringe privately owned rights. Reference herein to any specific commercial product, process, or service by trade name, trademark, manufacturer, or otherwise does not necessarily constitute or imply its endorsement, recommendation, or favoring by the United States Government or any agency thereof. The views and opinions of authors expressed herein do not necessarily state or reflect those of the United States Government or any agency thereof. 


\section{DISCLAIMER}

Portions of this document may be illegible in electronic image products. Images are produced from the best available original document. 
NOTICE

This report was prepared to document work sponsored by the United States Government. Neither the United States nor its agent, the United States Department of Energy, nor any Federal. employees, nor any of their contractors, subcontractors, or their employees makes any warranty, expressed or implied, or assumes any legal liability or responsibility for the accuracy, completeness, or usefulness of any information, apparatus, product or process disclosed, or represents that its use would not infringe privately owned rights.

\section{NOTICE}

Reference to a company or product name does not imply approval or recommendation of the product by KMS or the Department of Energy to the exclusion of others that may be suitable. Readers who desire further information on other KMSF documents should address their inquiries to KMS Fusion, Inc., Ann Arbor, Michigan 48106.

Printed in the United States of America

Available from:

National Technical Information Service

U.S. Department of Commerce

5285 Port Royal Road

Springfield, Virginia 22161

Price: Printed Copy $\$ 12.50$

Microfiche $\$ 3.00$ 


\title{
1978 ANNUAL REPORT \\ ON LASER FUSION RESEARCH
}

\author{
Fiscal Year 1978
}

Editor: R. R. Johnson

KMS FUSION, INC.

3621 S. STATE RD.

ANN ARBOR, MI 48104

PREPARED FOR THE

U. S. DEPARTMENT OF ENERGY

NEVADA OPERATIONS OFFICE

UNDER CONTRACT ED-78-C-08-1598 
A Statement for KMS Fusion, Inc.

We present herewith the report of KMS Fusion activity for FY 1978 under Contract ED-78-C-08-1598. The progress and results reported are an outgrowth of the ongoing KMS Fusion program initiated formally on February 3, 1971 with the signing of a "no-fund" contract between the U.S. Atomic Energy Commission and KMS Fusion, Inc., a subsidiary of KMS Industries, Inc. The laser-fusion program at KMS Industries started with theoretical studies initiated independently in 1969 at the KMS Technology Center. On May 19, 1975, a funded contract, E(11-1)-2709, was initiated between the United States Energy. Research \& Development Administration (ERDA) and KMS Fusion, which provided for the utilization of KMS facilities and manpower in support of the national laser-fusion program.

Over twenty million dollars of private funds were spent by KMS Fusion as part of its contribution to the creation of the field now known as Inertial Confinement Fusion (ICF). We believe ICF needs continuing and expanding involvement of the private sector. Government policy, however, has not yet attracted sufficient private involvement except for KMS Fusion, and, as a result, the development has moved since 1975 at a pace determined almost entirely by official government programs, with principal emphasis on the work at the nuclear. weapons laboratories. We wish to encourage more initiative on the part of the Government to mobilize untapped resources available in industry for timely development of ICF for civilian energy use. 
At this time, ten percent of the end use of energy, delivered to the home or factory, is in the form of electricity. About thirty percent of our fossil fuels go to supply electricity. The balance, or seventy percent is used directly for transportation, process heat, and so forth. This is where our national vulnerability is greatest and it is to this problem that we propose to apply nuclear energy from ICF for generating synthetic fuel. For that application, because of its geometry and engineering characteristics, the ICF system is uniquely suited.

One of the most sensitive questions raised relative to ICF is security. On the debit side, we are told, is the danger of protiferation; the teaching of others through pellet technology how to make weapons. We are aware of the problem, have studied it, and have testified before the Moe Committee on this subject. We-believe, as responsible citizens and scientists, that ICF can be developed under a special civilian program for power on an unclassified basis without contributing to weapons proliferation.

On the benefit side, if we develop an inexhaustible synthetic process to supply the needs now met by hydrocarbons, both domestic and imported, we will have made a major contribution to stabilizing the world economy and our own national strength.

We believe a National development program for ICF for which such goals can be demonstrated deserves support and effort beyond the "me-too" position it now occupies. We are proposing, therefore, the creation of an industry-based, energy-oriented, civilian ICF program to be carried out by KMS Fusion and additional industrial participants. 
The KMS Fusion experience is unique. It is a private company that undertook, on its own initiative, to enter a classified field to work on the basic science required to prove the feasibility of a new energy concept, recognizing the unprecedented promise of that concept and the role of private enterprise in fulfilling it. Operating in the private sector, the Company has today the potential and the plans for spearheading the introduction of laser fusion and its applications into the civilian economy.

We expect to pursue this role through continuing significant research as part of the national program in laser fusion and through growing activity in proprietary innovation and invention, with private funds, under our "parallel efforts" program.

Laser fusion, as a civilian energy source, must eventually stand the test of the competitive marketplace. KMS Fusion is working to hasten the day when that test will take place, and to being a leading participant in it.

H. J. Gomberg

Chairman and President KMS Fusion, Inc. 
Introduction

1.1 Spherical Shell Fuel Containers 1-4

1.1.1 Aspect Ratio Control 1-6

1.1.2 Shel1 Size Maximization 1-8

1.1.3 Glass She11 Strength $1-11$

$\begin{array}{ll}1.1 .4 & \text { Surface Finish } \\ 1-12\end{array}$

1.1 .5 Fuel Retention 1-15

1.1.6 Wal1 Uniformity 1-16

1.2 Polymer Research 1-19

1.2.1 Polymeric Foam Development 1-20

1.2.2 High Tensile Strength-Low Permeability 1-24

1.2.3 Deuterated Polymeric Shells $1-26$

$\begin{array}{ll}1.2 .4 & \text { Metal Loaded Polymers } \\ 1-27\end{array}$

1.2.5 Macro Polymeric Shells 1-27

References $1-31$

1.3 Cryogenic Technology Fabrication and Engineering 1-32

1.3.1 Liquid Layer Targets 1-32

1.3.2 Liquid Layer Target Shot Results 1-34

1.3.3 Limitation of the Technique Used 1-36

1.3.4 Extended Conduction Cooling Technique 1-37

$\begin{array}{ll}\text { 1.3.5 Solid Layer Targets } & \text { 1-4l }\end{array}$

1.3.6 Solid Fuel Layers Produced Under the 1-41

Radiation Shield
1.3 .7 Prndurtion of Uniform Solid Fuel

1.3.8 Equipment Required for Uniform Solid 1-47

1.3.9 Evaluation of Solid Fuel Layers $\quad$ 1-53

1.3.10 Target Chamber Experiments 1-60

(ASE Experiments on Sol id Fuel Layers)

1.3.11 Laser Irradiation of Uniform Sol id Fuel 1-61

Layer Targets

References $\quad 1-63$

1.4 Advances in Fabrication Technology 1-65

1.4.1 Application of Integrated Circuit Fabrication 1-65 Techniques to ICF Research Reactor Pellets 
1.4.2 Fabrication Techniques Development in Support 1-74

$\begin{array}{lll}1.4 .3 & \text { of Laser and Diagnostic Requirements } & 1-82\end{array}$

$\begin{array}{lll}1.4 .4 & \text { Injection } & 1-91\end{array}$

References $1-101$

\section{Section 2 Laser Fusion Experiments}

Introduction and Summary $2-1$

2.1 Implosion Physics 2-4

2.1.1 High Density Implosion Experiments 2-4

2.1.2 Analysis of Alpha-Particle Spectra 2-15

References 2-29

2.2 Fast Ion Measurements of Laser-Produced Spherical 2-30

Plasmas

References $2-47$

2.3 Absorbed Energy Measurements 2-49

References $\quad 2-57$

2.4 Diagnostic Development 2-58

2.4.1 X-Ray Streak Camera 2-58

2.4.2 Zone Plate Alpha. Particle and X-Ray 2-63

$\begin{array}{lll}2.4 .3 & \text { Coded Imaging } & \text { X-Ray Spectrography }\end{array}$

2.4.4 Self-Calibrating Differential Calorimeter $\quad 2-68$

2.4.5 Two Temperature Fit from X-Ray Diagnostics 2-71

$\begin{array}{ll}2.4 .6 & \text { Holographic Interferometry } \\ 2.77\end{array}$

$\begin{array}{ll}2.4 .7 & \text { The Data Acquisition System 2-81 }\end{array}$

References $\quad 2 \cdot 86$

\section{Section 3 Laser Fusion Theory}

Introduction 3-1

-3.1 Fast Ion Energy Loss in Dense Plasmas 3-5

3.1 .1 Introduction $3-5$

3.1.2 Ion Stripping in Solid Targets 3-6

3.1.3 Lnergy Loss 3-6

3.1.4 Results and Comparison with Experiment $3-12$

References 3-17 
3.2 Electron Transport: Fokker-Planck Calculations 3-18 References $3-21$

3.3 Ionization Equation of State 3-22 References 3-24

3.4 Profile Modification by Ponderomotive Forces: $3-25$ Equilibrium and Stability

3.4.1 Steady-State Equations 3-25

3.4.2 Stability of Profiles and Time-Dependent 3-29 Absorption

References 3-38

3.5 Ponderomotive Potential Effects on Ohm's Law 3-39 References 3-48

3.6 Effect of Flux-Limited Thermal Transport on 3-50

Critical Surface Jump Conditions

References 3-64

3.7 Spherical Rarefaction Shocks 3-66

References $3-73$

3.8 Explosively Heated Gaussian Objects $\quad 3-74$

References $\quad 3-86$

\section{SECTION 4 LASER AND OPTICS}

Introduction $4-1$

4.1 Randwidth Broadening - The Plasma Spatial Filter 4-3

4.2 Frequency Doubling Experiments 4-6

4.3. Advanced Laser Candidates 4-13

4.3.1 Chemically Pumped Iodine Laser (CPIL) 4-15

4.3.? The Free Electron Laser (FEL) 4-32

4.4 Glass Laser Operation 4-61

4.4.1 Power on Target 4-61

4.4.2 Far Field Data 4-64

4.4.3 Near Field Vata $4-67$

$\therefore \quad 4.4 .4$ Disc Thermal Heating Effects $\quad 4-72$

4.4.5 Laser System Cleanliness $4-74$ 
4.5 2 TW Laser Upgrade 4-84

4.5.1. Phosphate Laser Disc Cladding 4-86

4.5.2 Disc Amplifier Modifications 4-89

References $4-91$ 
PELLET TECHNOLOGY

Introduction

1.1 Spherical Shell Fuel Containers

1.2 Polymer Research

1.3 Cryogenic Target Fabrication and Engineering

1.4 Advances in Fabrication Technology 


\section{Introduction}

\section{E. Solomon}

The pellet technology program at KMSF has had as a goal since 1972 the development of a broad technology base capable of meeting the diverse and ever-changing requirements of the national inertial confinement fusion program. In the past year we have also provided pellets to meet the requirements of a few national laboratories in foreign countries (Italy and the United Kingdom). Four technical areas of continued concentration are 1) glass and plastic shells to serve as thermonuclear fuel containers and substrates for subsequent deposition of coatings, 2) thin and thick metal deposited coatings of varying density and thickness and sequence of deposition, 3) formation of uniform liquid and solid layers of thermonuclear fuel or of noble elements, and 4) microfabrication of symmetric, layered, nested or concentric pellet designs utilizing standard techniques, or in the most recent advance adapting integrated-circuit silicon technology to form the same types of pellet configurations but with silicon as one of the outer layers.

Installation of a new glass furnace in this reporting period has allowed us to extend the working temperature range, closely control the temperature and increase the dwell time of the glass-forming particles. The net result is that there has been a dramatic increase in overall yield and individual shell quality in the 400 to $500 \mu \mathrm{m}$ size range. Shells up to $1000 \mu \mathrm{m}$ in diameter have been blown in the furnace while other techniques have been used to form shells in the 4 to $5 \mathrm{~mm}$ range. Silica shells of greater than $99 \%$ purity have also been blown. Properties or parameters of the glass shells being methodically investigated are: tensile strength, hydrogen permeability, surface finish, diameter, wall uniformity, wall thickness and sphericity. The following sections of this report will 
describe the detailed way in which our overall understanding of the glass synthesis-blowing process has been improved, and has allowed us to develop an empirical model of the blowing process.

Research with polymeric materials has provided 1) high strength, low permeability polymer shells, 2) polymeric foam layers with densities between 0.01 and $0.1 \mathrm{~g} / \mathrm{cm}^{3}(1 \%$ to $10 \%$ of theoretical density) and with a cell size of order of $10 \mu \mathrm{m}, 3$ ) polymers in which hydrogen atoms have been replaced with deuterium atoms which become part of the TN-fuel, and 4) molecules of high atomic number elements incorporated into a pölymer she11. In Section 1.2 is the detailed description of the accomplishments in the polymer area.

Theoretical considerations suggest that high compressed fuel densities can be achieved if the fuel is initially condensed as a liquid or solid layer thus reducing the internal gas pressure against which the imploding shell must work. A liquid or solid layer of DT inside a target cannot respond quickly enough, upon absorption of fast electrons which are generated early in the laser pulse, to completery fill the interior volume of the target before the target undergoes significant volume reduction. Uniform solid DT layers are routinely formed by flash heating nonuniform DT layers with a laser, then permitting rapid resolidification in a uniform thermal environment. Such cryogenic pellet configurations have contributed to achieving a compressed fuel density which is thirty times liquid density. with only a $1 / 2$.TW laser driver.

Throughout the inertial fusion program there has been a mildly haunting question relative to the deleterious effect of the pellet mounting fiber. We have directly addressed that question by laser imploding a pellet with neither fiber nor membrane support. The result is 
a very symmetric spherical expansion as observed from holographic images, obviously without the asymmetry casued by the mass of the support. 'Details of how the "suspended" pellets were imploded is in Section 1.4.3.

Symmetric, nested pellet configurations of one sort or another are of great interest to the national inertial program. There is also general interest in being able ultimately to manufacture such pellet configurations at high rate $\left(\sim 10^{6} /\right.$ day) and at low cost ( $\sim 10 \phi /$ each). The transfer of billions of dollars worth of technology from the integrated circuit (IC) industry to the inertial confinement fusion (ICF) pellet tech-. nology area appears to be of inestimable importance to this objective. If this IC-ICF pellet technology transfer proceeds as anticipated (assuming that glass TN fuel shells can be manufactured and coatings can be applied to the interior of silicon hemispheres and to the exterior of TN fuel shells) then a break through in low cost, high rate pellet-manufacture will have been made. A description of the details of these processes can be found in Sections 1.4.1 through 1.4.2. 


\subsection{Spherical Shell Fuel Containers}

N. E. Doletzky, R. L. Downs, M. A. Ebner, P. J. Lenrion, A. J. Meyer, W. J. Miller, R. L. Nolen, Jr., J. J. Rolen, R. G.

Schneggenburger and D..E. Solomon

Glass shell research at KMS Fusion has continued along the dual path of improving shell quality and increasing shell size while concurrently furthering our understanding of the factors that dominate shell formation and shell properties. One important property of glass shells for laser fusion research is aspect ratio. Control of this property has been pursued by a parametric study of gel composition and shell blowing parameters. Gel composition (oxide equivalent) and furnace temperature were found to have major effects on aspect ratio, but the effect of the gel preparation method was minor. In the course of this study, much lower aspect ratio shells $(<100)$ were produced than previously encountered, demonstrating the metal-organic technique is capable of producing shells with a wide range of aspect ratios.

The installation of a new vertical tubular tower furnace during the past year extended both the temperature range and length of the hot zone: This has resulted in a dramatic increase in shell quality and yield in the moderate size $(400$ to $500 \mu \mathrm{m})$. The higher temperature capability also allowed the formation of one millimeter shells.

In a short term study, the basic techniques of blow-molding were developed. Shells, 4 to $5 \mathrm{~mm}$, were synthesized by blow-molding of sealed capillary preforms using spinning graphite molds in an induction furnace. Levitation of glass shells by gas jets was explored in another minor project aimed at developing techniques to manufacture and/or improve glass macroshells. Success was achieved after examining many nozzle designs, although more spatial stability is required for the technique 
to be practical. Sufficient heat transfer was achieved to soften and subsequently expand a glass macroshell.

A shell strength testing device and procedure has been developed and preliminary experiments conducted with compressive loads. Early results are consistent with expected strength differences based on differing glass compositions.

The effects of shell processing parameters on wall uniformity continue to be actively pursued. Wall uniformity was one shell property studied as a function of gel composition, gel particle size and tower furnace conditions. Some correlations have been observed, but the exact role of composition remains unclear. It is hoped that a more thorough statistical evaluation of the data presently available and additional complementary experiments will reveal the details of the composition effect on wall uniformity. Despite the puzzles that still exist, good wall uniformity has been observed for a wide range of shell sizes and aspect ratios.

Improvements in glass shell properties through composition changes have continued. The most dramatic achievement has been the recent synthesis of glass shells of $99 \%$ pure silica. These shells should have many superior properties. Their characterization is in progress.

The change in composition during gel to shell conversion, namely. alkali and boron loss, has been studied further with the new tower furnace. High lemperature $\left(>1400^{\circ}()\right.$ ) experiments have confirmed two earlier observations made at lower temperatures $\left(\leq 1250^{\circ} \mathrm{C}\right)$; the more $\mathrm{Na}_{2} \mathrm{O}$ present initially in the gel, the greater the percent loss and a greater $\mathrm{K}_{2} 0$ loss occurs, proportionately, than $\mathrm{Na}_{2} \mathrm{O}$ loss when both are present in the same gel. At high furnacc temperatures $\left(\sim 1500^{\circ} \mathrm{C}\right)$ essentially all 
boron is lost from the shells. The ability to reproduce shell composition despite relatively large alkali and boron losses has again been demonstrated using a potash soda-lime glass. Elemental analys is of individual gel particles and shells is still restricted to comparing relative amounts of various components from one shell to another. The difficulty of obtaining quantitative data on individual particles requires a greater experimental matrix than would otherwise be necessary to understand what changes are occurring during gel particle to shell transformations. The data base currently available is inadequate for developing correlations required for any conclusions on composition variations in shells or gel other than the general observation that intergel and inter-shell calcium variations are less than sodium. The recently acquired EDS capability for our scanning electron microscope will allow the collection of much more analytical data on individual shells and gel particles than has been possible in the past. An early example of the usefulness of this technique was its ability to show that cracked shells from a composition unusually high in calcium have relatively large intra-shell calcium variations. Shells from the same batch which were free of cracks had a much narrower spread in calcium content. Studies of this type will lead to the improvement of both gel synthesis techniques and improved control of shell-blowing parameters.

\subsubsection{Aspect Ratio Control}

A fairly wide range of shell aspect ratios (outside diameter/wall thickness) have been observed with dried gel starting materials. Earlier interest centered on shells with high (>100) aspect ratios as laser fusion pellets and consequently these have received the most attention. The use of metal-organic gel, which we have developed, seems particularly 
well suited as a starting material to produce high aspect ratio glass shells. It is not yet possible to synthesize shells with a predetermined narrow aspect ratio distribution. To better understand the effects of glass composition, gel preparation methods, gel particle size and tower furnace temperature on aspect ratio and the equally important property of wall uniformity, a series of shell synthesis experiments was conducted. A parallel objective was to produce low aspect ratio $(<100)$ shells and this was achieved in the course of the parametric study. Eleven compositions were examined in the soda-borosilicate and soda-lime systems. The tower furnace temperature was held constant for all the experiments while the gel size variation was minimized. The mean aspect ratios for the shells varied from 29 to 152 , but in our preliminary analysis there was no obvious correlation with glass composition other than the observation that the largest aspect ratio was associated with shells having the highest ( $(93 \%) \mathrm{SiO}_{2}$ content. In another experiment, the gel composition and furnace temperature were held constant and the gel particle size varied from 53 to $63 \mu \mathrm{m}$ (sieve cut range) to 150 to $180 \mu \mathrm{m}$. The aspect ratios were observed to increase with gel size to $125 \mu \mathrm{m}$ and then decrease. Changing furnace temperature over the range of 1000 to $1500^{\circ} \mathrm{C}$ ( 6 experiments), while holding composition and gel particle size constant, resulted in the lowest mean aspect ratio (83) for the lowest temperature and the highest mean ratio (273) for the highest temperature. Qualitatively, it appears that shells made from Acid method gel have smaller aspect ratios than those from Base method gel.

The spread (relative standard deviation) in shell aspect ratios observed for thirty-two experimental runs varied from a low of $20 \%$ to a high of $80 \%$ with an overall average of $44 \%$. The conditions required to 
control shell aspect ratio precisely remain elusive, but the results of this fairly extensive study suggest that the most critical parameters may reside in the physical and chemical make-up of the gel, an area that has not yet been studied in detail.

\subsubsection{She11 Size Maximization}

The new furnace has a maximum temperature of $1500^{\circ} \mathrm{C}$ compared to the previous upper temperature limit of $1250^{\circ} \mathrm{C}$. The older furnace hot zone was 2.1 meters while the new furnace is heated for $\sim 4.0$ meters and about $85 \%$ of its length can be held within $\pm 15^{\circ} \mathrm{C}$ of $1500^{\circ} \mathrm{C}$. Operating at maximum temperature resulted in the production of shell batches whose average shell diameters are 500um with excellent wall uniformity, e.g.,

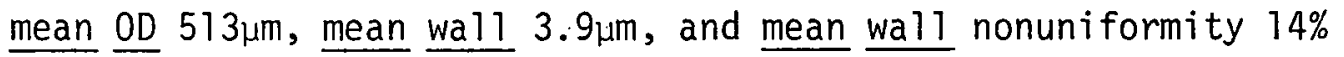
$\left(W N U=\left(W_{\max }-W_{\min }\right) / W_{\min }\right)$. This contrasts with earlier shell samples made at $\sim 1250^{\circ} \mathrm{C}$ with the older furnace where $500 \mu \mathrm{m}$ shells of fair to good quality were produced in only low yields, with the diameter average nearer $350 \mu \mathrm{m}$. The new furnace has produced even larger she11s. The over-all quality decreases with increasing shell size. With large gel particles 425 to $500 \mu \mathrm{m}$ for example, one glass composition was observed to give shells with an average diameter of $770 \mu \mathrm{m}$ and average wall of $12 \mu \mathrm{m}$; however, the wall uniformity was poor. Low yields of shells $1000 \mu \mathrm{m}$ in diameter and larger have also been produced. The combination of increased furnace temperature and length of the hot zone has about doubled the effective shell size attainable from gel particles dropped through the tower furnace. A mathematical model of gel particle to shell transformation is being developed. It is hoped this model will further our understanding of shell blowing and allow the prediction of furnace length and temperature requirements for still larger and higher quality glass shells. 
Two other complementary approaches to the production of large shells were initiated during the past year. One project was aimed at molding large ( 2 to $5 \mathrm{~mm}$ ) glass shells from preforms. Typically a handblown capillary buib was sealed as carefuliy as possible to minimize the amount of glass at the tip-off. This preform, with tip-off in the up position, was placed in high-quality graphite hemispherical molds which were heated inductively whereby the entrapped gases forced the softened glass to conform to the mold geometry. The molded shell was then inverted in the molds which were reheated, using a temperature gradient, while spinning about the $z$ axis. This resulted in redistribution of the glass and an improvement in wall uniformity. Molded shells 4 to $5 \mathrm{~mm}$ in diameter with aspect ratios to 200 were fabricated by this technique but wall nonuriformity was 50\% or more. The work thus far is only preliminary. It has become apparent that further success will necessitate the use of starting preforms which are more regular, i.e., possess an axis of symmetry, and whose shape and mass are more reproducible than hand-blown capillary bulbs. This regularity is required so temperature, temperature gradient, mold size and mold spinning rate witl not have to be changed from one preform to another. A machine-blown and sealed preform would be desirable. It was understood from the outset that even the best molded shells will have to undergo further refining to achieve the sphericity and surface finish required for ICF pellets. This refining can conceivably be achieved by dropping the molded shell through a vertical furnace or by reheating while levitating. The latter possibility was the impetus for a research project designed to test the feasibility of gas jet levitation of glass macroshells. 
Levitation by gas jets of submillimeter shells and beads has been demonstrated by workers at Bjorksten Research Laboratories and Los Alamos Scientific Laboratories. Our objectives were to levitate imperfect glass shells 2 to $5 \mathrm{~mm}$ in diameter and remold them into acceptable ICF pellet components. Ideally, the levitation should confine the shell to a restricted region of space for a sufficiently long time such that it can be heated to the softening point of the glass, thereby allowing more uniform distribution of the glass mass to occur as well as a smoothing of the shell surface. Numerous single-jet and multi-jet gas nozzles were examined.

Levitation of a perfect sphere whose center of gravity coincides with its geometric center is quite simple, but as asymmetry increases stable levitation also becomes increasingly difficult. Most molded glass shells prepared were too nonuniform to be levitated controllably for periods long enough to effect shell improvements. Some glass shells, however, were successfully levitated by gas jets at $\sim 4 \mathrm{~cm}$ above the nozzle surface. Air bearing style levitation was also occasionally observed at distances ranging from $1 \mathrm{~mm}$ to approximately one-half of the shell diameter above the nozzle. The extent of shell nonuniformity which can he tolerated was not quantified in this short-term study. The problem of providing adequate heat transfer to a levitated glass shell was solved by inductive heating of the gas jet nozzle. Feasibility of heating a molded shell in an air bearing type nozzle to the softening point of the glass was demonstrated by the expansion of a levitated glass shell. Additional work is required to properly maintain control of the motion of the shell as its density changes upon expansion in the hot gas stream. 


\subsubsection{Glass Shell Strength}

The successful manufacture of high strength glass shells requires both an understanding of the parameters that influence the attainment and maintenance of strength and the development of the capability to synthesize any preferred compositions. A successful strength testing procedure, the first step in the strength problem, has been developed. A simple device is used which allows the shells to be pressurized in individual cavities to prevent inter-shell effects, i.e., shattering of one she11 by fragments from another. The test procedure for she1.1 strength under compressive loads involves pressurizing the test device from a high pressure source, depressurizing and observing which shells survive. The procedure is then repeated several times with step-wise increase's in pressure. For tensile load effects shells can be filled in this same device using standard filling procedures and upon depressurization those shells which survived can be noted. A variety of parameters can be studied; glass shell composition, aspect ratio, wall thickness, wall. uniformity, temperature and humidity. Samples can be studied on a statistical basis or individual shells can be characterized prior to testing. Where shell aspect ratios are known the breaking (hoop) stress can be easily calculated. This test procedure should closely reflect the actual in-use conditions seen by glass shell components of ICF pellets. The limited number of tests conducted thus far have been restricted to compressive loading, but have shown that the test device and procedure work satisfactorily.

It is debatable whether glass composition per se has a significant impact on glass strength in an idealized environment. In a non-idealized actual use environitient, composition can play an indirect role in 
determining the practical strength of glass since it clearly affects other properties which dominate strength. The failure of glass by static fatigue is generally thought to occur by stress corrosion which in turn is influenced by chemical durability, a composition related property. Thus, those glass compositions which have superior chemical durability properties should be particularly resistant to stress corrosion. Pure silica glass meets this requirement while also being free of other possible strength-degrading conditions such as phase-separation.

The recent successful production of thin wall silica shells $\left(>99 \% \mathrm{SiO}_{2}\right)$ will provide an opportunity to test the assumptions stated above. Preliminary results from the strength testing device are encouraging in that for a given gas pressure (compressive loading), the number of silica shell survivors is several times that of sodium silicate shells with approximately the same aspect ratio. Actual glass strength calculations and quantitative comparisons between glasses await more detailed experiments with fully characterized shells, i.e., wall thickness values and uniformity specifications will be used. Once the effect of glass composition on shell strength is understood and applied to shell synthesis, other factors affecting strength, such as handling, abrasion protective coatings, etc., will be investigated.

\subsubsection{Surface Finish}

Earlier work has shown that most shell surface defects can be correlated to chemical durability of the glass comprising the shell. It was noted, for instance, that incorporation of calcium into a sodium silicate glass composition to produce a simple soda-lime glass resulted in glass shells with improved surface finish. The addition of small amounts of potassium was found to improve the soda-lime glass further 
to produce a shell whose surface was substantially free of surface defects observable by our scanning electron microscope ( $250 \AA)$. Subsequent experiments with this glass in the preparation of moderate size shells, e.g., $\overline{O D}=282 \mu \mathrm{m}$ and $\bar{W}=2.8 \mu \mathrm{m}$, has substantiated these earlier results. Larger, thicker wall she11s, $\overline{O D}=513 \mu \mathrm{m}$ and $\bar{W}=3.9 \mu \mathrm{m}$, with essentially the same composition were, however, observed to have isolated surface features (islands) which ranged in size up to $0.5 \mu \mathrm{m}$. Washing with $\mathrm{H}_{2} \mathrm{O}$, $\mathrm{HCl}$ and weak $\mathrm{HF}$ was ineffective in removing these features. The average mass of the latter shells is $i 5$ times that of the former, resulting in a very short residence time in the tower furnace. This shortened residence time may be insufficient to refine the glass and provide a smooth surface, although wall uniformity was not adversely affected. Additional work will be directed toward identification of these surface features, as well as trying additional washing schemes.

The poor chemical durability of shells made from simple sodium silicate glasses was again demonstrated even though the composition of the new she $11 \mathrm{~s}, 13 \% \mathrm{Na}_{2} \mathrm{O}$ and $87 \% \mathrm{SiO}_{2}$, was more sodium deficient than previous shells. It was observed that shells which were not desiccated had absorbed sufficient water vapor after 10 weeks to produce surface islands readily detectable by optical microscopy. In an effort to improve the surface finish of these shells, whose other properties, such as yield, uniformity and high aspect ratio, were excellent, experiments were conducted with potassium-containing gel; the addition of this element as noted above had improved the surface finish of the soda-lime glass shells. Shells of the same excellent quality were subsequently produced with the expected additional benefit of considerably enhanced chemical durdbilily. However, due to the high loss of potassium during 
shell formation, the composition of the shells was lower in alkali than expected, i.e., (wt. \%) $5.3 \% \mathrm{Na}_{2} \mathrm{O}, 1.8 \% \mathrm{~K}_{2} \mathrm{O}$ and $92.9 \% \mathrm{SiO}_{2}$. Thus, the improved durability may be simply due to a decrease in total alkali. Experiments to prepare shells with a glass composition having $\sim 13$ to $15 \%$ $\left(\mathrm{Na}_{2} \mathrm{O}+\mathrm{K}_{2} \mathrm{O}\right)$ are in progress and should resolve the question of whether the mixed alkali effect can be appropriated to this glass system.

A question has arisen from time to time regarding the nature of the surface defects seen on certain glass shells, particularly sodium silicate, but also some soda-lime glass shells. It has been our contention that in most instances these features can be ascribed to the effects of weathering as opposed, for example, to phase separation or devitrification. Nonetheless, as a further check to compare these features to other known types of well defined glass surface defects an attempt was made to produce glass shells with a high probability of phase separation. The surface features of a deliberately phase separated glass in a shell geometry could be used as a standard or model to aid in assessing whether surface features seen on previously prepared shells are possibly related to phase separation. This is an indirect test, but should further aid in removing the ambiguity which sometimes exists regarding the identity of shell surface defects. The approach taken was to prepare shells from a lithium silicate glass composition which is extremely prone to phase separation. Our preliminary efforts have not been successful, i.e., solid beads rather than shells were formed and the beads gave no evidence of phase separation. Analysis of the glass has shown some unexpected impurities so further work is necessary. Other easily phase-separated glasses will be examined if the lithium silicate system proves unsatisfactory. 


\subsubsection{Fuel Retention}

The 1977 Annual Report gave the results of deuterium permeability measurements on two experimental soda-lime glasses. One of these, the potassium-containing soda-lime glass shells, had excellent surface finish and good gas retention for the average shell. The shell-to-shell permeability, however, was noted to vary by as much as an order of magnitude. Although the worst case permeability was still acceptable, i.e., the out-permeation rate was very slow, some effort has been made to tighten the permeability spread. The approach taken has been to decrease the composition variability from shell to shell since this is felt to be the dominant factor in the permeability spread. (See Section 1.1.6 for a further discussion of this point.) Since the alkali losses during shell formation are so large, and presumably the major source of the she11 composition variability, a glass composition was prepared with a reduction in the total alkali from 15.1 wt \% to 7.7 wt. $\%$ and a corresponding increase in the calcium content from 9.9 wt. \% to 15.1 wt. \%. Calcium appears to undergo minor and in some cases no loss during shell formation. Unfortunately the shells produced from this gel were of very nonuniform quality with some exhibiting cracks through the shell wall. Static melts of buik samples of powdered gel have shown that this glass behaves very differently from other glasses studied thus far, i.e., no foaming or melting was observed below $1400^{\circ} \mathrm{C}$. No chemical analyses or gas permeability measurements were made on the glass shells, but the presence of cracked shells and crystalline-like patterns strongly suggest that an excursion was made outside of the practical glass forming region. Further work with this glass system is not planned since over-all permeability properties of the present shells are acceptable and some, if not all, ICF 
labs have used low temperatures to prevent any fuel loss by permeation during storage. Additionally, some glass shell'pellet designs require that thick walled shells be used and this means that the glass shells must have fairly high deuterium-tritium-permeability rates if they are to be diffusion filled in a reasonable time at acceptable temperatures. A glass which meets this other end of the fuel retention spectrum is pure silica which has recently been prepared.

The room temperature deuterium permeability of germania glass was determined from pressure tests of out-permeated germania glass she1ls. The test procedure has been described in the 1977 Annual Report. The permeability coefficient found was $5.70 \times 10^{-22} \frac{\mathrm{mol} \cdot \mathrm{m}}{\mathrm{m}^{2} \cdot \mathrm{s} \cdot \mathrm{Pa}}$ or $1.70 \times 10^{-16}$ $\frac{\mathrm{cm}^{3} \cdot \mathrm{cm}}{\mathrm{s} \cdot \mathrm{cm}^{2}}$ with a relative standard deviation of $22 \%$. Since the relative standard deviation is nearly within the combined measurement uncertainty of $\sim 16 \%$ we conclude that any she 11 -to-shell permeability differences can be ascribed to geometrical variations. This conclusion is consistent with the simple composition of the $\mathrm{GeO}_{2}$ glass and further supports our contention that the major portion of the shell-to-shell gas permeability variations seen in more complex glass systems arises from shell-to-shell composition variations.

\subsubsection{Wall Uniformity}

In an effort to further our understanding of the factors influencing shell aspect ratio and wall uniformity, shell samples from 32 experimental runs were examined in detail. These runs differed by gel composition, gel particle size, or furnace temperature. A random sample of shells was se:lected from each batch. Each shell was characterized by microradiography, permitting a diameter, wall thickness and percent wall uniformity to be assigned. The results have been only partially analyzed, but some general 
observations and conclusions which can be made are the following:

1) Batches of shells with both low (30 to:40) and high (270). mean aspect ratios were found with good wall uniformity; random samples of some batches has 40 to $60 \%$ of their shells with wall nonuniformities $<20 \%$.

2) At constant gel particle size (106 to $125 \mu \mathrm{m})$, the percent of she $17 \mathrm{~s}$ from a given sample having $W N U \leq 20 \%$ was observed to increase from 16 to $64 \%$ as the temperature was increased from 1010 to $1500^{\circ} \mathrm{C}$. The glass used was a soda-lime composition which also provided good surface finish.

3) A soda-potash-silicate glass with nominal gel composition (oxide equivalent' weight percent): $7.9 \% \mathrm{Na}_{2} 0 ; 12.1 \% \mathrm{~K}_{2} \mathrm{O} ; 7 \% \mathrm{~B}_{2} \mathrm{O}_{3}$; and $73 \% \mathrm{SiO}_{2}$ was found to produce very uniform shells. In a series of seven experiments, the tower furnace temperature was held constant at $1450^{\circ} \mathrm{C}$ while the gel particle size was varied from 53 to $180 \mu \mathrm{m}$ (seven sieve cut ranges). The mean wall nonuniformities of the randomly selected test samples all fell in the range of 32 to $38 \%$.

4) The effect of gel preparation method, i.e., using either metal acetates or metal alkoxides as glass component starting materials, was examined by making shells from gel, prepared by both methods, but having the same nominal oxide equivalent composition. Three composittons, duplicated by each method, Acid and Base, were used in three pairs of tower furnace runs. The uniformity of the shells from each batch was independent of the gel preparation method. One Acid-Base gel pair gave very good quality shells while another of a different composition gave very poor shells. A fourth composition was examined using both gel synthesis methods, but the 
both frit synthesis methods, but the shells were only characterized by optical inspection; both batches gave high quality shells in comparable yields.

5) All experimental runs showed high standard deviations for mean wall nonuniformity. The standard deviation was almost independent of the absolute value of the mean, i.e., both low quality and high quality shells showed a large spread in uniformity values.

6) The role of cobalt oxide in glass shelis in not yet clear, but some interesting observations were made. For example, a batch with one of the lowest mean wall nonuniformities (25\%) was for a soda-lime glass containing $\sim 1 \%$ cobalt oxide.

7) The composition of the glass comprising the shell and the gel from which the shell was made appears to have a strong influence on shell wall uniformity, but no particular functional relationship has yet been found between wall uniformity and compositional properties such as the presence or absence of either boron or calcium in the gel, or the amount of silica in the shells. The absence of more obvious correlations with composition hints of some indirect effects of composition on physical properties of the dried gel such as surface area, porosity, or degree of chemical molecular cross-linking. This is an area that will be examined more intently in the coming year. 


\section{$1.2 \quad$ Polymer Research}

N. E. Doletzky, M. A. Ebner, L. B. Kool, P. J. Lennon, R. L. Nolen, Jr., J. J. Rolen, D. E. Solomon and D. C. Wu

Polymers are of great utility in ICF targets. They take many different physical configurations and cover a wide range of chemical compositions. Polymeric foams have been designed as cushions to enhance elasticity between the outer layer of a spherical shell and the layer adjacent to the DT fuel and to decrease the problems arising from the lack of perfectly concentric layers. Our R\&D effort on polymeric foams has produced foam densities of 0.01 to $0.1 \mathrm{~g} / \mathrm{cm}^{3}$ and cell sizes of $10 \mu \mathrm{m}$ and 1 to $2 \mu \mathrm{m}$ wall thickness by two different techniques. Polymers with high tensile strength and low permeability to hydrogen and its isotopes are rare materials. We have determined that poly(vinyl alcohol) $P(V A)$ and some cellulose derivatives provide shells with these optimized qualities. Fuel containing compounds (e.g., deuterated $P(V A)$ ) have been of interest from the inception of ICF due to the ideality of carrying the fuel in a solid form without cryogenics. Model compounds (organic monomers) containing $96 \%$ deuterium have been synthesized in a three-step synthesis. Styrene and methyl methacrylate which has $96 \%$ of the hydrogen atoms replaced by deuterium atoms have been synthesized. The methyl methacrylate was in-situ microencapsulated into $1 \mathrm{~mm}$ shells. Metal containing (on molecular scale) polymers have been suggested to be thermal conduction barriers. Monomer synthes is of poly(vinyl hafneocene) $P(V H f)$ was started; the synthesis requires six steps. Monomer and poljmer synthesis of poly (p-iodostyrene) was completed and the polymer blown into shells. Macro polymeric shells ( $\sim \mathrm{mm}$ 's) are needed in electron and ion beam ICF as well as in target components of laser ICF. A droplet generator has been designed and fabricated which will generate 400 to $800 \mu \mathrm{m}$ polymeric solution 
droplets.

\subsubsection{Polymeric Foam Development}

Two different methods were developed for synthesizing foams with densities of 0.01 to $0.1 \mathrm{~g} / \mathrm{cm}^{3}$. One method produced $0.01 \mathrm{~g} / \mathrm{cm}^{3}$ density but very irregular cell sizes. This method employs a solution of $5 \%$ low molecular weight $P(M M A)$ in 1,2-dichloroethane combined with finely divided anhydrous sodium sulfate $\left(\mathrm{Na}_{2} \mathrm{SO}_{4}\right)$ to make a slurry. The slurry is then coated onto a substrate and the solvent allowed to evaporate. The sodium sulfate is then dissolved in water leaving a polymer film with an open cell structure as seen in Figure 1.2-1.

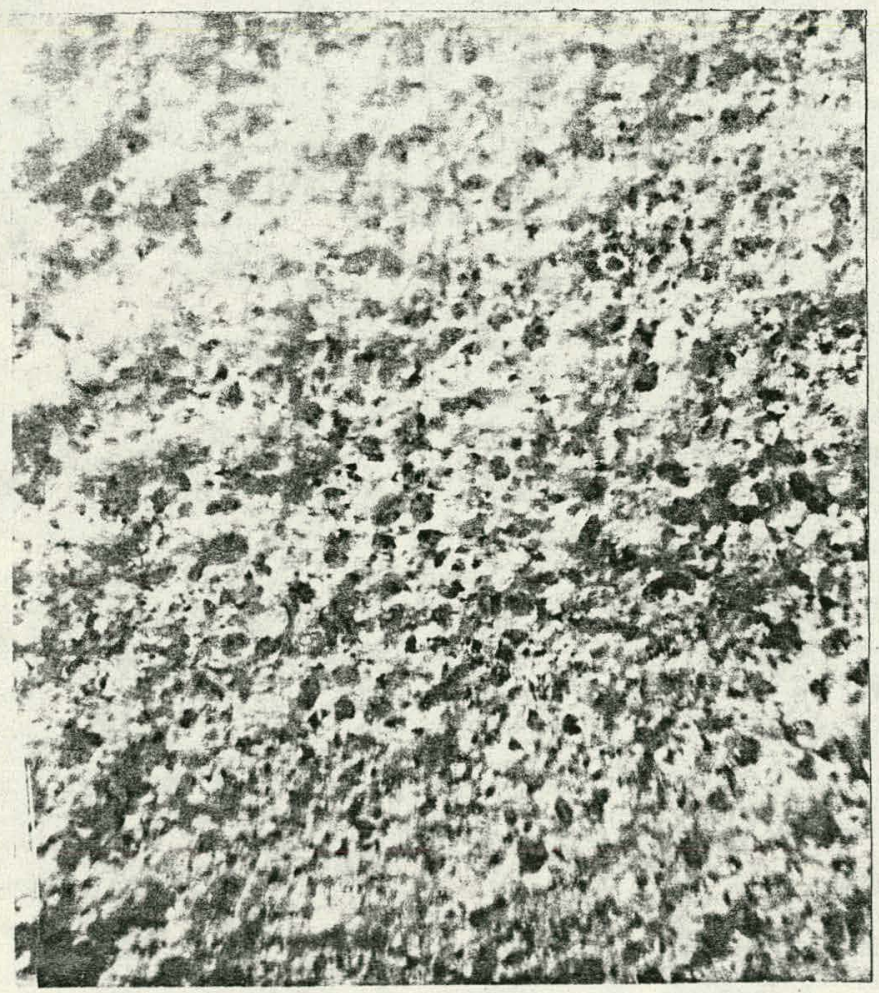

FIGURE 1.2-1 Low density $P(M M A)$ foam $30 \mathrm{X}$ 
The thickness of the planar foam is about $600 \mu \mathrm{m}$ and a density of $0.015 \mathrm{~g} / \mathrm{cm}^{3}$. In this method, the cell size and density control are highly dependent on the size distribution of the sodium sulfate particles. The second method is the more classical one for foam formation; i.e., an encapsulated blowing agent released at elevated temperature. Phenolic resin foams of $0.15 \mathrm{~g} / \mathrm{cm}^{3}$ were formed with 1 to $2 \mu \mathrm{m}$ cell sizes and 2 to 10um wall thicknesses as seen in Figures 1.2-2 and 1.2-3.

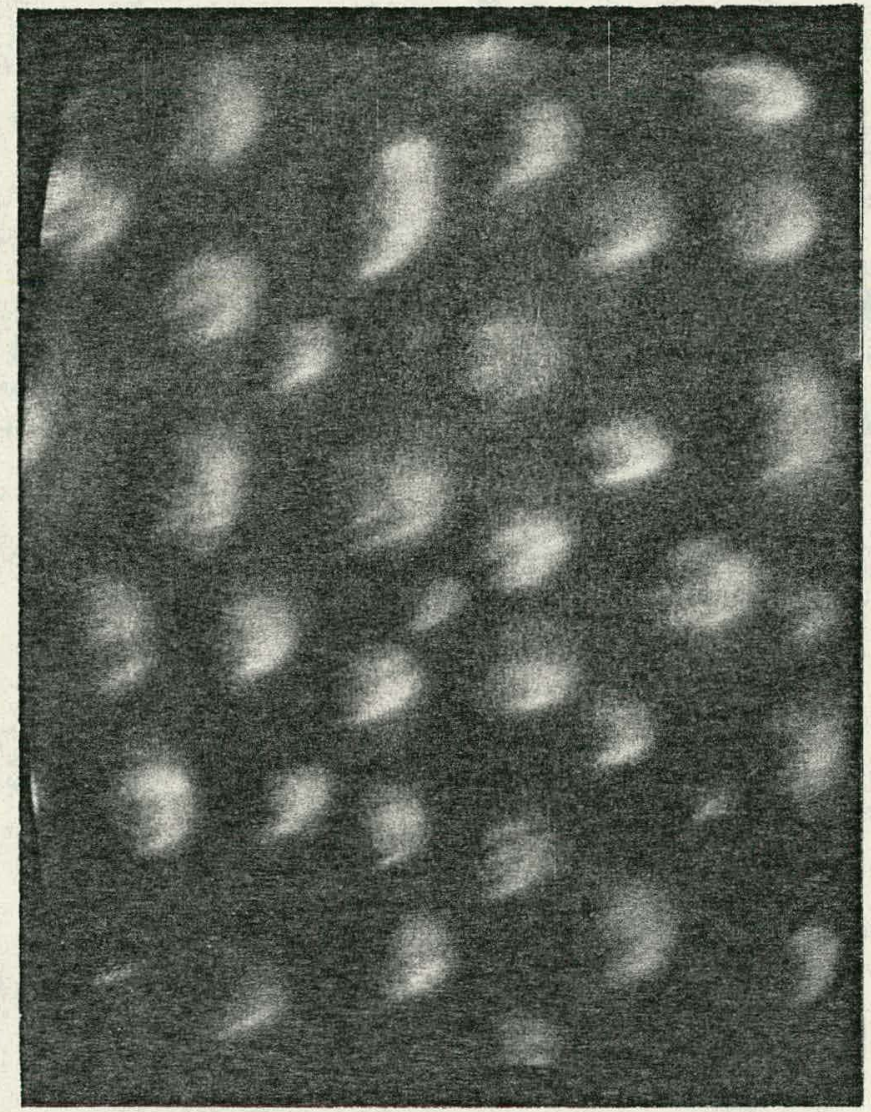

FIGURE 1.2-2 Foam: 45\% AROTAP, 5\% DKP, dried at 170 to $175^{\circ} \mathrm{C}$ for 2 minutes. $7000 x$ magnified, density $0.15 \mathrm{~g} / \mathrm{cm}^{3}$ 


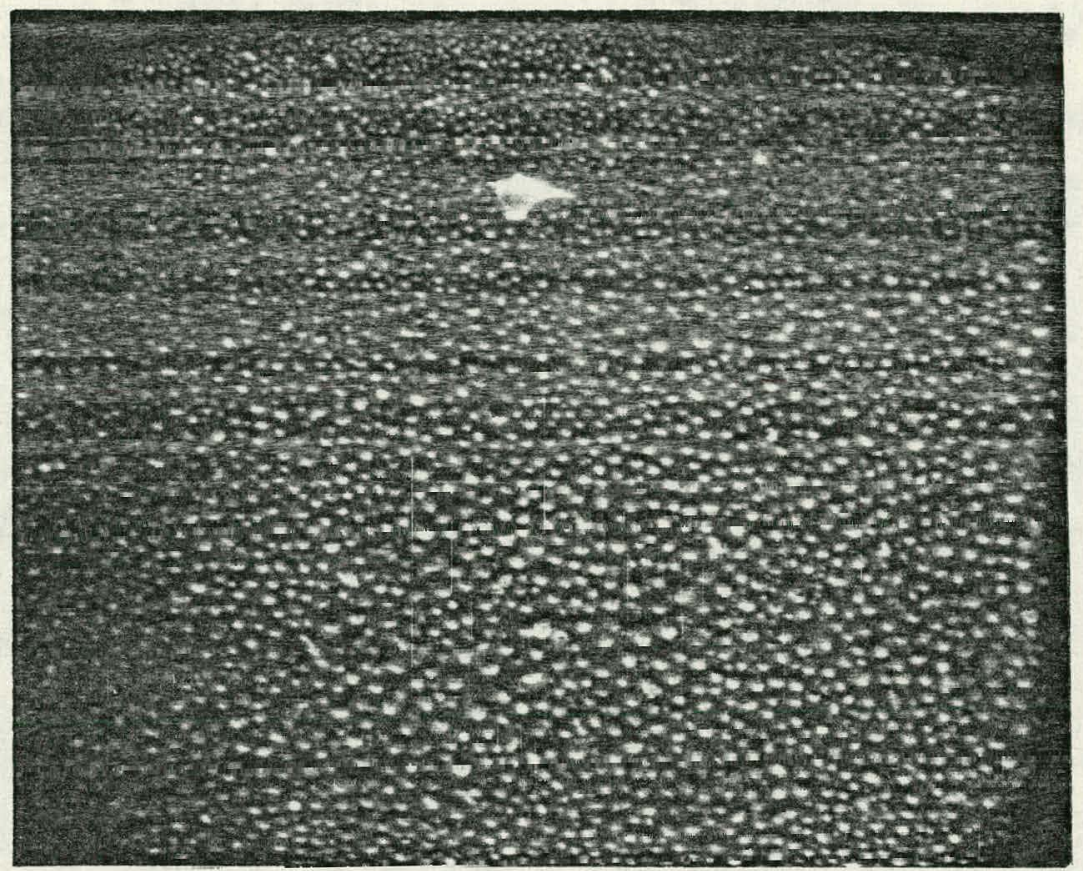

FIGURE 1.2-3 Foam: 45\% AROTAP, 5\% DKP, dried at 170 to $175^{\circ} \mathrm{C}$ for 2 minutes. $7000 \mathrm{X}$ magnified, density $0.15 \mathrm{~g} / \mathrm{cm}^{3}$

The use of a special organic expanding agent such as XP-143 (Polymerics, Inc.) or OBSH (Polyscience, Inc.) with phenolic resin AROTAP or AROFENE has resulted in reducing the density to about $0.1 \mathrm{~g} / \mathrm{cm}^{3}$ with cells in the ten micron and above range. The relative distribution of cell sizes largely depends on the mixing of phenolic resin with foaming agent XP-143 or $\mathrm{OBSH}$. Since neither XP-143 or $\mathrm{OBSH}$ is soluble in the phenolic resin, we can only obtain relatively uniform cell sizes after extensive ultrasonic treatment. In addition, the original size of $\mathrm{XP}-143$ or $\mathrm{OBSH}$ is about one micron, but is agglomerated. Thus, it is very difficult to further reduce the cell size using these foaming agents 
as they are. Although somewhat expensive, they do provide a convenient way of foaming with some control on the cell size.

Common practice of using surfactants and nucleating agents to control foam cell size in the micron or submicron range is not very effective. In order to obtain uniform micron cell size, the expanding agent should be soluble in a mutual solvent with the polymeric resin. Thus, the idea is to form a microemulsion with the expanding agent almost monodispersed in a polymeric solution. After heat treatment, the selected thermoset resin will polymerize and the expanding agent will sightly expand at the same time. The result should be a uniformly distributed foam of low density with controlled cell size.

Initial experiments involved simply mixing phenolic resin Plyophen 93-398 (Reichhold Chemicals, Inc.) and foaming agent Freon 113 (Fisher Scientific) in a blender. The coated emulsion is then cured in an oven at elevated temperature. The foam obtained is quite irregular in shape and size. The addition of surfactants and nucleating agents have not been effective in controlling the cells of this particular foam.

Mixing phenolic resin AROTAP-352-W-71 (Ashland Chemical Co.), foaming agent. Freon 113, surfactant DC 193 (Dow Corning Corp.) and colloidal silica (DuPont Co.) have met only limited success in controlling the uniformity of cell sizes.

The foaming system that has been extensively tested is phenolic resin AROTAP and expanding agent dipotassium hydrogen phosphate. Both components are water soluble which permits easy and uniform dispersion. In general, the foaming process consists of dissolving $5 \%$ dipotassium hydrogen phosphate in $50 \%$ de-ionized distilled water. To the completely dissolved solution, $45 \%$ AROTAP resin is added and all three components 
are thoroughly mixed in an ultrasonic bath for five minutes. The sonicated homogeneous solution is then coated on a microscope slide or a cover slide by painting or spraying. The slides are finally dried in an oven at approximately $150^{\circ} \mathrm{C}$ for 2 to 5 minutes. The drying time depends on the amount of coated polymer or the size of the slide.

Experimental results using this specific expanding agent have provided coatings with density close to $0.2 \mathrm{~g} / \mathrm{cm}^{3}$ and uniformly distributed one to two micron cells. This density appears to be the lowest density obtainable within a specific packing factor and polymeric system as predicted by a mathematical model.

\subsubsection{High Tensile Strength-Low Permeability Polymeric Materials} Poly(vinyl alcohol) $P(V A)$ is still the optimum polymer for free-standing shells. $P(V A)$ has the highest tensile strength, lowest permeability, and other necessary characteristics to form shells, of all polymers tested to date. (2) Even considering other polymer candidates, as in Table 1.2-1, there are only two of the three prerequisites met by any of the candidate polymers except sodium carboxymethyl cellulose, which does have low permeability, (2) high tensile strength, $\sim 9.2 \mathrm{kgf} / \mathrm{mm}^{2}$, and is shell-formable. However, sodium is present in $\sim 0.7$ atoms/glucose unit resulting in an average $Z$ higher than $P(V A)$. 
TABLE $1.2-1$

TENSILE STRENGTH AMD HYDROGEN PERMEABILITY COEFFICIENTS OF SOME COMMON POLYMERS

\begin{tabular}{|c|c|c|c|c|}
\hline Polymer & $\begin{array}{c}\text { Tensile Strength } \\
\text { kgf } \cdot \mathrm{mm}^{-2}\end{array}$ & $\begin{array}{r}\text { Hydro } \\
\text { Coeff } \\
\mathrm{cm}^{3} \text { (STP) }\end{array}$ & $\begin{array}{l}\text { ogen Permeab } \\
\text { ficient }{ }^{+}(x \\
\mathrm{cm}^{-2} \mathrm{~cm}^{-2} \mathrm{~s}^{-1}\end{array}$ & $\begin{array}{l}\left.10^{10}\right) \\
(\mathrm{cm} \mathrm{Hg})^{-1}\end{array}$ \\
\hline Polymethyl methacr.llate & 7 & & --- & $\left(0.1 N_{2}\right)$ \\
\hline Polyvinyl chloride. & 15 & & 1.70 & \\
\hline Epoxy resin & 8 & & --- & \\
\hline Polytetrafluorathy. ene film & $10.5-17.5$ & & 9.8 & \\
\hline Polyacrylonitrile fibers & $30-40$ & & --- & $\left(300 \mathrm{H}_{2} \mathrm{O}\right)$ \\
\hline Polyvinylidene chlcride fibers & $30-40$ & & --- & $(0.31 \mathrm{He})$ \\
\hline Polycarbonate îibers & 40 & & 5.4 & \\
\hline Polyethylene terephithalate fibers & $50-90$ & & 5.4 & \\
\hline Polyvinyl alcohol fibers, insolubilized & $50-105$ & & .009 & \\
\hline Nylon 11 & $5-6$ & & 1.8 & \\
\hline Na Carboxymethy' cellulose & 9.2 & \multicolumn{3}{|c|}{$0.0002\left(D_{2}\right)$} \\
\hline
\end{tabular}

*W. J. Roff, J. R. Scott and Pacitti, Handbook of Common Polymers CRC Prep., 597-604 (1971).

+J. Brandrup, E. H. Immergect, ed., Polymer Handbook, 2nd ed. III, 229-240. 


\subsubsection{Deuterated Polymeric Shells.}

Two highly deuterated (96\%) polymers were synthesized by polymerization of deuterated monomers. The two polymers are polystyrene PS and poly(methyl methacrylate) $P(M M A)$ as viewed in the following schemes. The MMA-D $D_{8}$ was polymerized in an in situ microencapsulation ${ }^{(2)}$ which provided $P(M M A)-D_{8}$ she $11 \mathrm{~s}, \sim 1 \mathrm{~mm}$ diameter.<smiles>[2H]c1c([2H])c([2H])c([2H])c([2H])c1[2H]</smiles>

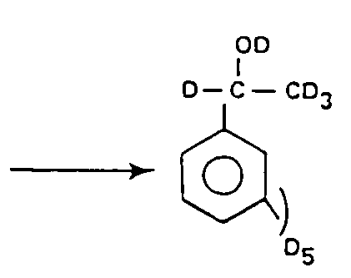

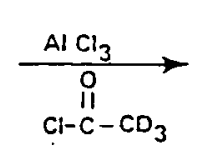

$99 \%$ YIELD $\mathrm{KHSO}_{4}$

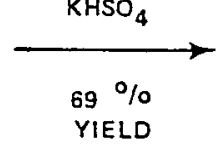

YIELD<smiles>CC(C)(C)C(=O)c1cccc(P)c1</smiles><smiles>O=C(O)c1cccc([18O])c1</smiles>

POLYSTYRENE - D

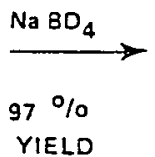

YIELD

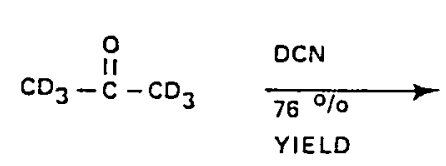

$$
\begin{aligned}
& \mathrm{CD}_{3}-\prod_{\mathrm{D}}^{\mathrm{C}}-\mathrm{CO}_{3} \\
& \mathrm{CO}_{3} \mathrm{OO} \\
& 66 \% \text { YIELD }
\end{aligned}
$$

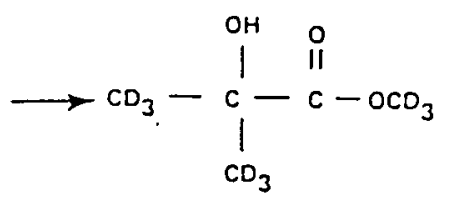

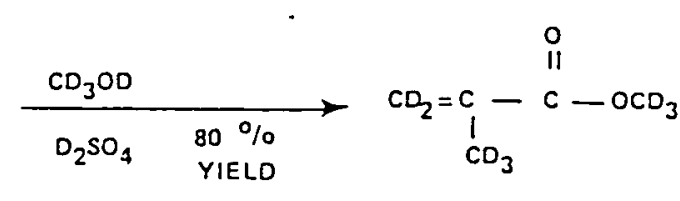

$$
\begin{aligned}
& 97 \% \text { DeUtealum }
\end{aligned}
$$


These molecules are models and primary starting materials for incorporating some finite quantity of tritium. Polymers containing deuterium and tritium might then be formed into a target. A further extension of this scheme is to incorporate deuterium and tritium into metal loaded polymers which would also be the fuel container for gaseous, liquid or solid DT.

\subsubsection{Metal Loaded Polymers}

Synthesis schemes for poly(p-iodostyrene) and poly(vinyl hafneocene) were designed. Poly(p-iodostyrene) was synthesized and blown into shells. Computer simulation predicts a high gain target consisting of a hollow spherical shell with $Z^{2} / \bar{Z} \sim 20$, approximately one atom of high $Z$ in 1000 atoms of $(\mathrm{CH})_{n}$, and $a 11$ hydrogen replaced by deuterium. The $z^{2} / \bar{Z} \sim 20$ is necessary to slow down thermal conduction from the container wall to the fuel. The reduction in $\bar{z}$ is necessary to avoid radiation losses.

Future plans are to synthesize poly(vinyl hafneocene) and incorporate it into polymers such as styrene, polyethylene, etc. This is planned to allow us greater flexibility in preparing various $\bar{Z}$ materials. The advantage of these mixtures provides greater flexibility in fabricating suitable shells.

\subsubsection{Macro Polymeric Shells}

Efforts toward producing macro polymeric shells ( $\sim 5 \mathrm{~mm}$ ) for uses as mandrils for coating, and electron and ion beam targets have been extended this year via design and fabrication of an "Acoustically. Modulated Fluid Jet AMFJ." A schematic of the vertically modulated jet is seen in Figure 1.2-4. The purpose of the device is to generate large droplets, millimeters in diameter, from polymer solutions and fabricale macro polymeric shèl1s. 


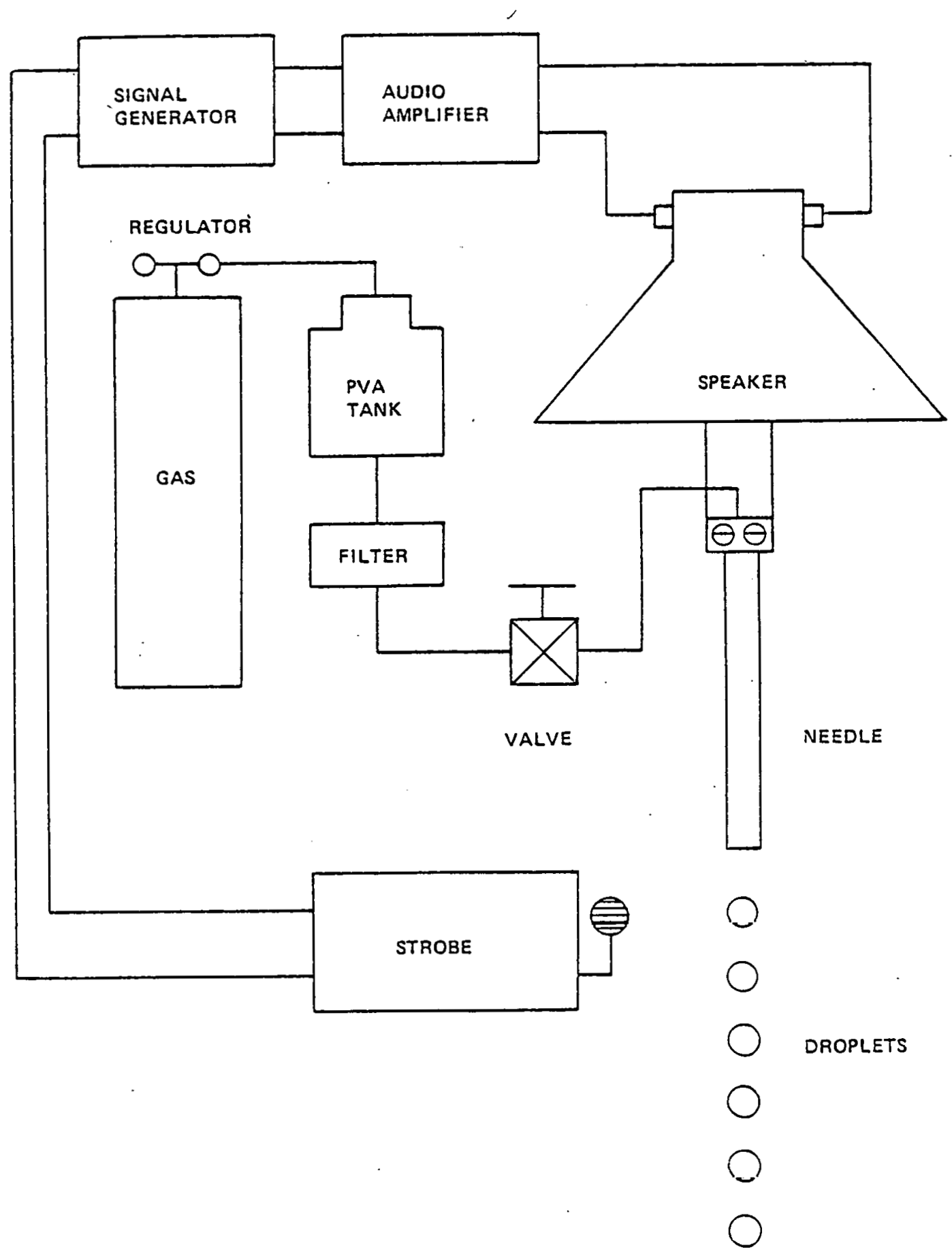

FIGURE 1.2-4 Schematic diagran of droplet generator 
The pressure from an argon gas cylinder serves to drive a $2.5 \%$ poly(vinyl alcohol) $P(V A)$ aqueous-methanol solution which is stored inside a stainless steel tank. The solution then passes through a polypropylene membrane filter and a Whitney micro-metering valve which feeds a hypodermic needle. The needle, in turn, is mounted on a 15 inch Cetec-Gauss loudspeaker ( 8 ohm impedance) driven by a Wavetek signal generator and a 100 watt Bogen audio amplifier. The sonic energy generated in the speaker is transmitted to the hypodermic needle which in turn breaks up the $P(V A)$ solution into uniformly sized droplets. The droplets can be monitored through a synchronized strobe or a camera.

It is now possible to produce uniformly sized ( $\sim 400 \mu$ to $800 \mu$ ) droplets, Figure 1.2-5, with good separation using the dilute $2.5 \% \mathrm{P}(\mathrm{VA})$ aqueous-methanol solution. However, the droplets generated are too numerous for drying in our spray drying facility. An experimental circuit for selectively charging droplets and removing them from the main stream has been designed and fabricated. 


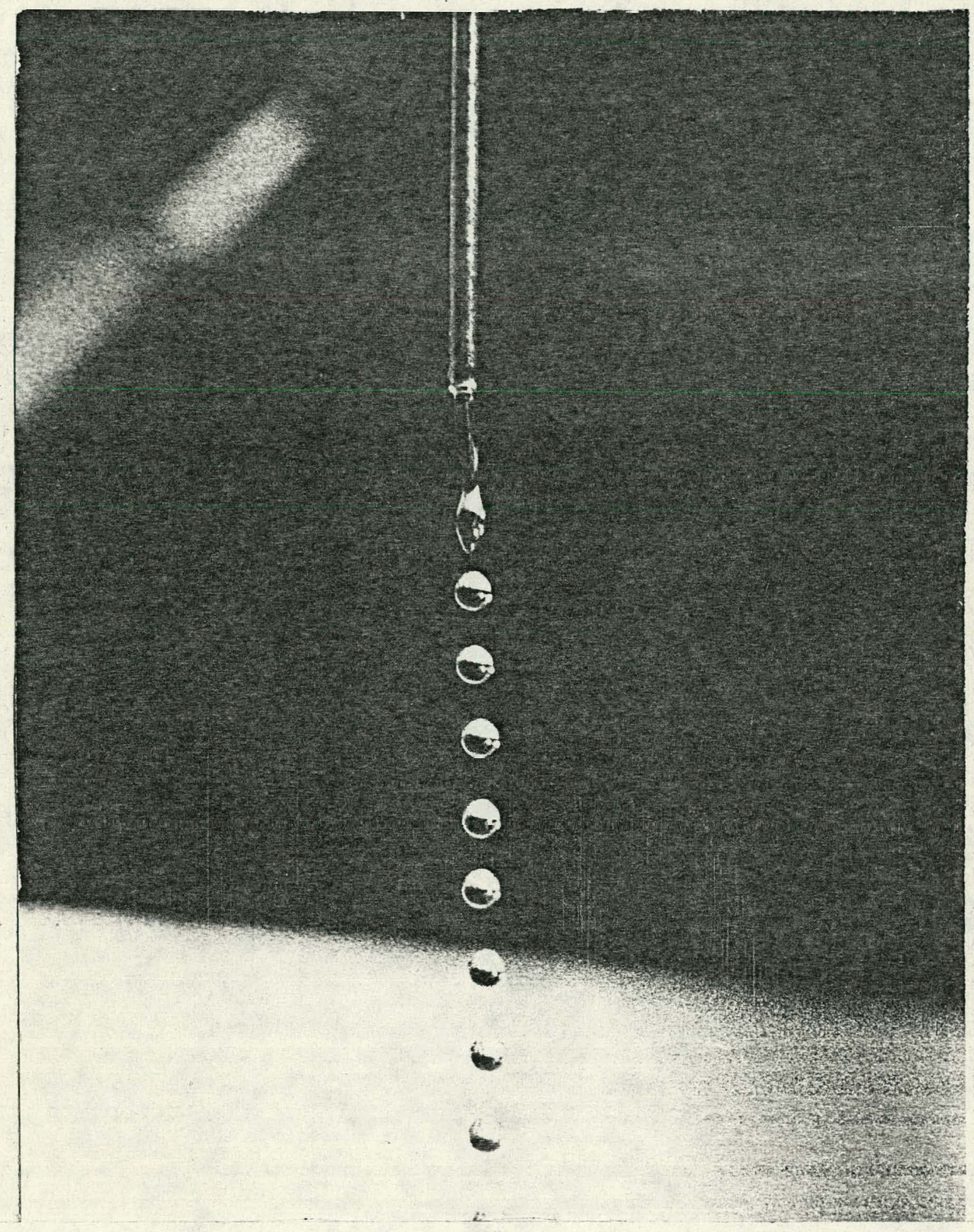

FIGURE 1.2-5 Droplets produced by audio-frequency vibration of fluid jet. 


\section{REFERENOES}

1. R. J. Simms (private communication).

2. KMS Fusion, Inc. Annual Report, U-762 (1977), Section 1.2, 25-34. 


\subsection{Cryogenic Technology Fabrication and Engineering}

E. J. Calabro, T. M. Henderson, D. L. Melmoth, D. L. Musinski, T. R. Pattinson, W. B. Rensel, R. J. Simms, D. E. Solomon and

J. A. Tarvin

The cryogenic target program continues at KMSF because theoretical (1) considerations suggest that the gain of a laser-fusion target can be enhanced if the deuterium-tritium fuel is condensed as a uniform liquid or solid layer on the inner surface of the shell at the time it is irradiated. During the implosion of the target, internal pressure within the target must be overcome if high fuel densities are to be achieved. Early in the time sequence of the laser/target interaction, fast electrons which are produced near the outer surface of the target deposit a fraction of their energy in the mass of fuel within the inter-

ior of the target. (2) This fast electron absorption causes pre-heating of the fuel and thus increases the internal fuel pressure. At a given level of laser power this increased fuel pressure limits the final fuel density. A liquid or solid layer target, however, may overcome this limitation. Fuel initially confined to the wall of the target cannot respond quickly enough upon absorption of fast electrons to fill the interior volume of the target. hefore the target begins to implode. Thus, at a given level of laser power a cryogenic liquid or solid layer target should compress to a higher fuel density and produce a higher yield than a target containing the same mass of fuel in the gaseous state.

\subsubsection{Liquid Layer Targets}

For current cryogenic experiments, targets are filled with a nominal 50:50 mixture of DT fuel to a pressure of at least 100 atmospheres measured at room temperature. This pressure fill assures that 
the thickness of the condensed fuel layer will be sufficient to be observed and quantified. (3) Although excessive by current standards for room temperature targets, a pressure fill of 100 atmospheres only approaches the requirements of future ICF reactor pellets.

The expected advantage of liquid layer targets over gaseous targets can be illustrated by considering the specific example of a 100 atmosphere filled target. If irradiated at room temperature, the entire amount of fuel is available to absorb fast electrons and impede the target implosion. However, if irradiated as a liquid layer target at $21 \mathrm{~K}$, approximately $1 \mathrm{~K}$ above the point where the fuel starts to solidify, only a small fraction of the fuel is in the gaseous state contained within the inner boundary of the liquid layer. The pressure of this fuel remaining in the gaseous state can be calculated by use of Raoult's law if the quantity of the various isotopes is known $\left(D_{2}\right.$, DT and $T_{2}$ plus some contamination of HD and HT). Applying the data for the vapor pressure of the individual isotopes as calculated by Souers et al ${ }^{(4)}$ to the proportions measured for one of the KMSF fills $18.4 \%$ HD, 9.3\% HT, $19.7 \% \mathrm{D}_{2}$, 46. $2 \%$ DT and $\left.19.0 \% \mathrm{~T}_{2}\right)^{(5)}$ gives. that the pressures within this liquid layer target is 293 torr. At room temperature, the pressure of this amount of gaseous fuel would be 5.5 atmospheres. Thus, the mass of gaseous fuel within this liquid layer target at $21 \mathrm{~K}$ that is available for absorption of fast electrons is $5.5 \%$ of the amount of fuel available if the target is irradiated at room temperature. For future targets which will be filled to higher pressures, the advantage of the liquid layer configuration increases since the pressure of the gaseous phase at $21 \mathrm{~K}$ remains at 293 torr. 
From an engineering point of view, the main advantage of the cryogenic liquid layer target is the relative simplicity with which it can be integrated into an experimental target chamber. The technique of pointcontact conduction cooling which we developed is illustrated in

Figure 1.3-1. The target is epoxied to the end of a $17 \mu \mathrm{m}$ diameter copper fiber which is one element of a direct conduction path from the target to a source of refrigeration at liquid helium temperatures. (A more complete description of this technique plus a detailed discussion of the liquid layers that are observed may be found in the 1977 Annual Report.) Once the liquid layer has been established and stabilized by setting and stabilizing the temperature of the cryostat, target manipulation and alignment within the experimental target chamber proceeds in the same manner as for a gaseous target at room temperature. Thus, this technique allows cryogenic experiments to be conducted with the minimum disruption to the normal routine of the target chamber.

\subsubsection{Liquid Layer Target Shot Results}

Liquid layer target shot experiments conducted during 1977 at KMSF, ${ }^{(6)}$ using the point-contact conduction cooling technique, have stimulated considerable interest throughout the laser fusion research community. For these liquid layer targets the highest compressed fuel density observed was $\left(\sim 7 \mathrm{~g} / \mathrm{cm}^{3}\right) .{ }^{(7)}$ This result obtained from $X$-ray pinhole photographs implies that cryogenic targets can indeed yield higher compressed fuel densities than gaseous targets. These high fuel densities are also inferred from downshifts in the alpha particle energy spectrum of $\sim 5 \pm 2 \%$ for these 1 iquid layer targets. Neutron yields from these liquid layer targets, al though not as clear cut as the results for fuel compression, also appear to be higher, in general, than for the gaseous targets. (3) 


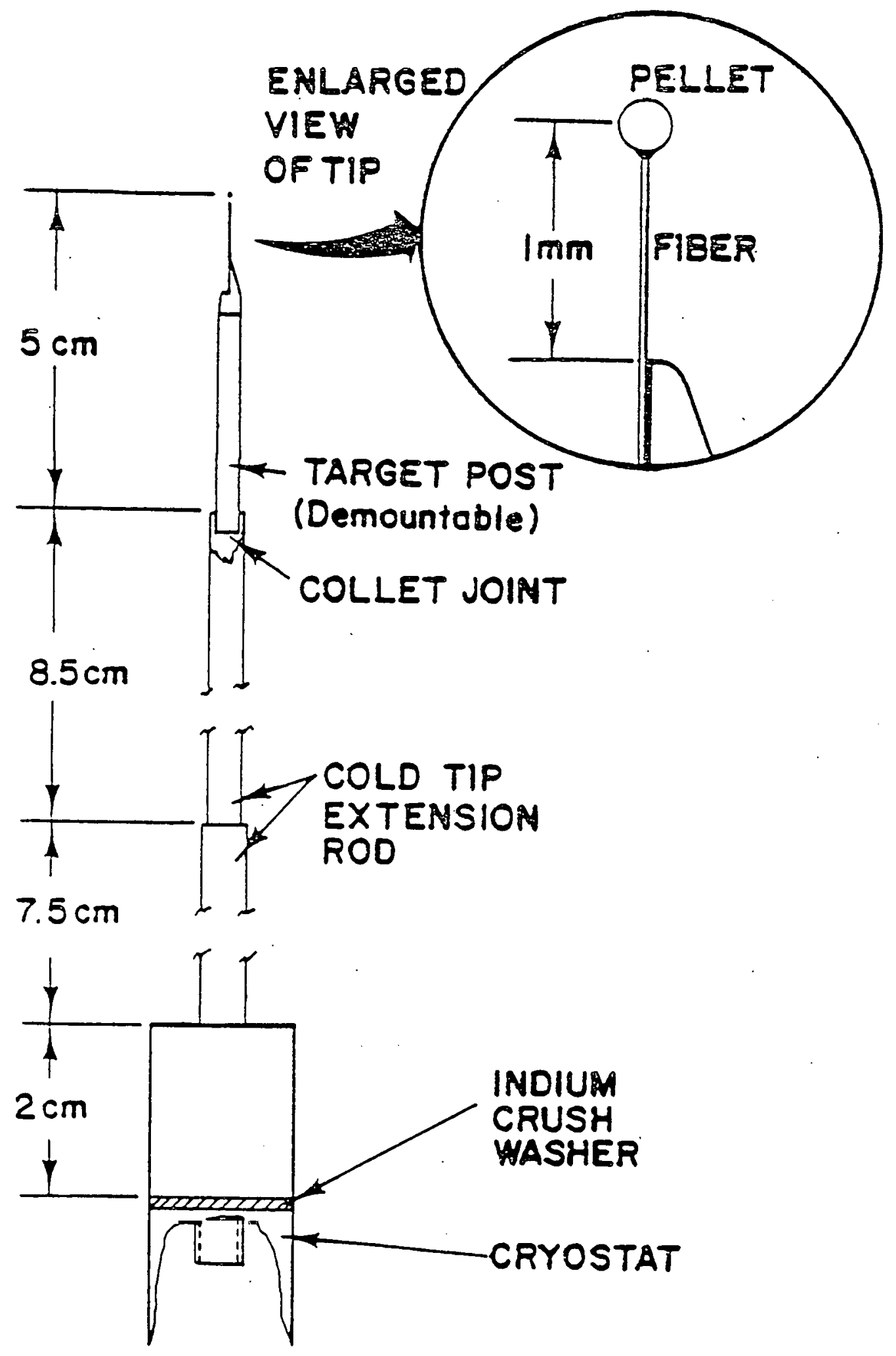

FIGURE 1.3-1 Point-contact conduction cooling. 
The higher fuel density and enhanced neutron output observed in implosions of liquid layer targets were obtained in spite of severe nonuniformities in the fuel layers. (Typically, the maximum thickness of the liquid layer was twice the minimum). Because of this nonuniformity, the liquid layer targets all compressed asymmetrically and model ing of the liquid layer experiments has been difficult. X-ray pinhole camera photographs for these liquid layer targets show the compression region to be displaced well away from the target center toward the region of the target where the liquid layer was thickest. (6) The correlation of asymmetric implosions with liquid layer asymmetries implies that uniformity of the fuel layer is a definite requisite for symmetrical target implosion. These encouraging trends observed for the asymmetrical liquid layer targets have placed significant emphasis on laser irradiation of more uniform fuel layer targets.

\subsubsection{Limitation of the.Technique Used for Liquid Layers}

The relatively simple technology of the point-contact conduction cooling technique is the basis for the ultimate limit on the uniformity obtained for deuterium-tritium mixtures of fuel. Absorption of room temperature radiation by the target forces the components ( $D_{2}$, DT and $T_{2}$ ) of the liquid fuel mixture to separate because of their different vapor pressures. A self consistent model of the liquid layer ${ }^{(8)}$ supported by experimental observations ${ }^{(9)}$ shows that the nonuniformity observed for mixtures of the hydrogen isotopes is due to this phase separation of the liquid mixture within the target. The steady state liquid configuration observed within the target is the liquid layer's response to the dynamics of the separation. (8) Thus, a DT cyrogenic liquid layer target which is 
maintained by the point-contact-conduction cooling technique is inherently nonuniform for steady-state conditions.

1.3.4 Extended Conduction Cooling Technique (Radiation Shièld)

During the experimental studies on liquid layer targets it became apparent that for non-steady-state conditions, there is the possibility that a more uniform liquid layer target can be illuminated by extending the basic technique of point-contact-conduction cooling. Figure 1.3-2a shows a liquid layer target exposed to room temperature radiation. The liquid fuel layer is thickest at the top. Figure 1.3-2b shows the same target under a cryogenic shield which reduces the rate of separation by reducing the radiation input to the target. The fuel layer for the shielded target is thicker at the bottom of the target. Removal of the shield by a retraction device allows the target to revert back to the configuration of Figure $1.3-2 \mathrm{a}$ in about $200 \mathrm{mill}$ iseconds. Since the configuration of the liquid layer changes continuously, laser irradiation can, in principle, be timed to occur when the liquid is most uniform. The additional equipment required for this experiment is a cryogenic shield with an associated retraction device timed to the laser firing. Thus, by incrementally increasing the complexity of a proven technique, an illurease in unitormity of the liquid layer is possible.

Figure 1.3-3 shows in schematic form the integration of a simple cryogenic radiation shield (or shroud) in the experimental target chamber. The sole purpose of this shield is to reduce the radiation energy absorbed by the target. The retraction device for this radiation shield is a gas driven piston on top of the target chamber (Figure 1.3-3). The speed with which this unit can retract the shield is shown in Figure 1.3-4. While the shield is in motion and before it reaches a position that puts 


$$
\text { PP }
$$




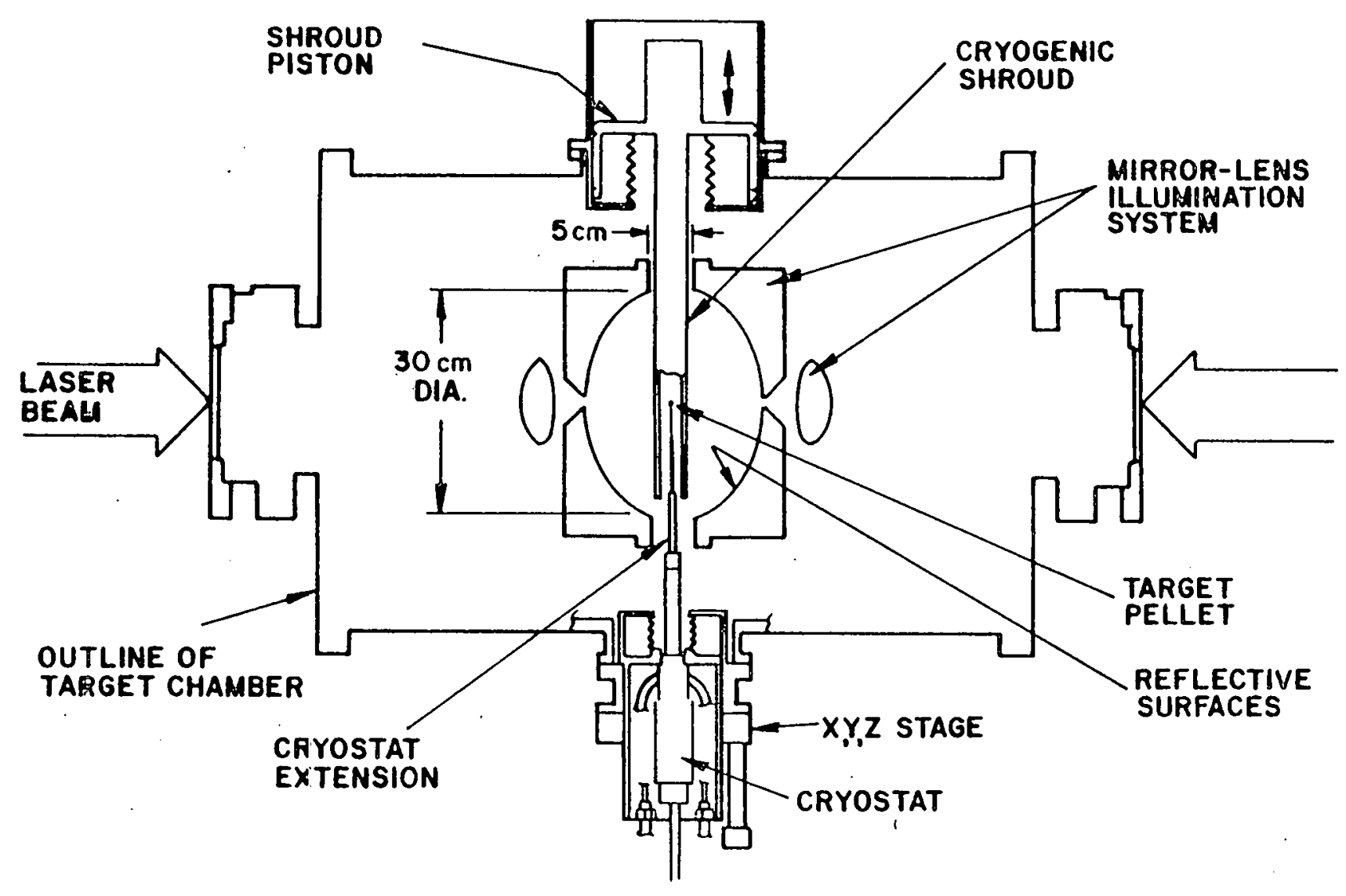

FIGURE 1.3-3 Conduction cooling system integrated in the laser fusion target chamber. 


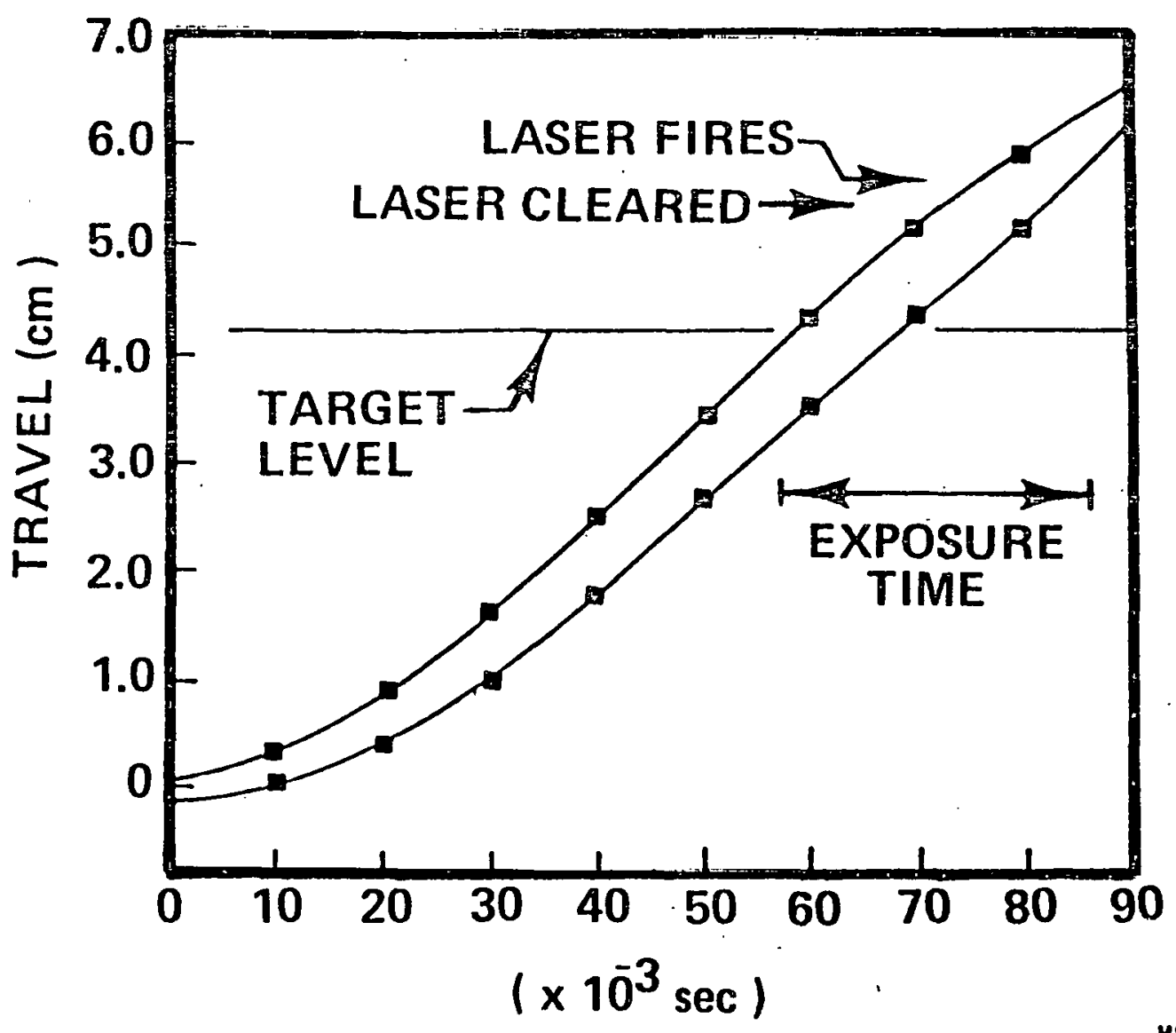

FIGURE 1.3-4 Radiation shield retraction. Position of shield vs time. Fastest and slowest curves.

it clear of the converging laser beam and all diagnostic equipment, the target is exposed to room temperature radiation. However, this exposure time, approximately 30 milliseconds, is shorter than the response time of the liquid layer in Figure 1.3-2b. Thus, the liquid layer can be irradiated at its most uniform stage. Although target irradiation experiments have not been conducted in fiscal year 1978 using this technique, it remains as a valid option for liquid layer targets.

The need to engineer a more complex system incorporating a radiation shield to increase the liquid layer uniformity precipitated a decision point for the KMSF cryogenic program: whether to continue with 
liquid layer target experiments or to redirect full effort to sol id layer targets. The decision to devote full effort to solid layer targets was based on the expected advantage of solid layers over liquid layers.

\subsubsection{Solid Layer Targets}

The detrimental effects of fast electrons preheating the gaseous fuel within the target can be minimized by using a solid layer target. If a target which is filled to a room temperature pressure of 100 atmospheres is irradiated at $21 \mathrm{~K}$ as a liquid layer target, $5.5 \%$ of the total fuel present is in the gas state available for absorption of fast electrons and the subsequent build-up of back pressure during the target implosion. However, if the same target is irradiated at $15 \mathrm{~K}$ as a solid fuel layer target, less than .02\% of the total fuel. is in the gas state available for absorption of fast electrons and the subsequent build-up of back pressure during the target implosion. (If the temperature of the solid fuel layer is less than $15 \mathrm{~K}$ when irradiated, the amount of fuel in the gas state is less than $.02 \%$ of the total fuel. However, $15 \mathrm{~K}$ serves to illustrate the point and is a temperature which can be reached without unduly stretching the engineering requirements.) Thus, since preheating of the gaseous fuel is indeed a significant limitation on the ultimate fuel compression, solid layer targets should yield the optimum results. The ability to irradiate solid fuel layer targets, however, is determined by the status of the engineering technology required to integrate a cryogenic system into the experimental target chamber.

1.3.6 Solid Fuel Layers Produced Under the Radiation Shield The radiation shield system discussed earlier for 1 iquid layer targets also allows the formation of a sol id fuel layer on the inside surface of the target. A solid layer of DT fuel that completely covers 
the inside surface of the target, Figure 1.3-5, can be formed by starting with the liquid layer of Figure 1.3-2b and quickly lowering the temperature of the shell/epoxy interface while the target remains under the shield. (9) The thermal gradient, imposed across the interior surface of the liquid layer by this procedure causes forced vaporization from the surface of the liquid layer. Since the heat of vaporization for the hydrogen isotopes is large compared to the heat of fusion (a factor: of 6.6 for $D_{2}$ ), forced boiling of $\sim 15 \%$ of the liquid causes the remaining liquid to freeze on the inner surface of the glass shell.

The temperature of the solid fuel layer is now pinned below the melting point of the fuel by the copper fiber. As a result, the solid layer of Figure 1.3-5 sublimes instead of melting when exposed to room temperature radiation. A fuel layer that must sublime rather than melt has a substantial lifetime, time when solid fuel remains as a continuous layer on the inner wall of the target. The amount of solid fuel remaining distributed on the inner surface of a given target at laser irradiation is determined by the time of exposure to room temperature radiation. The exposure time is determined by the retraction speed of the radiation shield (see Figure 1.3-4). This exposure time also sets the range of target size and fuel fill that can be used with this system. Figure 1.3-6 shows the amount of fuel remaining as a continuous layer on the inner surface of a target as a function of exposure time, for various size targets and fuel fills. The 30 millisecond exposure time of the piston retraction device allows a target as small as 70um in diameter with a fill pressure of $30 \mathrm{~atm}$ to be irradiated with an appreciable amount of fuel remaining as a continuous layer on the inner surface of the t.arget. 


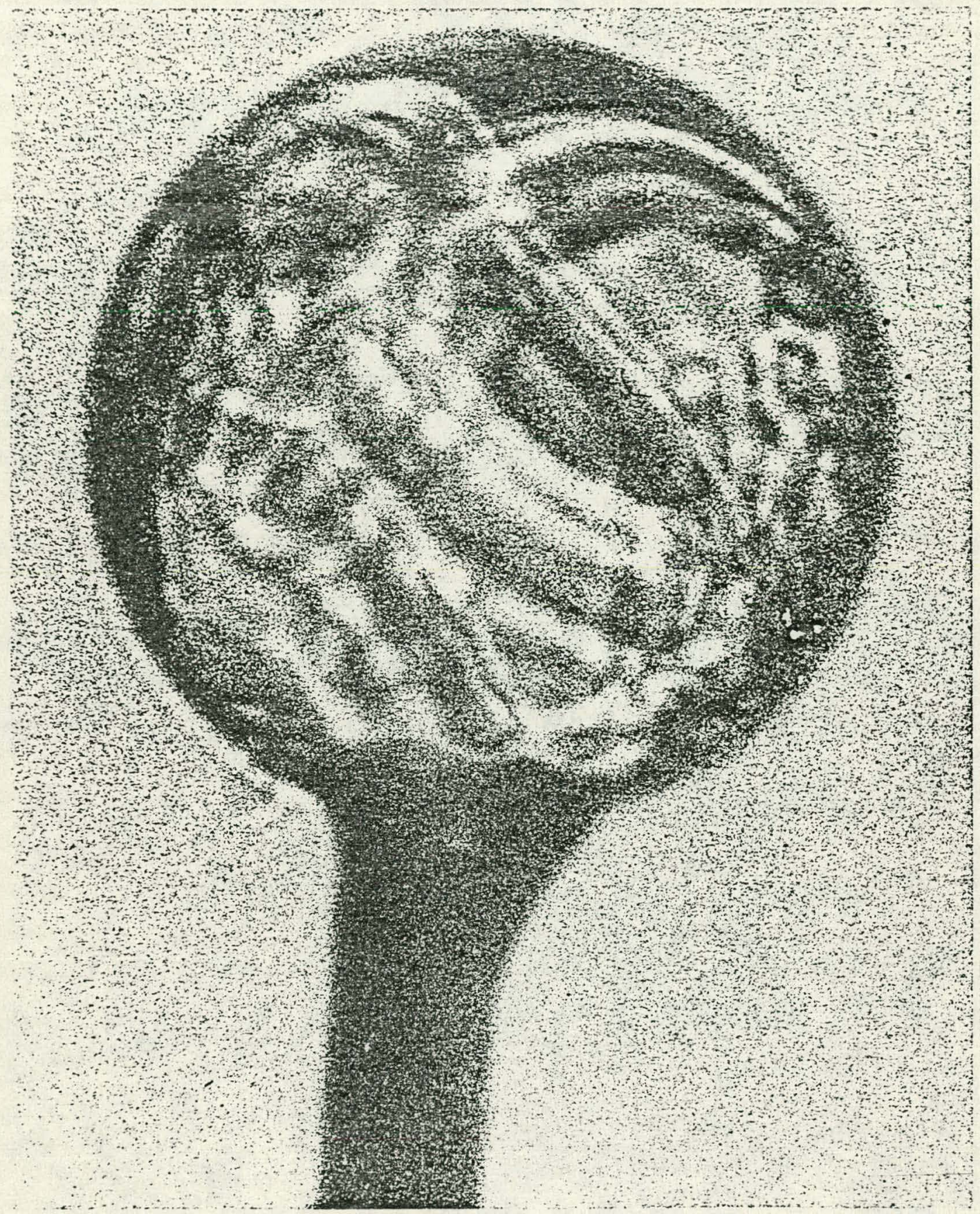

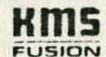

FIGURE 1.3-5 Solid layer of DT fuel. 


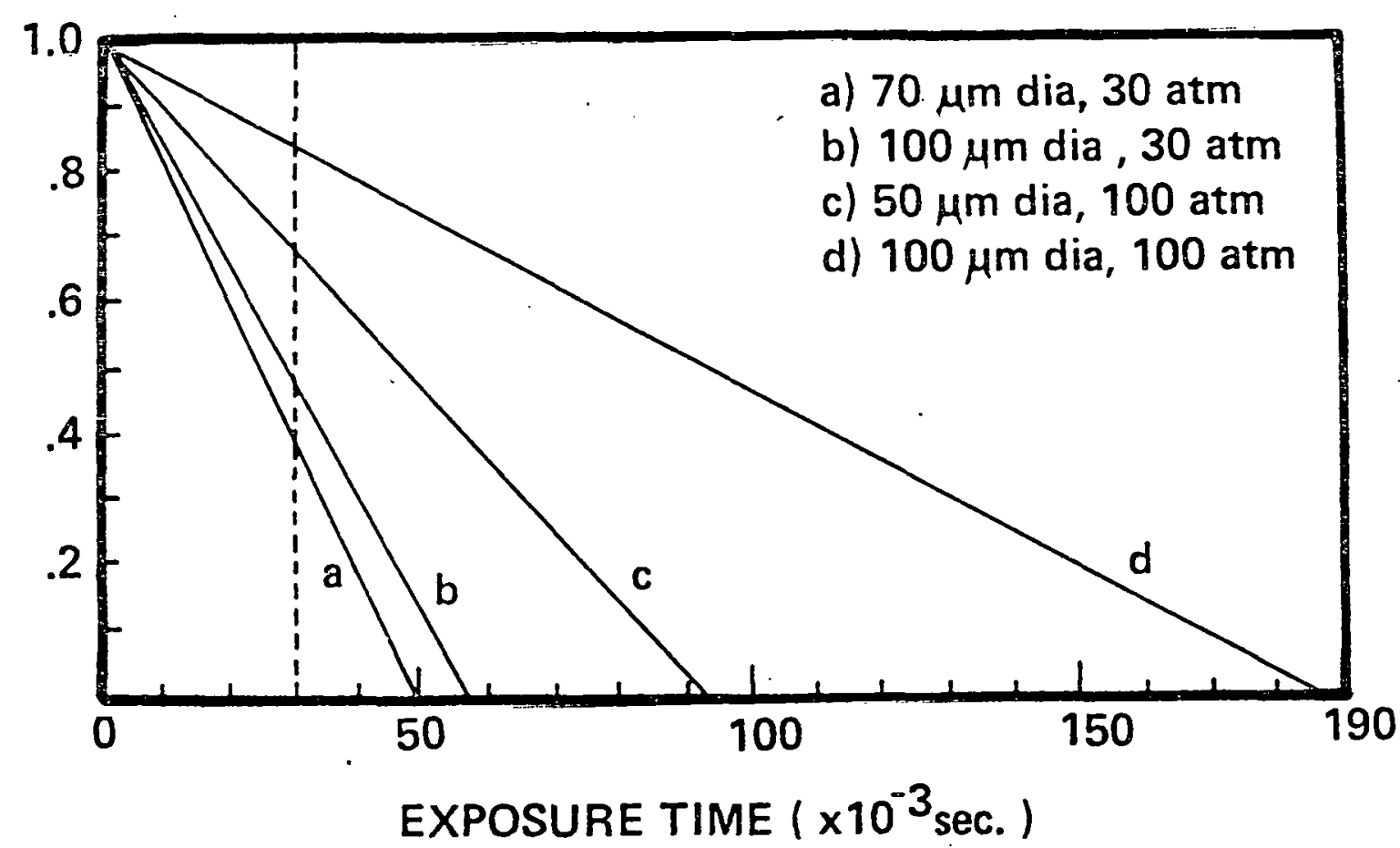

FIGURE 1.3-6 Solid fuel remaining on inner surface of shel1. (\%/100) Initial fuel $\approx 85 \%$ of available fuel:

$\underset{\text { KuSION }}{\text { KMIS }}$

Although this technique allows the formation and irradiation of solid fuel layer targets, these layers are inherently nonuniform. The 7iquid ( 15\%), which is forced to vaporize during formation of the solid layer ends up localized as a small solid spheroidal shape over the shell/ fiber interface. Also, the amount of fuel that sublimes during the exposure time is added to this localized mass. The asymmetric implosions observed for nonuniform liquid layer targets, therefore, argue against extensive use of this technique to produce solid fuel layer targets. However, integration of this system into the experimental target chamber afforded the opportunity to clearly identify and specify the problem areas involved with timing an active system (the retracting shield) to 
the laser firing sequence. The key requirements or problem areas were then addressed when designing the system for uniform solid fuel layer targets.

\subsubsection{Production of Uniform Sol id Fuel Layer Targets}

There are two separate aspects of producing a uniform solid fuel layer target for laser irradiation. First, the system and procedure must provide a source of refrigeration to form the fuel layer in a manner that maximizes its uniformity. Second, the system must be amenable to integration of the apparatus and procedures with the experimental target chamber. The constraints imposed by existing systems dictate, to a great extent, the options available.

Achieving uniformity in a solid fuel layer requires that two conditions ${ }^{(8)}$ be satisfied. First, any temperature gradient within the target must be small during the condensation and freezing of the fuel. Any temperature gradient.within the target is relieved by a redistribution of fuel, evaporation from the warm region and subsequent condensation at the cold region. This redistribution of fuel, mass per unit time, becomes negligible only below 10K. Therefore, while the fuel is condensing and freezing $(35 \mathrm{~K}$ to $18 \mathrm{~K}$ for the nominal $100 \mathrm{~atm} 100 \mathrm{um}$ diameter target), temperature gradients are intolerable. The second condition for achieving uniformity requires that the time for condensation and freezing be minimized. Under the influence of gravity the liquid and/or liquid and solid mixture can sag within the target. Minimizing the time minimizes the sag. A condensing and freezing time of $20 \mathrm{milli-}$ seconds permits a theoretical nonuniformity of less than $5 \%$. Cooling with gaseous helium can, in principle, satisfy both of the conditions. 
The most practical method of minimizing the condensation and freezing time is to heat the fuel in a previousiy frozen target, vaporize it and allow it to rapidly refreeze within a cold environment. In a series of well controlled bench experiments using an isothermal static hel ium environment, Miller ${ }^{(10)}$ has demonstrated the degree of uniformity that is possible with this method. He was ablje to produce targets that met the criterion of $20 \%$ WNU. In addition, he showed that a CW laser is an efficient means of coupling heat to the target. However, integration of a static isothermal environment with the experimental target chamber is a formidable task requiring several levels of complexity. (11) For our current experimental. chamber a static isothermal environment is not required.

The primary constraints imposed by our existing experimental chamber are that the main illuminating beams must not be blocked or degraded nor may any of the diagnostic equipment be precluded from operation. These constraints make it unavoidable that the target be exposed to room temperature radiation for at least a short time prior to the shot. This exposure to room temperature radiation is 1 imited for any particular target by both the amount and the physical properties of the fuel. Figure 1.3-7 illustrates the point. It shows the calculated 1 ifetime of the various phases of $\mathrm{D}_{2}$ in a typical size isolated target, initially at $10 \mathrm{~K}$, that is exposed to $300 \mathrm{~K}$ radiation. (The target is assumed to have an absorptivity of one for room temperature radiation.) After 10 milliseconds, the temperature of the fuel has reached the melting point where the uniformity of the fuel layer degrades as melting 


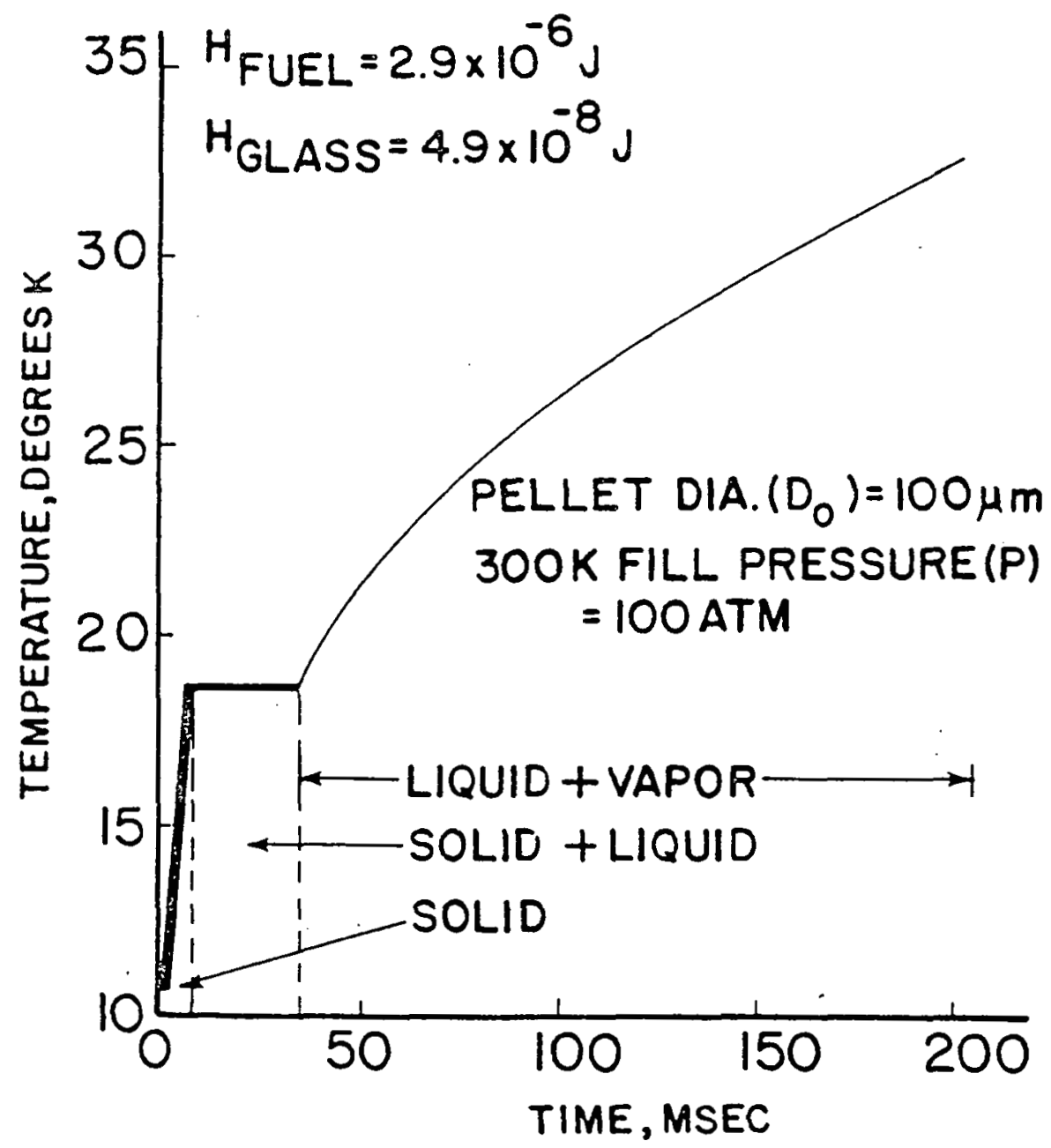

FIGURE 1.3-7 Pellet 1 ifetime 300K environment.

$\underset{f \cup S I O N}{\operatorname{Km}}$

commences. Thus, for a solid fuel layer target, the maximum exposure time is limited to less than 10 milliseconds. To minimize the amount of vaporizcd fucl (minimize prehed effects), an exposure time significantly less than 10 milliscconds is desirable.

1.3.8 Equipment Required for Uniform Sol id Fuel Layers

The system we have designed and constructed to meet the requirements of uniformity and compatibility with the target chamber uses a 
gaseous helium shroud and is shown in schematic form, integrated with the target chamber in Figure 1.3-8. Much like the radiation shield, it is lowered from the top of the experimental chamber to envelop the prealigned target and form the fuel layer. Just prior to the target shot the shroud is retracted uncovering the frozen target.

The details of the gaseous helium shroud are shown schematically in Figure 1.3-9. Simplicity of design is achieved by using a commercially available cryostat and allowing the shroud to continually supply gaseous helium at a constant rate through a pinhole orifice to the region around the target. Four windows are provided at the target level, two for observation and two for a heating laser (the CW alignment laser of the target chamber) used to vaporize the fuel in situ prior to the rapid refreeze. (10)

Alignment of the target at the focus of the laser system is critical ( $\pm 5 \mu \mathrm{m}$ permissible displacement). One must either attempt to prevent any displacement of a prealigned target while the fuel layer is being formed or provide for repositioning afterward. To avoid the complexity of alignment while the target is under the shroud, the system was designed to minimize target displacement during fuel-layer formation. The target is mounted on a copper post which fits into a collet joint in the extension of the lower cryostat. Before target alignment and formation of the fuel layer, the temperature of the lower cryostat is set at a value $<10 \mathrm{~K}$ to avoid subsequent thermal displacement of the target under the shroud. The target is mounted on a copper post as in previous cryogenic experiments (with liquid-layer targets) except that the target itself is thermally isolated from the post by a 200 - to $400-\mu m$ length of 10-im-diameler ylass ftber. This nounting arrangement prevents target 


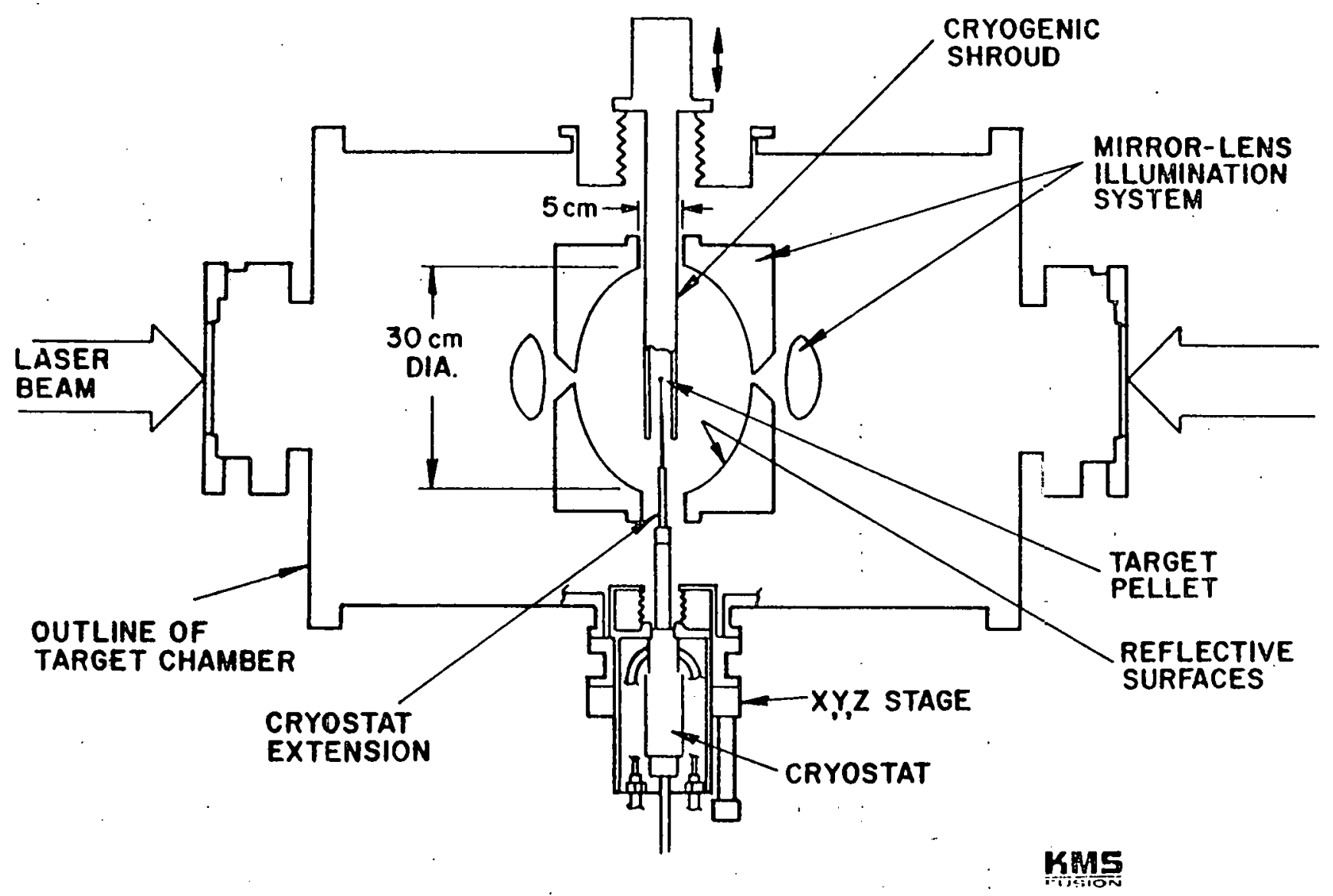

FIGUFE 1.3-8 Gasecus hel ium shroud integrated in the laser fusion target chamber. 


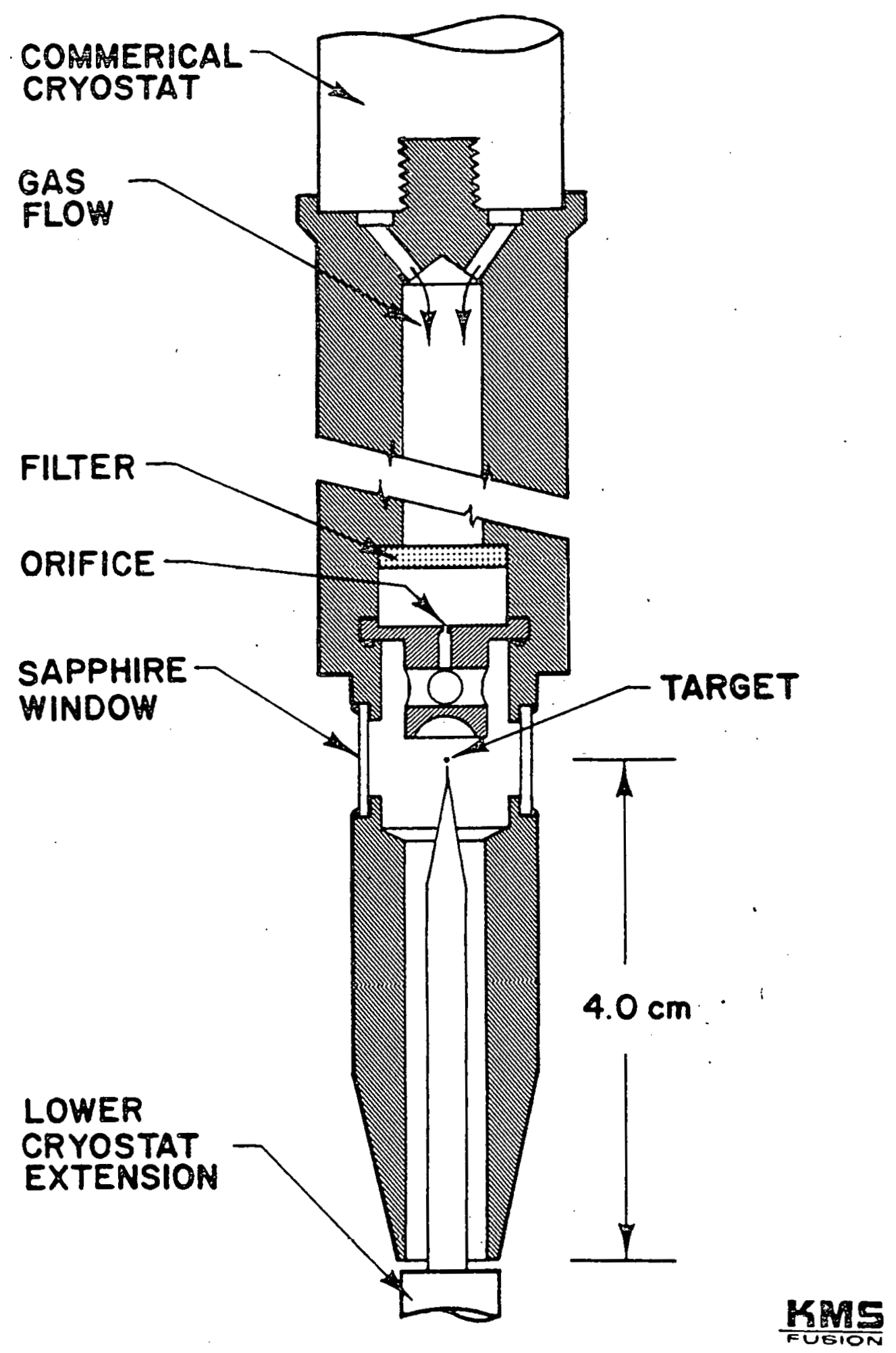

FIGURE 1.3-9 Gasenus hel ium shroud. 
motion due to cooling under the shroud yet assures that the cold gaseous hel ium is the dominant source of refrigeration for layer formation. Nonuniformities due to asymmetrical cooling by the fiber are thus avoided.

To prevent mechanical displacement of the target, the shroud does not touch the lower cryostat extension. The annular gap between the two acts as a variable impedance to the flow of gaseous helium from the target region inside the shroud to the rest of the target chamber. For maximum fuel layer uniformity, the pressure near the target needs to be maximized in order to minimize the condensation and freezing time. This pressure is determined by both the throughput of the pinhole and the size of the annular gas. The throughput into the target chamber is set by the size of the pinhole which must be chosen so that the pressure throughout the target chamber is no higher than $\sim 3$ millitorr. At higher pressures, the conductivity of the gas in the target chamber is sufficient to heat the cryostats above 10K. For this throughput, the pressure near the target is maximized by decreasing the gap to a minimum. Condensing and freezing of typical targets in this system occurs in $\leq 30$ milliseconds. The physical dimensions of the shroud plus the overlap of shroud and target post are dictated by considerations of shroud retraction. Exposure of the target to room-temperature radiation may be considered to start when the bottom of the shroud reaches the level of the target. From this position the shroud must be lifted clear of the path of the converging laser beams within $10 \mathrm{msec}$. The acceleration needed to accomplish lhis call be mintmized by maximizing the shroud overlap, i.e., the distance over which the shroud accelerates before exposing the target. The actual overlap chosen $(4 \mathrm{~cm})$ is a compromise between maximizing the overlap and assuring that the clearance (1. Gmm) between the instde of 
the shroud and the target post extension can be maintained during both initial alignment of the system and shroud retraction.

The retraction system is shown in schematic form in Figure 1.3-10. A primary design consideration, identified using the piston retraction system and subsequently applied to this system is the minimization of target motion due to vibration. Since any impulse applied to the target

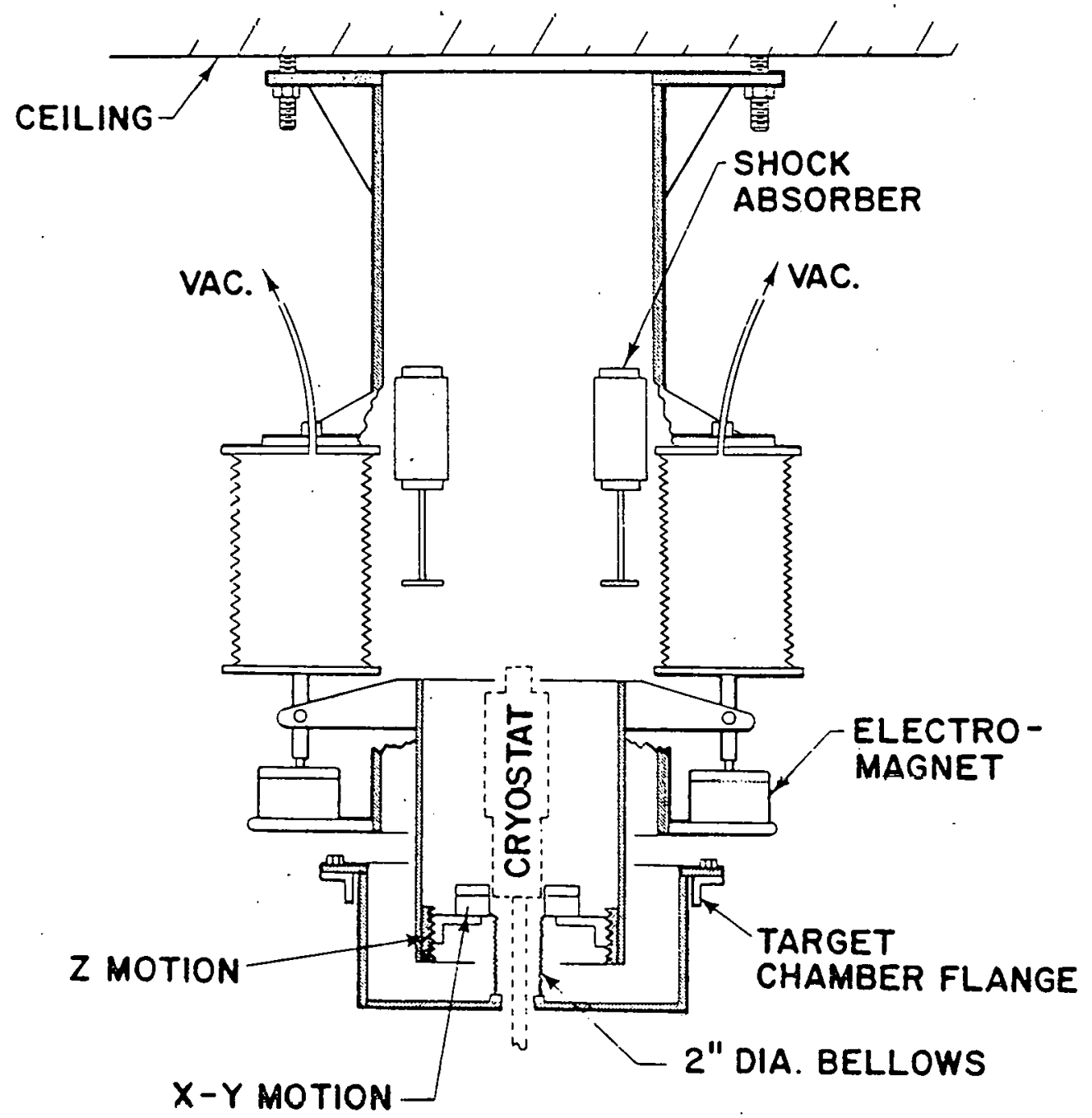

FIGURE 1.3-10 Shroud retraction device. 
chamber could displace the target, the retraction system is mounted on the ceiling, not on the target chamber, and the only mechanical coupling of the entire retraction device to the target chamber is through a 2 " diameter bellows, the minimum size that will permit $x, y$ alignment of the shroud through the vacuum seal (see Figure 1.3-8).

The force for retraction is provided by two evacuated $5^{\prime \prime}$ diameter bellows. This force is initially counterbalanced by flat-faced electromagnets. Retraction is triggered during the laser firing sequence by dumping the current in the magnetic field coils. Since the holding force of the magnets is very sensitive to gap, free acceleration of the shroud is achieved quickly and the release time is highly reproducible. Figure 1.3-11 shows the position of the shroud as a function of time during retraction. Time zero on the figure is the time when the magnet dumping circuit is triggered. The data shown were taken from multiple exposures on high speed polaroid film of an indicator flag attached to the system. The two data points at each time form the plot for the fastest and slowest curves for a series of 25 runs conducted over a two day interval. Total travel is $10.8 \mathrm{~cm}$ and occurs in $60 \mathrm{msec}$. Laser irradiation of the target is timed to occur at the point indicated on the figure before deceleration provided by two commercial shock absorbers begins. This eliminates the possibility of target vibration due to this impulse. With laser irradiation occurring at this point, the exposure of the target to room temperalure pradlation is kept to $4.0 \pm 0.35 \mathrm{msec}$.

1.3.9 Evaluation or Sulid Fuel Layers

To evaluate the quality of the fuel layers produced with this apparatus the interferometer shown schematically in Figure 1.3-12 was constructed and installed in our off-line simu?atiun chamber as well as 


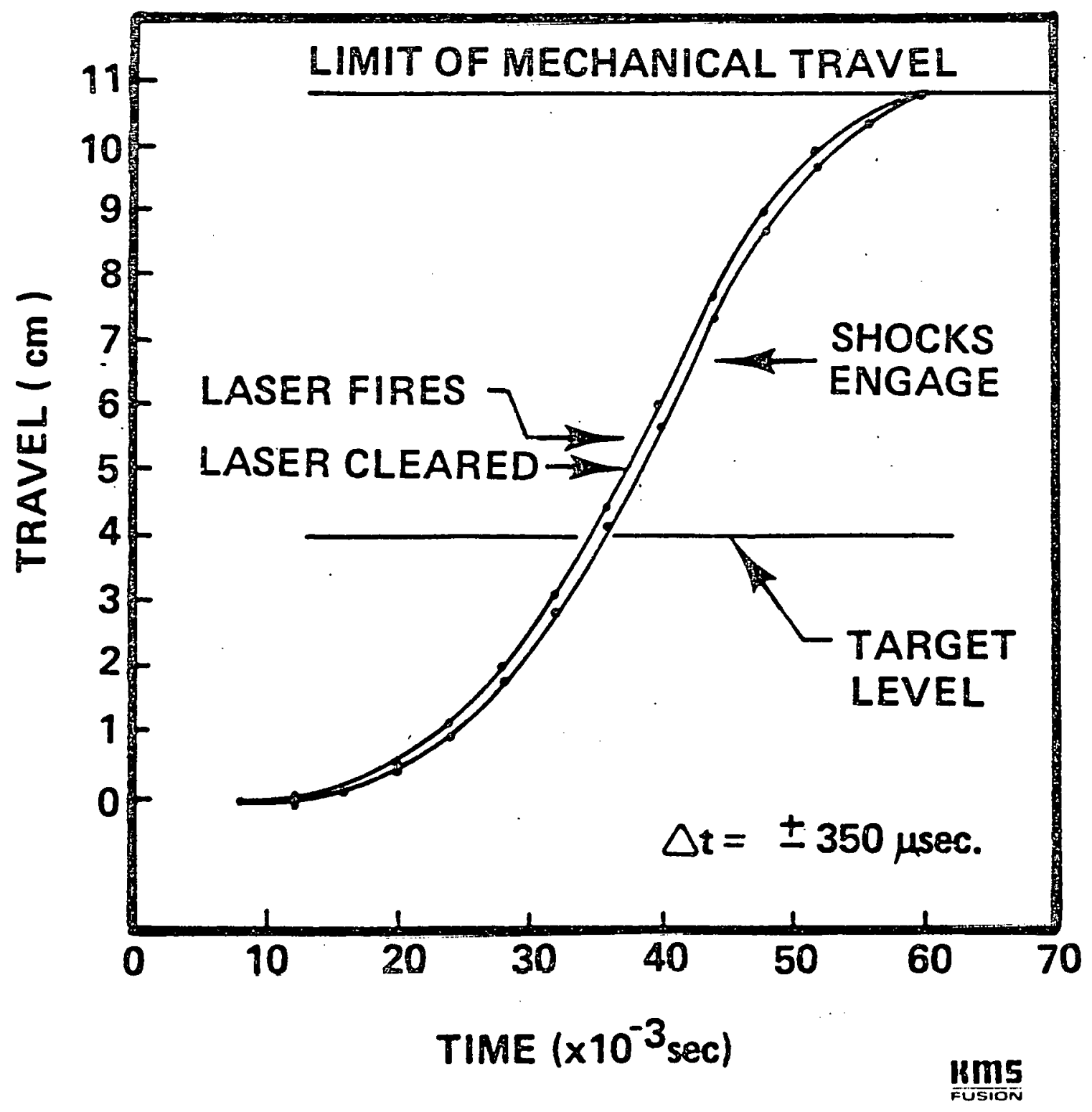

FIGURE 1.3-11 Shroud retraction. Position of shroud vs time. Fastest and slowest curves. 

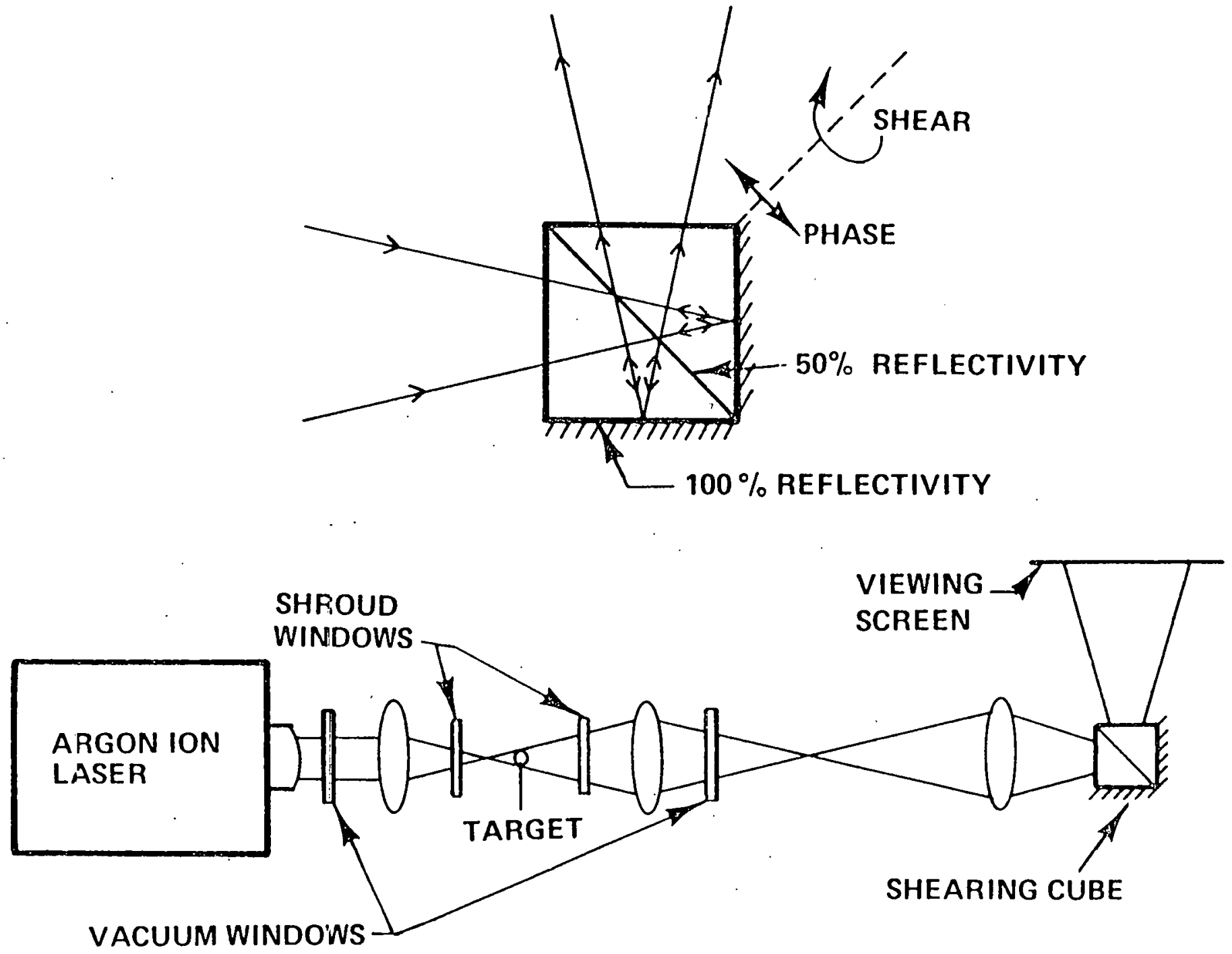

FIGUF:E 1.3-12 Shearinc cube interferometer. 
our target chamber. The major feature of this interferometer is the shearing cube. (12) With it both high contrast and stability are assured as well as the ability to continuously vary both the background phase and phase gradient. The illuminating lens is adjusted to focus the laser behind the target producing a diverging beam a few target diameters wide at the plane of the target. The imaging lens is set to focus the crossover of the laser beam on the back side of the shearing cube while focusing the target on the image plane. By limiting the focus spot on the shearing cube, extraneous phase shifts due to variations in the surface of the cube are eliminated. Phase shift and shearing are controlled by sliding and rotating one-half of the shearing cube relative to the other. The quality of targets that this system is capable of producing is shown in Figure 1.3-13. Figure 1.3-13a is the interferometric image of a DT-filled target when the fuel is in the gas phase. Figure 1.3-13b is an interferometric image of the same target with the fuel condensed out in the solid phase. Using a criterion based on computer modeling for these interferometric images $(10)$ the target shown in Figure $1.3-13 \mathrm{~b}$ meets the criterion of $\sim 20 \%$ WNU.

At the present time the reproducibility of the solid layer uniformity is not entirely satisfactory. A remelt of the target shown in Figure 1.3-13b may produce the target of Figure $1.3-13 \mathrm{c}$ as ofter as it reproduces the image of $1.3-13 \mathrm{~b}$. High speed movie films (500 fps) taken of the vaporize/refreeze cycle clearly show that during condensation, white lhe fuel is in the Tiquid state, the fuel layer is very uniform. The nonuniformities observed in the final stable layer occur either during or after freezing of the liquid. At present not all the factors 


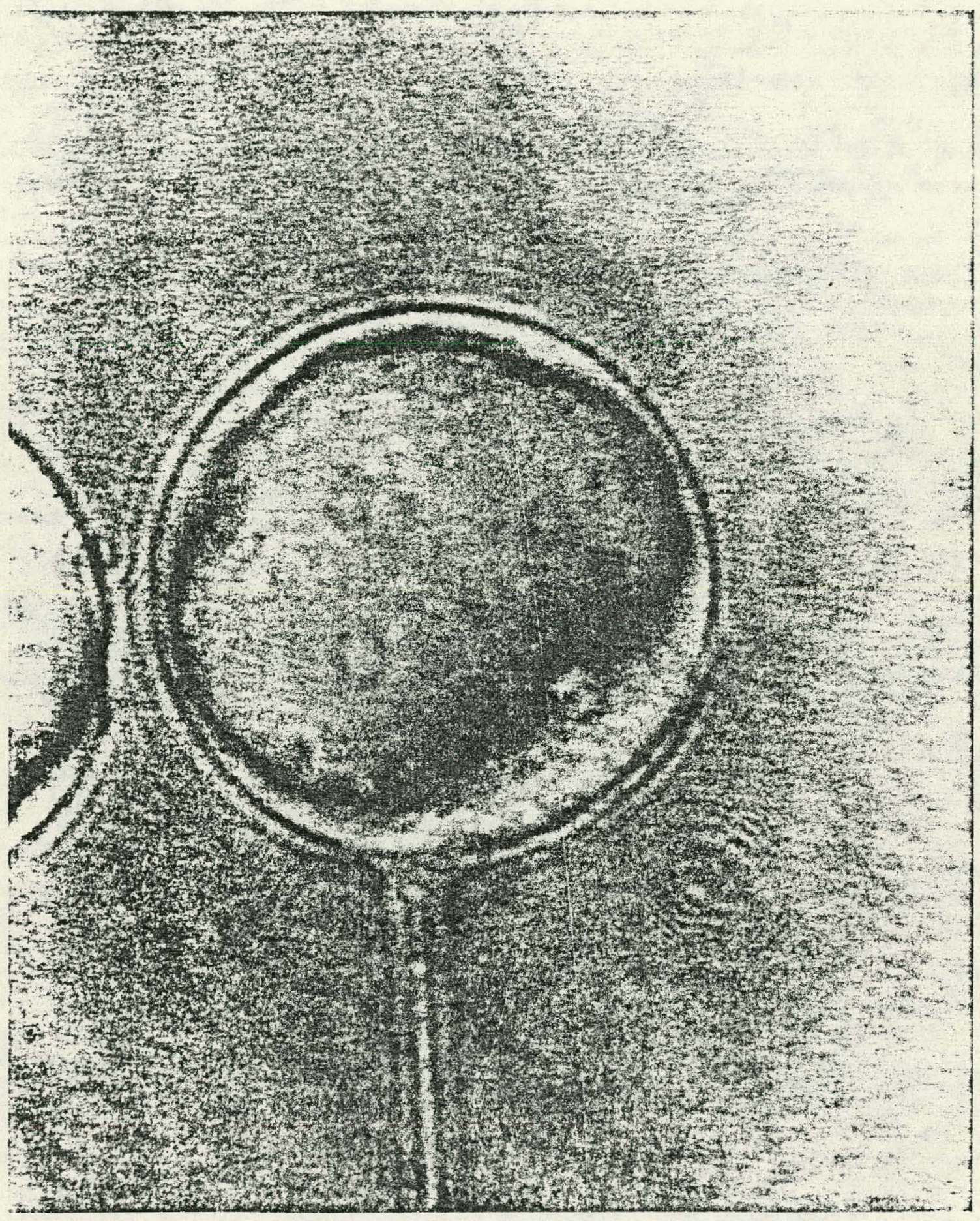

FIGURE 1.3-13a Interferometric image of target with fuel in gas phase. 


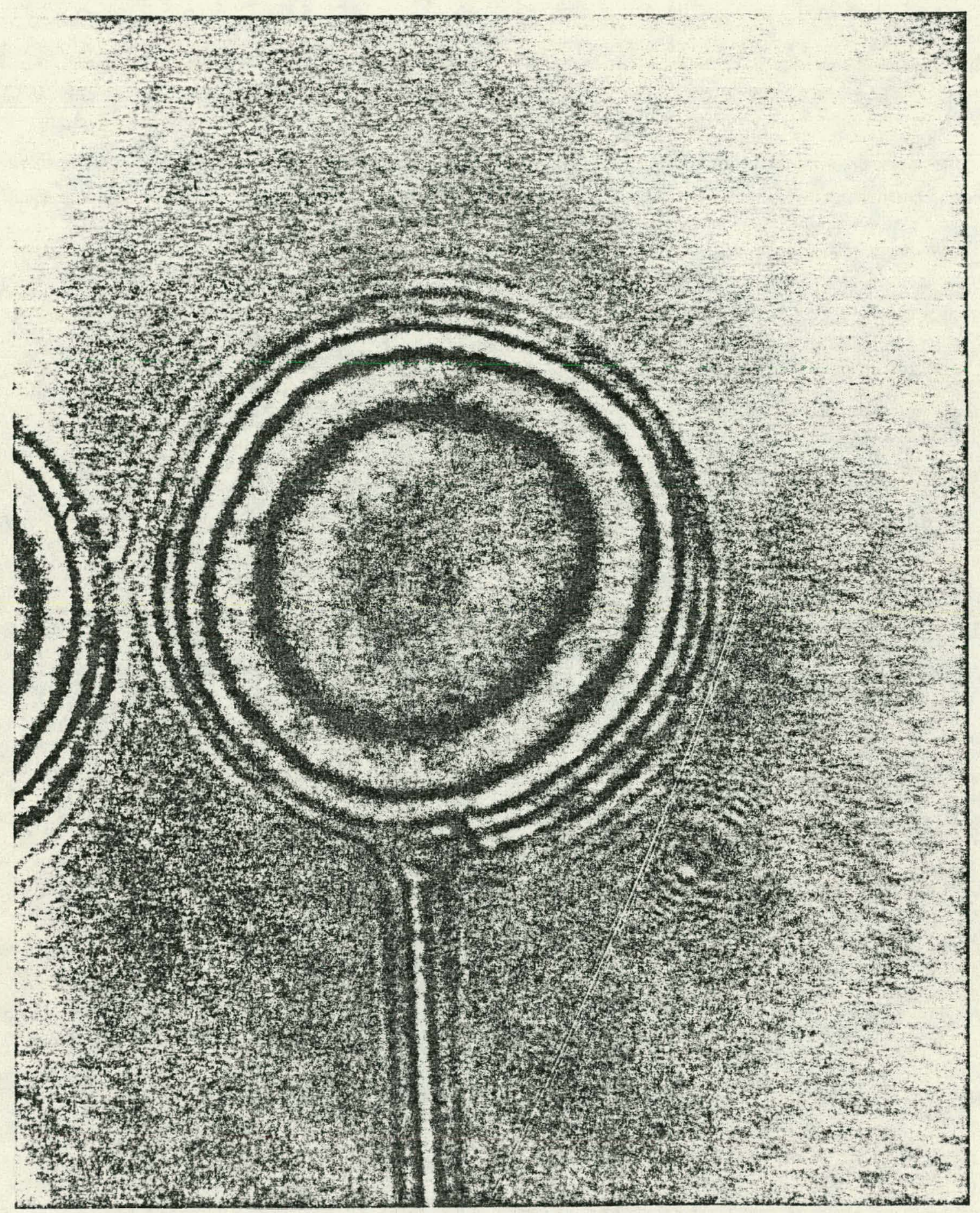

FIGURE 1.3-13b Interferometric image of uniform solid layer within the target. 


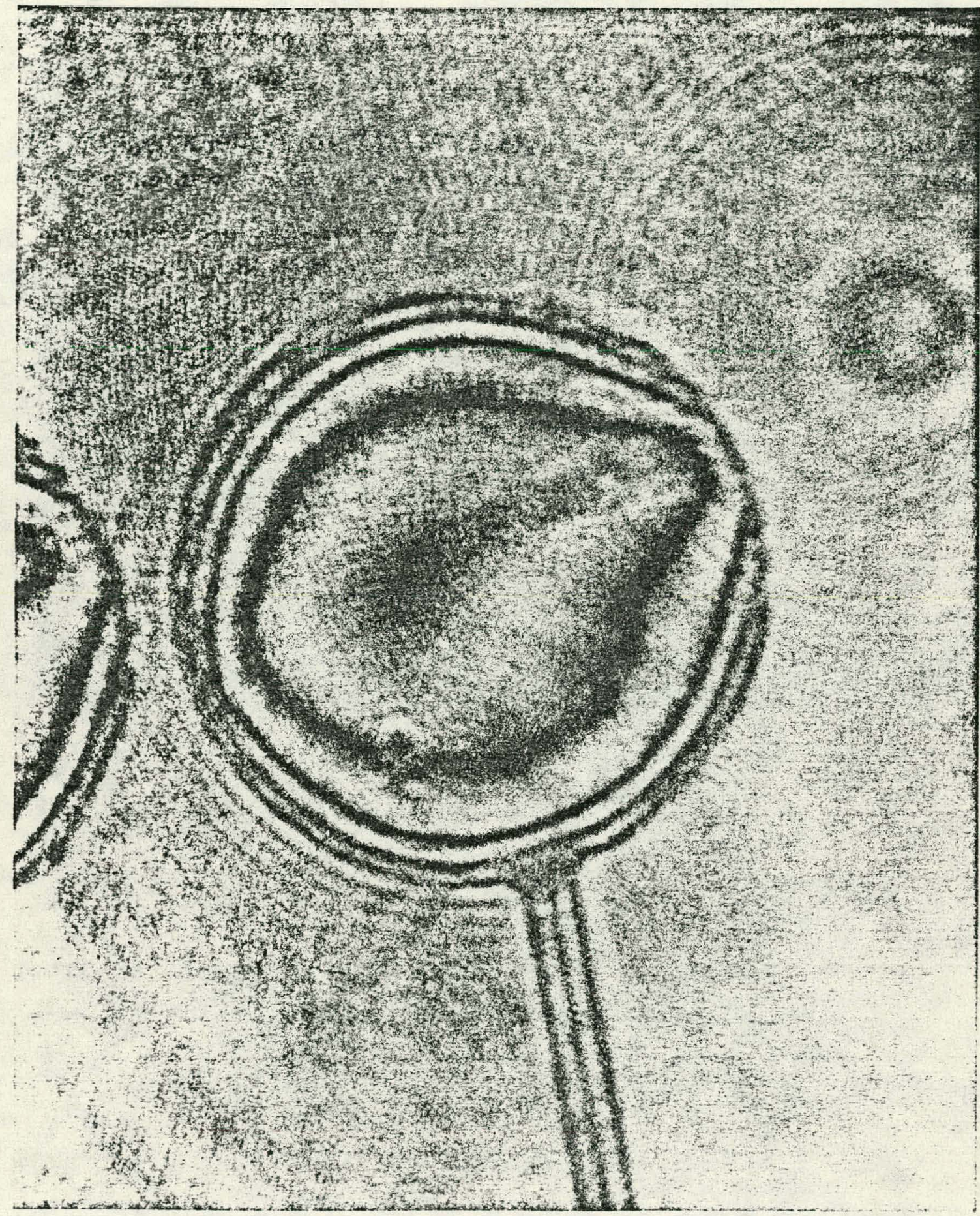

FIGURE 1.3-13c Interferometric image of nonuniform solid layer within the target. 
responsible for the uniformity or nonuniformities of the solid layer are understood. Further study is required to identify and understand these factors so that the reproducibility of the uniformity becomes a controlled parameter.

1.3.10 Target Chamber Experiments. (ASE Experiments on Solid Fuel Layers) The ability of cryogenic fuel layers to survive the energy which reaches the target prior to the main laser pulse is a dominant concern. (13) Amplified spontaneous emission (ASE) and pre-pulse energy are inherent problems for laser amplifier systems. Pre-pulse energy can be controlled electronically. However, approximately $100 \mu \mathrm{sec}$ before the arrival of the main pulse (the lead in flash lamp pump time for the KMSF ampl ifier system) ASE energy of 0.2 to $1.6 \mathrm{~mJ}$ is focused on target. $A b-$ sorption of only $\approx 3.2 \mu \mathrm{J}$ by a $100 \mathrm{~atm}, 100 \mu \mathrm{dia}$. target would completely melt and vaporize the fuel before the main laser pulse arrived.

To avoid ambiguity concerning the absorption of this ASE energy and thus the state of the fuel layer at the time the main pulse arrives, experimental verification of the layer's integrity is required. Ideally, one would like to photograph the condition of the fuel layer at the time that the main pulse arrives on target. However, a simpler method is to conduct ASE experiments, (13) fire the laser system but physically block the main laser pulse, preventing it from entering the first amplifier. By recording the condition of the fuel layer at the time when the main pulse would arrive on target had it not been blocked, the resporise of the fuel to various levels of ASE can be determined.

At a level of $0.20: \mathrm{mJ}$ of ASE energy, no change in the sol id fuel layer targets could be detected either by direct viewing or by interfer- . ometry. Data obtained al a level of $2.0 \mathrm{~mJ}$ of ASE energy showed that. at 
this higher level a small fraction of the solid fuel layer was melted. With this data it is possible to conduct laser irradiation experiments with a high degree of confidence that the fuel layer is undisturbed as long as the recorded ASE is $\lesssim 0.20 \mathrm{~mJ}$.

1.3.11 Laser Irradiation of Uniform Solid Fuel Layer Targets

The additional operational procedures required for these solid layer targets have been integrated into the normal routine of the experimental program at KMSF. The target is installed in the experimental chamber and aligned to the main laser using the same sequence of steps as used for the liquid layer experiments. ${ }^{(6)}$ The gaseous hel ium shroud is lowered over the target to initially freeze the fuel. The $\mathrm{CW}$ alignment laser which is coaxial with the main illuminating system is used to vaporize the fuel within the target. Because of the large angutar coverage of the target by our ellipsoidal-mirror illuminating system (see Figure 1.3-14) beam reducers are inserted into the path of the alignment laser. These allow the ellipsoidal-mirror illuminating system to focus all the power of the alignment laser onto the target through two of the four windows on the shroud. The power of the alignment laser is increased until the fuel within the target evaporates. An operator observing the interference pattern of the fuel layer forms solid layers by shuttering the $\mathrm{CW}$ alignment laser until the uniformity of the solid fuel layer satisfies his judgment. The interference pattern of the fuel layer is then photographed and the beam reducers are retracted from the path of the main beam. Charging of the shroud retraction device by energizing the holding magnets and evacuating the 5 " diameter bellow is the last additional step. Triggering of the retraction device is controlled automatically by 

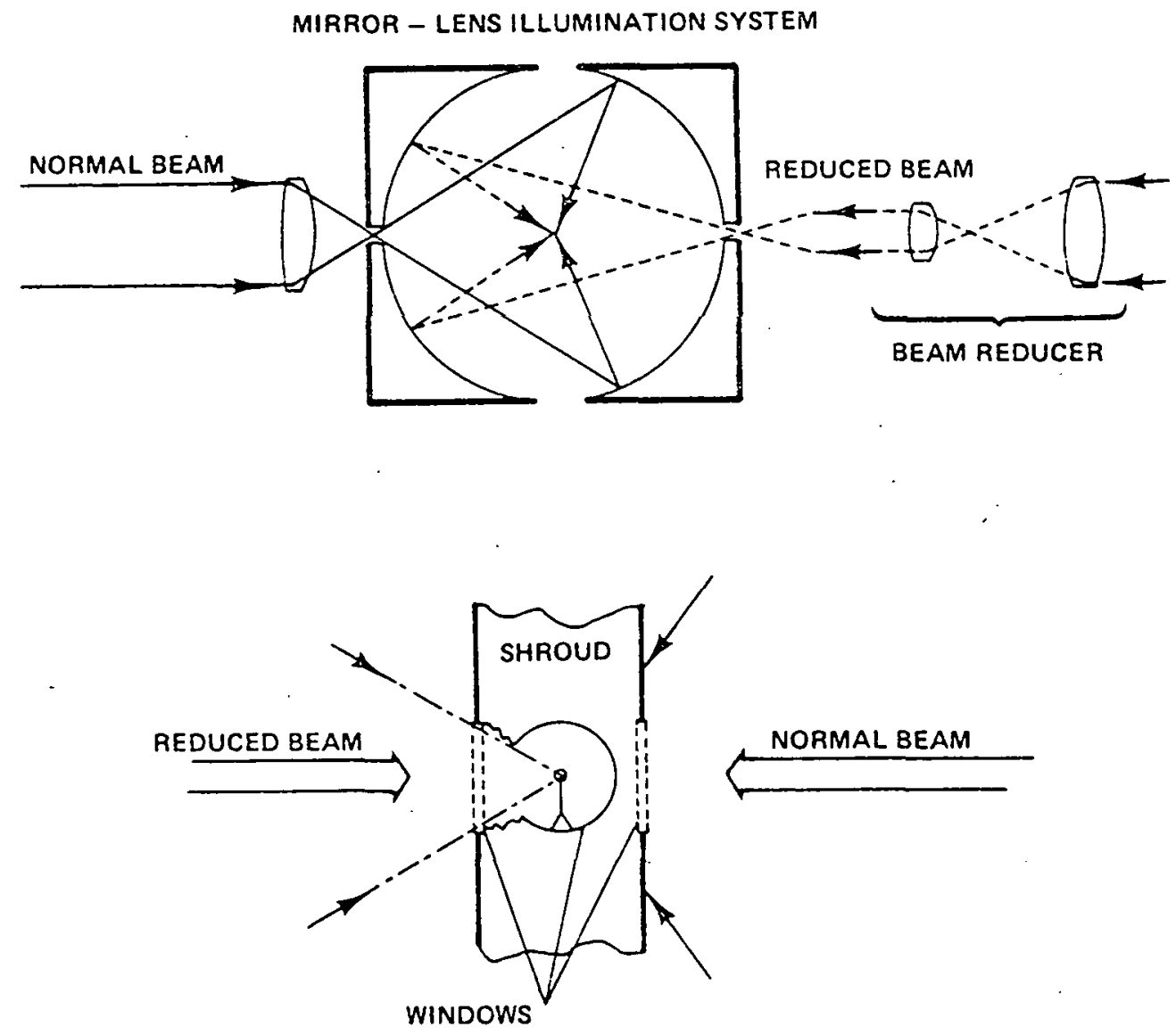

FIGURE 1.3-14 Laser heating shrouded target in target chamber.

\section{Kms}

appropriate timing circuits locked into the normal automated laser firing sequence.

A total of 49 laser irradiation shots at the 0.2 to 0.6 TW level have been conducted during 1978 on solid fuel layer targets to test the reliability of this system. X-ray photographs of target implosions have confirmed that the targets were uniformly illuminated, the alignment was held during the formation and mainenance of the fuel layer prior to the shot. Neutron production of $6 \times 10^{7}$ with 0.4 TW of power on target also 
confirms the reliability of the alignment. A more complete assessment of the plasma dynamics of these target shots requires more intensive data reduction in Section 2 .

The results of initial experiments using cryogenic liquid layer targets have been very encouraging and have led naturally to an interest in conducting experiments using uniform solid fuel layer targets. In an attempt to improve fuel layer uniformity, several successive stages of increased system complexity were added to the basic technique of pointcontact-conduction-cooling. The engineering and integration of these more complex systems identified the critical design features and procedural sequences required of a system that provides a uniform solid fuel layer target. Successful laser irradiation of these targets demonstrates that this system now offers the opportunity to experimentally study in a systematic way a new class of laser fusion targets, uniform sol id fuel layer targets.

\section{REFERENCES}

1. R. J. Mason, Nuclear Fusion 15, 1031 (1975).

2. G. S. Fraley and R. J. Mason, Phys. Rev. Lett. 35, 520 (1975).

3. T. M. Henderson, D. L. Musinski, R. B. Jacobs and R. J. Simms, Proceedings of Laser Fusion Symposium, 70th Annual Meeting of AIChe., New York, N.Y., November 13-77, 1977 (to be publ ished).

4. P. C. Souers, E. Kelly, P. E. Roberts, D. Fearan and R. T. Tsugawa, UCRL-79036 (preprint).

5. R. G. Schneggenburger (private communication).

6. T. M. Henderson and R. R. Johnson, Appi Phys. Letts. 31, 18 (1977). 
7. R. L. Berger et al. Proceedings of the 7 th International Conference on Plasma Physics and Controlled Nuclear Fusion Research, Innsbruck, Austria, August 23-30, 1978 (to be published).

8. T. M. Henderson, R. J. Simms and R. B. Jacobs, Adv. Cryog. Eng. 23, 682 (1978).

9. T. M. Henderson, R. B. Jacobs, D. L. Musinski, R. J. Simms and G. H. Wuttke, Adv. Cryog. Eng. 23, 690 (1978).

10. J. R. Miller, Adv. Cryog. Eng. 23, 669 (1978).

11. J. R. Miller, R. D. Day, E. H. Farnum, W. G. Hansen, H. E. Tucker and W. A. Teasdale. Technical Digest of the Topical Meeting on Inertial Confinement Fusion, ThEl0, San Diego, California, February 7-9, 1978.

12. B. J. Sanders, AppT. Optics $\underline{6}, 1581$ (1967).

13. D. L. Musinski, R. J. Simms and T. M. Henderson, J. Appl Phy. 48, 1176 (1977). 
S. M. Alexander, L. A. Burchett, D. A. Burgeson, D. E. Cielaszyk, R. L. Crawley, N. E. Doletzky, K. G. Doran, R. L. Downs, M. A. Ebner, W. J. Felmlee, B. D. Homyk, R. R. Kangas, D. L. Melmoth, R. L. Nolen, Jr., W. B. Rensel, R. J. Simms, D. E. Solomon, D. A. Steinman, S. C. Stephenson, L. J. Warren, D. C. Wu and G. H. Wuttke

1.4.1 Application of Integrated Circuit Fabrication Techniques to ICF Research Reactor Pellets

Initial investigations and laboratory experiments in the area of target fabrication using integrated circuit processing techniques to create special target components had shown the potential of a unique advanced manufacturing concept. The question of the applicability of integrated circuit technology to the fabrication of inertial confinement fusion targets was then posed. The combined efforts of the University of Michigan Electron Physics Laboratory and the Division of Material Sciences of KMSF have provided some relatively quick and very encouraging. answers and results.

Demonstration of electron beam lithographic etching of micron size characters on the walls of glass microballoons, fabrication of free standing thin-walled flanged hemispheres joined to form spherical structures and formation of a pellet support membrane were the proof of principle milestones and goals. Positive results in these areas suggested that the application of certain critical steps of current integrated circuit technology along with the development of some new and innovative concepts could be the basis for a manufacturing process to produce configurations of various reactor pellet designs of the future.

\section{Fabrication Techniques}

Various techniques have been developed for the fabrication of thin-walled hemispherical structures using different materials. These techniques have been summarized in a recent technical article ${ }^{(1)}$ and in 
a recently filed patent application. (2) Each of the fabrication techniques begins with the formation of etched hemispherical cavities in silicon. After the cavity etching step has been completed, the protective metal-oxide layers are stripped away leaving a two-dimensional array of hemispherical cavities in the silicon wafer. PMMA or metal hemispheres can then be formed by depositing a thin film of the material over the entire surface and subsequently peeling off the layer. This layer replicates the silicon surface and thus assumes the shape illustrated in Figure 1.4-7. The sequence of steps leading to the formation of the silicon hemispheres is depicted in Figure 1.4-2.

The etching step during which the hemispherical cavities are formed is critical and is undergoing considerable investigation. The basic procedure is to mount a wafer, into which appropriate diameter openings have been created in the protective metal-oxide layers, onto a support structure which is inserted into a beaker of etching solution. The etchant is agitated at a rate which ensures controlled stirring of the etching solution against the exposed silicon areas. The supply of etchant to the silicon and thus the frequency of the agitation is instrumental in controlling the shape of the etched cavities. As the frequency increases, the cavities change from bathtub shape at low frequencles to hemispherical (Figure 1.4-3a) and finally to bullet shape (Figure 1.4-3b) at high frequencies. By proper selection of the vibration frequency, it is possible to achieve nearly hemispherical structures with diameter variations across a given wafer of less than three percent. Variations in diameter between two separate wafer runs can be kept to less than five percent by careful mounting and proper frequency selection.

Two techniques have been used to form spherical structures. The first technique involves the joining of individual hemispheres by 


\section{REMOVE FILM FROM}

SILICON MOLD
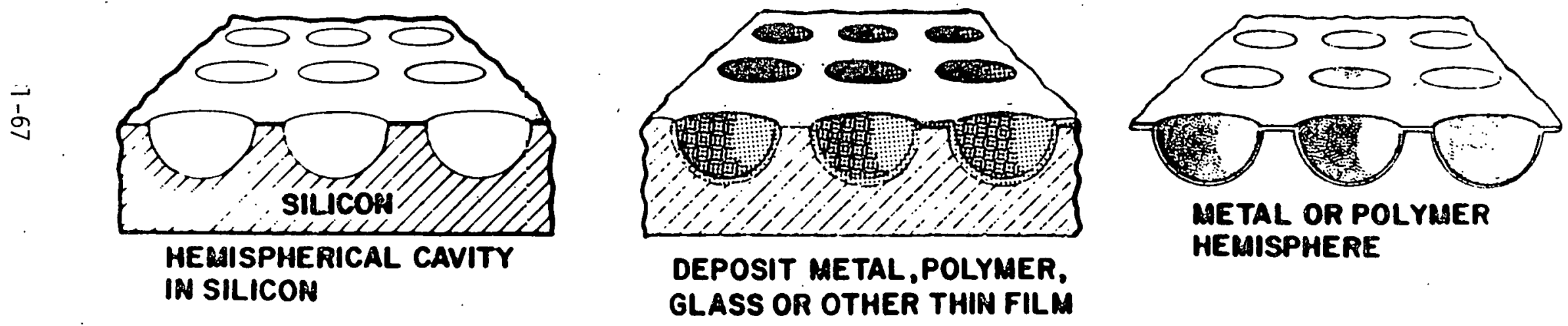

FIGURE 1.4-1 Fabrication sequence for PMMA or metal hemisphere formation. 
APPLICATION OF

INTEGRATED CIRCUIT TECHNOLOGY TO ICF PELLET MANUFACTURE

STARTING SILICON WAFER OXIOIZE AND ELECTRON BEAM OR

APPLY PHOTORESIST PHOTOLITHOGRAPHY
EXPOSE, DEVELOP
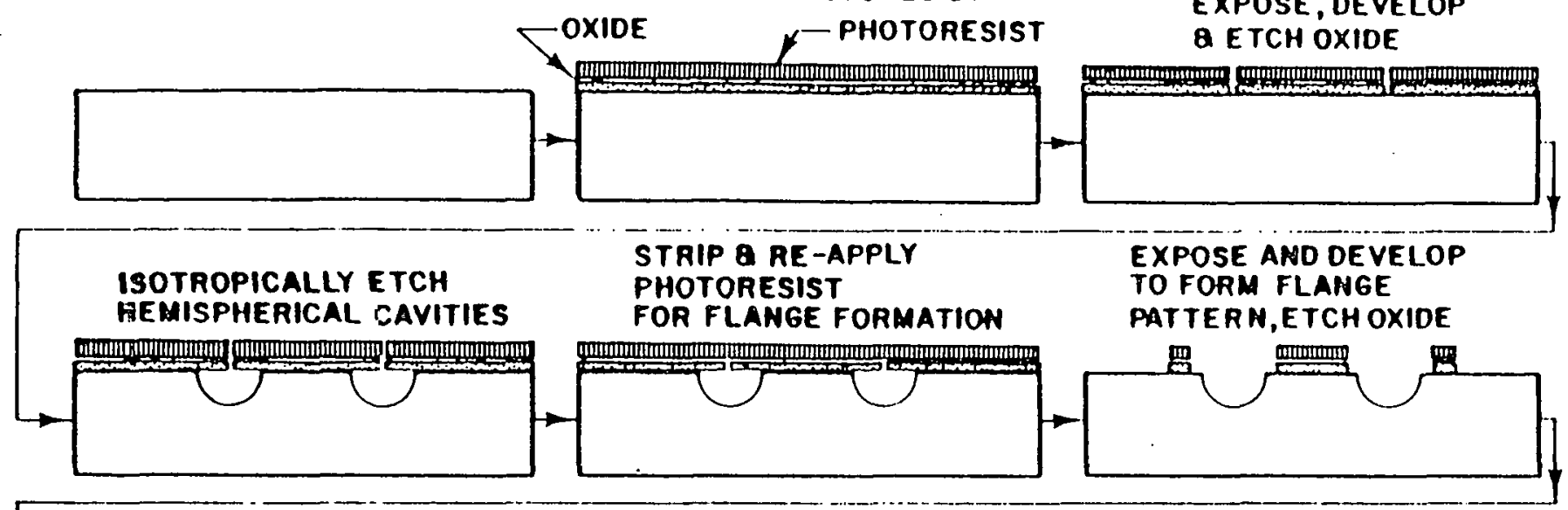

क̊
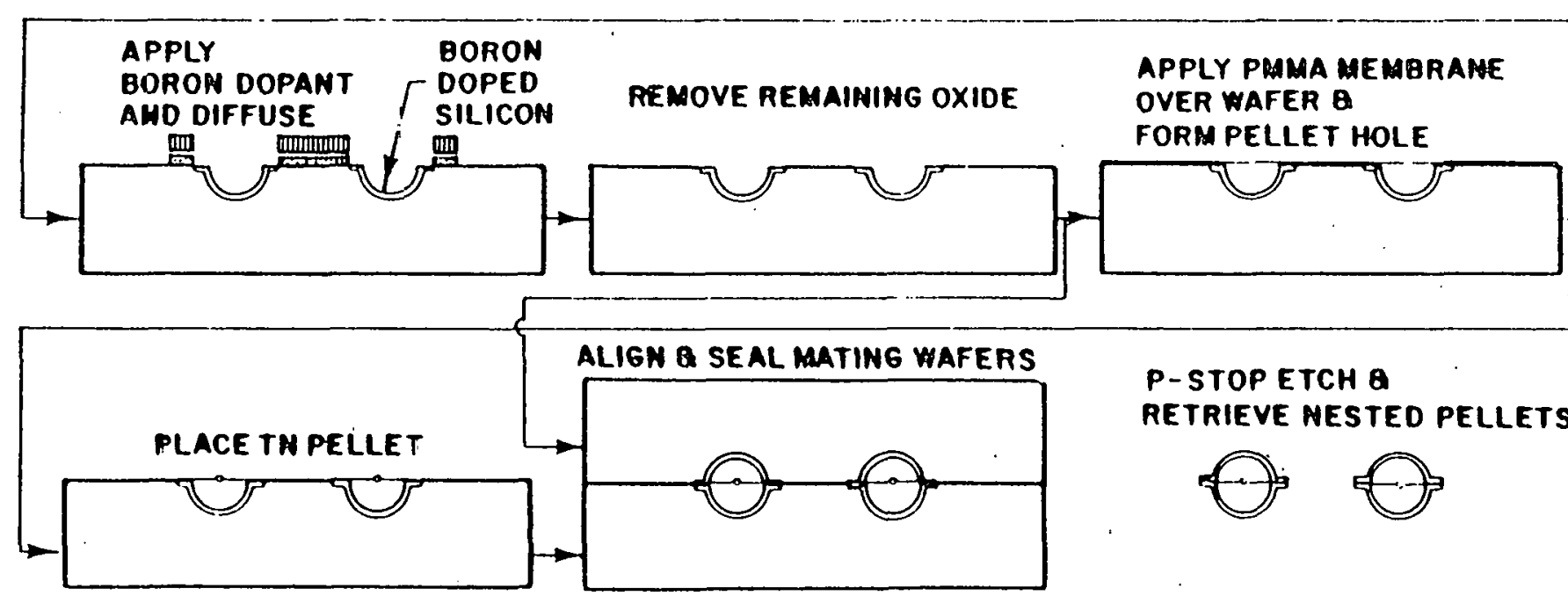

\section{P-STOP ETCH A}

RETRIEVE AESTED PELLETS

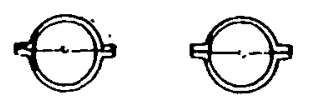

FIGURE 1.7-2 Fabrication sequence for hollow silicon pellet formation using the $p$-stop et:h technique. 
(a)

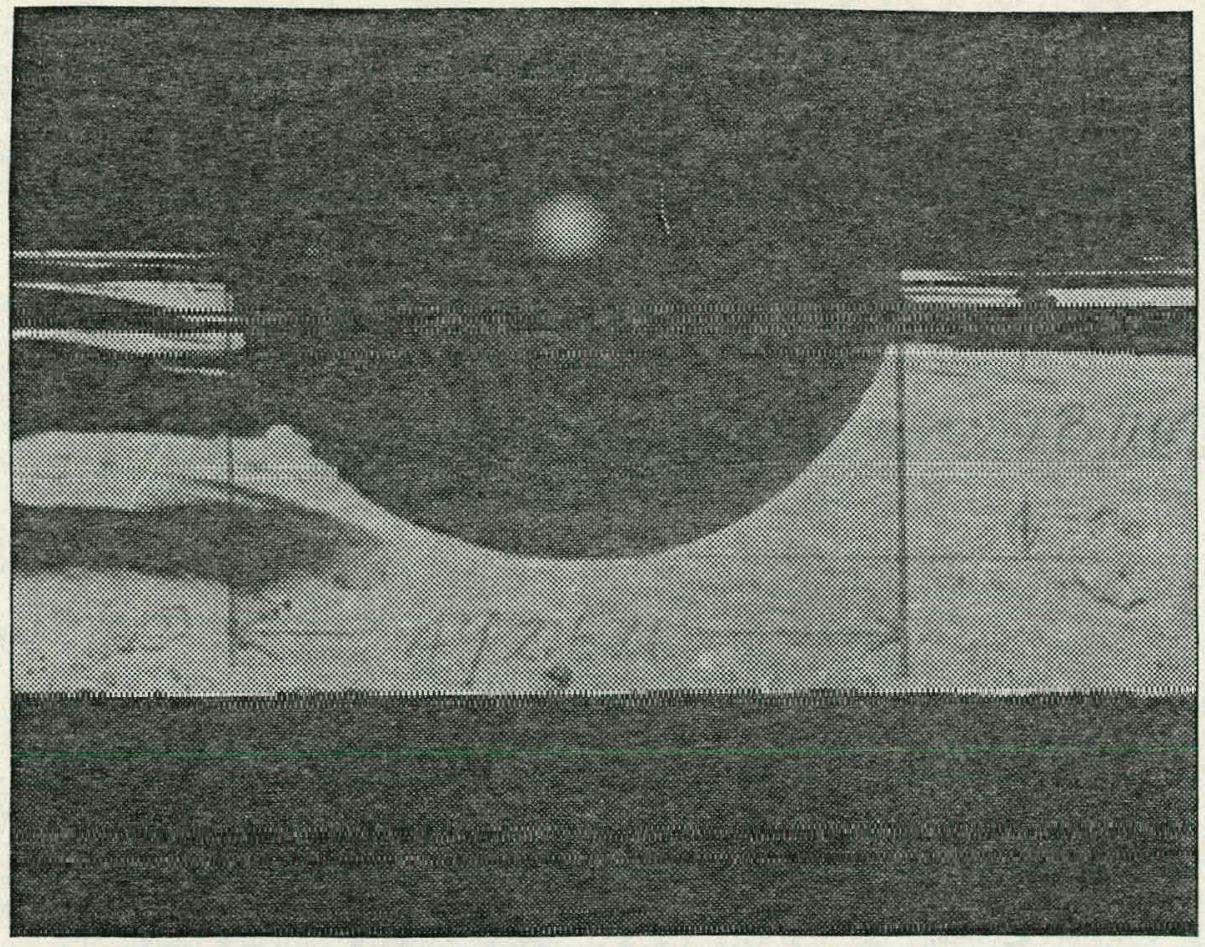

(b)

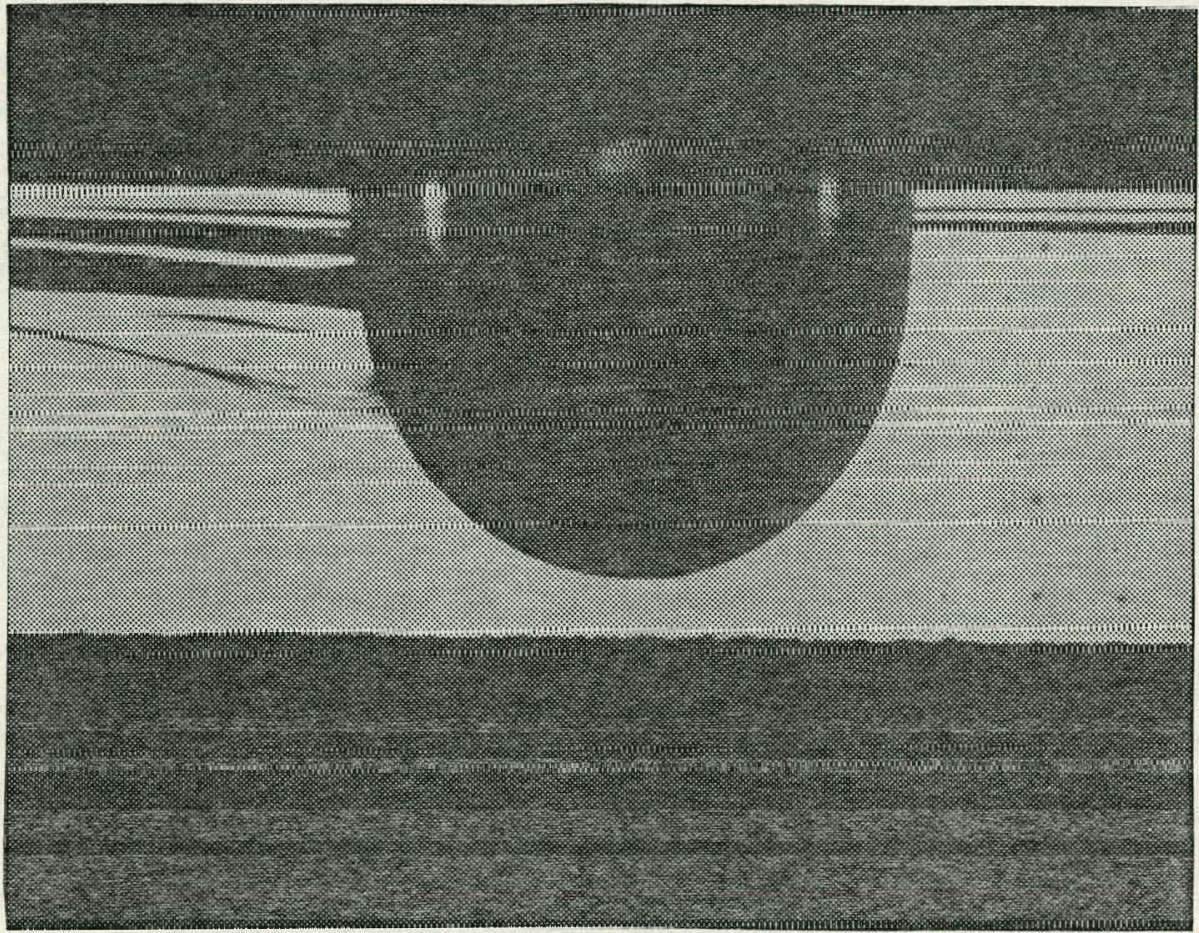

FIGURE 1.4-3 Etched cavity structures in silicon.

(a) Nearly hemispherical shaped cavity.

(b) Bullet-shaped cavity resulting from excessive agitation.

\section{Kms}


applying photoresist to the flanges and pressing two hemispheres into contact. The photoresist acts as the bonding agent holding the hemispheres together. This technique is satisfactory as a short-term exploratory effort but is not practical as a technique for the mass production of a large number of spheres.

The second technique involves the joining of two wafers at the diffused etched-cavity stage of Figure 1.4-2 and then subjecting the wafers to the p-stop etch step to free the spheres. Both Shipley and Kodak photoresist have been used as the glue to hold the wafers together during the etching step. Best results thus far have been obtained using Kodak 747 resist. Figure 1.4-4 illustrates an SEM photograph of a sphere formed in this manner. The hemispheres shown in Figure 1.4-4 are on the

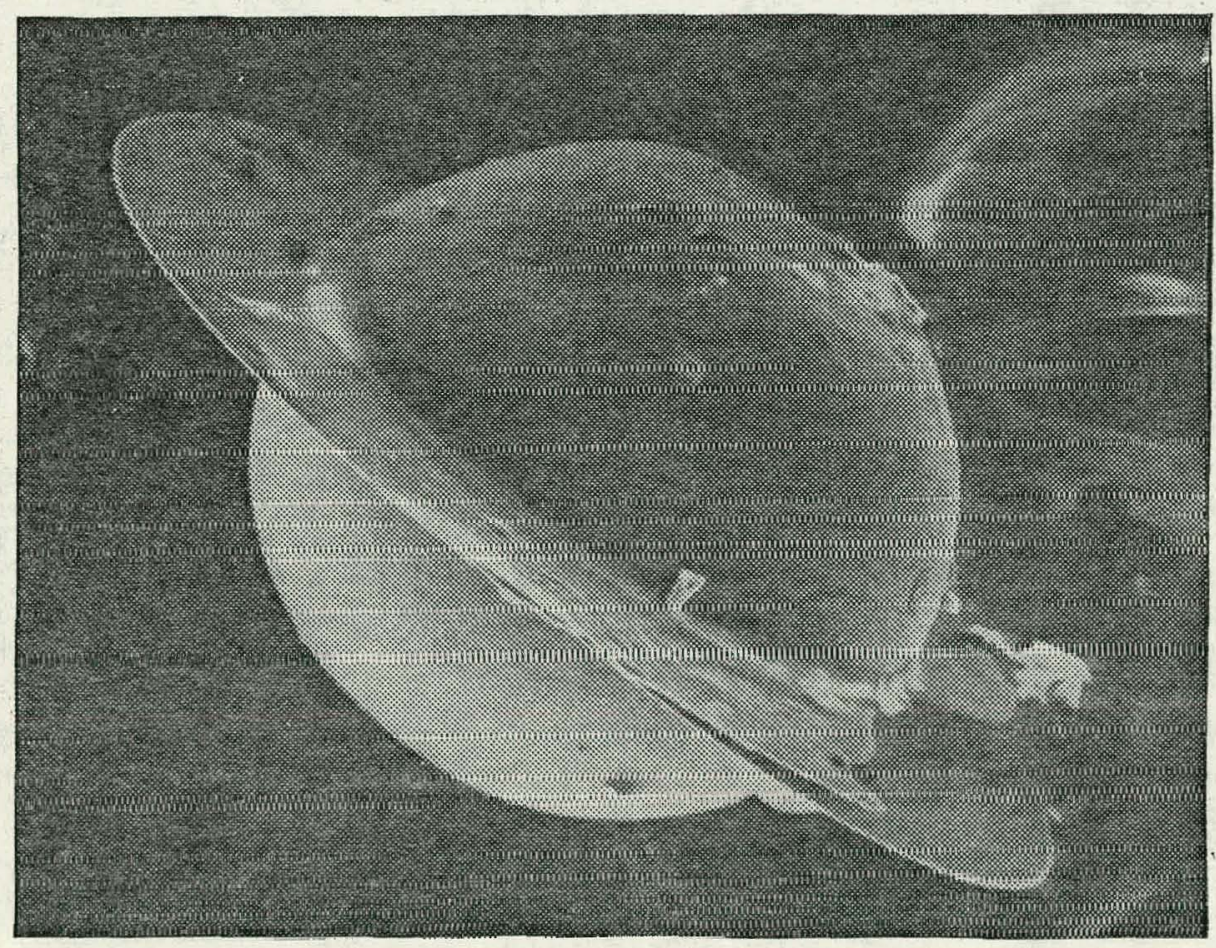

FIGURE 1.4-4 Two joined silicon flanged hemispheres. 
verge of separating due to the particular technique used in joining the wafers. The photoresist was spread uniformly over both wafers and then the two were aligned and joined together. After the silicon p-stop etch step there thus exists a matrix of flanged spheres embedded in the photoresist membrane. The spheres were then separated by immersing the membrane into a solution which dissolves photoresist. However, this step also results in softening the photoresist between the hemisphere flanges thus allowing them to separate. The problem can be alleviated by using circular photoresist patterns which are equal in diameter to the flanges. Thus, the spheres will automatically separate after the p-stop etch step and there will no longer be a need to subject the joined hemispheres to a final photoresist dissolution step.

Although the above alignment technique has been crude (manual alignment), it establishes the feasibility of aligning the cavity arrays of two separate wafers and then joining (gluing/bonding) and etching the wafers to achieve hollow spherical pellets. Although the alignment problem appears to be resolvable without undue difficulty, the bonding of two wafers may pose a more difficult challenge. Obviously, using photoresist as the bonding agent represents only a short-term solution and cannot be considered as the ultimate answer. Eventually better bonding techniques (e.g., electrostatic sealing) will have to be developed.

Recently, mask modifications have been made lo el Iminate the flange. Figure 1.4-5 illustrates the use of flangeless thin-wall hemispheres joined together to provide the outer shell of a double-shell target. The fiber mounted fuel pellet is 83 microns in diameter. 


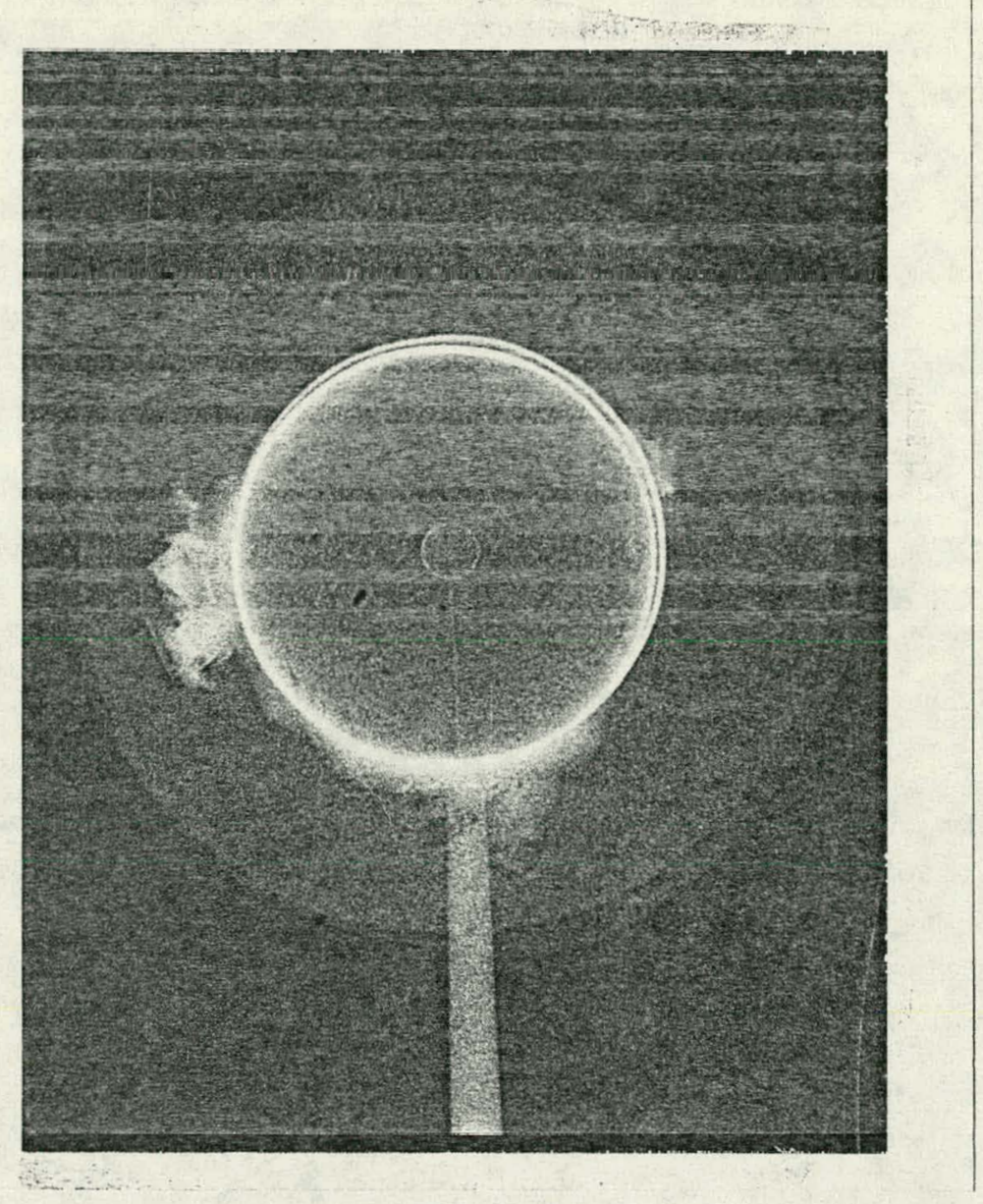

FIGURE 1.4-5 X-ray microradiograph of a double-shell fusion pellet.

$\operatorname{mins}_{\text {rusition }}$

Considerable effort has been expended in the evaluation of the various hemisphere fabrication techniques described above. Although the PMMA and metal hemisphere structures have also been investigated, most of the effort has involved the silicon structures. In particular, the SEM has been used to study wall thickness uniformity and the smoothness of the etched surfaces.

As described above, the net result of the silicon hemisphere fabrication process is the creation of numerous p-type hemispheres with wall thicknesses determined by the extent of the boron diffusion prior 
to the anisotropic p-stop etch step. The anisotropic etchant (ethylene diamine, pyrocatechol and water) is sensitive to the amount of boron in the silicon crystal. The etch rate drops nearly to zero when encountering boron levels exceeding approximately $7 \times 10^{19} \mathrm{~cm}^{-3}(\sim 0.1$ percent boron concentration within the silicon lattice). (3), (4) Thus, the etch first dissolves the $n$-type substrate until it reaches a p-type doping concentration of approximately $7 \times 10^{19} \mathrm{~cm}^{-3}$. This concentration is first encountered at the diffused region at the bottom of the cavity. The etchant then continues dissolving the n-type silicon around the B-doped hemisphere as it progressively moves toward the original silicon surface. Eventually the etch reaches the surface leaving only the ptype hemisphere as illustrated earlier in Figure 1.4-2.

Examination of the surface texture of the outer surface of a hemisphere revealed the flange edge to be rougher than the dome. The smoothness of the dome relative to that of the flange-hemisphere interface regions is understandable in view of the additional etching time which the dome receives compared to that of the flange. That is, since the radius of the hemisphere is approximately $200 \mu \mathrm{m}$ and the etch rate is approximately $0.3 \mu \mathrm{m} / \mathrm{min}$, the dome is exposed to the anisotropic etchant for considerably longer time than the interface region. At this point it is speculated that the smoother dome surface results from the p-stop etch slowly working away at the raised "hills" preferentially, thus smoothing off the surface. However, this can be verified only after doing a series of carefully controlled experiments in which the etching is stopped at varying times after first reaching the dome and investigating the surface roughmess of the dome at each step. 
It should also be noted that careful examination of the interior wall of the hemispheres has revealed a very smooth surface structure.

The hemisphere fabrication process described above shows promise as a technique for the mass production of spherical fusion pellets. With proper care, sphericity of the etched cavities, diameter uniformity and wall thickness uniformity appear to be quite adequate. Surface smoothness has shown considerable variation across the hemisphere. However, it appears that extended p-stop etching steps can be used to achieve surfaces which are quite smooth.

Wafer alignment does not appear to be a serious problem but considerable additional research will be required before adequate wafer-towafer sealing or bonding techniques can be achieved.

Future activities will be directed toward understanding the key fabrication process parameters which affect the uniformity and surface quality of the free standing hemispheres. The primary emphas is of the work will be to control the diameter, wall thickness and wall uniformity, to increase the wall thickness and to improve the surface quality of the she?ls.

1.4.2 Fabrication Techniques Development in Support of Laser and Diagnostic Requirements

In addition to the silicon and integrated circuit applications investigations, the ongoing target needs for the ICF program were satisfied. Thousands of Microshel ${ }^{\circledR}$ pellets were processed to yield suitable targets for experiments. Glass hemispheres were fabricated and mounted with and without coatings for layered target experiments using the Thompson Parabola. 
Preparation of targets for use in the cryogenic processing system included solid layer DT, solid layer neon, liquid layer DT and many configurations for laboratory investigations. Special large targets for large diameter/long pulse experiments were processed.

The fabrication of macroscopic bubbles ( 1 to $10 \mathrm{~mm}$ ) on capillary stems proved to be useful in several different areas of the NICFP. Glass bubbles were fabricated for the cast-reblow large shell fabrication technology area. Special large diameter glass bubbles with a liquid crystal. coating and DT-filled Microshel $1^{\circledR}$ pellets provided a diagnostic tool for absorption experiments.

Zone plate coded apertures were fabricated for target diagnostic analysis including the alpha particle measurements.

A microdrilling machine was used to fabricate quadrate pinhole ( 5 micron) arrays for the $X$-ray pinhole camera and various apertures for imaging devices.

Qua ? ity Assurance

Current and prospective pellet designs specify hollow spherical shells having layers of varying atomic number, density and thickness. To promote a symmetric implosion of the pellet upon-irradiation, the geometric qualities of the microstructure must be tightly controlled. Anticipated rates of use approaching millions of pellets per day will necessitate fully automated quality control. The cumbersome, labor intensive inspection methods presently employed must be replaced by simple, accurate techniques. which incorporate the automatic selection of acceptable pellets at high speed and with little or no operator assistance. Discussion follows of some of the novel approaches being 
developed in order to provide accurate quality assurance of present and future fusion pellets.

\section{Pellet Geometry Tolerances}

Pellet specifications are changing continuously, but some idea of the required specification tolerances can be inferred from the contemporary target design literature. Listed in Table 1.4-1 are our best estimates of spatial and topographical tolerances for the geometric qualities of a Microshel1 ${ }^{\circledR}$ pellet. Also listed are our current measuring capabilities and conceivable limits of capability using advanced automated techniques, some of which are described below.

TABLE $1: 4-1$

\section{TARGET SPECIFICATION TOLERANCES}

Feature

Outside Diameter Sphericity

Wall Thickness

Wa 11 Nonuniformity

surpace Finish

Localized Defects

( l umps, bumps, debris)
Requirements Now Proj (worst Case)
Measuring Capabilities Current Conceivable

$\begin{array}{rrrr}5.0 \% & ? & 2.0 \% & 0.1 \% \\ 5.0 \% & 0.3-0.5 \% & 5.0 \% & <2.0 \% \\ 3.0-5.0 \% & 0.4 \% & 2.0 \% & 0.3 \% \\ 3.0 \% & 1.0 \% & 5.0-7.0 \% & 0.2 \% \\ 100-200 \mathrm{~nm} & 3.10 \mathrm{~nm} & 50 \mathrm{~nm} & 1 \mathrm{~nm} \\ \text { none } & 0.3 \bar{W}<\text { La }<5 \bar{W} & 1 \mu \mathrm{m} & ? \\ & & & \\ & \text { La }=\text { defect 1ateral dimensions } \\ & \bar{W}=\text { average shell wall thickness }\end{array}$

\section{Diameter and Wall Thickness Mea surement}

We are developing a new mechanical method of diameter measurement which incorporates noncontact inspection and automatic selection at 
relatively high processing rates ( 10,000 pellets/hour). (5), (6)

The technique utilizes the wall effect phenomenon wherein the rise velocity of a buoyant object is attenuated by the close proximity of a surface or wall.

The final velocity of a Microshell ${ }^{\circledR}$ pellet rising through a fluidfilled tube will depend on the ratio of shell diameter to tube diameter, and the ratio of the shell's apparent density (a measure of its wall thickness) to the density of the fluid. If the pellet is electronically timed as it passes through two regions of differing tube diameter, we have a solvable system of two equations and two unknowns; namely, shell diameter and wall thickness.

Our studies. using lead shot of varying diameter and drilled nylon: balls of varying density have proven the sensitivity of wall effect terminal velocity attenuation and have shown agreement with theoretical calculations. Moreover, recent buoyancy tests on well characterized KMSF Microshel1 ${ }^{(\mathcal{B}}$ pellets show remarkably accurate correlation. Development is underway of a fully automatic device based on this technique, using microprocessor technology to characterize batches of pellets and select those of proper diameter and wall thickness.

\section{Wall Nonun iformity Mèasurement.}

An inclined plane sorting scheme has been developed which simply and quickly classifies Microshel $1^{\circledR}$ pellets for wall nonuniformity. (7), $(8),(9),(10)$ The technique takes advantage of the behavior of spherical shells rolling down an inclined plane. Those shells with uniform walls tend to roll straight down the plane while those with wall nonuniformities veer off a straight course. (See Figure 1.4-6) 


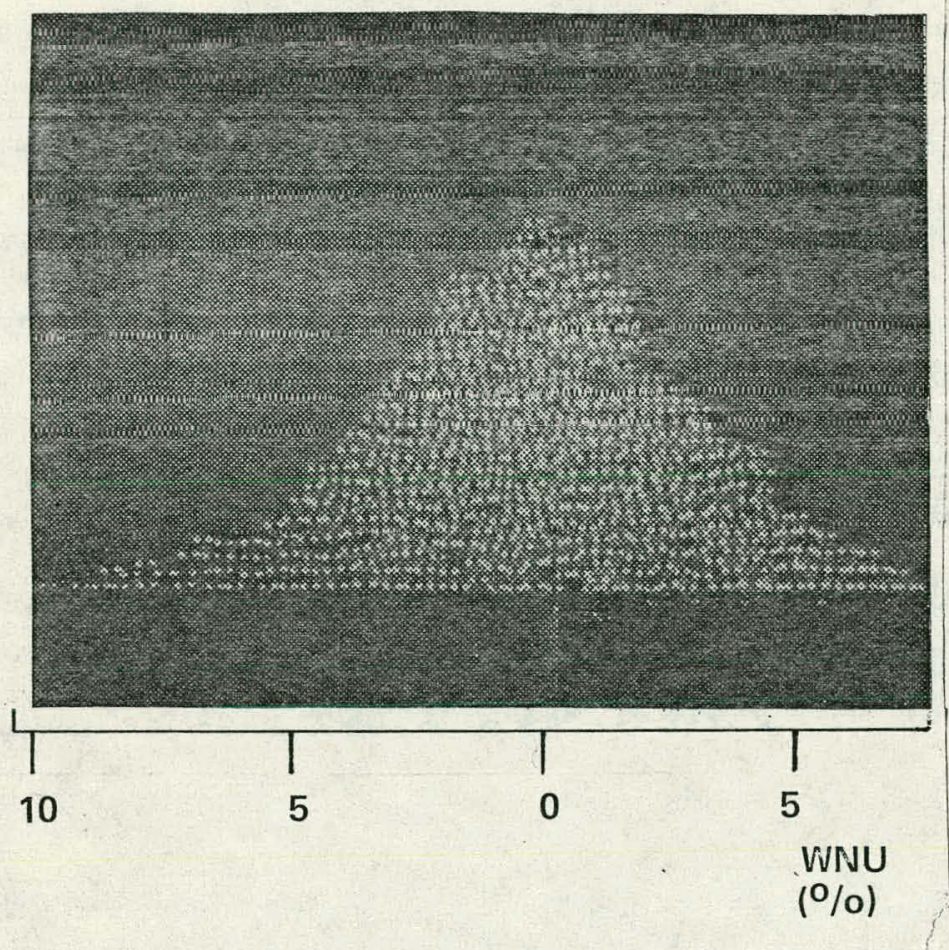

$\begin{array}{ll}\text { FIGURE } 1.4-6 & \text { Roll sorted KMSF Microshe } 11^{\circledR} \\ \text { pellets (batch T2R } 11 \text { ). }\end{array}$

The inclined plane technique also automatically spatially separates the specimens without the need for decision making electronics. Work is in progress to develop a smoother, conductive inclined plane surface which will decrease the minimum shel1 size which can be characterized by the rolling method. The method is currently limited to shells with diameters $\geq 200 \mu \mathrm{m}$.

Sphericity Measurement and the Detection of Localized Defects

Figure 1.4-7 shows interferograms of two KMSF Microshel1 ${ }^{\circledR}$ pellets. Irregularities in sphericity and local defects are delineated by changes in fringe spacing, breadth and contrast, or by the addition of new fringes. 


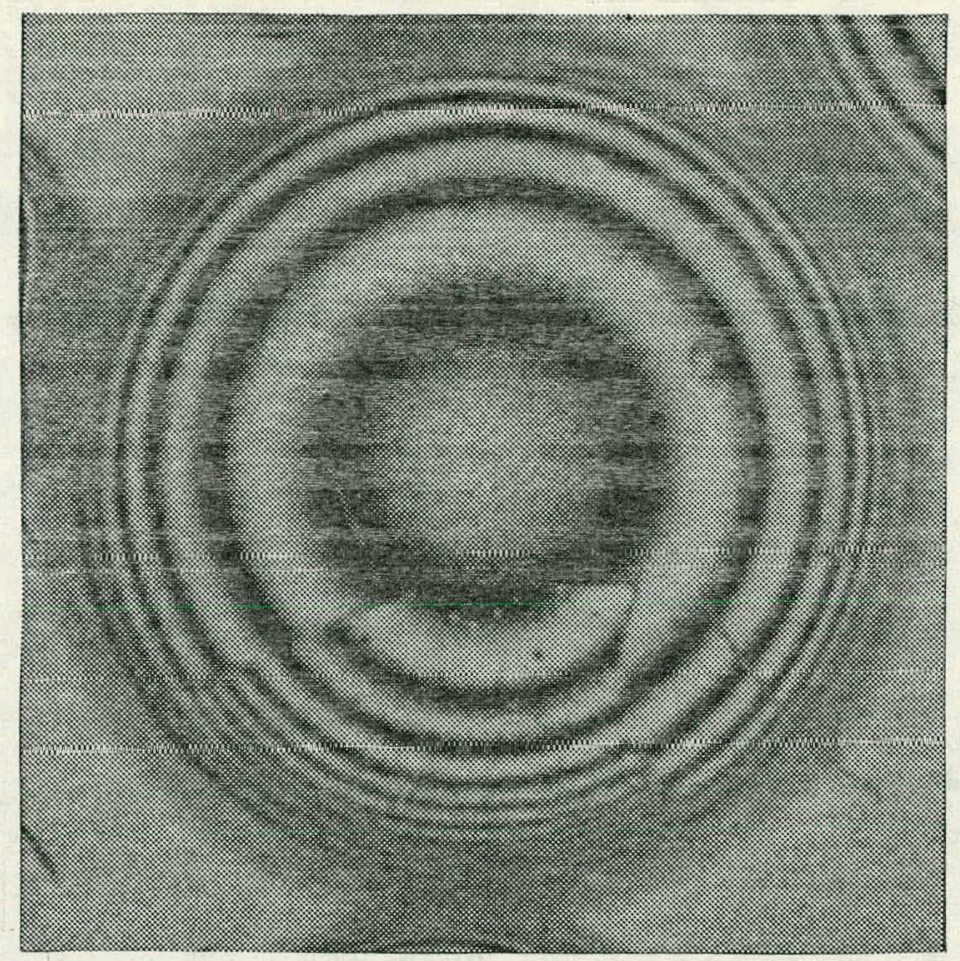

(a) $500 \mu \mathrm{m}$ O.D. ROLL SORTED 'POOR'

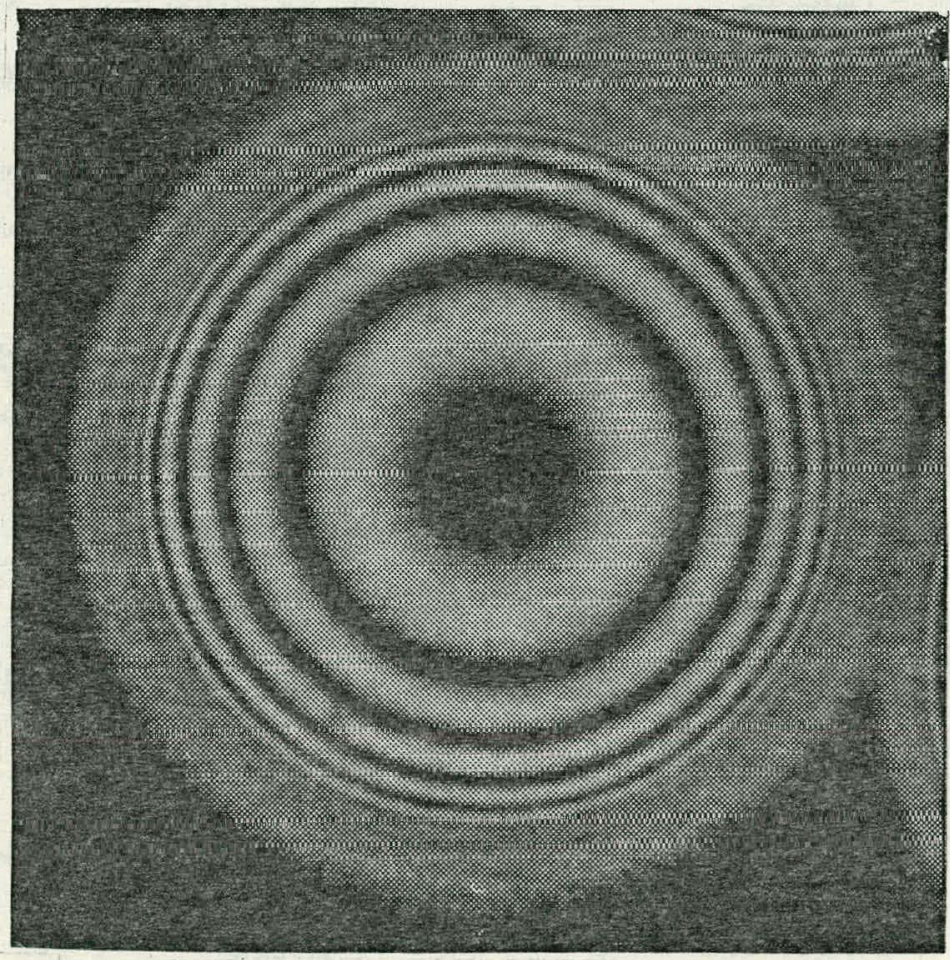

(b) $350 \mu \mathrm{m}$ O.D. ROLL SORTED 'GOOD'

FIGURE 1.4-7 Interferogram of KMSF Microshe11 pellets batch T2R70. 
Evaluation of these interferograms is both tedious and timeconsuming, even using digital processing. However, reactor grade pellets may not require specific characterization for quality assurance. At KMSF, we are investigating the use of a radial cross section interference fringe 'signature' of a perfect sphere as a reference for comparison with each pellet. In an industrial application an image dissecting vidicon would continuously monitor the radial fringe signature of each pellet during its passage under the camera and send the signal to a microprocessor for comparison with the recorded signature of a perfect sphere. Alternatively, holographic interferometry could be used to compare the moving pellet with the reference. Here, a holographic image of a perfect sphere is projected onto the surface of a (similarly illuminated) rotating pellet. Exceptionally bright interference effects occur at points of dissimilarity between the overlapping images. This process is becoming commercially available, but currently only for use on much larger objects.

\section{Surface Roughness Measurement}

Several years ago, fusion target theorists suggested that the physics of shell implosion stability required surface finishes smooth to one $\mathrm{nm}$. Later target designs have retreated from this requirement, but insufficient experimental data still leave wide open the question of maximum allowable surface texture. While KMSF Microshel1 ${ }^{\circledR}$ pellets have been made with surface finish smooth to the 1 imits of SEM examination $(25 \mathrm{~nm})$, one can imagine the fabrication problems associated with the joining of multishell hemispheres to a tolerance of one $\mathrm{nm}$.

Fourier transform surface analysis holds the most promise for the difficult task of automated surface finish quality assurance at 
nanometer resolution. It is a particular quality of the transform that small scale regular structure transforms onto large scale optical contrast. We can monitor the Fourier transform of the sphere's image at the focal plane of a simple lens. A scanning photometer and digital processing can compare the Fourier transform signal with that of a reference 'signature' for high speed inspection.

The viability of this technique has been proven in the recent scientific literature relating laser speckle (a Fourier transform phenomenon) to fine surface texture. (11)

Once pellet fabrication processes reach high degrees of reliability, statistical sampling inspection methods may be used. Military Sampling Standards (Mil. Stds. 105D) suggest that for 99\% assured good quality only 200 pellets out of every 10,000 need be inspected. In fact, General Electric's blow molding of glass light bulbs is controlled by a sampling inspection of only 5 out of every 75,000 bulbs, thereby yielding a consistently good quality glass bulb.

We have performed an extensive survey of existing and potential measuring devices. (12) Based on this review we feel confident that a mobile sized quality assurance instrument can be constructed within ten years of development startup. This instrument would be capable of inspecting 10,000 pellets per hour for al1 six geometric features currently of interest. For minimum development costs some pellet specifications must be known at startup, with most of the details required five years hence. A minimum knowledge of the pellet configuration would require parallel development of several inspection techniques, with accompanying higher costs. 


\subsubsection{Suspension}

The engineering prototype pellet inertial suspension apparatus(13) was installed and successfully tested in the target chamber. Two neutron producing target shots were performed utilizing ful1 laser system irradiation. The neutron yields were $1.1 \times 10^{5}$ and $3.1 \times 10^{5}$ respectively before testing was suspended. Figure 1.4-8 is a double-exposed holographic photograph showing:

1) the $3.1 \times 10^{5}$ neutron producing pellet on its supporting pedestal; and,

2) the plasma surrounding the pellet approximately 600 picoseconds before and after arrival of the main pulse train.

For comparative purposes a similar holographic photograph of an irradiated fiber mounted target is shown in Figure 1.4-9. A qualitative comparison of the two holograms reveals a substantial plasma nonuniformity in the region where the fiber and spherical pellet join. The plasma shown in Figure 1.4-8 reveals no such nonuniformity.

\section{Description of Pellet Inertial System}

The pellet inertial suspension system, proved in the simulation chamber, was adapted to the target chamber with only minor mechanical adjustments. The mechanical system is shown schematically in Figure 1.4-10. The Pellet Loading System

The pellet loading system was comprised of a vertical $350 \mathrm{\mu m}$ diameter bore capillary tube approximately $63 \mathrm{~cm}$ long which extended into the center of the taryel chamber. The target chamber was maintained under vacuum and air was allowed to leak through the capillary tube. Characterized spherical glass Microshe $17^{\circledR}$ pellets were introduced to the capillary tube by a microsyringe with an expulsion plunger. The 


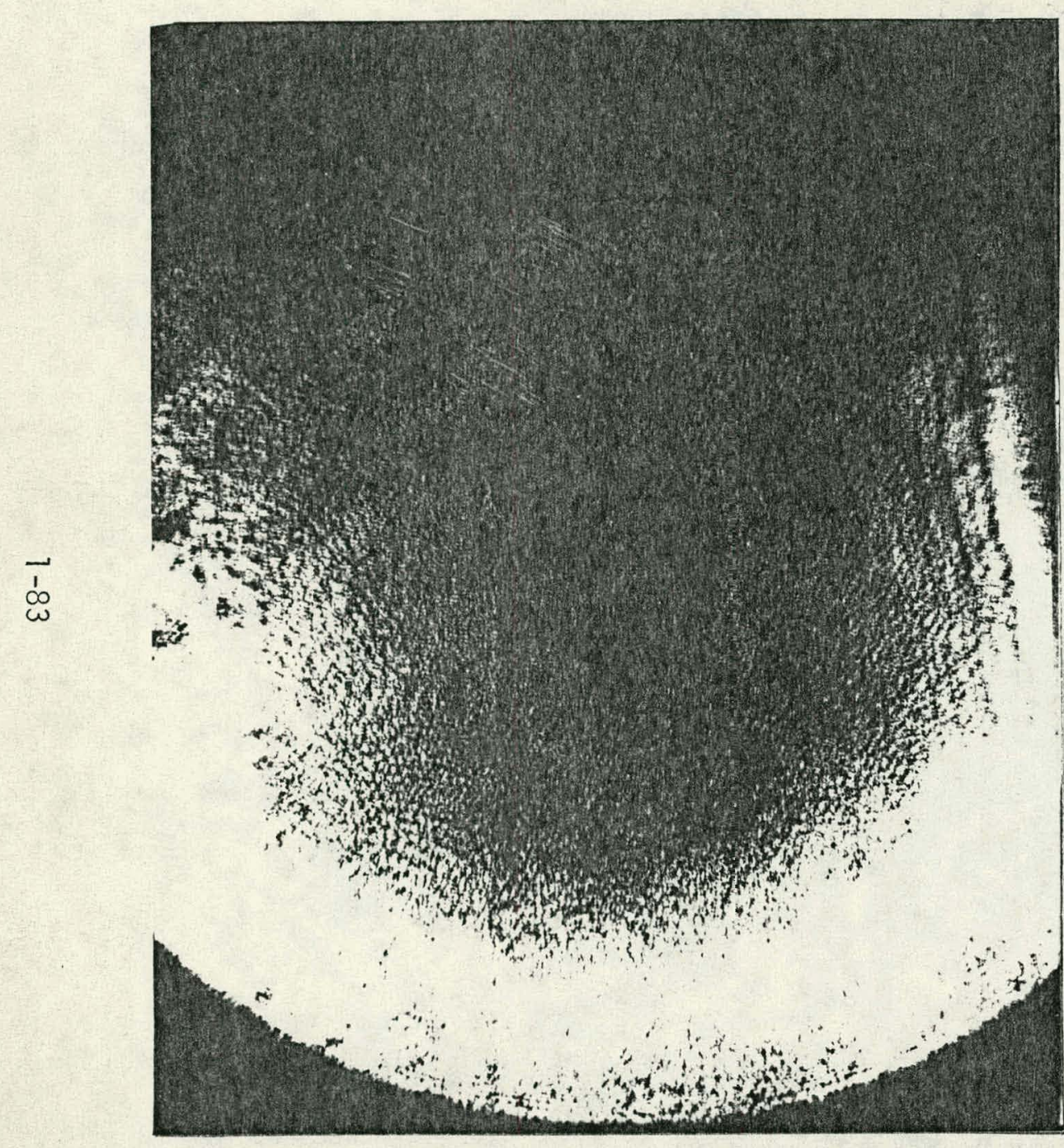

FIGURE 1.4-8 Suspended pellet neutron producing hologram.

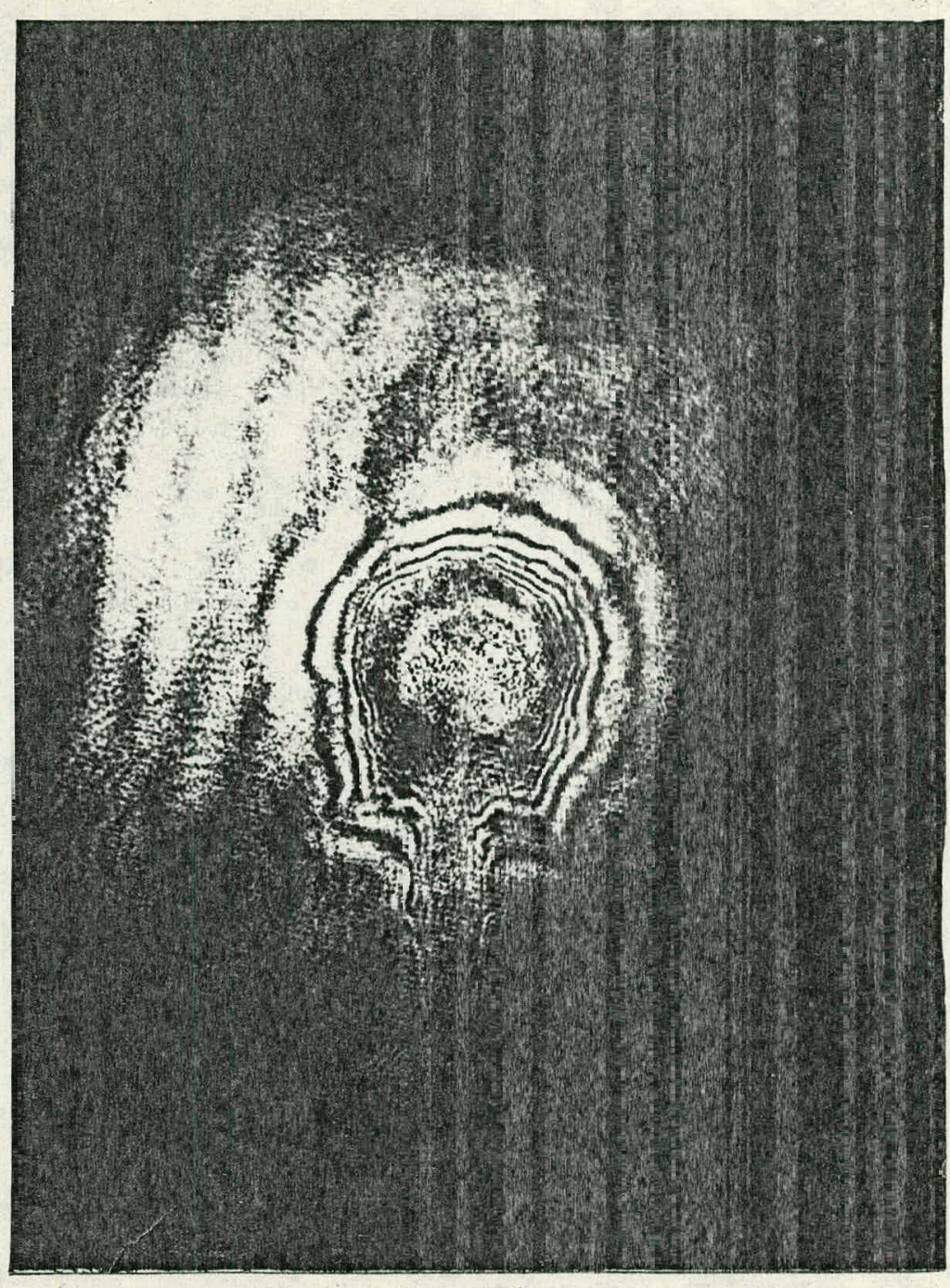

FIGURE 1.4-9 Post-mounted hologram.

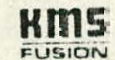




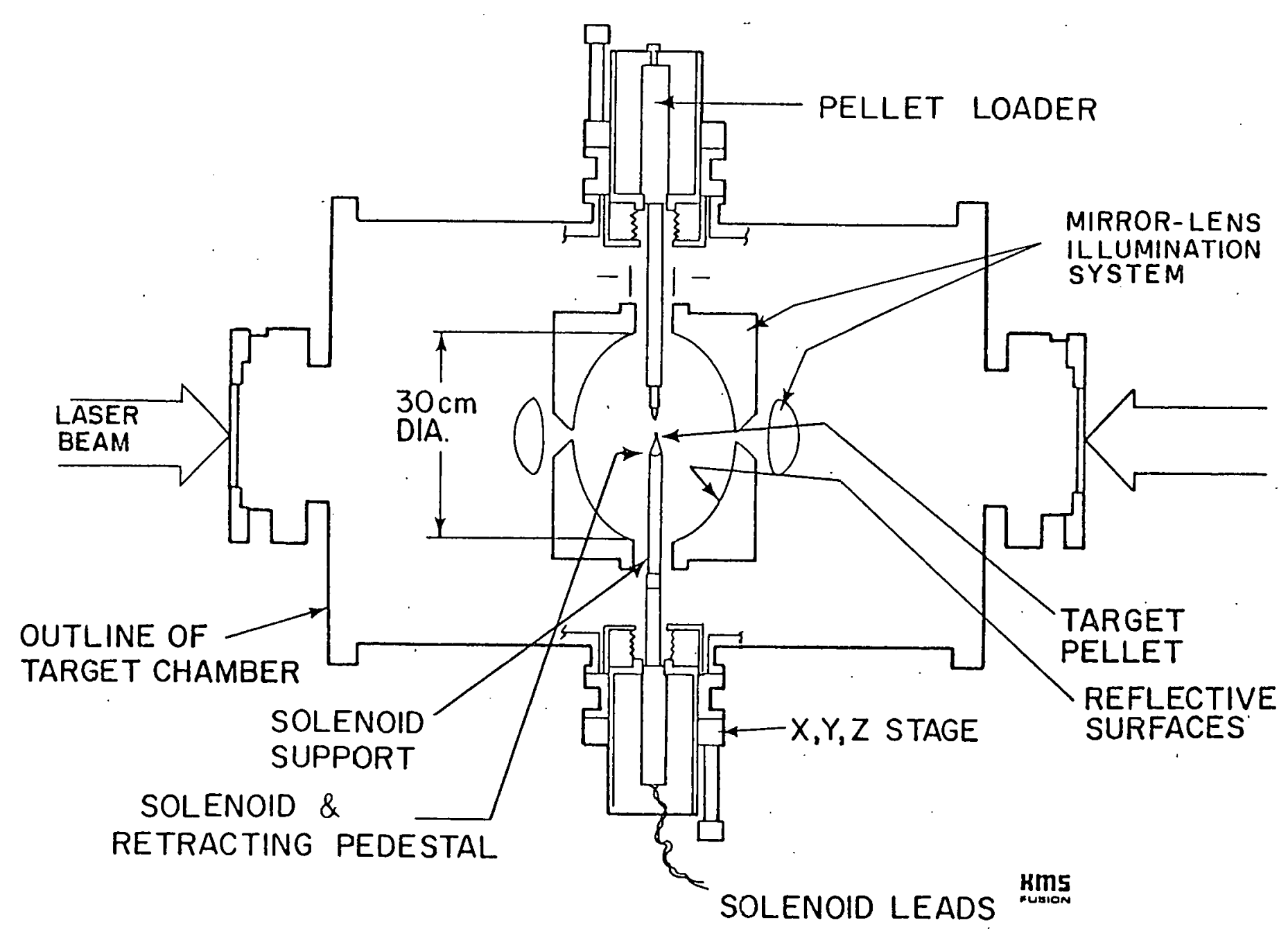

FIGUF:E 1.4-10 Suspension system integrated in the laser fusion target chamber. 
pellet was carried by the airflow into the target chamber. The pellet was prevented from leaving the capillary tube by a truncated conical graphite tip on the retracting pedestal assembly (Figure 1.4-11). Transfer and positioning of the pellet on the pedestal was accomplished by $x, y, z$ manipulation of the pellet at the tip of the capillary tube.

\section{The Support Pedestal}

Figure 1.4-12 shows schematically a cross sectional view of the mechanism used to retract the support pedestal from beneath the pellet. The mechanism features a two stage spring assembly to index the pedestal and solenoid slug against mechanical stops. The solenoid slug was designed with a hollow cage to allow contact with the support pedestal over an adjustable displacement range of $0-6 \mathrm{~mm}$. A momentum transfer between the solenoid slug and support pedestal occurs at the point of contact between them along the solenoid slug displacement path. The required velocity imparted to the support pedestal is that needed to overcome the forces coupling the fuel pellet to the support pedestal, while imparting an acceptably low initial velocity, $V_{0}$, to the pellet. Ideally, $V_{0}$ should equal zero, thereby placing the fuel pellet in free fall. Dwell times of the pellet vs. free fall distance is shown in Figure 1.4-13. Part of the mechanical design of the pedestal retraction mechanism was fixed by assuming a 30um diameter as the focal zone tolerance band. Thus defined, the center of an inertially suspended spherical pellet will be coaxial with the irradiating laser pulse(s) $\pm 15 \mu \mathrm{m}$ orthogonal to the beam axis. A beam/pellet coaxial tolerance zone of 50um was defined as an acceptable goal. A $2000 \mu \mathrm{m}$ pellet/pedestal separation at irradiation was assumed as the minimum desired pellet pedestal separation at irradiance. 


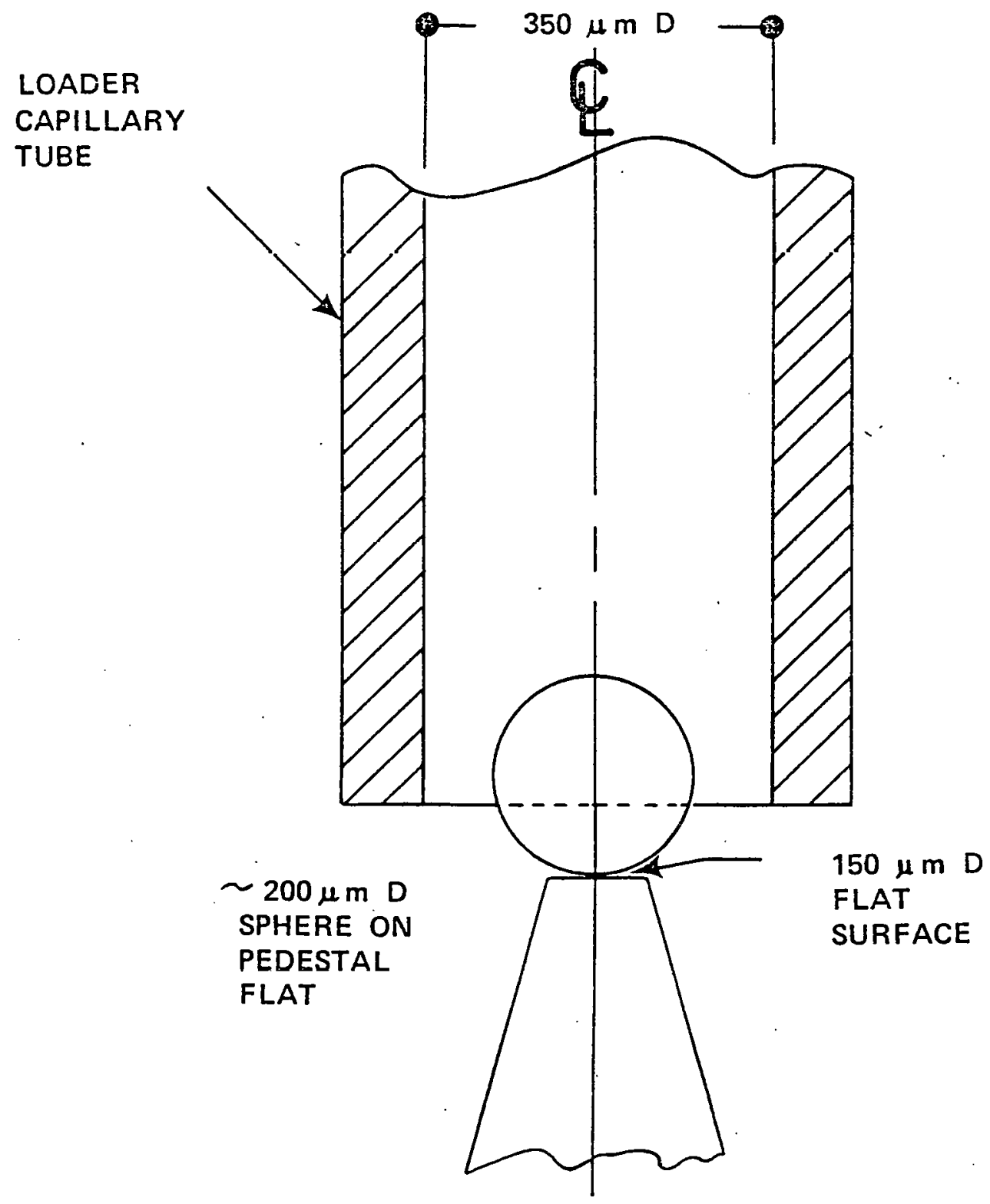

FIGURE 1.4-11 Section of capillary tubé pellet loader.

$\mathrm{hms}$ 


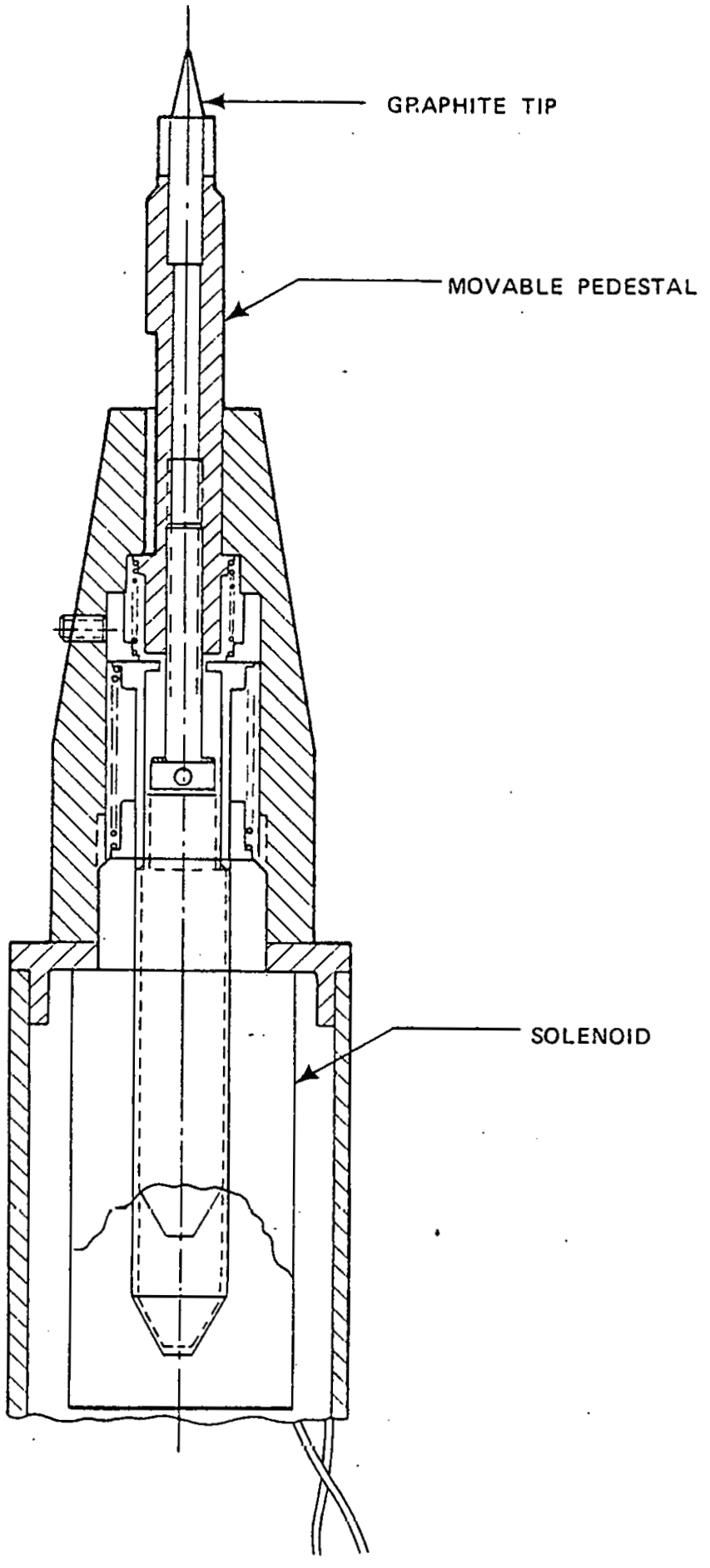

FIGURE 1.4-12 Section view of inertial platform device. 


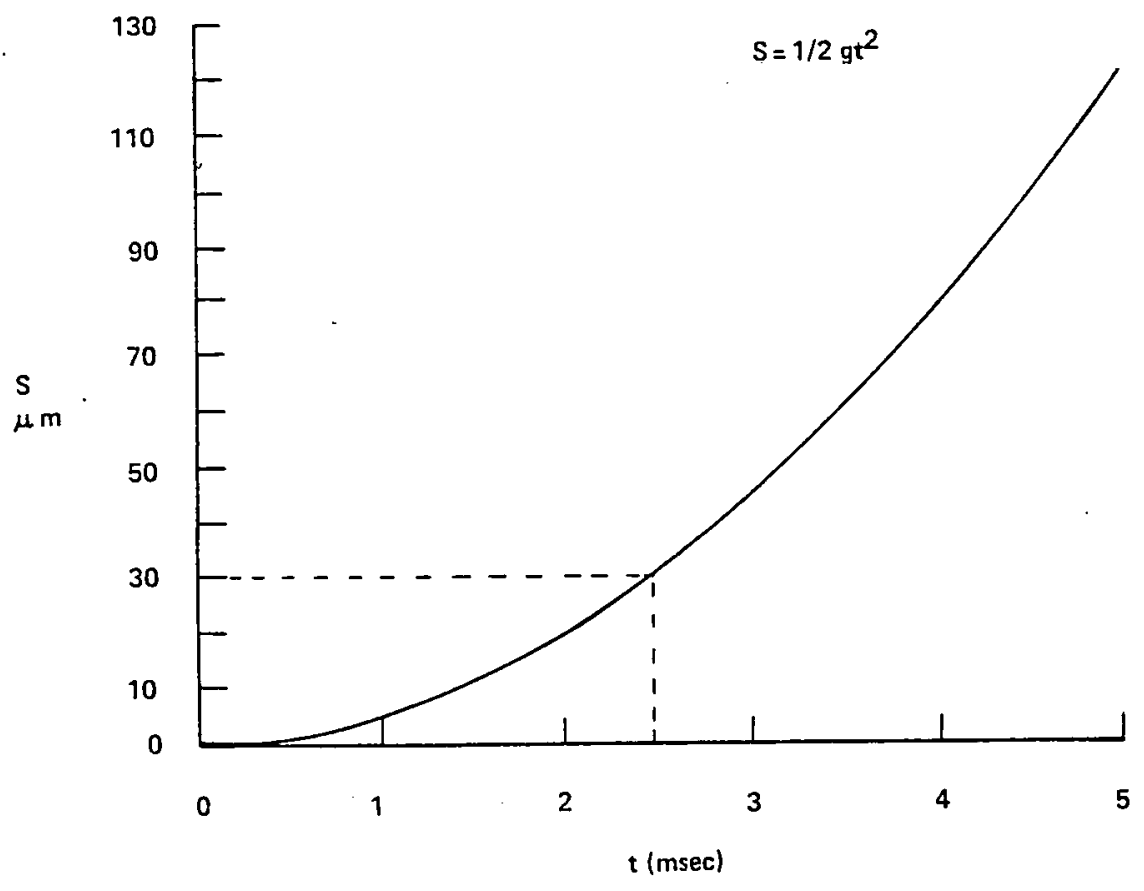

FIGURE 1.4-13 Free fall displacement vs, time plot.

Assuming a $30 \mu \mathrm{m}$ diameter tolerance zone and a pellet in free fall with zero initial velocity, the permissible timing window for pellet irradiation is $\sim 2.47 \mathrm{~ms}$ (Figure $1.4-13$ ). The average velocity of the pedestal under the desired separation conditions is $80 \mathrm{~cm} / \mathrm{sec}$.

\section{Timing and Control}

A series of tests on the engineering prototype unit in the simulation chamber revealed that the pedestal motion performed repeatably within 100 microseconds. This repeatability represented $4 \%$ of the allowable dwell time of $2.5 \mathrm{msec}$ and was deemed acceptable.

A schematic of the timing and control units used to synchronize the laser, solenoid and some diagnostics is shown in Figure 1.4-14. The low 


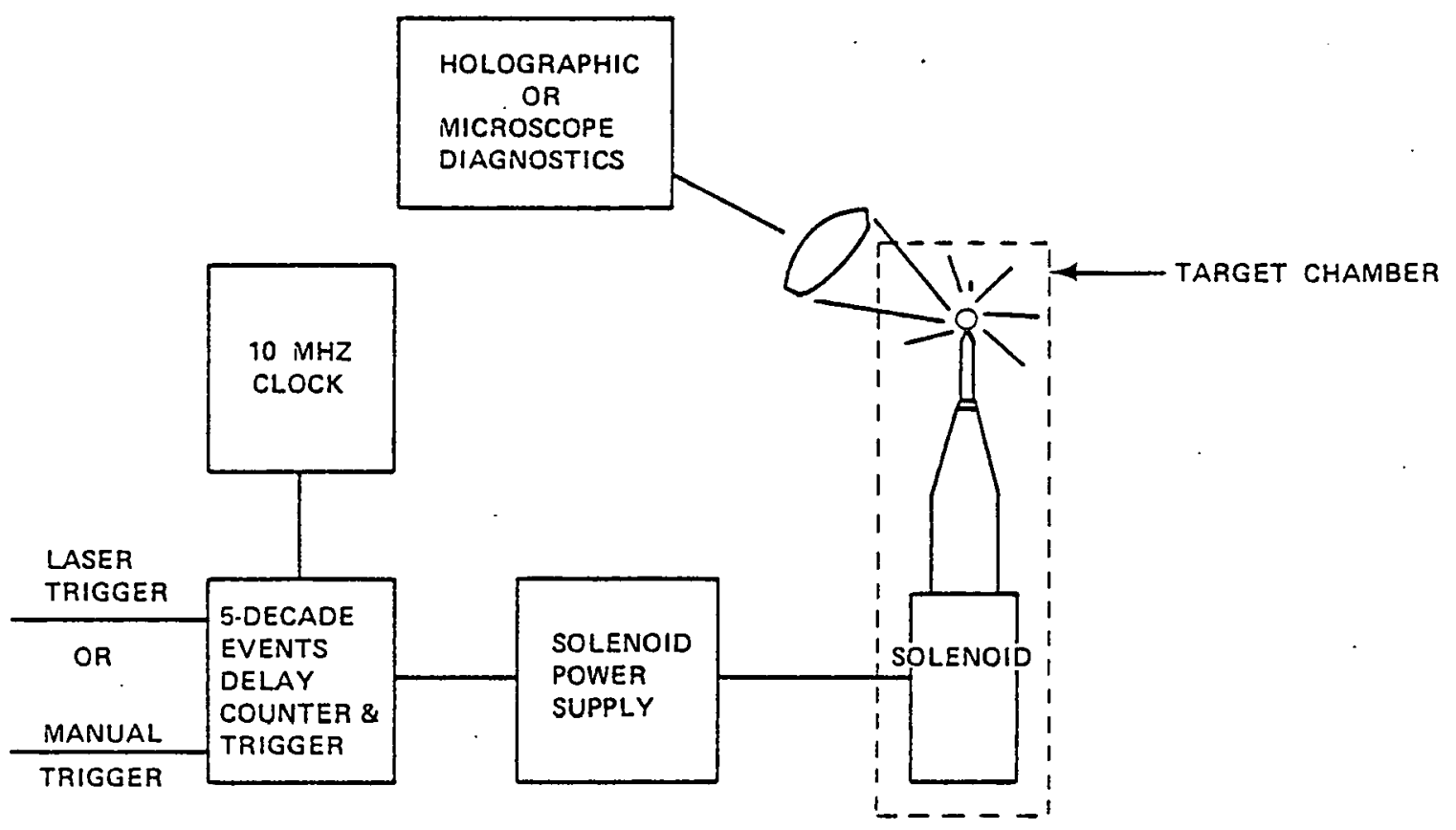

a) TARGET CHAMBER CONFIGURATION

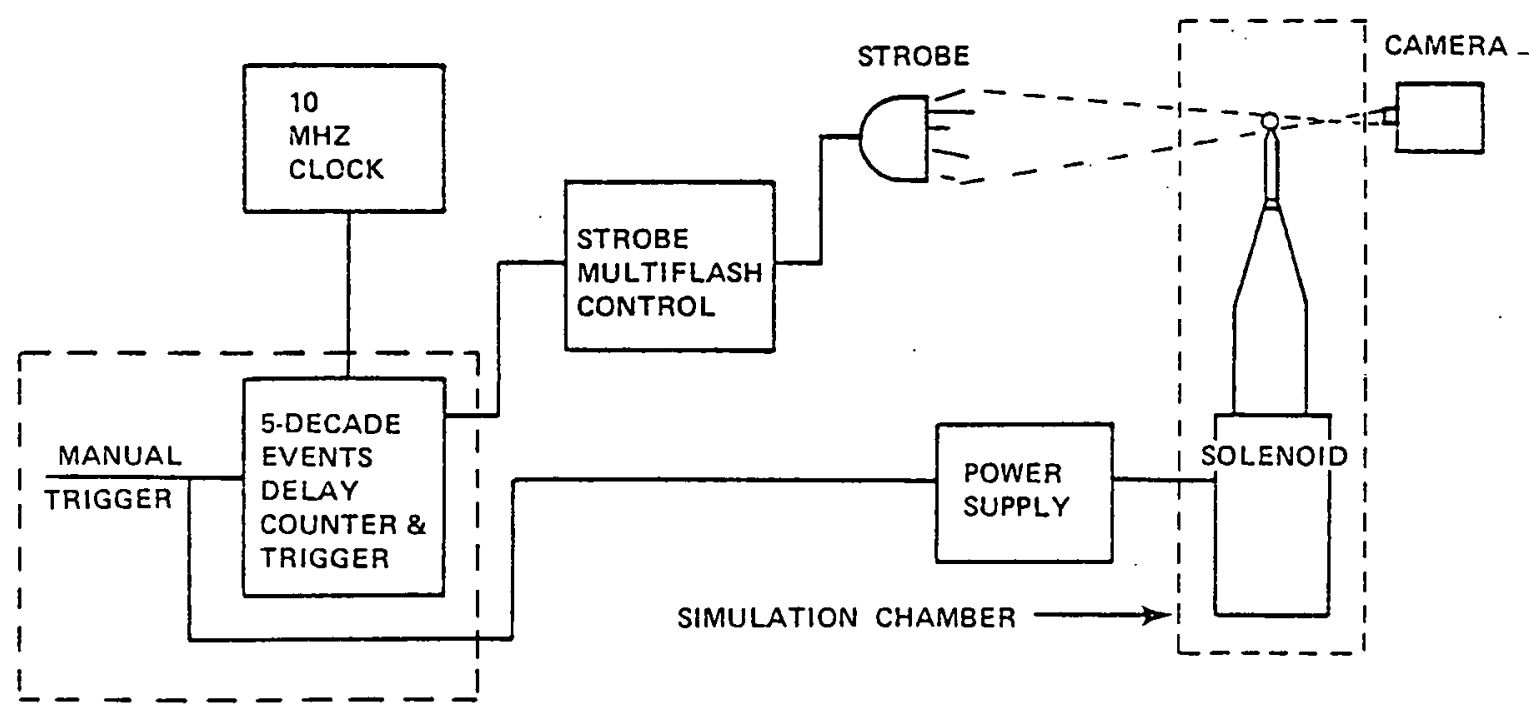

b) SIMULATION CHAMBER CONFIGURATION

FIGURE 1.4-14 Suspension system instrumentation and controls. 
power laser was used for the timing and calibration which synchronized the arrival of the main pulse laser with the retraction of the pedestal.

Separation of the shell and pedestal was sufficiently repeatable to achieve synchronization by adjusting the events timing delay alone.

Figure 1.4-15 shows the timing pulse sequence determined to achieve synchronism.

\section{Test Results}

The inertial suspension timing and control systems performed without any malfunction throughout the target chamber testing. We encountered some pellet handling difficulty with pellets less than 150 um diameter. because of geometrical clearances which existed between the the capillary tube and the graphite tip. Mechanical manipulations of the graphite tip during pellet positioning and transfer caused the loss of the smaller pellets. As a result, target shots on larger than optimum targets (160 $\mu \mathrm{m}$ diameter) were necessary. Neutrons were achieved on two of the large targets as reported above.

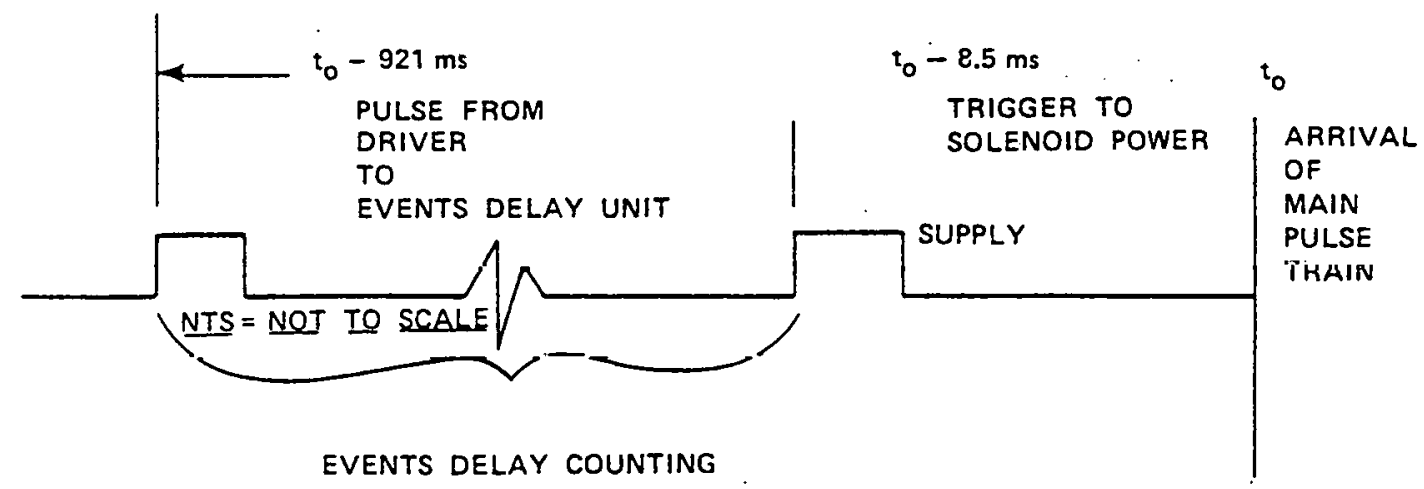

FIGURE 1.4-15 Laser suspension system pulse time control schematic. 


\section{Summary and Recommendations}

Inertial suspension of DT filled $160 \mu$ m diameter glass Microshe $11^{\circledR}$ pellets was accomplished. The suspension and timing sub-systems performed without flaw. Minor modifications to the pellet loading system will eliminate the existing geometric clearance problem. Further testing with smaller targets, siliilar sized targets or larger targets is possible at any time experiment priorities permit.

\subsubsection{Injection}

Our studies have addressed the injection of hollow glass spherical fuel pellets into a reactor.

We have assumed that the injection rate will range from one to fifty per second and the pellets will be two to five $\mathrm{mm}$ in diameter.

To remain oriented to our task, we made a heuristic analysis of some reactor injection conditions. We have assumed that the hostile atmosphere (high temperature $\sim 2000^{\circ} \mathrm{C}$, ion species, etc.) in a reactor precludes structures to guide the pellet into the focal zone of the reactor. Injection in present reactor concepts (e.g. Solase) requires typical ballistic path lengths of three to six meters outside of the injector. The assumption that structures will not be present in the reactor environment preciudes pellet trajectory sensing and correction in the reactor chamber. Under these assumptions, irradiation of a pellet in space and time coincidence requires accurate and precise orientation of the injector and irradiation source; or aiming of the irradiation source to irradiate the fuel pellet in a variable position space-time system.

In either of the above cases, any of several pellet injection mechanisms (see Table 1.4-2) are candidates for accelerating the pellet. The selection of a suitable injector mechanism is therefore primarily based on what driver effectively provides suitable pellet velocity and acceleration. 
TABLE $1.4-2$

CANDIDATE PELLET INJECTION SYSTEMS

\begin{tabular}{|c|c|c|}
\hline System & Advantages & Disadvantages \\
\hline Electrostatic & Field effect & $\begin{array}{l}\text { Undeveloped technology } \\
\text { Long charge time } \\
\text { Precise Q/M required } \\
\text { Path sensing } \\
\text { Vacuum } \\
\text { Charge destroys pellets }\end{array}$ \\
\hline Magnetic & Field effect & $\begin{array}{l}\text { Undeveloped technology } \\
\text { Magnetic materials required } \\
\text { on pellet } \\
\text { High currents, fast response }\end{array}$ \\
\hline Rail gun & $\begin{array}{l}\text { Electronic current } \\
\text { device }\end{array}$ & $\begin{array}{l}\text { Requires consumable carrier } \\
\text { Undeveloped technology } \\
\text { Vacuum }\end{array}$ \\
\hline Pneumatic & $\begin{array}{l}\text { Technology developed } \\
\text { (guns, loaders, etc.) }\end{array}$ & $\begin{array}{l}\text { Trajectory correction or } \\
\text { laser aiming required }\end{array}$ \\
\hline
\end{tabular}

As a result of our analysis of Table 1.4-2 we conclude that we would make an in depth study of pellet injection using pressurized gas as being the optimum system for a first analysis.

Studies of Pellet Injection by Means of Pressurized Gas

Pressurized Gas Pellet Injector. One of the candidate systems for injection of pellets into a fusion reactor is that involving a pressurized gas to propel the pellet through a close-fitting tube. Such an apparatus is shown schematically in the lower part of Figure 1.4-16. It was intuitively felt that this system would be capable of producing pellet velocities of the order of the speed of sound in the propellant gas. Since that speed is approximately 1280 meters per second for hydrogen gas at room temperature, with higher values at higher temperatures, the pressurized gas system seemed to offer the possibility of meeting requirements for a pellet injector in a large-scale fusion reactor. Accordingly, a study was initiated to determine the performance of the system. 


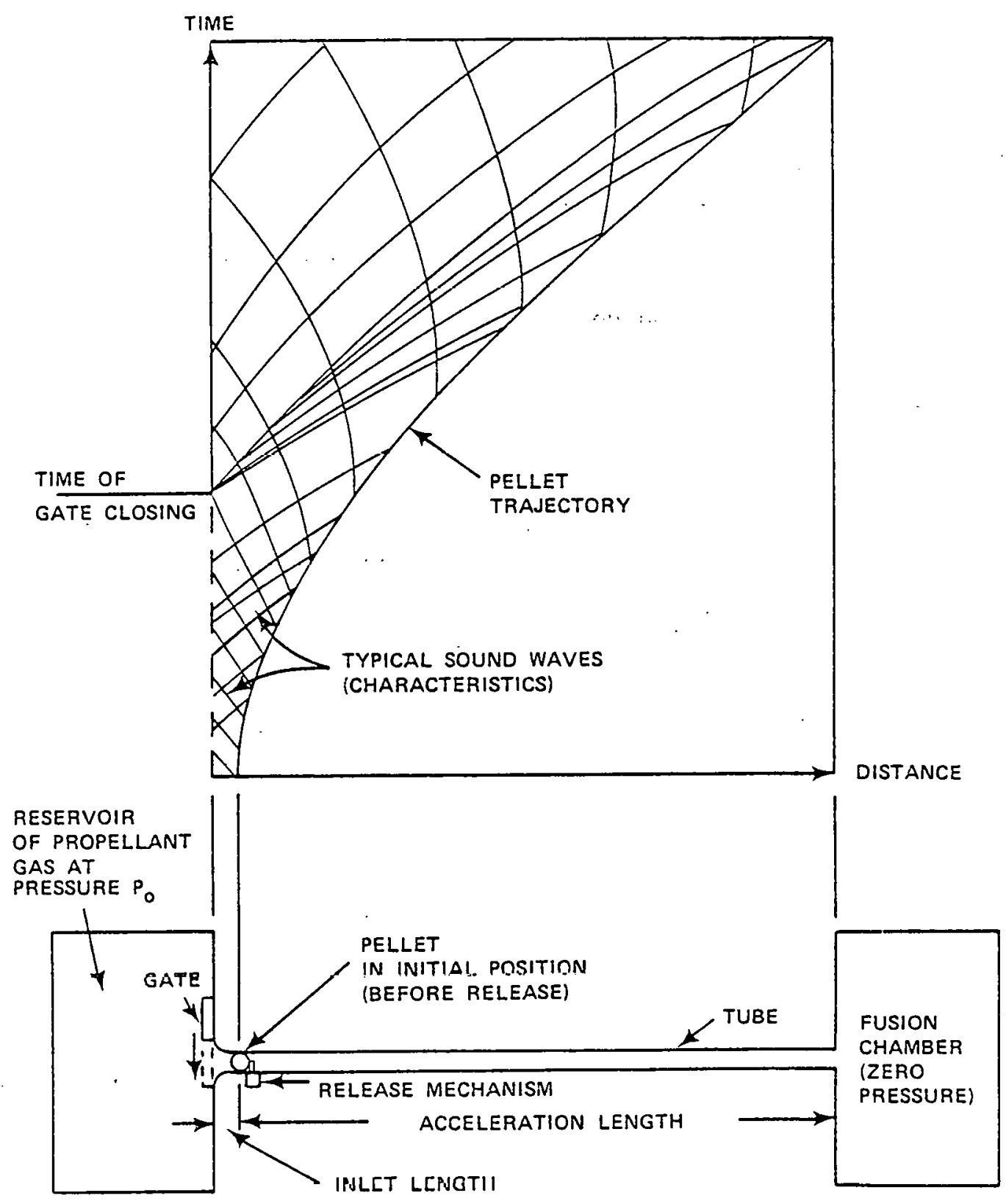

FIGURE 1.4-16 Schematic of pressurized-gas pellet injector with typical wave diagram.

Kms 
Theoretical Limits. A search of the literature revealed that the pellet speed need not be limited to the speed of sound. While the sonic speed would indeed be the rimiting speed if the pellet were entrained in a steady gas flow through a constant diameter tube, when the pellet acts as a piston in a nonsteady expansion into a vacuum the limit of its speed is actually five times the speed of sound in the pressurized gas reservoir, assuming a diatomic gas. (13) Thus, room-temperature hydrogen gas gives the highest possible potential pellet velocity of 640 meters per second, in an infinitely long tube because of its light weight per unit volume. In propelling a piston, it gives higher piston velocities because less energy is needed for accelerating the gas and therefore more energy is available for piston acceleration. Therefore, hydrogen gives highest performance in light-gas guns and gun tunnels. (13)

Theoretical Solutions. The movenent of a piston in a constant diameter tube under the influence of gas pressure can be understood by the characteristics theory of one-dimensional unsteady flow. (14) This theory develops equations for the variation of flow quantities along two families of characteristic curves, which are the traces of upstream and downstream... moving sound waves in a time-distance plot (as in Figure 1.4-16). When the inlet length is so long that waves reflected back from the inlet do not reach the pellet in the time period under consideration, the characteristic equations can be integrated anlaytically. (13) The resulting equation shows that, for example, a pellet velocity of 200 meters per second is produced in an acceleration length of 3.3 meters, when the propellant gas is hydrogen at room temperature and 10 atmospheres pressure. With an inlet length of 1.5 meters or longer, so that reflected waves do not reach the pellet before the end of the tube, experiments have shown reasonable agreement with the analytical solution. (15) 
The analytical solution for a long inlet case was of some interest, but it did not satisfy the need for a solution to the short inlet case, which would be of much greater practical use. Not only is a long inlet (i.e., greater than $10 \mathrm{~cm}$ ) awkward, occupying valuable space, but it reduces the pellet velocity that can be reached in a given acceleration length, by delaying the flow of propellant gas toward the pellet. Thus, it was decided to seek a solution to the short inlet case (i.e., less than $1 \mathrm{~cm}$ ), even though it was realized that much labor would be involved.

In the short inlet case, no analytic solution is possible. Instead, the characteristic equations must be integrated numerically by construction of a wave diagram, which traces the two families of waves in a time-distance plot, as in Figure 1.4-16.

To do the numerical integration, the relevant equations were first converted into a nondimensional form. A hand calculation was then performed for a nondimensional inlet length ratio of 0.1 . This calculation combined graphical construction of the wave diagram (see Figure 1.4-17) with numerical calculation of the flow variables at each wave intersection. The wave diagram contained thirty-five intersections. Each point on the pellet trajectory required an iteration between the graphical construction and a numerical calculation based on integration of second-order Lagrangian interpolated pressures.

The results of the hand calculation showed that, for the short inlet length ratio of 0.1 , an acceleration length of two meters would produce a pellet velocity of 200 meters per second, whereas 3.3 meters were required with the long inlet. This encouraging result justified further calculations for even shorter inlets, this time using a high-speed digital computer. The computer program was designed to handle both open and closed inlets, as well 
INLET .1

CLOSING $i$.

$N=10$

4

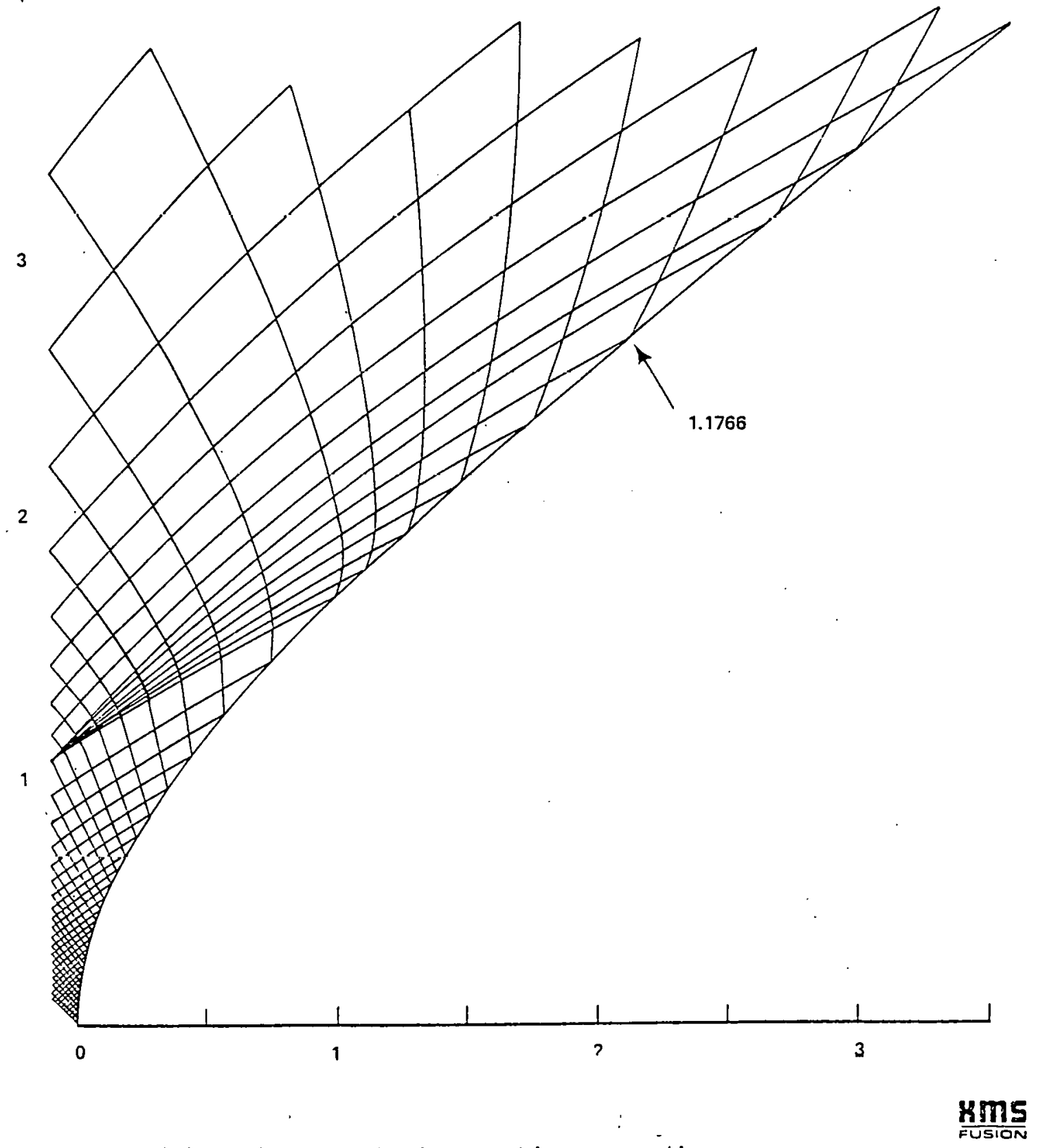

FIGURE 1.4-17 Characteristic equation wave diagram. 
as the mixed mode case where the inlet gate is closed while the pellet is in motion. This latter case is illustrated in the upper part of figure 1.4-16. Note that, in this particular case, the gate is closed when the pellet has traveled about one-quarter of the tube length, but the pellet trajectory is not affected until the midpoint is reached. Closing the gate reduces the amount of propellant gas that flows into the vacuum chamber, while also reducing the pellet acceleration beyond the midpoint. Results of the computer program are presented in Figure 1.4-18 to show how variation of the time interval between pellet release and inlet gate closing affects both pellet velocity and the mass of propellant gas injected into the vacuum chamber. Prompt closing of the inlet can minimize the mass of gas admitted, but the final pellet velocity can be quite sensitive to errors in timing.

Another presentation of the computer results is shown in Figure 1.4-19, for a short, open inlet of length ratio of 0.02 . The temperature of the propellant gas has a large effect on pellet velocity in the 2000 meter per second range, but not in the below 1000 meter per second range. In summary, pressurized gas pellet injection seems to have the potential for producing pellet velocities of 2000 meters per second in a two meter long tube using room temperature hydrogen at ten atmospheres as the propellant, or in a 0.5 meter long tube using 38 atmospheres. In either case, the mass of propellant gas injected can be of the same order as the mass of the pellet, if the inlet is closed promptly. 


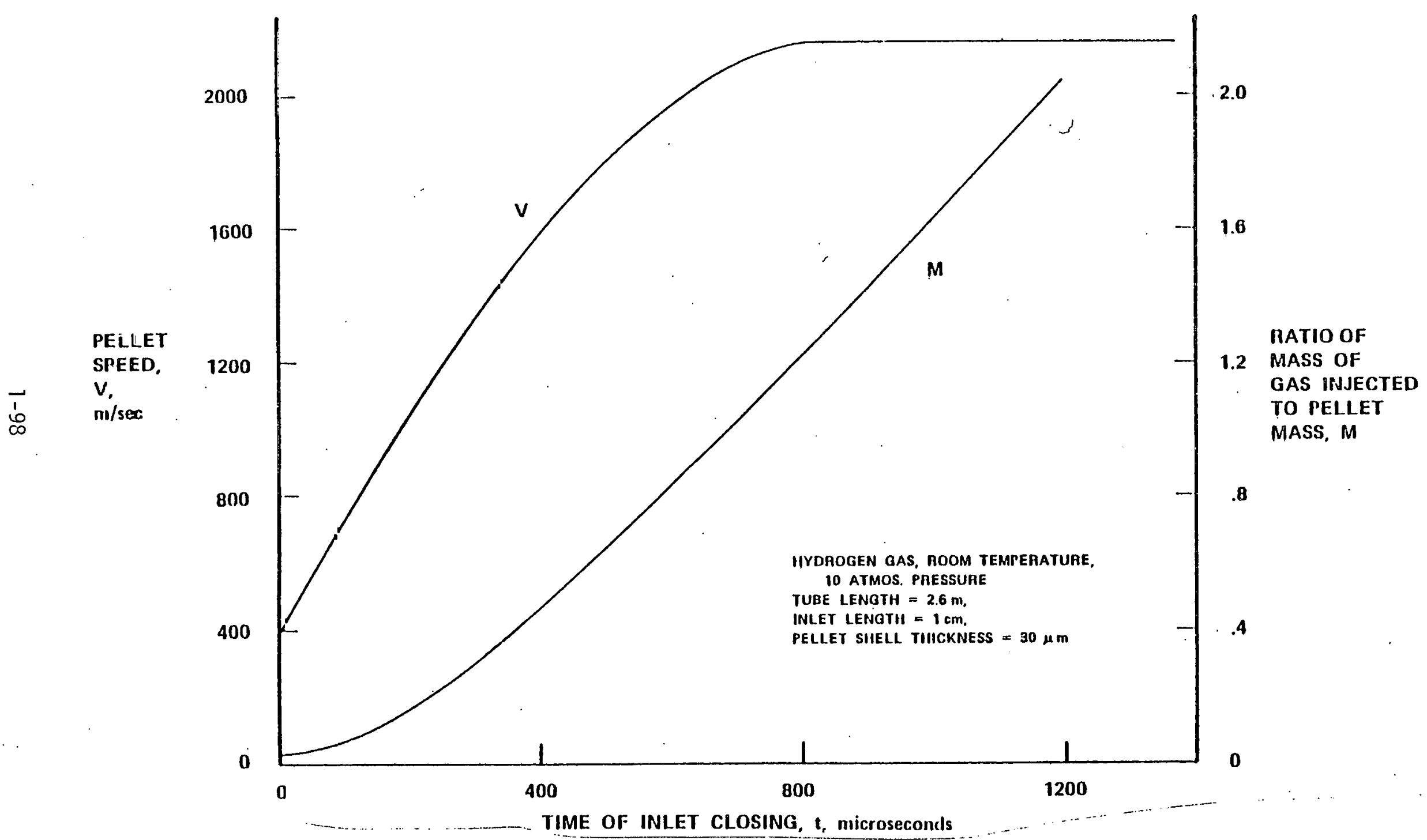

FIGURE 1.4-18 Effest of inlet gate closing on pellet speed and mass of gas injected into vacuum chamber. 


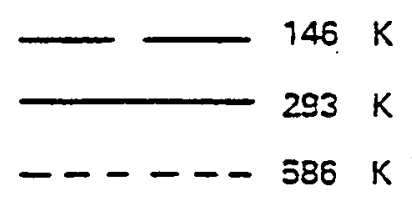

TUBE LENGTH, $m$

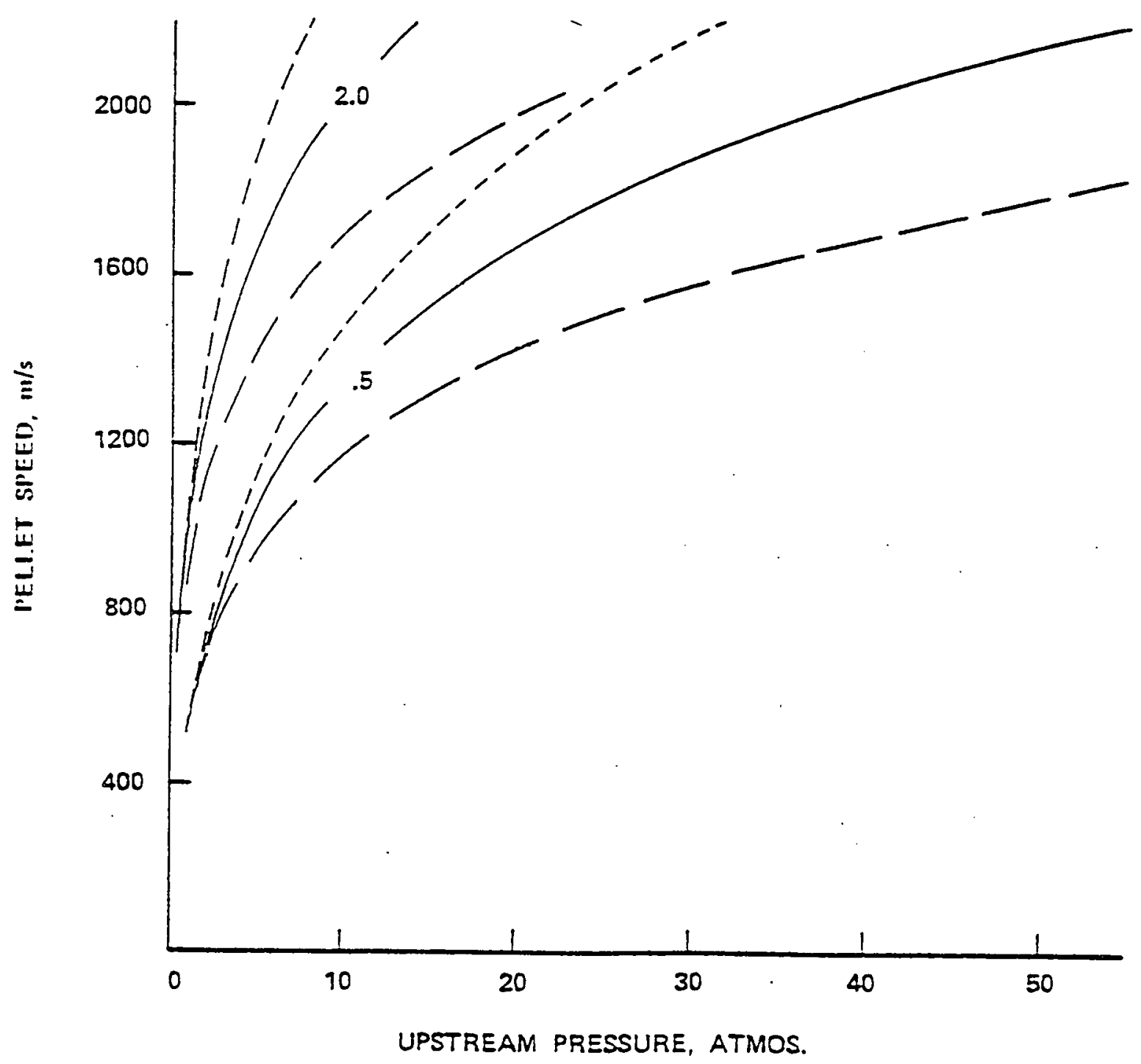

FIGURE 1.4-19 Effect of various temperatures of injectant hydrogen gas on pellet speed as a function of initial pressure. Zero downstream chamber pressure. Shell thickness, 30um. 


\section{REFERENCES}

1. K. D. Wise, T. N. Jackson, N. A. Masnari, M. G. Robinson, D. E. Solomon, G. H. Wuttke and W. B. Rensel, "Fabrication of Hemispherical Structures Using Semiconductor Technology for Use in Thermonuclear Fusion Research."J. Vac. Sci. and Tech. 16, (1979).

2. N. A. Masnari, W. B. Rensel, M. G. Robinson, D. E. Solomon, K. D. Wise and G. H. Wuttke, "Process of Manufacture of Inertial Confinement Fusion Targets and Resulting Product," Patent application $925,437,7-17-78$.

3. J. C. Greenwood, J. Electrochem. Soc. 116, 1325 (1969).

4. A. Bohg, J. Electrochem. Soc. 118, 401 (1971).

5. R. L. Crawley and D. A. Steinman, KMS Fusion Technical Memorandum, DMST-0132 (1978).

6. D. A. Steinman, KMS Fusion Internal Memorandum, DMS-2030 (1979).

7. R. L. Crawley and D. A. Steinman, KMS Fusion Technical Memorandum, DMST-0131 (1978).

8. R. L. Crawley and D. A. Steinman, KMS Fusion Technical Memorandum, DMST-0135 (1978).

9. R. L. Crawley, D. A. Steinman and D. J. Tanner, KMS Fusion Technical Memorandum, DMST-0147 (1978).

10. R. L. Crawley and D. A. Stejinman, KMS Fusion Internal Memorandum, DMS-1852 (1978).

11. T. Asakura, Speckle Metrology, R. Erf, ed. (Academic Press, New York, 1978) Chap. 3.

12. R. L. Crawley and D. A. Steinman, KMS Fusion Technical Memorandum, DMST-0166 (1979).

13. KMS Fusion, Inc. Annual Report, U-762 (1977), Section 1.4, 61-65.

14. A. C. Charters, B. P. Denardo and V. J. Rossow, NACA Technical Note 4143 (1957).

15. R. Courant and K. 0. Friedrichs, Supersonic Flow and Shock Waves (Interscience Publishers, New York, 1948) Chap. IIIB, p. 22.

16. R. J. Stalker, J. of Fluid Mechanics 22, 659 (1965). 


\section{LASER FUSION EXPERIMENTS}

Introduction and Summary

\subsection{Implosion Physics}

2.2 Fast Ion Measurements of Laser-Produced Spherical Plasmas

2.3 Absorbed Energy Measurements

2.4 Diagnostic Development 


\section{Introduction and Summary}

F. J. Mayer

The KMSF experimental program has continued to emphasize the implosion of spherical glass shell targets with the nearly spherical illumination of the ellipsoidal mirror focusing geometry. We have extended preliminary cryogenic liquid layer implosion experiments to experiments using solid cryogenic DT layers. These experiments have attained the highest DT fuel densities that have been produced at our modest laser power of $\sim 0.6 \mathrm{TW}$. The peak fuel density obtained in this nearly spherical implosion of a solid cryogenic layer target was approximately 7 grams per cubic centimeter. These experiments, in comparison with gas filled targets of similar DT fuel mass, have provided important data for the theoretical analysis of the implosion phenomena using hydrodynamic codes. These anatyses are still underway at this writing and some of the prerequisite work on the alpha particle energy spectra, $x$-ray pinhole photography and time-resolved measurements are described below. The cryogenic experiments are, of course, technically different from the standard gas filled implosion experiments, as it is imperative that the layer remain solid until the main laser pulse arrives. Part of our experimental plan is to acquire the technical capability to perform cryogenic experiments, as future experiments on higher gain targets will require this capability. The cryogenic experiments have led to some new laser hardware in the form of the flowing-gas-plasma-filter as described in Section 4.

of great importance in determining the compressed target core conditions, is the analysis of the alpha particle energy spectra from the DT burn. We have observed substantial differences in alpha spectra between imploded gas filled targets and cryogenic layer targets. These results 
have prompted a new examination of the alpha particle distribution which is expected under various core conditions. Both DT fuel doppler corrections and path length corrections to simple model calculations are found to be required when extracting temperature and density information from the alpha spectra.

We have continued to place emphasis on understanding the nature of the laser plasma corona. In particular, fast ion spectra have been examined under a variety of conditions. Attempts have been made to 1 ink the observed spectra to simple isothermal rarefaction models which generally have applicability to planar geometry. An examination using our hydrodynamic code TRHYD showed that this model is useful for spherical. corona expansions as we11, but with some small corrections.

A most important mechanism that can degrade target absorption is Stimulated Brillouin Scattering (SBS). SBS has been recognized as a potential problem for laser fusion for some time and some recent experiments at NRL and LLL have observed definite signatures of SBS. It has also been recognized for some time that a broad band laser pulse may be able to defeat SBS as a loss mechanism. We have done experiments at longer laser pulse lengths, where SBS should be more efficient, with both narrow and broad bandwidth laser pulses and found substantially better absorption for the broad band pulse. We believe that it may be possible to further broaden the incident laser pulse spectrum and to thereby increase the absorption fraction. Furthermore, the experiments using the flowing-gas in the plasma-filter (Section 4) as a long gradient length target, have shown clear evidence of SBS in the backscattered spectra. We expect to use the flowing-gas-target, in comparison experiments, with narrow and broad band laser pulses in the coming year. 
In the past year, we expanded our diagnostic capabilities with the addition of an x-ray streak camera, and a zone-plate imaging system. Also, we have begun development of a small x-ray spectrograph for making fuel density measurements in the x-ray region. The measurement of energy absorbed in a laser target experiment is very important and as the solid angle access is limited in our illumination system, it has been important to develop small total plasma energy calorimeters. A description of two calorimeter designs is given below. To examine the target ablation phenomena and to monitor the symmetry of corona expansion, we have developed a holographic interferometric probe. The present system operates at $2 \omega$, but our future system will operate in the ultraviolet allowing probing measurements in the target corona at higher electron densities. Our computerized data acquisition system has been upgraded with the addition of an image processing capability. The processing system provides a method for studying $x$-ray pinhole photographic images, compensation for noise from the images and determining the size of the core and irregularities in the compressed core $x$-ray emission.

We believe that, in the past year, the experimental program has been very successful. Results obtained in the coming year from cryogenic target implosions, short wavelength implosions and broad band laser pulse experiments will be very important in shaping laser fusion experiments in the future. 


\section{$2.1 \quad$ Implosion Physics}

\subsubsection{High Density Implosion Experiments}

A.H. Bennish, G. Charatis, R.R. Johnson, F.J. Mayer, D.C. Slater, and D. Sullivan

The most important experimental observations in laser fusion studies are those relating to the target implosion and fusion reaction processes. Experiments have been performed with the KMSF ellipsoidal mirror illumination system ${ }^{(1)}$ which produces nearly uniform illumination at near-normal incidence ${ }^{(2)}$ of DT-filled glass microshell targets. Measurements of neutron yield, alpha particle energy spectra, and $x$-ray pinhole camera images together with numerical simulations have been used to form a picture of the laser-driven implosion process. ${ }^{(3)}$

Experiments performed on gas-filled and liquid-1ayer cryogenic targets have been reported earlier $(4)$ in which:

--peak compressions were measured via time-integrated $x$-ray images of the implosion process using two orthogonally configured pinhole cameras,

--asymmetric liquid-fuel-layer cryogenic targets mounted on cryogenically controlled conductive fibers, gave asymmetrically compressed regions upon laser irradiation, resulting in compressed fuel density measurements of $7 \mathrm{~g} / \mathrm{cm}^{3}$ for a 90 atm cryogenic liquid-layer target, compared to $1 \mathrm{~g} / \mathrm{cm}^{3}$ for $10 \mathrm{~atm}$ gas-filled targets.

--a comparison of fuel-ion temperatures between those obtained from alpha-particle spectra and those inferred from model calculations (utilizing experimental measurements of neutron yield, compression ratios and fuel densities) gave fuel ion temperatures from the alpha spectrum measurements that were 
substantially larger indicating that other broadening mechanisms contributed to the alpha spectra,

--a measurement of the FWHM of the alpha particle energy distribution provided an upper limit to the ion temperature at peak compression,

--a measurement of the shift in energy of the peak of the alpha particle spectral distribution provided a measure of the $\int \rho d r$ for the fuel and the tamper

In order to get around the problem associated with the conducting fiber-cooled targets (i.e. non-uniform fuel distribution), a cryogenic shroud apparatus was developed which bathed the entire target in a helium gas atmosphere, at a controlled cryogenic temperature, thereby producing a more uniform solid-fuel layer (see Section 1.3). Sapphire windows in the walls of the shroud permitted the irradiation of the target with $\mathrm{CW}$ YAG laser power of sufficient intensity to momentarily vaporize the fuel and reform the solid layer. The flowing cold helium maintained the solid layer distribution until the shroud was retracted.

The shroud was fully retracted 4 milliseconds prior to the arrival of the laser pulse. This interval was sufficiently short to show that radiative heating from the surroundings did not vaporize the fuel. The surrounding helium pressure, upon removal of the shroud, also dropped to 0.035 mtorr during this same time interval -- a pressure sufficiently sma11, to cause no problem in irradiation of the target.

For all cryogenic layered targets it is important that the solid (or liquid) fuel layer is not disturbed by the amplified spontaneous emission (ASE) which is present just prior to the arrival of the main laser pulse. Insertion of dye cells, and other appropriate measures must be taken to reduce the ASE to acceptable levels. For the solid-layered 
target experiments we have found that the ASE must be kept below 200 microjoutes.

Shot 3723 is a typical example of the irradiation of a solid-layer cryogenic target. Table 2.1-1 lists experimental and inferred parameters that were obtained for this shot. Figure 2.1-1A is a pinhole picture of this shot taken through 2 mils of beryllium, with the outer circle showing schematically the initial position of the glass microshell. Figure 2.1-1B is a pinhole photograph of the $x$-ray image taken through 32 mils of beryllium. The location of the fuel region is clearly identified as the nonradiating area in the center contained by the radiating volume of the tamper. In general, the implosion symmetry was improved over the liquid-layer shots. Assuming cylindrical symmetry of the compressed fuel volume and that all of the initally-contained DT fuel was compressed, the peak fuel density was estimated to be $\sim 7 \mathrm{~g} / \mathrm{cm}^{3}$.

The high densities estimated from pinhole photographs of imploding targets are consistent with measurements of the energy loss of the alpha particles encountered in traversing the compressed fuel and tamper. If the alpha particles lost no energy, their spectra would be centered at $3.52 \mathrm{MeV}$. The evidence of increased fuel densities in cryogenic targets comes from alpha particle energy spectra which are downshifted from 3.52 MeV. This effect is shown in Figure 2.1-2, where alpha particle energy spectra from gaseous, liquid-layer, and solid-layer targets are compared.

The gaseous target in Figure 2.1-2A (Shot 2833) was a $52 \mu \mathrm{m}$ diameter microshell with a $0.7 \mu \mathrm{m}$ wall and $10 \mathrm{~atm}$ DT fill pressure. The laser energy was $31 \mathrm{~J}$ in $90 \mathrm{psec}$, and $1.9 \times 10^{7}$ neutrons were produced. The mean a) pha particle energy was downshifted by $0.85 \% \pm 1.9 \%$.

The cryogenic liquid layer target in Figure 2.1-2B (Shot 3046) was a $68 \mu \mathrm{m}$ diameter microshell, with $0.7 \mu \mathrm{m}$ wall and 81 atm DT fill pressure. 
TABLE 2.1-1

EXPERIMENTAL PARAMETERS FOR TARGET SHOT 3723

$\begin{array}{lc}\text { Shell Diameter } & 88 \mathrm{\mu m} \\ \text { Shell Thickness } & 0.8 \mu \mathrm{m} \\ \text { Shell Mass } & 45 \mathrm{ng} \\ \text { DT-Fill Gas Density } & 18.4 \mathrm{mg} / \mathrm{cm}^{3} \\ \text { DT Shell Thickness } & 1.2 \mu \mathrm{m} \\ \text { Laser Energy on Target } & 60 \mathrm{~J} \\ \text { Pulse Length } & 132 \mathrm{psec} \\ \text { Absorbed Energy } & 8.9 \mathrm{~J} \\ \text { Neutron Yield } & 1.5 \times 10^{7} \\ \text { Al.pha Particle Spectrum } & \\ \text { FWHM } & 375 \pm 25 \mathrm{keV} \\ \text { Mean Peak Shift } & -8.5 \% \pm 1.4 \% \\ \text { Reduced Volume Diameter } & 12 \pm 3 \mu \mathrm{m}\end{array}$

\section{INFERRED PARAMETERS FOR TARGET SHOT 3723}

From Reduced Volume Diameter Measurement

$\begin{array}{lc}\text { Compression } & 380 \\ \text { Compressed Fuel Density } & -174 \\ & 7 \pm 9 \mathrm{~g} / \mathrm{cm}^{3} \\ \langle\rho R\rangle & 4.3 \pm 3.2 \mathrm{mg} / \mathrm{cm}^{2}\end{array}$

From FWHM of Alpha Spectrum

Ion Temperature less than $4.5 \pm 0.6 \mathrm{keV}$ *

From Mean Peak Shift of Alpha Spectrum

$$
\begin{array}{ll}
\langle\rho R>\text { if electron temp }=2 \mathrm{keV}, & \\
\text { and shift due all to glass, } & 2.2 \times 10^{-3} \mathrm{~g} / \mathrm{cm}^{2} \\
\text { and shift due all to fuel, } & 2.8 \times 10^{-3} \mathrm{~g} / \mathrm{cm}^{2}
\end{array}
$$

* See Section 2.1.2 


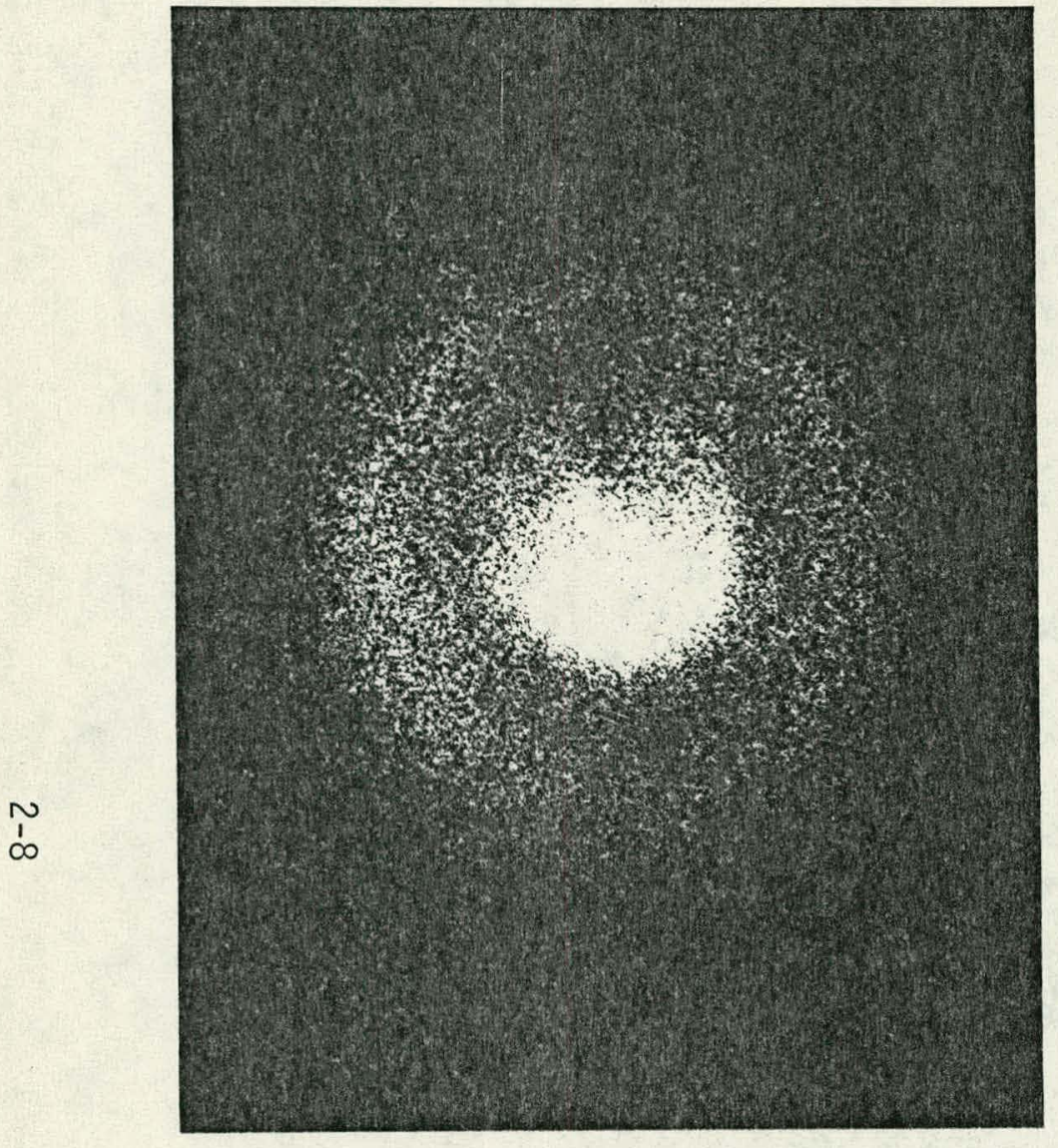

(a)

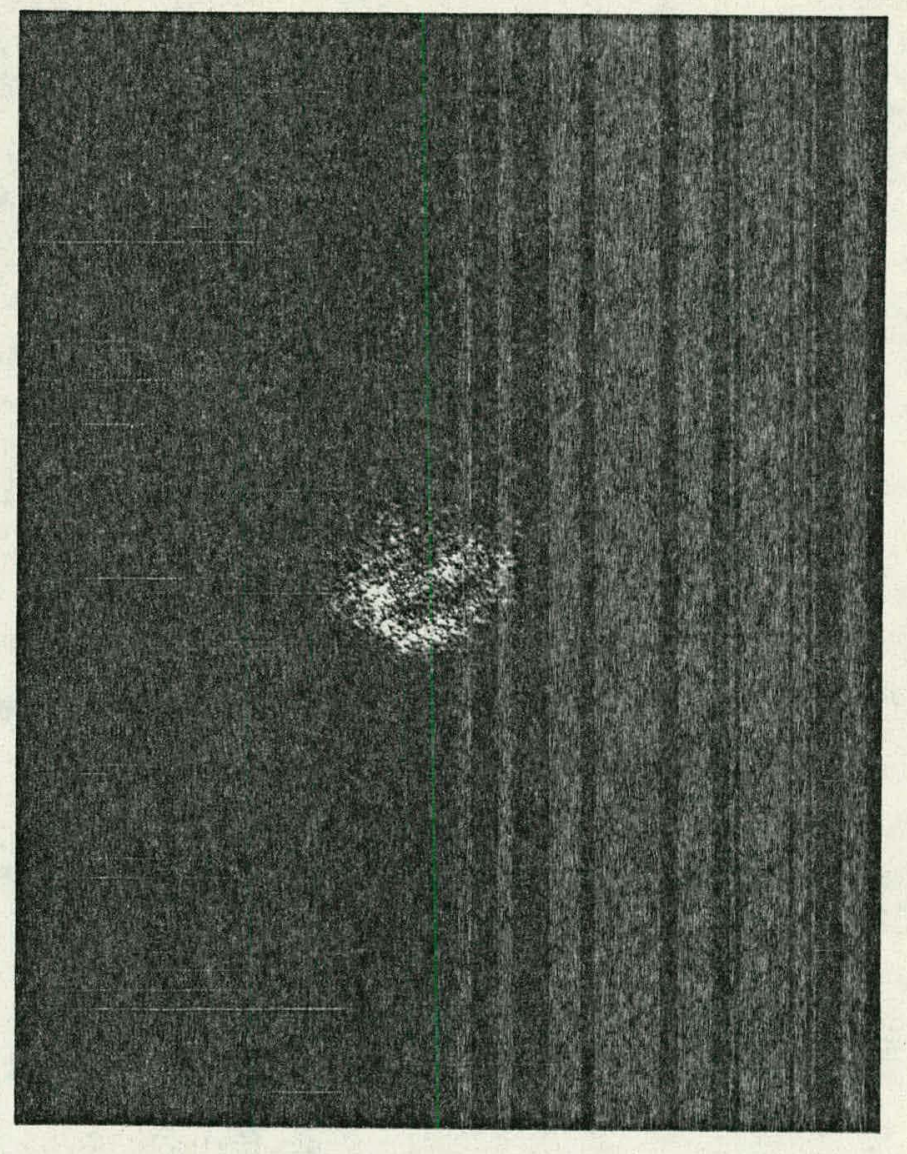

(b)

Fig. 2.1-1 Pinhole pictures of cryogenic solid layer shot 3723. (a) is taken through 2 mils of beryllium, with the outer circle indicating the initial position of the microshe11. (b) is taken through $32 \mathrm{mils}$ of beryllium, with the compressed non-radiating fuel region indicated by the central dark area. 


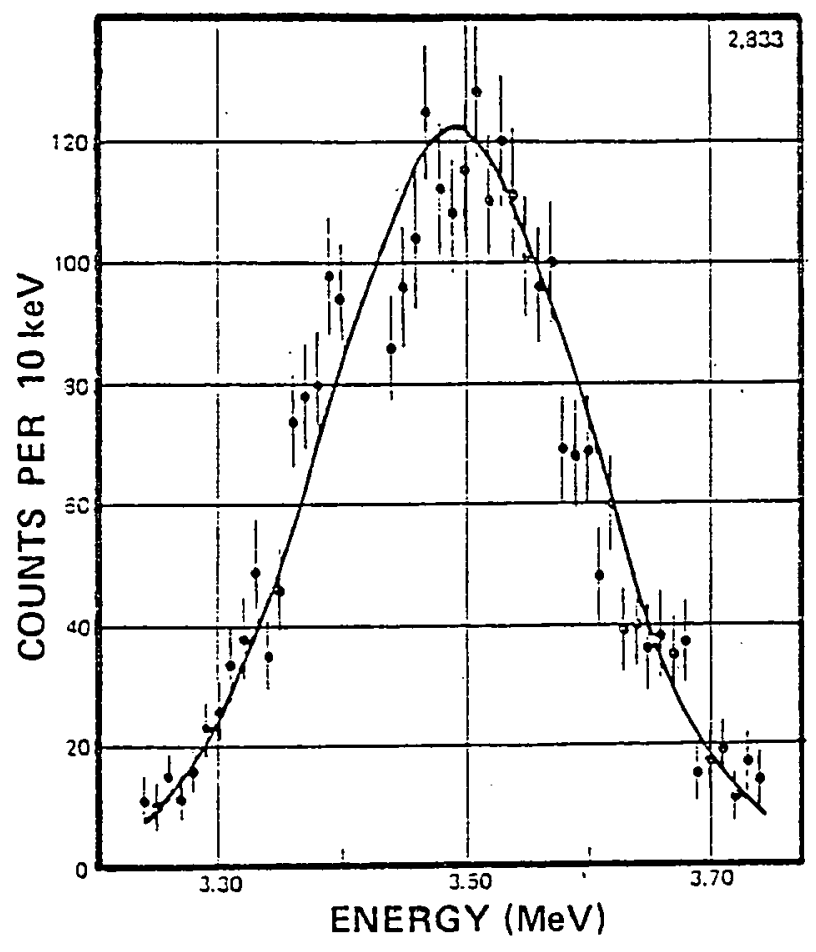

(A)

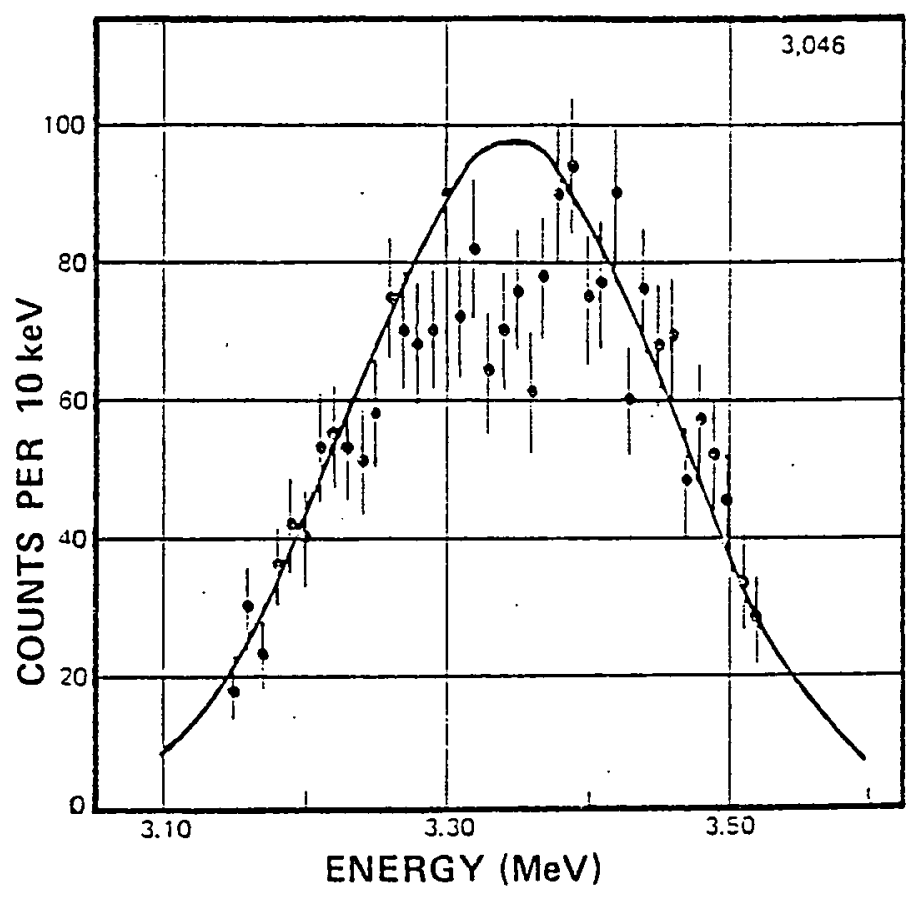

(B)

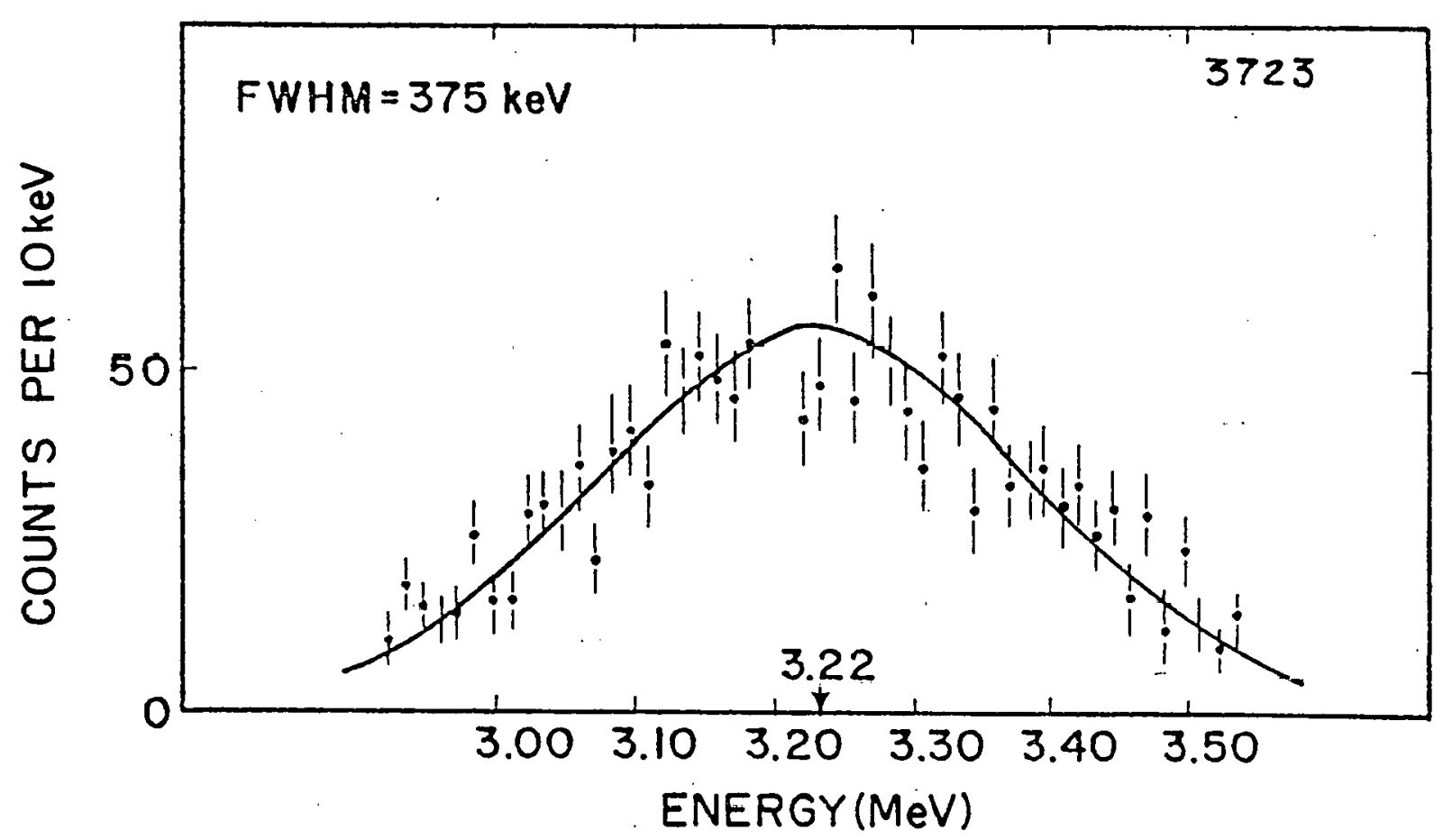

(C)

Fig. 2.1-2 Alpha particle energy spectra or representative (A) gas, (B) liquid-layer, and (C) solid-layer target shots. 
The laser energy was $86 \mathrm{~J}$ in $138 \mathrm{psec}$, and $3.1 \times 10^{7}$ neutrons were produced. The alpha-particle energy spectrum shows a measured downshift of $5 \% \pm 2 \%$. For the solid-DT-layer target shots the neutron yields were comparable to those of the liquid-layered targets at equivalent laser power on target. The solid-layer cryogenic target in Figure 2.1-2C (Shot 3723) was a $88 \mu \mathrm{m}$ diameter microshell with a $0.8 \mu \mathrm{m}$ wall and a $92 \mathrm{~atm}$ DT fill pressure. The laser energy was $60 \mathrm{~J}$ in $132 \mathrm{psec}$ and $1.5 \times 10^{7}$ neutrons were produced. The alpha-particle spectrum showed a measured downshift in energy of $8.5 \% \pm 1.4 \%$.

Computer code simulations of the implosion of cryogenic targets indicate that a $5 \%$ energy loss for the:liquid layer targets, and a $8.5 \%$ loss for the solid layer targets is consistent with compressed fuel densities of a few $\mathrm{g} / \mathrm{cm}^{3}$. The same calculations show that alpha particles should lose energy not only in the compressed fuel but also in the surrounding glass, so that the $\int \rho d r$ of the fuel cannot be obtained directly from the energy-loss measurement.

Alternatively, the energy loss rate can be calculated for both glass and DT fuel as a function of the fodr of the material. It also depends on the electron temperature and density of the medium, as shown in Figure 2.1-3. Assuming an electron temperature of $1 \mathrm{keV}$, which is consistent with the measurement of the compressed core electron temperature obtained from beryllium-filtered pinhole pictures, the $\int \rho d r$ for the liquidlayer targets would be $6 \times 10^{-4} \mathrm{~g} / \mathrm{cm}^{2}$, if $10 \%$ to $20 \%$ of the glass remained at peak compression. For the DT fuel $\int \rho d r$ would be $9 \times 10^{-4} \mathrm{~g} / \mathrm{cm}^{2}$, assuming that all of the DT fuel was compressed and an average alpha-particle path length of $\frac{3}{4} r_{0}$.

Similarly, for the solid-layer target shot considered above, the 
ALPHA PARTICLE ENERGY LOSS IN $\mathrm{SiO}_{2}$

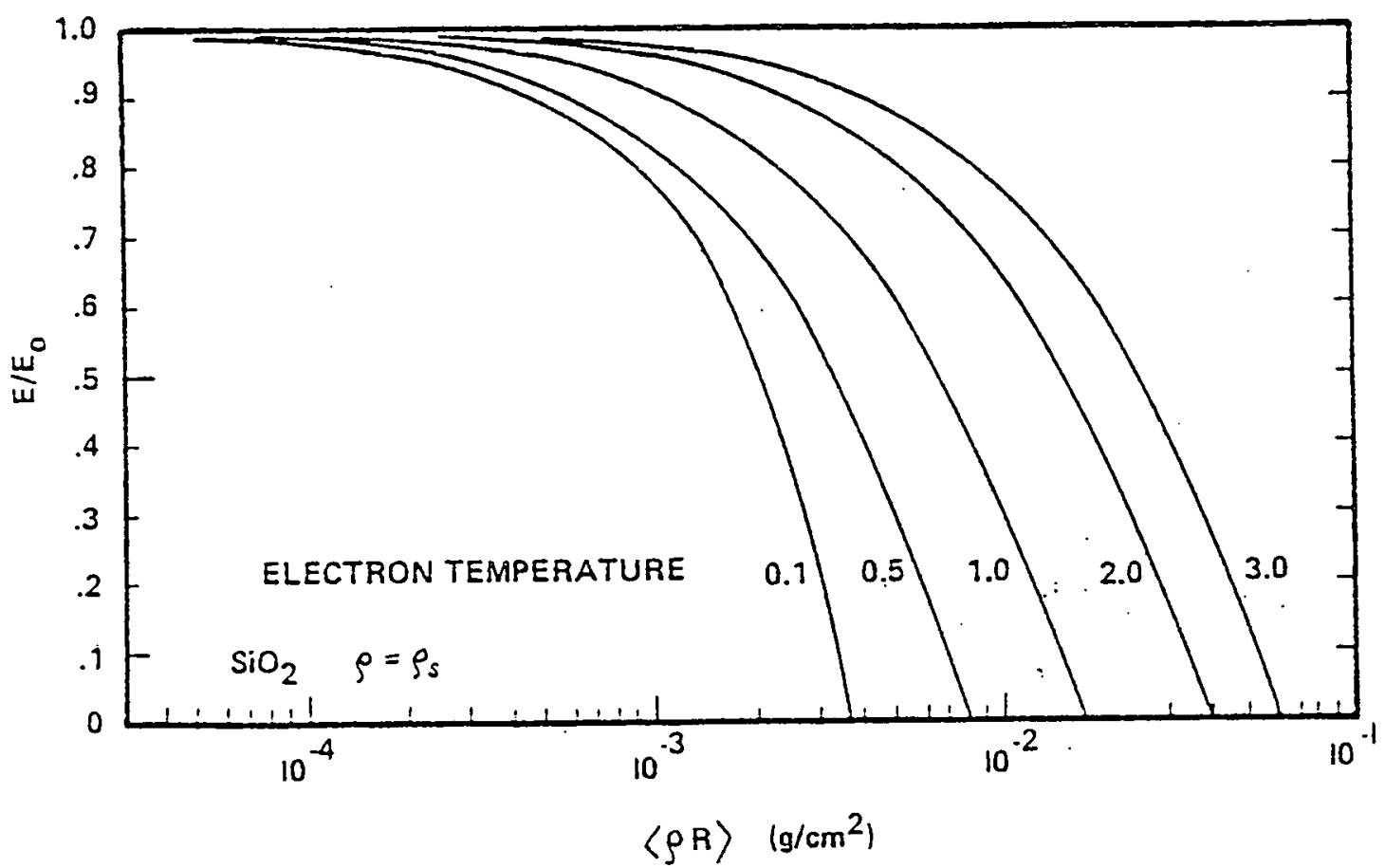

ALPHA PARTICLE ENERGY LOSS IN D-T

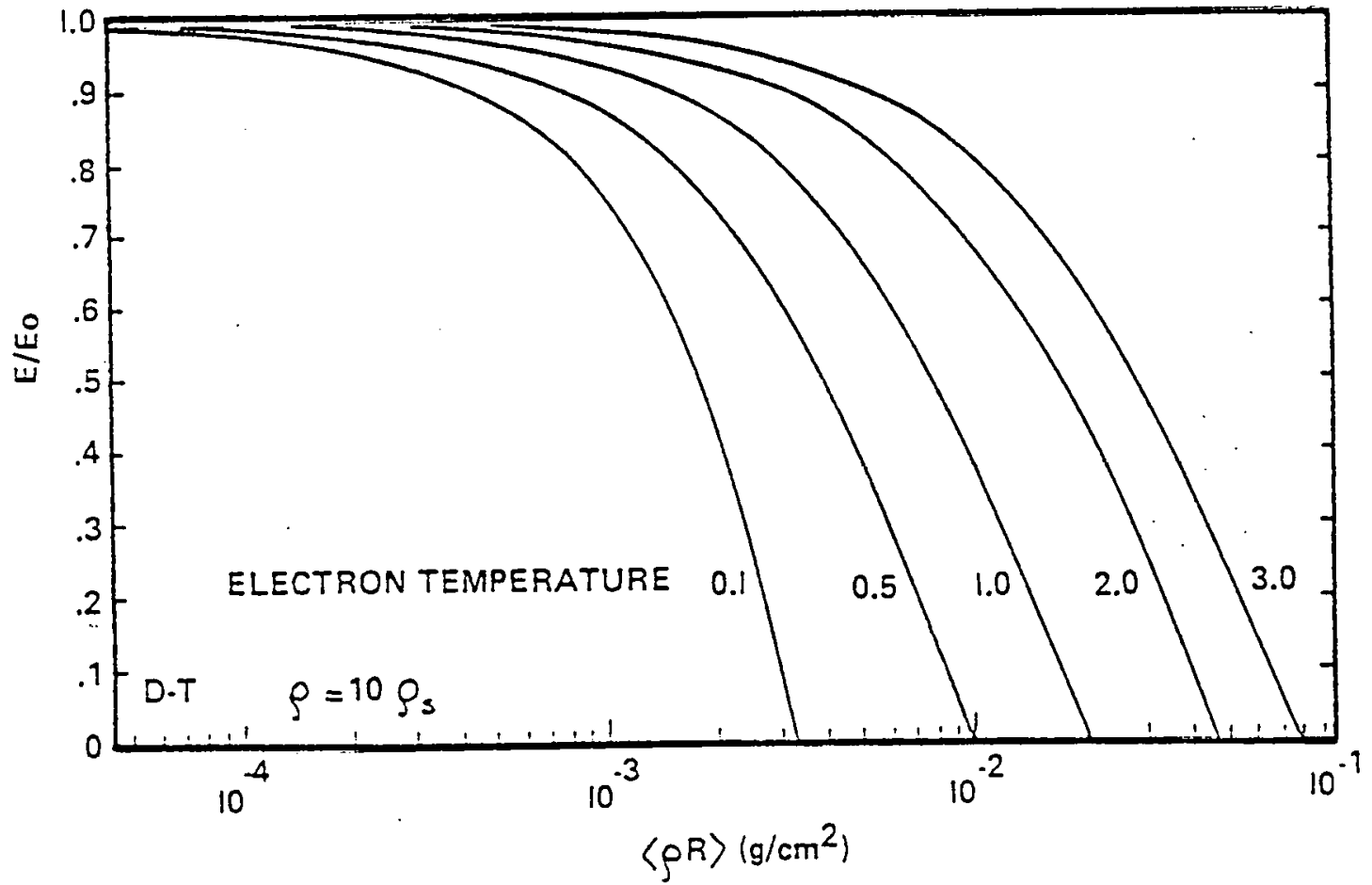

Fig. 2.1-3 Mean alpha particle energy loss vs $\langle\rho R\rangle$ in a $\mathrm{SiO}_{2}$ tamper and DT fuel, for electron temperatures of $0.1,0.5,1.0,2.0$ and $3.0 \mathrm{keV}$. 
larger downshift $(8.5 \%)$, corresponds to $\int \rho d r$ values (at a measured 2 keV electron temperature) of $2.2 \times 10^{-3} \mathrm{~g} / \mathrm{cm}^{2}$, if all of the loss was in the glass, and $2.8 \times 10^{-3} \mathrm{~g} / \mathrm{cm}^{2}$, if all of the energy loss was due to the fuel. In comparison the measured volume reduction of 380 obtained from the pinhole photograph results in $\int \rho d r$ in the fuel of $4.3 \times 10^{-3} \mathrm{~g} / \mathrm{cm}^{2}$.

Several target shots have been observed to result in mean alpha particle energies which are greater than the zero-shift value of $3.52 \mathrm{MeV}$, as can be seen in Figure 2.7-4. There, for shot 3900, a gas DT target, the mean energy was $3.70 \pm .08 \mathrm{MeV}$, an upshift of $5.1 \pm 1.5 \%$, which can be compared with a downshifted mean energy of $3.36 \pm 0.5 \mathrm{MeV}$ (i.e. $4.5 \pm 1.4 \%$ ) for shot 3905, a solid layer DT target. Mean energy upshifts have been observed at LLL ${ }^{(5)}$ when the laser pulse length has been longer than the collapse time of the target. We will be able to verify this phenomenon when our recently acquired x-ray streak camera (see Section 2.4.1) becomes fully operational.

In Figure 2.1-5 the number of laser-target events is plotted vs the mean alpha energy for room temperature gas filled, cryogenic liquid and solid layer DT targets. As can be seen, the solid and liquid-layer target shots, in general, exhibited mean alpha energies which were lower than the gas filled target shots. 


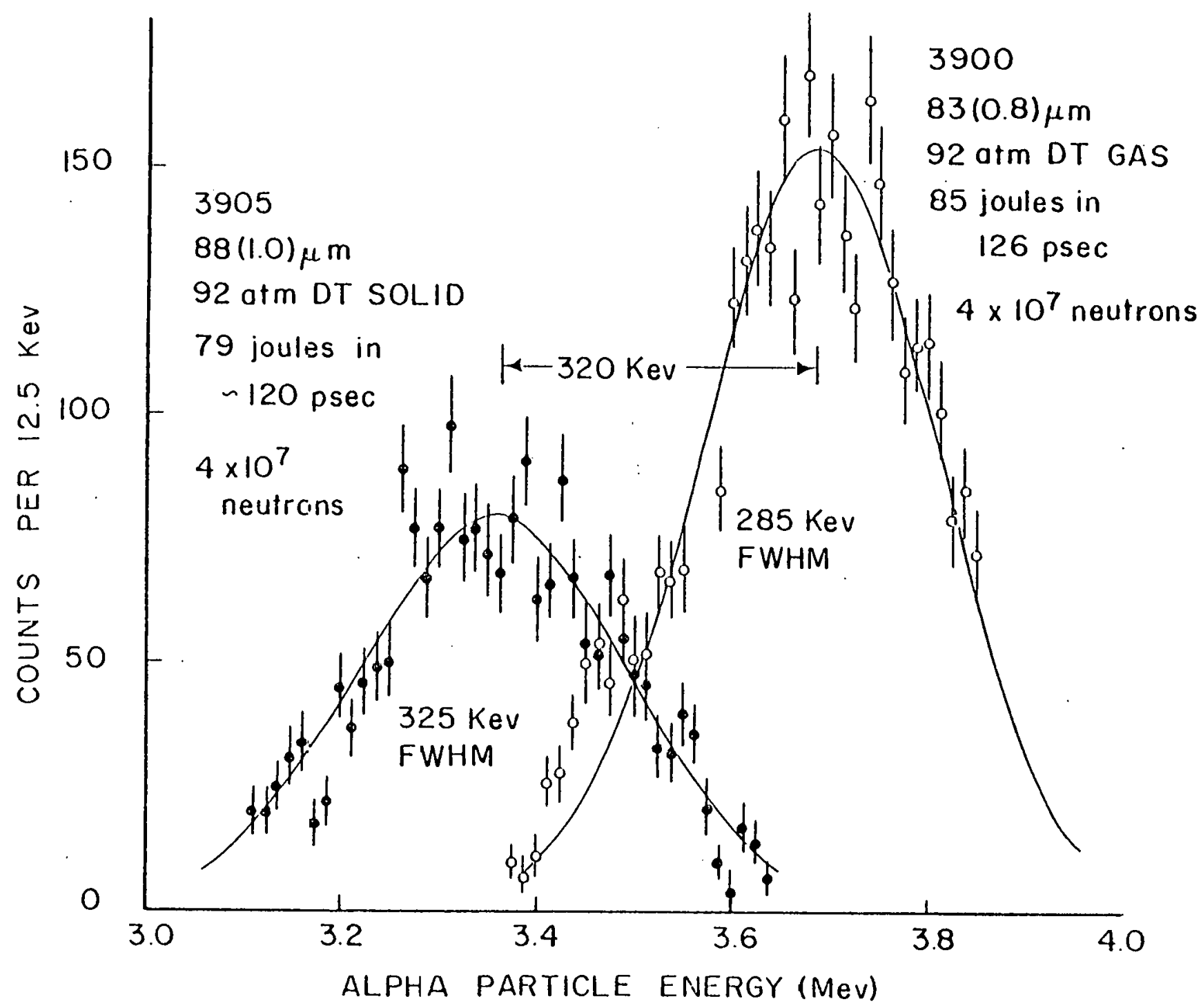

Fig. 2.1-4 Comparison of an alpha energy spectrum, for which the mean energy was greater than the zeroshift value of $3.52 \mathrm{MeV}$, with the alpha spectrum from a typical solid-layer DT target shot. 


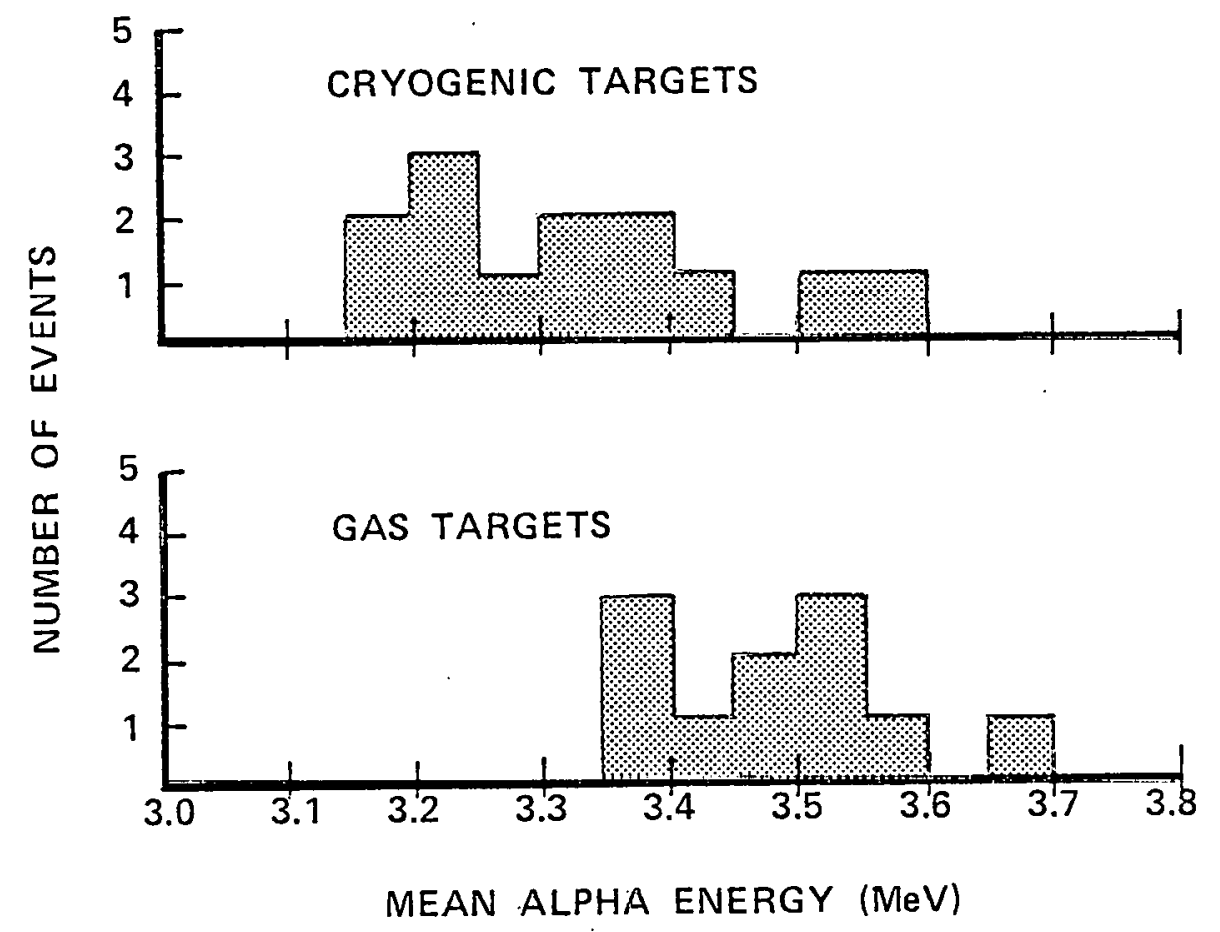

Fig. 2.1-5 A comparison of mean alpha energies for gas and cryogenic target shots. 


\subsubsection{Analys is of Alpha-Particle Spectra}

\section{R.R. Johnson and D.C. Slater}

The DT Fusion reaction produces an alpha particle with nominal energy of $3.52 \mathrm{MeV}$. The measured energy spectra of alpha particles have full-width at half-maximum (FWHM) spreads of a few hundred keV, and mean energies in the range 3.2 to $3.7 \mathrm{MeV}$. Previous analyses have emphasized that the energy spread results largely from thermal motion of the fusing

ions and therefore can be used as a measure of the fuel ion temperature. $(6,7)$ Energy loss from the nominal reaction energy is expected because the alphaparticles suffer collisions as they pass through the fuel and tamper material. Energy gain has been observed when the alpha-particle emission occurs during the laser pulse, ${ }^{(8)}$ but can probably be neglected if the laser pulse terminates prior to peak compression.

For exploding pusher targets in which the fuel density is low and the fuel temperature high, other mechanisms which might influence the alpha particle energy spectra can generally be ignored. At higher fuel densities and lower fuel temperatures, however, these other effects become larger. We have studied some of the effects which we believe contribute to the alpha-particle spectra from cryogenic target experiments.

Doppler Broadening:

Brysk $^{(9)}$ has determined the alpha particle energy spectrum when the reacting DT pair has a center of mass velocity $V$ to be

$$
r(E \alpha) \mathrm{d} E \alpha-\mathrm{d} E_{\alpha}\left[\exp -\left(E_{u}-E_{\alpha}>\right)^{2} / D\right]
$$

where $\quad D=\frac{1 M_{n} M_{\alpha}<V^{2}>Q}{3\left(M_{n}+M_{\alpha}\right)}$

and $Q$ is the reaction energy. When the fuel is in a Maxwellian distribution at temperature $\theta$, he shows 


$$
\left\langle V^{2}>=3 \theta /\left(M_{\alpha}+M_{n}\right)\right.
$$

This result can be extended to the case where the DT pair are in a fuel element moving with velocity $U$. Then $V=V^{\prime}+U$, where $V^{\prime}$ is the centerof-mass velocity of the pair in the rest frame of the fuel element. $\quad V$ ' then replaces $V$ in equation $(3)$.

$$
\begin{aligned}
& \text { Evaluating }\left\langle V^{2}\right\rangle \\
& \left\langle V^{2}\right\rangle=\left\langle\left(V^{\prime}\right)^{2}\right\rangle+U^{2}+2 U\left\langle V^{\prime} \cos \phi\right\rangle
\end{aligned}
$$

The last term vanishes in the spherically symmetric case, so that equation (2) becomes

$$
D=\frac{4 M_{n} M_{\alpha} Q}{3\left(M_{n}+M_{\alpha}\right)}\left[\left\langle\left(V^{\prime}\right)^{2}\right\rangle+u^{2}\right]
$$

Replacing $\left\langle\left(V^{\prime}\right)^{2}\right\rangle$ as in equation ( 3 ) gives $D$ in terms of $U$ and $\theta$. The FWHM of the energy spectrum thus becomes

$$
\Gamma=177\left[\theta+1.57 \times 10^{6}(U / C)^{2}\right]^{1 / 2} \mathrm{keV}
$$

with $\theta$ in $\mathrm{keV}$ and $\mathrm{C}=3 \times 10^{10} \mathrm{~cm} / \mathrm{sec}$

A complete description of the spectrum therefore requires knowledge of $U$, appropriately averaged over the reaction rate and integrated over the reaction time.

The quantity $\Delta \theta=1.57 \times 10^{6}(\mathrm{U} / \mathrm{C})^{2}$ is plotted in Fig. 2.7-6 as a function of $U$. TRHYD code simulations show significant fusion yield can occur for zones moving with velocities of $1-2 \times 10^{7} \mathrm{~cm} / \mathrm{sec}$ which would give $\Delta \theta$ around $0.5 \mathrm{keV}$.

Fnergy I nss:

The energy loss of alpha particles traversing the fuel or glass plasma depends on the temperature and $\rho R$ integral of the plasma, with only weak dependence on the plasma density. (10) The energy loss affects the 


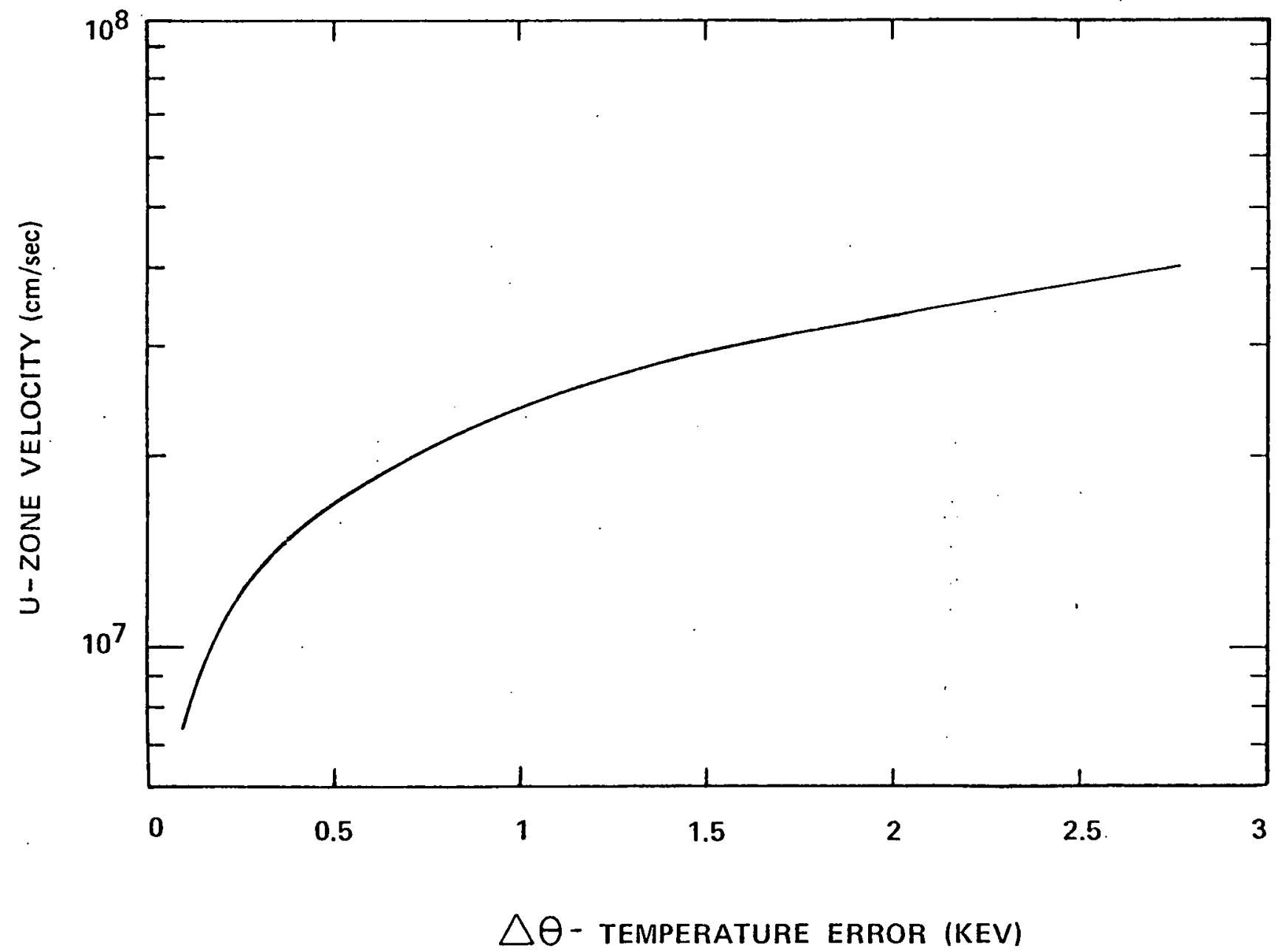

Fig. 2.1-6 Apparent temperature shift $\Delta \theta$ due to Doppler broadening of the alphaparticle spectrum. $U$ is the velocity of the fuel elements. 
alpha particle energy spectrum in two ways. First, the average alpha particle energy is lowered so the entire spectrum is downshifted. Second, since alphas suffer differing amounts of energy loss, the spectrum is broadened.

We can estimate these effects by considering only the geometry which determines the path length through the medium, while neglecting temperature and density variations along the path.

Considering a spherical glass shell of inner radius $\mathrm{R}$ and outer radius $R+\delta$, and a source point at a distance $r$ from the center. (Fig. 2.1-7) A straight line path from this point at an angle $\theta$ with the radial direction will include path length $f$ through the fuel and $g$ through the tamper. Then

$$
\begin{aligned}
& f+g=r \cos \theta+\sqrt{r^{2} \cos ^{2} \theta-r^{2}+(R+\delta)^{2}} \\
& f=r \cos \theta+\sqrt{r^{2} \cos ^{2} \theta-r^{2}+R^{2}}
\end{aligned}
$$

The average paths lengths are given by

$$
\bar{f}=\frac{1}{2} \int_{-1}^{1} f(\cos \theta) d \cos \theta
$$

and

$$
\dot{g}=\frac{1}{2} \int_{-1}^{1}[(f+g)-f] d \cdot \cos \theta
$$

Evaluating the integrals gives

$$
\begin{gathered}
\bar{f}=\frac{R}{2}+\frac{R^{2}-r^{2}}{4 r} \ln \left[\frac{\dot{R}+r}{R-r}\right] \\
\bar{g}=\frac{\delta}{2}+\left[\frac{(R+\delta)^{2}-r^{2}}{4 r}\right] \ln \left(\frac{R+\delta+r}{R+\delta-r}\right)-\left[\frac{R^{2}-r^{2}}{4 r}\right] \ln \left(\frac{R+r}{R-r}\right)
\end{gathered}
$$




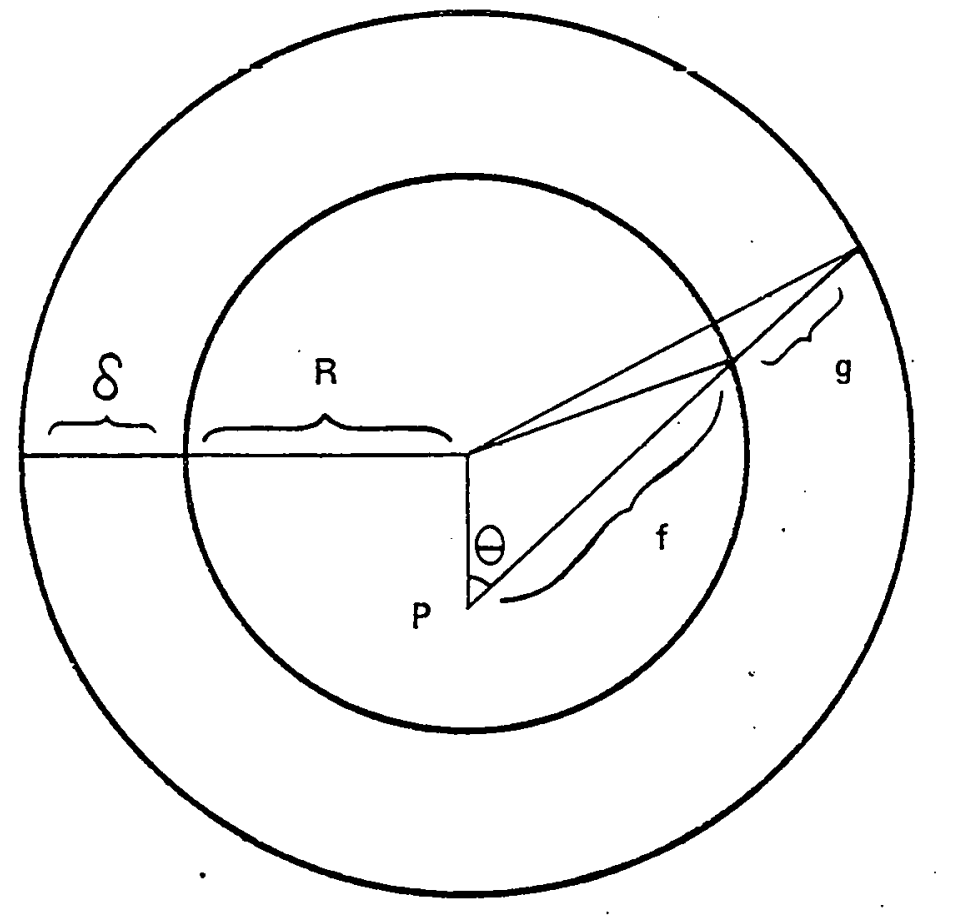

Fig. 2.1-7 Schematic representation of a glass shell of inner radius $\mathrm{R}$ and wall thickness $\delta$. An alpha particle created at point $P$ moving at an angle $\theta$ with respect to the radial direction if through the fuel and $g$ through the tamper. 
In the limit $\delta \ll R$,

$$
\bar{g}=\frac{\delta}{2}\left[\frac{R}{r} \ln \left(\frac{R+r}{R-r}\right)\right]
$$

Equations (11), (12) and (13) can be used to determine the average path length of an alpha particle originating at a distance $r$ from the origin. By averaging $\bar{f}$ and $\bar{g}$ over the spherical fuel volume, the mean path lengths for all alphas can be found. These can then be used to estimate the mean alpha particle energy downshift.

The contribution to the energy spread can be estimated as well. The spectrum is given by

$$
\frac{d N}{d E}=\frac{d N}{d r}\left(\frac{d E}{d r}\right)^{-1}=\frac{d N}{d r}\left(\frac{d E}{d \bar{f}} \frac{d \bar{f}}{d r}\right)^{-1}
$$

In the simplest approximation, $\frac{d N}{d r}=4 \pi r^{2}, d E / d \bar{f}$ is assumed constant, and $d \bar{f} / d r$ can be found from equation (11). The same procedure can be followed for energy loss in the glass.

The possibility of several factors contributing both to the spectral width and downshift makes it impossible to obtain unambiguous fuel ion temperature and tamper $<\rho R>$ values from the alpha spectrum alone.

As shown above, the specific location for the origin of an alpha particle and its direction can play a significant role in the amount of energy loss it sustains in leaving the compressed core. This is particularly true if the $\langle\rho R>$ of the fuel is appreciably high or if the fuel region large compared to the tamper region. The $\langle\rho R>$ that determines the energy that an individual alpha particle loses is given by $\rho_{f} f+\rho_{t} g=\rho_{f}\left\{r \cos \theta+\sqrt{r^{2} \cos ^{2} \theta-r^{2}+R^{2}}\right\}+\rho_{t}\left\{\sqrt{r^{2} \cos ^{2} \theta-r^{2}+(R+\delta)^{2}}-\sqrt{r^{2} \cos ^{2} \theta-r^{2}+R^{2}}\right\}$ 
where $\rho_{f}$ and $\rho_{t}$ are the densities in the fuel and tamper regions, respectively. For the present calculations they will be considered to be uniform.

It is convenient to normalize the quantities in Equation (15) as $y=\frac{r}{R} \cos \theta, x=\frac{r}{R}$, and $\Delta=\frac{\delta}{2 R}$. Furthermore, we set $\alpha=\frac{\rho_{t}}{\rho_{f}}$ and $A=\frac{\rho_{f} f+\rho_{t} g}{R \rho_{f}}=\frac{f+\alpha g}{R}$ and then rewrite Equation (15) as

$$
A-y-\sqrt{y^{2}-x^{2}+1} \quad(1-\alpha)=\alpha \sqrt{y^{2}-x^{2}+(1+2 \Delta)^{2}} .
$$

This equation is solved for $y$ as a function of $x$ to determine the angular dependence of the energy loss.

The energy loss can be calculated for a Gaussian distribution of alpha particles generated at a given ion temperature. (io) The energy loss per unit length of an incident particle, with subscript "l", to a uniform Maxwellian plasma of field particles with subscript "2" is given by

$$
\left\langle\frac{\mathrm{dE}}{d s}\right\rangle=2 \pi n_{2} e^{4} Z_{1}^{2} Z_{2}^{2} \ln \lambda_{2}\left(\frac{m_{1}}{m_{2}} E_{1}\right) F\left(\frac{V_{1}}{V_{\Theta}}\right)
$$

where

$$
v_{\Theta}=\left(\frac{2 \Theta_{2}}{m_{2}}\right)^{1 / 2}
$$

and the function $F$ is. given by

$$
F(x)=\operatorname{erf}(x)-\frac{2}{\sqrt{\pi}}\left(1+\frac{m_{2}}{m_{1}}\right) x e^{-x^{2}}
$$

and the remaining quantities have their usual significance.

As shown by Cover, (11) Equation (17) can be inverted to determine the path length as a function of the electron temperature and density of the medium, as shown by 


$$
\Delta S=\frac{1}{n_{2}} \int_{V_{0}}^{V_{f}} G\left[\theta, \ln \left(n_{2}\right), V_{1}\right] d V_{1}
$$

with $V_{f}$, the velocity of the alpha particle leaving the given region.

The previous calculations have assumed that all alpha particles were detected with the same $v_{f}$ for a given density and electron temperature. We now specify that $V_{f}$ will vary, depending on the given coordinates, $r$ and $\theta$, where the alpha particle was created. Thus, the $v_{f}$ will have a distribution, and, hence, an alpha particle energy spectrum will be generated. It should be noted that many particles will have the same $V_{f}$ even though they are created with different values of $r$ and $\theta$ in the fuel. The energy spectrum defined by Equation (20) is divided up into equal energy intervals, noting that $\Delta S$ is proportional to $A$. Therefore, the exiting alpha energy $E_{k}$ is a given value for $A_{k}$.

Thus, the number of alpha particles within the energy range $E_{k+1}$ $-E_{k}$ is found by integrating the volume between $A_{k+1}$ and $A_{k}$. This can be expressed by the volume integral,

$$
\Delta v_{k+\frac{1}{2}}=v_{k+1}-v_{k}=\int_{r_{k}}^{r_{k+1}} \int_{\Theta_{k}}^{\theta^{k+1}} r^{2} \sin \theta d r d \theta
$$

where $A_{k}$ is implicit in the integration.

A number of simplifying assumptions will be made to make the calculations easier. The first is that the functional dependence of $f$ and $g$ on $\langle\rho R>$ are the same for both the fuel and the tamper. An examination of Figures 2.1-8 and 2.1-9 shows that this is approximately the case, with electron differences playing a more significant role. This allows us to treat $A_{k}$ as a single variable for determining the energy loss. The solution of Equation (16) results in a cubic equation in $y$ which, when solved, can 


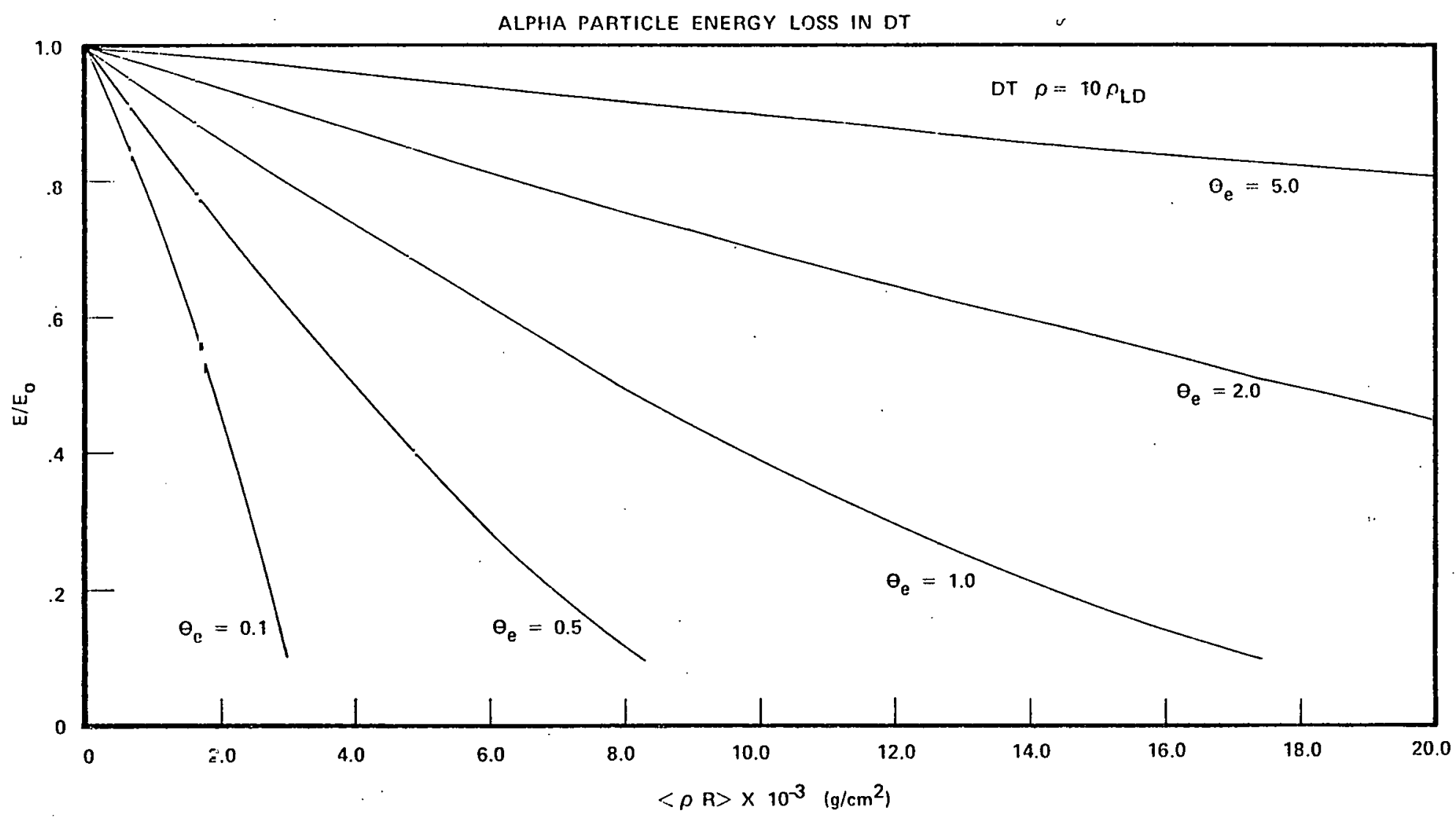

Fig. 2.1-8 Alpha particle energy loss in DT fuel vs $\langle$ oR $\rangle$, for electron temperatures of 0.1 , $0.5,1.0,2.0$ and 5.0 , with $\rho=10 \rho_{\text {LD }}$ 


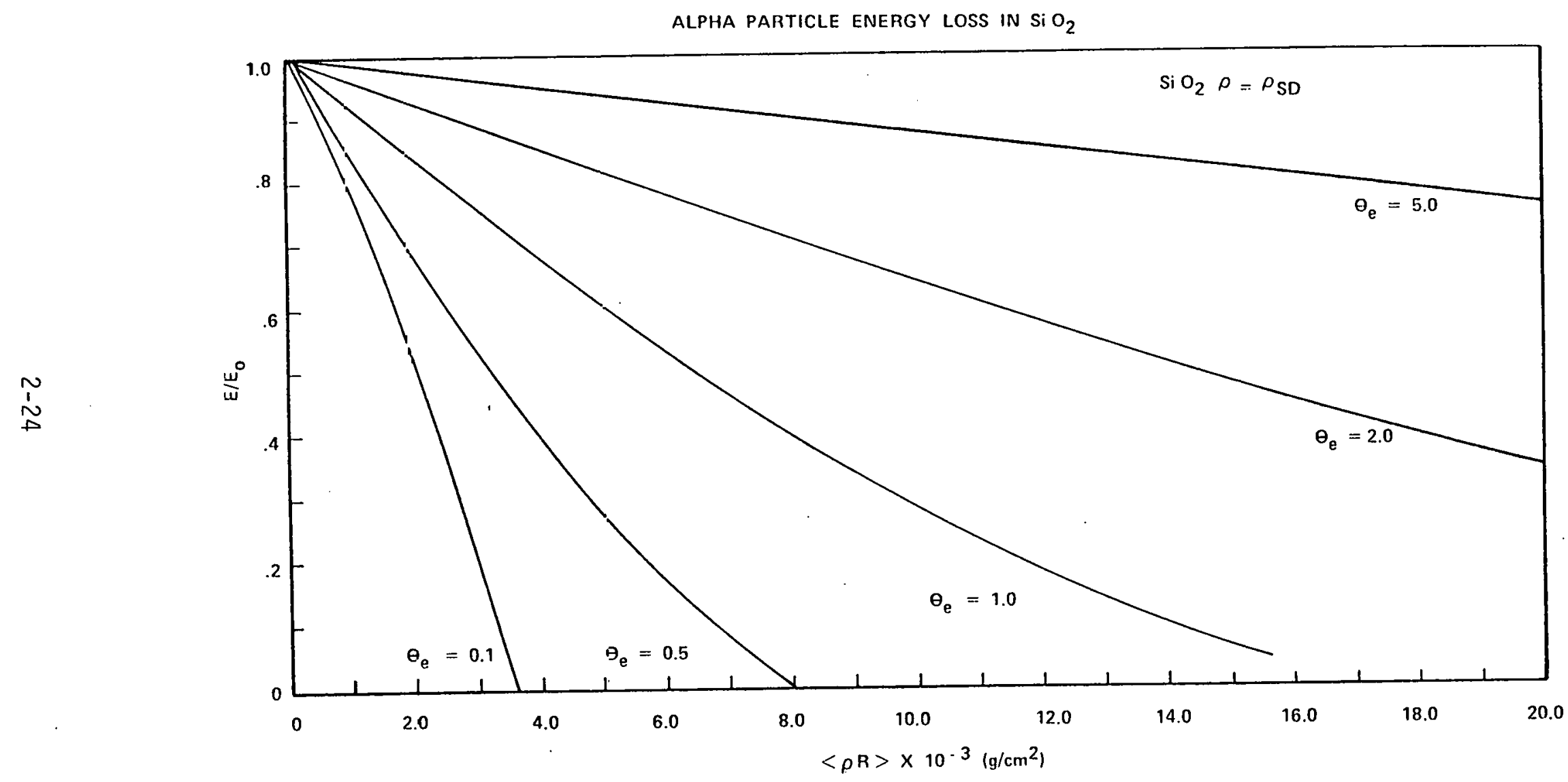

Fig. 2.1-3 Alpha particle energy loss in $\mathrm{SiO}_{2}$ tamper vs $\left\langle{ }_{\mathrm{p}} \mathrm{R}\right\rangle$, for electron temperatures of $0.1,0.5,1.0,2.0$ and 5.0 , with $\rho=\rho_{S D}$. 
be used in Equation (21). A further simplifying assumption is made by setting $\rho_{f}=\rho_{t}$, which is approximately correct for most cases of interest. Equation (16) is then solved for $y$ and we find

$$
\cos \Theta=\frac{A^{2}+x^{2}-(1+2 \Delta)^{2}}{2 A x} \text {. }
$$

Substitution of Equation (22) into Equation (21) and carrying out the integration gives

$\Delta V_{k+\frac{1}{2}}=V_{k+1}-V_{k}=R^{3}\left\{\frac{A_{k+1}^{3}-A_{k}^{3}}{24}-\frac{\left(1+2 \Delta+2 \Delta^{2}\right)}{2}\left(A_{k+1}-A_{k}\right)-2 \Delta^{2}(1+\Delta)^{2}\left(\frac{1}{A_{k+1}}-\frac{1}{A_{k}}\right)\right\}$.

The spectrum is generated using Equation (23) with the minimum energy loss in the tamper, the aspect ratio, and the electron temperature as parameters. Figure 2.1-10 shows a series of alpha spectra for an electron temperature of $1 \mathrm{keV}$ and $a \Delta=0.25$, for different values of the minimum energy loss in the tamper. It is observed that the FWHM of the spectrum is related to the mean energy loss of the aipha particles. This is plotted in Figure 2.1-11 for an electron temperature of $1 \mathrm{keV}$ and for various values of $\Delta$. It is found that for $\Delta=0.25$ or $\delta=R / 2$, the width of the alpha spectrum is comparable to that measured in the cryogenic target experiments. This value of $\Delta$ is consistent with the pinhole pictures and the computer simulations.

The measured alpha spectrum for target shot 3723 in Figure 2.1-12 is superimposed on a calculation of the alpha particle spectrum, with $\Delta=$ $0.25, \theta_{\mathrm{e}}=1 \mathrm{keV}$ and minimum tamper energy loss of $.04 \mathrm{E}_{0}$. As can be seen the correlation is rcasonably good.

The results of these calculations show that it is possible to determine unambiguously the simultaneous contribution of both the fuel and the tamper to the alpha particle energy loss. This will eventually make 


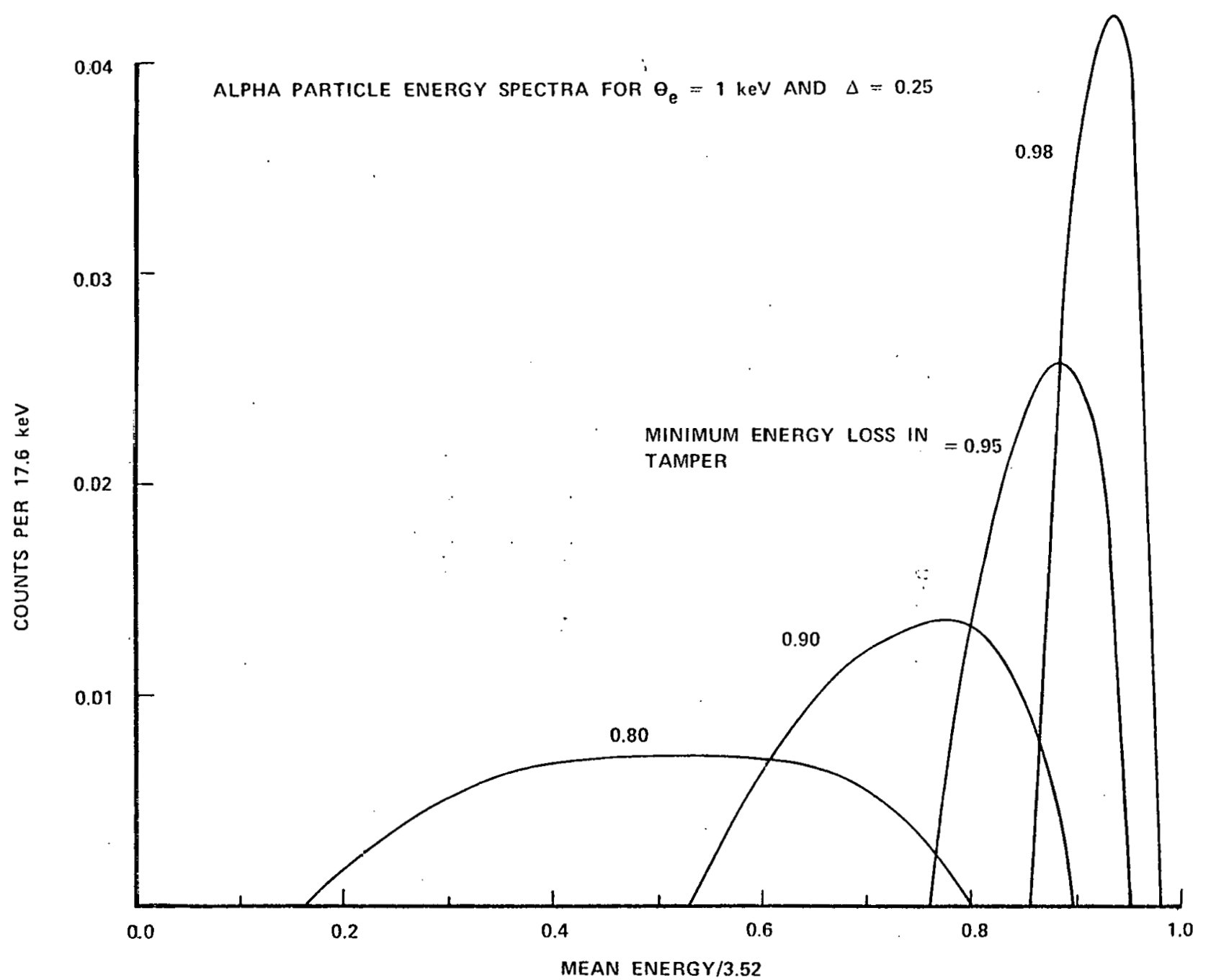

Fig. 2.1-10 Calculated alpha particle energy spectra for an electron temperature of $1 \mathrm{keV}$ and $\Delta=0.25$, for minimum energy losses in the tamper of $0.80,0.93,0.95$ and 0.98 . 


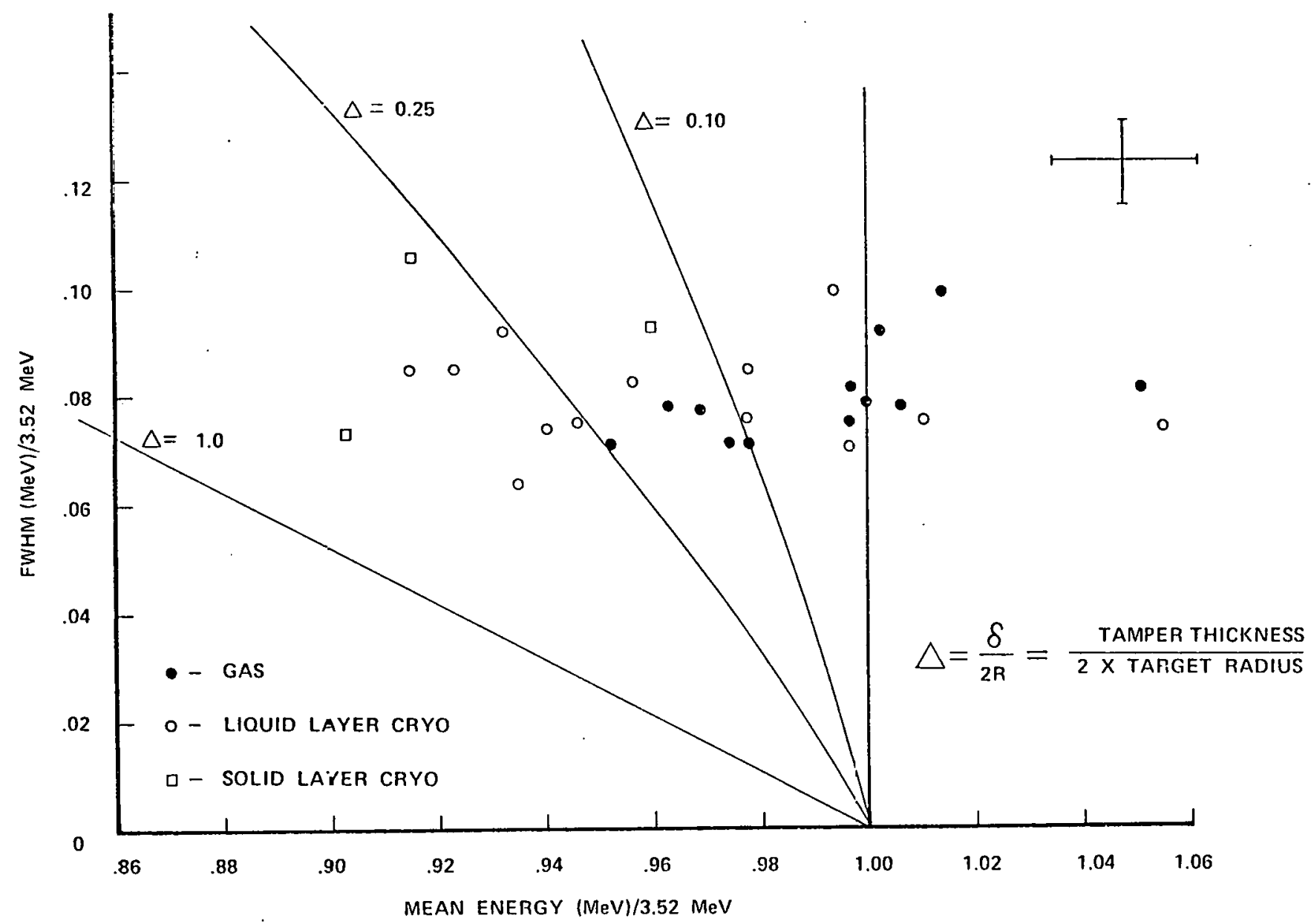

Fig. 2.1-11 FWHM of calculated alpha spectra vs their mean energy for an electron temperature of $1 \mathrm{keV}$ and $\Delta=0.10,0.25$, and 1.0 . 


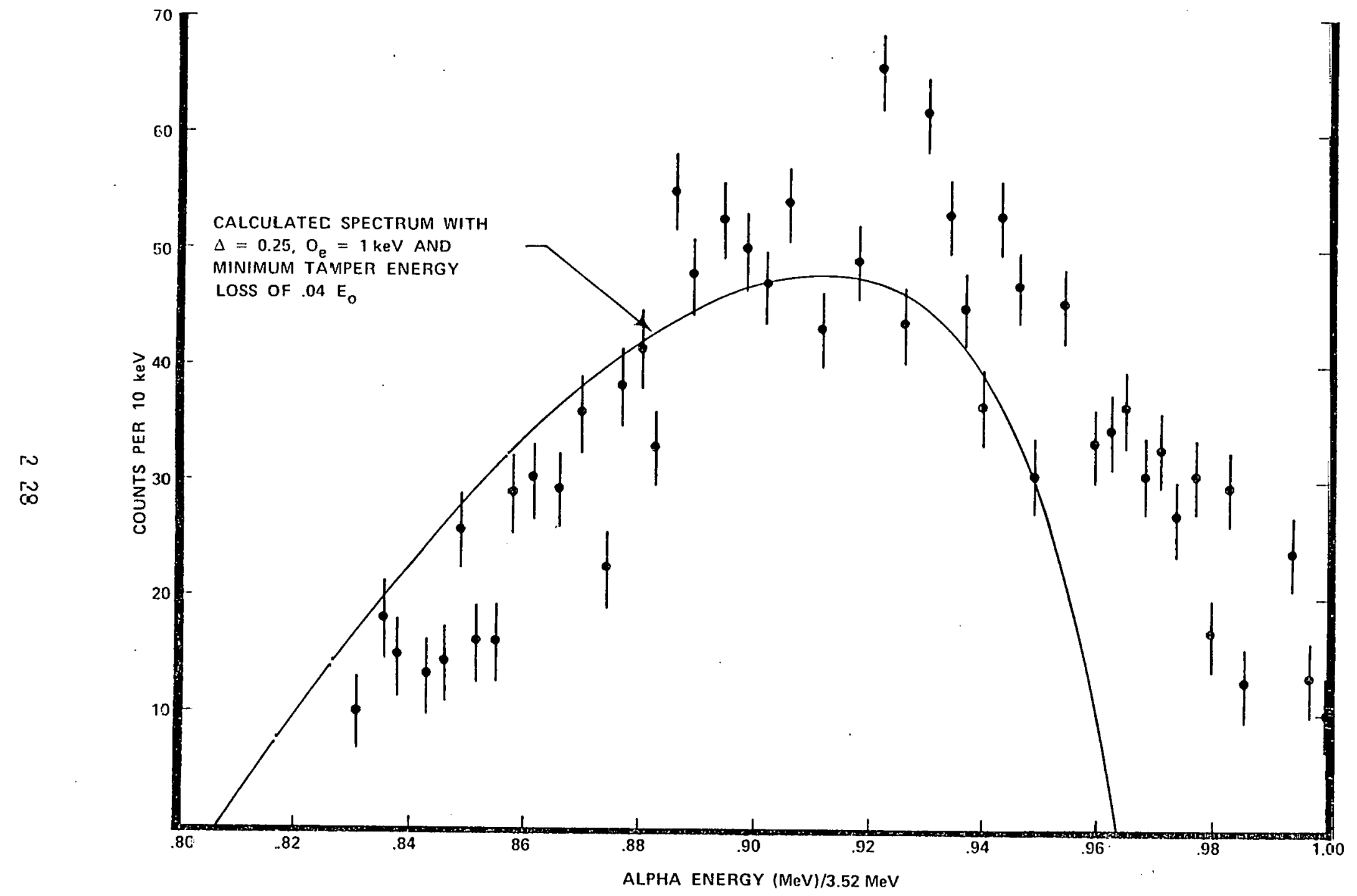

Fig. 2.1-12 Measured alpha spectrum for shot 3723 superimposed on a calculation of the alpha particle spectrum, with $\Delta=0.25, \theta_{e}=1 \mathrm{keV}$ and a minimum tamper energy loss of $.04 \Sigma_{0}$ 
it possible to determine the $\langle\rho R>$ of the fuel from a radiochemistry measurement of the $\langle\rho R>$ of the tamper. This will provide a direct density measurement, since $\rho_{f}=\left\langle\rho R>^{3 / 2} / \rho_{0}{ }^{1 / 2} R_{0}{ }^{3 / 2}\right.$.

Independent measurements of the fuel ion temperature and the neutron yield, which determine the compression, are not as sensitive as this but will be used for self-consistency. The fuel ion temperature will have to. be measured from the dispersion of the neutron signal since the above effects limit the usefulness of the ion temperature determination from alpha particle energy dispersion.

\section{REFEREI:CES}

1. C. E. Thomas, App1. Opt. 14, 1267, (1975).

2. K. A. Brueckner and J. E. Howard, App1. Opt. 14, 1274, (1975).

3. P. M. Campbeli, P. Hammerling, R. R. Johnson, J. J. Kubis, F. J. Mayer and D. C. Slater, "Plasma Physics and Controlled Nuclear Fusion Research" (Proc. Int. Conf., Berchtesgaden, 1976), IAEA, Vienna (1977).

4. 1977 Annual Report on Laser Fusion Research, KMS Fusion, Inc., Section 2. Laser Fusion Experiments.

5. See Section 2.1.2, reference 3 .

6. V. W. Slivinsky, H. G. Ahlstrom, K. G. Tirsell, J. Larsen, S. Glaros, G. Zimmerman and H. Shay, Phys. Rev. Lett. $\underline{35}, 1083,(1975)$.

7. R. R. Goforth, F. J. Mayer, H. Brysk and R. A. Cover, J. Appl. Phys. 47, 4850, (1976).

8. M. J. Boyle, D. T. Attwood, K. M. Brooks, E. M. Campbell, L. W. Coleman, L. N. Koppel, H. N. Kornblum, J. T. Larsen, P. H. Y. Lee, D. W. Phillion, R. H. Price, V. C. Rupert, V. W. Slivinsky, E. K. Storm and K. G. Tirsell, Lawrence Livermore Laboratory, UCRL-79779, November 1977.

9. H. Brysk, Plasma Phys. 15, 611, (1975)

10. K. A. Brueckner and H. Brysk, KMS Fusion Report U7, (1971).

11. R. A. Cover, J. J. Kubis, F. J. Mayer and D. C. Slater, Appl. Phys. Lett. 33, 222, (1978). 


\subsection{Fast Ion Measurements of Laser-Produced Spherical Plasmas}

\section{D.C. Slater}

The efficient compression of spherical laser-fusion targets requires the largest possible momentum in the blowoff plasma to drive the implosion. In present day experiments a substantial fraction of the absorbed laser energy is carried away by a relatively small number of energetic ions. These ions impart substantially less recoil momentum to the target than would a larger. number of ions sharing the same energy. Reliable measurements of the ion spectra over a broad range of laser and target parameters offer the possibility to understand better the nature and origin of the hot electrons which drive the ion acceleration and to improve further the efficiency of target compression and fusion energy release.

The targets used in these experiments were glass microshells 50$90 \mu \mathrm{m}$ in diameter with wall thicknesses of 0.7-1.1 $\mu \mathrm{m}$, containing approximately $2 \mathrm{mg} / \mathrm{cm}^{3}$ of deuterium and tritium gas. The Nd:glass laser was operated with up to 0.53 TW on target and with pulse length varied between 50 and $300 \mathrm{psec}$. The lens-mirror illumination system provides almost uniform illumination intensity of the spherical target surface at near-normal incidence. Measurements were made using KDP crystals to halve the wavelength of the incident light from 1.06 to $0.53 \mu \mathrm{m}$. (1)

The laser intensity at the target surface is böth $i 11$ defined and difficult to measure in these experiments. Power-dependent focusing effects in the laser and optics can lead to variations in laser intensity over the target surface and occasionally to focal volumes larger than the target size. In the pulse stacker system used to generate longer pulses from a series of short pulses, uncontrolled coherence effects can lead to substantial temporal variation of the intensity. Finally, the critical 
density surface where most of the light absorption occurs can move outward during the pulse, effectively lowering the irradiance. Despite these uncertainties we nevertheless follow standard practice by defining an irradiance I as the peak laser power divided by the original target surface area. The peak laser power is obtained from calorimeter measurements of the total laser energy and a streak camera record of the temporal history of the laser pulse. For several shots with pathological pulse shapes no irradiance was calculated.

The fast ions are detected on Kodak LR-115 Type II cellulose-nitrate film in a Thomson parabola spectrograph. (2) The combination provides resolution and sensitivity adequate to determine the velocity and charge state of oxygen, silicon, and carbon ions with velocities greater than $2 \times 10^{8}$ $\mathrm{cm} / \mathrm{sec}$. Hydrocarbon contamination of the target surface also introduces a number of protons into the plasma. These are detected only in the 100$200 \mathrm{keV}$ energy range, so that their spectra are not fully determined. Because they are few in number, it is not likely that they represent a substantial energy loss, in contrast to plastic foil target experiments. (3)

A typical ion velocity spectrum is shown in Figure 2.2-1. Here ion spectrograph data are combined with measurements from an adjacent charge collector to display the spectrum over a broad energy range. A transition region around $2 \times 10^{8} \mathrm{~cm} / \mathrm{sec}$ separates two exponential portions of the spectrum. The data presented below deal only with the high velocity ion group, i.e. those with sufficient velocity to be detected in the Thomson parabola spectrograph. Only the fully-stripped ions are analyzed. A previous study of charge state distributions obtained under similar experimental conditions showed that fully-stripped ions comprise about $40 \%$ of the total number of fast ions and have the same velocity distribution. (4) 


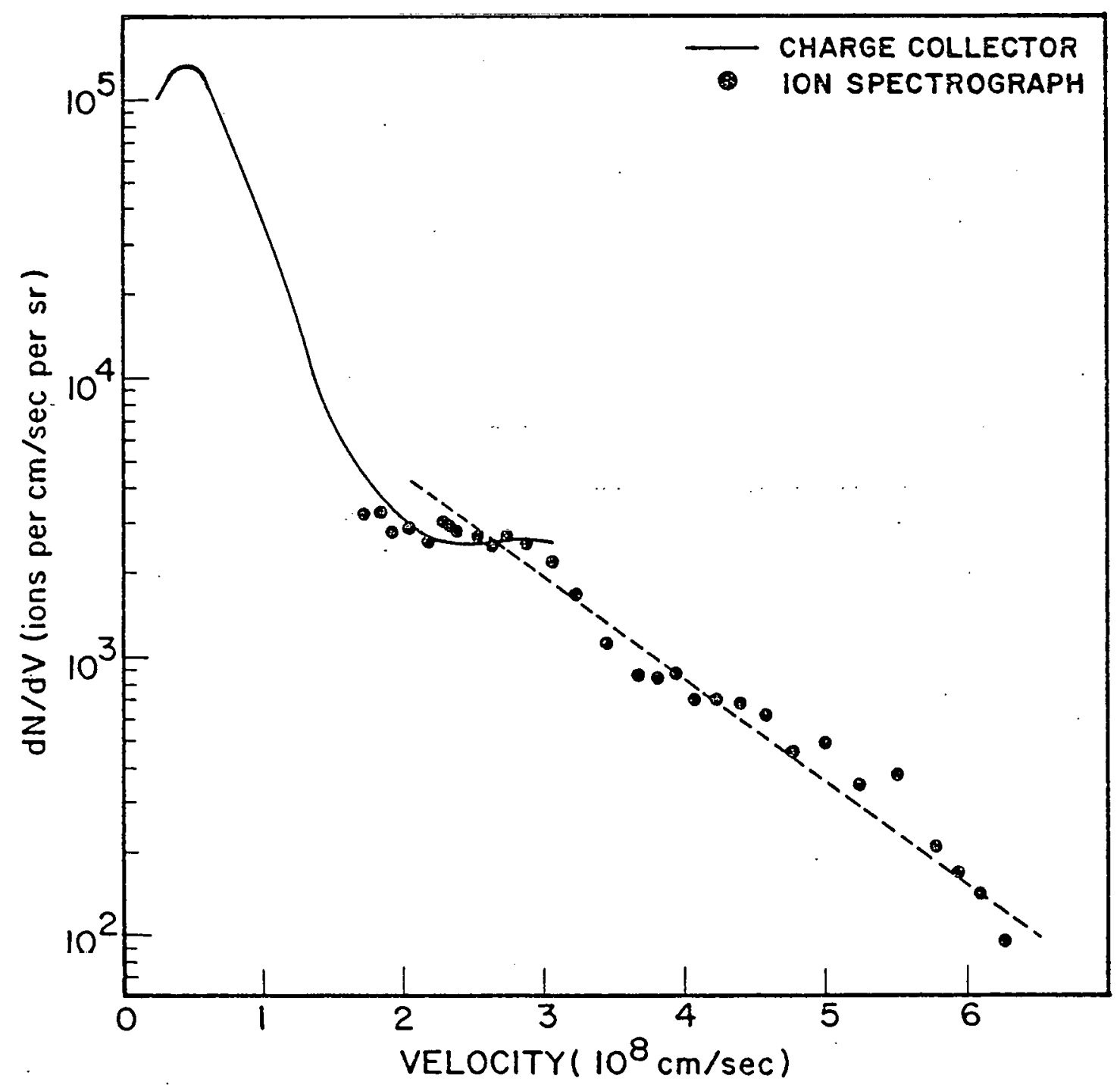

Fig. 2.2-1 - Ion Velocity spectrum from a spherical glass target $53 \mu \mathrm{m}$ in diameter with $0.7 \mu \mathrm{m}$ wall, irradiated with 34 joules in a pulse of approximately $90 \mathrm{psec}$. The dashed line has the form $d N / d v=A \exp \left(-v / v_{0}\right)$, with $v_{0}=1.2 \times 10^{8} \mathrm{~cm} / \mathrm{sec}$ and $A=2.3 \times 10^{4}$ ions per $\mathrm{cm} / \mathrm{sec}$, per $\mathrm{sr}$. 
The inverse exponential character allows most of the fast ion spectra to be fully characterized by two parameters; the extrapolated intercept $A$ and the scale velocity $v_{0}$, such that

$$
\frac{d N}{d v}=A \exp \left(-v / v_{0}\right)
$$

A strong inverse correlation between these two parameters is evident in Figure 2.2-2, in which $A$ is plotted against $v_{0}$ for all spectra with well defined values of these parameters, regardless of laser energy or pulse length. This correlation can be parametrized in a number of ways. For example, if we hypothesize that the number of ions $N^{*}$ with velocities greater than some value $v^{\star}$ is fixed, then

$$
A=\frac{N^{*}}{v_{0}} \exp \left(v^{*} / v_{0}\right)
$$

The solid line in Figure 2.2-2 has this form, with $v^{*}=2 \times 10^{8} \mathrm{~cm} / \mathrm{sec}$ and $N^{*}=2 \times 1011$ fully-stripped ions per steradian. Although equally good fits can be obtained with other parametrizations, the properties of $N^{*}$ and $v *$ make Equation (25) a useful representation.

When the data in Figure 2.2-2 are grouped according to the incident laser energy (but still with varying power), better fits are obtained by making $N^{*}$ proportional to this energy. Two such groupings are shown in Figure 2.2-3. The solid circles correspond to shots with 23-27 joules on target and are fitted with $N^{*}=2 \times 10^{11}$ ions per steradian. The open circles are for shots of 13-16 joules and are better matched with $N^{*}=1 \times 10^{11}$. In both cases, $v^{*}=2 \times 10^{8} \mathrm{~cm} / \mathrm{sec}$, indicating that this parameter is relatively insensitive to the laser pulse details.

The $x$ 's on Figure $2.2-2$ represent data taken with $0.53 \mu \mathrm{m}$ wavelength illumination. The energy on target for these shots ranged from 11 to 29 joules, but at powers of only 0.1 to $0.2 \mathrm{TW}$. These data suggest that fewer 


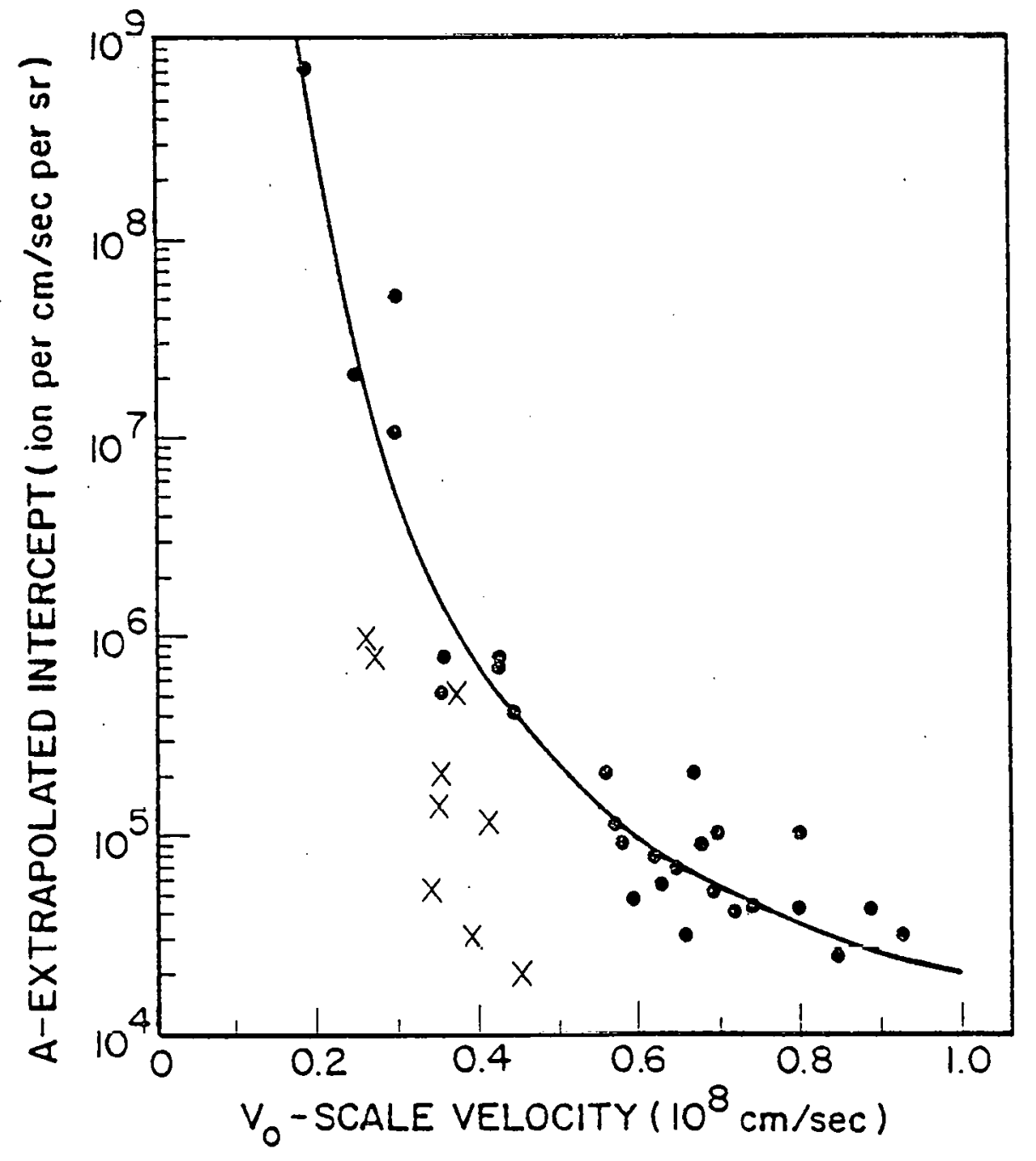

Fig. 2.2-2 - The parameter $A$ versus $v_{0}$ for all spectra of fully-stripped fast ions. The solid line has the form $A=\left(N^{*} / v_{0}\right) \exp \left(v^{*} / v_{0}\right)$ with $N^{*}=2 \times 10^{11}$ ions per $\mathrm{sr}$ and $\mathrm{v}^{*}=2 \times 10^{8} \mathrm{~cm} / \mathrm{sec}$. The $x$ 's are data obtained with $0.53 \mu \mathrm{m}$ illumination. 


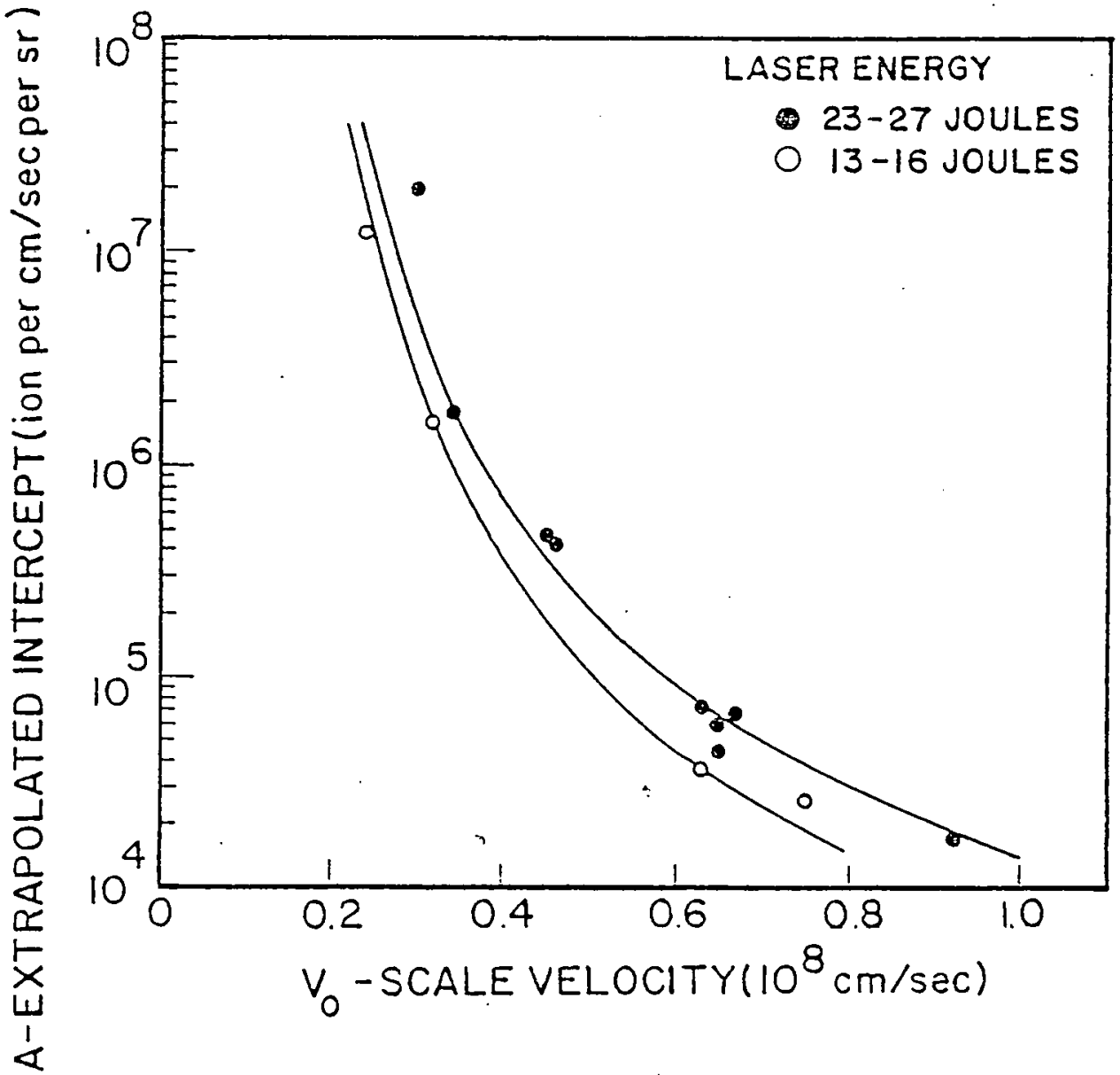

Fig. 2.2-3 - A versus $v_{0}$ for two values of incident laser energy. The curves have the form given in Figure 2.2-2, with $N^{*}=2 \times 10^{11}$ ions per $s r$ for the solid line and $N^{*}=1 \times 10^{8}$ for the dashed line. In both cases $\mathrm{v}^{*}=2 \times 10^{8} \mathrm{~cm} / \mathrm{sec}$. 
fast ions are created in green-light experiments. This conclusion must be considered only preliminary, however, because no accurate measure of absorbed energy was available for these shots. The decrease in fast ion yield might result from a decrease in absorption due to an increase in the focal volume rather than a true wavelength effect.

The data in Figure 2.2-4 represent a set of $1.06 \mu \mathrm{m}$ shots with the irradiance fixed at $(1.4-1.7) \times 10^{15} \mathrm{~W} / \mathrm{cm}^{2}$ but with various pulse lengths and energies. $N^{*}$ is plotted as a function of incident laser energy in Figure 2.2-4A. The data suggest that $N^{*}$, the number of ions with velocity greater than $2 \times 10^{8} \mathrm{~cm} / \mathrm{sec}$, is approximately proportional to the total laser energy, at fixed intensity. The scale velocity $v_{0}$, plotted in Figure 2.2-4B, is almost constant for these shots, decreasing slightly for the longer pulses. This decrease might result from a decrease in the timeaverage irradiance as the critical density surface expands.

The scale velocity $v_{0}$ is largely determined by the irradiance I. This dependence is demonstrated by plotting $v_{0}$ versus $I$ in Figure 2.2-5. The closed circles represent shots with 23-27 joules of laser energy, while the open circles correspond to all other values in the range 13-32 joules. Despite some scatter in the plot, it is evident that $v_{0}$ is essentially independent of laser energy. The dependence on laser irrádiance is approximately as the square-root, which is illustrated by the straight line. The three triangular points are shots in which the laser output was frequency doubled so that the target was illuminated with $0.53 \mu \mathrm{m}$ green light. These limited data are essentially indistinguishable from the $1.06 \mu \mathrm{m}$ wavelength shots, showing no strong wavelength dependence in the fast ion scale velocity at $\sim 6 \times 10^{14} \mathrm{~W} / \mathrm{cm}^{2}$.

Accurate determination of the fraction of the absorbed energy which 

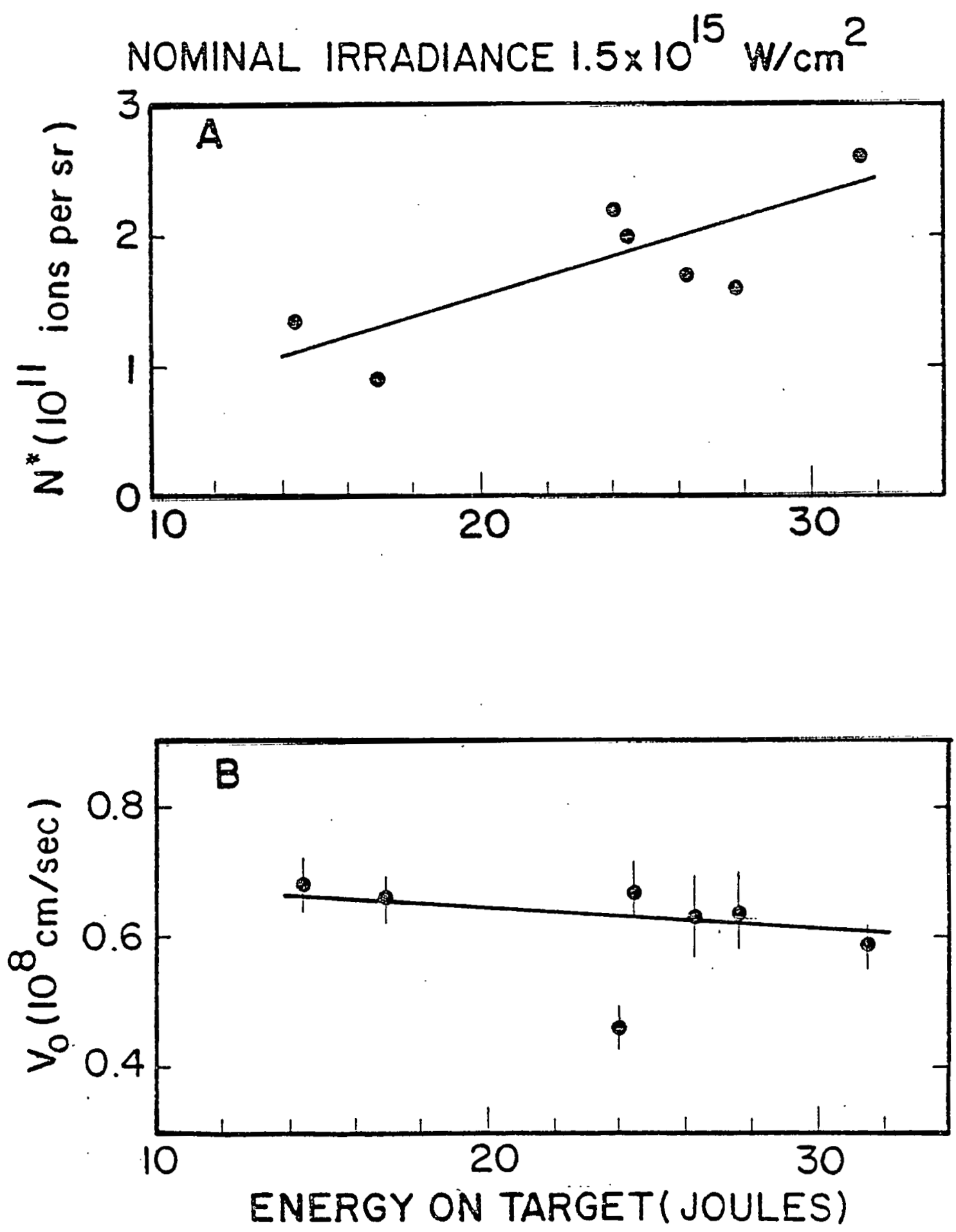

Fig. 2.2-4 - (A) Number of ions with velocity greater than $2 \times 10^{8} \mathrm{~cm} / \mathrm{sec}$, and (B) the exponential scale velocity versus incident laser energy. For all these shots the laser intensity at the target surface was about $1.5 \times 1015 \mathrm{~W} / \mathrm{cm}^{2}$. 


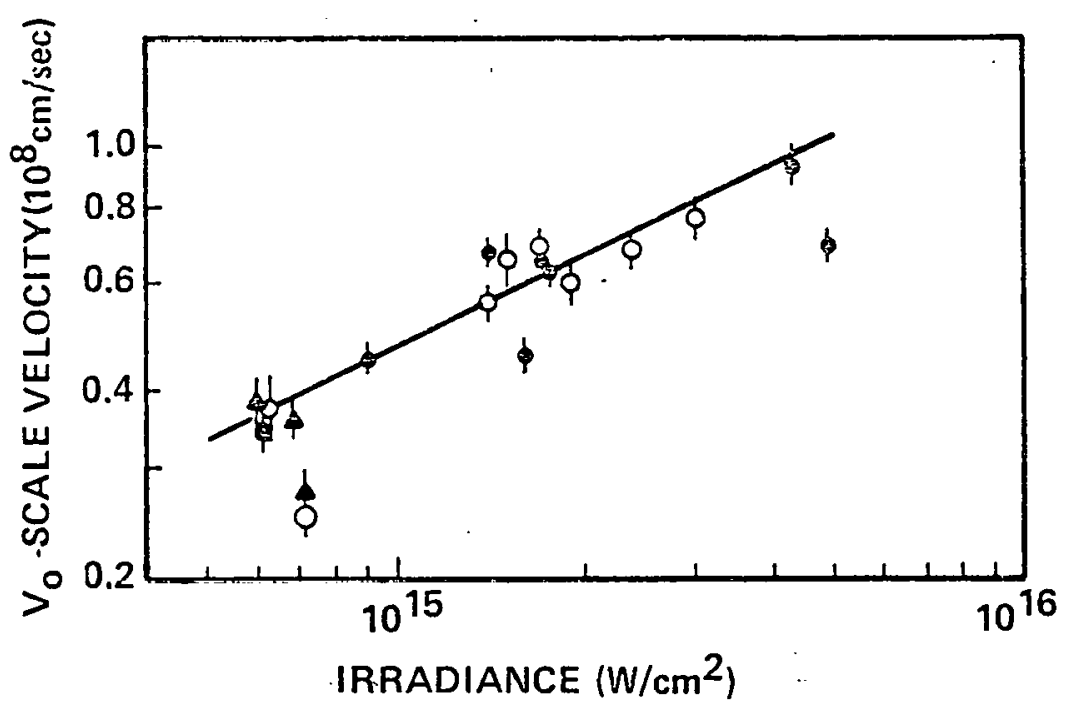

Fig. 2.2-5 - Exponential scale velocity versus irradiance. The solid circles represent shots with 23-27 joules on target, the open circles all other incident energies. The triangles show data with $0.53 \mu \mathrm{m}$ wavelength incident light obtained by frequency doubling the laser output.

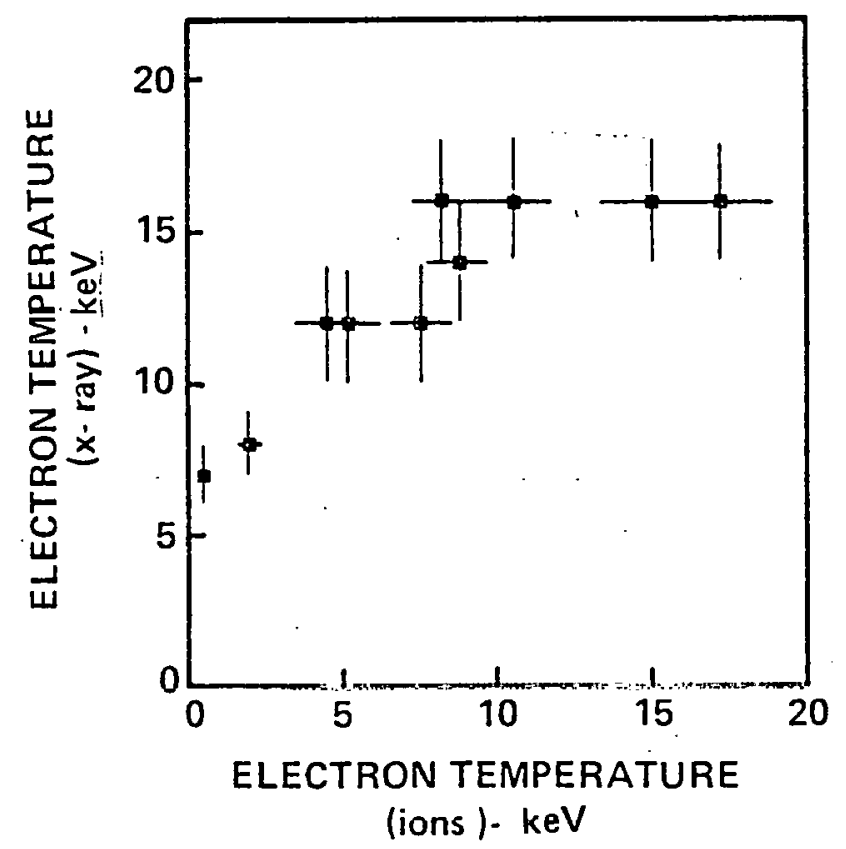

Fig. 2.2-6 - Hot electron temperatures obtained from x-ray spectra compared with those obtained from fast-ion spectra. 
is carried by the fast ions has not been made over a wide range of laser energy and irradiance. To do so would require a complete accounting for a 17 heavy ion charge states and for the protons. Estimates of the total number and energy of fast ions can be made by integrating the appropriate function with empirical values of $N^{*}$ and $v_{0}$, then multiplying the results by a factor of 2.5 to account for partially stripped ions. For the shots with $I \sim 1.5 \times 10^{15} \mathrm{~W} / \mathrm{cm}^{2}$ this procedure gives an average of $20 \%$ of the absorbed energy in ions with velocities in excess of $2 \times 10^{8} \mathrm{~cm} / \mathrm{sec}$. When $N^{*}=2 \times 10^{11}$ ions per sr, the total number of fast ions in all charge states corresponds to $0.2 \mathrm{ng}$ of glass, which is less than $0.5 \%$ of an $85 \mu \mathrm{m}$ diameter, $1 \mu \mathrm{m}$ wall target.

The relation of the scale velocity $v_{0}$ to the corona electron temperature has been studied with the TRHYD simulation code. The occurence of hot electrons in laser fusion plasmas is generally considered detrimental to the implosion process which compresses the fuel. Hot electron preheat substantially decreases the achievable compressed fuel density, and the concentration of a substantial fraction of the absorbed energy in a small number of particles diminishes the efficiency of the ablation process. One source of these electrons is the resonance absorption process, although others may exist as well. The behavior of hot electrons, once created, is a subject of intense current interest.

Direct measurements of the spectra of energetic electrons have been made, $(5,6)$ but with detectors situated far from the laser-irradiated target. It is not clear how these measurements relate to the plasma conditions at the time of irradiation. The traditional technique for deducing hot electron temperatures and numbers is via the energy spectrum of $x$-rays emitted from the target. Assuming the many-keV radiation is generated by brems- 
strahlung, the slope of the x-ray energy spectrum is a measure of the electron energy spectrum and hence of the electron temperature. More recently, the velocity spectra of high energy ions have been shown to have the form expected from the self-similar solutions of an isothermal rarefaction model. (7) This correspondence suggests that the fast-ion velocity spectrum may provide a good measure of the corona electron temperature. A comparison of $x$-ray-deduced with ion-deduced electron temperatures, shown in Figure 2.2-6, shows a clear correlation but not detailed agreement. Here we explore more fully the relationship between the fast ion velocity spectrum and the corona hot electron temperature.

In the isothermal rarefaction model, mass flows from the ablation front into an isothermal corona whose temperature is maintained by electron conduction, with energy absorbed from the incident laser flux. The similarity solutions of the hydrodynamic equations in planar geometry give an exponentially falling ion velocity distribution $d N / d v \sim \exp (-v / c)$, where $c$ is the sound speed at the isothermal electron temperature, given by $c=\sqrt{Z \Theta T}$. The parameters $Z$ and $M$ are the ionic charge state and mass. This distribution obtains at the end of the laser pulse, long before the ions have reached any detector. At the end of the laser pulse, 3/8 of the ablation energy is still thermal and will presumably be converted to additional ion kinetic energy as the plasma expansion continues adiabatically. We therefore take the experimentally determined velocity scale factor to be greater than $c$ by a factor of $8 / 5$. Other investigators prefer not to make this modification. (8) This model has been extended to include the possibility of two coexisting electron groups with different temperatures. $(8,9)$ The velocity spectrum of the fastest ions retains its exponential character with the scale velocity given by the sound speed at the tempcrature of the 
hotter electrons.

In applying this model to experiments with spherical illumination of spherical targets, two questions immediately arise. First, is the isothermal condition met, and secondly, does the spherical divergence of the flow affect the character of the velocity distribution? Since no simple similarity solutions have been found for spherical geometry, we have attempted to answer both questions with the help of our one-dimensional, spherically-symmetric hydrodynamic simulation code TRHYD.

Our purpose is to establish the relation between the fast ion velocity spectrum and the corona temperature during the laser pulse. The first question of paramount interest is whether the corona is characterized by a single temperature in both space and time. We have used a flux-limited model of electron energy transport and a combination of inverse bremsstrahlung absorption and resonance absorption. Resonance absorption is assumed to absorb fifteen percent of the incident energy, in rough agreement with what is needed to match experimental observations.

The flux limiting has been treated in two ways. In each case the value of the flux limit parameter is arbitrary. In the first method, three zones on each side of critical density are flux limited and the rest aren't. In the second method, the flux limiter is applied whenever the electron-toion temperature ratio exceeds four. The free-streaming limit on the electron thermal conduction is defined here to be

$$
F_{\max }=f(0.64) \frac{n_{e^{\theta} e^{3 / 2}}}{m_{e^{1 / 2}}{ }^{1 / 2}}
$$

Energy deposition into fast electrons is also done in two ways. In one method this energy is deposited uniformly per unit mass in the first three zones on each side of critical density radius. In the second, the 
energy is deposited uniformly per unit mass in zones up to one hybrid mean free path away from the critical density zone. The hybrid mean free path is defined to be $\lambda_{H}=\left(\lambda_{\text {ei }} \lambda_{\text {ee }}\right)^{1 / 2}$, where $\lambda_{\text {ei }}$ and $\lambda_{\text {ee }}$ are the electron-ion and electron-electron mean free paths, respectively. The electron velocity is taken to be three times the velocity of a thermal electron in the critical zone. $\lambda_{H}$ is the distance travelled by an average fast electron making $\lambda_{\mathrm{ee}} / \lambda_{\mathrm{ei}}$ steps of length $\lambda_{\mathrm{e}} \mathrm{i}$ in a random-walk fashion.

The flux limiter $f$ was varied from 1 to 0.005 . The incident laser power was varied between 0.1 and 2 TW, the pulse length from 40 to 120 psec, and the target size between 40 and $80 \mu \mathrm{m}$ in diameter. The number of zones at the outside of the target was increased so that smatr mass increments could be tracked as they move-away from the target. The evolution of the system was followed for one nanosecond. At this point, very little thermal energy remains in the outer zones and the ion velocity distribution is essentially frozen. The outermost zone is typically several millimeters from the origin.

It is important to understand that the ion acceleration in this model is due only to the pressure of a high-temperature thermal-electron group. The plasma is assumed everywhere quasi-neutral. We are not confronting the much more difficult problem of the behavior of a non-thermal electron group and the associated electric fields.

The first question we can ask of the simulation results is the extent to which the corona can be characterized by a single temperature. Figure 2.2-7 shows the electron temperature as a function of position and time for a simulation of a $0.5 \mathrm{TW}, 40 \mathrm{psec}$ laser pulse on an $80 \mu \mathrm{m}$ target, with the flux limiter $f=0.01$. In this case, a temperature of $\approx 6 \mathrm{KeV}$ is reached in less than $10 \mathrm{psec}$ and remains nearly constant for the remainder 


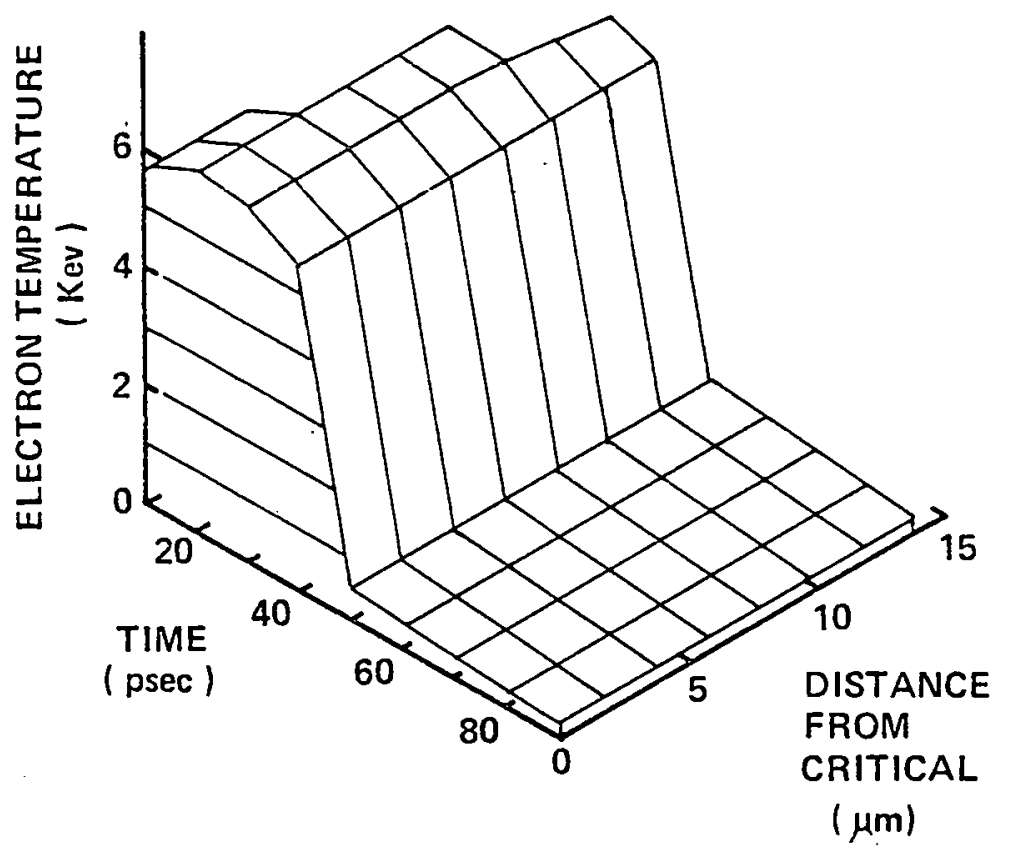

Fig. 2.2-7 - Calculated electron temperatures versus time from start of laser pulse and distance from critical-density radius. The laser irradiance was $2.5 \times 10^{15} \mathrm{~V} / \mathrm{cm}^{2}$ and the pulse length was $40 \mathrm{psec}$ in this computer simulation.

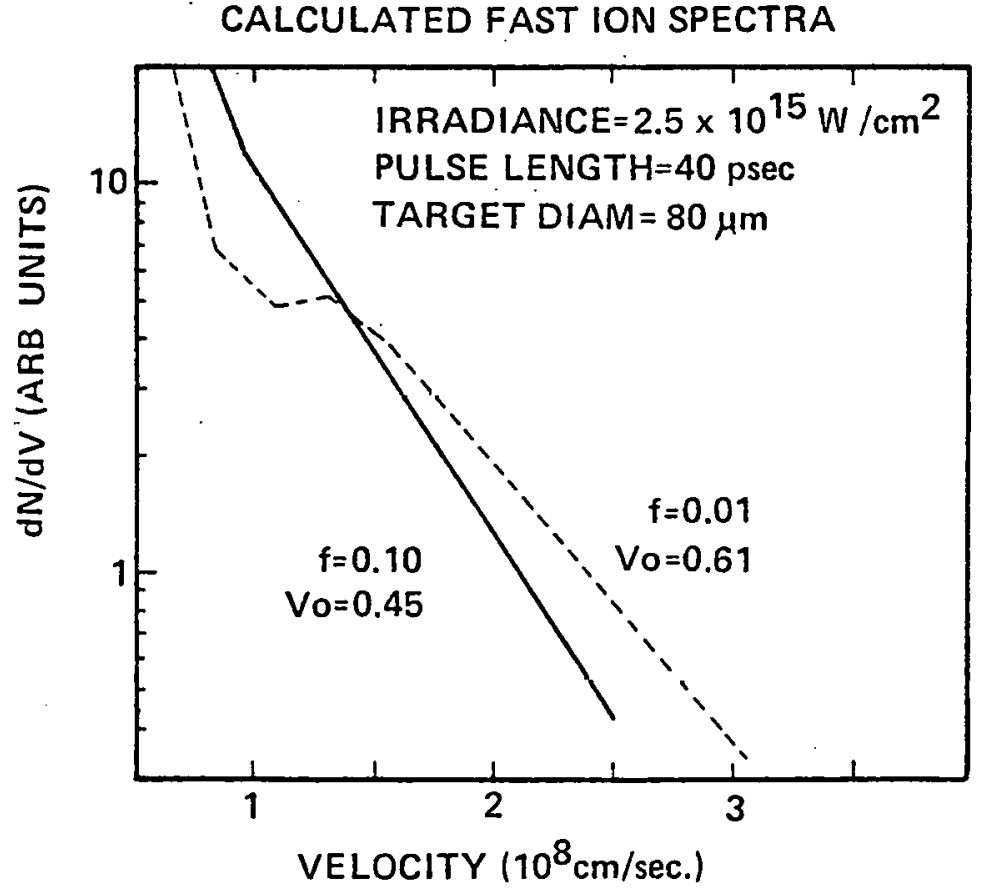

Fig. 2.2-8 - Computed fast-ion spectra for two simulation runs with different values of the flux limit parameter $f$. 
of the laser pulse. With no flux limiting $(f=1)$, the corona temperature generally increases gradually during the laser pulse. For strong flux limiting $(f<0.01)$ at higher laser intensity, the peak temperature is reached very early in the laser pulse. In these extreme cases, it is not possible to characterize the corona electrons with a single temperature. The isothermal nature of the corona did not depend on the choice of absorption and flux-limit models, except that the relation between the temperature and f-value changer.

The calculated ion velocity spectra from two simulations are plotted in Figure 2.2-8. The $f=0.01$ line gives the spectrum for the simulation described in Figure 2.2-7. The other curve results when the flux limiter is changed to 0.7 . The corona electron temperatures are 6.0 and $3.5 \mathrm{KeV}$, respectively. For each example, the spectrum of the fastest ions is well represented by an inverse exponential, with scale velocities of $6.3 \times 10^{7}$ $\mathrm{cm} / \mathrm{sec}$ and $4.6 \times 10^{7} \mathrm{~cm} / \mathrm{sec}$ respectively. If we let $\theta=k \frac{M v_{o}{ }^{2}}{Z}$, then $k=0.73$ and 0.80 for the two cases. Recall that in the planar isothermat rarefaction model, we choose $k=0.625$ as our normalization factor.

The relationship between the scale velocity and electron temperature for a variety of simulation runs is plotted in Figure 2.2-9. These runs span a broad range of laser intensity and pulse length, for flux-limiter values $0.01 \leq f \leq 0.1$ : The vertical error bars indicate the extent of the temporal variation of the electron temperature. The straight line, of the form given above, has $k=0.76$. This plot confirms that in this model the electron temperature can be determined from the slope of the fast-ion velocity spectrum. The data in Figure 2.2-5 are replotted in Figure 2.2-10 using the relationship between hot electron temperature and $v_{0}$ obtained from the code runs. The solid line is a general fit to a variety of data, obtained 


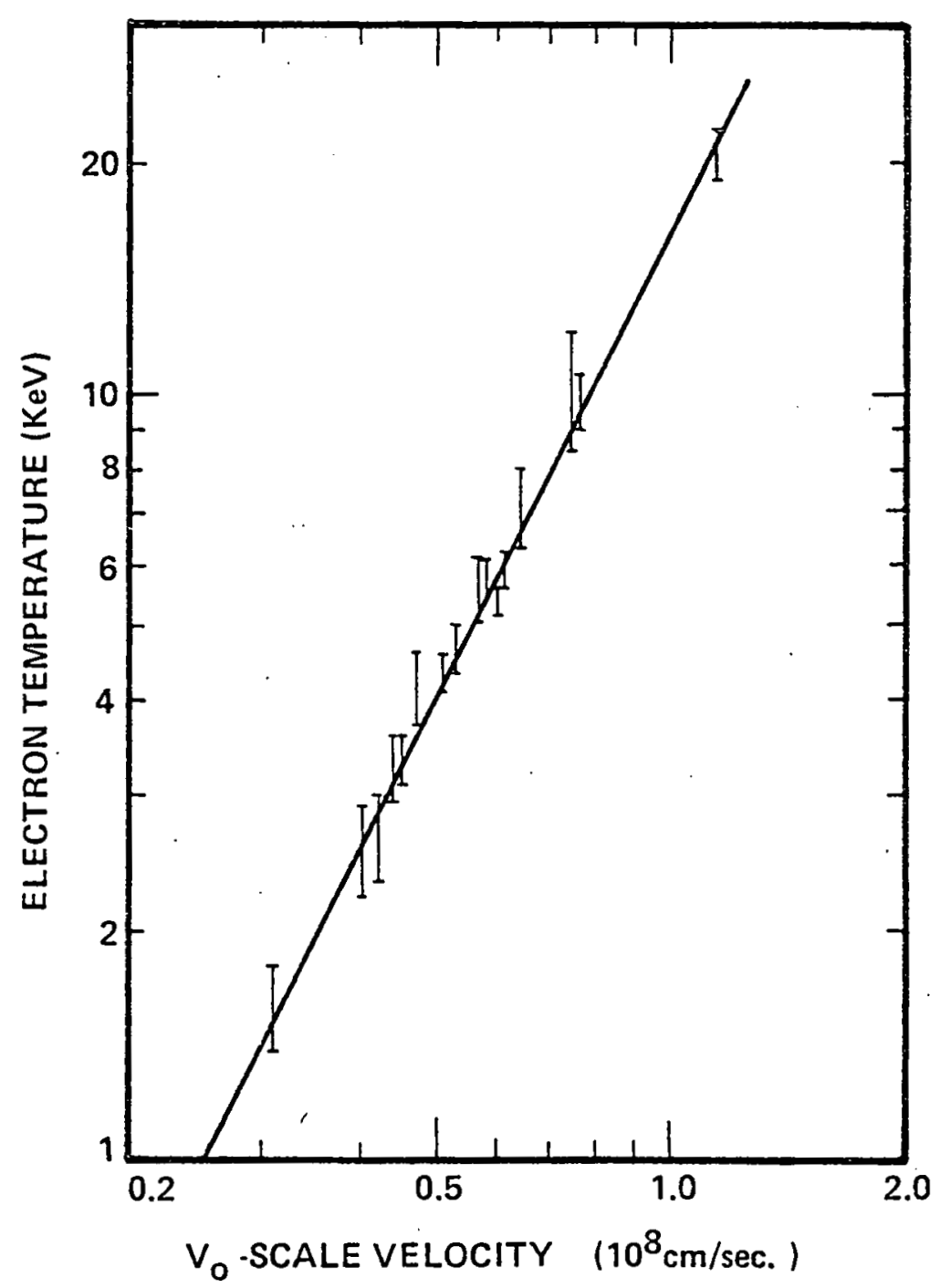

Fig. 2.2-9 - Electron temperature at the critical-density radius versus fast-ion velocity scale factor obtained from a variety of simulation runs. The straight line has the form $\theta_{e}=0.76 \frac{M}{Z^{v}} o_{0}^{2}$. 


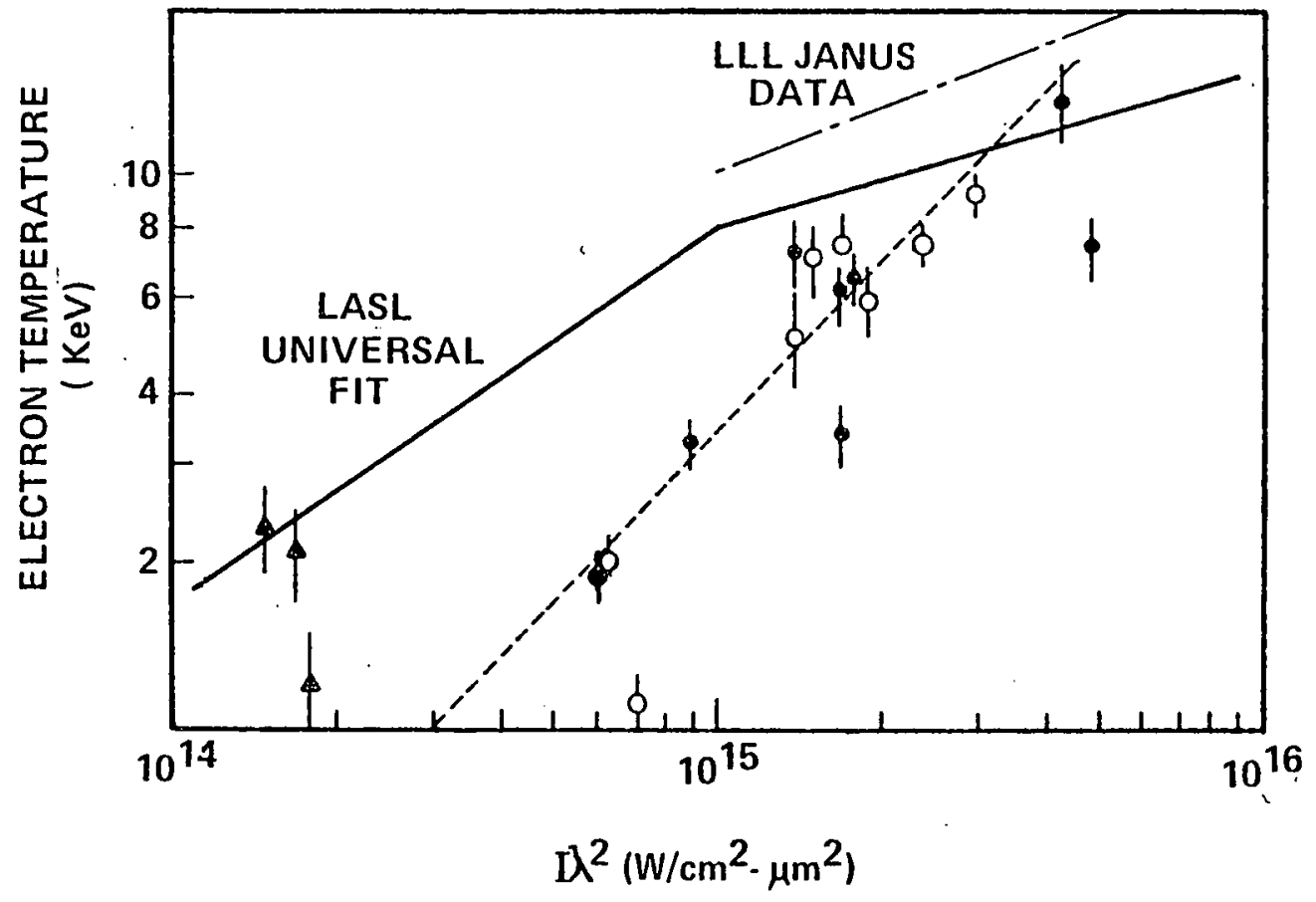

Fig. 2.2-10 - Hot electron temperatures deduced from the data of Figure 2.2-5, compared to data compiled from $x$-ray spectra at other labs. 
primarily from $x$-ray spectra, compiled at LASL. (10) The dot-dash line shows $x$-ray-determined temperatures obtained from glass-shell targets with the JANUS laser at LLL. (11) The dashed Iine approximates the fast-ion data taken with $1.06 \mu \mathrm{m}$ illumination, and defines a temperature proportional to the first power of the intensity. Above $10^{15} \mathrm{~W} / \mathrm{cm}^{2}$ these data are essentially consistent with the $x$-ray determined temperatures, but at lower intensity. values the discrepancy is greater. A similar result was seen in Figure 2.2-6, where the $x$-ray data were substantially above the fast ion data for temperatures below $5 \mathrm{keV}$.

In summary, we have shown that in one-dimensional, spherical hydrodynamic simulations with a flux-limit value of $0.01-0.1$, the scale velocity of the fast ion spectrum provides a good measure of the corona electron temperature. Hot electron temperatures obtained in this manner from measured ion spectra differ from those deduced from hard $x$-ray spectra. At low intensity the fast-ion method gives temperatures which scale proportionally with the laser irradiance. Preliminary results show similar temperatures for $1.06 \mu \mathrm{m}$ and $0.53 \mu \mathrm{m}$ light at about $6 \times 10^{14} \mathrm{~W} / \mathrm{cm}^{2}$ irradiance.

\section{REFERENCES}

1. T.A. Leonard, R.R. Johnson, D.C. Slater, S.B. Segall, KMS Fusion, Inc. Report, KMSF-U762, p. 2-17, (1978).

2. D.C. Slater, Rev. Sci. Instr. 49, 1493, (1978).

3. R. Decoste and B.H. Ripin, Appl. Phys. Lett. 31, 68 (1977)

P. Wagli, T.P. Donaldson, and P. Ladrach, App I. Phys. Lett. 32, 638 (1978) J.S. Pearlman and J.P. Anthes.

4. D.C. Slater, F.J. Mayer, and R.R. Johnson, Technical Digest of Topical Meeting on Inertial Confinement Fusion, San Diego, CA, (Optical Society of America), 1978, paper TuDlo.

5. D.V. Giovanielli, J.F. Kephart, and A.H. Williams, Los Alamos Scicntific Laboratory Report, LA-UR76-88. 
6. K.G. Tirsell, H.C. Catron, S.S. Glaros, H.N. Cornblum, and V.W. Slivinsky, Bul1. Ain. Phys. Soc., 22, 1195, (1977).

7. P.M. Campbell, P. Hammerling, R.R. Johnson, J.J. Kubis, F.J. Mayer, and D.C. Slater, Plasma Physics and Controlled Nuclear Fusion Research, (IAEA, Vienna, 1977), Vol. 1, p. 227.

8. L.M. Wickens, J.E. Allen, and P.T. Rumsby, Phys. Rev. Lett. 41 , 243 (1978).

9. Erick L. Lindman, Los Alamos Scientific Laboratory Report LAUR-77-1007, (1975).

10. D.W. Forslund, J.M. Kindel, and K. Lee, Phys. Rev. Lett., 39 , 284, (1977).

11. K.R. Manes, H.G. Ahlstrom, R.A. Haas, and J.F. Holzrichter, J. Opt. Soc. Am., 67, 717, (1977). 


\subsection{Absorbed Energy Measurements}

A.H. Bennish, S.B. Segall, and D.C. Slater

The energy absorbed by spherical targets has been studied as a function of four parameters of the incident laser light: the wavelength, pulselength, intensity, and bandwidth. The primary diagnostic device used to measure absorbed energy is the thermopile differential calorimeter described in the 1977 Annual Report. The differential calorimeter is positioned in the gap between two ellipsoidal mirrors which focus the laser light onto the target. Total absorbed energy values are obtained by assuming a uniform plasma blowoff and extrapolating the energy measured in the acceptance angle of the detector to $4 \pi$ solid angle. For the relatively uniform illumination pattern produced with the lens-ellipsoidal-mirror focusing system, this is a good approximation. For the long-pulse experiments, a second differential calorimeter was added. In addition, an energy calibration for the unfiltered TLD chips was obtained by comparison with the differential calorimeters over a large number of shots. While the TLD determination of absorbed energy showed a $\pm 20 \%$ uncertainty, it provides a good consistency check on the symmetry of the plasma blowoff:

Most of the target shots are taken using normal inciderce focusing for which the focal positions of the two beams are coincident with the center of the target. For some of the data the focal position of each beam was axially displaced 5 to $10 \mu \mathrm{m}$ relative to the center of the target in order to produce a more uniform illumination pattern on the surface of the target. No significant difference in absorption was noted in these cases compared with normal-incidence focusing.

Laser power for the wavelength comparison shots at short pulselength $(-100 \mathrm{psec})$ was in the range 0.070 to $0.160 \mathrm{TW}$ both at $1.06 \mu \mathrm{m}$ and 
$0.53 \mu \mathrm{m}$. Corona electron temperatures determined from ion velocity spectra using an isothermal model of the plasma blowoff indicated electron temperatures in the range of 1 to $3 \mathrm{keV}$. The experiments indicate that the fractional absorption was $21 \pm 4 \%$, at $1.06 \mu \mathrm{m}$ with and $20 \pm 3 \%$ without the plasma spatial filter, as shown in Figure 2.3-7. The experiments at 0.1 TW and $0.53 \mu \mathrm{m}$ using the plasma spatial filter showed a fractional absorption of $34 \pm 4 \%$, whereas without the plasma spatial filter the fractional absorption was $22 \pm 2 \%$. The data are shown in Figure 2.3-2.

A series of 62 target shots using $\sim 1$ ns laser pulses was performed in December 1978. Targets were spherical glass microshells ranging in diameter from 109 to 290 microns, and in wall thickness from 0.8 to 2.5 microns. The irradiance, obtained by dividing the laser power (averaged over the FWHM pulse time) by the target surface area, ranged from $2 \times 10^{13}$ to $5 \times 10^{14} \mathrm{~W} / \mathrm{cm}^{2}$. The measured energy absorption fractions are plotted against irradiance in Figure 2.3-3. Each point represents one target shot. The error bars are the standard deviation of a weighted average of the three absorbed energy measurements.

Two sets of points are shown in Figure 2.3-3. The solid-circle data were taken with the plasma spatial filter (PSF) installed in the laser system. The open-circle data, taken with the PSF removed, show lower absorption at all measured intensities. The PSF device changes the character of the laser beams in three ways. (1) First, it smoothes the time-averaged spatial intensity pattern, effectively removing the hot spots typical of an unfiltered beam. Second, it broadens the bandwidth of the light incident on the target from about $1 \AA$ to approximately $30 \AA$. The broadening does vary shot-to-shot. Finally, the beam is slightily less focusable with the PSF installed, so that the diameter of the volume enclosing $80 \%$ of the laser 
$\stackrel{\sim}{\prime}$

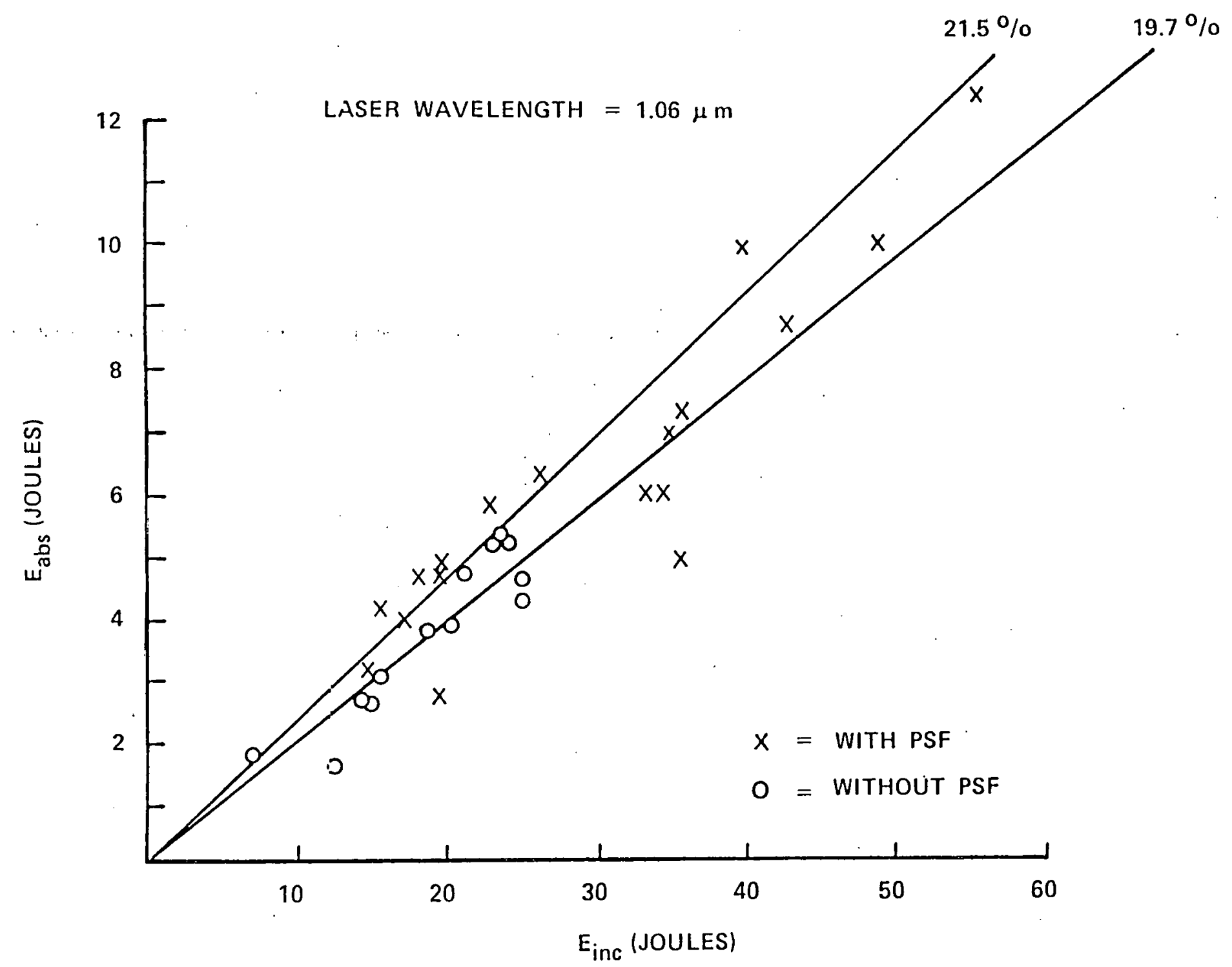

Fig. 2.3-1 Fractional absorption at laser wavelength of $1.06 \mu \mathrm{m}$, from $E_{\text {abs }} v E_{\text {inc }}$, with and without the plasma spatial filter. 


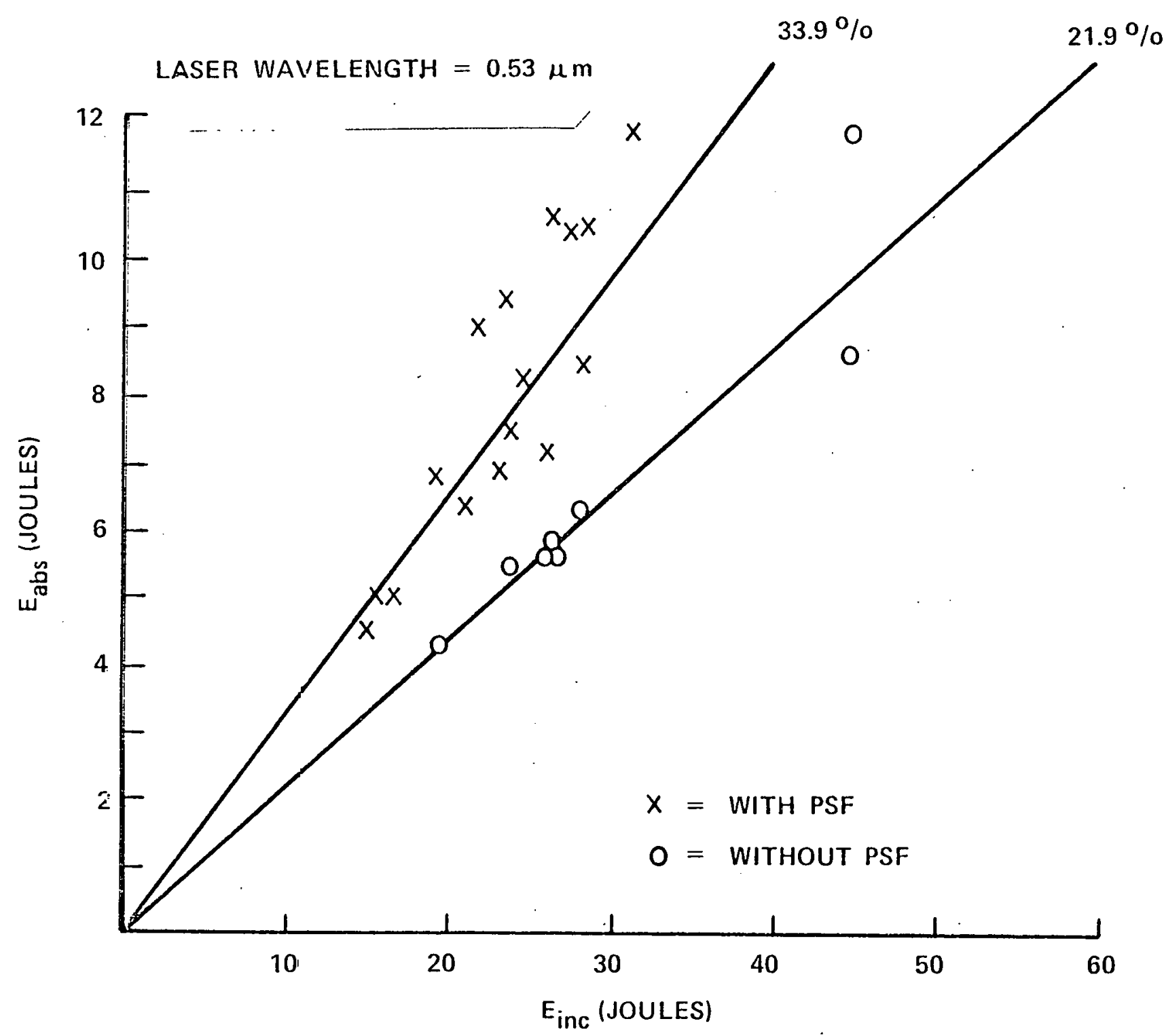

Fig. $2.3-2$ Fractional absorption at laser wavelength of $0.53 \mu \mathrm{m}$, from $E_{\text {abs }}$ vs $E_{\text {inc }}$, with and without the plasma spatial filter. 


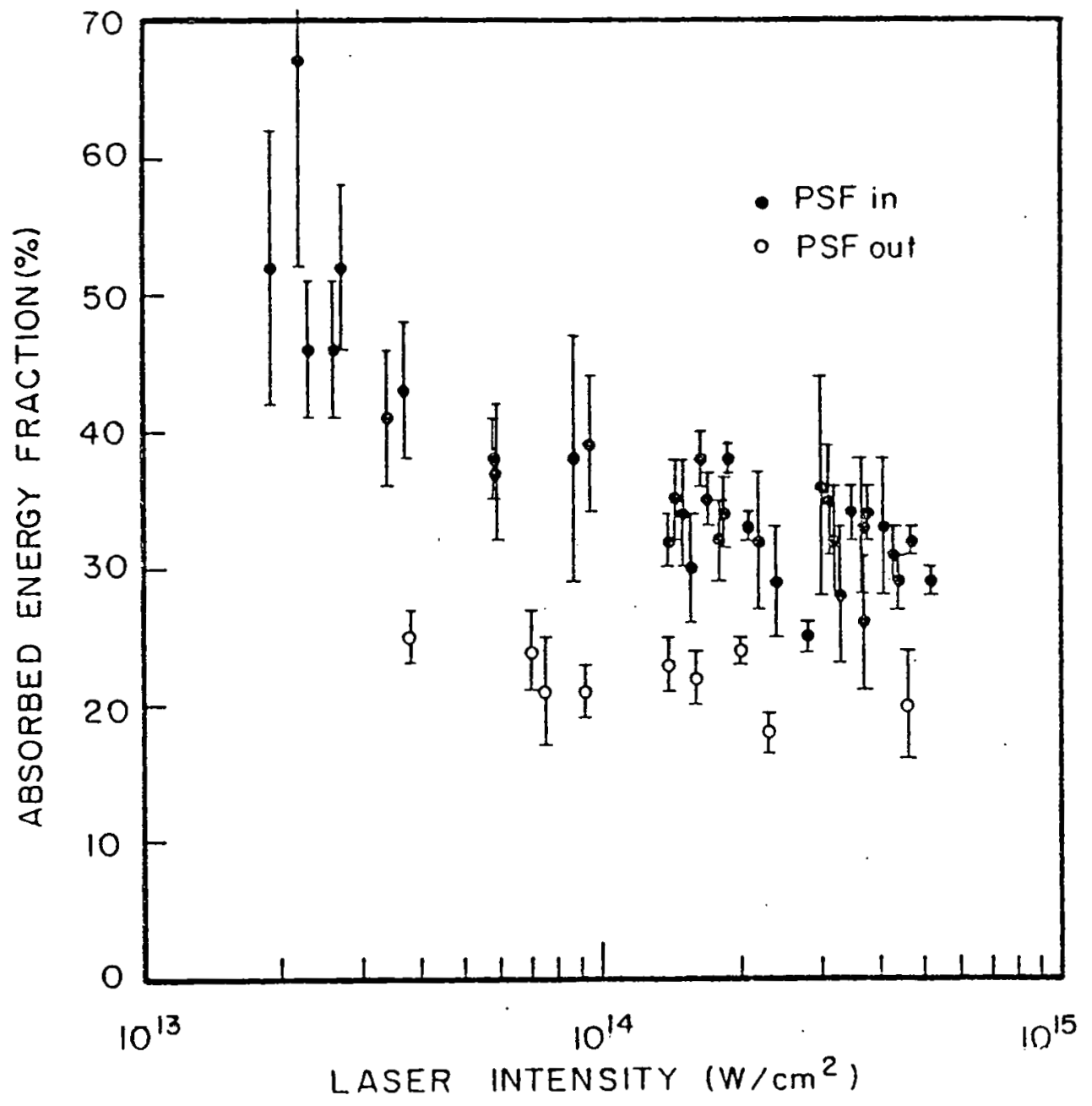

Fig. 2.3-3 Measured energy absorption fractions vs irradiance, with and without the plasma spatial filter. 
energy is about $30 \mu \mathrm{m}$ on average. Each of these three effects might cause a change in absorption, but we beleive the increase in absorption with the PSF is due largely to the increase in laser bandwidth.

If the absorption were a strongly decreasing function of laser irradiance, then a beam with substantial intensity fluctuations might show a lower absorption than a more uniform beam with the same average irradiance. Absorption measurements at the Lawrence Livermore Laboratory covering a range from $10^{14}$ to $10^{17} \mathrm{~W} / \mathrm{cm}^{2}$ show no such dependence on irradiance. (2). The data in Figure 2.3-3 also show that the absorption differences are somewhat greater at $8 \times 10^{13}$ than at $5 \times 10^{14} \mathrm{~W} / \mathrm{cm}^{2}$. Presumably the intensity fluctuations due to nonlinear self-focusing would be diminished at the lower irradiance, so that absorption differences due to intensity fluctuations would be less.

With the plasma filter installed, the beam focal volume is increased somewhat. This might cause a reduction in absorption if it increases refraction losses. However, as the focal volume diameter is several times smaller than the target diameter in all cases, the changes in refraction should be small.

This leaves the increase in laser bandwidth as the most likely reason for the improved absorption when the PSF was installed. Qualitatively, this agrees with one's expectations if stimulated Brillouin scattering (SBS) is effectively limiting the amount of energy reaching the critical density surface. At fixed intensity, the saturated SBS reflection is reduced by a factor proportional to the bandwidth if the bandwidth is sufficiently greater than the growth rate. (3)

A signature of SBS is that the reflected light should be red-shifted. Since the spectrum of the reflected light is already Doppler blue shifted, 
ABSORPTION BY SPHERICAL GLASS TARGETS AT $3 \times 10^{14} \mathrm{w} / \mathrm{cm}^{2}$ LASER INTENSITY

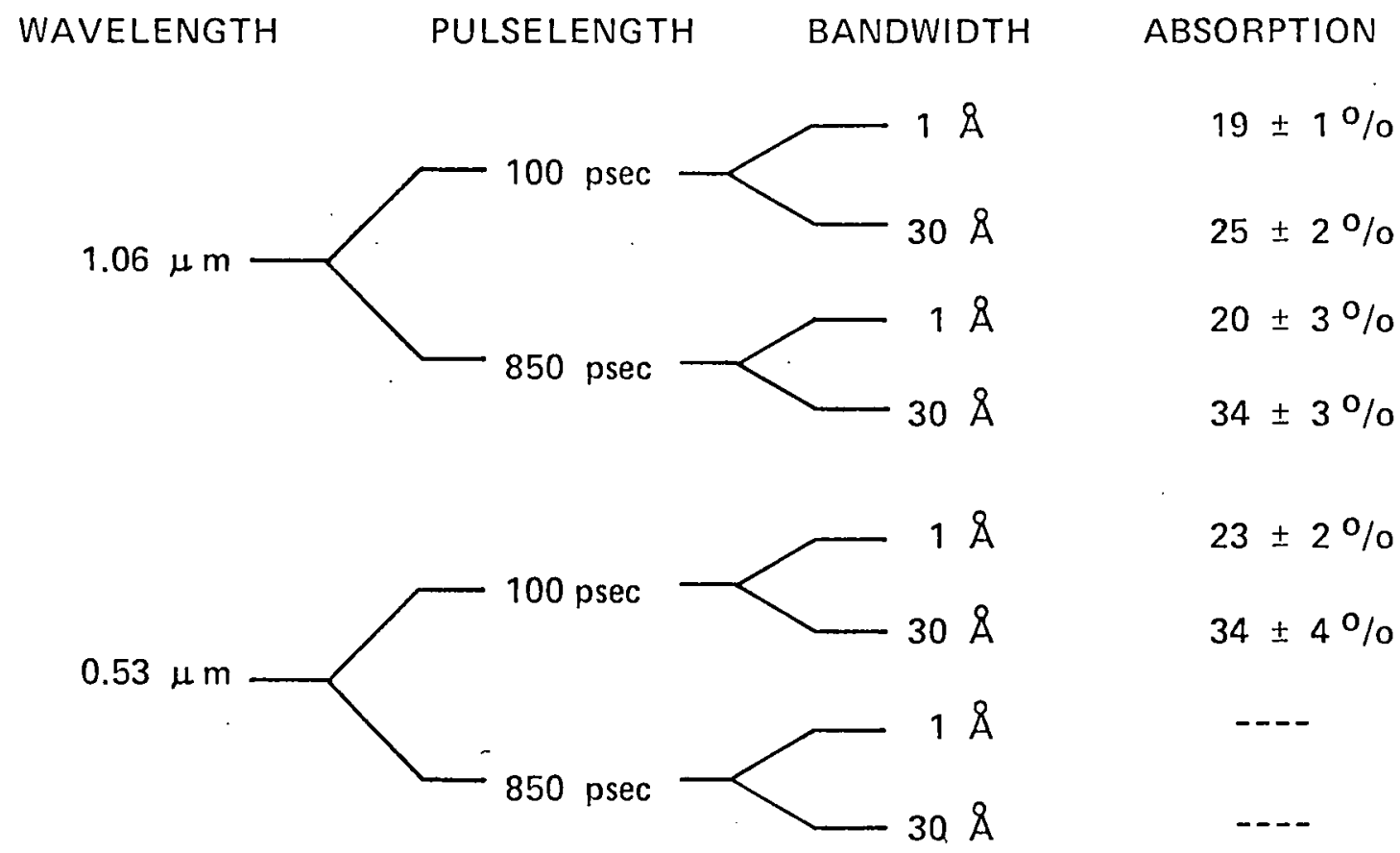

Fig. 2.3-4 Absorption by spherical glass targets at an irradiance of $3 \times 10^{14} \mathrm{~W} / \mathrm{cm}^{2}$, with wavelengths of $1.06 \mu \mathrm{m}$ and $0.53 \mu \mathrm{m}$, pulse lengths of $100 \mathrm{psec}$ and $850 \mathrm{psec}$, and bandwidths of $1 \AA$ and $30 \AA$. 
TABLE 2.3-1

SPECTRAL SHIFT OF $1.06 \mu \mathrm{m}$ RADIATION

RETURNED THROUGH FOCUSING OPTICS

WITH PLASMA FILTER

Shot

3582

3584

3597

3599

3560
$(\AA)$

$-16$

$-13$

$-16$

$-13$

$-8$
WITHOUT PLASMA FILTER

Shot

$(\AA)$

3613

3617

3614

3618

3622

3623
$-9.6$

$-4.8$

0.0

$-3.2$

$-6 . \overline{4}$

$-3.0$ 
one would expect a smaller blue shift with the PSF than without. Table 2.3-1 shows data on spectral shifts for targets irradiated with short pulse $1.06 \mu \mathrm{m}$ light. On the average, target shots for which the PSF was on-line produced larger blue shifts than target shots without the PSF, indicating that some of the laser light is being lost due to SBS and that use of the plasma spatial filter reduces this loss. Spectral data on the long-pulse shots would be expected to show an even greater difference, but these data are not yet available.

The results of these absorption measurements are summarized in Figure 2.3-4. The most striking result is the absorption improvement due to the PSF. We believe these data demonstrate that laser bandwidth can be used to reduce stimulated Brillouin scattering and allow greater absorption to occur.

\section{REFERENCES}

1. N.K. Moncur, Appl. Opt. 16, 1449, (1977).

2. K.R. Manes, H.G. Ahlstrom, R.A. Haas, and J.F. Holzrichter, J. Opt. Soc. Am. $\underline{67}, 717,(1977)$.

3. Kent Estabrook, Lawrence Livermore Laboratory Report UCRL-81017 (1978) and references therein. 


\section{$2.4 \quad$ Diagnostic Development}

\subsubsection{X-Ray Streak Camera}

G. Charatis and D. Sullivan

Implosion dynamics of laser irradiated targets, to be properly assessed, must be examined with an x-ray streak camera, (1) capable of resolving the entire implosion process.

KMSF has procurred from G.E.A.R., Inc. an x-ray proximity-focused streak camera capable of resolving 3 to 5 psec $x$-ray emission features. Employing a $100 \mu \mathrm{m}$ wide slit at the camera's gold photo-cathode it is also possible to obtain space-time $(x-t)$ information of the implosion process, if a pinhole is used for imaging the target at the slit. Specifications of the camera are listed in Table 2.4-1.

Figure 2.4-1 is a photograph of the camera, which is designed to interface with a flange of the target chamber. The camera slit, the target and the pinhole are aligned with a specially designed opto-mechanical system shown in Figure 2.4-2. As can be seen schematically in Figure 2.4-3 the telemicroscope is positioned to define the line between the center of target and the center of the camera slit. The pinhole mounted on a movable snout is then also positioned on this same center-line.

Figure 2.4-4 shows typical time-signatures of $x$-ray emission from a $76 \mu \mathrm{m}$ diameter glass shell target as recorded by the camera through $1 \mathrm{mil}$ and 3 mils of berylluim.

Attempts to obtain $x-t$ measurements with target imaging via a $25 \mu \mathrm{m}$ diameter pinhole were not successful. Since the target-illuminating ellipsoidal mirrors do not allow the camera photo-cathode to be placed closer than $30 \mathrm{~cm}$, it will not be possible to operate the camera in $x$-t mode unless more $x$-ray flux is concentrated at the camera slit with an x-ray microscope. 
TABLE 2.4-1

OPERATING SPECIFICATIONS OF X-RAY STREAK CAMERA

Temporal Resolution (claimed)

Spatial Resolution

Trigger:

Triggering Jitter

Trigger Delay (at highest sweep velocity)

Inverse Sweep Velocity

- with $7.6 \mathrm{kV}$ across trigger gap

- with $4.4 \mathrm{kV}$ across trigger gap

Record Length

- at sweep velocity of $8.3 \mathrm{psec} / \mathrm{mm}$

- at sweep velocity of $28 \mathrm{psec} / \mathrm{mm}$
3 psec at $1 \mathrm{keV}$

3 line pairs/mm

laser-triggered dielectric gap

less than $\pm 30 \mathrm{psec}$

$20 \mathrm{psec}$

$8.3 \mathrm{psec} / \mathrm{mm}$

$28 \mathrm{psec} / \mathrm{mm}$

150 psec

$350 \mathrm{psec}$ 


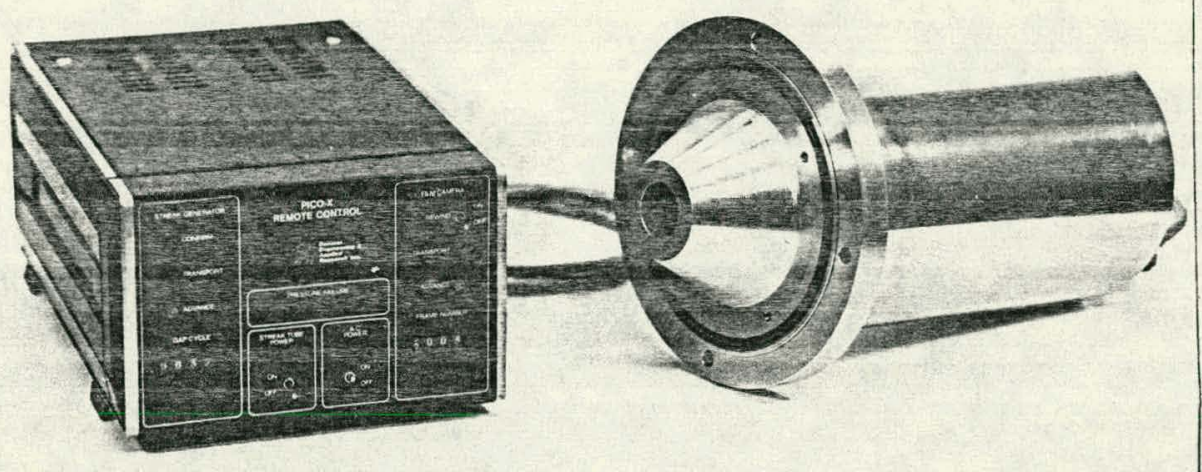

Fig. 2.4-1 - X-Ray streak camera with control electronics.

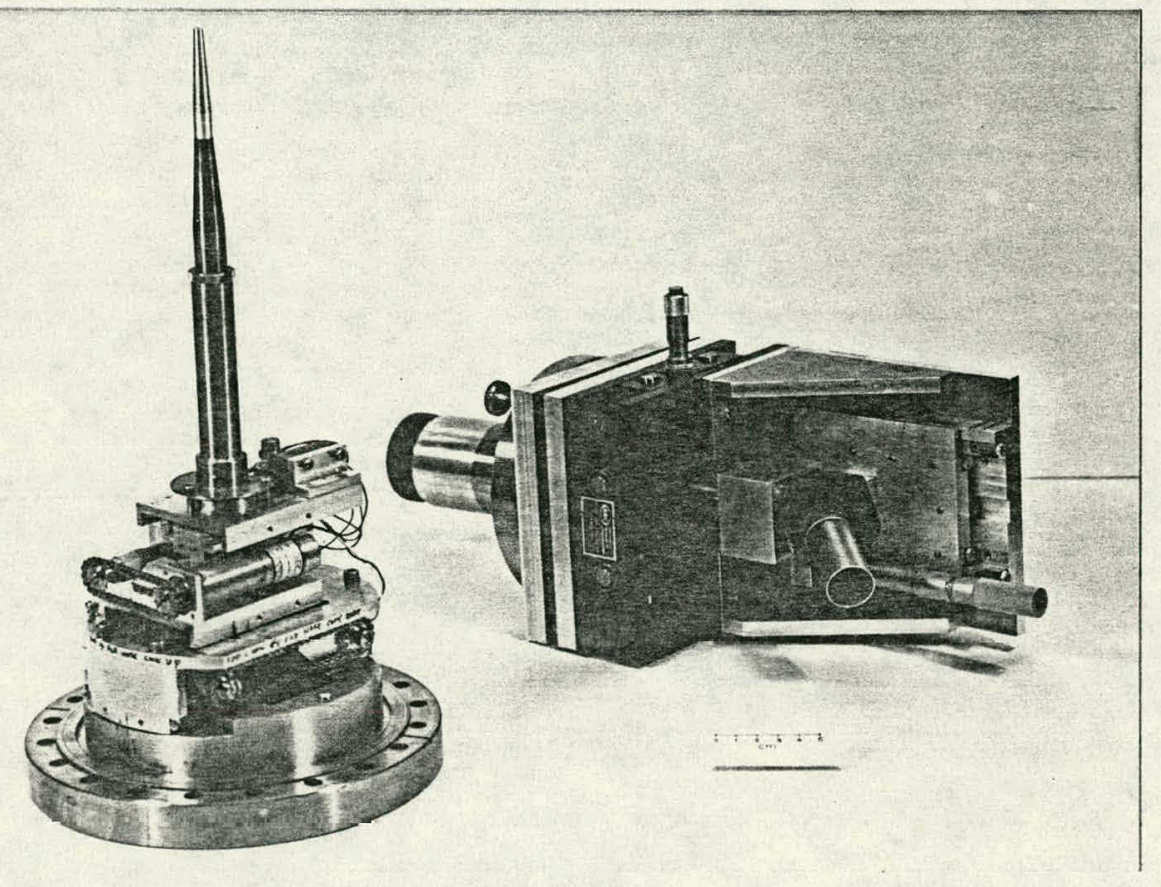

Fig. 2.4-2 - Opto-mechanical alignment system. 


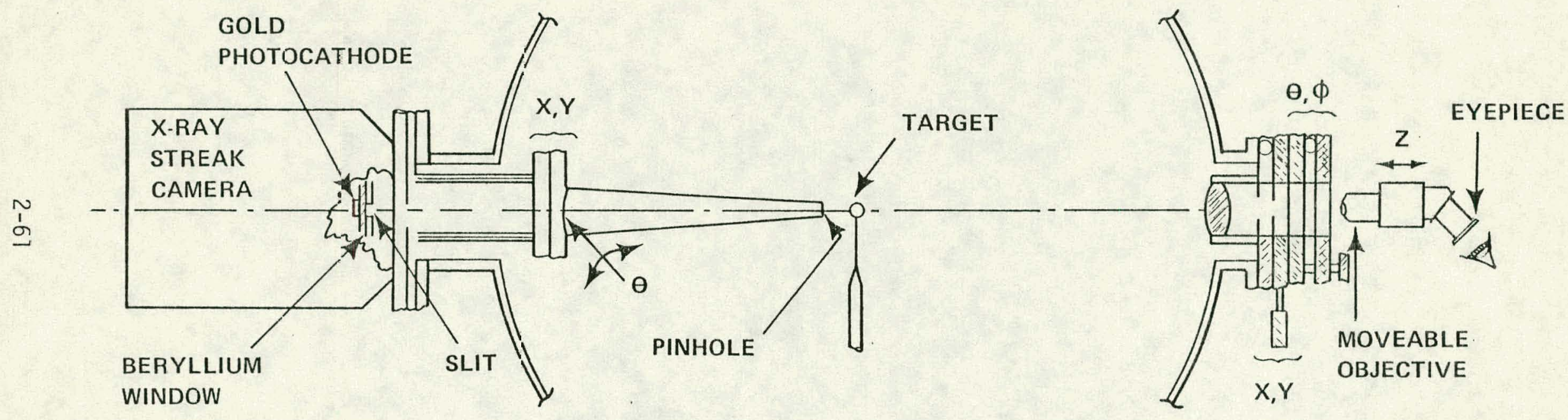

$=$ ig. 2.4-3 - Schematic of the optical alignment of the X-Ray streak camera. 
Shot $3902-76 \mu \mathrm{m}$ dia., $0.8 \mu \mathrm{m}$ wall

$92 \mathrm{~atm}$ DT gas, 67 joules on target, $156 \mathrm{psec}$ pulse length

Densitometer Scans

X-ray Streak Camera (G.E.A.R., Inc.)

Photozathode - $210 \mathrm{Au}$

Sweep Speed - $25.3 \mathrm{ps} / \mathrm{mm}$

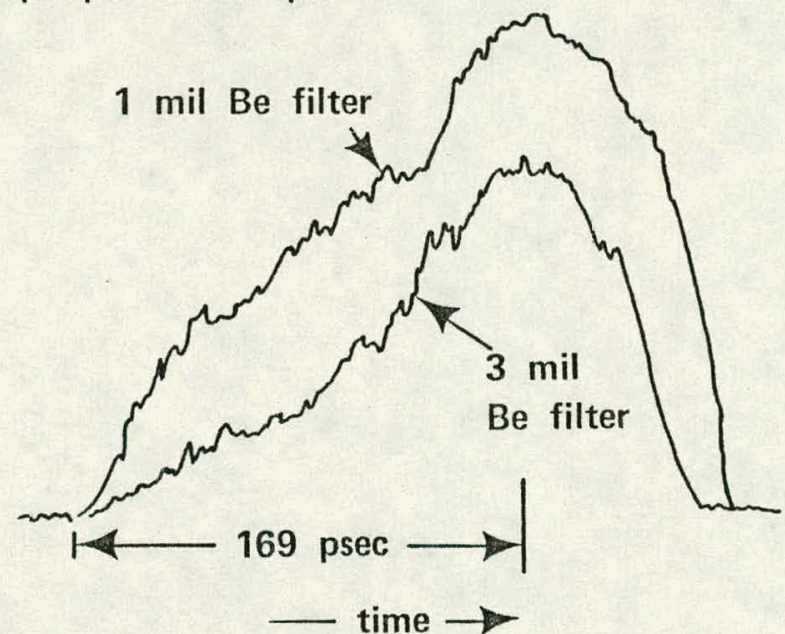

$\underset{\text { Kusion }}{\text { Kums }}$

Fig. 2.4-4 - Microdensitometer traces of the X-Ray emission from an $80 \mu \mathrm{m}$ diameter glass shell target, through $1 \mathrm{mil}$ and 3 mils of beryl1ium. 


\subsubsection{Zone Plate Alpha Particle And X-Ray Coded Imaging}

G. Charatis and D.C. Slater

To study the spatial extent of the thermonuclear burn volume of a laser fusion target it is desirable to produce an image of a thermonuclear reaction product. Recently, a new diagnostic device, a Fresnel zone-plate camera utilizing coded imaging techniques has been used to reconstruct the image of the alpha particle distribution within the fusion reaction region. ${ }^{(2)}$ A pinhole camera can also be used for alpha-particle imaging but it does not have one important feature available with zoneplate coded imaging; tomography - the ability to reconstruct two-dimensional slices of the 3-dimensional imploding core.

We have designed and built a zone-plate camera, shown schematically in Figure 2.4-5A and photographically in Figure 2.4-5B. Zone plates for this camera were fabricated via photo-1 ithographic techniques, $(3,4)$ of 5 to $6 \mu \mathrm{m}$ thick gold and are free standing, to permit the passage of alphaparticles through the annular openings without scattering and dispersion. A special feature of the camera is that it can record zone-plate coded images of both alpha-particles and $x$-rays in in-line registration, thereby permitting the direct comparison of the alpha-particle spatial distribution within the containing volume of the x-ray emitting tamper.

With a 2-mm diameter zone plate placed $1.5 \mathrm{~cm}$ from the target, the size of the alpha particle shadowgram is $5.6 \mathrm{~mm}$ diameter, and for the $x$-ray, $6.7 \mathrm{~mm}$ diameter. At that distance about $5 \times 10^{-4}$ of the alphas will be included in the coded image. Since approximately $10^{5}$ alphas are required for a good reconstruction, a minimum yield of $2 \times 10^{8}$ neutrons is necessary to use this technique.

Using cellulose nitrate film for detection behind a $6 \mu \mathrm{m}$ thick 


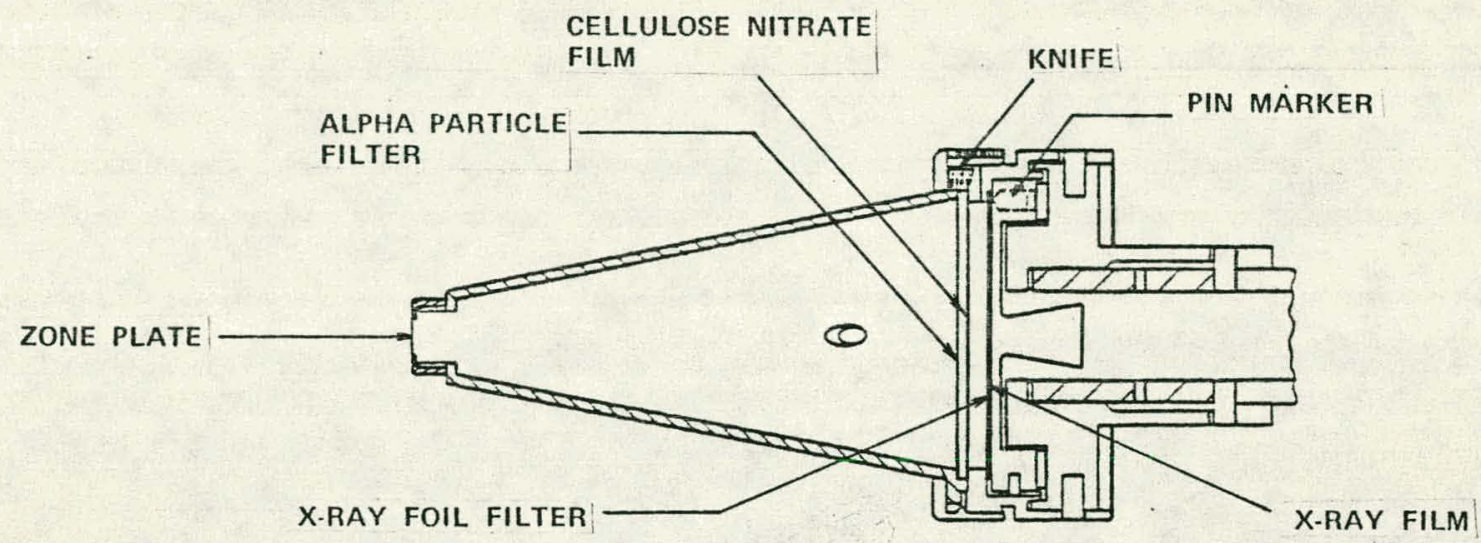

Fig. 2.4-5A - Schematic of the Zone Plate Camera. With the zone plate at $1.5 \mathrm{~cm}$ from the target, the size of the alpha particle shadowgram is $5.6 \mathrm{~mm}$ in diameter, and for the $\mathrm{x}$-ray, $6.7 \mathrm{~mm}$ in diameter.

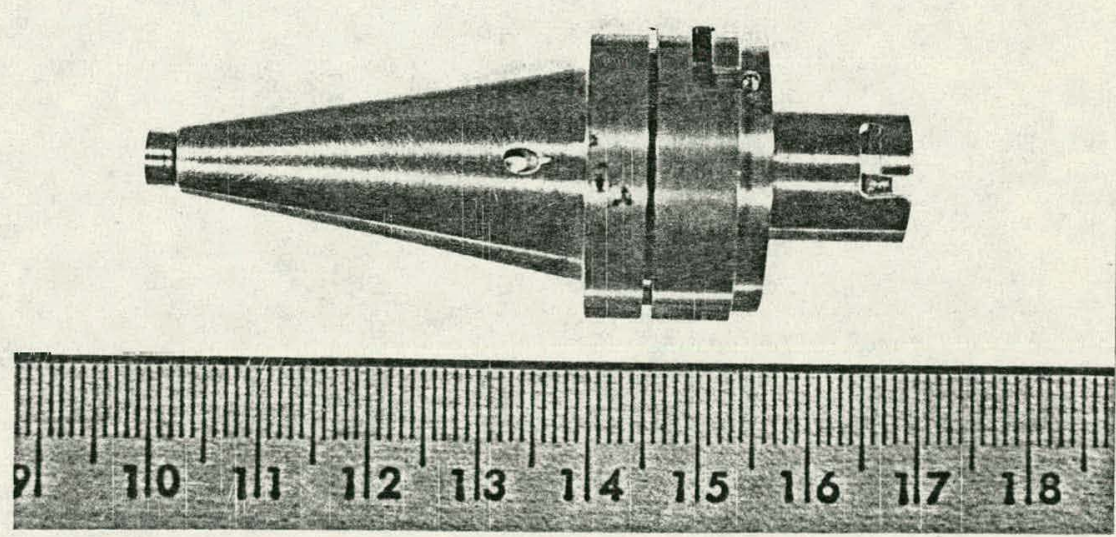

Fig. 2.4-5B - Photograph of the assembled zone plate camera. 
mylar foil, very good alpha particle tracks have been obtained on a shot with a yield of $3 \times 10^{7}$ neutrons. While the zone plate shadow was distinguishable on the $f i 1 m$, there were too few tracks to reconstruct the image. 


\subsubsection{X-Ray Spectrography}

\section{G. Charatis}

The seeding of DT gas-filled glass microshell targets with neon has been used ${ }^{(5)}$ as a way of making the compressed fuel volume luminous, since DT fuel mixtures do not emit sufficient $x$-ray photons at thermonuclear temperatures and densities to be detectable.

A well known technique, the spectroscopy of $x$-ray emission from imploding targets can be used to determine the electron density of the ablator, the tamper and the fuel. Yaakobi, et al., ${ }^{(6)}$ Key, et al., (7) and Mitchell et al. (8) have reported spatially and spectrally resolved Stark-broadened neon lines which have been used to determine the compressed core size, its density and its temperature.

As a means of verifying our high compression cryogenic layer experiments spectroscopically we have designed and built a bent crystal spectrograph covering the spectral range from $0.9 \mathrm{keV}$ to $2.6 \mathrm{keV}$. Design criteria were based on (a) low crystal efficiency $3 \times 10^{-5}$ radians for KAP, and (b) minimal target irradiation obscuration within the ellipsoidal mirrors. A slit of $15 \mu \mathrm{m}$ width situated between the target and the crystal, provides a means of discriminating between coronal and compressed core $x$-radiation in one-dimension normal to the plane of energy dispersion. A photograph of the spectrograph is shown in Figure 2.4-6.

Preliminary measurements of narrow SiXIII and SiXIV lines indicate at $2 \mathrm{keV}$ that the instrumental width is less than $2 \mathrm{eV}$ and the reciprocal dispersion is $0.2 \mathrm{keV} / \mathrm{mm}$. 


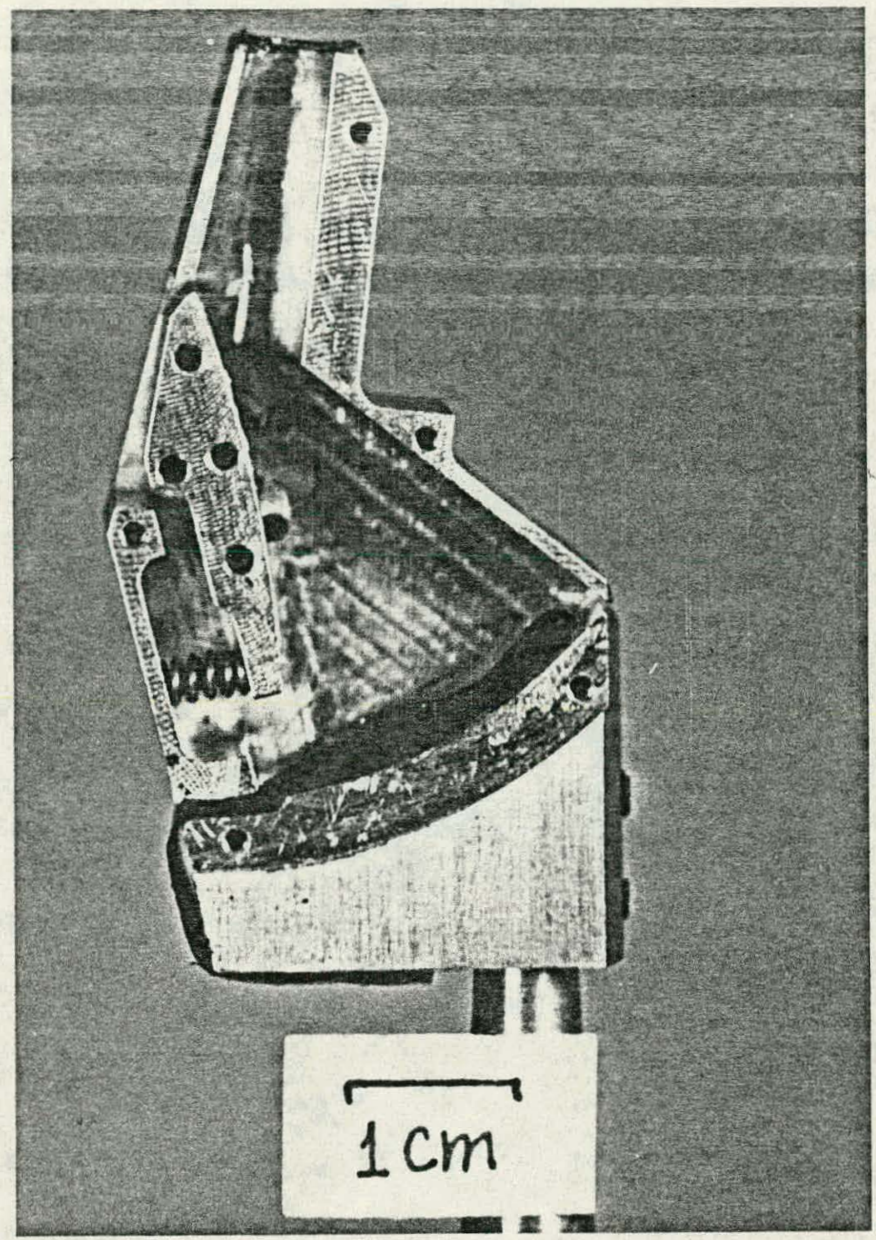

Fig. 2.4-6 - X-Ray KAP Crystal Spectrograph. Spectral range: $0.8-2.8 \mathrm{keV}$. Reciprocal dispersion: $\sim 0.2 \mathrm{keV} / \mathrm{mm}$. Instrumental width at $2 \mathrm{keV}$ : less than 2 ev. 


\subsubsection{Self-Calibrating Differential Calorimeter}

J. D. Simpson

During laser-fusion experiments some fraction of the incident $1.06 \mu \mathrm{m}$ laser energy is absorbed by the target and re-emitted primarily as plasma ions and $x$-rays while the rest of the incident light is scattered from the target without being absorbed. Calorimeters used to measure the absorbed fraction have been designed to discriminate between the scattered $1.06 \mu \mathrm{m}$ radiation and the ion/ $\mathrm{x}$-ray fluxes, and are ordinarily designated as differential calorimeters.

There have been a variety of instruments designed and built to make absorbed energy measurements, with an apparent tradeoff between device sensitivity and ease and convenience of calibration. S.R. Gunn and V. Rupert of LLL have done considerable work with ion, x-ray, and scattered $1.06 \mu \mathrm{m}$ radiation calorimetry, ${ }^{(9)}$ using electrical heating for calibration. Their work is encouraging and the convenience of electrical calibration is attractive but the relatively low sensitivity and large size of the LLL instruments are definite disadvantages in the. KMSF system. Thin film thermopile detectors ${ }^{(10)}$ have been used quite successfully in calorimeters developed by S. Segall at the KMSF laboratories. The features of small size and good sensitivity are an advantage in the KMSF system. However, the calibration of these instruments is done by comparison with other calibrated instruments and is therefore not absolute nor as convenient as the electrically calibrated types. We will discuss the development of a third type of ion/x ray calorimeter that uses thin film thermistor flakes as detector elements that can be self-calibrated. 
Thermistors are devices, generally made of semiconductor materials, whose resistance varies as a function of temperature on the order of $=4 \%$ per ${ }^{\circ} \mathrm{C}$. The type that we are using as calorimeter detector elements are an alloy of nickel oxide and magnesium oxide. They are $2 \mathrm{~mm}$ square and $25 \mu \mathrm{m}$ in thickness. There are two flakes per detector unit which are mounted side by side on a T0-5 header. Figure $2.4-7$ is a $7 x$ view of the flakes mounted on the T0-5 header.

The high resistivity and large alpha (temperature coefficient of resistance) of thermistor materials makes them attractive for use as temperature/energy sensing devices. Characteristically, $I^{2} R$ heating can be induced in the thermistor which will in turn cause a resistance change which therefore enables the device to be self-calibrated electrically.

Presently, the thermistor calorimeter is under development and as yet no experimental data has been collected. During other experiments at KMSF with semiconductor materials as energy transducers, large noise currents were observed as a result of ionizing radiation creating electronhole pairs in the material. The time scale of this transient effect is in milliseconds which is small compared to the time scale of the thermal decay which is several seconds. This effect will clearly disappear long before the thermal effect and is not expected to interfere with the measurement.

The sensitivity of the thermistor flakes in the bridge configuration is expected to be on the order of .33 millivolts per joule of absorbed $1.06 \mu \mathrm{m}$ energy. Temperature sensitivity is nearly 15 millivolts per ${ }^{\circ} \mathrm{C}$ or 42 volts per joule at the detector, a significant increase over other detectors prosently in use. 


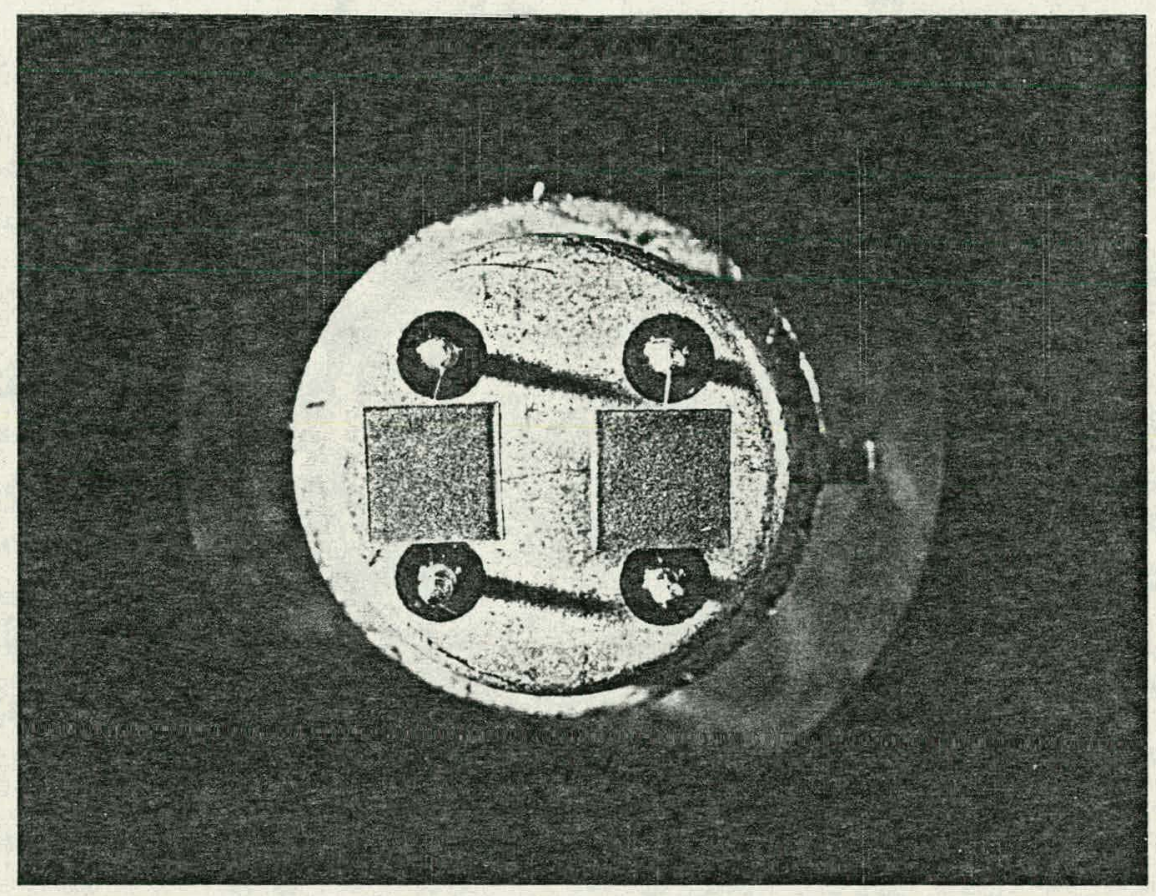

Fig. 2.4-7 - Self-calibrating thin film thermistor flakes arranged in a differential pair configuration on a TO-5 header (7x magnification). 


\subsubsection{Two Temperature Fit from $X$-Ray Diagnostics}

A. H. Bennish and S. B. Segall

A computer code fit is routinely obtained for target shots by measurement of the $x$-ray spectrum above $3 \mathrm{keV}$. In a previous report, (11) it has been shown that the observed data are consistent with bremsstrahlung $x$-ray emission at two temperatures.

The instrumentation used has been developed from an earlier system. $(11,12,13)$ Major efforts have been made to optimize the existing detectors and to develop new ones. Each detector is filtered by a foil of a different element.

The primary detectors are $x$-ray photo-diodes ( $p-i-n$ diodes). Eight diodes are used to measure the spectrum from 3 to $25 \mathrm{keV}$ (see Table 2.4-2). The filter thickness and the diode thickness are computer calculated to maximize the fraction of the response in the spectral region immediately below the absorption edge of the filter. This is important since each diode is then sampling a different region of the spectrum. Larger diameter diodes are used in the more energetic spectral region, where the flux is down by several orders of magnitude, to provide increased sensitivity.

The fast charge pulse from each diode is measured by a gated integrator. The voltage levels at each integrator are then digitized by the analog-to-digital converter (ADC), connected to the PDP $11 / 45$ computer.

A major goal is to add measurements of the $x$-ray spectrum at energies above $25 \mathrm{keV}$ using filters of high atomic number. Since the x-ray flux drops off rapidly with energy, a detector with greater sensitivity than a $x$-ray photodiode is required. Sodium iodide (NaI) detectors were chosen, since by the use of a photomultiplier tube very high sensitivities are 
TABLE 2.4-2

DIODES AND FILTERS SINCE 14 NOVEMBER 1978

\begin{tabular}{|c|c|c|c|c|}
\hline Filter & $\begin{array}{l}\text { Absorption } \\
\text { Edge (keV) }\end{array}$ & $\begin{array}{c}\text { Filter Thick- } \\
\text { ness (mil) }\end{array}$ & Area $\left(\mathrm{mm}^{2}\right)$ & $\begin{array}{l}\text { Detector } \\
\text { Depth }(\mu \mathrm{m})\end{array}$ \\
\hline Sn & 3.93 & .476 & 50.0 & 75 \\
\hline $\mathrm{Ti}$ & 4.97 & 3.0 & 25.0 & 125 \\
\hline $\mathrm{Fe}$ & 7.11 & 2.2 & 25.0 & 125 \\
\hline $\mathrm{Cu}$ & 8.98 & 2.08 & 25.0 & 250 \\
\hline $\mathrm{Ge}$ & 11.10 & 6.16 & 25.0 & 250 \\
\hline $\mathrm{Zr}$ & $18.0^{\circ}$ & 5.62 & 25.0 & 250 \\
\hline Mo & 20.0 & 6.8 & 100.0 & 500 \\
\hline $\mathrm{Ag}$ & 25.5 & 7.93 & 100.0 & 500 \\
\hline
\end{tabular}


obtained. The data from the NaI detectors as yet have not employed on the two-temperature fit.

\section{Computer Analysis}

The computer code to obtain two-temperatures is based on an algorithm which minimizes the squared differences between the actual detector responces (normalized) and the calculated responses. (14) Four fitted parameters may be varied: the high and low temperatures, and the energy emitted by each of the two populations. The total energy is constrained to be less than the energy on target. At least seven good diode readings are required to obtain a fit:

\section{Experimental Results}

Results have been obtained for spherical glass shell targets at intensities ranging from $2 \times 10^{13} \mathrm{Wcm}^{-2}$ to $5 \times 10^{15} \mathrm{Wcm}^{-2}$. All the data at intensities below $1 \times 10^{15} \mathrm{Wcm}^{-2}$ were from the long-pulse-length experiments, with pulse lengths from 700 ps to $1.4 \mathrm{~ns}$. The data at higher intensities were from shots with pulse lengths from 60 to 160 ps.

For the majority of shots with long pulse lengths the laser spatialintensity profile was smoothed and its bandwidth increased by the inclusion of a plasma spatial filter. All the high temperatures are in the range of 4.5 to $8.0 \mathrm{keV}$ with no apparent intensity dependence (see Figure 2.4-8). other studies have shown a marked intensity dependence at these intensities.

Some target shots with long pulse lengths were performed without the plasma spatial filter. The high temperatures for these shots are markedly higher, in the range of 7 to $14 \mathrm{keV}$. Although only 6 of the 31 long pulse results graphed in Figure 2.4-8 are from shots without the plasma spatial filter, the three highest temperatures achieved in the long-pulse-length experiments were all without the plasma filter. An increased fraction 


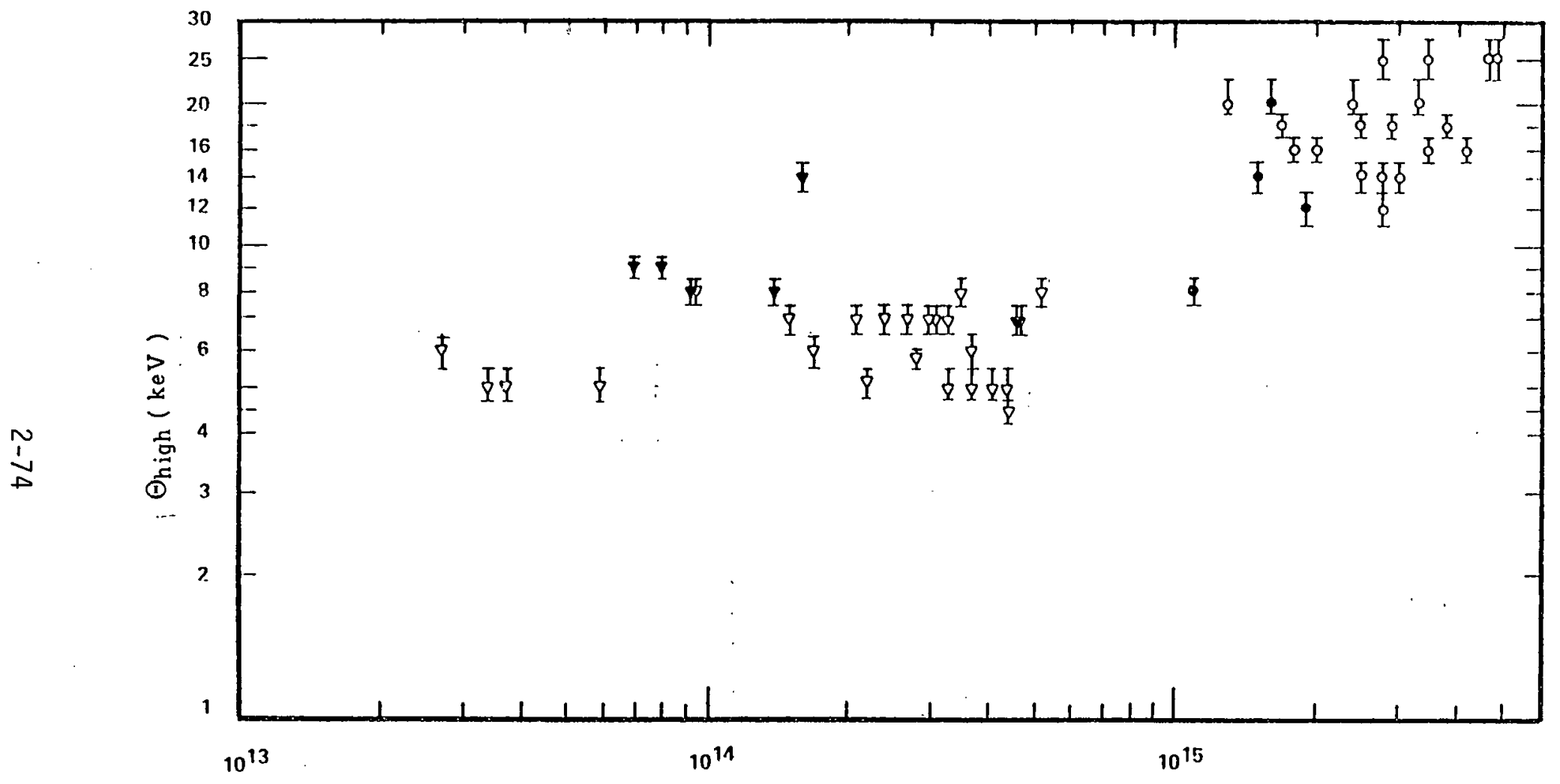

Fig. 2.4-8 $\Theta_{\text {high }}$ vs IRRADIANCE $\left(W / \mathrm{cm}^{2}\right)$ for GLASS SHELL TARGETS
Triangles
-.- long pulse experiments
Circles
-..- short pulse experiments
Closed Symbols --- without plasma spatial filter
Open Symbols ‥- with plasma spatial filter 
of the total absorption coming from resonance absorption (since the beam now has "hot spots" and a smaller bandwidth) could account for the increase in the high temperature.

The data with pulse lengths from 60 to 160 ps at intensities above $1 \times 10^{15} \mathrm{Wcm}^{-2}$ show considerable scatter, with high-temperatures from 8 to $25 \mathrm{keV}$. Only four shots without the plasma spatial filter graphed, with intensities below $2 \times 10^{15} \mathrm{Wcm}^{-2}$. The lowest intensity short-pulse-length shot is one of these and had the lowest high temperature, $8 \mathrm{keV}$. All the other short-pulse-length shots had high temperatures at or above $12 \mathrm{keV}$. Above $3 \times 10^{15} \mathrm{Wcm}^{-2}$ intensity, all shots had high temperatures at or above $16 \mathrm{keV}$.

To produce a good two-temperature fit, the observed energy spectrum cannot have significant amounts of line radiation. Since all silicon radiation is below $3 \mathrm{keV}$, for shells made of borosilicate glass no line radiation was observed. Some of the glass shells used in the long-pulse-length experiments had large amount.s of calcium, which has lines above $3 \mathrm{keV}$. However, the calcium line radiation never prevented a successful two-tem-* perature fit. Most cryogenic glass targets had a $17 \mu \mathrm{m}$ copper fiber support outside the focal volume. On a few shots very large amounts of the copper line radiation were noticed. Several of the target shots using a polylodustyrene plastic target produced more line radiation than had ever been measured before. The results graphed in Figure 2.4-8 are all from glass shell targets with no noticeable copper line radiation.

The two series of experiments which have the most data are the two using glass shell targets with the plasma spatial filter - one series at intensities below $10^{15} \mathrm{Wcm}^{-2}$ (long-pulse-length experiment) and the other at intensities above $10^{15} \mathrm{Wcm}^{-2}$ (pulse length from 60 to $160 \mathrm{ps}$ ). 
For the long-pulse-length data, the geometric-mean intensity is $2.1 \times 10^{14}$ $\mathrm{Wcm}^{-2}$, and the geometric-mean high temperature is $6.2 \mathrm{keV}$. For the shortpulse-length data, the geometric-mean intensity is $2.8 \times 10^{15} \mathrm{Wcm}^{-2}$ and the geometric-mean high temperature is $17.6 \mathrm{keV}$. Assuming that the high temperature is proportional to the $m$ th power of the incident laser intensity, a fit to the above yields $m=.40$. These results are in agreement with other results which show the high-temperature increasing with intensity. (16) 


\subsubsection{Holographic Interferometry}

Garland E: Busch

Holographic interferometry, in tens of picoseconds time scales and less, is used to "snapshot" the plasma density profile. In addition to measuring the electron density the reconstructed image can provide information on illumination symmetry and timing or test for pre-pulse effects. The technique used is similar to that of Attwood, et al.

The $40 \mathrm{psec}$ probe pulse is generated in the same laser oscillator, and at the same time as the main oscillator pul'se, so that a precise time reference is established. The probe pulse is amplified and doubled in frequency. Figure 2.4-9A shows schematically the layout of the optical system. The lens used to collect the probe light refracted by the plasma was limited in aperture to $f / 4.2$ due to constraints imposed by the available opening between the main ellipsoidal target illuminating mirrors. Since the lens is large and removed from the target by $24 \mathrm{~cm}$ the resulting hologram is small $(<1 \mathrm{~cm}$ in diameter). This is a distinct advantage, as the resolution of the system is limited by the optical uniformity of the photographic plate within the aperture of the hologram. The spatial resolution in object space at the $0.53 \mu \mathrm{m}$ wavelength is 3 to $4 \mu \mathrm{m}$.

The present system provides automatic double-pulse operation. Double-pulse holography produces interference fringes when optical changes occur between the pulses. The first pulse set arrives $3 \mathrm{nsec}$ before the arrival of the leading edge of the main target-irradiating pulse. The second pulse set can be timed to probe the plasma at a selected interval after the arrival of the target-irradiating pulse, by the optical arrangement shown in Figure 2.4-9B. Since the two pulse probe sets occur at an exceptionally small interval, the fringes they produre are unaffected by 


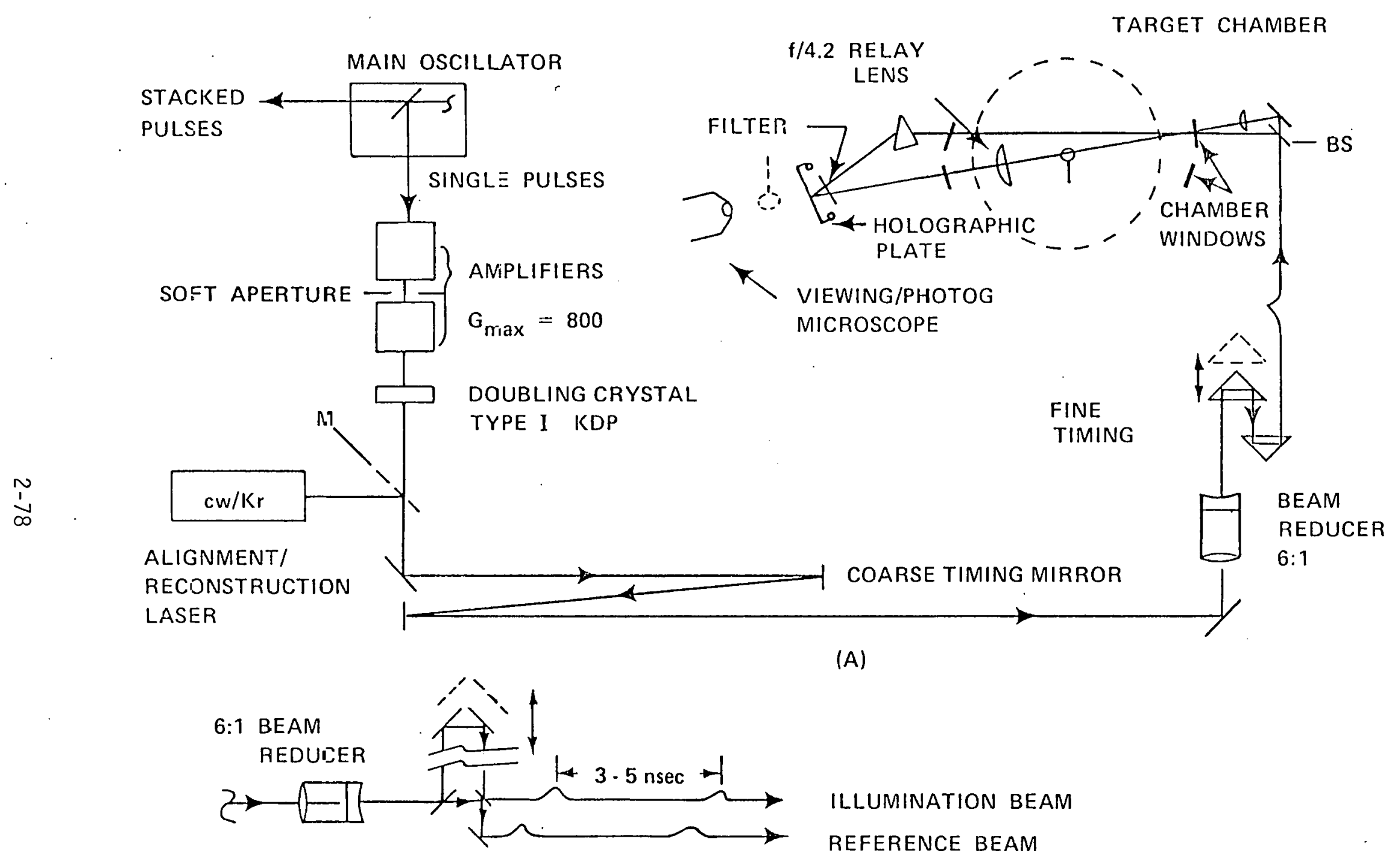

(B)

Fig. 2.4-9 Schematic of the optical layout of $(A)$ the holographic interferometer probe, with (B) the double pulse set optics. 
mechanical vibration, atmospheric turbulence and oscillator intensity variations.

The holographic probe system has operated routinely during many laser target interaction experiments. Examples of reconstructed images are presented in Section 1.6. Additional interferograms are shown in Figures 2.4-10, and 2.4-11.

The squareness of the image in Figure 2.4-10 was predicted and is evidence of the sensitivity of the technique to illumination nonuniformities. In this target shot, \#3688, an annular irradiance profile was induced, resulting in a cylindrical corona development. In the second example, shot \#4006, the target is highly visible due to the 3 nsec early probe pulse. Also, the opacity of the central plasma and the uniformity of the interference fringes in the corona is clearly visible, (except in the supporting stem region). None of the holograms has yet been subjected to the Abel inversion techniques for piasma electron profile characterization.

Several improvements in the system are being readied for future experiments. First, the optical system is being converted into a frequencyquadrupled (UV) probe beam. This, together with an $f / 2$ UV collection optical system, will allow probing to higher electron density in the plasma, as well as providing a system which can be used when the main laser pulse is frequency-doubled. Finally, a spatially and temporally resolved multipulsed system is being developed. With it four to six discrete interferograms would be generated on a given target shot, representing sequentially timed slices of the evolving corona. 


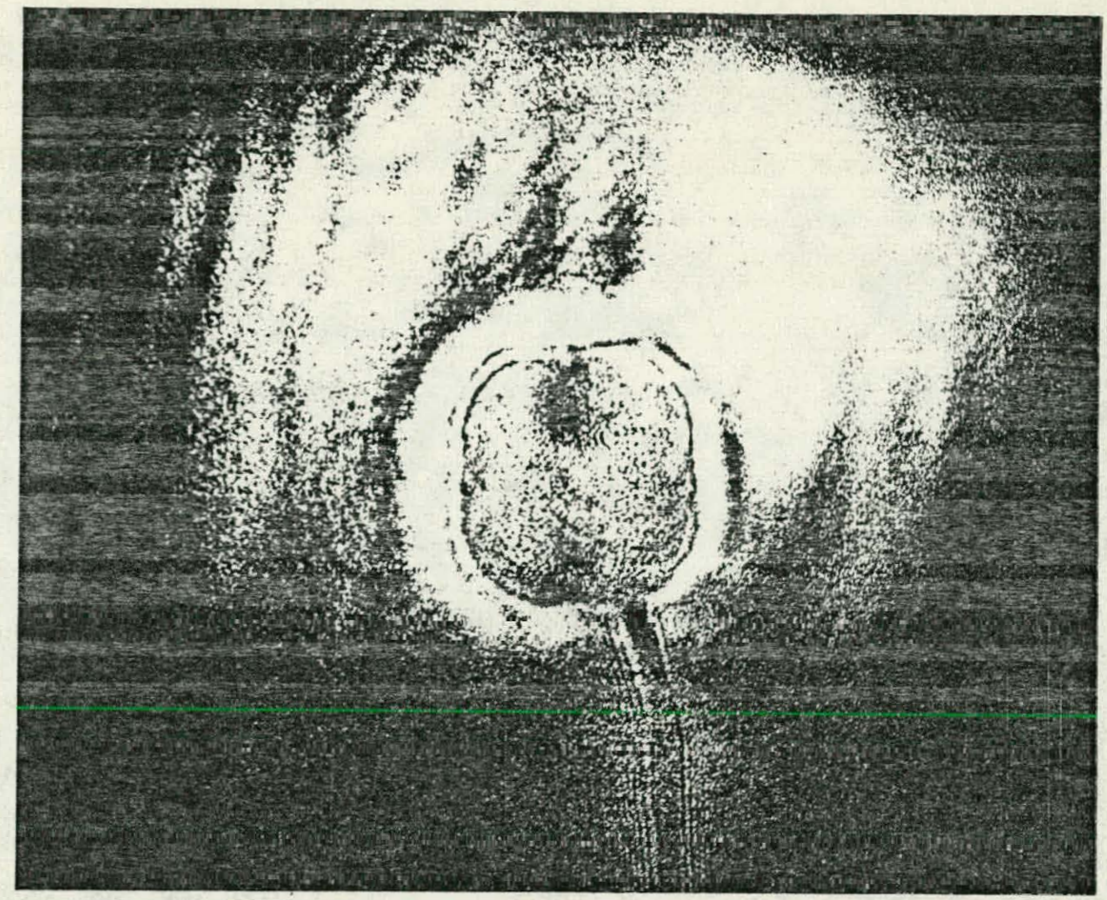

Fig. 2.4-10 Reconstructed image from an interferometric hologram for shot 3688 , where the target irradiance profile was annular.

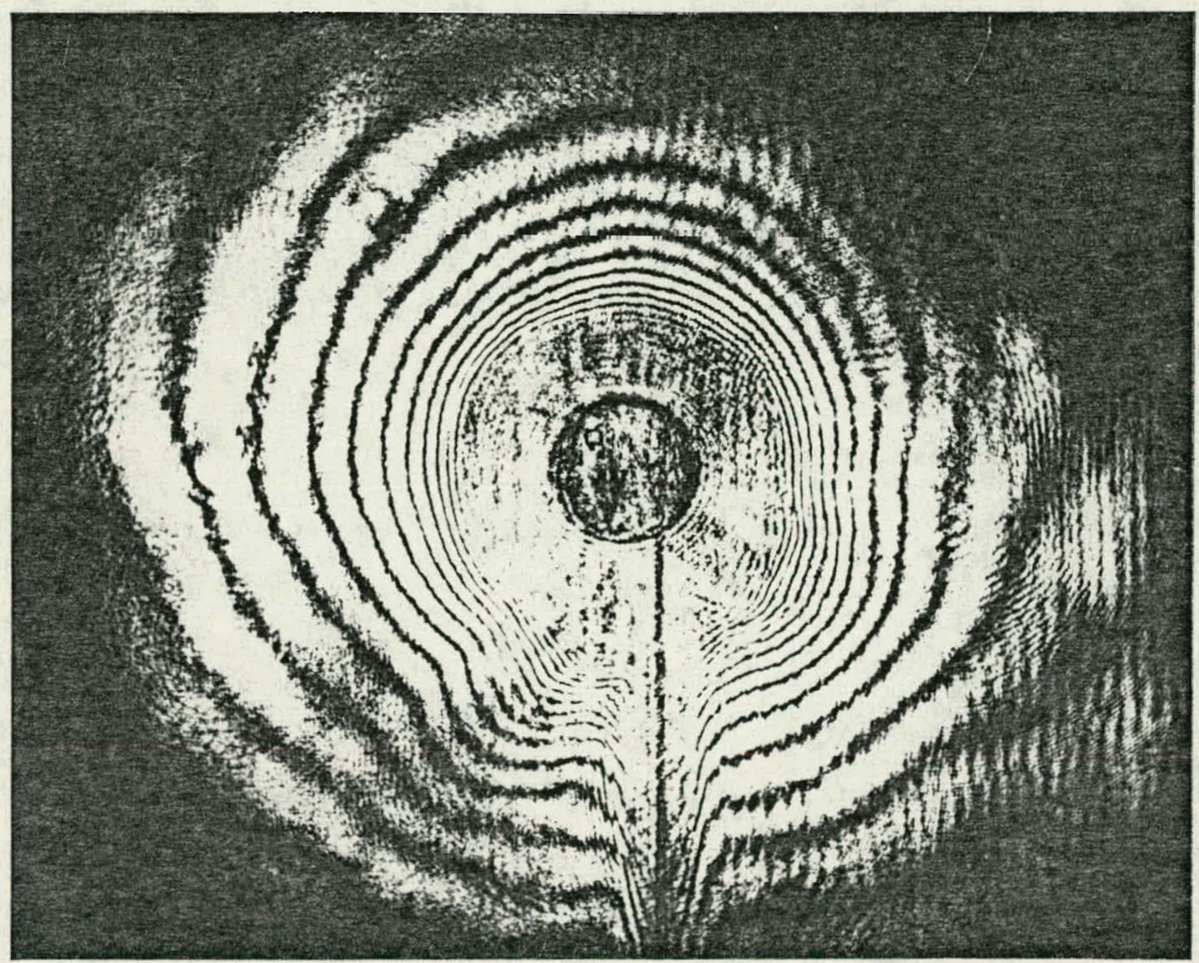

Fig. 2.4-11 Reconstructed image from an interferometric hologram for shot 4006 . Note visibility of original target, and circular uniformity of the interference fringes, except in the supporting stem region. 


\subsubsection{The Data Acquisition System}

M.H. Barron, D.W. Daniels, and J.G. Downward IV

Work on the KMS Fusion DATA ACQUISITION SYSTEM has been concentrated on improving the functionality and reliability of the existing system. The current schematic of the system is shown in Figure 2.4-12. The operating system (RSX IIM V3.1) was modified to enable efficient utilization of our DEC PDP $11 / 45$ for an ever increasing number of users and data acquisition tasks.

In addition to data acquired directly during target shots by either the Analog Multiplexer or the Digital Input and Output Processor (DIOP), an increasing amount of data judged to be important for analyzing a target shot is entered into the data files by an operator. To accommodate the expanded goals of the data files, second generation data entry software has been implemented which allows for rapid update of single elements within the data files.

The major increase in system functionality is the result of integrating an Image Processor System with our PDP 11/45. Photographic data is digitized into the DeAnza IP5532 Image Processor's memory using a vidicon TV camera specifically adapted for image metrology. The ITM vidicon camera has a dynamic range in density of 3.0 or greater, and outputs data through an antilog amplifier. This allows approximately equal steps in density to output as linear changes in voltage output to the digitizer, insuring equal digitizing accuracy over the entyre dynamic range.

The image processor incorporates a real time digitizer, which can take the output of a vidicon TV camera and digitize it to 8 bits accuracy into its $512 \times 512$ memory in two video frame times. This feature was a primary factor in selecting the IP5532 Image Processor to he used with the 


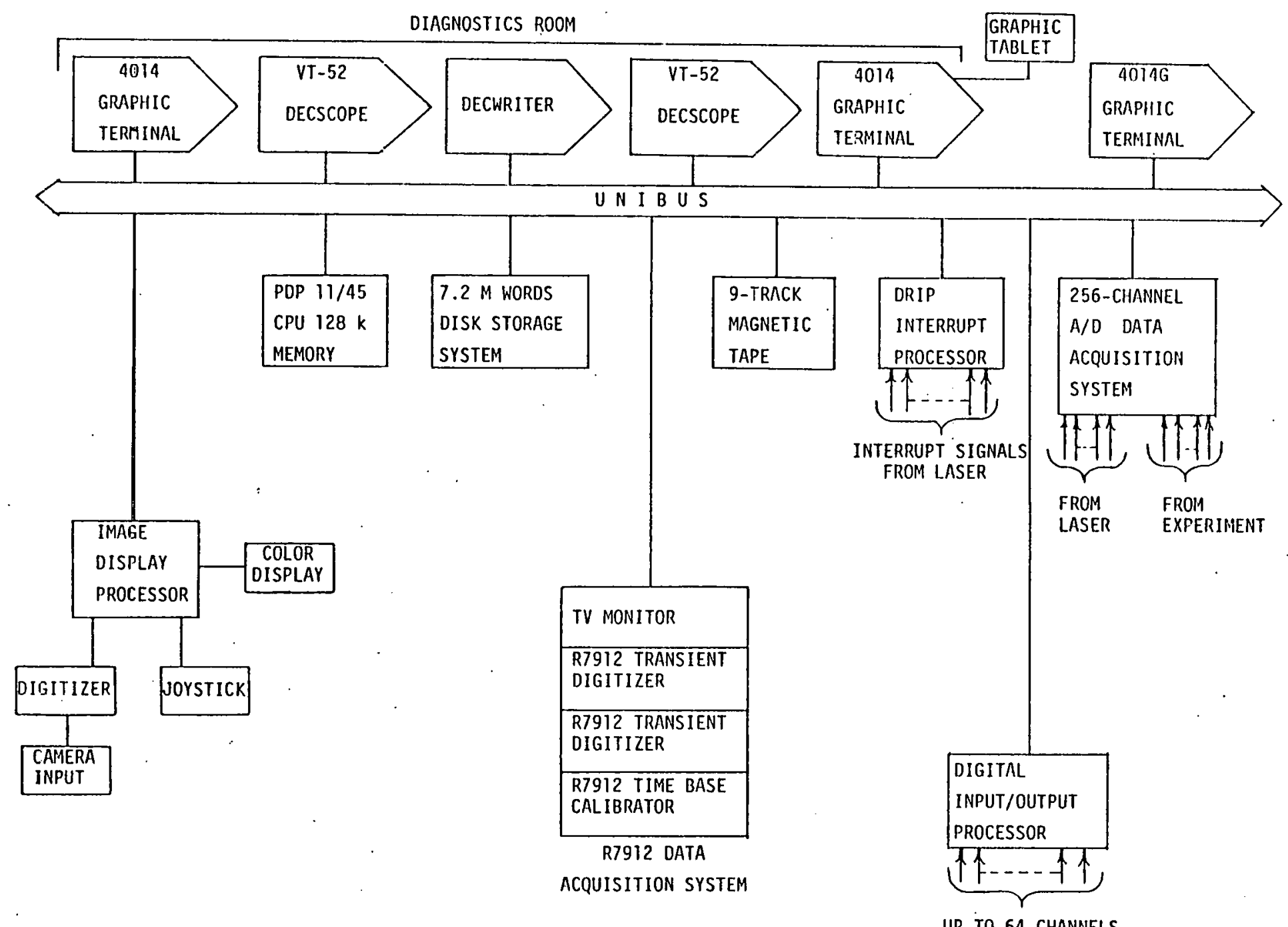

Fig. 2.4-12 Schematic layout of the Data Acquisition System. 
PDP 11/45. In addition to being much faster than other commerical TV video digitizers, the IP5532 image digitizer, operates without computer intervention, thus freeing the CPU for other use while digitization is taking place.

The IP5532 Image Processor itself is designed to offload as much processing as possible from the PDP 11/45. It is an 8 bit pipeline processor containing dual arithmetic logic units. It is capable of performing a large number of arithmetic operations on any two image memories in one video frame time $(1 / 30 \mathrm{sec})$. For example, two images may be added together or subtracted, or a constant offset can be added to an image in one frame time. This allows very powerful image processing to be done with a minimum of CPU intervention. In addition to the arithmetic logic unit, the image processor has graphic and alphanumeric capability. It can display data ejther on a $B / W$ monitor, or a RGB color monitor. The data can be selectively transformed via intensity transform tables for $B / W$, red, green, and blue. This allows simple false density color slicing of an image to be done. A block diagram of the Image Processor is shown in Figure 2.4-13. The first task committed for use with the Image Processor, has been to provide quasi-real-time analys is of $x$-ray pinhole images of target implosions. While a target shot can be done every 1-1.5 hours, traditional data analysis techniques on the $x$-ray pinhole photographs take a day or more to accomplish. The image analys is system is now being used to provide a rapid first cut analysis of the photograph as soon after the target shot as possible. This provides the scientists with more timely information with which to judge the success or failure of a given experiment.

The image processor is inherently a complicated device (32 control registers). It is essential to make the interface between the operator 


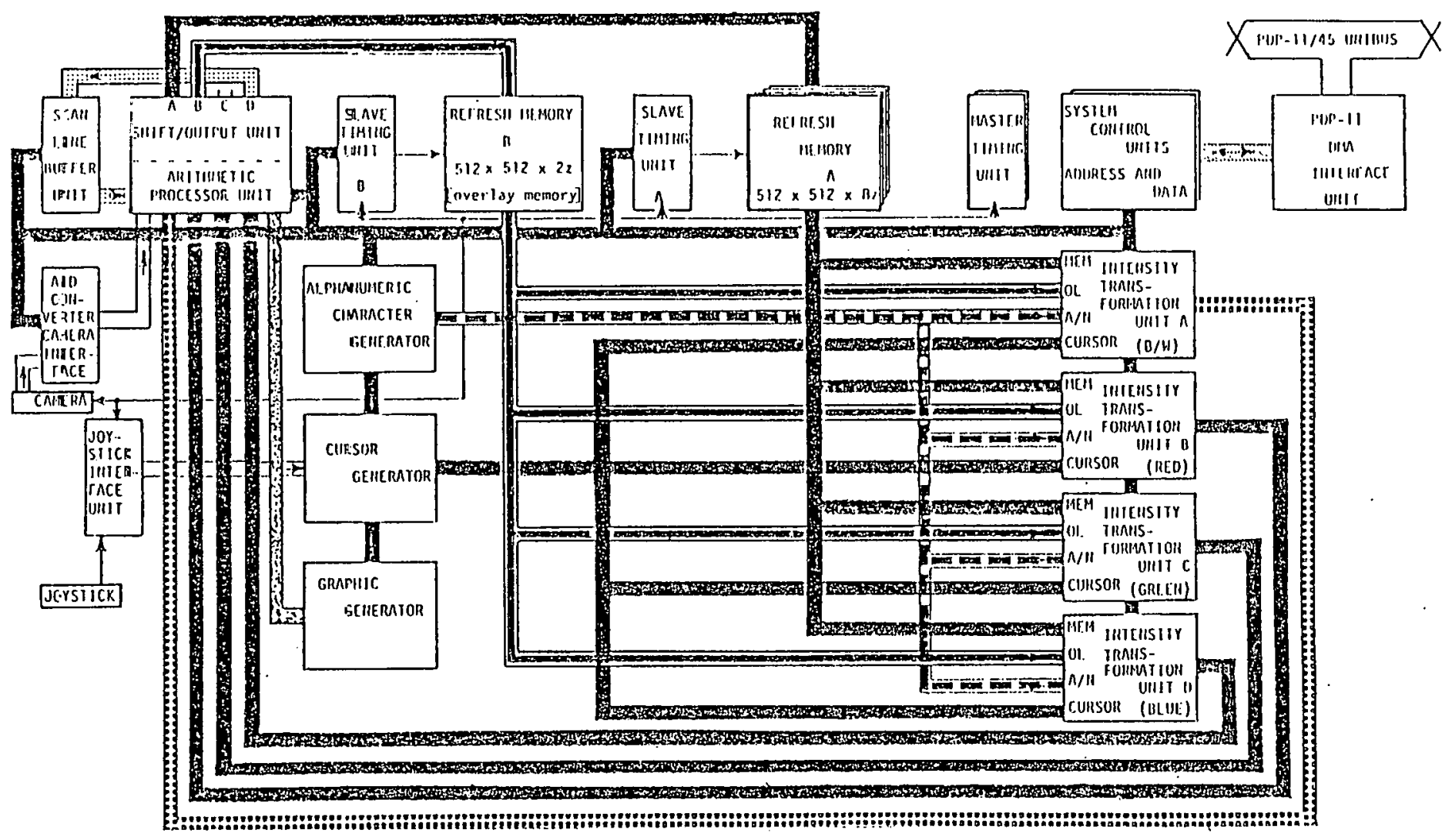

Fig. 2.4-13 Block Diagram of Image Array Processor. 
and processor simple, keeping the programming time to a minimum, with the control software as modular as possible. Since different tasks, and in the future, a variety of devises, will require new control software, a tailorable Console Command Language (CCL) has been implemented under RSXI1M. $\mathrm{CCL}$ is a table driven command interpreter and task dispatcher which is used to process commands not normally recognized as system commands. For example, the command OFFSET 50, will run a task OFF and pass the number 50 to it. In this manner a control language for the image prociessor has been developed as a collection of modular, easily debugged, tasks, dispatched by CCL with english mnemomics.

A normal sequence of analysis is for the user to "take a picture" of the pinhole photograph with the TV camera. The picture output to the processor for digitization is first passed through an antilog amplifier to linearize video output with film density. The picture is then corrected for any possible illumination nonuniformities or vidicon sensitivity variations. A calibration image of a "uniformly" illuminated field is used to provide a map of correction values on a pixel by pixel basis. After correction, some of the grain noise can be filtered out of the picture, the contrast adjusted (stretched), unwanted parts of the display removed, offsets to the picture added or subtracted, density traces displayed, through selected parts of the image, and the picture color enhanced, to look for iso-density contours. 


\section{REFERENCES}

1. Laser Program Annual Report - 1975, Lawrence Livermore Laboratory, Rept. UCRL-50021-75 (1976), p. 418.

2. N. M. Ceglio, J. Appl. Phys. 48, 1563, (1977).

3. Fabricated by the University of Michigan Electron Physics Laboratory.

4. Zone-plate mask supplied by N. M. Ceglio of Lawrence Livermore.

5. G. Charatis, J. Downward, R. Goforth, B. Guscott, T. Henderson S. Hildum, R. Johnson, K. Moncur, T. Leonard, F. Mayer, S. Sega 11, L. Siebert, D. Solomon and C. Thomas, KMS Fusion, Inc., "Experimental Study of Laser Driven Compression of Spherical Glass She11s", IAEA-CN-33/F-1, IAEA Conf., Tokyo, Japan, (1975).

6. B. Yaakobi, D. Steel, E. Thorsos, A. Hauer and B. Perry, Phys. Rev. Lett. 39 , 1526, (1977).

7. M. H. Key, R. G. Evans, D. J. Nichols, F. O'Neill, P. T. Gremsby, I. N. Ross, W. T. Toner, P. R. Williams, M. J. White, C. L. S. Lewis, J. G. Lunney, A. Moore, J. M. Ward, D. Brown, P. Carter, T. A. Hall, T. P. Hughes, P. Slater, E. Frabikesi, A. Raven, J. Murdoch and J. Kilkenny, Central Facility Report RL-77-122/B, Rutherford Laboratory.

8. K. B. Mitche11, D. B. van Hulsteyn, G. H. McCall, Ping Lee,

H. R. Griem, LA-UR-78-1708 Report, LASL.

9. S. R. Gunn and V. C. Rupert, Rev. Sci. Instrum. 48, 1375, (1977).

10. S. B. Sega11, Bu11. Am. Phys. Soc. 22, 1113, (1977); KMSF Report U675 (Nov. 1977).

11. S. B. Segall and T. A. Crispin, KMSF Report U274.

12. S. B. Segall, G. Charatis, R. R. Johnson, F. J. Mayer and P. M. Campbe11, KMSF Report U120.

13. S. B. Segall, KMSF Report U375.

14. Henry Brysk, unpublished.

15. D. W. Forslund, J. M. Kindel and K. Lee, Phys. Rev. Lett. $\underline{39}$, 284, (1977).

16. K. R. Manes, H. G. Ahlstrom, R. A. Haas and J. F. Holzrichter, J. Opt. Soc. Am., 67, 717, (1977).

17. D. T. Attwood, E. L. Pierce, D. W. Sweeney, L. W. Coleman, Laser Program Annual Report - 1976, Lawrence Livermore Laboratory, UCRL-50021-76 (1977), p. 3-34. 


\section{LASER FUSION THEORY}

Introduction

3.1 Fast Ion Energy Loss in Dense Plasmas

3.2 Electron Transport: Fokker-Planck Calculations

3.3 Ionization Equation of State

3.4 Profile Modification by Ponderomotive Forces: Equilibrium and Stability

3.5 Ponderomotive Potential Effects on Ohm's Law

3.6 Effect of Flux-Limited Thermal Transport on Critical Surface Jump Conditions

3.7 Spherical Rarefaction Shocks

3.8 Explosively Heated Gaussian Objects 


\section{Introduction}

R. L. Berger and P. M. Campbell

In the past year, the KMSF Theory Group has incorporated new models of physical processes into its basic hydrodynamic simulation code and has improved the existing models of physical processes. At the same time research in the plasma physics of the laser-plasma interactions has continued with the aim of identifying the most important effects to include in the simulation codes.

One of the most promising alternatives to a laser driver within the context of inertial confinement fusion is ion beams. In order to design targets for ion beam fusion, the ion energy range in dense plasma is of utmost importance. Since there is only limited experimental and theoretical work on the stopping power of dense plasmas, the starting point for our calculations is classical range formulae for which good agreement with data on unionized material exists. For heavy ions, which are of most interest for accelerator efficiency, the energy loss is almost entirely to electrons until near the end of the ion's range. Our simulations show that, once the plasma has been heated, the energy loss jer unit length of the ion's range is almost constant. This results in a less favorable deposition pattern.

The equation of state model equations have been modified to include radiative recombination within a local equilibrium assumption. This effect is important to include at low densities $\left(n<10^{20} \mathrm{~cm}=3\right.$ ) arid for moderate temperatures $\left(\theta_{e}<1 \mathrm{keV}\right)$. Its principal effect is to reduce the average ionization below that predicted by the Saha model for thermodynamic equilibrium. 
Numerical work is continuing on an electron transport model that can treat the limit of long mean free path electrons. For a homogeneous plasma this reduces to solving the Fokker-Planck equation for electrons. Solving this latter equation has provided some interesting features that the inhomogeneous problem should also reproduce. For example, the model demonstrates that work done by the electrons in driving the expansion comes mainly from the high energy electrons. Thus, the tail of the electron distribution is depleted by this process as well as by the process of slowing down on colder electrons.

Density profile modification due to the ponderomotive force effects in the ion momentum equation has been studied extensively in both particle and fluid simulations. Steady-state isothermal hydrodynamics and the wave equation in spherical geometry predict, in the absence of absorption, two kinds of profiles. Which profile obtains depends on whether the flow is subsonic or supersonic inside the critical surface. The former case results in the well-known density step. The latter results for normally incident light in slightly subcritical density plateaus just outside the critical surface. We have generalized these latter solutions to obliquely incident p-polarized light. For these steady-state profiles, the percentage of resonance absorption is not as large as the typical percentage in density step profiles. However, for large incident intensities $\left(|E|^{2} / 4 \pi n_{c}{ }^{\top} e^{\sim 1)}\right.$ no steady solution exists in this case. Moreover, we have shown that these profiles are unstable for moderate intensities and studied their long time unsteady behavior. One interesting feature is the generation of multiple critical surfaces where conversion of light to electron plasma waves and the inverse process occur. The resultant 
time-averaged absorption rate is larger than results from a steady-state analysis.

The effects of the ponderomotive force on the density profile can be found from a fluid equation analysis. If one looks at the particle equations of motion, one sees that the ponderomotive force is just the time averaged Lorentz force on the electrons. From this viewpoint, it is easy to see that this force can trap low velocity electrons in an 'electromagnetic well'. Near the critical surface, this effect is of particular importance since it can prevent cold electrons inside the critical surface from sharing the energy with hotter ones outside the critical surface, i.e., a natural flux inhibition occurs. The modification of Ohm's Law due to this effect has been worked out. The main effect is to make the electron pressure a function of the radiation pressure.

Most calculations of profile modification assume infinite thermal conductivity, i.e., zero temperature gradient across the critical density. Most hydrodynamic simulations however use strongly flux-limited thermal transport and local energy deposition to model experimental results. Under these conditions we have solved the steady-state equations of continuity, momentum and energy transport and found that profile modification does indeed occur but mainly as a result of the hydrodynamic forces, not of the ponderomotive force. In addition we have shown that, with absorption, the flow can remain subsonic while passing through the critical region. However, a transition to supersonic flow is more in agreement with existing experimental results. The predicted density step from this model agrees quite well with existing data.

In addition to the density jumps produced by light pressure, shocks can result from a two-temperature electron distribution as shown in planar 
geometry by Bezzerides, et al. We have studied the formation and behavior of these rarefaction shocks in spherical geometry with and without the assumption of quasi-neutrality. The model chosen corresponds closely to that of Bezzerides but the results differ in some respects. For example, no downstream shelf is observed to form. However, good qualitative ex-. planation of phenomena commonly observed in experiments such as fast ions is found without involving anomalous flux limiting.

As a model of an exploding pusher target, we studied the selfsimilar expansion of a uniformly-heated, gaussian-shaped object. Our simulation code has verified that a uniformly heated box of plasma will quickly evolve into such a shape and will evolve as predicted by the self-similar solution. The maximum temperature achieved scales as $T_{e} e^{\alpha \sqrt{P}} \tau$ where $P$ is the power and $\tau$ the pulse length.

Clearly the interrelationship of the phenomena discussed above and their eventual importance in inertial confinement fusion is the subject of ongoing research. Many of the improvements in the simulation code have helped diagnose experiments as is demonstrated in that section of this report. As effects are discovered and proven important, an attempt will be made to simulate them in the fluid hydrocode where their interplay with other effects will influence target design. 


\subsection{Fast Ion Energy Loss in Dense Plasmas}

P. M. Campbell

\subsubsection{Introduction}

The energy loss of heavy ions in dense material is of interest for the design of targets for inertial confinement fusion. There is much experimental data on the ion stopping power of gases and solids, but apparently no measurements of energy loss in dense plasmas. Range calculations based on classical effects give good agreement with existing data for unionized materials! (1) These formulae provide a natural starting point for designing experiments for ion beam heating of plasmas.

Energy deposition by fast heavy ions has been added to the radiation, hydrodynamic, and equation of state models in the laser fusion code TRHYD. The energy loss of a fast ion is almost entirely to electrons until near the end of the ion's range. Thus a heavy particle follows essentially a straight line path. In the calculations described here, the ion trajectories are assumed to be straight lines with no deflections due to ion-ion scattering. Ion beam accelerators require for efficiency high ion energies at reasonably low charge states. (2) Under these conditions the net mass, momentum and charge delivered to the target per unit of energy is smal1. In the present calculations, mass and momentum deposition are neglected in the hydrodynamics, and the incident beam is assumed quasi-neutral. Possible anomalous mechanisms neglected in the model are beam-produced electric and magnetic fields and plasma collective effects. The calculations, therefore, give the results to be expected in ion beam heating of ICF targets if the stopping power of a plasma obeys the classical formulae. 


\subsubsection{Ion Stripping in Solid Targets}

The charge states of fast heavy ions penetrating gaseous and solid targets have been studied for some time. (3) As a fast ion travels through the medium, it assumes an equilibrium charge state determined by the competing effects of ionization and electron capture. It is possible to predict equilibrium charge states from semiempirical relationships with an average uncertainty of approximately \pm 1 unit of charge for both gaseous and solid targets. The relative charge of different fast ions in solid targets as a function of velocity is well represented by the formula $(3)$

$$
\bar{z}_{f}=z\left(1-1.032 \mathrm{e}^{-v / v^{\prime}}\right),
$$

where

$$
v^{\prime}=\frac{e^{2}}{\hbar} z^{0.69}=2.188 \cdot 10^{8} z^{0.69} .
$$

The use of such a formula for predicting the stopping power of heavy ions in solids and gases has been demonstrated by Nesbet and Ziegler. (4)

There is at present no comprehensive theory for the charge states of fast ions in either unionized materials or in plasmas. In the absence of such a theory we use Equation (1) to obtain $\bar{Z}_{f}$ in all cases, recognizing that there is considerable uncertainty as to its accuracy in a plasma.

\subsubsection{Energy Loss}

\section{Free Electrons and Ions}

In order to obtain the energy loss of an incident fast ion to free electrons and ions in the target material, one must first find a general expression, in the laboratory frame, for scattering of an incident particle 
$m_{1}, \vec{v}_{1}$ by a target particle $m_{2}, \vec{v}_{2}$. The resulting expression for the energy transfer is then integrated over a Maxwellian velocity distribution of target particles at temperature $\theta$. These calculations have been done by Brueckner and Brysk ${ }^{(5)}$ with the result

$$
-\frac{d E_{1}}{d s}=2 \pi N_{2} e^{4} z_{1}^{2} z_{2}^{2} \ln \Lambda \frac{m_{1}}{m_{2}} \frac{F(x)}{E_{1}},
$$

where

$$
\begin{aligned}
F(x) & =\operatorname{erf}(x)-\frac{2}{\sqrt{\pi}}\left(1+\frac{m_{2}}{m_{1}}\right) x e^{-x^{2}}, \\
x & =\sqrt{\frac{m_{2}}{m_{1}} \frac{E_{1}}{\theta}} .
\end{aligned}
$$

In this expression $s$ is the distance along the particle path, and en $\Lambda$ is the Coulomb logarithm given by

$$
\ln \Lambda=\frac{1}{2} \ln \left(1+\frac{b_{\text {max }}^{2}}{b_{\text {min }}^{2}}\right) \text {, }
$$

where $b_{\max }$ and $b_{\min }$ are the maximum and minimum impact parameters.

For the case of scattering by ions

$$
\begin{aligned}
& m_{1} \simeq m_{2}, \quad E \gg \theta \\
& x \gg 1, \quad F \simeq 1,
\end{aligned}
$$

and the energy loss formula becomes

$$
\left.\frac{d E_{f}}{d s}\right)_{i}=2 \pi N_{i} \bar{Z}^{2} \bar{z}_{f}^{2} \frac{A_{f}}{A} e^{4} \ln \Lambda_{i} E_{f}^{-1} .
$$


In this expression $N_{i}$ is the number density of ions in the target, $\bar{Z}$ is the mean charge of target ions $(Z, A), \bar{Z}_{f}^{2}$ is the stripped charge state of the incident fast ion $\left(Z_{f}, A_{f}\right)$ given by Equation (1), and $\ln \Lambda_{i}$ is the Coulomb logarithm for ion-ion scattering.

For the case of scattering by free electrons, $m_{2} \ll m_{1}$, but since $E \gg \theta$ no approximation to $F(x)$ can be made in general. The energy loss formula becomes

$$
\begin{aligned}
\left.-\frac{d E_{f}}{d s}\right)_{e} & =2 \pi N_{i} \bar{Z} \bar{Z}_{f}^{2} A_{f} \frac{m_{p}}{m_{e}} e^{4} \ln \Lambda_{e} F(x) E_{f}^{-1}, \\
F(x) & \simeq \operatorname{erf}(x)-\frac{2}{\sqrt{\pi}} \times e^{-x^{2}} \\
x & =\sqrt{\frac{m_{e}}{A_{f} m_{p}} \frac{E_{f}}{\theta_{e}}},
\end{aligned}
$$

where $\theta_{e}$ is the electron temperature, and $\& n \Lambda_{e}$ is the Coulomb logarithm for ion-electron scattering.

In order to determine $\ln \Lambda_{e}$ and $\ln \Lambda_{i}$ we note that $b_{\max }$ is set by screening. (6) The minimum impact parameter for ion-electron scattering is given by (7)

$$
b_{\min }^{e}=\operatorname{MAX}\left[b_{c}^{e}, b_{q}^{e}\right] \text {, }
$$

where the classical value, $b_{c}{ }^{e}$, is set by the maximum allowable energy transfer,

$$
b_{c}{ }^{e}=\frac{\bar{z}_{f e^{2}}}{m v_{f}^{2}}
$$


and the quantum mechanical value, $b_{q}{ }^{e}$, is set by the de Broglie wavelength of an electron as seen from the fast ion,

$$
b_{q}^{e}=\frac{\hbar}{2 m v_{f}} \text {, }
$$

The corresponding value of $b_{\min }$ for ion-ion scattering is

$$
b_{\min }^{i}=\operatorname{MAX}\left[b_{c}^{i}, b_{q}^{i}\right],
$$

where

$$
\begin{aligned}
& b_{c}^{i}=\frac{\bar{z}_{f} \bar{z} e^{2}}{m_{r} v_{f}^{2}}, \\
& b_{q}^{i}=\frac{\not}{2 m_{r} v_{f}},
\end{aligned}
$$

and $m_{r}$ is the reduced mass of the target ion.

\section{Plasma Oscillations}

The effect of binary collisions represented by Equations (3) and (4) is limited by screening through the maximum impact parameter contained in $\ln \Lambda$. For almost all cases where the material exists as a plasma, the screening distance is the electron Debye length $\lambda_{e}$. For distances small compared to $\lambda_{e}$, the incident particle interacts with the plasma predominantly through close binary encounters. Beyond $\lambda_{\theta}$ the plasma acts as a continuous medium, and the effect of distant collisions is the loss of energy by the excitation of plasma oscillations. The excitation energy appears as an oscillating "wake" trailing behind the particle. The electrostatic field of this polarization wake acts to retard the motion of the ion. 
If the plasma is considered as a polarizable medium, one can use the expression ${ }^{(7)}$ originally due to Fermi ${ }^{(8)}$

$$
-\frac{d E}{d s}=\frac{2}{\pi} \frac{z_{f}^{2} e^{4}}{v_{f}^{2}} \operatorname{Re} \int_{0}^{\infty} \frac{i \omega}{\varepsilon(\omega)}\left[\frac{\lambda_{e} \omega}{v_{f}} k_{1}\left(\frac{\lambda_{e} \omega}{v_{f}}\right) k_{0}\left(\frac{\lambda_{e} \omega}{v_{f}}\right)\right] d \omega,
$$

where $k_{0}$ and $k_{1}$ are modified Bessel functions. Jackson ${ }^{(7)}$ chooses for the dielectric constant

$$
\varepsilon(\omega)=1-\frac{\omega_{p}^{2}}{\omega^{2}+i \omega \cdot \Gamma},
$$

and obtains the energy loss formula

$$
-\frac{d E}{d s}=2 \pi N_{i} \bar{Z} \bar{Z}_{f}^{2} A_{f} \frac{m_{p}}{m_{e}} e^{4} \ln \left(\frac{1.123 v_{f}}{\lambda_{e} \omega_{p}}\right) E_{f}^{-1}
$$

This result is essentially the same as that obtained by Pines and Bohm (9)

$$
\left.-\frac{d E_{f}}{d s}\right)_{p o}=\pi N_{i} \bar{Z} \bar{z}_{f}^{2} A_{f} \frac{m_{p}}{m_{e}} e^{4} \ln \left(1+4 x^{2}\right) E_{f}^{-1},
$$

where $x$ is given in Equation (4). In the present calculations we use Equation (5) for the energy loss to plasma oscillations. Equations (4) and (5) together give the net loss rate to free electrons in the target material.

\section{Bound Electrons}

In computing the energy loss to bound electrons, we must adopt a method that can be applied easily to partially ionized atoms imbedded in a plasma. The energy loss to bound electrons in both solids and plasmas can be ublained in terms of the dielectric properties of the 
medium and the polarization wake induced by the fast ion. $(4,7,10)$ However, the generalization of these methods to the present problem is somewhat difficult. In the calculation described here we use Bethe's quantum mechanical result corrected for screening by free electrons with a semi-classical prescription for the mean binding frequencies.

The energy loss formula is written (7)

$$
\left.-\frac{d E_{f}}{d s}\right)_{b e}=2 \pi N_{i} \bar{Z}_{f}^{2} A_{f} \frac{m_{p}}{m_{e}} e^{4}[(Z-\bar{z}) \ln \Lambda] E_{f}^{-1},
$$

where

$$
(Z-\bar{Z}) \ln \Lambda=\sum_{j} n_{j} \frac{1}{2} \ln \left(1+\frac{b_{\text {max }}^{2}}{b_{\text {min }}^{2}}\right) \text {, }
$$

with

$$
\begin{aligned}
& b_{\min }=\frac{\hbar}{2 m_{e} v_{f}}
\end{aligned}
$$

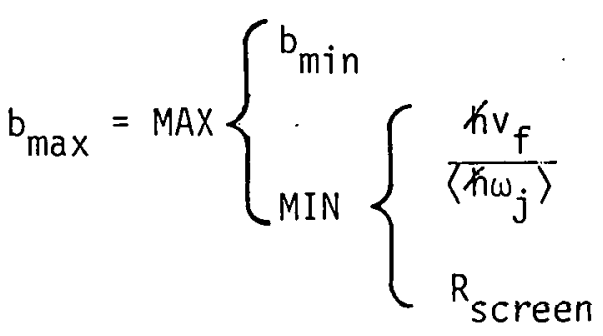

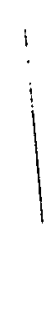

In these expressions, $n_{j}$ is the number of bound electrons in level $j$ of the target atom, and $\omega_{j}$ is the mean harmonic binding frequency of electrons in level $j$. Equation (6) reduces to Bethe's formula except where $v_{f} /\left\langle\omega_{j}\right\rangle$ exceeds the screening distance.

Since the radiation treatment in the hydrodynamic code uses Mayer's independent electron approximation for the atomic structure, $(12,13)$ we use this model to compute the $\left\langle\hbar_{\omega_{j}}\right\rangle$. 


\subsubsection{Results and Comparison with Experiment}

There are no accurate data presently available for the stopping of ions in dense plasmas. It is possible, however, to check the calculational model in the room temperature 1 imit. In Figures 3.1-1 and 3.1-2 are shown proton range measurements in $\mathrm{Al}, \mathrm{Cu}$, and $\mathrm{Pb}$. The corresponding ranges calculated by the methods described above are shown for proton energies of $1 \mathrm{MeV}, 10 \mathrm{MeV}$, and $100 \mathrm{MeV}$. The agreement is good, especially in view of the simplicity of the atomic model that is used. This calculation does not involve the question of ion stripping, however, since protons are completely ionized at $1 \mathrm{MeV}$ and above.

In Table 3.1-1 are shown the calculated ranges for $1.6 \mathrm{GeV} \mathrm{Hg}$ and Xe ions in different materials at normal density and various temperatures up to $1 \mathrm{keV}$. These calculations show that, according to the classical expressions, there is a relatively small effect on the range of these ions due to the ionization state of the material.

The energy deposition profile of protons or fully stripped low-Z ions exhibits the "Bragg peak" at the end of the ion range. This deposition profile is a very favorable one for driving ICF target implosions, hecause the energy is deposited deeply in the target shell leading to an efficient ablation drive. The deposition profile for high-Z ions is not as favorable, however, because as the ion slows down the effective charge, and hence the deposition rate, is reduced. Deposition profiles for $\mathrm{Hg}$ ions slowing down in $\mathrm{Pb}$ at normal density and different temperatures are shown in Figure 3.1-3. The stripped ion charge is given by Equation (1) and leads to a fairly uniform deposition profile. In contrast, the deposition profile for a $\mathrm{Hg}$ ion with a fixed charge $\left(\bar{Z}_{F}=46\right)$ is also shown in Figure 3.1-3 and exhibits the usual Bragg peak. 


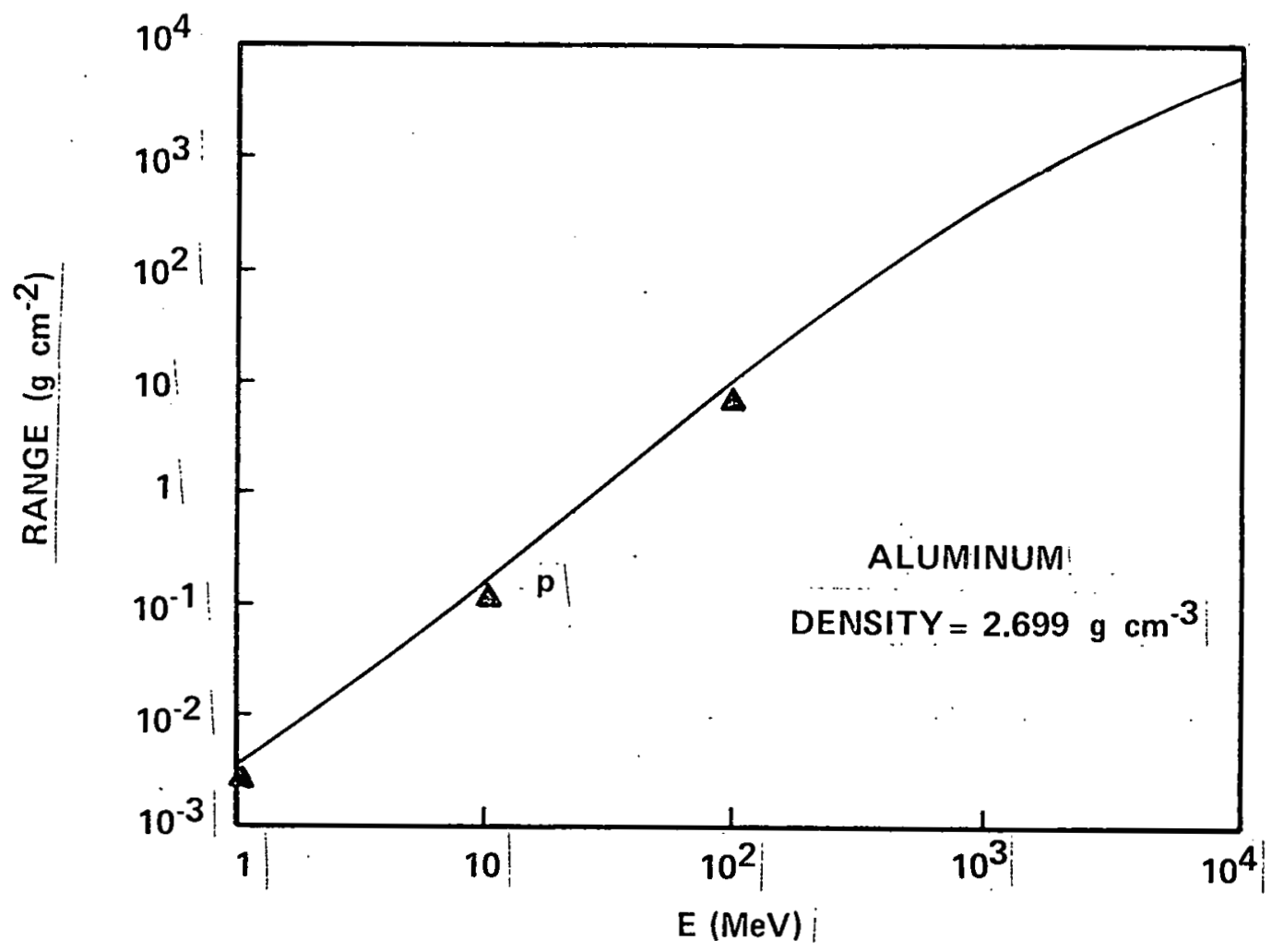

FIGURE 3.1-1 Calculated proton ranges ( $\mathbf{A}$ ) in aluminum as compared to expcrimental data. 

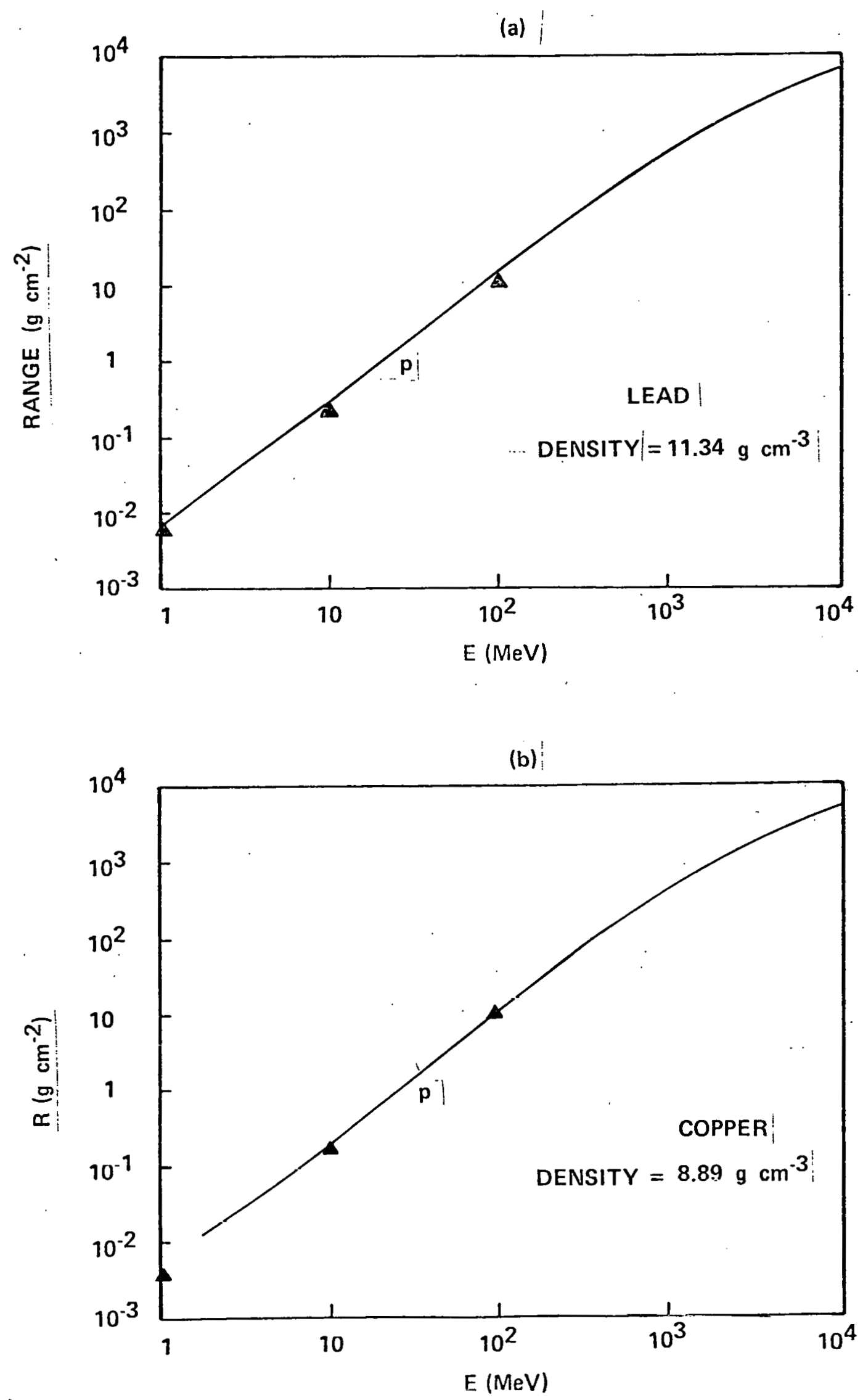

FIGURE 3.1-2 Calculated proton ranges (A) in copper and lead as compared to experimental data. 
TABLE 3.1-1

HEAVY ION RANGES CALCULATED FOR MATERIALS AT DIFFERENT TEMPERATURES

\begin{tabular}{|c|c|c|c|c|c|}
\hline Material & Temperature & $\mathrm{N}_{i}$ & $\bar{Z}$ & $\begin{array}{r}1.6 \mathrm{GeV} \text {. } \\
\text { Hg Range }\end{array}$ & $\begin{array}{r}1.6 \mathrm{GeV} \\
\times \mathrm{e} \text { Range } \\
\end{array}$ \\
\hline $\begin{array}{l}\mathrm{Pb} \\
\left(\rho=11.3 \mathrm{~g} / \mathrm{cm}^{3}\right)\end{array}$ & $\begin{array}{r}0 \mathrm{eV} \\
100 \\
1000\end{array}$ & $3.3 \cdot 10^{22} \mathrm{~cm}^{-3}$ & $\begin{array}{c}0 \\
16.4 \\
54.8\end{array}$ & $\begin{array}{l}26 \mu \mathrm{m} \\
29 \\
38\end{array}$ & $\begin{array}{l}38 \mu \mathrm{m} \\
42 \\
45\end{array}$ \\
\hline$\left.\stackrel{N_{j}}{\rho}=8.9 \mathrm{~g} / \mathrm{cm}^{3}\right)$ & $\begin{array}{c}0 \mathrm{eV} \\
100 \\
1000\end{array}$ & $9.1 \cdot 10^{22} \mathrm{~cm}^{-3}$ & $\begin{array}{c}0 \\
7.7 \\
24.0\end{array}$ & $\begin{array}{l}21 \cdot \mu \mathrm{m} \\
29 \\
27\end{array}$ & $\begin{array}{l}33 \mu \mathrm{m} \\
43 \\
37\end{array}$ \\
\hline $\begin{array}{l}\mathrm{S}_{i} \mathrm{O}_{2} \\
\left(\rho=2.4 \mathrm{~g} / \mathrm{cm}^{3}\right)\end{array}$ & $\begin{array}{c}0 \mathrm{eV} \\
100 \\
1000\end{array}$ & $3.0 \cdot 10^{22} \mathrm{~cm}^{-3}$ & $\begin{array}{l}0 \\
6.0 \\
9.7\end{array}$ & $\begin{array}{l}60 \mu \mathrm{m} \\
65 \\
65\end{array}$ & $\begin{array}{l}100 \mu \mathrm{m} \\
105 \\
100\end{array}$ \\
\hline $\begin{array}{l}\mathrm{C}_{2} \mathrm{H}_{4} \mathrm{O} \\
\left(p=1.0 \mathrm{~g} / \mathrm{cm}^{3}\right)\end{array}$ & $\begin{array}{c}0 \mathrm{eV} \\
100 \\
1000\end{array}$ & $9.6 \cdot 10^{22} \mathrm{~cm}^{-3}$ & $\begin{array}{l}0 \\
2.4 \\
3.4\end{array}$ & $\begin{array}{l}120 \mu \mathrm{m} \\
125 \\
140\end{array}$ & $\begin{array}{l}200 \mu \mathrm{m} \\
210 \\
235\end{array}$ \\
\hline
\end{tabular}




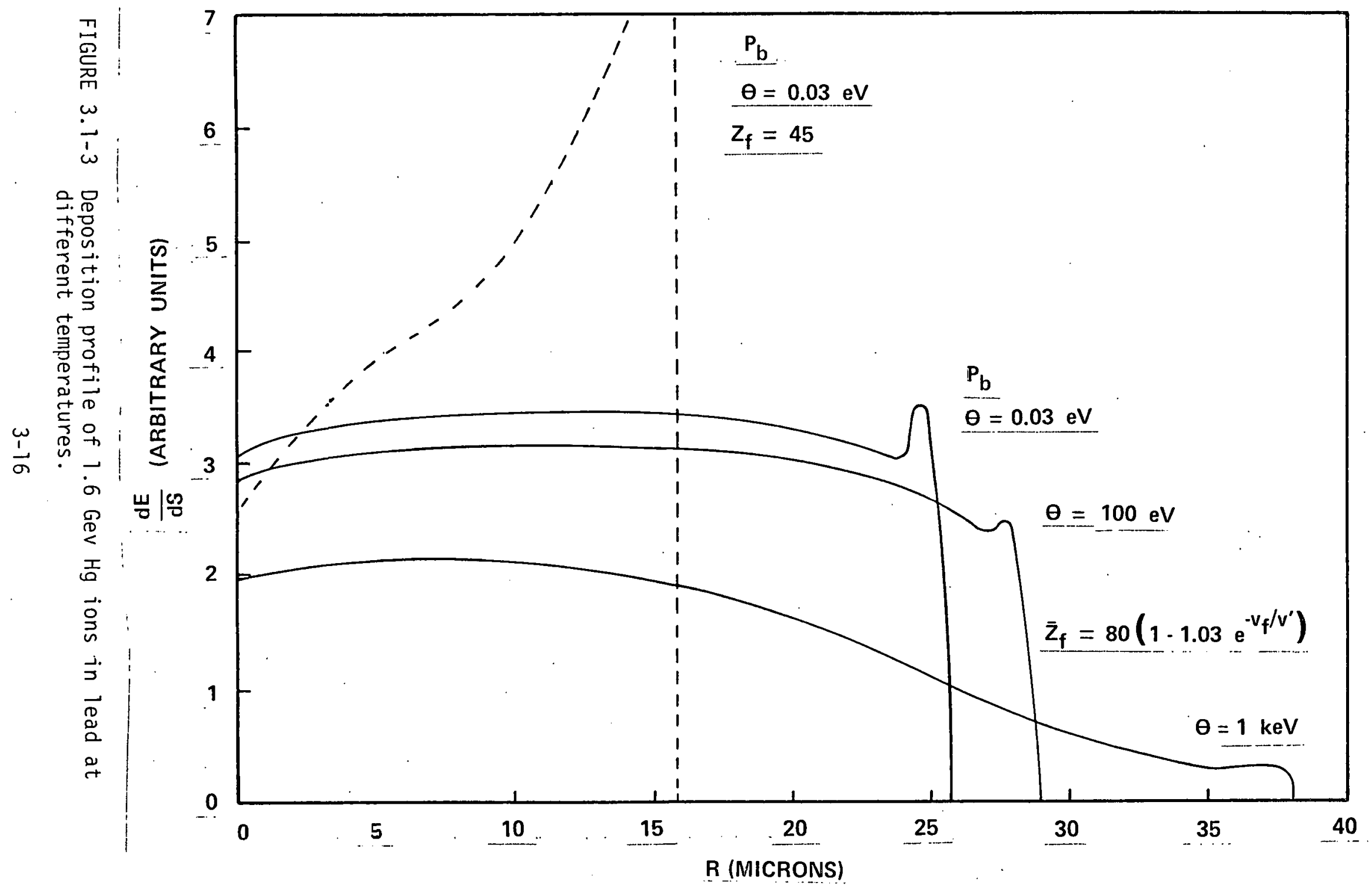




\section{REFERENCES}

1. H. Anderson (Ed.), The Stopping and Ranges of Ions in Matter, Pergamon Press, New York, Five Volumes (1977).

2. R. C. Arnold, "Heavy-Ion Beam Inertial-Confinement Fusion", Nature 276, 19 (1978).

3. H. Betz, "Charge States and Charge-Changing Cross Sections of Fast Heavy Ions Penetrating Through Gaseous and Solid Media", Revs. Mod. Phys. 44, 465 (1972).

4. R. Nesbet and J. Ziegler, "Low Velocity Stopping of Heavy Ions", App1. Phys. Lett. 31, 810 (1977).

5. K. A. Brueckner and H. Brysk, "Energy Loss of a Charged Particle in a Plasma", KMSF Document KMSF-U7 (1971).

6. E. E. Salpeter, "Electron Screening and Thermonuclear Reactions", Austr. J. Phys. 7, 373 (1954).

7. J. D. Jackson, Classical Electrodynamics, John Wiley and Sons, New York, Section 13.5, (1962).

8. E. Fermi, "The Ionization Loss of Energy in Gases and in Condensed Materials", Phys. Rev. 57, 485 (1940).

9. D. Pines and D. Bohm, "A Collective Description of Electron Interactions II", Phys. Rev. 85, 338 (1952).

10. Z. Vager and D. Gemme11, "Polarization Induced in a Solid by the Passage of Fast Charged Particles", Phys. Rev. Lett. 37, 1352 $(1976)$.

11. E. Nardi, E. Peleg, and Z. Zinamon, "Energy Deposition by Fast Protons in Pellet Fusion Targets", Phys. Fluids 21, 574 (1978).

12. H. Mayer, "Methods of Opacity Calculations", Los Alamos Report LA-647 (1947).

13. B. Kivel and H. Mayer, "Opacity of Highly Ionized Air", J. Quant. Spectr. and Radiat. Transfer 5, 13-38 (1965). 


\subsection{Electron Transport: Fokker-Planck. Calculations}

R. A. Grandey

Whenever the laser field is depositing energy into the piasma, and/or work is being done on the ions, or transporting electrons are depositing energy into the body of the pellet faster than the electrons in the vicinity of the critical surface can collisionally exchange energy, the quasi-equilibrium electron distribution can become very nonMaxwellian. (1) The electron distribution in a homogeneous plasma has been calculațed numerically in the Fokker-Planck approximation for a situation typical of current experiments. The calculation simulates. deposition of $3 \times 10^{15} \mathrm{~W} / \mathrm{cm}^{2}$ in electrons with an energy 10 times the plasma "temperature" to simulate resonant or parametric decay deposition and deposition of $1 \times 10^{15} \mathrm{~W} / \mathrm{cm}^{2}$ by inverse bremsstrahlung. This is balanced by allowing $3 \times 10^{15} \mathrm{~W} / \mathrm{cm}^{2}$ to be lost by PdV work and $1 \times 10^{15}$ $\mathrm{W} / \mathrm{cm}^{2}$ to be lost by frictional energy exchange to the body of the pellet. The problem describes a corona with a "temperature" of $10 \mathrm{keV}$ with $3 / 4$ of the energy being deposited in $100 \mathrm{keV}$ electrons.

The resultant quasi-equilibrium normalized number and energy density in energy space are plotted in Figure 3.2-1. A Maxwellian distribution is shown in Figure 3.2-2. The Fokker-Planck energy distribution is depressed relative to a Maxwellian distribution by PdV and frictional losses between 2 and 5.5 times the "temperature", with the result that the transporting part of the distribution (beyond $W \equiv E / \theta=3$ ) has approximately the same energy content for both distributions. The integrated rate of frictional energy loss (preheating) is proportional to the integral of the velocity times the energy distribution times the frictional cross section, i.e., to $\int F d W$, the integral in this case 


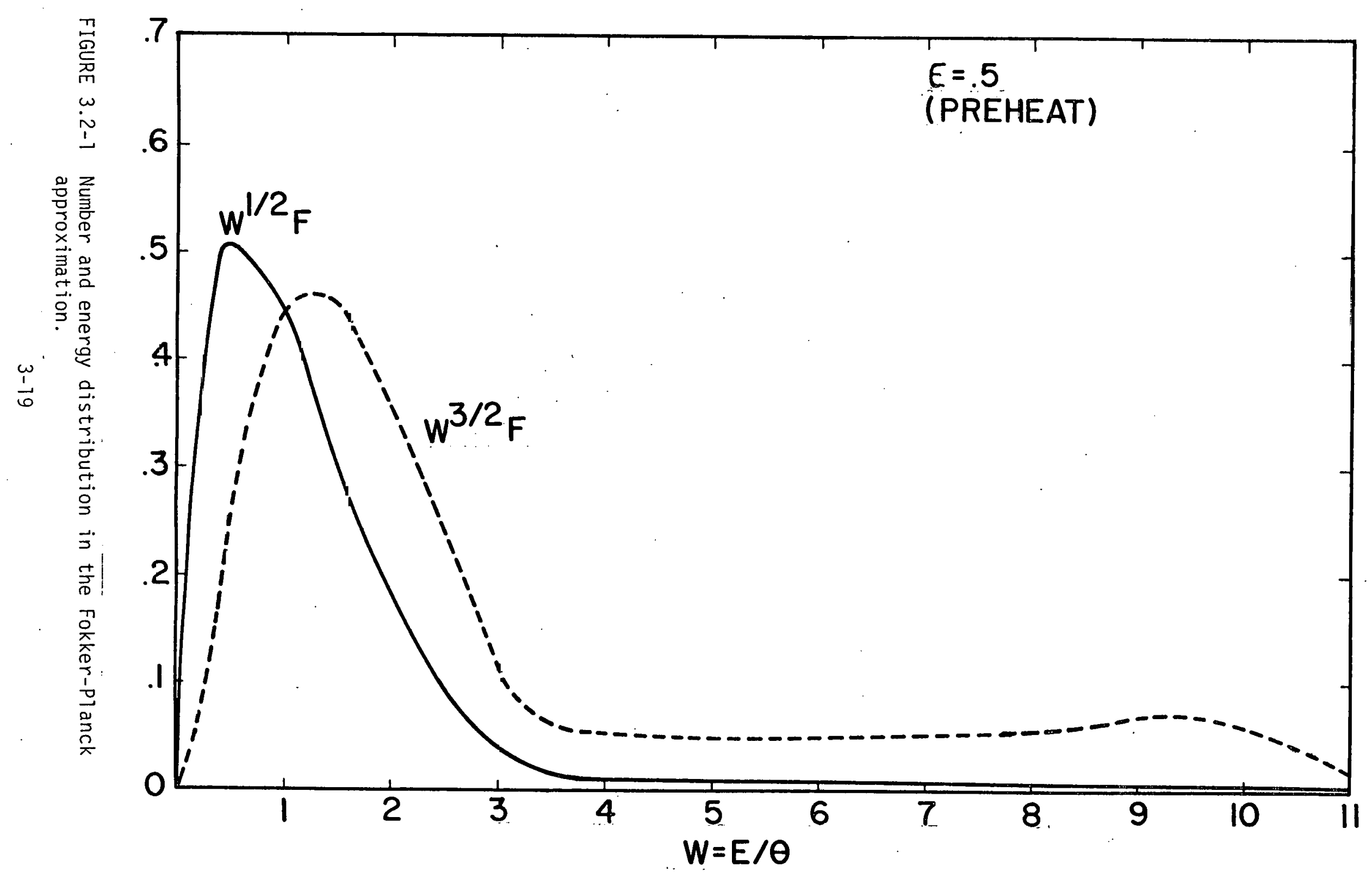




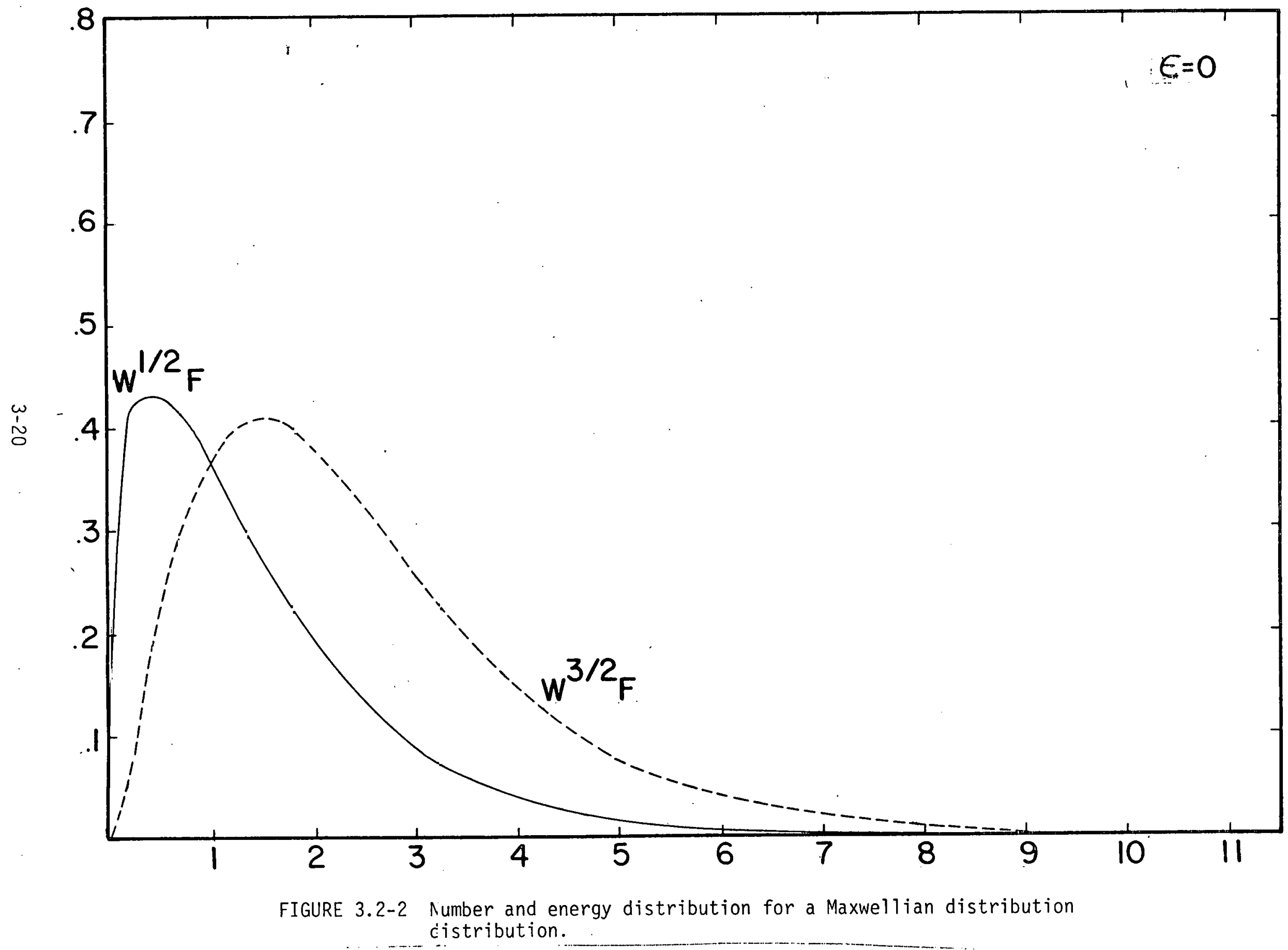


extending from $W \approx 3$ to $\infty$. The amount of preheating in the Fokker-Planck distribution is about a factor of two less than for a Maxwellian distribution.

The various transfer coefficients (thermal conduction, free-free and free-bound radiative transitions, etc.) for the two distributions are markedly different. The non-Maxwellian nature of the electron distribution function in the vicinity of the critical surface and the inability to approximate it even as the superposition of two Maxwellians must be recognized in evaluating the experimental data and in the numerical models.

\section{REFERENCES}

1. R. A. Grandey, "Status of Laser Fusion", edited by G. Kalman, Plenum Press, 1978. 


\subsection{Ionization Equation of State}

J. J. Kubis

The principal research work on the equation of state during 1978 has been the inclusion of radiative effects in the model for steady state solutions. The model equations may be expressed, in general form, as

$$
\frac{n(j+1) n_{e}}{n(j)}=\frac{I_{c}}{R_{c}+R_{r}}
$$

where $n(j)$ is the ion number density in the $j$-th ionization state, $n_{e}$ is the number density of free electrons, $I_{C}$ is the collisional ionization rate from state $j$ to state $j+1, R_{c}$ and $R_{r}$ are the collisional and radiative recombination rates, respectively, from state $j+1$ to state $j$. This is a generalization of the set of model equations listed in the 1977 Annual Report. (1) In that work we had not included $R_{r}$, the radiative recombination rate.

The physical assumptions of the model are contained in the expressions for the ionization and recombination cross sections and in the choice of ion and electron distribution functions. We assume, firstly, that the ion distributions are Maxwellian, and that the electrons form a Fermi gas whose degeneracy parameter ranges from a dilute Maxwellian gas to a very dense, completely degenerate, zero-temperature gas. We assume, secondly, that the collision cross sections for both ionization and recombination have the form of the Thomson ionization cross section. (2) We average the cross sections over the electron Fermi distribution, rather than over the Maxwellian one, to get the collisional rates. We assume, thirdly, that the radiation distribution has its equilibrium forlll, at the same temperature of the electrons and ions. For the radiative recombination cross section we take the kramers formula ${ }^{(3)}$, 
assuming that our system is transparent to its own photon radiation. We average this expression over the Fermi electron distribution to get the recombination rate. These assumptions define all the necessary cross section parameters. The solution of this system of model equations is a steady state one, since we balance both ionization and recombination rates. But it is not necessarily the state of local thermodynamic equilibrium. Because radiation energy escapes, we implicitly require an external energy source to maintain constant temperature. If we omit the radiative terms, then we recover the usual Saha model for thermodynamic equilibrium.

The effects of the radiative recombination term in the model are important for electron number densities below $10^{20} / \mathrm{cm}^{3}$. The radiative term is larger than the collisional term, and the ionization model reduces to the astrophysical "corona" model. We have ignored the radiative ionization term in our model, because it is always much smaller than the collisional term. As we decrease the ion density to the very dilute or "ideal gas" region, the radiative model leads to an average ionization value 1 or 2 charge units lower than the value gotten from the purely collisional model. The assumption of full ionization at low density is not true below a temperature of $1 \mathrm{keV}$; some $\mathrm{K}$-shell electron population remains.

For electron number densities above $10^{20} / \mathrm{cm}^{3}$, the collisional recombination dominates over the radiative rate, and we return to the collisional model of thermodynamic equilibrium, regardless of the photon distribution function. The values of average ionization from the radiative approach those of the collisional model. 
We have retained the model of ionization potential lowering taken from the work of Stewart and Pyatt ${ }^{(4)}$ and generalized to an arbitrary Fermi distribution. There has been a great deal of research work on this subject ${ }^{(5)}$, but no general formulation has emerged that can span a wide range of electron densities and temperatures.

\section{REFERENCES}

1. J. J. Kubis, "Ionization Equation of State", KMS Fusion, Inc. 1977 Annual Report on Laser Fusion Research, Chapter 3, pp. 21-23.

2. E. M. Dewan, Phys. Fluids 4 , 759 (1961).

3. G. Elwert, Zeit. f. Naturforschung 7a, 432 (1952).

G. Elwert, Zeit. f. Naturforschung $\overline{7 a}, 703$ (1952).

4. J. C. Stewart and K. D. Pyatt, Astrophys. J. 144, 1203 (1965).

5. B. H. Armstrong, et. al.., "Opacity of High-Temperature Air", Progress in High Temperature Physics and Chemistry, ed. C. A. Rouse (.0xford: Pergamon Press, 1967). Vol. 1, pp. 139-242. 
3.4 Profile Modification by Ponderomotive Forces: Equilibrium and Stability

R. L. Berger, G. R. Montry and L. V. Powers

Light pressure has been shown to have a significant effect on the plasma profile near the critical density in both particle and fluid simulations. (1-4) The equilibrium profiles without absorption for normal incidence have been found in both planar and spherical geometry. $(6,7)$ We have extended these results to include obliquely incident light in spherical geometry for the special case that the flow velocity is everywhere supersonic. ${ }^{(8)}$ These latter equilibria are not stable however for moderately strong laser fields, i.e., $|E|^{2} / 4 \pi_{n_{c}}{ }_{e} \geq .25$. The time evolution of these unstable profiles shows that the absorption varies markedly in time and can instantaneously be less than zero because of trapping of the radiation by density cavities. For obliquely incident light, such cavities and the concomitant multiple critical surfaces. leads to an increase in the amount of resonance absorption over that predicted by the steady state equations. (9)

\subsubsection{Steady-State Equations}

The basic equations we consider for normaliy incident light are the continuity, momentum, and wave equations:

$$
\begin{aligned}
& N V \xi^{2}=N_{0} V_{0} \xi_{0}{ }^{2} \\
& \frac{V^{2}}{2}+\ln N+\frac{1}{2}|\hat{E}|^{2}=\frac{V_{0}^{2}}{2}+\ln N_{0}+\frac{1}{2}\left|\hat{E}_{0}\right|^{2} \\
& {\left[\frac{d^{2}}{d \xi^{2}}+(1-N)\right] U=0}
\end{aligned}
$$


where $E=\xi_{0} U / \xi, \hat{E}=|E| /\left(4 \pi_{C_{c}}{ }^{\top}\right)^{1 / 2}, \xi=\omega r / c, V=v / C_{S}, N=n / n_{c}$, and $c_{s}=\left(Z T_{e} / m_{j}\right)^{1 / 2}$. Here $n$ and $v$ are the fluid density and velocity respectively. The electric field intensity is normalized to the plasma thermal energy $4 \pi n_{c} T_{e}$. For obliquely incident light the wave equation is generalized to

$$
\underline{\nabla}_{\xi}^{2} \underline{E}-\underline{\nabla}_{\xi}\left(\underline{\nabla}_{\xi} \cdot \underline{E}\right)+\varepsilon(\xi) \underline{E}+\beta(\xi) \gamma \cdot \underline{\nabla}_{\xi}\left(\underline{\nabla}_{\xi} \cdot \underline{E}\right)-\underline{\nabla}_{\xi} \underline{\underline{E}} \frac{\nabla_{\xi}^{n_{0}}}{n_{0}}=0
$$

which reduces to the former case for normal incidence and electron temperature, $T_{e}=0$. Here we have defined

$$
\varepsilon(\xi)=1-N(\xi)(1+i \nu / \omega)
$$

and

$$
\beta(\xi)=\left(T_{e} / m_{e} c^{2}\right) /\left(1+i \nu_{L} / \omega\right)
$$

where the absorption coefficients $\nu$ and $\nu_{L}$ are used to model inverse bremsstrahlung and Landau damping of the resonantly-excited electron plasma wave respectively. For our purposes, we have chosen $v$ constant and small but have chosen $\nu_{L}$ as given by Forslund, et al ${ }^{(3)}$, that is,

$$
\nu_{L}=\omega\left(\frac{\pi}{8}\right)^{1 / 2}(\gamma / \varepsilon)^{5 / 2} \exp (-\gamma / 2 \varepsilon)
$$

This form of $\nu_{L}$ is motivated by the WKB expression for the wave number and the kinetic theory expression for Landau damping in a homogeneous plasma and for a Maxwellian electron velocity distribution. It is important that $\nu_{L}$. be large enough and the plasma be large enough that the 
electron plasma wave is completely damped before it has propagated to the plasma boundary (or equivalently, the point where the wave equation boundary conditions are applied). Otherwise a spurious reflection of the electron plasma wave component will occur at the boundary.

For normal incidence in spherical geometry without absorption, Mulser and Vankessel ${ }^{(7)}$ have shown that a plateau density profile as shown in Figure 3.4-7a is the only steady state solution of the hydrodynamic equations of continuity and momentum combined with the wave equation if the flow velocity into the critical density is supersonic. No steady flow exists once the incident field exceeds a value $\left|E_{S}\right|$ such that the flow becomes subsonic in the plateau region. We have integrated the wave equation (11). for oblique incidence together with Equations (8) and (9). Of course the absorption is no longer zero, but the important difference is that the ponderomotive force near the plateau region for the same incident intensity is larger for obliquely incident light than for normally incident light. Moreover this maximum occurs very near the critical density $n_{c}$ instead of about one light wavelength away. In Figure 3.4-1b, we show a steady state profile for light incident at an angle $\theta=20^{\circ}$. The plateau density has been raised to above critical and is monotonically decreasing with radius. The electron plasma wave slows the flow into the plateau region which is everywhere near critical. If the plateau density were below critical and increasing with radius, the electron plasma wave would be very large in this region. Thus, the plasma adjusts by raising the plateau density and thus reducing the maynitude of $|E|^{2}$. 
(a)

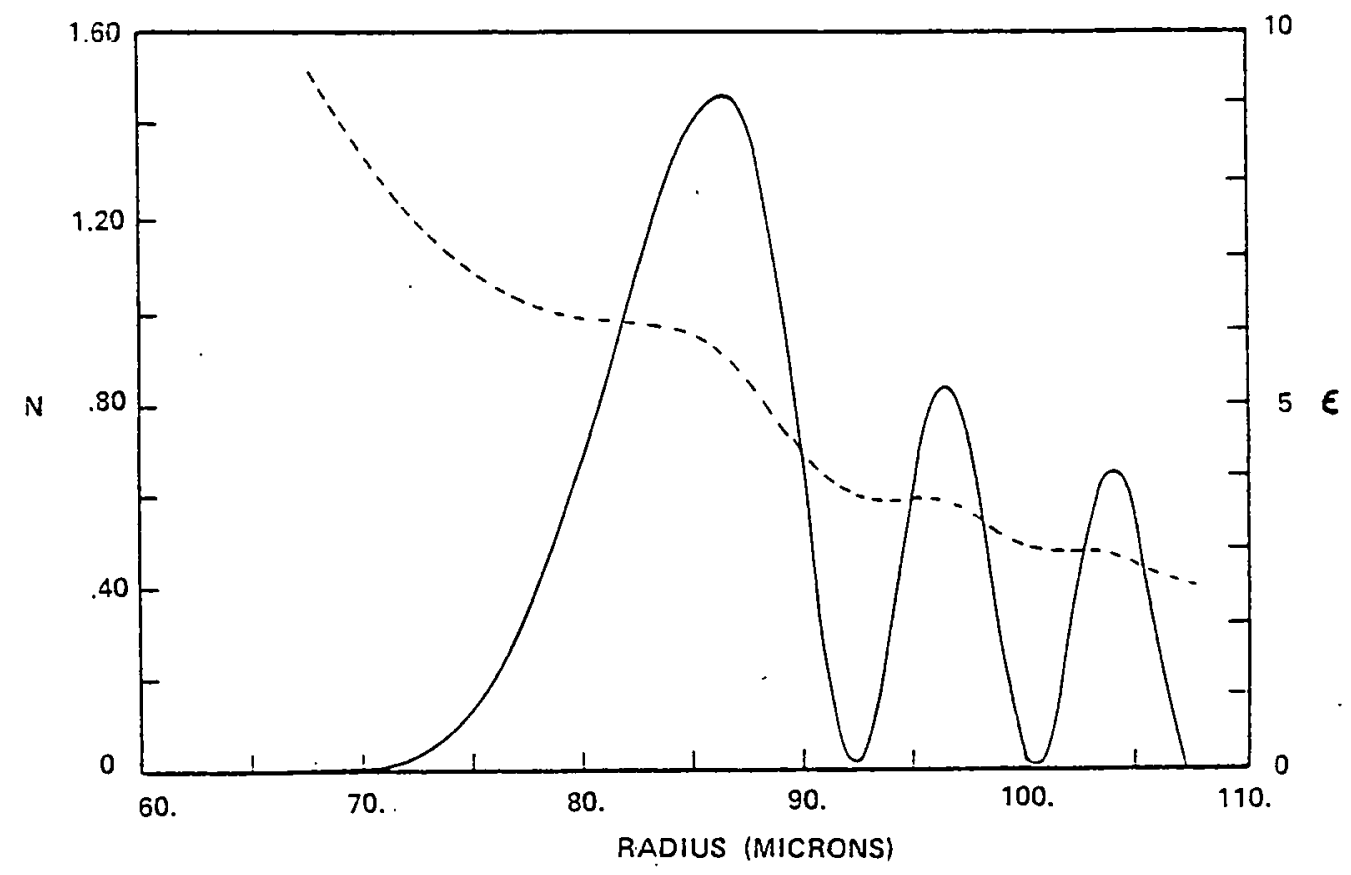

(b)

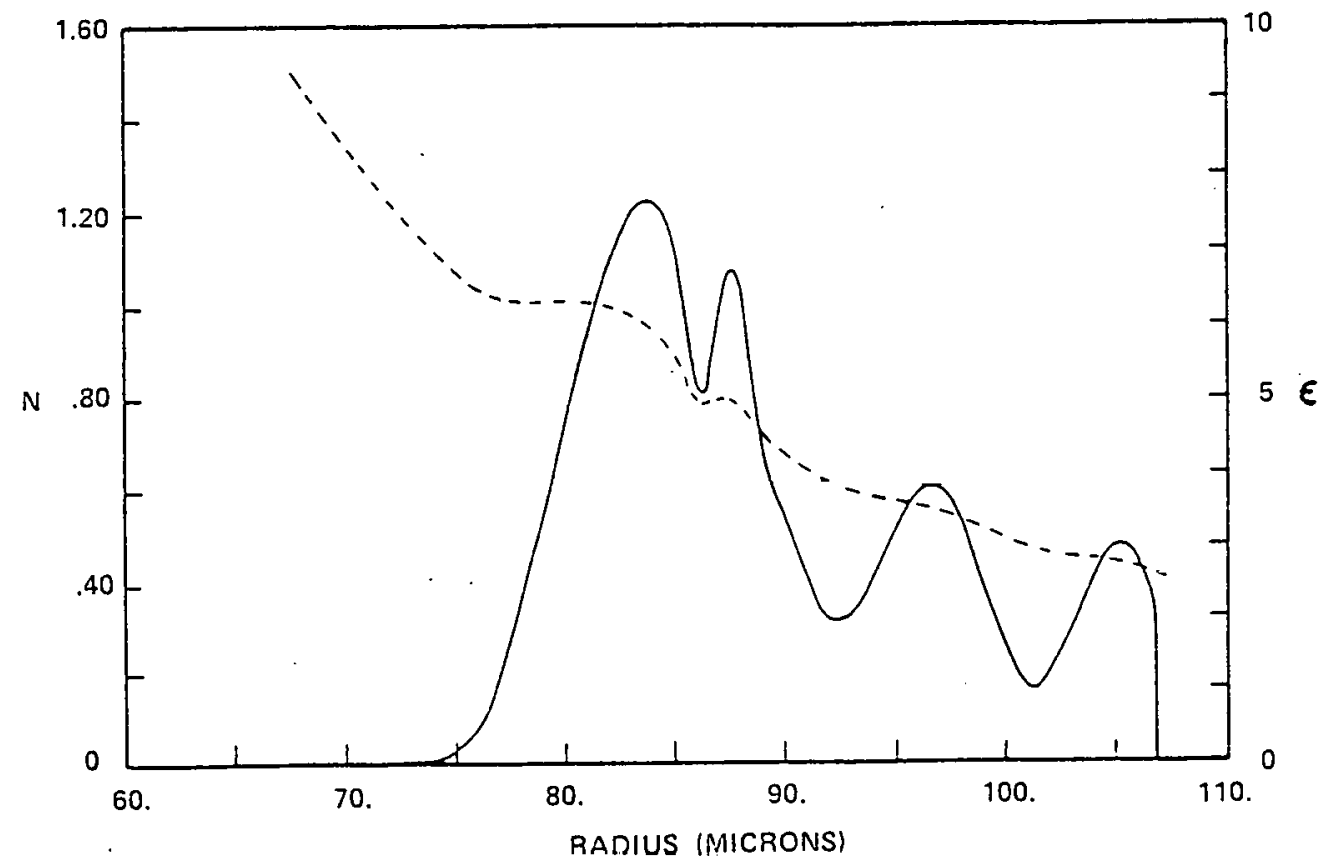

FIGURE 3.4-1 Examples of Steady-State Density Profiles for Incident Intensity $|E| 2 / 4 \pi n{ }^{T}=0.45:$ a) normal incidence, b) angle of incidence $0=20^{\circ}$ : The scalc on the left refers to the normalized density (dashed curve) and that on the right to the normalized electric field intensity $\varepsilon=|E|^{2} /\left|E_{o}\right|^{2}$ (solid curve). 
As the incident field increases the plateau density adjusts to reduce the penetration of $E$ past the turning point density $n_{t}$ and reduces the percentage absorption as shown in Figure 3.4-2 for $\theta=20^{\circ}$. The absorption still peaks as a function of angle of incidence. The angle dependence is similar in shape to subsonic flow profiles, but the amount of absorption is lower in magnitude.

One interesting feature we observed is that if the incident field is composed of several angles of incidence (as might occur in many beam systems), the absorption percentage can be higher than the absorption percentage on a self-consistent profile by any one of the components with all the energy. Thus large focal spots on many beam systems may lead to increased absorption even if refractive losses are increased.

\subsubsection{Stability of Profiles and Time-Dependent Absorption}

As mentioned previously, the steady-state plateau type density profiles are unstable to radial density perturbations for incident fields in excess of a threshold field, $\hat{E}_{t h}{ }^{(10)}$ This threshold field depends on the incident flow velocity $V_{0}$, on the radius of the critical surface $\xi_{0}$, on the wavelength $\lambda$, and, for obliquely-incident, p-polarized light, on the angle of incidence. This instability which occurs in a one-dimensional treatment is different from the instability that ripples the critical surface. $(11,12)$ This latter instability is sensitive to the angle of incidence and plane of polarization, and thus, it appears to require a threedimensional treatment. To our knowledge, the stability of plateau density profiles to rippling has not been established. 


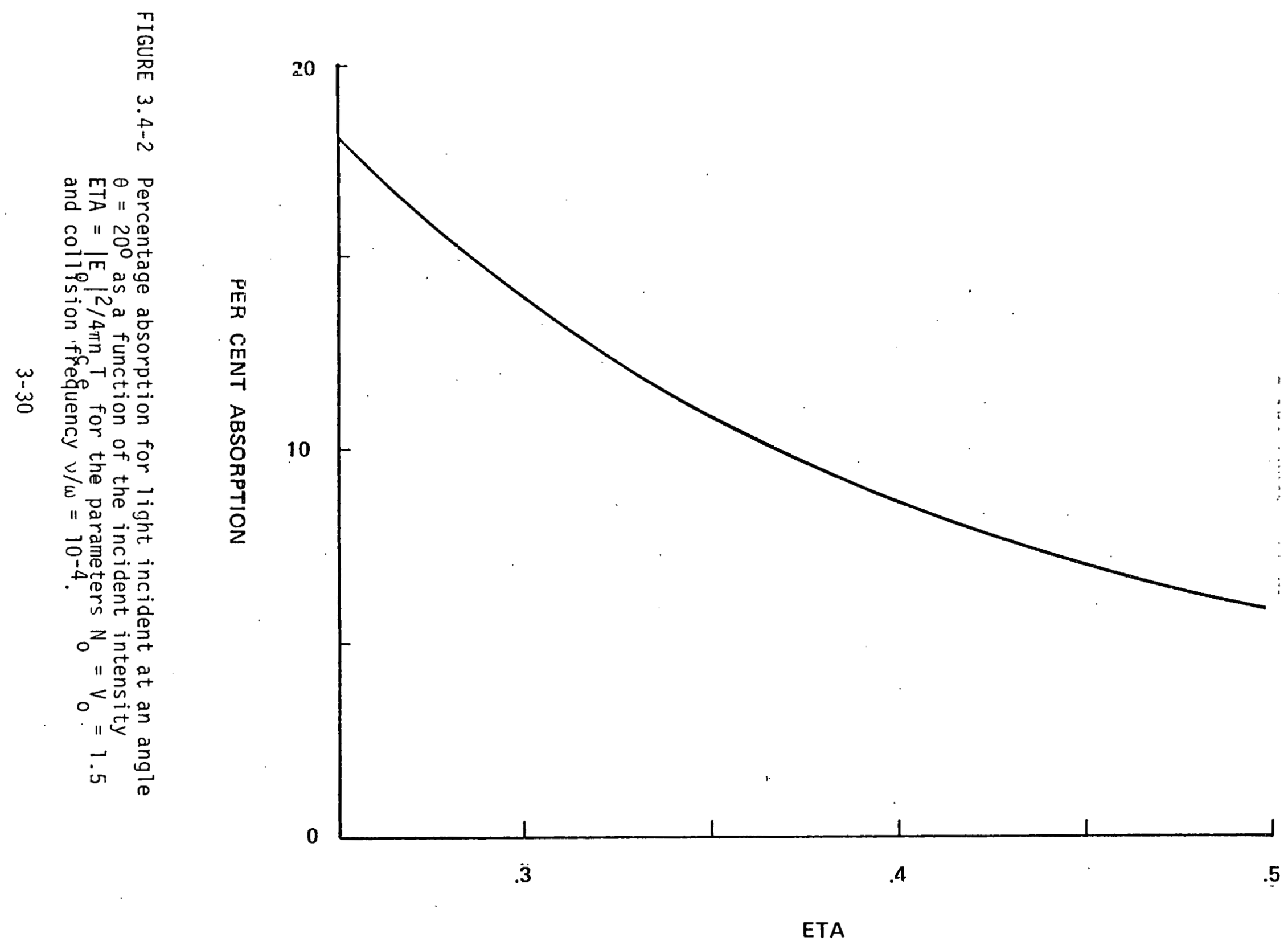


Hormal Incidence: Radial Stability

The stability of plateaus to radial perturbations is examined in this section. In Figure 3.4-3, we show for a fixed flow velocity, $V_{0}$, and critical surface radius $\xi_{0}$ the variation of the density profile with the incident intensity $\left|\hat{E}_{0}\right|^{2}$ (the incident intensity is computed at the critical radius for light in a vacuum). As the intensity increases, the length of the plateau increases and the density gradient decreases and eventually changes sign at $\hat{E}=\hat{E}_{B}$. Finally an intensity is reached for which no solution exists. The ponderomotive force in the plateau region retards the flow. The end of the plateau occurs when this force changes sign, accelerates the flow and thus causes a density drop. It seems clear for the $E \geq E_{B}$ that a density perturbation that enhances the density in the front of the plateau and decreases the density in the rear will cause the maximum of the field to move inside the plateau. Once this has occurred, the equilibrium is lost, and a hole is dug by the light pressure with the displaced plasma accumulating in a density bump at the front edge of the plateau. This effect is depicted in Figure 3.4-4 for a simulation 100 picoseconds in duration starting from an equilibrium. In this example, the time-dependent, wave equation versions of Equations (10) and (11) were solved, i.e., a term $(2 i / \omega) \partial \underline{E} / \partial t$ appears on the right hand side. Note the fast time variation is removed by the assumption that $\underline{E}$ varies as $e^{i \omega t}$. Simulations in which the wave equation is time independent show basically the same evolution. In particular. the incident field for which instability occurs is found to be the same and to correspond to that field for which plateaus have a positive density gradient, i.e., $\hat{E}_{\text {th }}=\hat{E}_{B}$. 


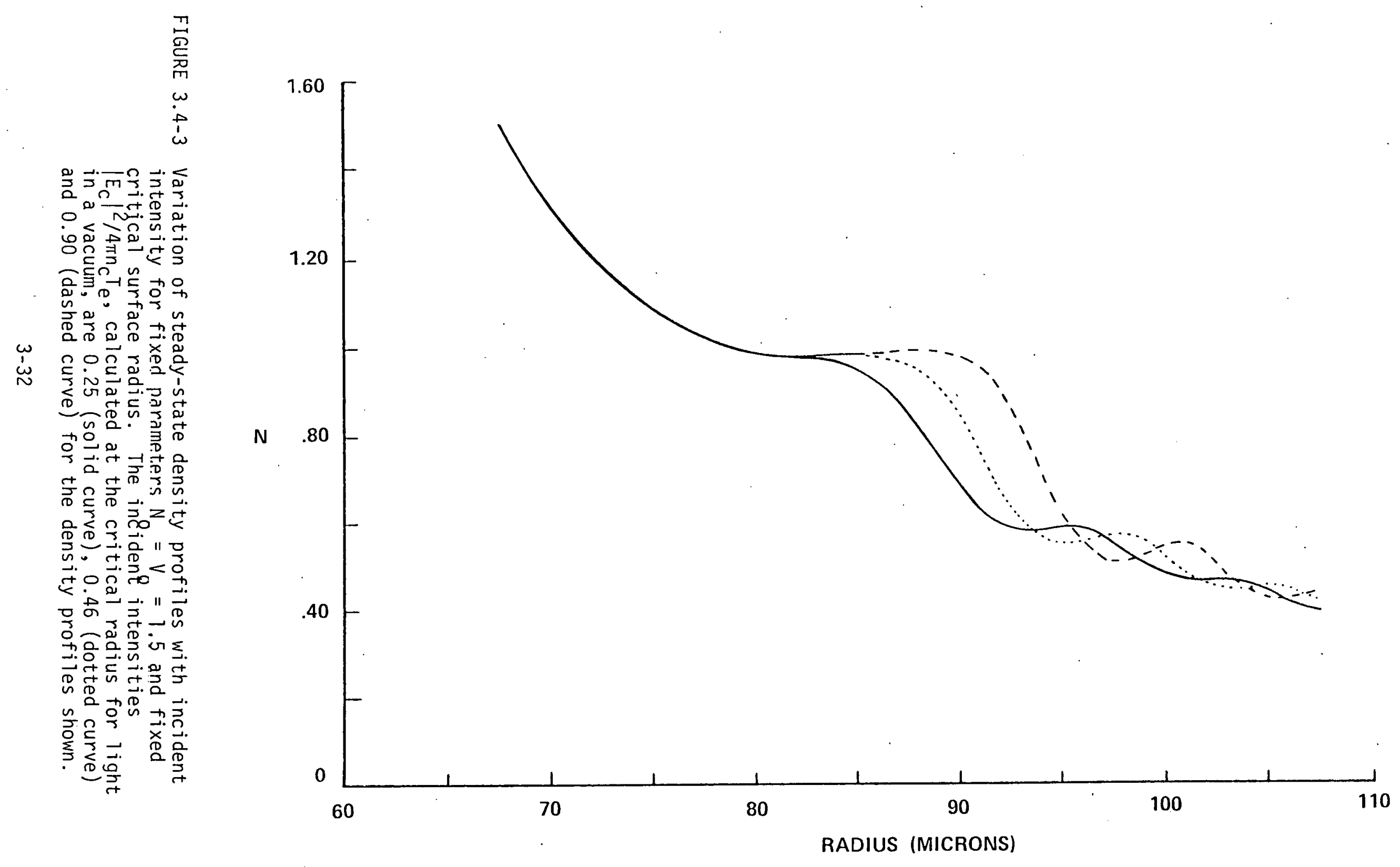


The long time behavior of unstable profiles has been found - an example of which is shown in Figure 3.4-4. The initial density perturbation grows and propagates outward at a speed somewhat less than the sound velocity. The density bump becomes supercritical and traps light in the cavity in this one-dimensional model. (13) Later this bump drops in density and a new bump begins to develop near the original surface. No time-independent flow develops but rather a repetitive unsteady flow pattern emerges. Because the profile can form cavities that trap light, the instantaneous reflection can be very small or larger than one. However, for normal incidence, the time average absorption is zero because no effective absorption mechanism is included.

A number of simulations have been done to establish the dependence of the instability growth rate on the intensity. The dependence of the growth rate, $\gamma$, on intensity was predicted by Virmont, et al, to be $\gamma \sim\left(1-|\hat{E}|^{2} /\left|\hat{E}_{t h}\right|^{2}\right)^{1 / 2}$. This dependence could not be verified from the simulations, due to the small range of intensities for which unstable steady-state profiles exist and the uncertainty in the data from which the growth rate was calculated. The threshold however is in good agreement with this theory.

Oblique Incidence: Radial Stability

We have extended our stability studies to include obliquelyincident, p-polarized light. The equilibrium self-consistent profiles were discussed previously. One new feature here is that the density perturbations strongly influence the amount of absorption, and a small change 

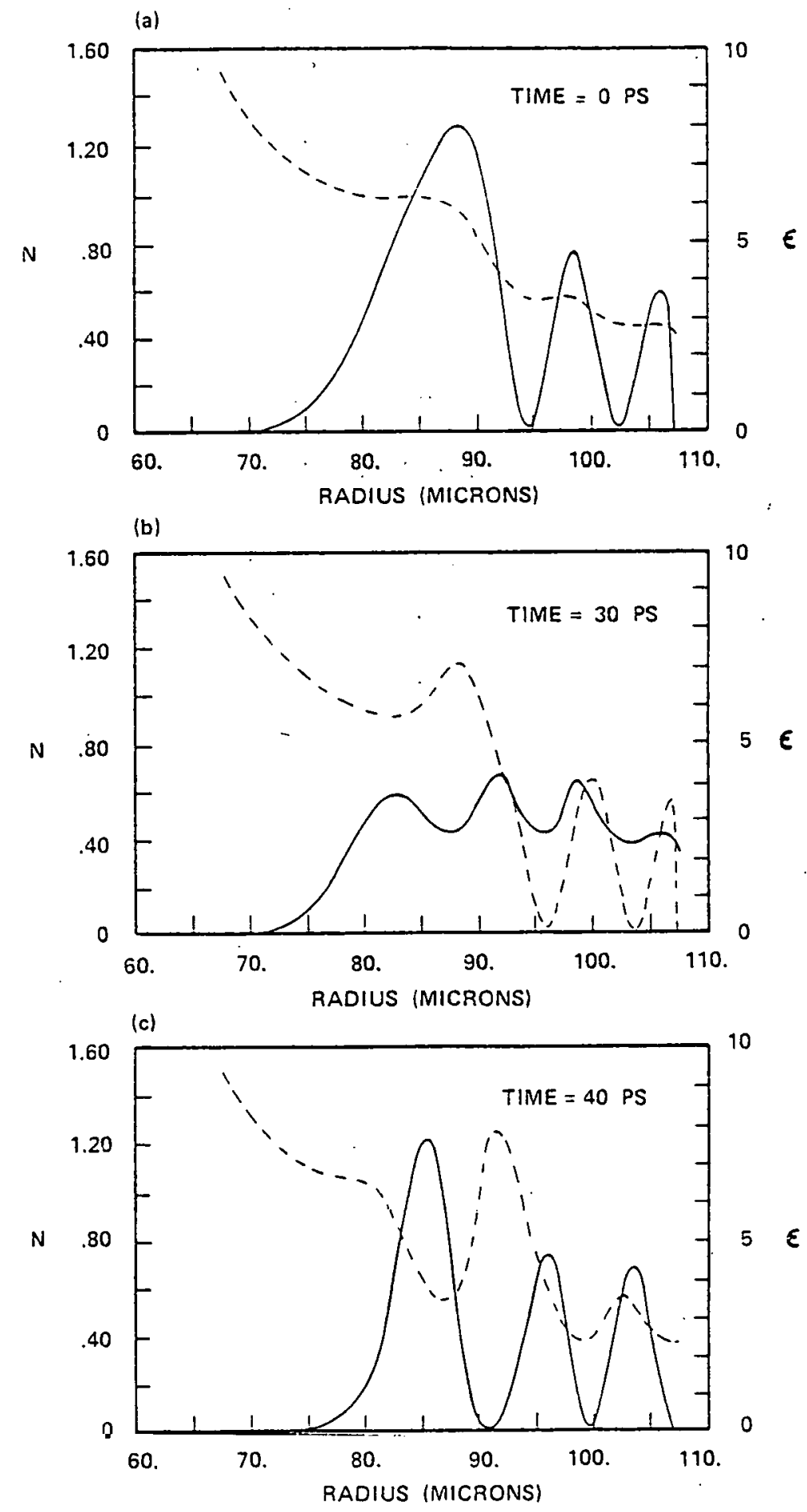

FIGURE 3.4-4 Time Evolution of Unstable Density Plateau for Incident Intensity $|\vec{E}|^{2}=0.67$ : a) $t=0$, b) $t=30 \mathrm{psec}$, and c) $l=40 \mathrm{psec}$. The scale on the left refers to the normalized density (dashed curve). The scale on the right refers to the normalized electric field intensity $\varepsilon=|E|^{2} /\left|E_{0}\right|^{2}$ (solid curve). 
in the profile can have a large effect on the strength of the ponderomotive force. As a result the threshold electric field for which the profile is unstable is smaller for oblique incidence than for normal incidence. Once again cavities are produced but now the average absorption is affected because there are several critical surfaces where resonant absorption can occur. To further complicate matters the light absorbed near the innermost critical surface can propagate outward, be partially absorbed and tunnel out to the outermost critical surface and there reconvert to light by the process inverse to resonant absorption. In this case a time-dependent wave equation solution is essential to find all these features, although the threshold for instability can still be obtained from a steady-state treatment. Of course this follows from the fact that the two treatments give the same results for stable profiles. The growth rate of the instability for light incident at an angle $\theta \simeq 20^{\circ}$ is larger than in the normal incidence case for the same incident intensity. Again no clear dependence of the growth rate on incident intensity can be observed, for the same reasons cited in the normal incidence case.

The absorption rate varies dramatically with time as shown in Figure 3.4-5 for $\theta=20^{\circ}$ and $\hat{E}_{0}^{2}=.45$. This absorption rate includes inverse bremsstrahlung, resonance absorption, and the 'fake' absorption of light trapping. We have no easy way to isolate each part, especially because, in this case, the inverse bremsstrahlung rate is anomalously high, i.e., $v / \omega \simeq .01$. The time averaged absorption versus time is shown as a mcasure of the true absorption. The absorption rate in the initially self-consistent profile is clearly lower than the time averaged one once the instability is fully developed. 


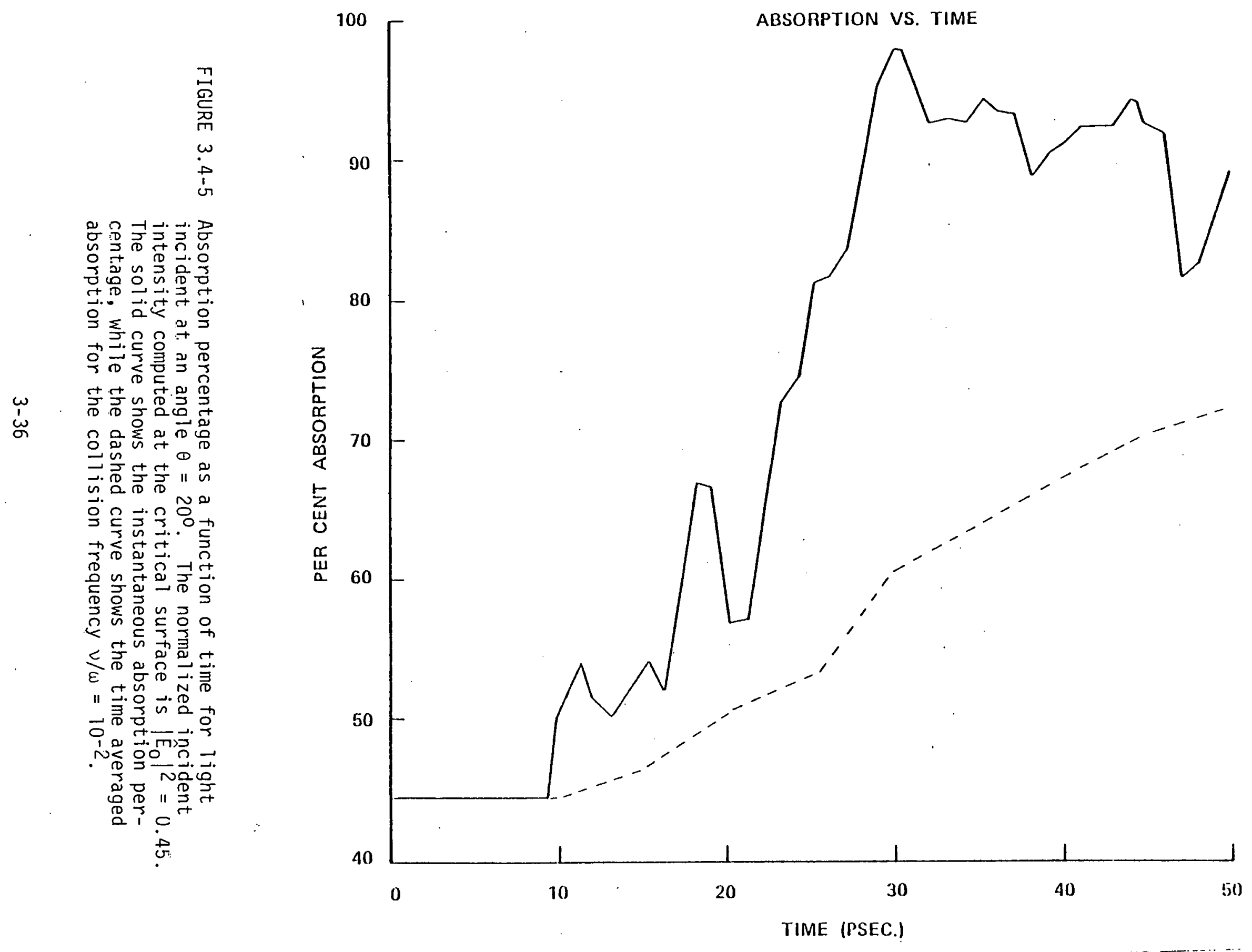


We are currently testing the dependence of the instability on wavelength for obliquely incident light. Even for stable profiles, there is the question of accessibility of that equilibrium from an initial state. Bandwidth may play an important role for unstable profiles although the definition of and solution to equilibrium is difficult in that case. However, for small bandwidth, the 'equilibrium' may be close to the zero bandwidth case. Bandwidth also effects the wave damping process but this is more important in describing the effects of resonant absorption on the electron distribution function than in accounting for the momemtum reaction on the profile (which is taken into account by the wave damping, i.e., an average over the distribution).

We have shown that for supersonic flow into the critical density and modest but realistic intensities (for $\lambda \sim 1$ micron), the profile near the critical density is not necessarily step-like and stationary. The absorption rate may vary significantly in time due to the variation of the density gradient near the critical surface. Trapping of radiation requires at least a two-dimensional treatment since the light could escape by moving sideways. However, this instability grows on a time scale of the same order or less than the rippling of the critical surface and thus must be considered if the flow is supersonic in the frame of the critical surface. 


\section{REFERENCES}

1. D. W. Forslund, J. M. Kinde1, K. Lee and E. L. Lindman, Phys. Rev. Lett. 36,35 (1976).

2. K. G. Estabrook, E. J. Valeo and W. L. Kruer, Phys. Fluids 18, $1151(1975)$.

3. D. Forslund, J. Kinde1, K. Lee, E. L. Lindman and R. L. Morse, Phys. Rev. A 11, 679 (1975).

4. W. L. Kruer and K. Estabrook, Phys. Rev. Lett. 40, 42 (1978).

5. K. A. Brueckner and R. S. Janda, Nuc. Fusion 17-3, 451 (1977).

6. K. Lee, D. W. Forslund, J. M. Kindel and E. L. Lindman, Phys. Fluids 20, 51 (1977).

7. P. Mulser and C. VanKesse1, Phys. Rev. Lett. 38, 902 (1977);

D. Mitrovich, "Effect of Density Plateau on Inverse Bremsstrahlung", KMSF-U706, September 1977.

8. G. R. Montry and R. L. Berger, Eighth Annual Conference on Anomalous Absorption of Electromagnetic Waves, Tucson, Arizona, April 19-21, 1978.

9. L. V. Powers, G. R. Montry, D. J. Tanner and R. L. Berger, Bul1. Am. Phys. Soc. 23-7, 857, September 1978.

10. J. Virmont, R. Pellat and A. Mora, Phys. Fluids 21 (4), 567 (1978).

11. E. J. Valeo and K. G. Estabrook, Phys. Rev. Lett. 34, 1008 (1975),

K. Estabrook, Phys, Fluids 19, 1733.

12. LASL Progress Report, January 1 - June 30, 1976, pp. 87.

13. This mechanism may in two dimensions drive a sausage instability as described in Ref. 11. 


\subsection{Ponderomotive Potential Effects on Ohm's Law}

R. L. Berger

The effect of the ponderomotive force on the density profile near the critical surface is now well established. These results can be derived from a kinetic equation that includes the Lorentz force of the light wave as has been shown elsewhere ${ }^{(1,2)}$ and will be considered here in the special case of planar geometry and normal incidence. Considered from a particle viewpoint, the ponderomotive potential, $\Phi=|\mathrm{e} E / \mathrm{m} \omega|^{2}$, where $\underline{E}$ is the light electric field, acts as a potential barrier to low velocity electrons. Thus, near the maximum of the potential, $\Phi_{m}$, the current is inhibited, and the temperatures on either side of $\Phi_{m}$ are related by the heat flow which involves, therefore, the high velocity electrons only. For large enough $\Phi_{m}$, the scattering rate of these 'suprabarrier' electrons is small enough that a substantial temperature jump across critical can be maintained. Here we do not self-consistently determine this temperature 'jump' but assume independent electron distribution functions exist on either side of $\Phi_{m}$ for $v^{2}<2 \Phi_{m}$.

The Boltzmann equation for electrons including the Lorentz force of the light wave, collisions with infinitely massive ions of charge $Z$, and electron self-collisions is

$$
\begin{aligned}
\left(\frac{\partial}{\partial t}+\underline{v} \cdot \frac{\partial}{\partial \underline{\partial}}\right) F & -\frac{e}{m}\left(\underline{E}-\underline{\nabla} \phi(x)+\frac{\underline{v}}{c} \times \underline{B}\right) \cdot \frac{\partial}{\partial \underline{v}} F \\
& =\Gamma_{e} \frac{\partial}{\partial \underline{v}} \cdot\left(\frac{v^{2} \underline{\underline{I}}-\underline{v} \underline{v}}{v^{3}} \cdot \frac{\partial}{\partial \underline{v}} F\right)+\left.\frac{\partial F}{\partial t}\right|_{e e}
\end{aligned}
$$


where $\underline{E}$ is the laser electric field, $\underline{B}$ the laser magnetic field, $\phi(x)$ is a slowly varying in time electrostatic field, $\partial F /\left.\partial t\right|_{e e}$ is the electronelectron collision term, and $\Gamma_{e}=2 \pi Z^{2} e^{4} N_{j} \ln \Lambda / m_{e}^{2}$.

We wish to solve for the slowly time-varying behavior of the distribution function and thus we separate $F$ into slow and fast time varying parts, i.e.,

$$
F=f+\left(\delta f e^{-i \omega t}+c \cdot c .\right)
$$

Substituting (16) into (15), we find for the slow (time averaged over a wave period) part of $F$ the equation,

$$
\begin{aligned}
\left(\frac{\partial}{\partial t}+v_{x} \frac{\partial}{\partial x}\right) f & +\frac{e}{m} \frac{\partial}{\partial x} \phi \frac{\partial}{\partial v_{x}} f-\frac{e}{m}\left\{\left(\underline{\hat{E}}+\frac{\underline{v}}{c} x \underline{\hat{B}}\right) \cdot \frac{\partial}{\partial \underline{v}} \delta f^{\star}+c . c .\right\} \\
& =\Gamma_{e} \frac{\partial}{\partial \underline{v}} \cdot\left(\frac{v^{2} \underline{\underline{I}}-\underline{v} \underline{v}}{v^{3}} \cdot \frac{\partial}{\partial \underline{v}} f\right)+\left.\frac{\partial f}{\partial t}\right|_{e, e}, \mid
\end{aligned}
$$

and for the fast varying part

$$
-i \omega \delta f=\frac{e}{m}\left(\underline{\hat{E}}+\frac{\underline{v}}{c} \times \underline{\hat{B}}\right) \cdot \frac{\partial}{\partial \underline{v}} f .
$$

In obtaining (18) we are deliberately neglecting absorption. In (17) and (18)

$$
\begin{aligned}
& E=\hat{E} e^{-i \omega t}+c \cdot c . \\
& B=\hat{B} e^{-i \omega t}+c \cdot c .
\end{aligned}
$$

and we will make use of the Maxwell equation,

$$
\underline{\nabla} \times \underline{E}=-\frac{1}{c} \frac{\partial}{\partial t} \underline{B} \simeq i \omega \underline{B} / c .
$$


Substituting (18) in (17) and using (19), we find the desired equation, i.e.,

$$
\left(\frac{\partial}{\partial t}+v_{x} \frac{\partial}{\partial x}\right) f-\frac{\partial}{\partial x} \tilde{\Phi} \frac{\partial}{\partial v_{x}} f=\left.\frac{\partial}{\partial t} f\right|_{e j}+\left.\frac{\partial}{\partial t} f\right|_{e e}
$$

where

$$
\left.\frac{\partial f}{\partial t}\right|_{e i}=\Gamma_{e} \frac{\partial}{\partial \underline{v}} \cdot\left(\frac{v^{2} \underline{\underline{I}}-\underline{v} \underline{v}}{\dot{v}^{3}} \cdot \frac{\partial}{\partial \underline{v}} f\right) \text { and } \tilde{\Phi} \equiv \Phi-e \phi / m_{e} \text {. We now. }
$$

assume that $\left|\frac{1}{f} \frac{\partial f}{\partial t}\right| \sim\left|\frac{1}{f} \frac{\partial f}{\partial t}\right|_{e e}\left|\simeq z^{-1} \frac{1}{f} \frac{\partial f}{\partial t}\right|_{e, i}$. and that $z>>$. Thus: to order $z^{-1}$, we solve (20). Without collisions, i.e., setting the RHS of (20) equal to zero, the solution of (20) is any function of $v_{x}^{2} / 2+\tilde{\Phi}$ and $v_{y}$ whereas the solution to $\partial \mathrm{f} /\left.\partial \mathrm{t}\right|_{\mathrm{ei}}=0$ is any isotropic distribution. We are motivated therefore to choose the coordinates

$$
W=\frac{v^{2}}{2}+\tilde{\Phi}
$$

and $v_{y}$ and $x$ so that Equation (20) becomes

$$
\pm \frac{\partial}{\partial x} f^{ \pm}=v_{e i}(W, x) \frac{\partial}{\partial v_{y}}\left(\left|v_{x}\right| \frac{\partial f^{+}}{\partial v_{y}}\right)
$$

where the \pm refers to the sign of $v_{x}$ and $\left|v_{x}\right|=\sqrt{2}\left(w-\tilde{\Phi}-v_{y / 2}^{2}\right)^{1 / 2}$. We are interested in solving (21) in the vicinity of the critical surface and assume $\tilde{\Phi}$ has a single maximum just outside the surface as depicted in Figure 3.5-1. Analytic solutions can be sought in two limits, i.e., when the mean free path, $\lambda$, is much less than the ponderomotive potential scale length, $L_{\Phi}$, i.e., $\lambda \ll L_{\Phi}$ and in the limit $L_{\Phi} \ll \lambda$. For low velocity electrons, that constitute the bulk of the electrons reflected by the potential, the former limit is more appropriate. An estimate of the electron energy $E^{*}$ at which these two length scales are equal is found by setting $I_{\phi} \simeq c / \omega_{\text {pe }} n, c / \omega$ and then 


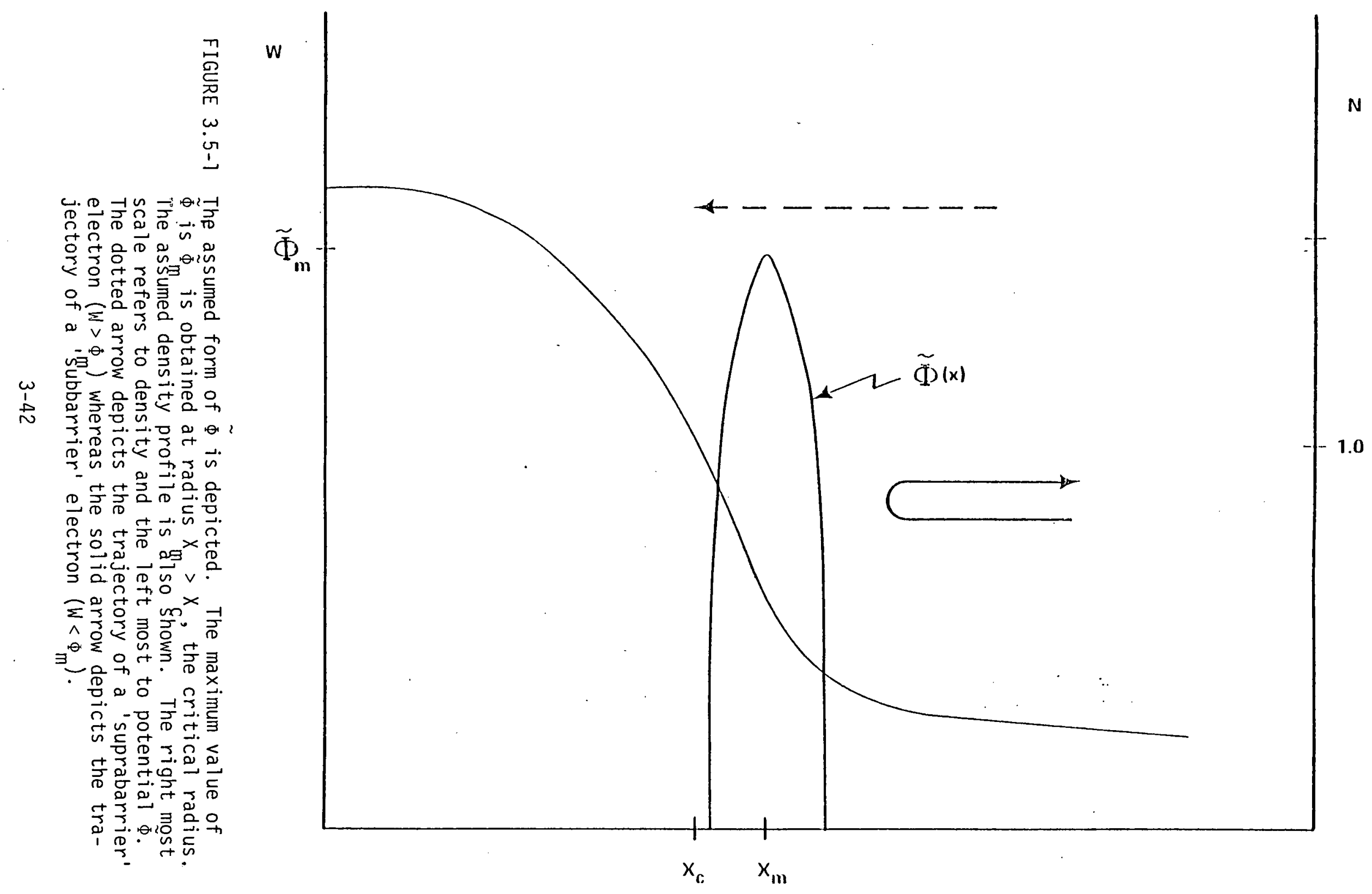




$$
E^{*}=\frac{1}{2} m v^{2}=\frac{1}{2} m_{e}\left(\frac{2 \pi z^{2} N_{i} e^{4} \ln n c^{2}}{\omega m_{e}^{2}}\right)^{1 / 2} \simeq 500 \mathrm{ev}
$$

The latter estimate is obtained by setting $Z$ en $\Lambda=100, \lambda=1$ micron and $\mathrm{Zn} \mathrm{n}_{i}=\mathrm{n}_{\mathrm{c}}$, the critical density.

When $\lambda \ll L_{\Phi}$, we can make a subsidiary expansion in $\varepsilon=\lambda / L_{\Phi}$, that is

$$
f=f_{0}+\varepsilon f_{1}+\ldots
$$

and find $f_{0}=f_{0}(W, x)$

$$
f_{1}=\mp \frac{v_{x}\left(W, v_{y}, x\right)}{v_{e j}(W, x)} \frac{\partial}{\partial x} f_{0}(W, x)
$$

It is now an easy matter to find the current,

$$
\begin{aligned}
J_{x} & =-e \int d^{2} \underline{v} v_{x} f, \\
& =-e \int d^{2} \underline{v} v_{x} f_{1}, \\
& =\frac{2^{5 / 2} \pi e}{c n_{i} z^{2}} \int_{\tilde{\Phi}}^{\infty} d W(W-\tilde{\Phi})^{5 / 2} \frac{\partial}{\partial x} f_{0}(W, x)
\end{aligned}
$$

where we have defined $\dot{c}=2 \pi$ ln $\Lambda \mathrm{e}^{4} / \mathrm{m}_{\mathrm{c}}{ }^{2}$ for convenience. For comparison with the usual case we assume $f_{0}$ is composed of Maxwellians, i.e.,

$$
f_{n}^{\sigma}=\hat{n}_{\sigma} \exp \left(-\frac{m W}{T_{u}}\right) \text {, }
$$

where $\sigma=1,2$ refers to 'subbarrier' electrons on the high and low density sides of $\Phi_{m}$ respectively and $\sigma=3$ refers to suprabarrier electrons. We impose the constraint that $f_{0}(W, x)$ be continuous at $W=\tilde{\Phi}_{m}$ with the results that 


$$
\hat{n}_{\sigma}=\hat{n}_{3} \exp \left(\frac{m \omega}{T_{\sigma}}\left[1-\frac{T_{\sigma}}{T_{3}}\right]\right), \sigma=1,2 .
$$

The general expression for the current is then

$$
\begin{aligned}
& J_{\sigma}=\frac{\omega_{p e^{2}}}{\nu_{e i}{ }^{\sigma}}\left(\begin{array}{l}
\hat{n}_{\sigma} T_{\sigma}^{2} \\
e_{i} m Z
\end{array}\right) \exp \left(-\frac{m}{T_{\sigma}} \tilde{\Phi}\right) \\
& \left\{\left[\frac{1}{\hat{n}_{\sigma}} \frac{\partial \hat{n}_{\sigma}}{\partial x}+\frac{m}{T_{\sigma}^{2}} \tilde{\Phi} \frac{d T_{\sigma}}{d x}\right] S_{1 \sigma}\left(z_{\sigma}\right)\right. \\
& \left.+\frac{1}{T_{\sigma}} \cdot \frac{\mathrm{dT}}{\mathrm{dx}} \mathrm{S}_{2 \sigma}\left(\mathrm{z}_{\sigma}\right)\right\}
\end{aligned}
$$

where

$$
\begin{aligned}
& \nu_{e i}^{\sigma}=c n_{i} z^{2}\left(m / T_{\sigma}\right)^{3 / 2} \\
& s_{11}(z)=s_{12}(z)=\frac{15 \sqrt{\pi}}{8} \operatorname{erf}(z)-z \exp \left(-z^{2}\right)\left[\frac{15}{4}+\frac{5}{2} z^{2}+z^{4}\right] \text {, } \\
& S_{21}(Z)=S_{22}(z)=\frac{3 \cdot 5 \cdot 7}{2^{4}} \sqrt{\pi} \operatorname{erf}(z)=Z \exp \left(-z^{2}\right)\left[\frac{3 \cdot 5 \cdot 7}{2^{3}}+\frac{35}{4} z^{2}\right. \\
& \left.+\frac{7}{2} z^{4}+z^{6}\right] \\
& S_{13}(Z)=\frac{15}{8} \sqrt{\pi}-S_{17}(Z), \\
& S_{23}(Z)=\frac{3 \cdot 5 \cdot 7}{2^{4}} \sqrt{\pi}-S_{27}(Z)
\end{aligned}
$$

and $z_{\sigma}^{2}=m\left(\tilde{\Phi}_{m}-\tilde{\Phi}\right) / T_{\sigma}$. The current on the low density side is $J=J_{S B}+J_{2}$ and on the high density side is $J=J_{S B}+J_{1}$. At $\Phi=\Phi_{m}$, there are no 'subbarrier' electrons and thus $\mathrm{J}_{1}=\mathrm{J}_{2}=0$ as can be seen by inspection of Equations (27) through (31), that is, $S_{1 \sigma}(0)=S_{2 \sigma}(0)=0$ for $\sigma=1,2$. To complete the description we impose quasineutrality, i.e., 


$$
\begin{aligned}
Z n_{i} & =\int d^{2} \underline{v} f_{0} \\
& =\sqrt{2} \frac{\pi \hat{n}_{\alpha} T_{\alpha}}{m} \exp \left(-\frac{m}{T_{\alpha}} \tilde{\Phi}\right)\{1 \\
& \left.+\left(\frac{T_{3}}{T_{\alpha}}-1\right) \exp -z_{\sigma}^{2}\right\} .
\end{aligned}
$$

In obtaining (32), we have used (26). Equations (29) through (32) can be examined in various limits, e.g., if $T_{1}=T_{2}=T_{3}$ then

$$
J=\frac{15}{8 \sqrt{2 \pi}} \frac{\omega_{\text {De }}^{2}}{v_{e i}} \frac{T}{e}\left\{\frac{1}{n T} \frac{d n T}{d x}+\frac{m}{T} \frac{d \tilde{\Phi}}{d x}+\frac{5}{2} \frac{1}{T} \frac{d T}{d x}\right\},
$$

which is of the usual form except for the numerical factor due to our two-dimensional model. However, no assumption about the magnitude of $m \Phi / T$ has been made in obtaining this result.

Now we restrict our discussion to piecewise isothermal plasmas, that is, $d T_{\alpha} / d x=0$ but $T_{1} \neq T_{2} \neq T_{3}$. Assuming no static magnetic field exists, Ampere's law requires $J=0$ which by inspection of Equations (27) through (32) requires $\partial \hat{n}_{\sigma} / \partial x=0$

or

$$
\frac{d}{d x}\left\{z n_{i}(x)\left[1+\left(\frac{T_{3}}{T_{\alpha}}-1\right) \exp -z_{\alpha}^{2}\right]^{-1} \exp \left(\frac{m \tilde{\Phi}}{T_{\alpha}}\right)\right\}=0
$$

This equation can be solved for the electrostatic field $\phi=m(-\ddot{\Phi}+\Phi) / e$ in terms of $n_{j}(x)$ and $\Phi$ at least in principle. The task is easy in the limit that $\left|\mathrm{e} \phi / T_{\alpha}\right| \ll 1$ in which case, 


$$
\begin{gathered}
\frac{e}{m_{e}} \frac{\partial \phi}{\partial x}=\frac{\partial}{\partial x} \Phi+\frac{T_{\alpha}}{m_{e}} \frac{1}{n_{i}} \frac{\partial n_{i}}{\partial x}\left\{1+\left(\frac{T_{3}}{T_{\alpha}}-1\right)\right. \\
\left.\quad \exp \left(-\frac{m}{T \alpha}\left[\Phi_{m}-\Phi\right]\right)\right\} .
\end{gathered}
$$

The hydrodynamic fluid equation follows immediately by combining Equation (35) with the ion momemtum equation

$$
m_{i} n_{i}\left(\frac{\partial}{\partial t}+v \frac{\partial}{\partial x}\right) v=-Z_{i} n_{i} \frac{\partial}{\partial x} \phi
$$

in which we have neglected ion pressure assuming $2 \mathrm{~T}_{\mathrm{e}} \gg \mathrm{T}_{j}$. Note that the effect of the ponderomotive force 'barrier' is to modify the electron pressure term, but not the wave pressure term.

Finally, we consider how the steady-state versions of Equations (35) and (36) combined with the steady-state continuity equation and wave equation alter the picture of profile modification in plane geometry. We can combine (35), (36) and the continuity equation to form the one equation,

$$
\frac{d u}{d \xi}\left(u-\frac{\vec{G}}{u}\right)=-\frac{d}{d \xi} \hat{\Phi}
$$

where

$$
G=\frac{T_{\alpha}}{T_{3}}+\left(1-\frac{T^{\alpha}}{T_{3}}\right) \exp \left[-\frac{T_{3}}{T_{\alpha}}\left(\hat{\Phi}_{m}-\hat{\Phi}\right)\right] \text {, }
$$

and $u=v /\left(Z T_{3} / m_{i}\right)^{1 / 2}$ and $\hat{\Phi}=|E|^{2} / 4 \pi_{c}{ }_{c}{ }$. The wave equation is, of course,

$$
\left[\frac{d^{2}}{d \xi^{2}}+\left(1-\frac{N}{N_{c}}\right)\right] \hat{E}=0 .
$$


where $\xi=\omega x / c$. It is well-known that the density step profiles for an isothermal plasma are characterized by a transition from subsonic to supersonic flow where the sonic point occurs at the maximum of $\Phi$, i.e., $\Phi_{m}$. That feature is unchanged by the present analysis except that the sound speed is that of the 'suprabarrier' electrons, i.e., $c_{s}=\left(Z T_{3} / m_{i}\right)^{1 / 2}$. This is true even though the number of 'suprabarrier' electrons can be quite small once $\Phi \neq \Phi_{\mathrm{m}}$. The ratio of the number density of sub- to suprabarrier electrons is $\left(T_{\alpha} / T_{3}\right)\left[\exp \left(T_{3}\left(\hat{\Phi}_{m}-\hat{\Phi}\right) / T_{\alpha}\right)-1\right]$ which is very large if $\hat{\Phi}_{\mathrm{m}} \sim 1$ and $T_{3} \gg T$ provided $\Phi \neq \Phi_{\mathrm{m}}$.

Since $T_{3} \neq T_{1}, T_{2}$ in general one might expect a rarefaction shock to exist as noted previousiy. (3) This does not occur at the sonic point and, because the number of suprabarrier electrons is independent of $\phi$, our model does not go over to the two-temperature isothermal fluid model. We now examine one consequence of this procedure. The usual assumption is that profile modification can be explained as a balance of radiation pressure, particle pressure of thermals, and ion flow and that the electrons in the tail of the distribution do not play a significant role. Here we compare two cases where the subbarrier electrons have the same isothermal temperature on both sides of the sonic point but in one $T_{3}=5 T_{1}$, whereas in the other $T_{3}=T_{1}$. In both cases the radiation pressure is the same; that is when $T_{3}=5 T$, the normalized electric field intensity at the sonic point, $\Phi_{m}$, is taken to be $1 / 5$ the value of $\Phi_{m}$ when $T_{3}=T_{1}$. The ratio of supra- to subbarrier electrons is small $(\underset{\sim}{<} 7 \%)$ in both cases when $\Phi \rightarrow 0$, e.g., in the overdense plasma, where $G \simeq T_{7} / T_{3}$. Here, Equation (37) reverts to its usual form, and $T_{3}$ becomes as an overall scale factor. Near the sonic point however, the pressure is entirely determined by the suprabarrier electrons and, since the thermal pressure 
is five times larger in one case, dramatic differences result.

These differences are illustrated in Figure 3.5-2 where the density dependence on radius is shown for the two cases. Note the lower density shelf is lower and the higher density shelf is much higher when $T_{3}=T_{1}=T_{2}$

The analysis given here is strictly valid only for zero absorption but clearly, inverse bremsstrahlung and parametric instabilities are not as strongly suppressed by profile modification when $T_{3}>T_{1}, T_{2}$.

A more complete description is necessary before meaningful conclusions can be drawn. The most straightforward extension of the ideas detailed here is to derive a thermal transport equation. Other necessary ingredients are absorption processes, and obliquely incident light, and the treatment of long mean free path electrons $\left(\lambda>L_{\Phi}\right)$.

\section{REFERENCES}

1. B. Bezzerides, D. F. Duboi's, D. W. Forslund and E. L. Lindman, Phys. Rev. Lett. 38, 495, (1977).

2. I. B. Bernstein, C. E. Max and J. J. Thomson, Phys. Fluids 21, 905 (1978).

3. B. Bezzerides, D. W. Furslund and [. L. Lindman, "The Existence of Rarefaction Shocks in a Laser-Plasma Corona", submitted to Phys. Fluids, December, 1977; Los Alamos Report LAUR-78-12. 


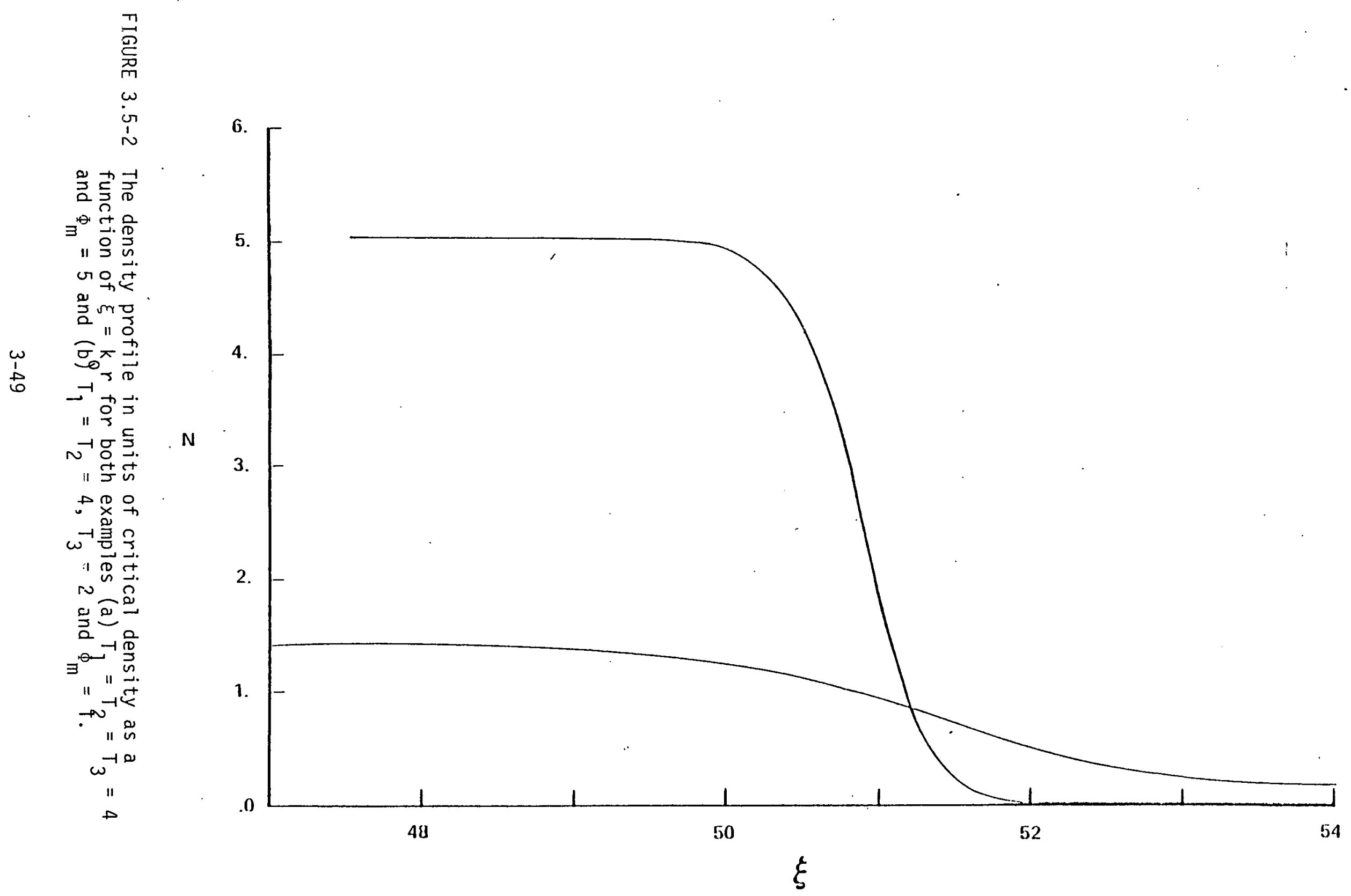




\subsection{Effect of Flux-Limited Thermal Transport on Critical Surface}

Jump Conditions

R. L. Berger and F. J. Mayer

There is now considerable theoretical literature ${ }^{(1,2)}$ on the nature of the plasma density profile near the critical surface in laser irradiated plasmas. Experiments ${ }^{(3)}$ have recently observed the predicted density steps produced by the laser radiation pressure. In a recent paper, Max and McKee ${ }^{(4)}$ analyzed the effects of flow and radiation pressure on the jump conditions across the critical-density surface. Specifically, they determined which plasma density profiles were consistent with conservation of mass and momentum across the critical surface with radiation pressure included. Here, we examine the additional constraints on the plasma density profile and jump conditions when laser energy deposition and flux-limited electron thermal conduction are included in the steady-state plasma hydro model. We assume both momentum and laser energy are deposited at the critical surface, and that the density gradient scale length is short compared to the hydrodynamic scale lengths. The laser energy, is assumed to be deposited in a singletemperature electron distribution. Our model of hydrodynamics and energy transport is identical to that of Max and Mckee but augmented by a result . of Lee, ${ }^{(2)}$ et al., which makes explicit use of the wave equation. We find, within the context of this model, that the size of the plasma-density jump is determined by the energy equation when thermal transport is inhibited and that the ratio of radiation pressure to thermal pressure is relatively small for laser intensities in present experiments. The size of the density step is shown to be related to the reduction of the electron thermal conduction ${ }^{(5)}$ from its classical value. Since the size of the flux-limit parameter or the relation of this 
parameter to physical variables is not firmly established (experiments indicate a small value ${ }^{(\overline{6})}$ ), we treat the flux-limit parameter itself as a model variable.

Finally, we show that the density jump at critical, the upper and lower shelf densities, the Mach numbers on both sides of critical, and the electron temperature are determined once the flux-limit parameter, the laser intensity and wavelength, and the absorption fraction have been specified.

The steady-state equations conserving mass, momentum, and energy result in the following relations between upstream density $\rho_{1}$ and Mach number $M_{1}$ and downstream density $\rho_{2}$ and Mach number $M_{2}$ provided the temperature is continuous across this critical density interface (see Figure 3:6-1):

$$
\begin{aligned}
& (R-1)\left(\frac{1}{R}-M_{1}^{2}\right)=(2-f) \frac{I_{0}}{c n_{c} \theta_{e} \rho_{1}} \equiv \beta / \rho_{1}, \\
& M_{1}\left(M_{1}^{2}+5\right)-10 \phi=M_{1}\left(M_{1}^{2} R^{2}+5\right)+\frac{10 \phi}{R}-\frac{2 f}{2-f} \frac{\beta_{g}}{\rho_{1}},
\end{aligned}
$$

We have defined a density ratio $R=\rho_{1} / \rho_{2}$, the absorption function $f$, the incident laser flux $I_{0}=c\left|E_{0}\right|^{2} / 8 \pi$, and the flux-limit paramter $\phi=\left(m_{j} / Z m_{e}\right)^{1 / 2} F / 5 \sim 12 F$ where $F$ is the ratio of the heat flow to the free streaming flux, $n_{e} \theta_{e}\left(\theta_{e} / m_{e}\right)^{1 / 2}$. The density $\rho$ is in units of the critical density; $\beta$ is the ratio of radiation pressure to thermal pressure; and $g \equiv\left(m_{i} c^{2} / \theta_{e}\right)^{1 / 2}$. Equation (40) expresses the combination of mass and momentum conservation; Equation (41) is the conservation of energy.

As was discussed by Max and Mckee, Equation (40) admits four solutions. Two of these correspond to a condition of decreasing density 


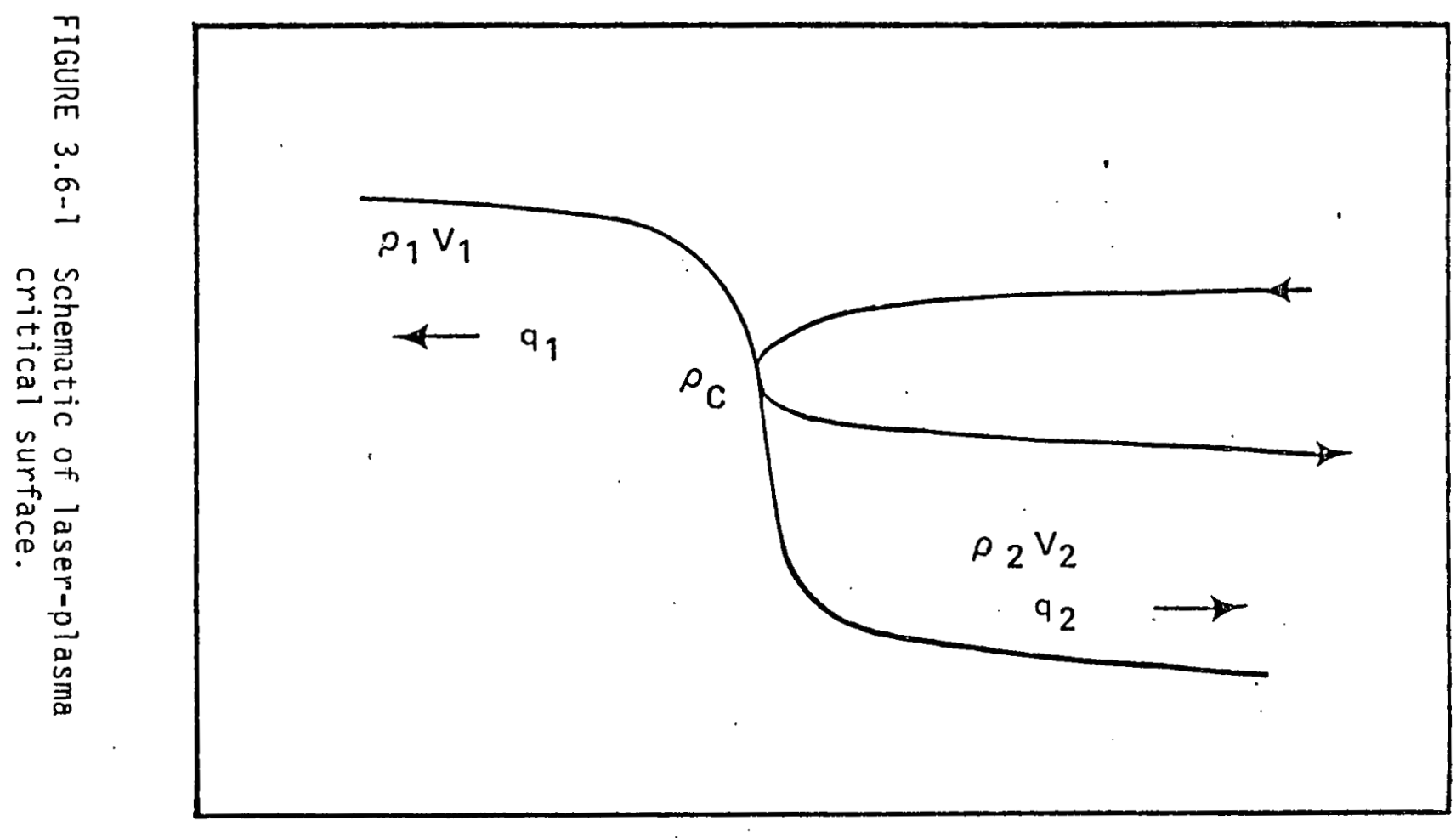


across the critical-density surface and have the flow on the upper density-shelf subsonic. The other two correspond to a condition of increasing density across the critical-density surface and have supersonic flow into the critical surface. These latter two solutions require weak flux-limiting $(\phi>0.6)$ and require a second downstream critical surface. Thus, we choose to focus only on the first two cases. Plateau type solutions which require supersonic flow into the critical density surface are not amenable to jump condition analysis. These solutions fall into the class where jump conditions predict no solution in steady state. Figure 3.6-2 is a plot of $R$ vs. $M_{1}$ in Equation (40) for $\dot{B}=0.1 \rho$. The two solutions for the condition of decreasing density $\left(M_{1}<1\right)$ have been characterized as 1) a weak D-front, which is a subsonicto-subsonic flow transition, and 2) a strong D-front, which is a subsonicto-supersonic flow transition. Notice that the weak D-front is the root of Equation (40) for which $R \simeq 1$, and the strong $D$-front has $R \simeq \frac{1}{M_{T}^{2}}$. (7) Moreover, in a spherical geometry the flow velocity must become supersonic as $r \rightarrow \infty$ and thus weak $D$-fronts require a sonic point at densities below critical density. We will return to this point later. It is easy to show that Equation (40) possesses solutions for $M_{1}{ }^{2}<1$ only $i+M_{1}{ }^{2}<1-B / \rho_{1}$. As $\beta$ decreases the density-increasing solutions and the density-decreasing solutions coalesce at $M_{1}=1$.

Notice that Equations (40) and (41) have vastly different numerical coefficients multiplying the incident flux, that is, the coefficient $g$ in the energy equation is larger by a factor $g \approx 10^{3}$ at $\theta=1 \mathrm{keV}$. This large factor forces $\beta$ to be small for moderate intensities and temperatures, as will be seen below. 


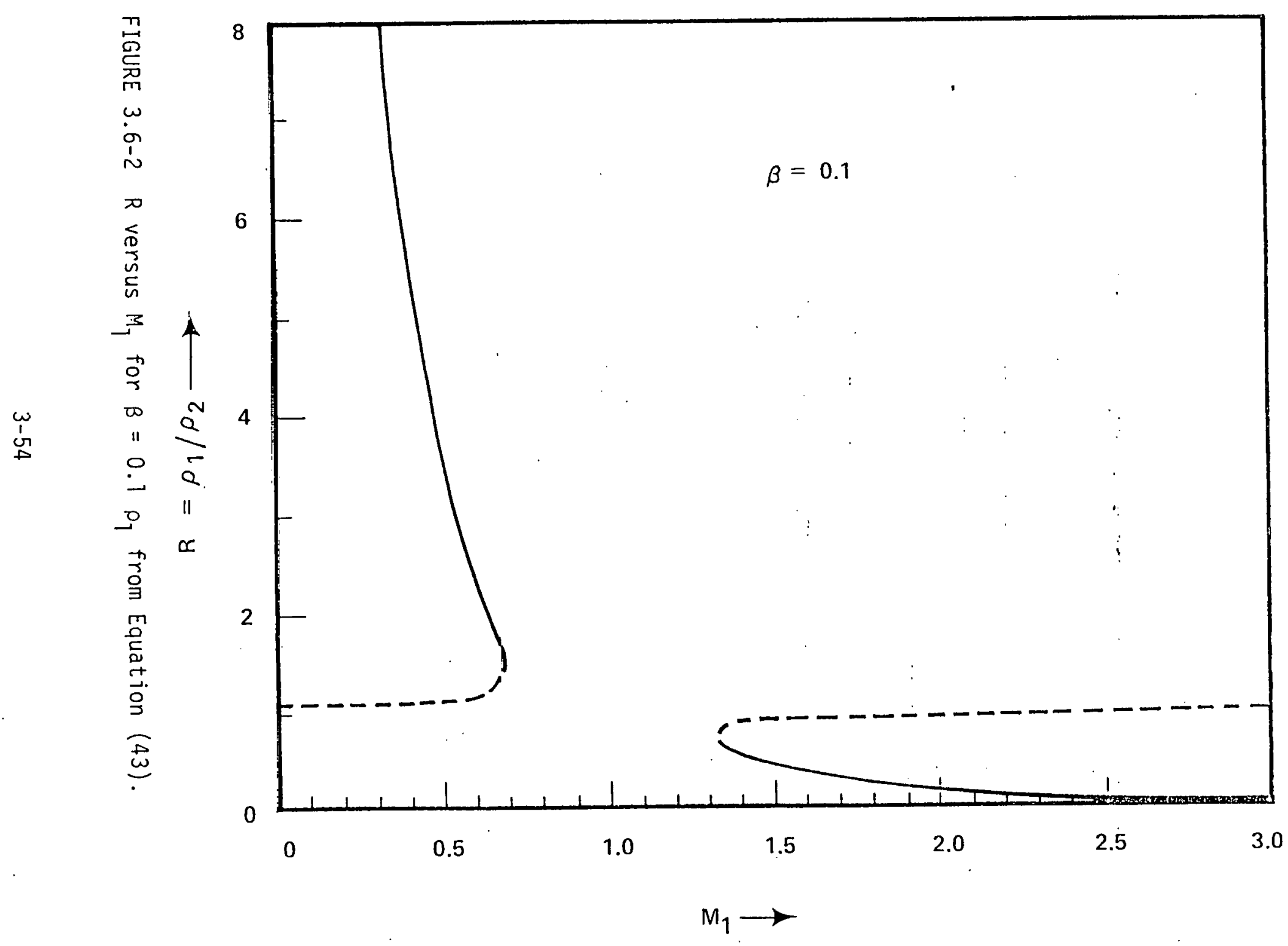


The first term on the left hand side of Equation (41) is just the hydro-energy flux into the critical surface, and the second term is the electron thermal flux into the high-density side. In the steady state these two terms are equal, as noted by Max and McKee. This may be seen by referring to figure 3.6-1. Since there are no sources or sinks of energy far upstream of critical, the electron thermal flux flowing into region 1 must be balanced by the hydrodynamic flux flowing out of region 1 . Hence, the following very simple relationship between the flux-limit parameter and the Mach number on the high density side is obtained:

$$
M_{1}\left(M_{1}^{2}+5\right)=10 \phi
$$

Weakly flux-limited thermal conduction corresponds to $\phi^{\prime} s \geq 1$ and therefore to Mach numbers $M_{1}>1$, while strongly flux-limited thermal conduction corresponds to $\phi<0.6$, and $M_{1}<1$. Notice here that if $B$ (the ratio of radiation pressure to plasma pressure) is small and $\phi<0.6$, then the root $\mathrm{R} \approx \frac{1}{M_{1}^{2}}$ corresponds to large density jumps. In what follows, we will show that energy balance does give small $B(z 0.1)$ for moderate intensities.

Lee ${ }^{(5)}$, et al., have recently shown that the wave equation may be integrated to obtain an important relationship between the density and the Mach number on the upper density shelf for the subsonic-tosupersonic transition. A generalization of their Equation (10), in our notation, reads

$$
\rho_{1}=-\frac{2\left(M_{1}^{2}-M_{c}^{2}\right)-4 \ln M_{1} / M_{c}}{4 M_{1}\left(M_{c}+\frac{1}{M_{c}}-M_{1}-\frac{1}{M_{1}}\right)},
$$


where $M_{c}$ is the Mach number of the flow at the maximum of the laser electric field. Equation (43) is strictly valid only for no absorption but is a good approximation as long as $f \ll 1$. The neglected term is proportional to the product of the absorption fraction and the jump in the derivative of the electric field amplitude due to this localized absorption. The validity condition can be shown to be $f<(2 / \phi)^{1 / 2(8)}$. This relation is required to fully constrain the solutions of the jump equations across critical. We point out that, given $\rho_{1} v s . M_{1}$, it is possible to calculate the mass ablation rate $\rho_{7} M_{7}$ as a function of $M_{7}$, or as a function of $\phi$, by using Equation (42). Figure 3.6-3 shows a plot of both $\rho_{1}$ and $\rho_{1} M_{1}$ as functions of $\phi$. Notice that the mass ablation rate ${ }^{\prime} \rho_{1} M_{1}$ becomes very small for strong flux-limiting, i.e., small $\phi$. of course, we need to know the electron temperature (in the sound speed) to convert to dimensional units. The value of $M_{c}$ lies between $M_{1}$ and $M_{2}$; so for the purpose of calculating $\rho_{1}$, we approximate:

$$
M_{C}=\frac{M_{1}+M_{2}}{2}=\frac{M_{1}(1+R)}{2}
$$

for subsonic to subsonic transitions and $M_{C}=1$ for supersonic to supersonic transitions. Figure 3.6-4 is plot of $\rho_{1}$ and $\rho_{1} M_{1}$ for a density jump of $R=1.06$, a representative value for the subsonic-to-subsonic transition. Notice that here the upper shelf density $\rho_{1}$ is very close to critical. It is important to realize that for both weak and strong D-fronts the mass ablation rate decreases with decreasing flux-limit parameter $\phi$ in accordance with Equation (42). Also, it is rather important to realize that the above relations between the upper shelf density and the Mach number do not depend on the laser intensity. We point out that the ablation rates given by the above equations (43) and (44) are quite 


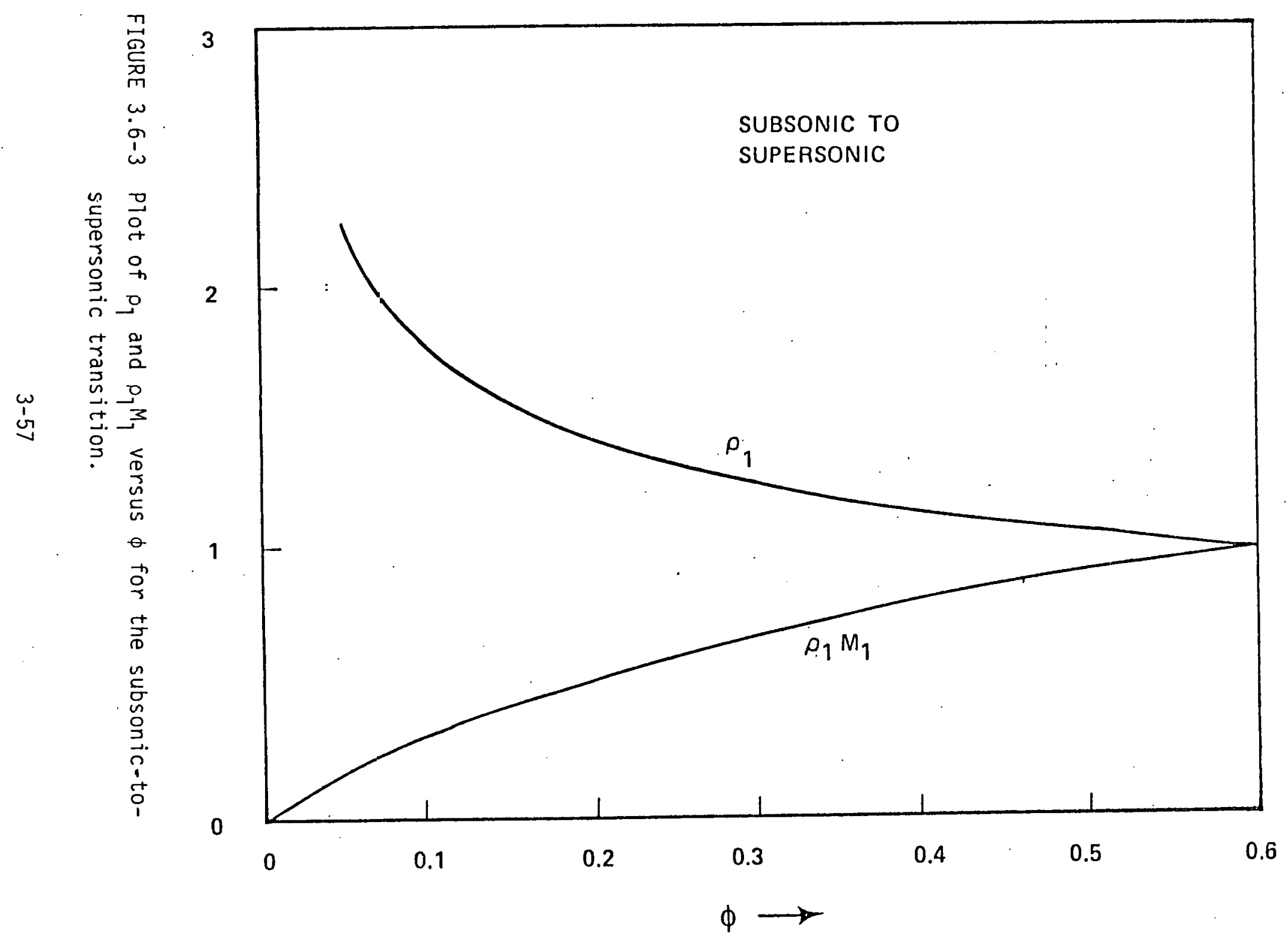




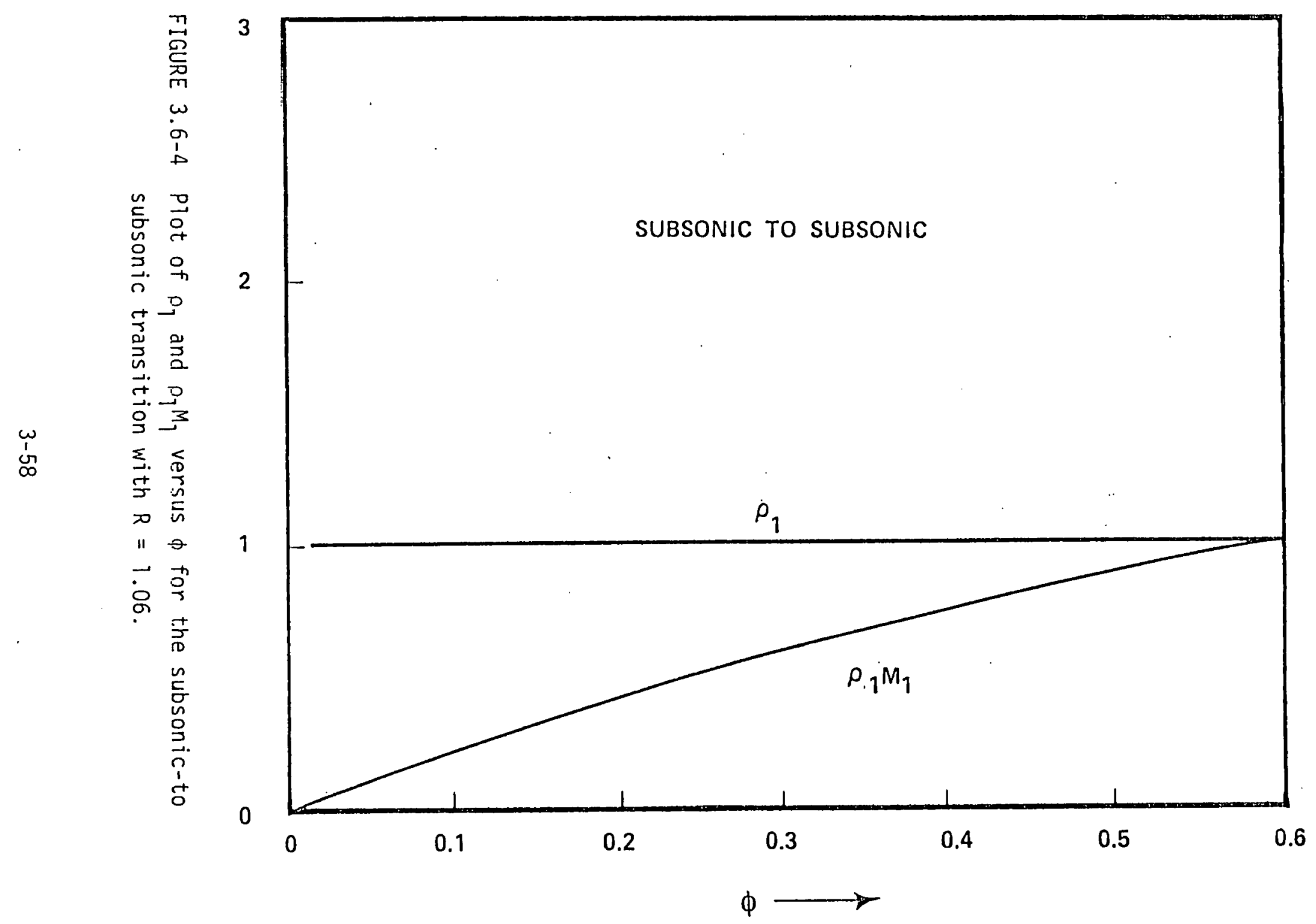


small compared to a classical ablation rate where Mach numbers are typically $\sim 1$ to 2 . The classical ablation solutions, however, are either 1) not steady-state, or 2) they may require the density plateaus, or 3 ) they may require the critical surface to move relative to the ablation surface. It is of course the Mach number in the frame of the critical surface that is of interest.

The right hand side of Equation (41) is equal to zero since the left hand side is. Hence,

$$
M_{1}\left(M_{1}^{2} R^{2}+5\right)+\frac{10 \phi}{R}=\frac{2 f g}{2-f} \beta \rho_{1}^{-1}
$$

We may rearrange our equations for the electron temperature by el iminating $B$ in the momentum Equation (40) by using Equation (45) to find

$$
g^{-2}=\frac{\theta_{e}}{m_{i} c^{2}}=\frac{2 f}{(2-f)} \frac{(R-1)\left(1 / R-M_{1}^{2}\right)^{2}}{M_{1}\left(M_{1}^{2} R^{2}+5\right) \frac{10 \phi}{R}}
$$

Equation (45) is just the usual flux-balance relation expressed in terms of the density ratio, $R$, the absorption fraction, $f$, and the flux-limit parameter $\phi$. Notice that $B g \sim I_{0} \lambda^{2} / \theta_{e}^{3 / 2}$. We seek a solution to the set of Equations (42), (43), (45) and (46) by specifying $f$ and $\phi$. Once $\phi$ is given, $M_{1}$ is given by Equation (42). For subsonic-to-supersonic flows, $f$ and $\phi$ are thus sufficient to also determine $\rho_{1}$ from Equation (46). The next step is to assume a value for $R$ so that $\rho_{1}$ is determined for subsonic-to-subsonic profiles from Equation (44) and the temperature is determined from Equation (46). Using this value of $g$, we can find $\beta$ from Equation (45). The variable $R$ can be used as a parameter relating $B\left(\sim I_{0} \lambda^{2}\right)$ and $\theta_{e}\left(\sim g^{-2}\right)$ as shown in. Figure 3.6-5 for subsonic-tosupersonic transitions. The different curves are labeled by the value 


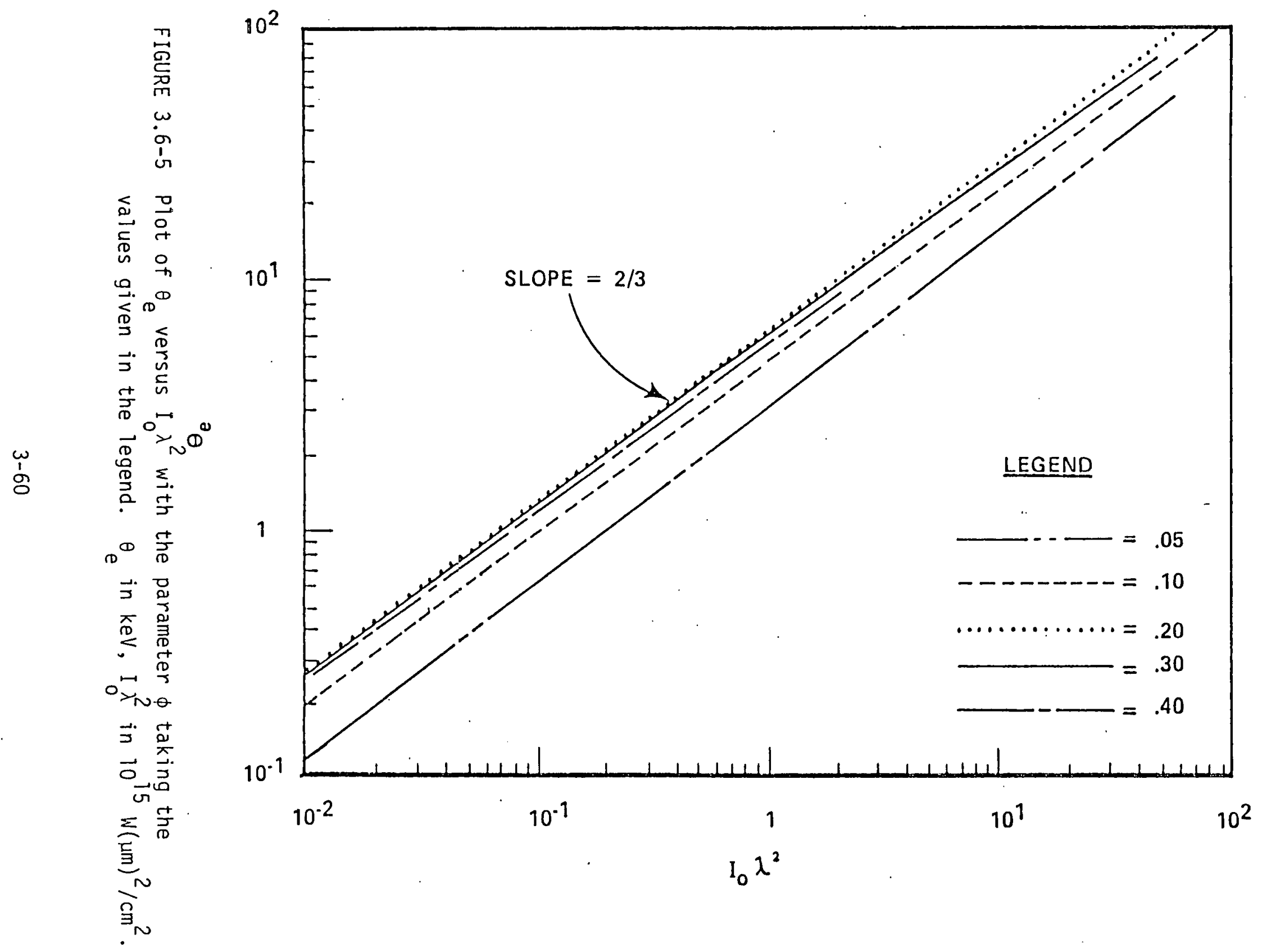


of the flux-limit. For $0.1 \leq \phi \leq 0.4, B \leq 0.1 \rho_{1}$ except at intensities such that $I_{0} \lambda^{2} \sim 10^{9}$ watts. Ponderomotive force by itself in the momentum equation would result in little profile modification for such small $\beta$.

For a given value of $I_{0} \lambda^{2}$, there is a maximum $\phi$ for which solutions exist. That this is true can be seen from taking the limit $\phi \rightarrow 0.6$. In this limit the only root to Equation (42) is $M_{1}=1$ so that the two roots to Equation (40) coalesce. The term $(R-1)\left(\frac{1}{R}-M_{1}^{2}\right)$ in Equation (46) is arbitrarily smal1 and thus $\beta \rightarrow 0$. But if $I_{0} \lambda^{2} \neq 0$, then $\theta_{e} \rightarrow 0$ by Equation (46) and $\beta \rightarrow \infty$ violating the assumption in Equation (40).

Figure $3.6-6$ is a plot of the density ratio across critical and the self-consistent electron temperature as functions of the flux-limit parameter for the case where $f=0.2$, and $I_{0} \lambda^{2}=10^{7}$ watts. Notice, the behavior of the density ratio for the two different transitions (subsonicto-supersonic, and subsonic-to-subsonic), as a function of the flux-limit parameter. The subsonic-to-subsonic transition has $R$ very close to one, whereas, the subsonic-to-supersonic transition has $R \simeq \frac{1}{M_{1}^{2}} \simeq \frac{1}{4 \phi^{2}}$ for small $\phi$. Also, notice the decrease in $\theta_{\mathrm{e}}$ with increase in $\phi$ for the subsonic-to-subsonic transition. This is expected. Since the density jump is close to one, the velocity jump is close to one; hence, the reduced thermal conduction must raise the temperature at constant absorbed intensity. On the other hand, for the subsonic-to-supersonic transition, the temperature decreases (after reaching a maximum) with decreasing flux-limiter at constant absorbed intensity. Because of the large density jump, a corresponding large velocity jump is produced, and the reduced thermal conduction is accommodated by an increase in flow on the low density side, allowing the temperature actually to decrease. 


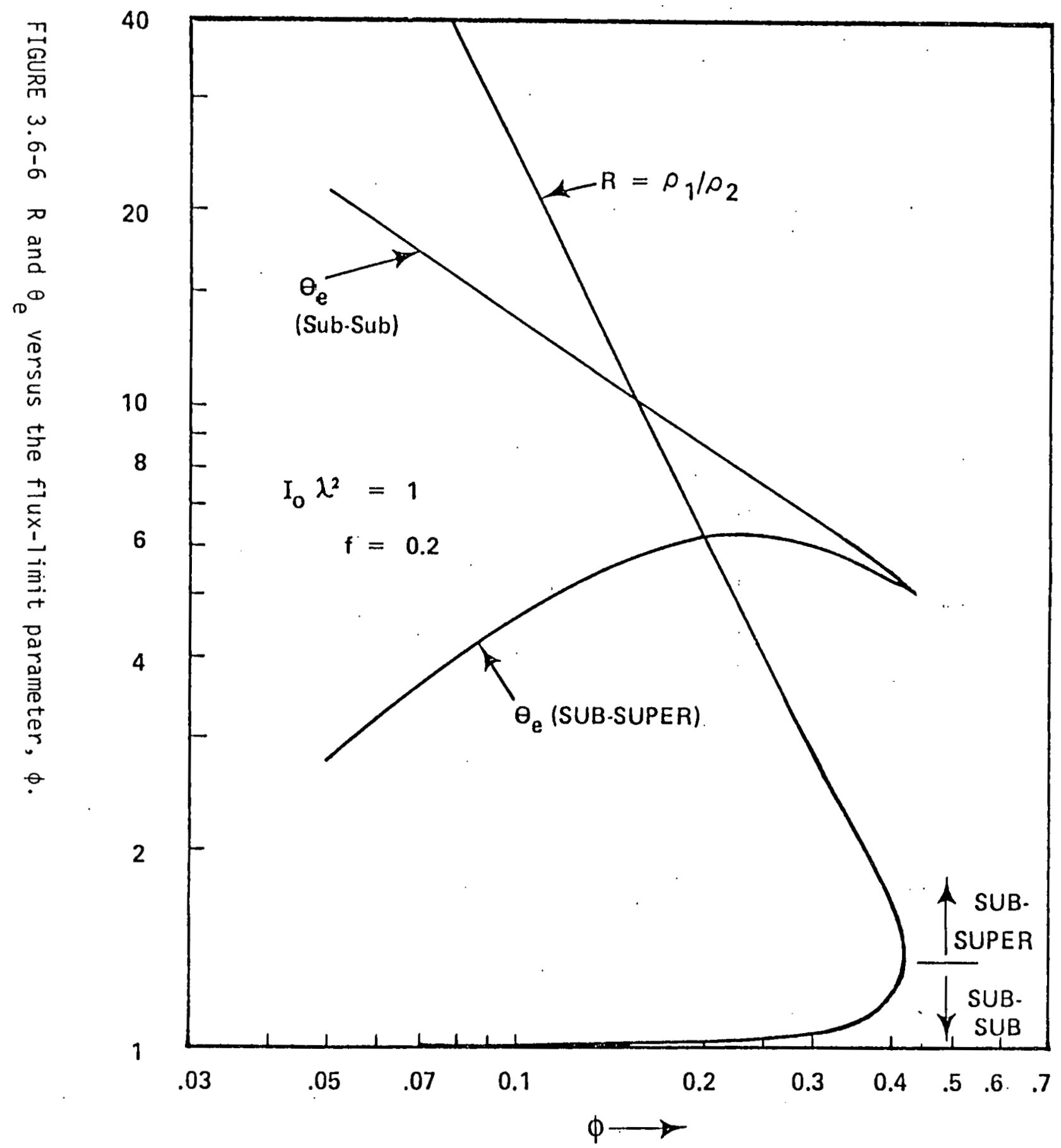


The $R$ vs. $\phi$ curve for $I_{0} \lambda^{2}=10^{8}$ lies very close to the $R$ vs. $\phi$ curve of Figure 3.6-6 due to the fact that $B$ in Equation (40) is still small over most of the range of $\theta_{e}$; hence $R$ is still close to one of the two roots $R=1, R=\frac{1}{M_{1}{ }^{2}}$.

Finally, we point out that, although the conservation equations and the wave equation fully determine the jump conditions when given a flux-limit parameter, the solution is not unique since there are two possible solutions corresponding to the subsonic-to-subsonic and subsonicto-supersonic transitions. It is clear that the flow must asymptotically be supersonic in an isothermal corona. If the radiation pressure is a maximum at the critical surface then no downstream transition from subsonic to supersonic flow will be allowed by the conservation of mass and momentum. (1) Therefore, except for pathological conditions (strong Brillouin scattering or strong inverse Bremsstrahlung absorption), which would alter the momentum equation significantly, only the subsonic-tosupersonic transition is physically acceptable. In fact, it can be shown using only the steady-state continuity and momentum equations that the flow velocity in the corona must be supersonic provided one assumes that the temperature has its maximum value and the ponderomotive furce has its maximum value near the critical density. (9)

The size of the density jump across critical for the case of a strong D-front, (subsonic-t.n-supersonic transition) depends mostiy on the size of the flux-limit-parameter scaling as $R \sim \frac{1}{4 \phi^{2}}$. Also, if the absorbed flux is carried away by a one-temperature electron group in steady state, the flux balance makes the temperature high enough to keep the ration of radiation pressure to plasma pressure small ( 0.1 ) at moderate intensities. 
It is instructive to analyze the recent experimental results of Attwood ${ }^{(3)}$ on the basis of this model. Attwood measured a density-jump ratio of about 4 to 5 , at an intensity of $10^{14} \mathrm{~W} / \mathrm{cm}^{2}$. For this case, we find that the flux-limit parameter $\phi$ is about 0.2 to 0.4 , and the electron temperature about $1 \mathrm{keV}$. This value of the flux-limit parameter is close to the value used in simulations $(5,6)$ which fit other experimental results. Thus, strongly flux-limited thermal conduction may be playing a role in producing the observed density jumps.

Finally, we observe that the temperature at critical is a maximum for a particular value flux-limit $\phi^{\star}$. For $\phi>\phi^{\star}$, the heat flow is more efficient and lowers $\theta_{e}$ for a fixed absorbed flux. For $\phi<\phi^{\star}$, the energy is carried off by acceleration of ions since the heat flow is so inhibited. An analogous effect has been observed before for classical heat conduction. $(10)$ Since this value of $\phi^{*}(\sim 0.24)$ corresponds to that needed to mode 1 experiments, one can speculate whether the plasma chooses $\phi=\phi^{*}$ to avoid microinstabilities.

\section{REFERENCES}

1. R. E. Kidder, in Proceedings of Japan-U. S. Seminar on Laser Interactions with Matter, edited by C. Lamanka (Tokyo International Book Co., Tokyo, p. 331 ) (1975).

K. G. Estabrook, E. J. Valeo, and W. L. Kruer, Phys. Fluids 18, 1151 (1975).

K. A. Brueckner and R. J. Janda, Nuclear Fusion 17, 451 (1977).

J. Virmont, R. Pellet and P. Mora, Phys. of Fluids, 21, 567 (1978).

P. Mulser and C. Vankesscl, Phys. Rev. Lett 38, 902 (1977).

2. K. Lee, D. W. Forslund, J. M. Kindel, and E. L. Lindman, Phys. Fluids 20, 51 (1977).

3. D. T. Attwood, D. W. Sweeney, J. M. Auerbach, and P. H. V. Lee, Phys. Rev. Lett 40, 184 (1978).

4. C. E. Max and C. F. McKee, Phys. Rev. Lett 39, 1336 (1977). 
5. R. C. Malone, R. L. McCrory, and R. L. Morse, Phys. Rev. Lett 34 , 721 (1975).

6. P. M. Campbe11, R. R. Johnson, F. J. Mayer, L. V. Powers, D. C. Slater, Phys. Rev. Lett 39, 274 (1977).

7. These two solutions have been independently derived by J. Albritton.

8. F. J. Mayer, R. L. Berger, and C. E. Max, to be published.

9. Claire Max, private communication.

10. R. L. Morse, private communication. 


\subsection{Spherical Rarefaction Shocks}

D. Mitrovich

Resonance absorption in a plasma can create a second electron population whose effective temperature is many times the nominal electron temperature. If these two electron populations are in equilibrium with the potential field, they will distribute themselves such that the cold electrons predominate in the denser plasma while the hot electrons populate the low density plasma, the density at the transition being determined by the relative abundances of the two populations. When the plasma is flowing supersonically down the density gradient, Bezzerides, Forslund and Lindman $(1)$ showed that in planar geometry a rarefaction shock forms in the transition region if the hot- to cold-electron temperature ratio $\alpha>5+\sqrt{24}$. They also concluded that the shock attachment to the downstream plasma is inherentiy time dependent and occurs by way of an everlengthening density and velocity shelf.

Hydrodynamic code calculations, both including and excluding charge separation effects, were undertaken to study the formation and behavior of rarefaction shocks in spherically symmetric configurations.

In the initial calculation charge separation was neglected, so that detailed shock structure could not be determined. The expressions for equilibrium between hot and cold electron densities,

$$
{ }_{{ }_{C}} / \tilde{n}=\exp \left(\frac{C S}{\theta_{C}}\right)=\exp \left(\alpha \frac{\theta R}{\theta_{H}}\right)=\left(n_{H / n}\right)^{\alpha}
$$

and quasi-neutrality, $n_{C}+n_{H}=Z n_{j}$, were adjoined to the hydro equations, with the cold-electron temperature $\theta_{C}$, the temperature ratio $\alpha$, and the reference electron density $\tilde{n}$ at which hot and cold electron densities are equal, all held fixed. A rarefaction shock was observed to form in the 
expanding blow-off for temperature ratios a exceeding $\sim 10$. However, instead of connecting with a shelf, the shock merged gradually into the downstream plasma, as shown in Figure 3.7-1.

In these isothermal calculations energy had to be supplied continuously to the configuration in order to maintain the temperature at its fixed value. To investigate energy partitioning between hot and cold electrons in the more realistic situation of a finite amount of energy absorbed over a finite time, the hydro code was arranged to calculate the following model. The temperature ratio is kept fixed " $(\alpha=25)$ but the temperatures themselves vary according to internal energy. All the energy is absorbed by hot electrons, which transfer it collisionally to the cold electrons and the ions. Both electron populations do expansion work. The number of hot electrons, and therefore the reference density $\tilde{n}$, adjusts itself according to energy flows into internal energy, into expansion work, and between populations. Figures 3.7-2a and 3.7-2b show the evolutions of ${ }^{\theta} \mathrm{C}$ and $\tilde{n}$ for an $80 \mu \mathrm{m}$ glass shell with $1 \mu \mathrm{m}$ wall filled with 50 atmospheres of DT, absorbing $30 \mathrm{~J}$ at a constant rate over 100 psec. Figure $3.7-2 c$ shóws energy partitioning between hot and cold eletron internal energies and expansion work. Note that in this example in which all energy input is by resonant absorption, about $60 \%$ of the energy ends up, via hot electrons, as fast ions, corresponding, according to Figure $3.7-2 b$, to 6 to $7 \%$ of the total mass. The consistence of these numbers with what is commonly measured experimentally suggests that such a hot electron mechanism may explain the fast ion phenomenon. Note that this model bears some dynamical similarity to the heat-flux-inhibition model insofar as the effective electron temperature, defined as

$$
\theta_{e f f}=\frac{n_{H} \theta_{H}+n_{C}{ }^{\theta} C}{n_{H}+n_{C}}
$$


(a)

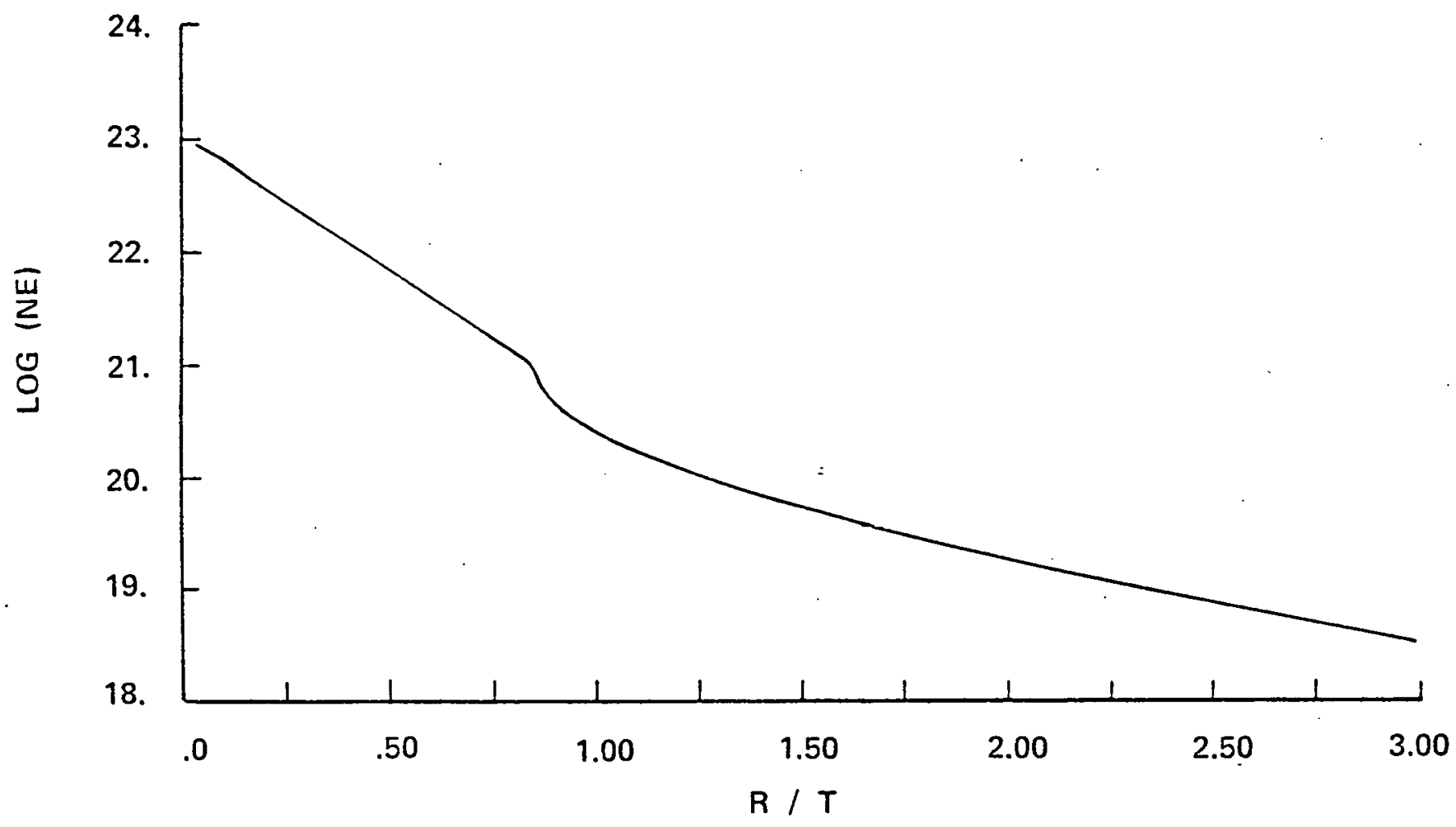

(b)

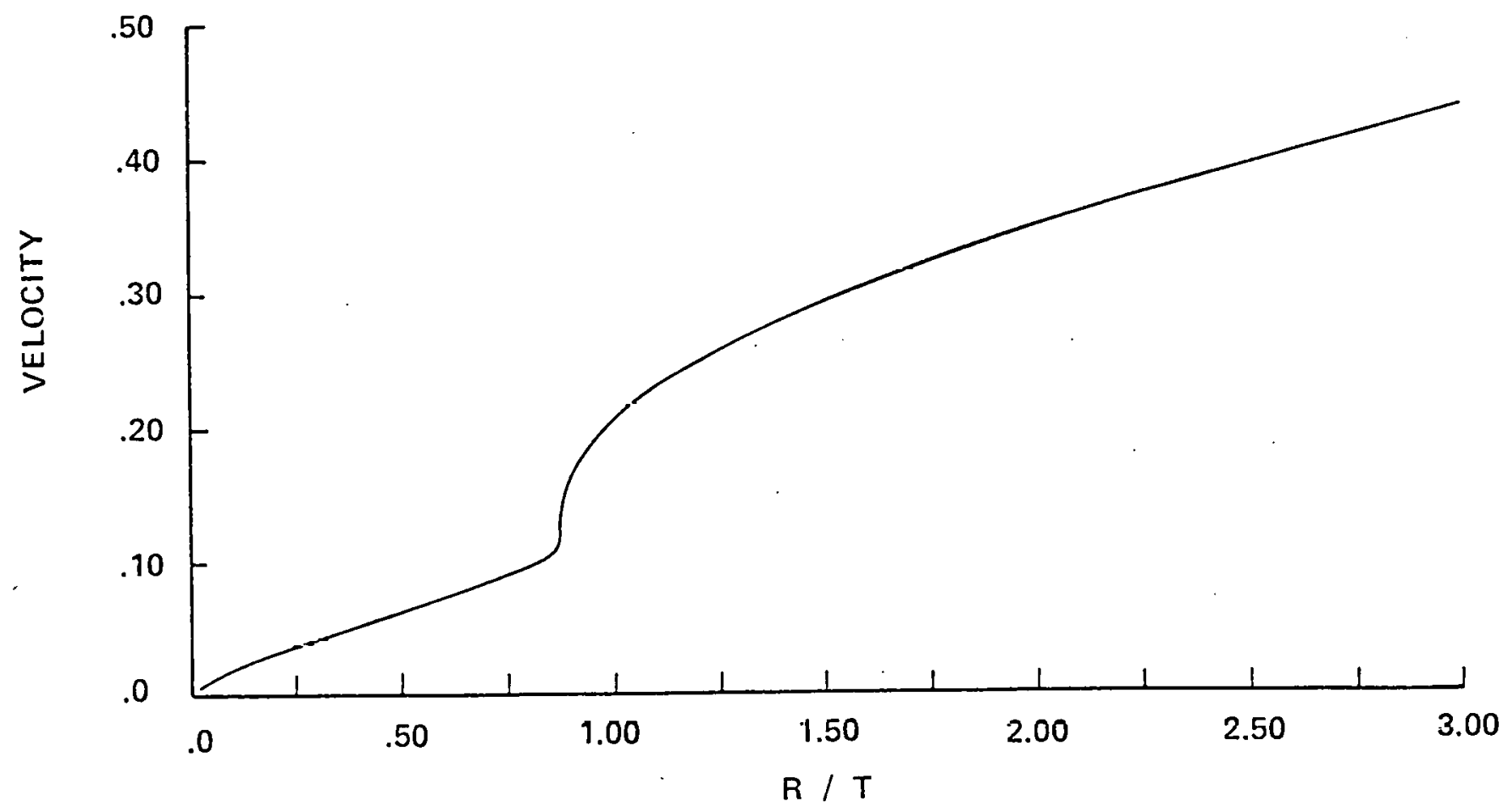

FIGURE 3.7-1 Density (a) and velocity (b) distributions $150 \mathrm{psec}$. after start of disassembly of an $80 \mu \mathrm{m}$ diameter DT sphere whose isothermal cold-electron temperature is maintained at $1 \mathrm{keV}$. The hot-to-cold electron temperature $\alpha=25$, and the reference electron density $\tilde{n}=10^{21} \mathrm{~cm}^{-3}$. 
(c)
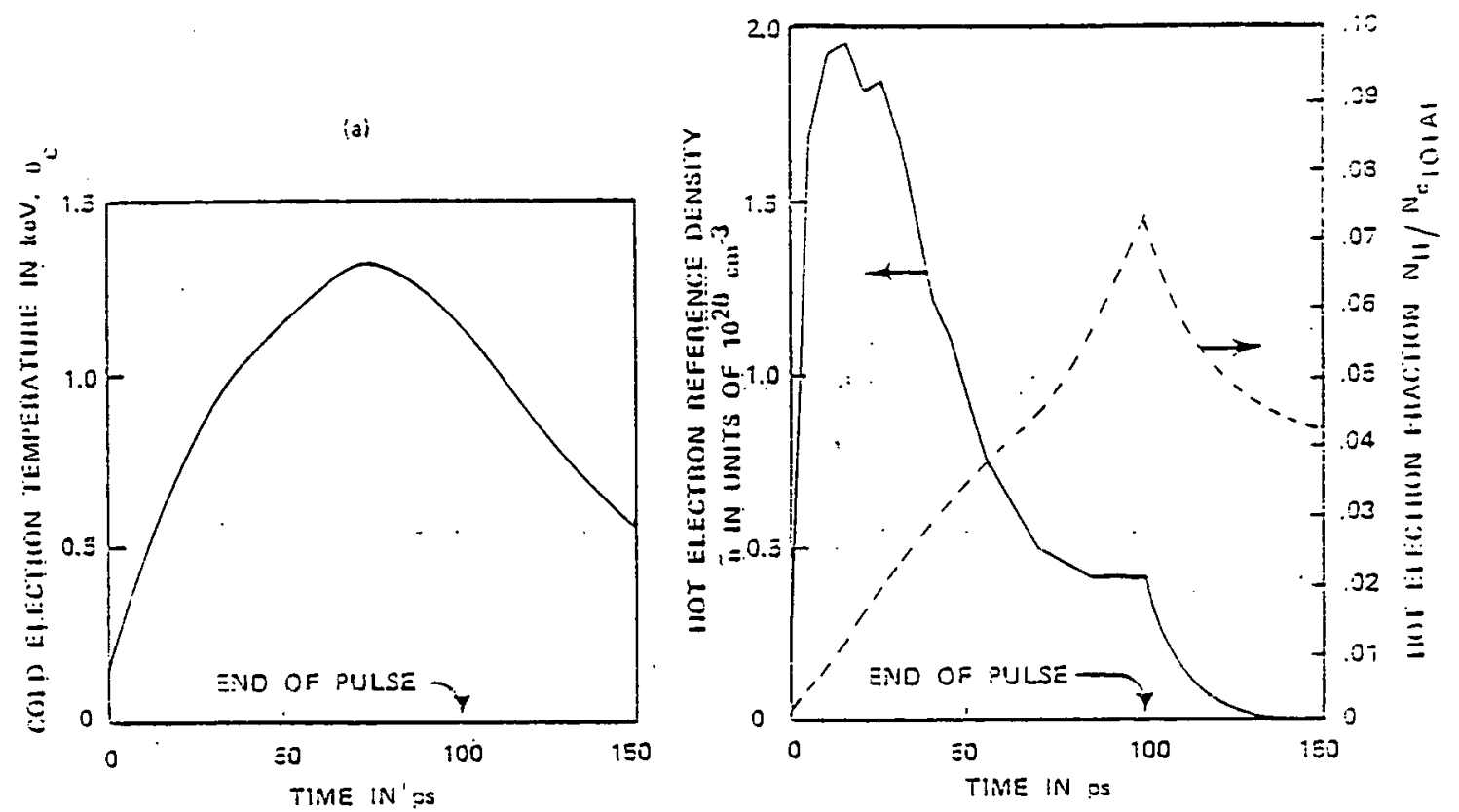

(c)

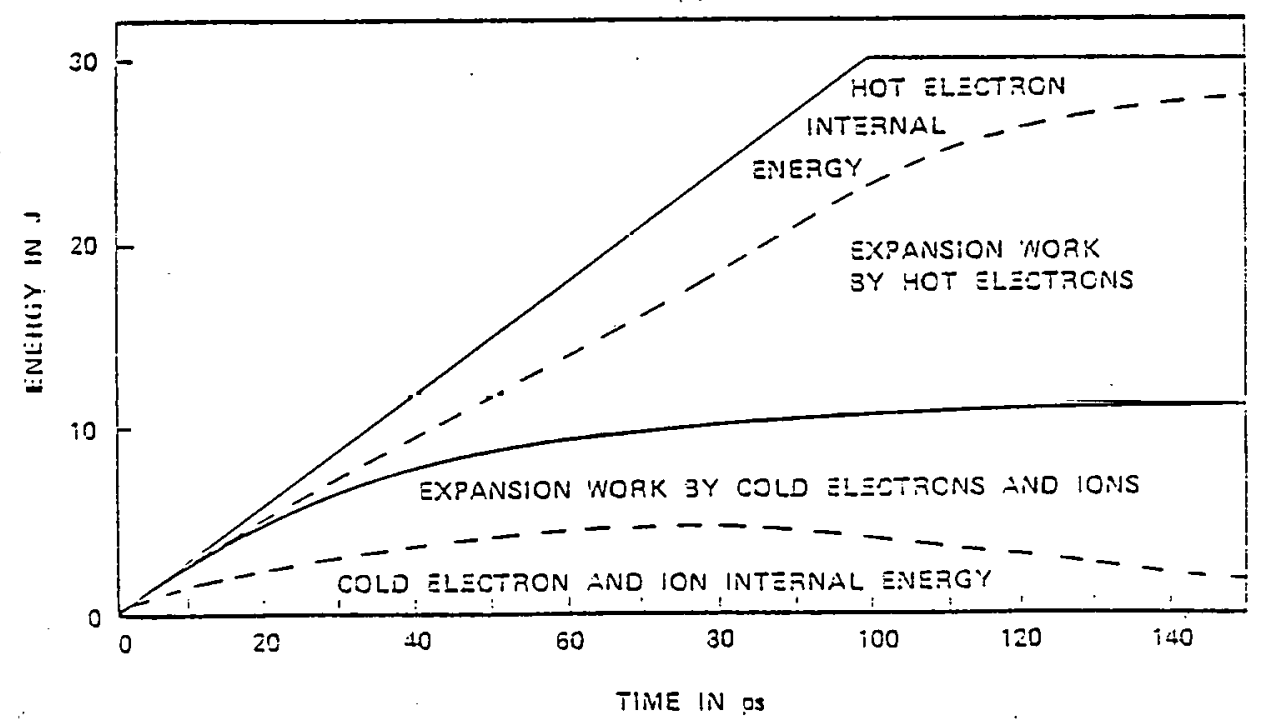

FIGURE 3.7-2 Dynamic evolution of an $80 \mu \mathrm{m}$ diameter glass shell with a $1 \mu \mathrm{m}$ wall filled with 50 atmospheres of DT gas when $30 \mathrm{~J}$ are absorbed by hot electrons at a constant rate over $100 \mathrm{psec}$. The temperature ratio $\alpha=25$. Energy transfer from hot to cold electrons (and to ions) is by collisions. (a) Cold electron temperature history, (b) Hot electron population and reference density histories, (c) Histories of energy partitioning between internal and kinetic energies for all components. 
increases from $\sim{ }_{C}$ inside the shock to $\sim \theta_{H}$ outside of $i t$, simulating heat-flux-inhibition in the shock region.

To study shock structure in detail, charge separation has to be included in the hydro equations, requiring parallel solution of the Poisson equation in the shock regions: This has been implemented in a special, fine zone, spherical hydro code. Isothermal expansion of a she11, again with the temperature ratio $\alpha=25$, with the Poisson equation solved in the shock regions, is shown in Figure 3.7-3. Since hot electrons have long mean free paths even in dense plasma, they penetrate easily to the inside of a thin shell and produce an inner as well as an outer rarefaction shock. The inward (converging) and outward (diverging) spherical flows appear nearly symmetrical because of the large shell radius $\left(10^{3}\right.$ reference Debye lengths). After an initial phase in which the shocks are close to and interact with the plasma boundaries, the shocks settle into diffuse dipole layers that merge gradually into the quasi-neutral downstream plasma. The shock structures begin evolving in a self-similar manner after $t \approx 0.4 \mathrm{psec}$. Note that at $t=0.8 \mathrm{psec}$, after the shocks are well established, there is still no sign of the shelf feature predicted by Bezzerides, et al. However, if the same shell configuration is recomputed neglecting charge separation, shelves do develop on the downstream sides of both inner and outer shocks, as seen in Figure 3.7-4, the shelf lengths increase linearly with time. The discrepancy may come from the fact that in the latter calculation the shock is very thin, consistent with the assumption made by Bezzerides, et al, whereas the charge separation shock region in the former calculation is evolving in time and is always at least severäl Debye lengths thick. 


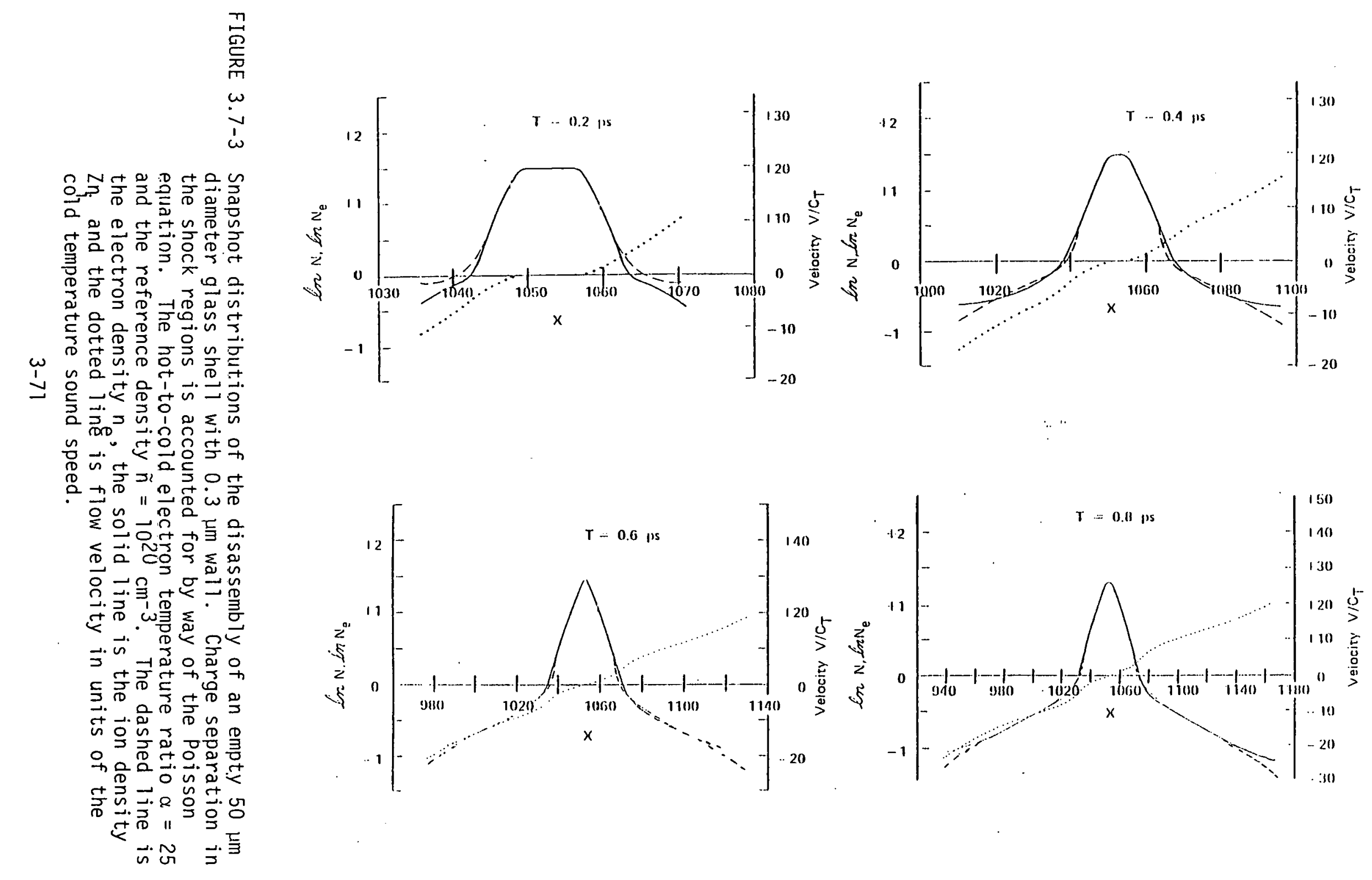




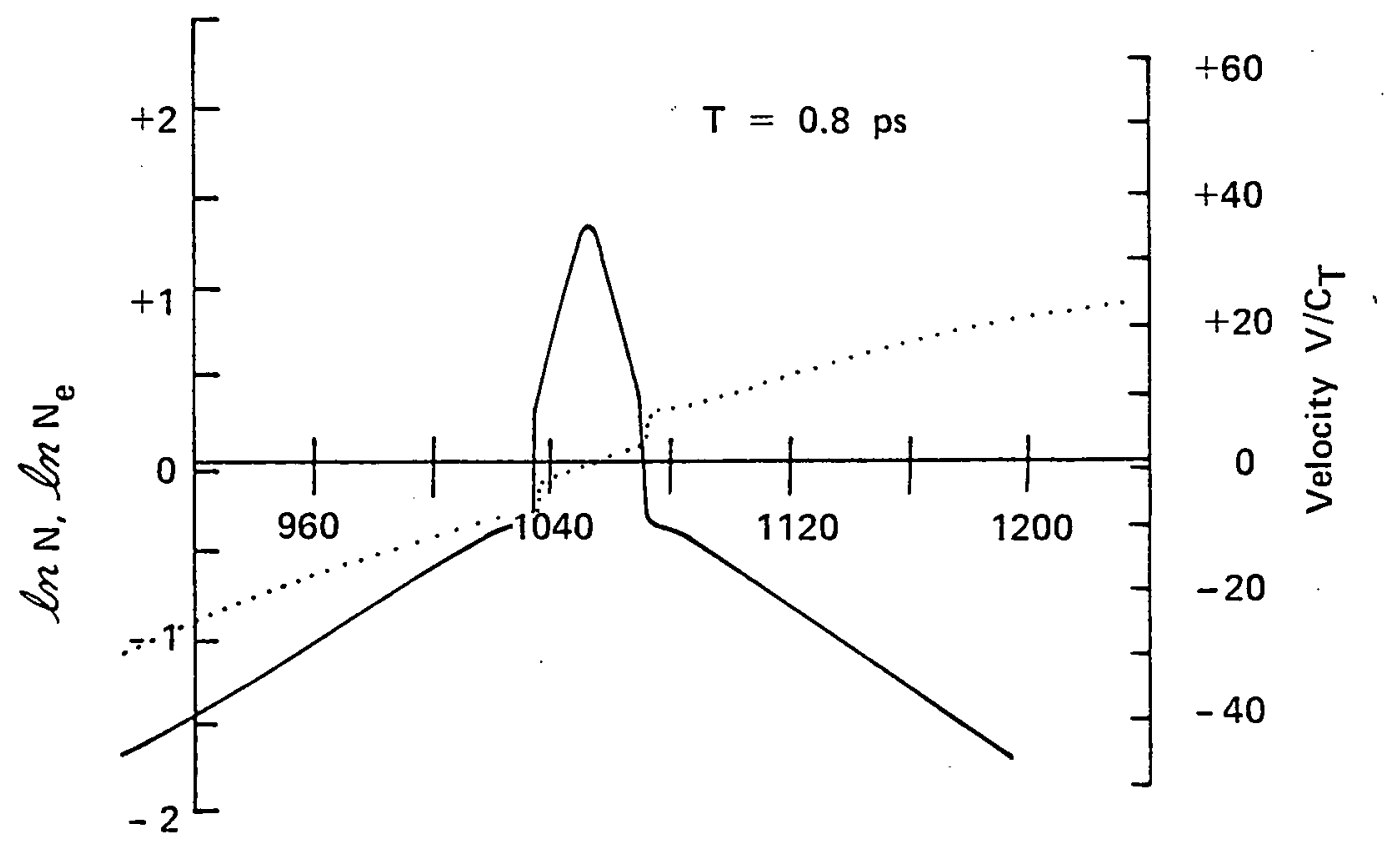

FIGURE 3.7-4 Snapshot at $t=0.8$ psec of the disassembly of the same configuration as in Figure 3.7-3, except with charge separation ignored. Note the density and velocity shelves downstream of the shocks. 
Diffuse dipole layers appear at the plasma boundaries, Figure 3.7-3, the ion excess being upstream. Because of the discrete nature of the mass zones in the Lagrangian code calculation, the details of the plasma edges are not certain. The existence of the boundary dipole layer, and hence of the outer ion group does not appear in doubt. Slater. (3) observed experimentally a separate fast ion group that may be related to the boundary ion group predicted by the code calculations.

Study of these shock structures is continuing, as is study of spherical similarity flows and their relation to the two-electron-temperature plasma flows. The interaction of rarefaction shock and ponderomotive forces will also be investigated.

\section{REFERENCES}

1. B. Bezzerides, D. W. Forslund and E. L. Lindman, "The Existence of Rarefaction Shocks in a Laser-Plasma Corona", Los Alamos Scientific Laboratory LA-UR-78-12, December 1977.

2. J. E. Crow, P. L. Auer and J. E. Allen, J. Plasma Physics 14, p 65-76 (1975).

3. KMS Fusion 1977 Annual Report, Sec. 2.2 by D. C. STater. 


\subsection{Explosively Heated Gaussian Objects}

F. J. Mayer and D. J. Tanner

There are three energy delivery mechanisms currently being examined or proposed for inertial confinement fusion: lasers, electron beams and ion beams. Preliminary experiments usually involve delivering large amounts of energy in a short time to simple targets: foils, wires and spheres. This energy is rapidiy distributed through the material and "explodes" the target. These explosions are usually modelled with complicated heat transport, hydrodynamic codes which are not easily accessible to those actually doing the experiments. As a hopefully usable tool, we have developed a similarity solution to the one-fluid hydrodynamic system to obtain some simple but quantitative estimates of the hydrodynamic behavior of these targets in this exproding mode. We assume that the target has a uniform temperature (isothermal approximation) and that the power absorbed from the beam is proportional to the local matter density. We expect the expansion to remain nearly one-dimensional for foils and wires until the thickness has expanded to approximately the depositing beam spot size.

The similarity method for solving the fluid equations is discussed extensively by Sedov. (1) Previously, similarity solutions to the hydrodynamic equations have been used in laser-fusion research to calculate basic plasma properties, (see, for example, the early work of Dawson (2), Basov and Krokhin ${ }^{(3)}$ and Haught and Polk $\left.{ }^{(4)}\right)$. Our present approach differs in that we assume uniform energy deposition and a one-temperature isothermal fluid. Some features of this model have been worked out for spherical symmetry by various authors ${ }^{(5-9)}$ and for planar symmetry by Nemchinov. (10) 
We will first develop the solution for a foil and then generalize to cylindrical and spherical geometry. The one-fluid isothermal hydrodynamic equations in planar geometry are given by

$$
\begin{aligned}
& \frac{\partial n}{\partial t}+\frac{\partial}{\partial x}(n v)=0 \\
& m_{i} n\left(\frac{\partial v}{\partial t}+v \frac{\partial v}{\partial x}\right)=-\frac{\partial p}{\partial x} \\
& n\left(\frac{\partial \theta}{\partial t}+v \frac{\partial \theta}{\partial x}\right)+\frac{2}{3} p \frac{\partial v}{\partial x}=\frac{2}{3} P_{a}(x, t)
\end{aligned}
$$

where $n$ is the number density of plasma ions, $\theta=\bar{Z} \theta_{e}+\theta_{i}, p=n \theta, n_{0}$ is the central ion density at $t^{\prime}=0$, and $P_{a}(x, t)$ is the deposited ion beam power per unit volume.

We take the absorbed power per unit volume $P_{a}(x, t)$ to be given by $P_{a}(x, t)=\left.\frac{N_{0} f(t)}{A_{b} n_{0}} \frac{d E_{1}}{d x}\right|_{0} n(x, t)=P_{0}(t) n(x, t)$,

where $N_{0} f(t)$ is the number of beam ions per unit time being absorbed, $f(t)$ is the temporal shape of the ion beam (normalized to one), $A_{b}$ is the beam area, and $\left.\frac{d E}{d x}\right|_{0}$ can be a function of time (we will assume it to be constant) but not space. Although the relations (47, 48 and 49 ) can be solved for arbitrary $f(t)$, we simplify the subsequent analys is by choosing a simple pulse shape,

$$
\begin{aligned}
f(t) & =\sin ^{2} \pi \tau, & 0 \leq \tau \leq 1 \\
& =0 \quad, & \tau>1
\end{aligned}
$$

where $\tau=t / t_{p}$, and $t_{p}$ is the pulse length. 
We choose the following notation and look for solutions to Equations (47-49) of the form,

$$
\begin{aligned}
& \theta=\theta(\tau) . \\
& n=n_{0} h\left(\frac{x}{L}\right) \psi(\tau) \\
& p=n \theta \\
& v=\frac{x}{t_{p} L} i \\
& L=L_{0} y(\tau)
\end{aligned}
$$

where $\theta$ is independent of $x$, the dot signifies differentiation with respect to $\tau$ and $L_{0}$ is the scale length at $\tau=0$. Substituting these into Equation (47), and integrating with the boundary condition, $y(0)=1$, we find,

$$
\psi y=1
$$

This result and the momemtum equation (48) result in the usual separation of variables:

$$
(h \xi)^{-1} \frac{d h}{d \xi}=\alpha \text { and } y \ddot{y}=-\frac{\alpha \cdot \theta t_{p}{ }^{2}}{m_{i} L_{0}^{2}}
$$

where $\xi=\frac{X}{L}$, and $\alpha$ is a constant.

Without loss of generality we can take $\alpha=-2$ and integrate the spatial part of Equation (53) to give

$$
h=\exp -\left(\frac{x}{L}\right)^{2} \text {. }
$$

We see that the conditions imposed on the form of the solution result in a gaussian profile in space where the central density, $n_{0} \psi$, and the 
characteristic thickness, L, vary with time. Kidder $^{(7)}$ has noted previously that energy deposition proportional to mass and a gaussian shape are required for a self-similar solution in spherical geometry.

Substituting $\mathrm{n}$ and $\mathrm{v}$ from Equation (51) into Equation (49) and using Equations (52) and (53) to el iminate $\psi, \theta$, and $L$,- we have the following ordinary differential equation:

$$
y \ddot{y}+\frac{5}{3} \dot{y} \ddot{y}=A \sin ^{2} \pi \tau \text {. }
$$

where $A=\frac{2 P_{0} t_{p}}{3 \theta_{0}}, \quad P_{0}=\frac{\left.N_{0} \frac{d E}{d x}\right|_{0}}{A_{b} n_{0}}$ and $\theta_{0}=\frac{m_{i} L_{0}^{2}}{2 t_{p}^{2}}$ is a scale temperature; when $\tau>1$, the right hand side is zero.

The constant $A$ is more meaningfur when written in terms of the $a b-$ sorbed energy per ion. The total absorbed energy $E_{a}$, is the integral of $\mathrm{P}_{\mathrm{a}}$ over space and time

$$
E_{a}=t_{p} \int_{0}^{1} d \tau \int P_{a}(x, \tau) d^{3} x=\left.\frac{\sqrt{\pi}}{2} L_{0} t_{p} N_{0} \frac{d E}{d x}\right|_{0},
$$

and the number of ions absorbing energy from the beam is the integral of n

$$
N_{i}=\int n d^{3} x-\sqrt{\pi} n_{0} \Lambda_{b} L_{0} .
$$

Using these relations $A$ is seen to be

$$
A=\frac{4 E_{a}}{3 N_{i} \theta_{0}}=\frac{4}{3} \frac{\theta_{a}}{\theta_{0}}
$$

where $\theta_{a}=E_{a} / N_{i}$ is the temperature rise of the plasma if the energy were absorbed instantaneously.

Equation (55) can be checked by looking at the kinetic and thermal energy in the flow. Integrating $\frac{1}{2} m_{i} n v^{2}$ over space gives the kinetic energy 


$$
E_{k}=\frac{1}{2} N_{i} \theta_{0}(\dot{y})^{2}
$$

Likewise the thermal energy is the integral of $\frac{3}{2} p$ over space,

$$
E_{t h}=\frac{3}{2} N_{i} \quad \theta_{0} \ddot{y} y
$$

Integrating Equation (55) to a time greater than $t_{p}(\tau>1)$ we have

$$
E_{k}+E_{t h}=\frac{3}{4} N_{i} \cdot \theta_{0} A=E_{a}
$$

showing that Equation (55) is simply a statement of conservation of energy.

It is simple to generalize the analysis presented above for exploding planar objects to include explosive heating of cylindrical and spherical objects as well. Equation (52) becomes,

$$
\psi y^{d}=1
$$

where $d=1,2$, or 3 for planar, cylindrical or spherical symmetry, and Equation (55) becomes

$$
y \ddot{y}+\left(\frac{3+2 d}{3}\right) \dot{y} \ddot{y}=A \sin ^{2} \pi \tau \text {. }
$$

Since $n \sim \psi=y^{-d}$, the first relation is just the effect of spherical or cylindrical divergence on the density. The value of $d$ has a much smaller affect on $y$ in Equation (60) than on $\psi$ in Equation (59). As seen below, the velocity, determined by $y$, is weakly dependent on $d$ while the density, determined by $\psi$, is strongly dependent on $d$. Several authors $(5 ; 6,8)$ have obtained Equation (60) for the spherical case; Nemchinov $(10)$ has derived the planar case. The expression (58) for the constant A remains unchanged, although the calculation of the integrals introduce different geometric factors in $N_{j}$ and $E_{a}$. The kinetic energy is modified to 


$$
E_{k}=\frac{1}{2} d N_{i} \theta_{0}(\dot{y})^{2}
$$

while the thermal energy is unchanged. As before, adding these together produces the first integral of (60), demonstrating energy conservation.

Now let us proceed to the results. Equation (60) was integrated numerically to produce the graphs of Figure 3.8-1. In Figure 3.8-1 (a through c) we present as a function of time the normalized temperature $\theta / \theta_{0}$, the normalized central density $n(0) / n_{0}$ and the normalized velocity $v / v_{0}$ at $x=L$ in each kind of symmetry for three values of $4 \theta_{a} / 3 \theta_{0}$. The scale velocity $v_{0}$ is $L_{0} / t_{p}$. The effects of cylindrical and spherical divergence on the density are clearly seen. In fact the densities for cylindrical and spherical symmetry are approximately the square and cube of the density for planar symmetry. As expected, the velocity is much less sensitive to geometry. The decrease in temperature in going from planar to spherical geometry occurs because a greater fraction of the energy is kinetic as seen in Equation (61). The maximum in temperature occurs roughiy mid-way between the maximum in the pulse at $\tau=0.5$, and the pulse cutoff. For a given energy input maximum thermal energy density occurs for planar targets. Figure 3.8-1(d) presents normalized temperature as a function of time with $4 \theta_{a} / 3 \theta_{0}$ as a parameter. Above a vaiue of $10^{2}$, the ratio of the temperature for planar geometry to that for spherical geometry is independent of $\theta_{a} / \theta_{0}$.

Figure 3.8-2 has the dependence of the maximum temperature and the central density at that temperature as a function of $4 \theta_{a} / 3 \theta_{0}$. At large deposited energies, it is clear that exploding foils have much larger densities than cylinders or spheres. The slope of the maximum normalized temperature line is nearly one. Since $\hat{\theta}_{m}=\frac{\theta_{m}}{\theta_{0}}$ and $A=\frac{4 \theta_{a}}{3 \theta_{0}}$ 

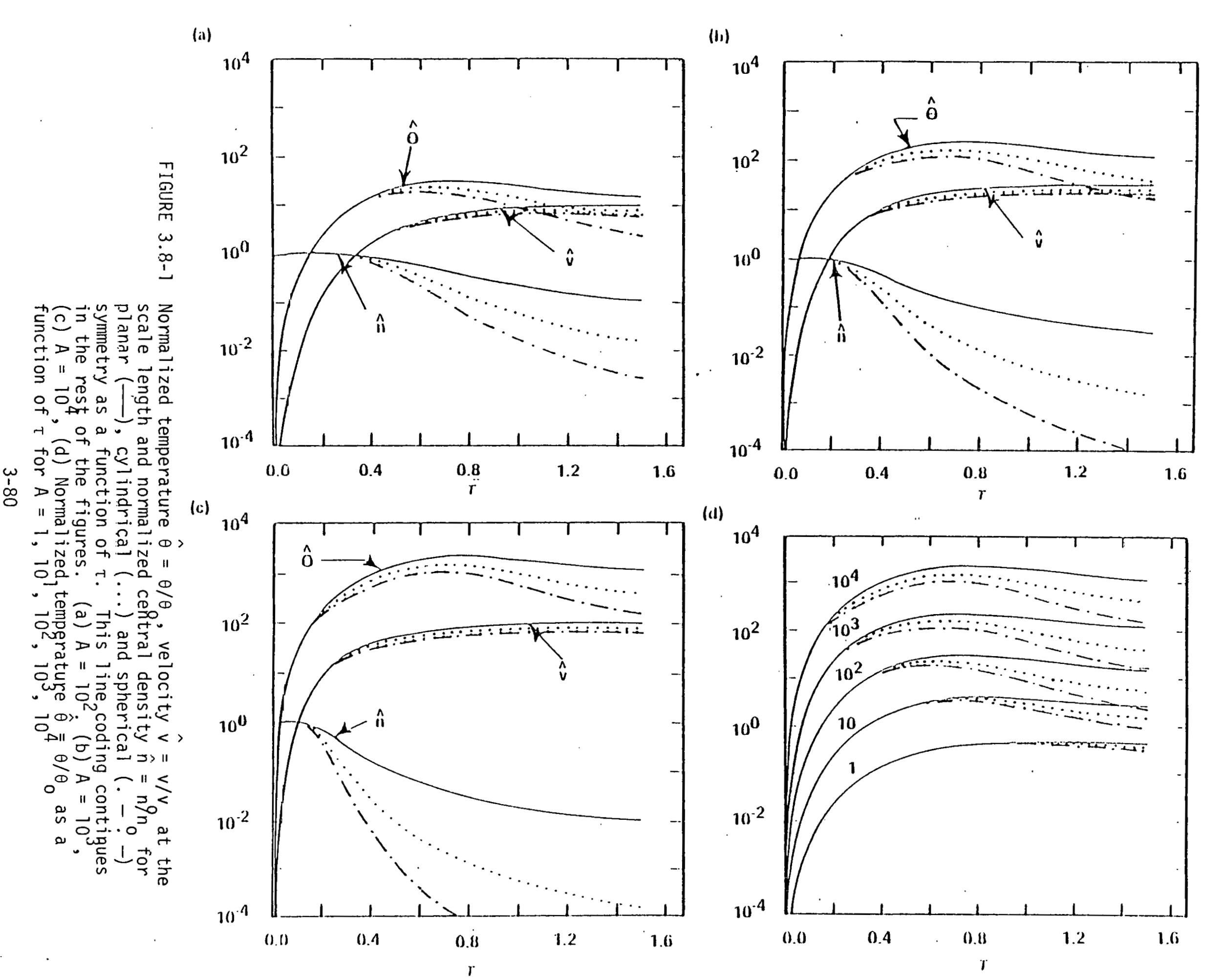
(a)

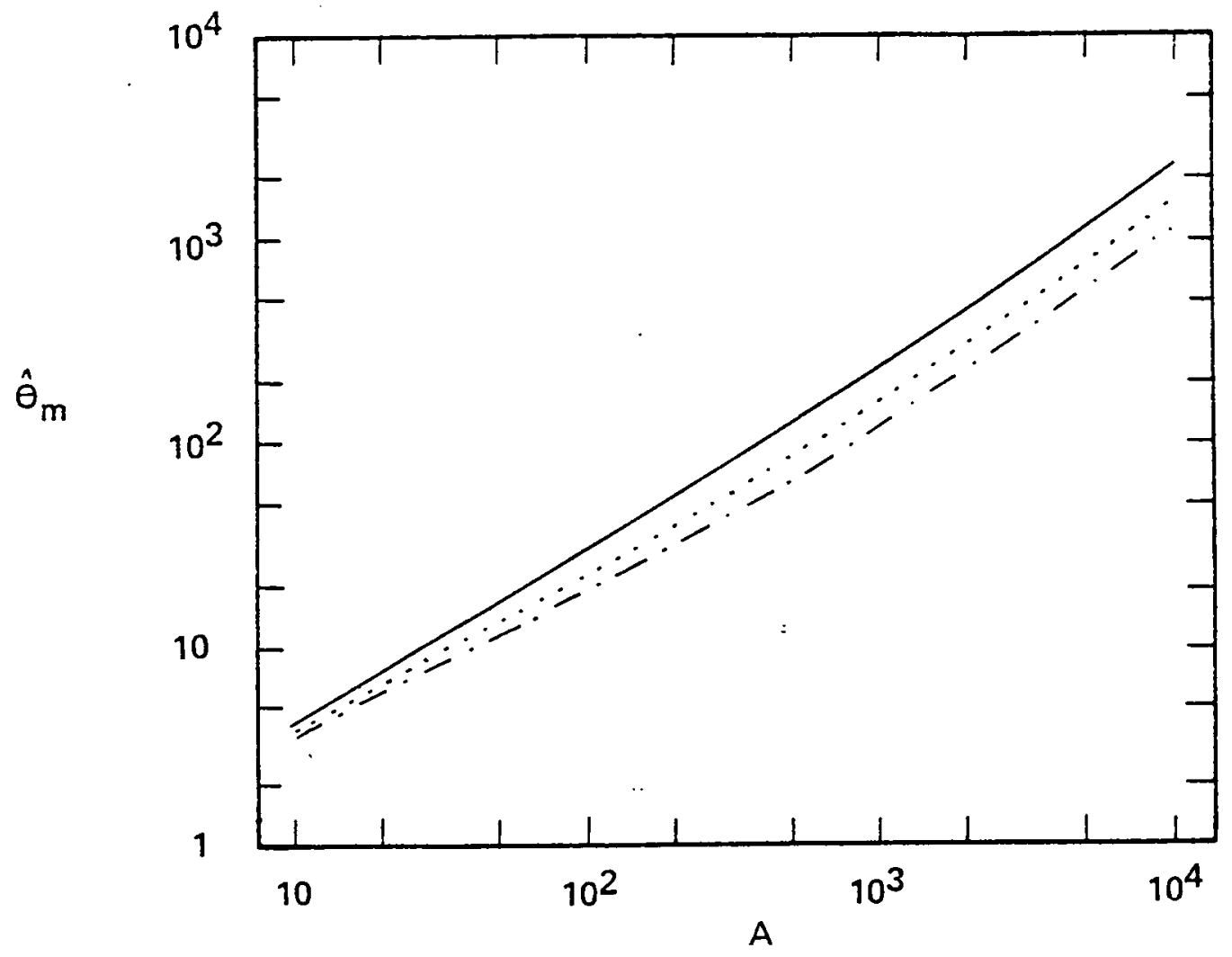

(b)

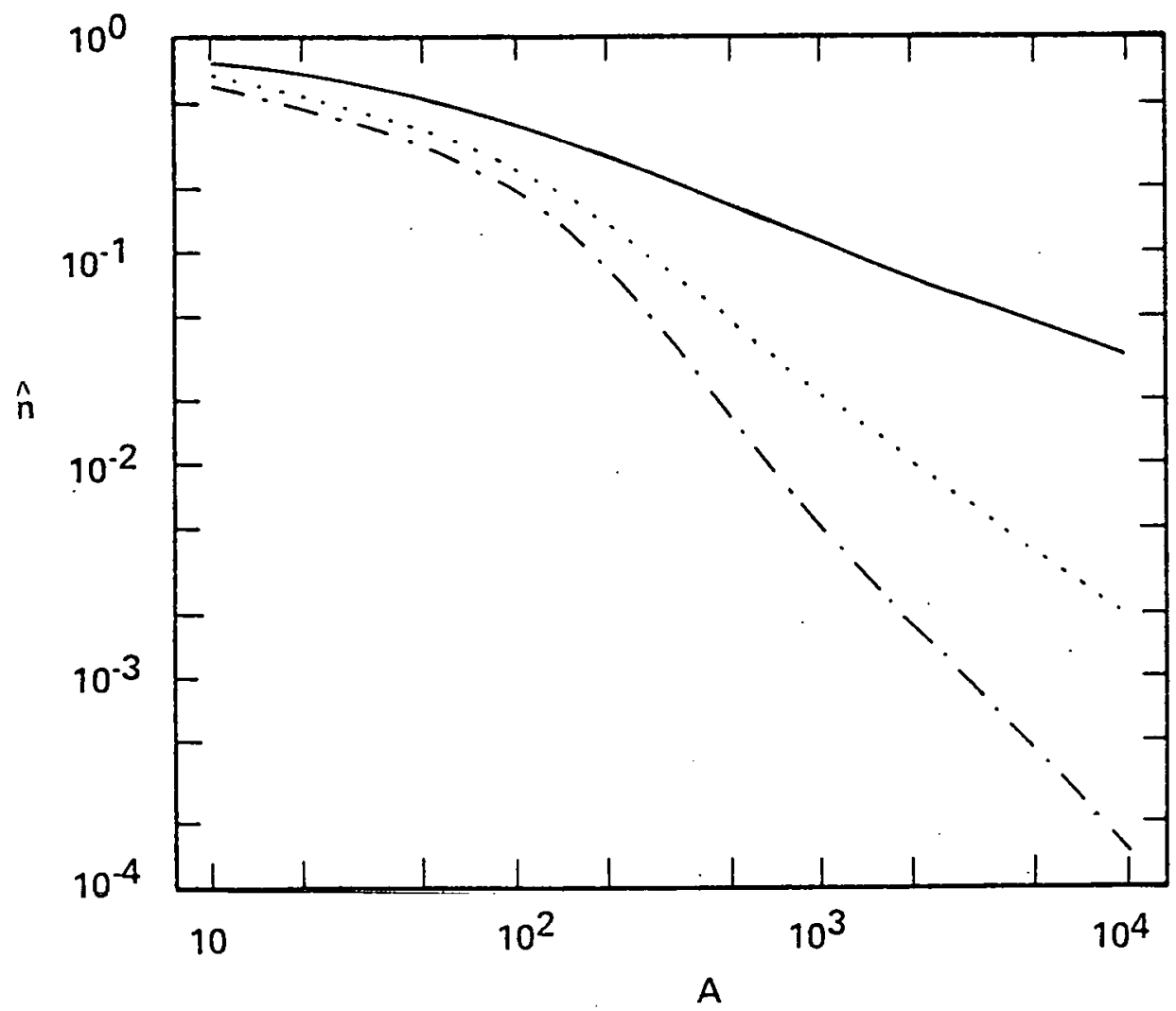

FIGURE 3.8-2 (a) The maximum temperature as a function of $A$.

(b) The normalized central density at the time of maximum temperature. 
then $\theta_{m} \sim \theta_{a}=E_{a} / N_{i}$. The maximum temperature achieved is then proportional to the energy deposited per ion and independent of the pulse length. This is true as long as $\theta_{a} / \theta_{0}$ is large. However, if higher densities are desired, $\theta_{a} / \theta_{0}$ should be kept small.

The isothermal explosion of any foil, wire or sphere is represented by the solution of Equation (60) if the density profile is gaussian. The solution is determined by the ratio of two parameters: $\theta_{a}$, the absorbed energy per ion, and $\theta_{0}$ the kinetic energy of an ion which moves through the scale length, $L_{0}$, in the scale time, $t_{p}$. In Table 3.8-1 we present formulae for calculating $\theta_{0}$ and $\theta_{a}$ from experimental parameters in practical units. We also present the relationship between the characteristic length $L_{0}$ and the dimension of a physical object of uniform density. This was obtained by taking a gaussian object with a central density $n_{0}$ equal that of the physical object, integrating the density $n$ from Equations (51) and (54) over. space and requiring the resulting mass to be equal to the mass of the physical object.

We now proceed to a practical example. Consider a polyethylene foil $20 \mu \mathrm{m}$ thick struck by a heavy ion beam with a diameter of $2 \mathrm{~mm}$ which deposits $0.5 \mathrm{~kJ}$ in the foil. The scale temperature $\theta_{0}$ is $2.5 \mathrm{eV}, \theta_{a}$ is $400 \mathrm{eV}$ and $A$ is 200. Referring to Figure 3.8-2 we have $\theta_{\max }=50 \theta_{0}$ or $100 \mathrm{eV}$ and the central density at this temperature is $20 \%$ of the initial density.

In real experiments, targets do not initially have a gaussian shape. An important question to ask is: when does an exploding foil, wire or sphere obtain the gaussian shape and how is the parameter $L_{0}$ related to the initial half-thickness of the target? We have examined this question with our one-dimensional heat transport, hydrodynamic code TRHYD. The code 
Table I

\begin{tabular}{|c|c|c|c|}
\hline & Foil & Wire & Sphere \\
\hline$\theta_{\mathrm{a}}$ & $\frac{E_{a} A_{n}}{0.3 \rho t_{d} r_{d}^{2}}$ & $\frac{E_{a} A_{n}}{3 \cdot 10^{-4} \rho 2 r_{r}^{2}}$ & $\frac{2.5 \times 10^{-6} \cdot E_{a} A_{n}}{\rho r_{s}^{3}}$ \\
\hline$L_{0}$ & $1.13 t_{d} / 2$ & $r_{r}=$ & $0.75 r_{s}$ \\
\hline$\theta_{0}$ & $\frac{A_{n} L_{o}^{2}}{200 t_{p}^{2}}$ & & . \\
\hline
\end{tabular}

Characteristic quantities for real (square) objects. $\theta_{a}$ in keV, $\theta_{0}$ in $e V, L_{0}$ in $\mu m, t_{p}$ in $n s e c$, and $E_{a}$ in $k J . A_{n}$ is the atomic number, $\rho$ is in $\mathrm{g} / \mathrm{cm}^{3}$, $t_{d}$ is the thickness of the foil in $\mu \mathrm{m}, r_{d}$ is the radius of the spot size on the foil in $\mathrm{mm}, r_{r}$ is the radius of the wire in $\mu \mathrm{m}$, $\ell$ is the length of the spot on a wire in $\mathrm{mm}, r_{s}$ is the radius of a sphere in $\mu \mathrm{m} . \theta_{0}$ is independent of geometry. 
calculations were made for lead objects having a density of $19 \mathrm{~g} / \mathrm{cm}^{3}$, a thickness of $10 \mu \mathrm{m}$ and absorbing $40 \mathrm{~kJ}$ in $1 \mathrm{nsec}$ from a beam $2 \mathrm{~mm}$ in diameter. Energy was deposited proportional to mass and the electron and ion conductivities were artificially made large enough to keep the plasma isothermal to within about $5 \%$. Figure $3.8-3$ shows the density at $\tau=1$ and $\tau=2.5$ plotted against the square of the distance from the center normalized by the initial thickness. A gaussian would be a : straight line. In Figure 3.8-3(a). we see that, at $\tau=1$, the density profile is not gaussian when the central density is at $0.7 \mathrm{~g} / \mathrm{cm}^{3}$ or $3 \%$ of the initial density. However, in Figure $3.8-3(b)$ when $\tau=2.5$, the gaussian profile is evident except for the outer two zones. Here, fine zoning and longer times may be required to find the outer edge following the gaussian shape. In any case, most of the sphere is gaussian at this time. Based on 14 code runs in planar, cylindrical and spherical symmetry, we conclude that the density profile becomes gaussian by the time the central density is $1 \%$ of the original density, and remains so thereafter.

The slope of the curve in Figure 3.8-3(b). gives a value for $L$ from Equation (54) and the central density gives a value for $\psi$ and, from Equation (59), y. The resultant value for $L_{0}=L / y$ agrees with the value calculated from the formulae in Table 3.8-1 to within $1 \%$.

In conclusion, we have developed a simple similarity solution which may be very useful for predicting some properties of early ion beam experiments. All uniformly heated explosions are characterized by just two parameters $-\theta_{a}$ and $\theta_{0}$. With these parameters, it is possible to calculate how far one is from the ideal case wherein all the deposited energy is thermal at temperature $\theta_{a}$. The similarity solutions show that explosions of various sized object with appropriate deposited energies are 
(a)

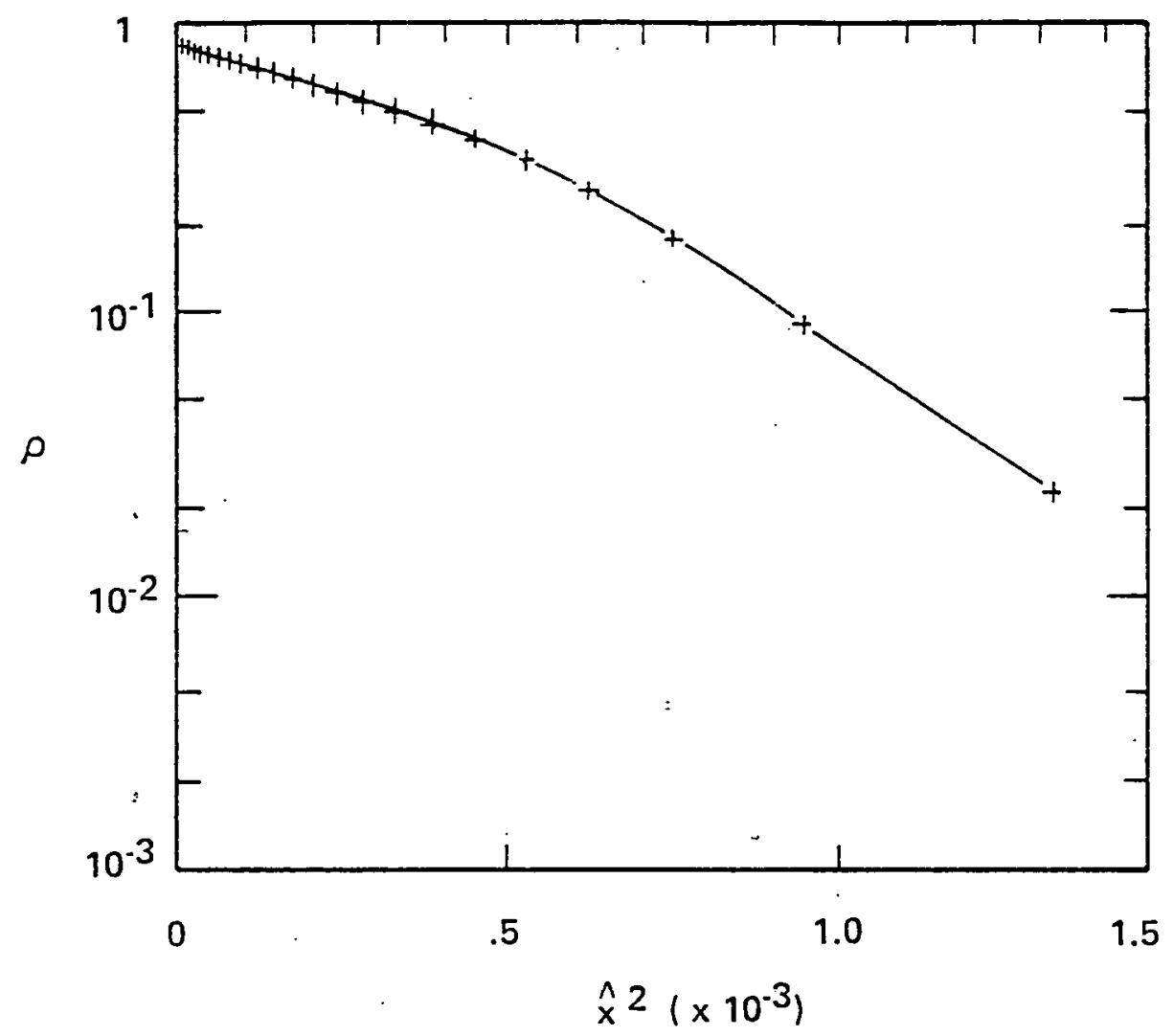

(b)

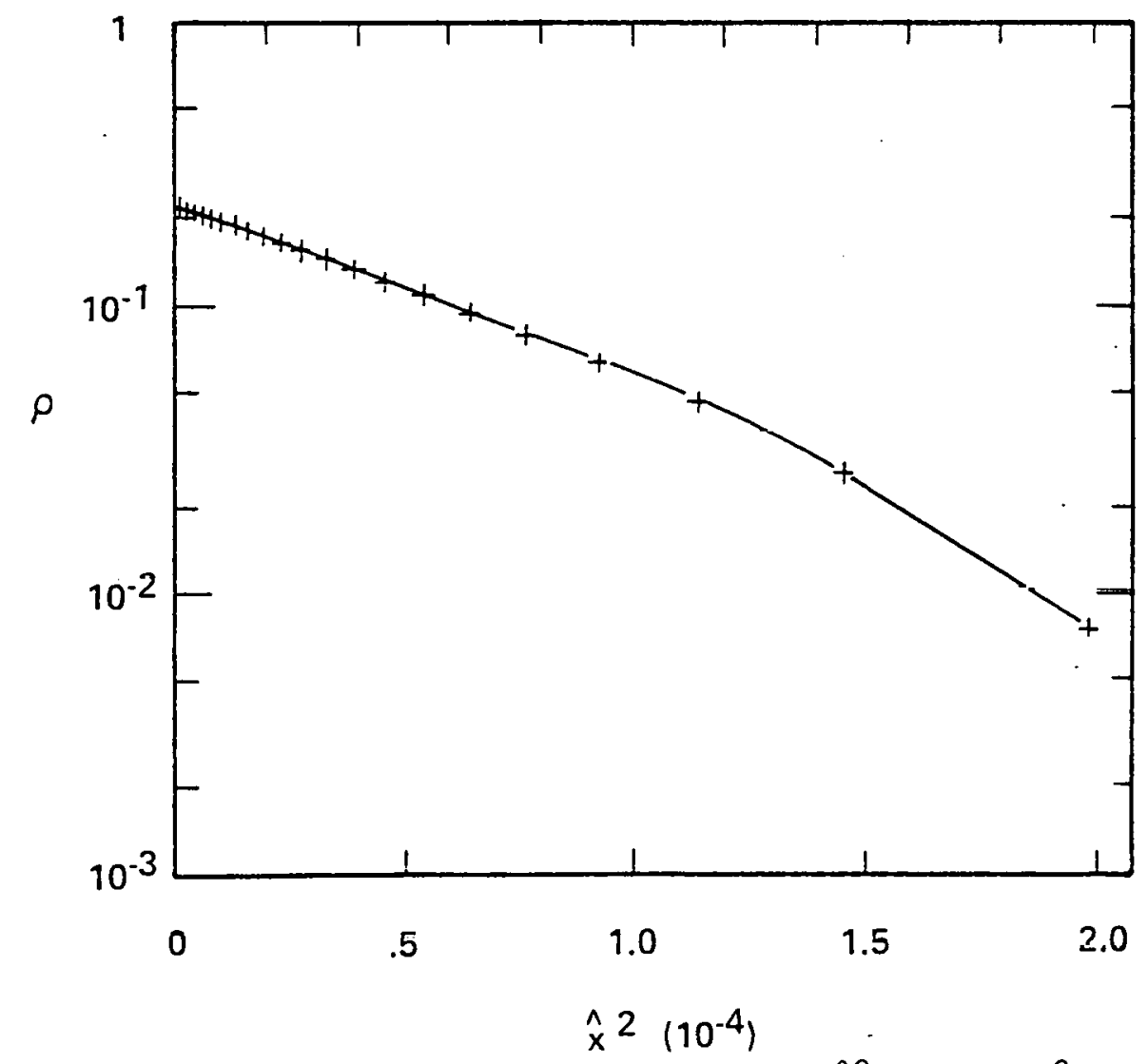

FIGURE 3.8-3 The density as a function of $\hat{x}^{2}=\left(x / x_{0}\right)^{2}$ at (a) $\tau=1$ and (b) $\tau=2.5$ for the lead foil disclssed in the text. Note the different. horizontal scale between the two figures. 
hydrodynamically identical. These similarity solutions may also be useful as routine test problems for one-dimensional hydrodynamic codes.

\section{REFERENCES}

1. L. I. Sedov, "Similarity and Dimensional Methods in Mechanics" (Academic, New York, 1959) Chap. IV.

2. J. M. Dawson, Phys. Fluids $\underline{7} ; 981$ (1964).

3. N. G. Basov and O. N. Krokhin, Sov. Phys. JETP 19, 123 (1964).

4. A. F. Haught and D. H. Polk, Phys. Fluids 9, 2049 (1966).

5. W. J. Fader, Phys. Fluids 11, 2200 (1968).

6. A. F. Haught and D. H. Polk, Phys. Fluids 13, 2825 (1970).

7. R. E. Kidder, Proceedings of the International School of Physics $\underline{49}, 306$ (1971).

8. L. S. Dzung and Z. Angew, Math. Phys. 23, 301 (1972).

9. L. Lengyel and M. Salvat, Zeit. Naturforsch 30a, 1577 (1975).

10. I. V. Nemchinov, Zh. Prik1. Mekh. Tekh. Fiz. 1, 17 (1961). 


\section{LASER AND OPTICS}

Introduction

4.1 Bandwidth Broadening - The Plasma Spatial Filter

4.2 Frequency Doubling Experiments

4.3 Advanced Laser Candidates

4.4 Glass Laser Operation

4.52 TW Laser Upgrade 


\section{Introduction}

\section{E. Thomas}

Three aspects of the KMS laser system are unique to the National ICF program: short wavelength (visible) implosion experiments, variable bandwidth laser output, and symmetrical target illumination. Work proceeded in all three areas in 1978. Experimental results indicate that the combination of short wavelength $(532 \mathrm{~nm})$ and broad bandwidth $(30 \AA)$. improves target absorption compared to narrow band IR $(1.06 \mu \mathrm{m})$ radiation. Green light experiments were performed with $10 \mathrm{~cm}$ KDP doubling crystals, and $14 \mathrm{~cm}$ crystals were obtained in preparation for the 2 TW laser upgrade experiments. The laser bandwidth was increased with the KMS plasma spatial filter ${ }^{(1)}$, and new spatial filter targets were investigated to control bandwidth.

The Nd:glass laser system was operated for target interaction experiments throughout most of the year. The $10 \mathrm{~cm}$ glass laser system delivered an average of 502 GW on target immediately after clean-up and restaging last year, ${ }^{(2)}$ but excessive damage required the addition of a plasma spatial filter to retard further damage. As a result, the average power-on-target in July-August of 1978 dropped to $354 \mathrm{GWN}$, but there was no significant damage with the time-averaged beam smoothing by the plasma filter. During September, two $14 \mathrm{~cm}$ disc amplifiers were added to each laser chain, and the average power on target with the plasma spatial filter was $576 \mathrm{GW}$.

DOE funds were provided to begin the Phase I laser upgrade to $2 \mathrm{TW}$ (3). Changes include an actively mode locked YLF oscillator, improved amplifiers, phosphate laser glass, and an all-reflective target illumination system. 
Two new advanced laser candidates for fusion reactor drivers are currently under investigation at KMSF. The free electron laser, developed at Stanford, offers the potential of high efficiency and operation in complete vacuum. The chemically-pumped iodine laser, demonstrated at Kirtland Air Force Base, offers high efficiency, but is also the only known advanced laser candidate which does not require a pulsed power supply, which is a major engineering concern of other drivers. 


\subsection{Bandwidth Broadening - The Plasma Spatial Filter}

N. K. Moncur, G. E. Busch and C. M. Kinzer

The plasma spatial filter was originally invented at KMS to smooth the laser beam intensity distribution, thereby retarding component damage. Subsequently we found that this filter also increases the laser bandwidth, (Figure 4.1-1) and this phenomenon may be very important for understanding and for increasing pellet absorption. The

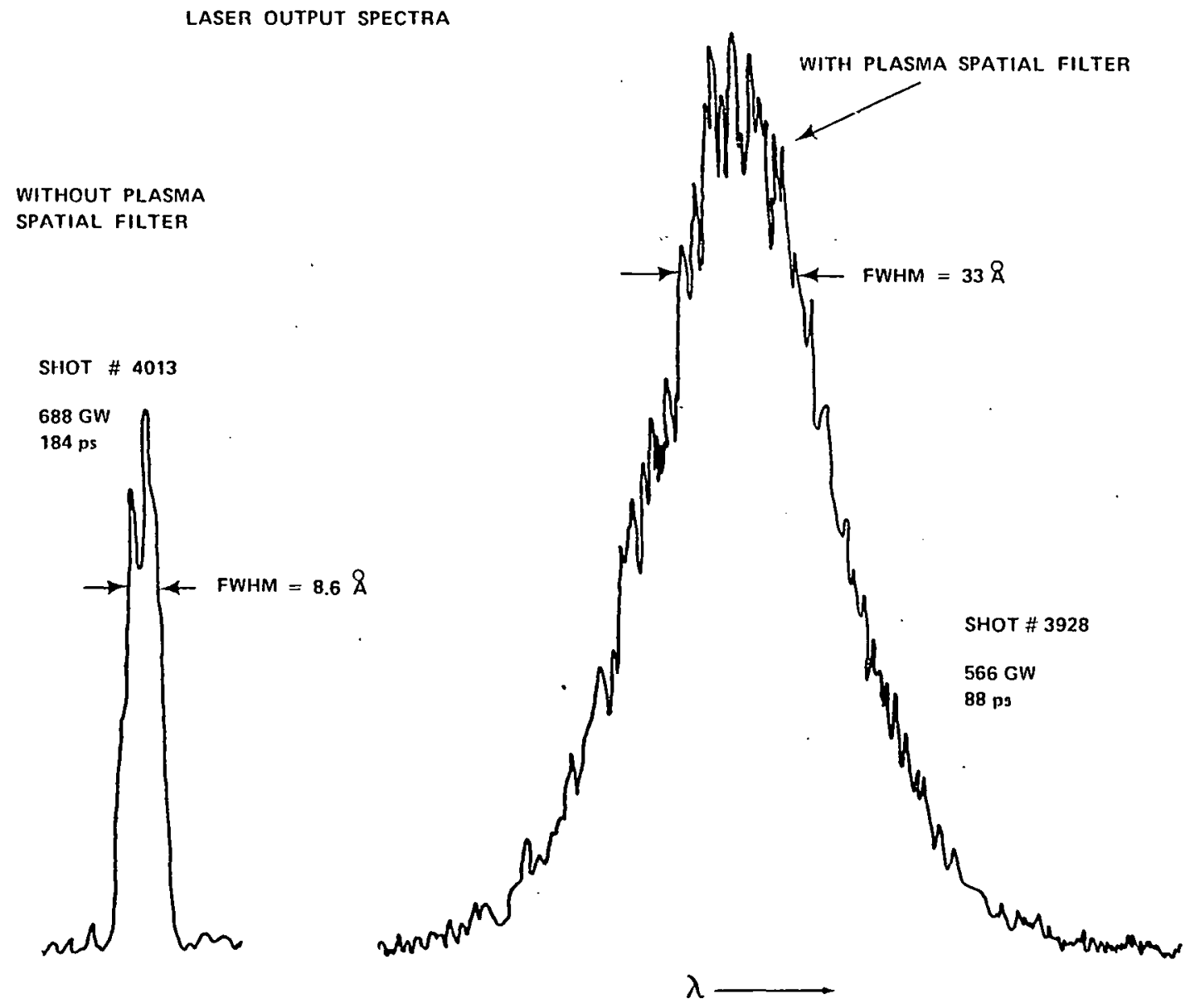

FIGURE 4.1-1 Laser Output Spectra With and Without The Plasma Spatial Filter 
original targets in the plasma spatial filter were smooth discs of polyethylene. We have since tested several different materials as targets including different metals and glass, and have found little difference in the pattern or spectrum of the reflected light. The current target is a glass slide (Figure 4.1-2) which is tilted so that the specular reflection does not return down the laser beam path. Since there is no reflection returning from the target until a plasma is formed, the target receives no amplified spontaneous emission (ASE) from rod amplifiers in front of the plasma spatial filter.

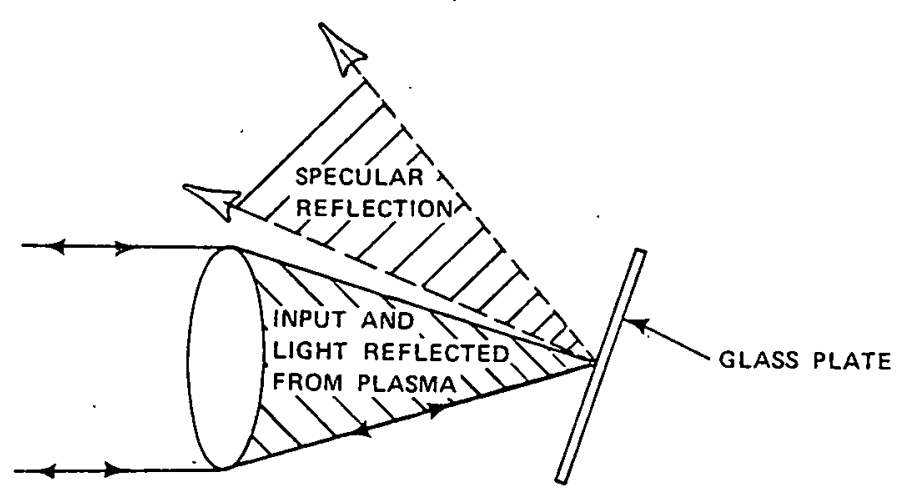

FIGURE 4.1-2 Tilted glass slide target for the plasma spatial filter 
Another target being tested for the plasma spatial filter is a jet of high pressure gas (Figure 4.1-3) flowing out of a small (100 $\mu \mathrm{m})$ pinhole. This gas target has provided some interesting results. First, the amount of light reflected back into the collecting lens increased from 1 to $5 \%$ with the solid target to 40 to $60 \%$ with the gas target. Second, the spectrum of the gas reflected light was not broadened as much as the reflected light from the solid target. The incident laser beam has a bandwidth of $\sim 4 \AA$. The solid target broadens this bandwidth to 40 to 60.9 , whereas the gas target broadens the bandwidth to only 10 to $15 \AA$. These results were obtained with pulse widths from 40 to $120 \mathrm{psec}$, power at $10^{9}$ to $10^{11}$ watts and an $\mathrm{f} / 3.0$ focusing lens. With a solid target, the reflected light was generally shifted towards the blue by 5 to $10 \AA$ except when a prepulse or multiple pulse occurred; then the shift was towards the red and reflectivity increased by a factor of 2 to 5 . With the gas target the reflected light was shifted towards the red by 5 to $10 \AA$. With both targets, the bandwidths tended to increase with increased input power.

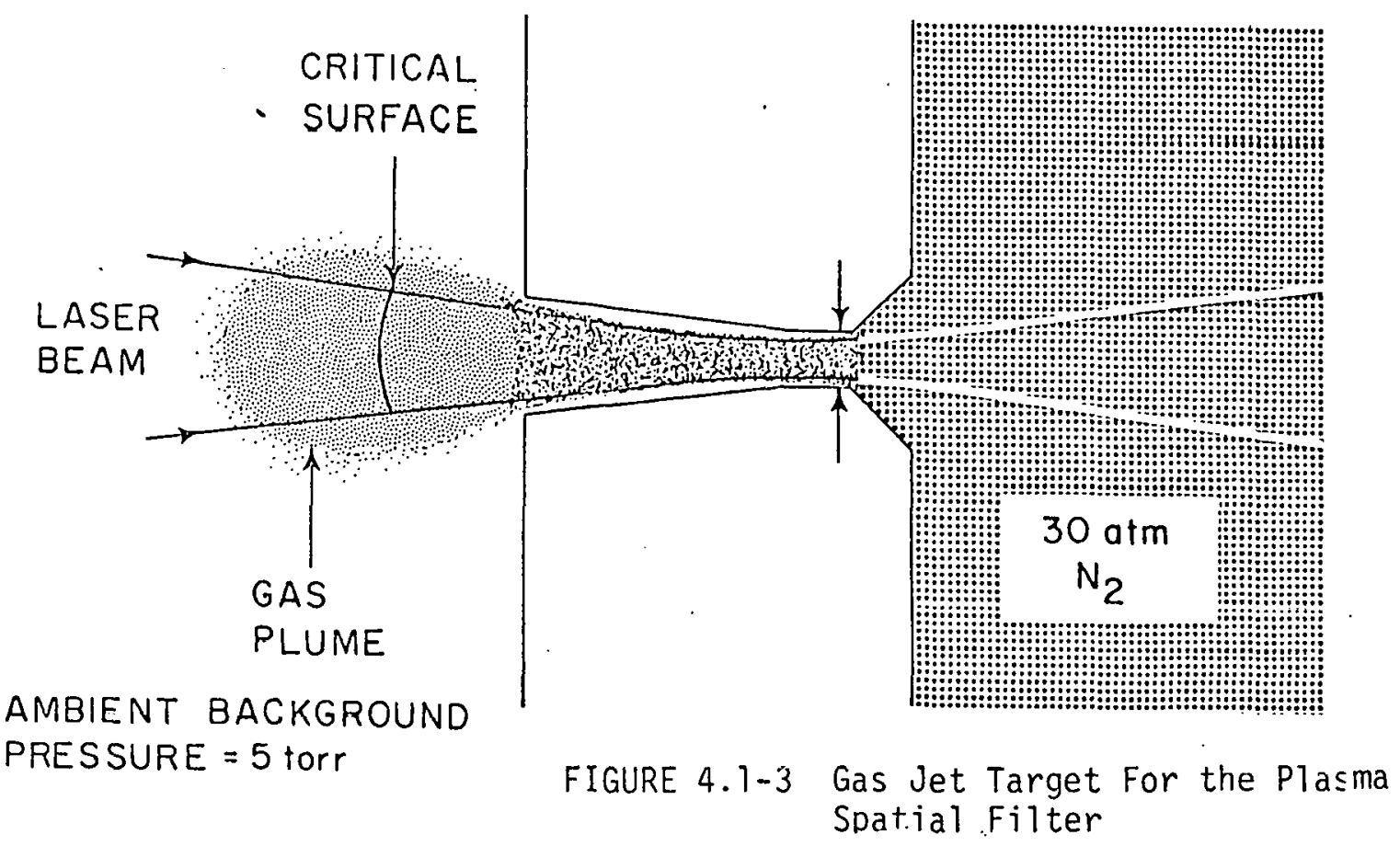




\subsection{Frequency Doubling Experiments}

L. D. Siebert

The $1.064 \mu \mathrm{m}$ output from the $10 \mathrm{~cm} \mathrm{Nd:glass} \mathrm{laser} \mathrm{was} \mathrm{frequency}$ doubled with KDP crystals to conduct short wavelength $(532 \mathrm{~nm})$ target interaction experiments (see Section 2.3). As analyzed in the 1977 Annual Report ${ }^{(4)}$, the doubling efficiency depends on the crystal thickness, the input power density, the angular laser spread, and the laser bandwidth. Prior to conducting the target experiments, we measured the high power doubling efficiencies of a $6 \mathrm{~mm}$ thick and a $12.8 \mathrm{~mm}$ thick crystal, both with and without the plasma spatial filter.

The doubling efficiency was measured with the apparatus shown in Figure 4.2-1, using one of the $10 \mathrm{~cm}$ laser beams. The first shots were made without the doubling cell or the KG3 filter in the beam to obtain a relative calibration. The previously measured calibration of the Scientec SN 106 calorimeter $(.599 \mathrm{~J} / \mathrm{mv}$ ) was used as the absolute calibration. All data were taken with four 40 ps pulses stacked 40 ps apart; hence the pulse shape was approximately square.

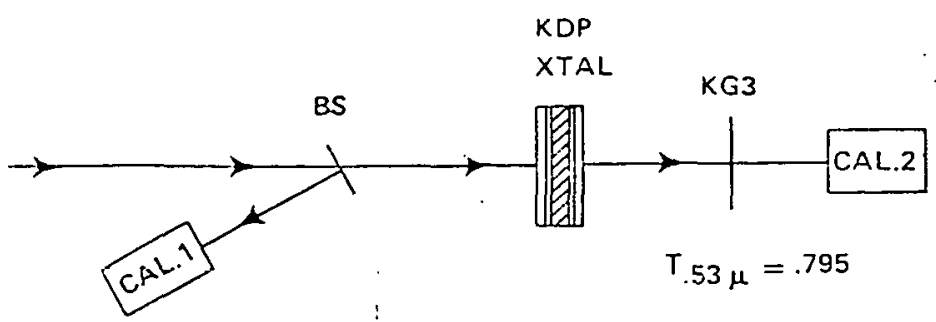

FIGURE 4.2-1 Experimental arrangement for measuring doubling efficiency. Cal. 1 = Scientec SN105, Cal. 2 = Scientec SN106, BS = beamsplitter (1/2" thick, 8" dia. uncoated fused quartz), KDP crystal = type II KDP, and KG3

$=1.06 \mu \mathrm{m}$ rejection filter 
The results of these efficiency measurements are summarized in Table 4:2-1 The average input and output energy density listed in Table 4.2-1 is estimated by dividing the two calorimeter readings by the nominal beam area. The average power densities are then derived by dividing the energy densities by the FWHM laser pulse length, which is estimated from the Imacon 600 streak camera record of the input $1.064 \mu \mathrm{m}$ pulse for each shot. The maximum output green power density (2.71 $\mathrm{GW} / \mathrm{cm}^{2}$ ) for the thin $6 \mathrm{~mm}$ crystal was obtained on shot 3395 , but the $4.54 \mathrm{GW} / \mathrm{cm}^{2}$ input power density on this shot also produced three pairs of damage sites in the $1.064 \mu \mathrm{m}$ AR coating on the front window of the crystal cell assembly.

We conclude from the data of Table 4.2-1 that the plasma filter significantly reduces doubling efficiency (from about $58 \%$ to $34 \%$ on the average), but, on the other hand, we can increase the input $1.064 \mu \mathrm{m}$ power density to higher levels without suffering damage to optical components. As a result, the safe operating green output from the crystal is about $2 \mathrm{GW} / \mathrm{cm}^{2}$ either with or without the plasma spatial filter.

The choice between thick and thin crystals is less conclusive. The theoretical predictions of doubling efficiency versus input power density are shown in Figure 4.2-2 for the thin $(6 \mathrm{~mm})$ crystal and on Figure 4.2-3 for the thicker crystal; the parameter $K$ is the phase mismatch, which combines angular mismatch and frequency mismatch. On the basis of these curves, we had expected better over-all. doubling efficiency with the thinner crystals, particularly for power densities above $3 \mathrm{GW} / \mathrm{cm}^{2}$; the predicted decrease in efficiency with increased power density did not occur, as shown by the experimental data on Figure $4.2-2$ and $4.2-3$ 
TABLE 1.2-1 Frequency Doubling Experimental Results

\begin{tabular}{|c|c|c|c|c|c|c|c|}
\hline $\begin{array}{l}\text { Experimental } \\
\text { Configuration }\end{array}$ & $\begin{array}{c}\text { Shot } \\
\|\end{array}$ & $\begin{array}{l}1.06 \text { unl } \\
\text { Input; Energy } \\
\mathrm{J} / \mathrm{cul}^{2}\end{array}$ & $\begin{array}{l}\text { Pulse } \\
\text { Width } \\
\text { ns }\end{array}$ & $\begin{array}{l}1.06 \text { "In } \\
\text { Input Power } \\
\text { GiN/ } \mathrm{cm}^{2}\end{array}$ & $\begin{array}{l}.53 \mathrm{H} \text {."I } \\
\text { Output Energy } \\
\mathrm{J} / \mathrm{cml}^{2}\end{array}$ & $\begin{array}{l}.53 \mathrm{um} \\
\text { Output Power } \\
\text { Cilv/cin? }\end{array}$ & $\begin{array}{c}\text { Douliting } \\
\text { Efficiency } \\
\%\end{array}$ \\
\hline Calibration & 3389 & .405 & .151 & 2.53 & .405 & 2.53 & 100 \\
\hline $\begin{array}{l}\text { Gomm Crystal } \\
\text { \& Plasilia } \\
\text { Filter }\end{array}$ & $\begin{array}{l}3391 \\
3392 \\
3393\end{array}$ & $\begin{array}{l}.199 \\
.833 \\
.922\end{array}$ & $\begin{array}{l}.154 \\
.174 \\
.184\end{array}$ & $\begin{array}{l}1.29 \\
1.79 \\
5.02\end{array}$ & $\begin{array}{l}.030 \\
.270 \\
.312\end{array}$ & $\begin{array}{l}.195 \\
1.55 \\
1.69\end{array}$ & $\begin{array}{l}15 \\
32 \\
34\end{array}$ \\
\hline $\begin{array}{l}6 \text { mun Crystal } \\
\text { Plo Plasma } \\
\text { Filter }\end{array}$ & $\begin{array}{l}3394 \\
3395 \\
3396\end{array}$ & $\begin{array}{l}.413 \\
.752 \\
.466\end{array}$ & $\begin{array}{l}.156 \\
.166 \\
.154\end{array}$ & $\begin{array}{l}2.65 \\
4.54 \\
3.03\end{array}$ & $\begin{array}{l}.198 \\
.449 \\
.210\end{array}$ & $\begin{array}{l}1.27 \\
2.71 \\
1.36\end{array}$ & $\begin{array}{l}48 \\
60 \\
45\end{array}$ \\
\hline $\begin{array}{l}12.8 \text { min Crystal } \\
\text { Ho Plasina } \\
\text { Filter }\end{array}$ & $\begin{array}{l}3397 \\
3398 \\
3400 \\
3401 \\
3402\end{array}$ & $\begin{array}{l}.210 \\
.134 \\
.218 \\
.251 \\
.505\end{array}$ & $\begin{array}{l}.156 \\
.154 \\
.158 \\
.156 \\
.156\end{array}$ & $\begin{array}{r}1.35 \\
.87 \\
1.38 \\
1.61 \\
3.24\end{array}$ & $\begin{array}{l}.130 \\
.063 \\
.127 \\
.156 \\
.372\end{array}$ & $\begin{array}{r}.836 \\
.408 \\
.805 \\
1.00 \\
2.38\end{array}$ & $\begin{array}{l}62 \\
47 \\
58 \\
62 \\
71\end{array}$ \\
\hline $\begin{array}{l}12.8 \text { min Crysta } \\
\text { and Plasira } \\
\text { Filter }\end{array}$ & $\begin{array}{l}3403 \\
3404 \\
3405 \\
3407\end{array}$ & $\begin{array}{l}.259 \\
1.00 \\
.569 \\
.197\end{array}$ & $\begin{array}{l}.156 \\
.156 \\
.156 \\
.086\end{array}$ & $\begin{array}{l}1.66 \\
6.44 \\
3.65 \\
2.29\end{array}$ & $\begin{array}{l}.081 \\
.375 \\
i .93 \\
.070\end{array}$ & $\begin{array}{r}.519 \\
2.40 \\
1.24 \\
.82\end{array}$ & $\begin{array}{l}31 \\
37 \\
34 \\
36\end{array}$ \\
\hline
\end{tabular}




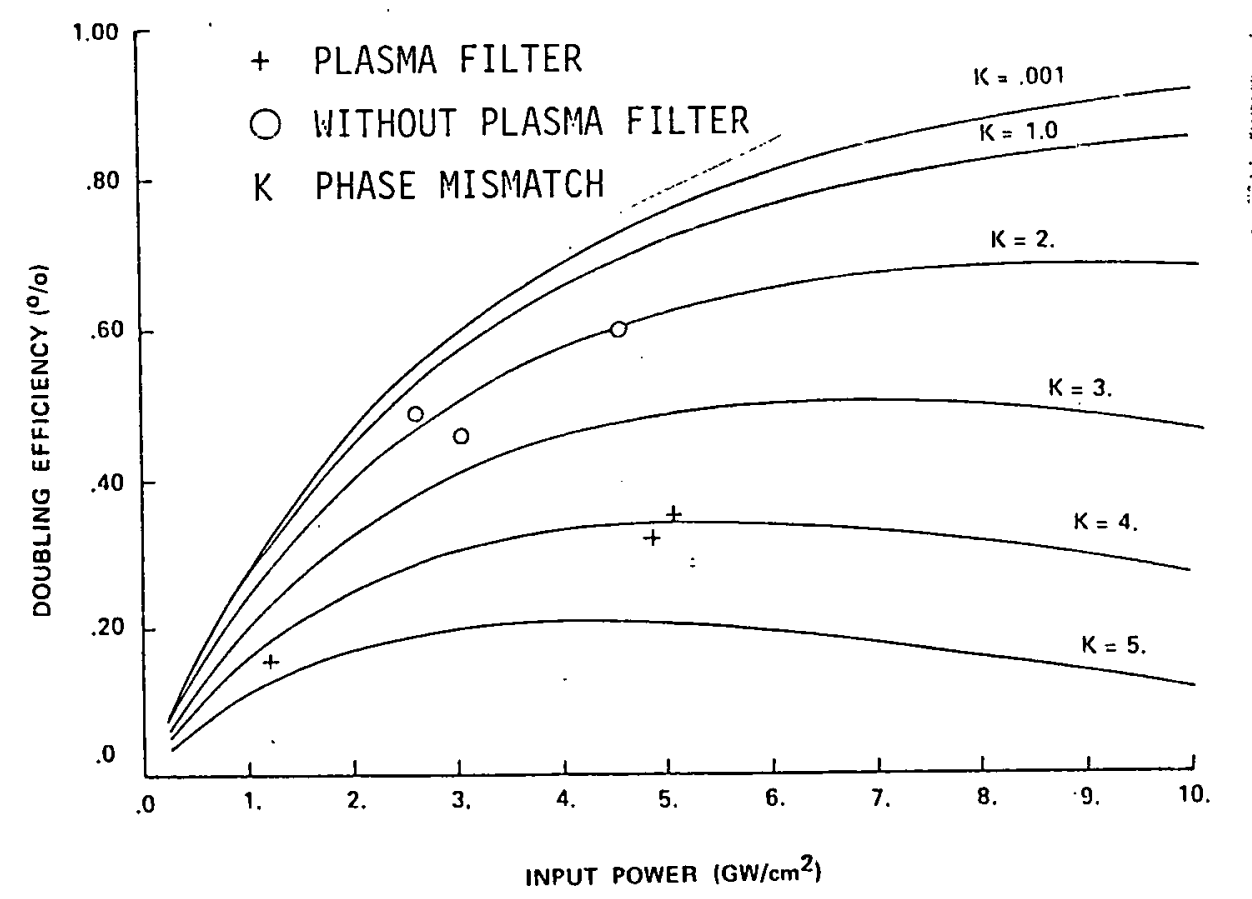

FIGURE 4.2-2 Doubling Efficiency For the $6 \mathrm{~mm}$ Crystal

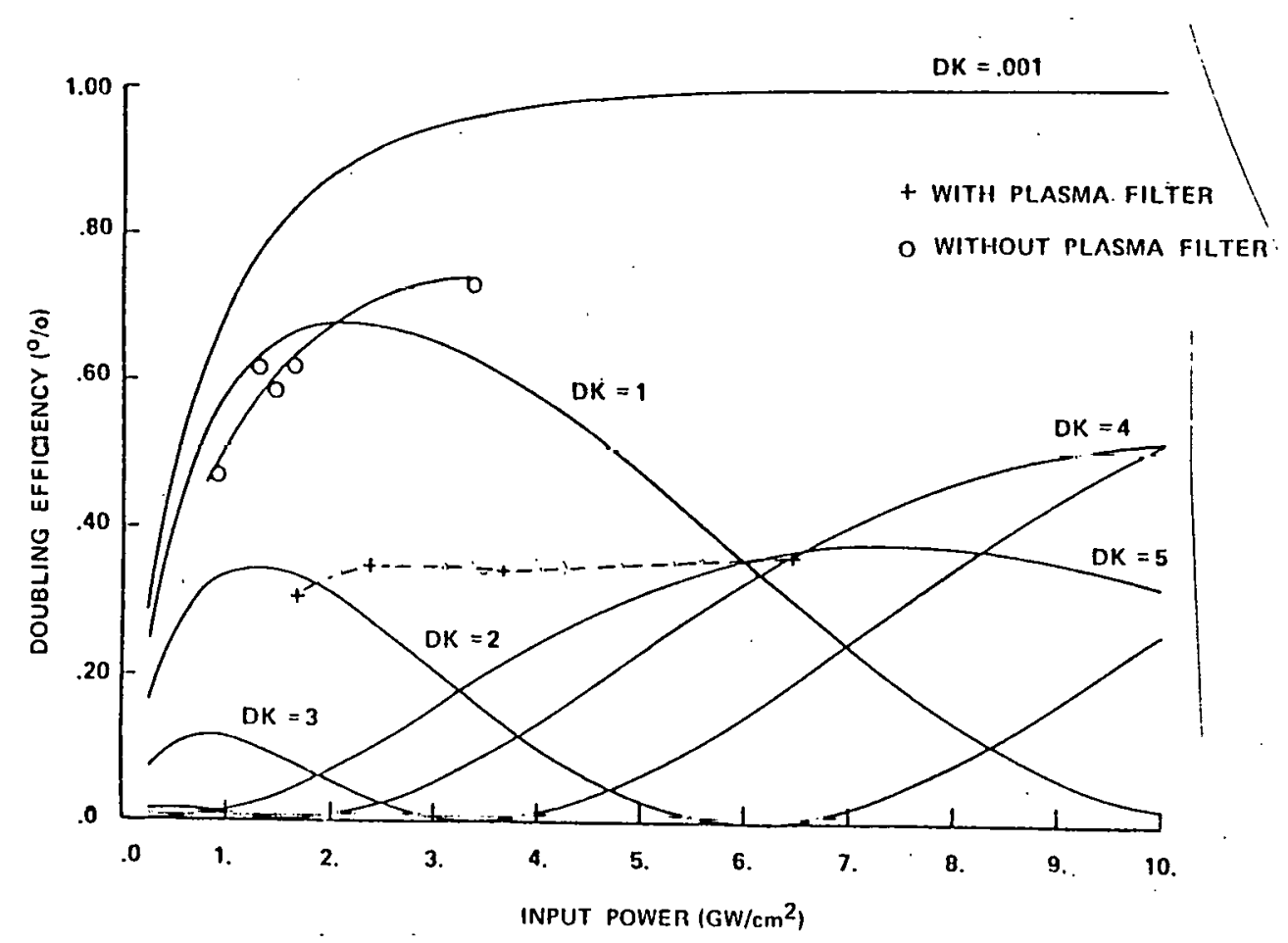

FIGURE 4.2-3 Doubling Efficiency for the $12.8 \mathrm{~mm}$ Crystal 
Note that the doubling efficiencies for both crystals without the plasma filter generalty increase with increasing input power density. Hence, higher doubling efficiencies may be obtained with high laser output power densities. These results are in contrast to the efficiency curve for tests on the previous $10 \mathrm{~cm}$ laser configuration where the efficiency decreased for input power densities greater than $1 \mathrm{GW} / \mathrm{cm}^{2}$ :

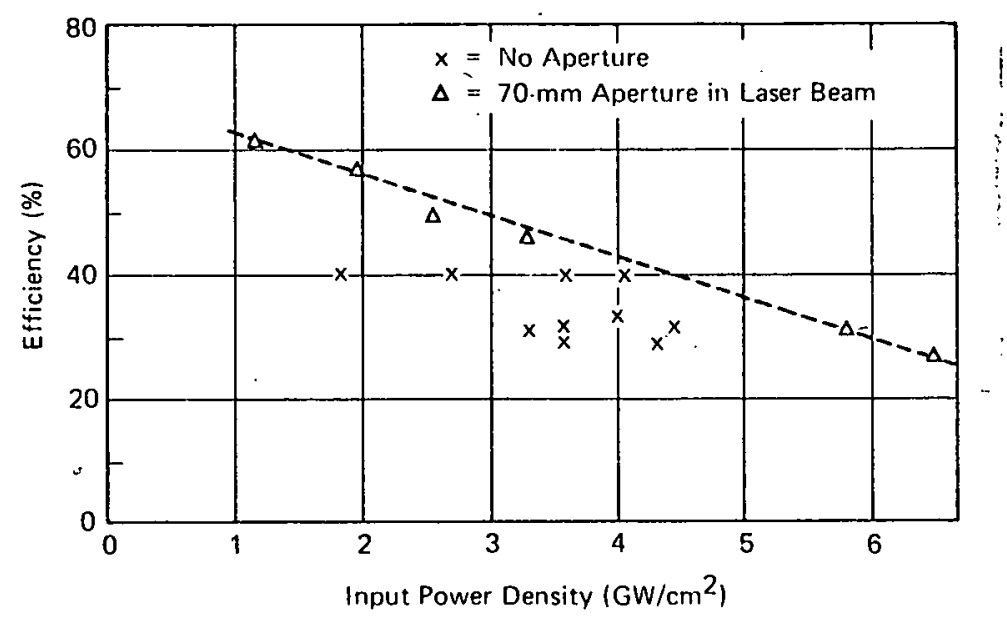

FIGURE 4.2-4 Doubling efficiency from a previous $10 \mathrm{~cm}$ laser system ulilizing a 1.5 $\mathrm{cm}$ thick by $10 \mathrm{~cm}$ diameter type II KDP crystal 
Figure 4.2-5 and 4.2-6 show the output green light power density versus the input $1.064 \mu \mathrm{m}$ power density. The solid line is the theoretical maximum efficiency (i.e. zero phase mismatch). These curves indicate more clearly that higher laser powers would still produce higher green light powers.

A total of 82 full-power green-light target interaction experiments were conducted in 1978 using the $12.8 \mathrm{~mm}$ thick KDP doubling crystals. The plasma spatial filter was used in 54 of these shots, the average green power on target was $90 \mathrm{GW}$, with the maximum power of $163 \mathrm{GW}$. For the other 28 green shots, the plasma spatial filter was by-passed, and the first two $10 \mathrm{~cm}$ disc amplifiers were removed to avoid severe damage; the average green power on target was $98 \mathrm{GW}$, with a maximum power on one shot of $162 \mathrm{GW}$. 


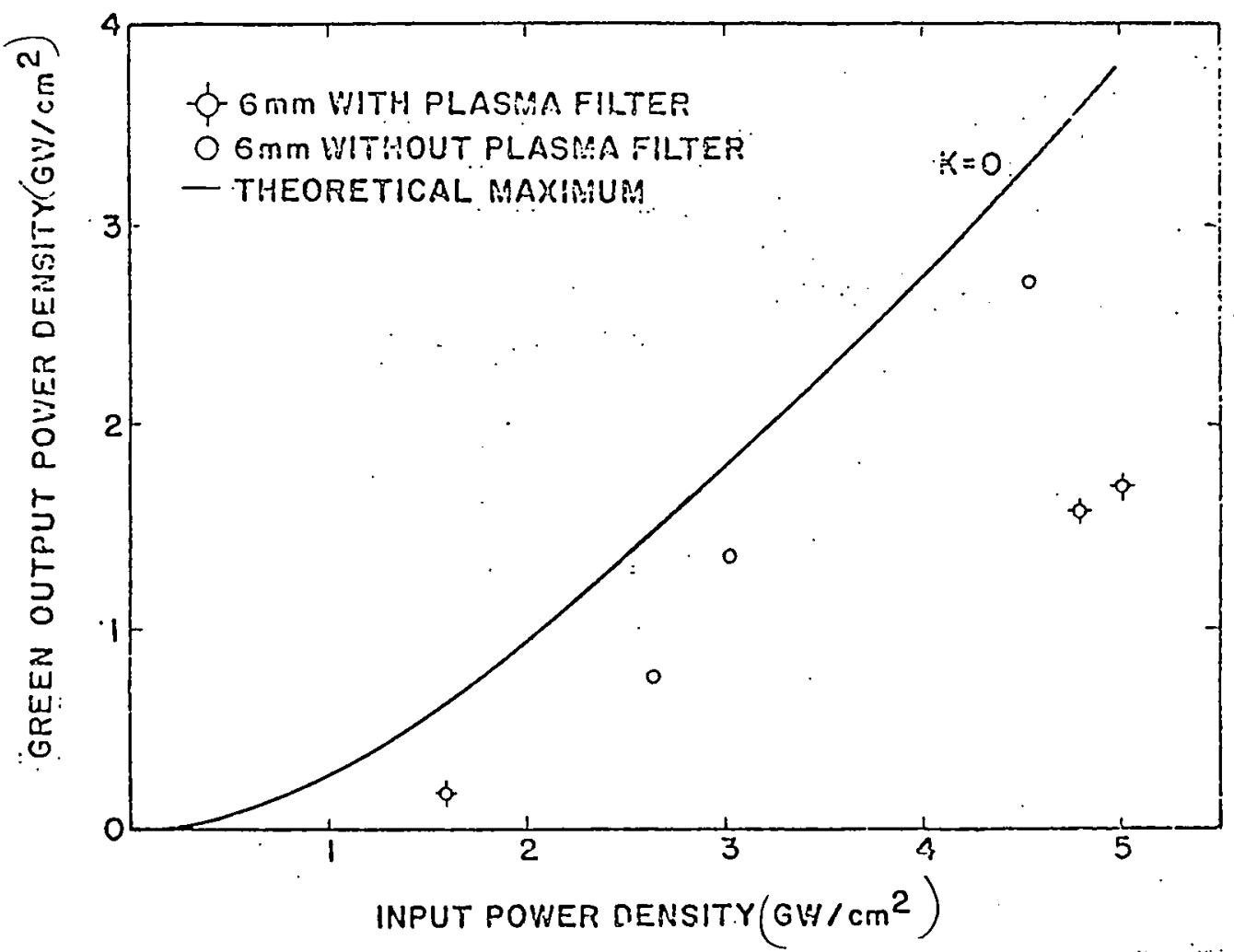

FIGURE 4.2-5 Green output power density for the $6 \mathrm{~mm}$ crystal

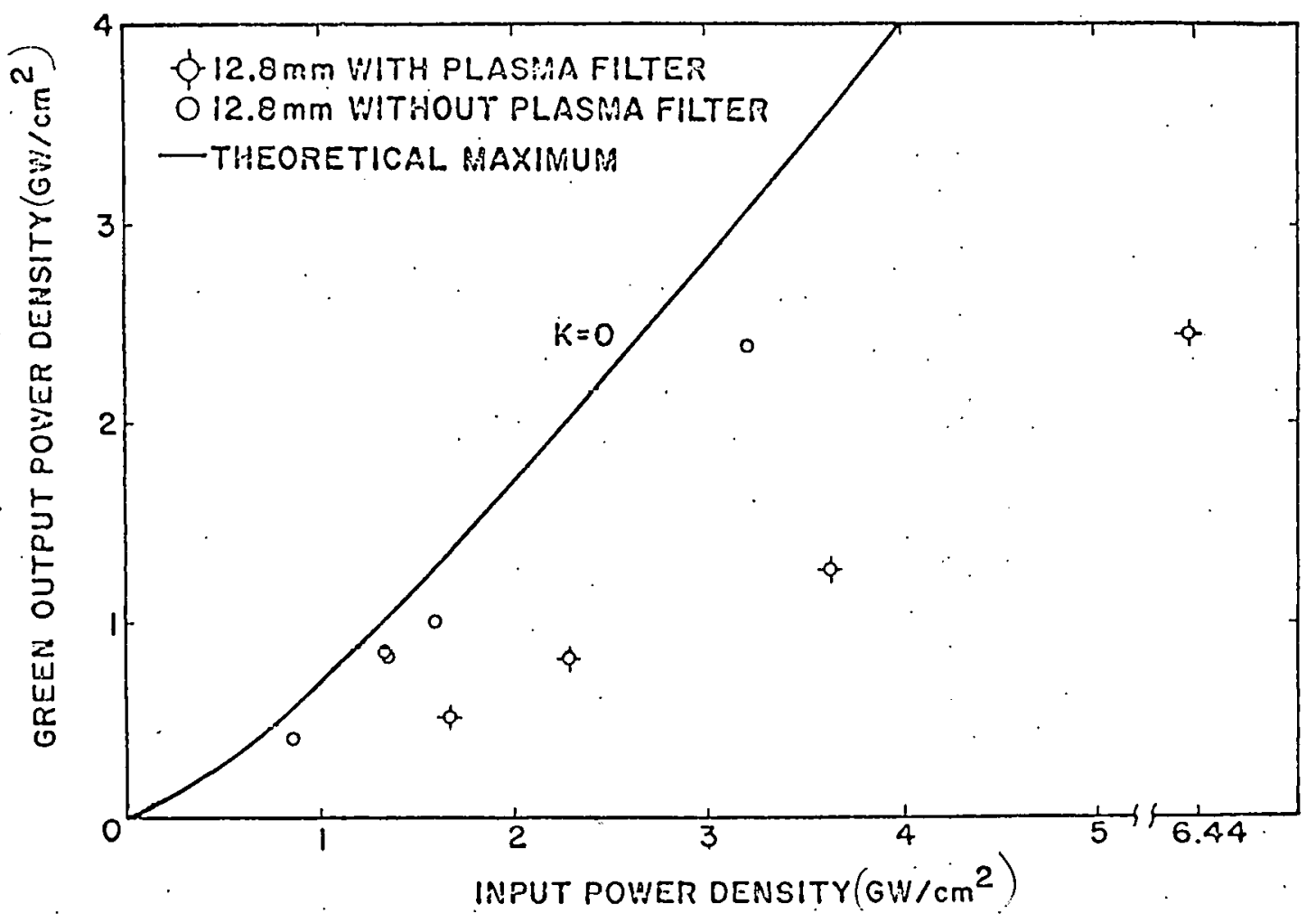

FIGURE 4.2-6 Green output puwer density for the $12.8 \mathrm{~mm}$ crystal 


\subsection{Advanced Laser Candidates}

The laser which will eventually drive a fusion power plant will have far different characteristics than current research lasers (see Table 4.3-1). In addition to high power and energy, the power plant driver must have high efficiency and high repetition rate. The $\mathrm{CO}_{2}$ laser is the primary existing candidate which might be scaled to reactor levels, but its wavelength may be too long, and it faces serious engineering difficulties in achieving reliable, cost-effective operation at 1 to 10 pulses per second. Indeed, the pulsed power supply for high repetition rate operation may be the engineering limitation for all currently proposed advanced laser systems. In view of these difficulties, we believe entirely new laser concepts should be explored now, to maximize the probability of having a satisfactory laser driver available when needed.

KMS Fusion's committment to develop the appropriate technology for laser fusion has therefore included evaluations of new laser sources that might provide a back-up or perhaps even a replacement for the carbon dioxide laser. During the past year two outstanding driver candidates have been identified. Both of these laser's, the chemically pumped iodine laser (CPIL) and the free electron laser (FEL), were developed at other laboratories for non-fusion applications. In both cases, KMS scientists recognized the potential of these lasers for fusion, and have bequn analyses to judge their suitability. Some of the important results of these exploratory studies are given below. 
TABLE $4.3-1$

\section{ICF LASER DRIVER PARAMETERS}

1. Efficiency

2. Total Energy on Target

3. Pulse Length

4. Pulse Repetition Rate

5. Pulse Shape

6. Wavelength
$>3 \%$

$1 \mathrm{MJ}$

10 nsec

1 to $10 \mathrm{~Hz}$

Variable

$\lambda=0.2$ to $3.0 \mu \mathrm{m}$ 


\subsubsection{Chemically Pumped Iodine Laser (CPIL)}

G. E. Busch

Early in 1978 a chemical reactor was developed by the Air Force Weapons Lab (AFWL) which produced a high yield of oxygen molecules in an electronically excited state. (5) This permitted them to demonstrate the first chemically pumped laser operating on an electronic transition. KMS then began an evaluation of the laser to determine if it could be converted from the $\mathrm{CW}$ mode of operation used by AFWL to the pulsed, high power mode needed for laser fusion. The results of these preliminary studies are very encouraging.

From a power plant laser driver viewpoint, the CPIL has great appeal since it completely eliminates the pulsed power supply. All energy is stored in electronically excited oxygen molecules which have very long lifetimes. Prior to entering the laser amplifier, the excited oxygen is mixed with and excites iodine, and the laser operates on the excited iodine $1.314 \mu \mathrm{m}$.1 ine, which can be frequency converted to the visible if necessary. The total system efficiency of a CPIL power plant driver could be as high as $50 \%$ while 10 to $20 \%$ efficiencies appear readily achievable. Since a11 other laser driver candidates have estimated efficiencies of 1 to $10 \%$, the CPIL combines the highest potential efficiency with the only known total solution to the pulsed power supply problem.

The transfer of electronic excitation from molecular oxygen to atomic iodine is depicted by the reaction:

$$
0_{2} *\left({ }^{\prime} \Delta_{g}\right)+I\left({ }^{2} P_{3 / 2}\right) \rightarrow O_{2}\left({ }^{3} \Sigma_{g}\right)+I *\left({ }^{2} P_{1 / 2}\right)
$$

The $0_{2} *\left({ }^{\prime} \Delta \mathrm{g}\right)$ on the left side of equation 1 is currently produced by the reaction of chlorine with alkaline hydrogen peroxide: 


$$
2 \mathrm{NaOH}+\mathrm{H}_{2} \mathrm{O}_{2}+\mathrm{Cl}_{2} \rightarrow 2 \mathrm{NaCl}+2 \mathrm{H}_{2} \mathrm{O}+\mathrm{O}_{2} *\left({ }^{\prime} \Delta \mathrm{g}\right)+\left[\mathrm{O}_{2}\left({ }^{3} \Sigma \mathrm{g}\right)\right] .
$$

The best yield of electronically excited oxygen (i.e., $0_{2}{ }^{*}\left({ }^{\prime} \Delta g\right)$ ) obtained to date is $40 \%$, with the remainder of the oxygen being produced in the ground state (i.e. $\mathrm{O}_{2}\left({ }^{3} \Sigma_{g}\right)$ ). Work is under way at AFWL to increase the reaction efficiency to at least $80 \%$ excited oxygen.

\section{CPIL Reaction Rate Computations}

At KMS we have assumed that the performance of the excited oxygen generator will continue to improve, and have directed our attention toward identifying and examining any additional parameters which would influence the efficiency and scalability of the laser when operated as a fusion driver. Two interrelated questions which have been evaluated in some detail are the energy storage density and extraction efficiencies which can be achieved.

Whereas high $\mathrm{CW}$ power from a chemical laser requires large mass flow rates, high pulse power demands high energy storage density, since flow is insignificant on the time scale of energy extraction. In principle, the energy storage density could be selected at will, simply by raising the pressures of $0_{2} *\left({ }^{\prime} \Delta_{g}\right)$ and iodine. Since 1 Torr of $0_{2} *\left(\Delta_{g}\right)$ contains $5.3 \mathrm{~J} / \ell$, an amplifier operating at atmospheric pressure could conceivably store $4 \mathrm{~kJ} / l$, i.e. the amplifier would only need to be $5 \mathrm{~cm}$ long to reach the $20 \mathrm{~J} / \mathrm{cm}^{2}$ fluences corresponding to damage levels extrapolated for optical materials. In practice, however, parasitic oscillation in the amplifier would prevent such short amplifiers from being useful. But even more importantly, quenching processes in the laser medium increase rapidly as pressure is 
increased, such that the proper balance must be selected to achieve maximum energy storage while maintaining an acceptable quenching rate.

Fortunately, computations can be used to guide the optimization of parameters. Most of the kinetic processes likely to occur within the laser medium have been studied and values for rate constants (or at least estimates for them) exist in the literature. A computer model of an amplifier has been developed which includes most of the energy transfer and chemical reactions fast enough to be important (see Table 4.3-2). The rate equations for these reactions are numerically integrated to give the concentrations of the known important species as a function of time. Uniform mixing of reactants is assumed at $t=0$.

Some results of computer computations for an amplifier with reactant partial pressures in the range of 1 Torr at room temperature are shown in Figures 4.3-1 and 4.3-2. In addition to the partial pressures of the six species: $\mathrm{O}_{2}, \mathrm{O}_{2}{ }^{*} \mathrm{O}_{2}{ }^{*}, \mathrm{I}_{2}, \mathrm{I}$ and $\mathrm{I}^{*}$, the extractable energy (expressed as the partial pressure of excited iodine atoms that could be made to lase) is plotted vs time. For these conditions the reactants and products rapidly establish a steady state which then prevails for milliseconds. After that time the quenching reactions mentioned previously begin to deplete the energy storage within the amplifying medium. Increases in concentrations of reactants tend to move the steady state region toward shorter times while decreases extend the energy storage times. The length of time for which energy storage remains high is an important parameter, since it determines the flow speed and mixing times required for the reactant gases. Sufficient time must be available to fill the amplifying volumie and obtain uniform mixing before the energy can be extracted. Otherwise 
TABLE 4.3-2

REACTIONS AND RATE CONSTANTS INCLUDED

IN COMPUTER MODEL
REACTION
1. $\mathrm{I}+\mathrm{O}_{2}^{*}\left({ }^{\mathrm{I}} \Delta\right) \longrightarrow \mathrm{I} *+\mathrm{O}_{2}\left({ }^{3} \Sigma\right)$
RATE CONSTANT $^{\mathrm{a}}$ TORR $^{-\mathrm{N}} \mu \mathrm{SEC}^{-1}$
2. $\mathrm{I}^{*}+\mathrm{O}_{2}^{*}\left({ }^{\top} \Delta\right) \longrightarrow \mathrm{I}+\mathrm{O}_{2}{ }^{* \star}\left({ }^{\mathrm{l}} \Sigma\right)$
2.7
3. $0_{2}^{\star}\left({ }^{1} \Delta\right)+M \longrightarrow 0_{2}^{\star *}\left({ }^{3} \Sigma\right)+M$
$9.4 \times 10^{-4}$
4. $0_{2}^{\star \star}\left({ }^{1} \Sigma\right)+M \longrightarrow 0_{2}^{*}\left({ }^{1} \Delta\right)+M$
$3.5 \times 10^{-10}$
5. $I^{*}+\mathrm{O}_{2}\left({ }^{3} \Sigma\right) \longrightarrow I+0_{2}^{*}\left({ }^{1} \Delta\right)$
$5.3 \times 10^{-7}$
6. $\mathrm{I}^{*}+\mathrm{O}_{2}\left({ }^{3} \Sigma\right) \longrightarrow \mathrm{I}+\mathrm{O}_{2}\left({ }^{3} \Sigma\right)$
$9.4 \times 10^{-1}$
7. $\mathrm{I}+\mathrm{O}_{2} *\left({ }^{\mathrm{I}} \Delta\right) \longrightarrow \mathrm{I}+\mathrm{O}_{2}\left({ }^{3} \Sigma\right)$
$2.0 \times 10^{-3}$
8. . $\mathrm{I}^{*}+\mathrm{M} \longrightarrow \mathrm{I}+\mathrm{M}$
$4.7 \times 10^{-3}$
9. $\mathrm{O}_{2} *\left({ }^{1} \Delta\right)+\mathrm{O}_{2}^{*}\left({ }^{1} \Delta\right) \longrightarrow \mathrm{O}_{2} * *\left({ }^{1} \Sigma\right)+\mathrm{O}_{2}\left({ }^{3} \Sigma\right)$
$5.9 \times 10^{-8}$
10. $0_{2} * *\left({ }^{1} \Sigma\right)+\mathrm{I}_{2}\left({ }^{1} \Sigma\right) \rightarrow \mathrm{O}_{2}\left({ }^{3} \Sigma\right)+2 \mathrm{I}$
$7.0 \times 10^{-7}$
11. $\mathrm{I}^{*}+\mathrm{I}_{2} \longrightarrow \mathrm{I}+\mathrm{I}_{2}$
12. $\mathrm{O}_{2}{ }^{*}\left({ }^{1} \Delta\right)+$ wall $\longrightarrow \mathrm{O}_{2}\left({ }^{3} \Sigma\right)+$ wall
8.2
13. $\mathrm{O}_{2}^{* *}\left({ }^{\top} \Sigma\right)+$ wall $\rightarrow \dot{O}_{2}\left({ }^{\top} \Delta\right)+$ wall
$1.5 \times 10^{-1}$
14. I + wall $\longrightarrow 1 / 2 \mathrm{I}_{2}+$ wall
$2.0 \times 10^{-5} \mathrm{~b}$
15. $\mathrm{I}^{*}+$ wall $\longrightarrow \mathrm{I} / 2 \mathrm{I}_{2}+$ wall
$1.0 \times 10^{-2} \mathrm{~b}$
16. I* + wall $\longrightarrow \mathrm{I}+$ wall
$1.0 \times 10^{-3} \mathrm{~b}$
17. $\mathrm{I}^{*} \longrightarrow \mathrm{I}+\mathrm{hv}$
$1.0 \times 10^{-3} \mathrm{~b}$
18. $\mathrm{O}_{2}^{* *}\left({ }^{1} \Sigma\right) \longrightarrow \mathrm{O}_{2}\left({ }^{3} \Sigma\right)+\mathrm{h \nu}$
1.0
$6.0 \times 10^{-6}$
19. $2 \mathrm{I}+\mathrm{I}_{2} \longrightarrow 2 \mathrm{I}_{2}$
$7.7 \times 10^{-8}$
20. $2 \mathrm{I}+\mathrm{O}_{2} \longrightarrow \mathrm{I}_{2}+\mathrm{O}_{2}$
$4.8 \times 10^{-3}$
21. $2 \mathrm{I}+\mathrm{M} \longrightarrow \mathrm{I}_{2}+\mathrm{M}$
$5.2 \times 10^{-5}$
$3.5 \times 10^{-6}$

a. $\quad \mathrm{N}=0,1,2$ for first, second, and third order reactions, respectively, at $300^{\circ} \mathrm{K}$

b. Rate constants for wall reactions are expressed as probability/collision. 


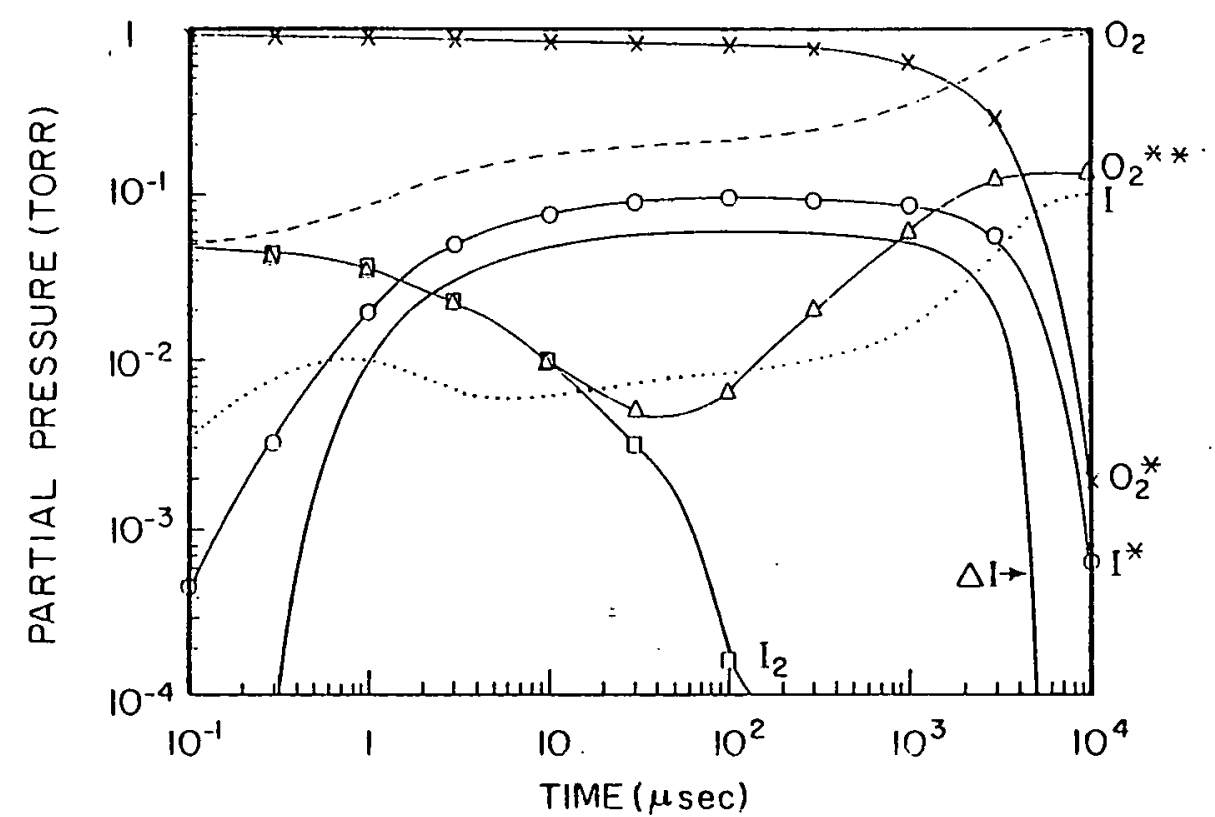

FIGURE 4.3-1 Partial pressures of chemical species in a CPIL versus time after complete mixing. $\Delta I=\left(21^{*}-I\right) / 3$ is the partial pressure of excited iodine atoms which can be made to lase, and is a measure of the extractable energy density. Initial conditions: 0.9 torr $\mathrm{O}_{2} * \ldots 05$ torr $\mathrm{O}_{2}, \mathrm{O}_{2}$ ** and $\mathrm{I}_{2} ;$ no $\mathrm{I}$ or $\mathrm{I}^{\star}$.

CHEMICAL KINETICS IN CPIL AMPLIFIER

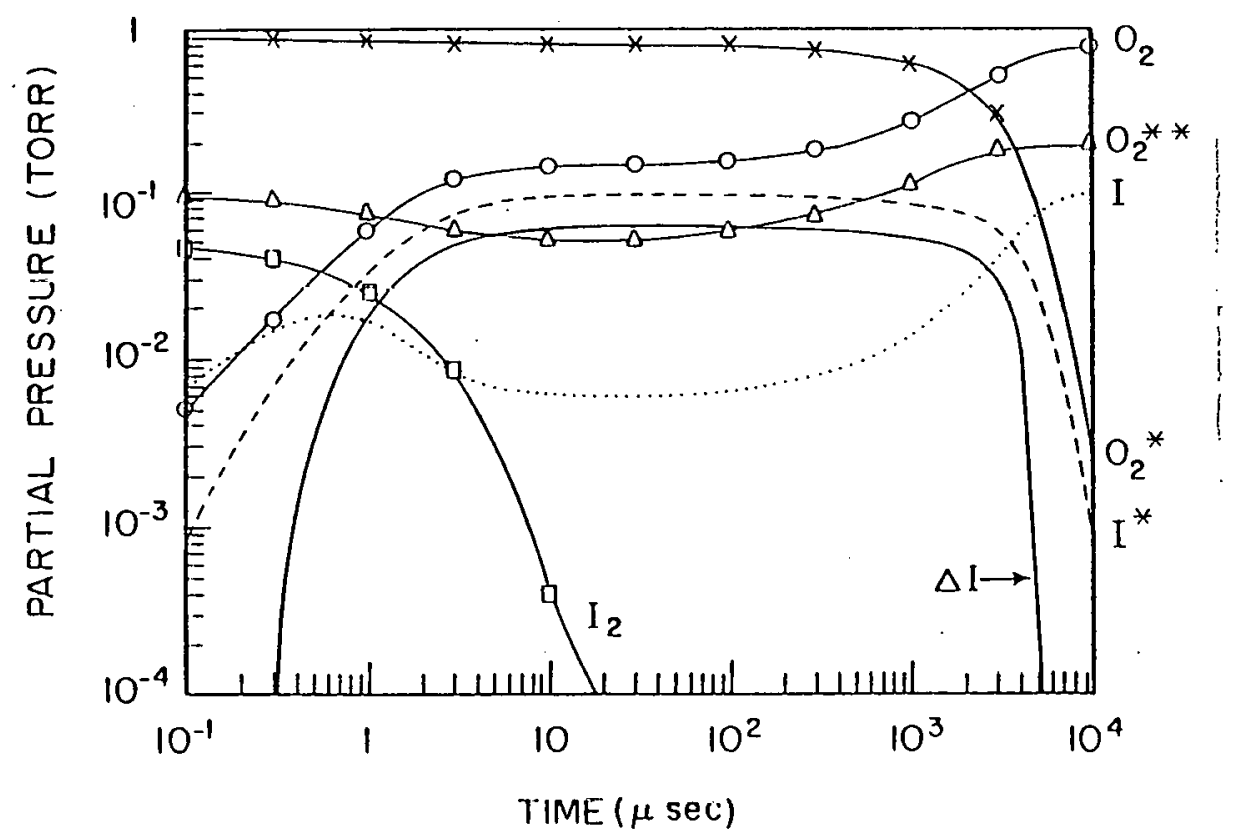

FIGIIRF 4.3-2 Same as Figure 4.3-1, but with initial conditions: 0.9 torr $0_{2}^{*}, 0.1$ torr $\mathrm{U}_{2}^{* *} ; .05$ torr $\mathrm{I}_{2}$; and no $\mathrm{O}_{2},{ }^{*} \mathrm{I}$ or $\mathrm{I}^{*}$. 
laser beam quality will be degraded due to refractive index and gain nonuniformities. Computer simulations currently aid in the selection of energy storage densities, energy storage times, and amplifier lengths appropriate for CPIL fusion drivers.

\section{CPIL Steady State Approach}

The existence of steady state conditions within the amplifying medium permits substantial simplification in calculations describing the characteristics of an amplifier under various conditions. Steady state approximation can be applied without solving the full kinetics on the computer, saving computation time. This technique has been utilized to obtain the maximum amplifier energy extraction efficiencies as a function of initial concentrations of reactants. The latter results have been used to predict optimum reactant concentration ratios and to predict amplifier performance in multiple pass applications.

We want to model the case when the output of an excited oxygen generator, with known partial pressures of $\mathrm{O}_{2}{ }^{*}$ and $\mathrm{O}_{2}$ (and sufficient $0_{2}$ ** to dissociate all $I_{2}$ via reaction (10) in Table 4.3-2) is mixed with a particular amount of $\mathrm{I}_{2}$, to give a steady state mixture of the species: $0_{2}, 0_{2}^{*}, I$ and $I^{*}$. The steady state condition has been soived for the concentration of $\mathrm{I}^{*}$ in terms of the initial concentrations of $\mathrm{O}_{2}, \mathrm{O}_{2}{ }^{*}$ and $\mathrm{I}_{2}$, giving the quadratic expression:

$$
\begin{aligned}
& I^{*}=\left(B-\sqrt{B^{2}-4 A C}\right) / 2 A \\
& A=K-1 \\
& B=K\left(O_{2} *\right)+O_{2}+2 I_{2} \\
& C=2 K\left(I_{2}\right)\left(O_{2}^{*}-2 I_{2}\right)
\end{aligned}
$$


in which $K$ is the quasi equilibrium constant for the steady state condition, given by the ratio of the forward and reverse reactions (1) and (5) in Table 4.3-2, i.e. $k=k_{7} / k_{5}$. It should be emphasized that $I^{*}$ is the steady state partial pressure, while all other partial pressures in Equation (2) are those of initial reactants.

The expression in (2) for $I^{*}$ is useful for computing the gain and the maximum energy that can be extracted from an amplifier medium with a short laser pulse. The gain $G$ is related to $I$ and $I^{*}$ by the relation

$$
\ln G=\sigma L\left(I^{*}-\frac{I}{2}\right)
$$

where $\sigma$ is the transition cross-section and $L$ is the amplifier length. The factor 2 arises because $I^{*}$ has half the level degeneracy of I. Gain saturation occurs, and thus energy extraction ceases, when $2 I^{*}=I$. Using this as the final condition for the amplifying medium, it is easy to show that the decrease in the excited state population, $\Delta \mathrm{I}$, brought about by a laser pulse producing total gain saturation is $\Delta I=\left(2 I^{*}-I\right) / 3$, or in terms of $I^{*}$ and the original $I_{2}$ added, $\Delta I=I^{*}-2 I_{2} / 3$. Equation (2) can then be substituted for $I^{*}$ to obtain $\Delta$ I completely in terms of the initial reactant partial pressures $\mathrm{O}_{2}{ }^{*}, \mathrm{O}_{2}$, and $\mathrm{I}_{2}$. Finally, if we divide $\Delta \mathrm{I}$ by the initial $0_{2}$ partial pressure, the net single-pass extraction efficiency, $\varepsilon$, for a totally saturating pulse is:

$$
\varepsilon=\frac{\Delta I}{0_{2}^{*}}=I_{r}^{*}-2 I_{2 r / 3}
$$

where ${ }^{\mathrm{I}^{*}}{ }_{r}$ and $\mathrm{I}_{2 r}$ are the normalized variables $\mathrm{I}_{1} / \mathrm{O}_{2}{ }^{*}$ and $\mathrm{I}_{2} / \mathrm{O}_{2}{ }^{*}$. For a given efficiency of production of $\mathrm{O}_{2}{ }^{*}$ (and thus a given $\mathrm{O}_{2} / \mathrm{O}_{2}{ }^{*}=\mathrm{O}_{2} \mathrm{r}$ ratio) there will be an optimum $I_{2 r}$ which will maximize the amplifier 
extraction efficiency. This value of $\mathrm{I}_{2 r}$ can be obtained from (3) by differentiating with respect to $I_{2 r}$; however, it is more informative just to plot (3) vs $I_{2 r}$ for chemical generator efficiencies of interest, as shown in Figure $4.3-3$

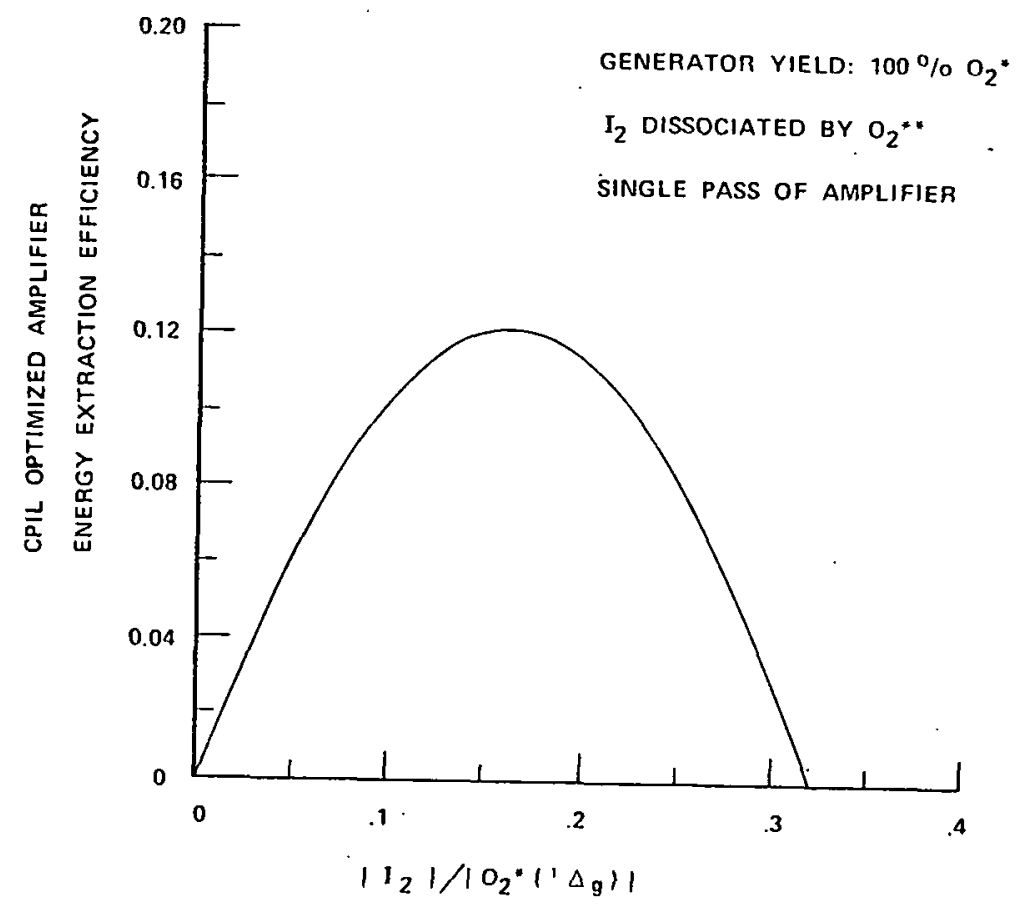

FIGURE 4.3-3 CPIL single -pass fractional energy extraction efficiency.

\section{Multiple Passing of CPIL Amplifiers}

The above steady state treatment can be extended to multiple passes of an amplifier. Consider that passage of the pulse through the laser medium removes a population of excited atoms $\Delta I$ given by (2). Immediately after the pulse the system will begin to relax toward a new steady state, the steady state reached by mixing the same reactants together, but with different initial concentrations: $0_{2}{ }^{\prime}=O_{2}{ }^{*}-\Delta I$ and $\mathrm{O}_{2}^{\prime}=\mathrm{O}_{2}+\Delta \mathrm{I}$. Thus, if the light pulse is passed back through the amplifier after the system has relaxed to the new steady state, then 
energy can again be extracted; the extracted energy can be computed by substituting $\mathrm{O}_{2}{ }^{\prime \prime}$ and $\mathrm{O}_{2}$ ' into (2) to get a new $\Delta I^{\prime}$. This procedure can be iterated on a computer. Figure 4.3-4 shows computed extraction efficiencies vs number of passes of an amplifier for several initial iodine concentrations for the case in which the chemical generator produces $100 \% 0_{2} *$. Figure $4.3-5$ shows the results with $50 \% 0_{2}$ * generation efficiency. It is seen that the curves cross at some intermediate number of passes, such that higher $\mathrm{I}_{2}$ concentrations give the best extraction efficiencies for small number of passes. However, the highest extraction efficiencies are obtained at low $\mathrm{I}_{2}$ concentrations and large number of passes. The advantage of operating an amplifier in a $\mathrm{CW}$ to quasi-CW mode is clearly evident. Since a fusion driver

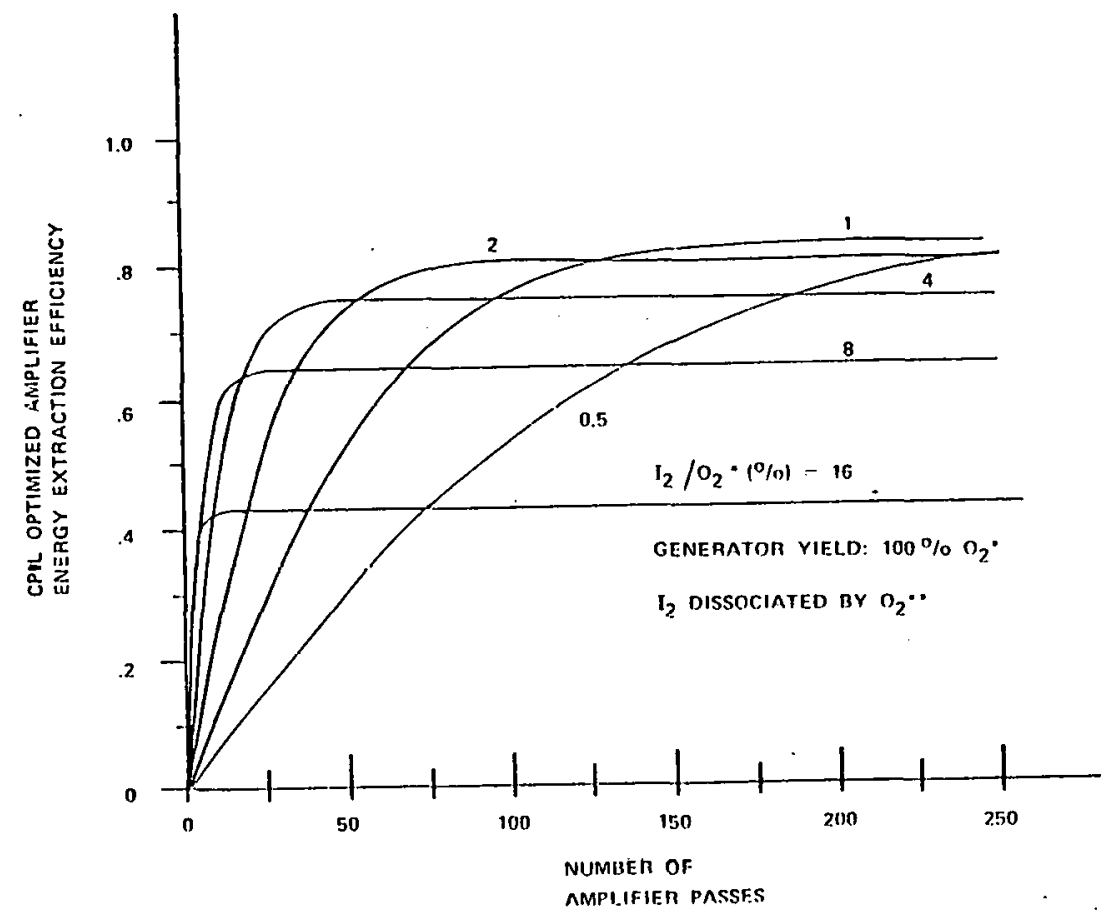

FIGURE 4.3-4 CPIL fractional energy extraction efficiency versus number of passes for different iodine concentrations 


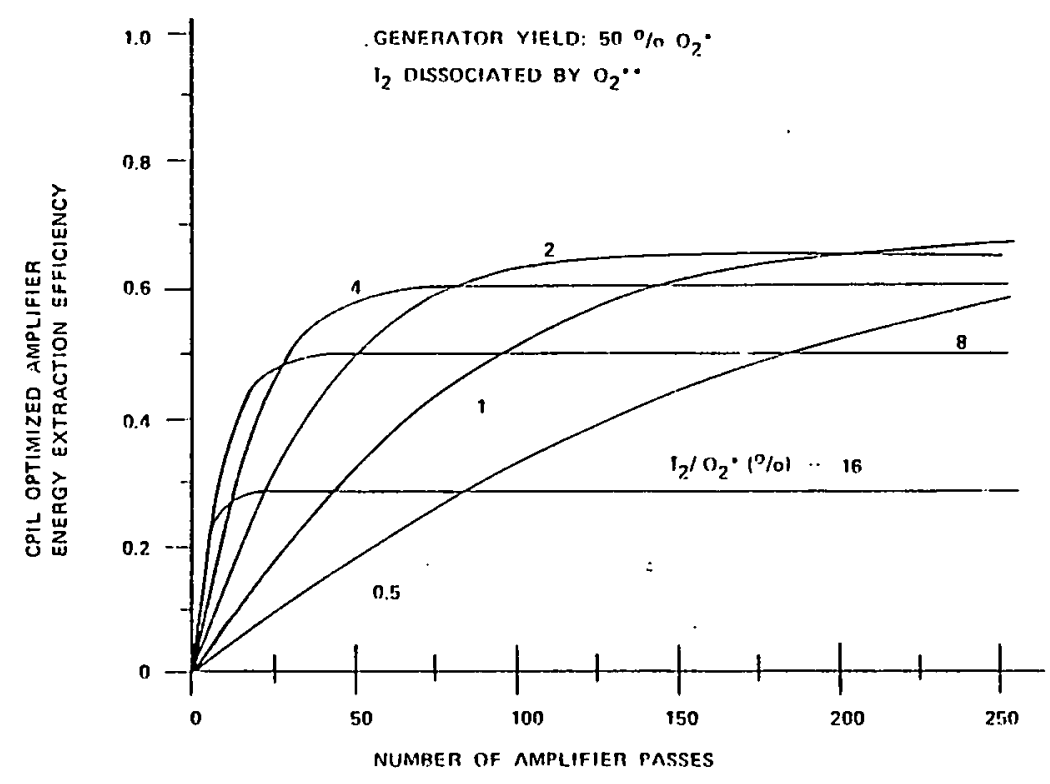

FIGURE 4.3-5 CPIL fractional energy extraction efficiency assuming $50 \%$ excited oxygen generation efficiency

amplifier would probably operate with a small (fixed) number of passes it is also instructive to replot the data of Figures $4.3-4$ and 4.3-5 as shown in Figure 4.3-6 and 4.3-7. so that optimum concentrations of $I_{2}$ may be selected.

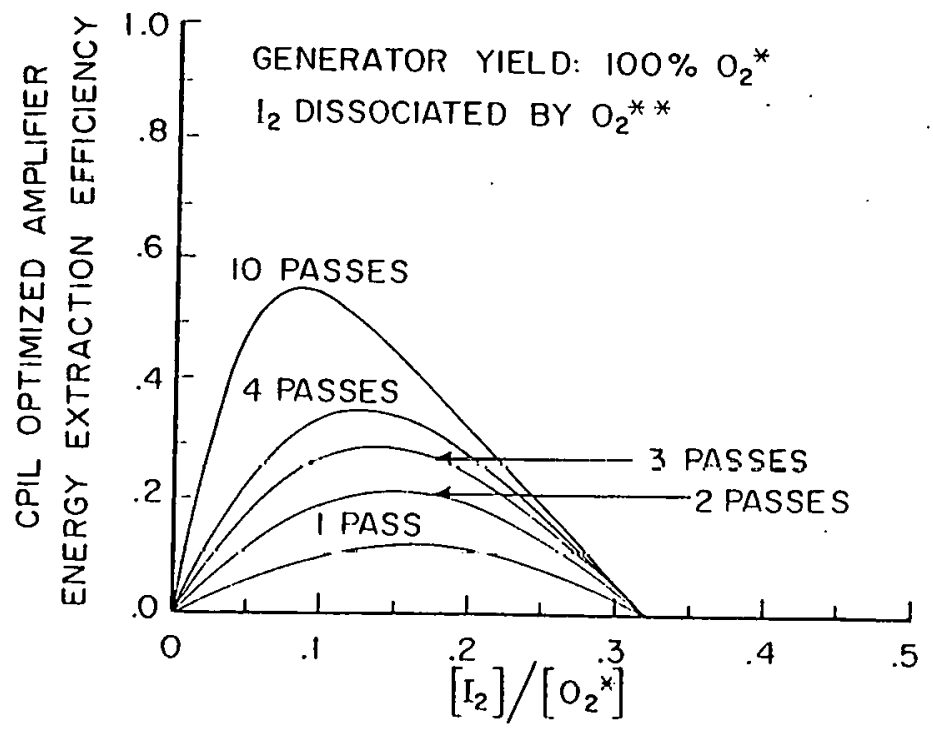

FIGURE 4.3-6 CPIL fractional energy data of Figure 4.3-4 replotted as a function of iodine concentration 


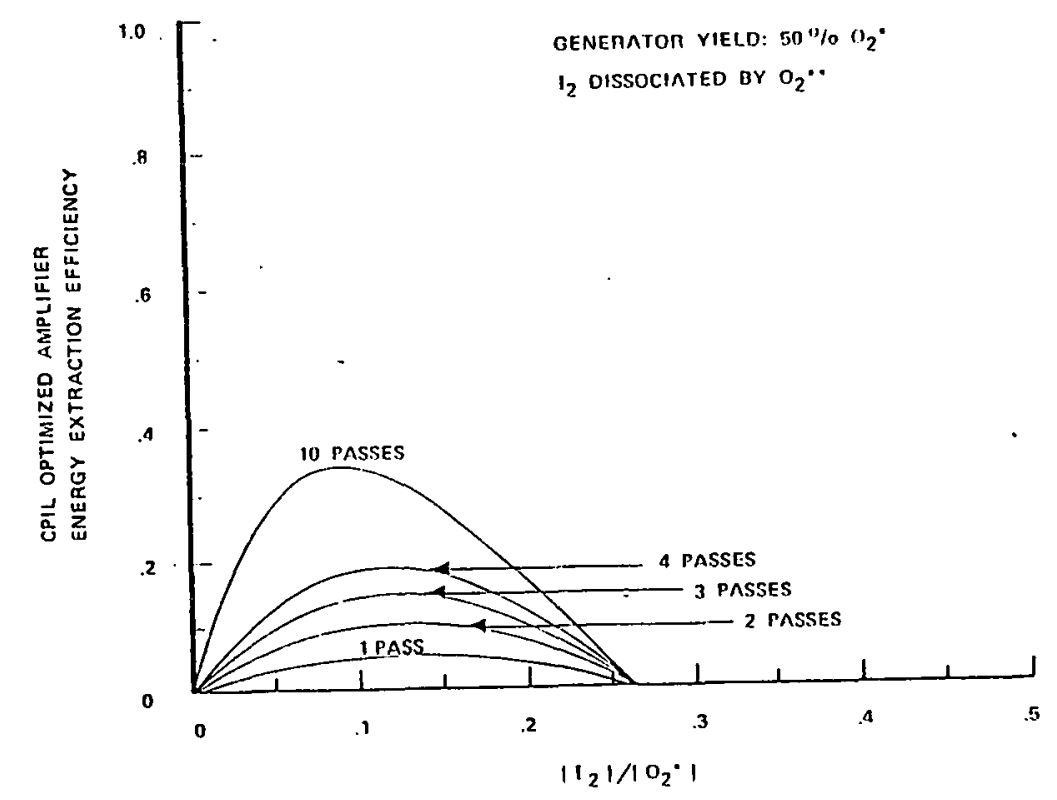

FIGURE 4.3-7 CPIL fractional energy extraction efficiency data of Figure 4.3-5 replotted as a function of iodine concentration

\section{Thermal Iodine Dissociation}

Much energy is required to dissociate molecular iodine, thereby lowering efficiency. Reaction (10) in Table 4.3-2 provides this dissociation, but the $0_{2}{ }^{\star *}$ is produced at a cost of two quanta of electronic excitation vid reaclivil (9). Since the electronic excitation is a high-grade form of energy, produced at a wall plug efficiency of about $15 \%$ and a net thermal energy efficiency of less than $6 \%$ it is worth exploring more efficient methods of dissociating $I_{2}$. For example, at pressures less than an atmosphere, iodine is almost totally dissociated at temperatures in the range of $1000^{\circ} \mathrm{C}$. Thus it may be possible to use direct thermal output from a fusion reactor to produce I atoms at high efficiency prior to mixing with excited oxygen. 
The impressive improvements in amplifier energy extraction efficiency when electronic excitation is not consumed for $I_{2}$ dissociation is shown in Figure 4.3-8. In making comparisons with Figure 4.3-6, it should be noted that the horizontal axis in that figure is a function of partial pressure of $\mathrm{I}_{2}$ added, while the partial pressure of added I atoms is plotted in Figure 4.3-8.

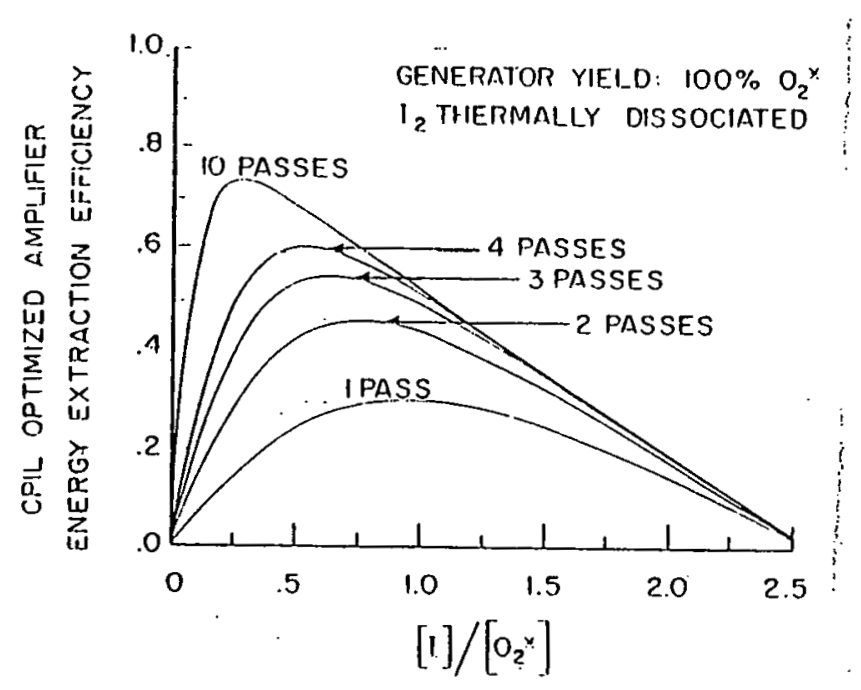

FIGURE 4.3-8 CPIL fractional energy efficiency assuming thermal dissociation of iodine. molecules

\section{Low Temperature CPIL}

It is currently anticipated that lowering the temperature of the reaction mixture in a CPIL amplifier would produce substantial improvements in performance. The basis for this extrapolation is that reaction rates generally decrease as temperatures are reduced. The Arrhenius relationship for rate constants is often a useful description:

$$
k=A e^{-\Delta E / K T}
$$


where $\kappa$ is Boltzmanns constant, $T$ is the absolute temperature, and $\Delta E$, called the activation energy, is interpreted as the minimum energy which molecules must have to react. The factor $A$ is only weakly temperature dependent and for most reactions is approximately equivalent to a collision cross section or rate constant. Typical values for $A$ are in the range of 1 to $10 \mathrm{Torr}^{-1} \mathrm{sec}^{-1}$ for second order rate constants. Since most of the rate constants for deactivation reactions such as (2), (3) and (6) through (11) in Table 4.3-2 are at least $10^{3}$ times slower than the collision rate at room temperature, this implies the exponential factor in Equation (4) is $\leq 10^{-3}$ for these rate constants. Thus if the temperature could be cut in half to $\sim 150^{\circ} \mathrm{K}$, we would expect this factor to be squared to give a reaction rate which is only $\leq 10^{-6}$ of the collision rate. Similarly, if the temperature were further reduced to $\sim 100^{\circ} \mathrm{K}$, this factor would become $\leqslant 10^{-9}$. Thus substantial reductions in quenching rates should be achievable at reduced temperatures. Significant cooling of the gas mixture is expected to occur during adiabatic expansion through mixing nozzles, thus lower temperatures may occur automatically with a CPIL.

In addition to the benefits of reduced quenching rates at lower temperatures, a further improvement in amplifier performance is expected due to a shifting of the steady state condition to favor energy storage in excited iodine atoms. Because the $I^{*}\left({ }^{2} \mathrm{P}_{1 / 2}\right)$ state is $279 \mathrm{~cm}^{-1}$ lower in energy than $0^{*}{ }_{2}\left({ }^{\prime} \Delta g\right), K$ in Equation (2) should change from $\sim 3$ at $300^{\circ} \mathrm{K}$ to $\sim 10$ at $150^{\circ} \mathrm{K}$ and $\sim 40$ at $100^{\circ} \mathrm{K}$. The improvement in energy extraction efficiency of an amplifier in which $K=30$ (i.e. $-110^{\circ} \mathrm{K}$ ) is shown in Figure 4.3-9 (c.f. Figure 4.3-6). Single pass extraction efficiencies as high as $50 \%$ may thus be realizeable at low temperatures. 


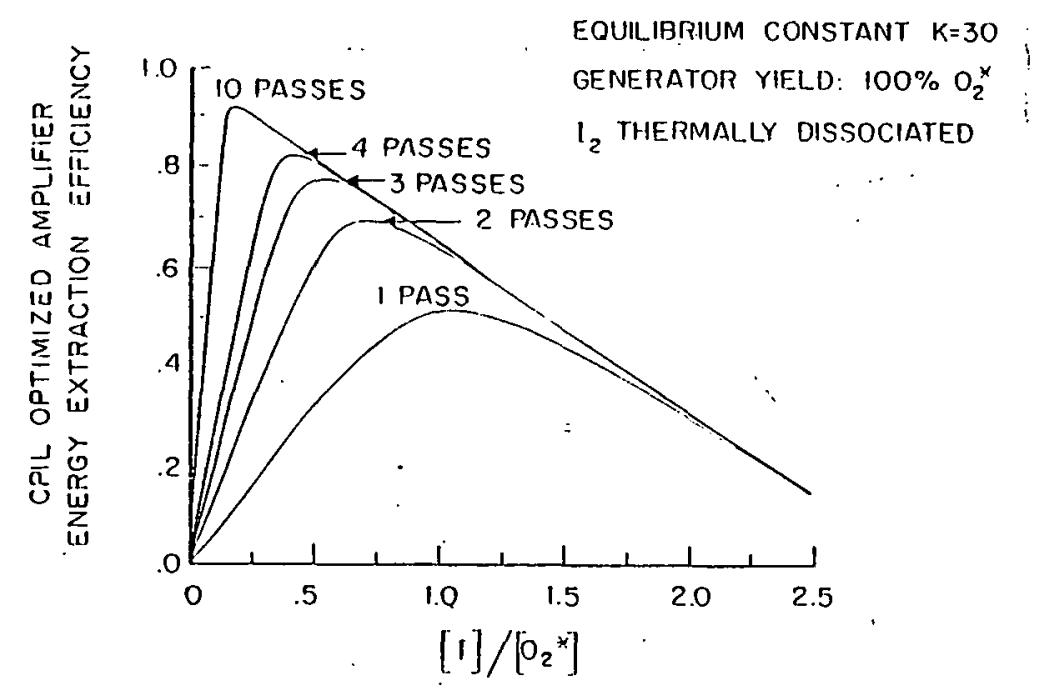

FIGURE 4.3-9 CPIL fractional energy extraction efficiencies for low temperature operation and thermal dissociation of iodine

\section{Chemical Regeneration and Total Energy Efficiencies}

The overall chemical sequence within the CPIL, in its present form, converts chlorine, hydrogen perioxide, and sodium hydroxide to sodium chloride, water, and oxygen as illustrated in Figure 4.3-10. The reactants are readily regenerated from products by standard, large-scale industrial processes, but the energy efficiency applicable to the CPIL (i.e. the energy stored in singlet oxygen divided by the energy consumed in industrial regeneration) is presently only 15\%. If singlet oxygen could be produced in acid, rather than alkaline solution, it is conceivable that this efficiency could be increased to greater than $30 \%$ due in part to the improved energetics of industrial electrolysis in acid solutions. 


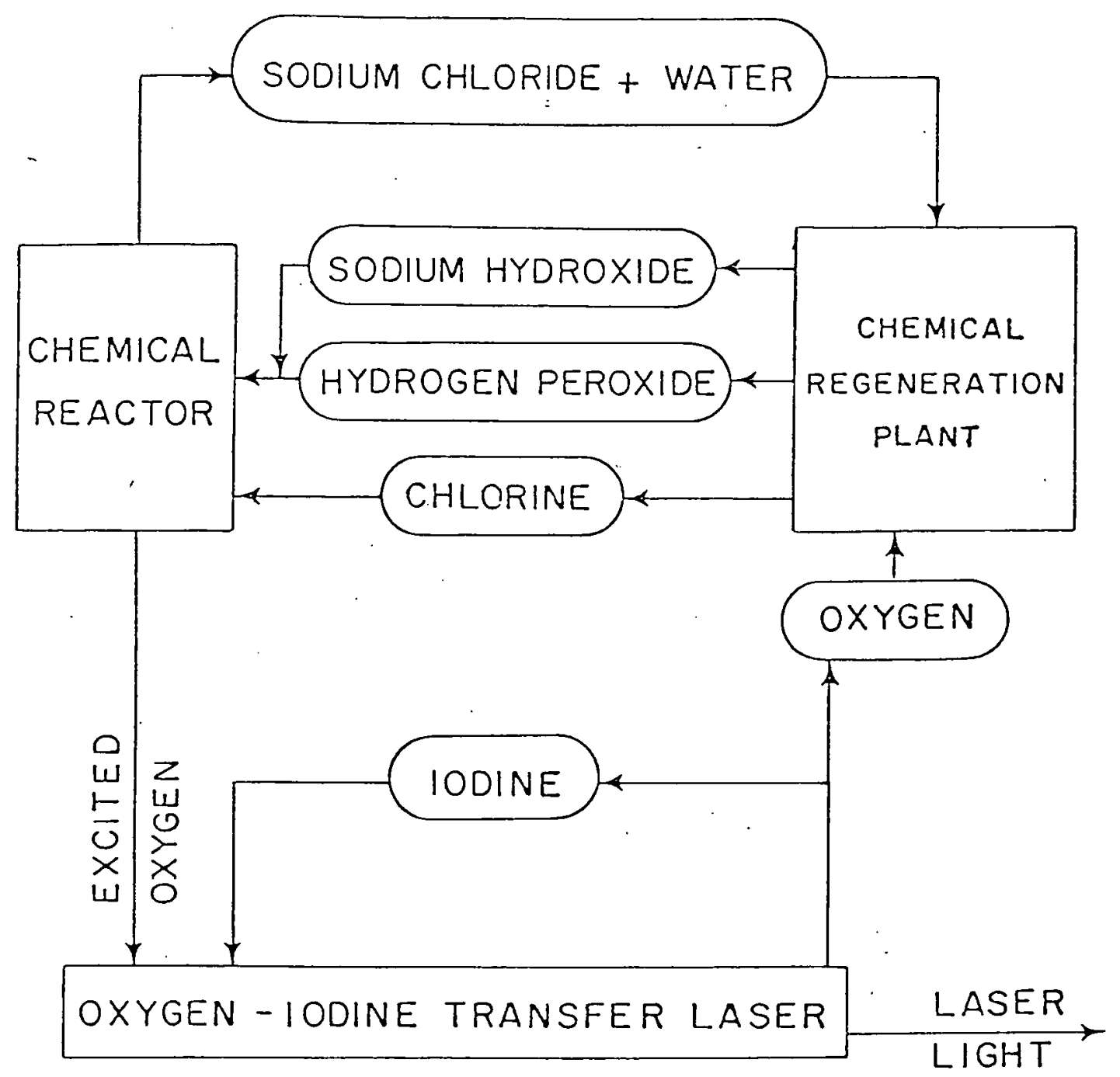

Figure 4.3-10 Chemical Flow In A Closed-Cycle Iodine Laser System 
Alternative chemical reaction sequences leading to the production of singlet oxygen are known and some of these are currently being evaluated by KMS scientists in collaboration with A. Paul Schaap of Wayne State University. Thermal decomposition of organic endo-peroxides looks particularly promising, but still other processes might be developed. Since there are no thermodynamic limitations (beyond entropy/work considerations, and many of these will be included within separate flow analyses of the (PIL) to obtaining efficiencies approaching $100 \%$, it is conceivable that chemical regeneration techniques yielding efficiencies greater than $50 \%$ may be found.

Because the amplifier portion of the CPIL is potentially very efficient (see Figure 4.3-9), the total system efficiency is determined by the chemical regeneration efficiency to a much greater extent than for gas laser pumped by pulsed power supplies. Three possible regeneration efficiencies, ranging from that corresponding to present industrial practice to a highly optimistic figure of $60 \%$, are utilized in Table 4.3-3 to illustrate the potential total energy efficiencies that may be achievable with various configurations of a CPIL. It has been assumed for the purposes of the table that pressures and flow speeds of gases within the CPIL will be sufficiently low that power consumption for pumps will be low compared with chemical regeneration. 
TABLE $4.3-3$

\section{POTENTIAL CPIL DRIVER SYSTEM EFFICIENCIES}

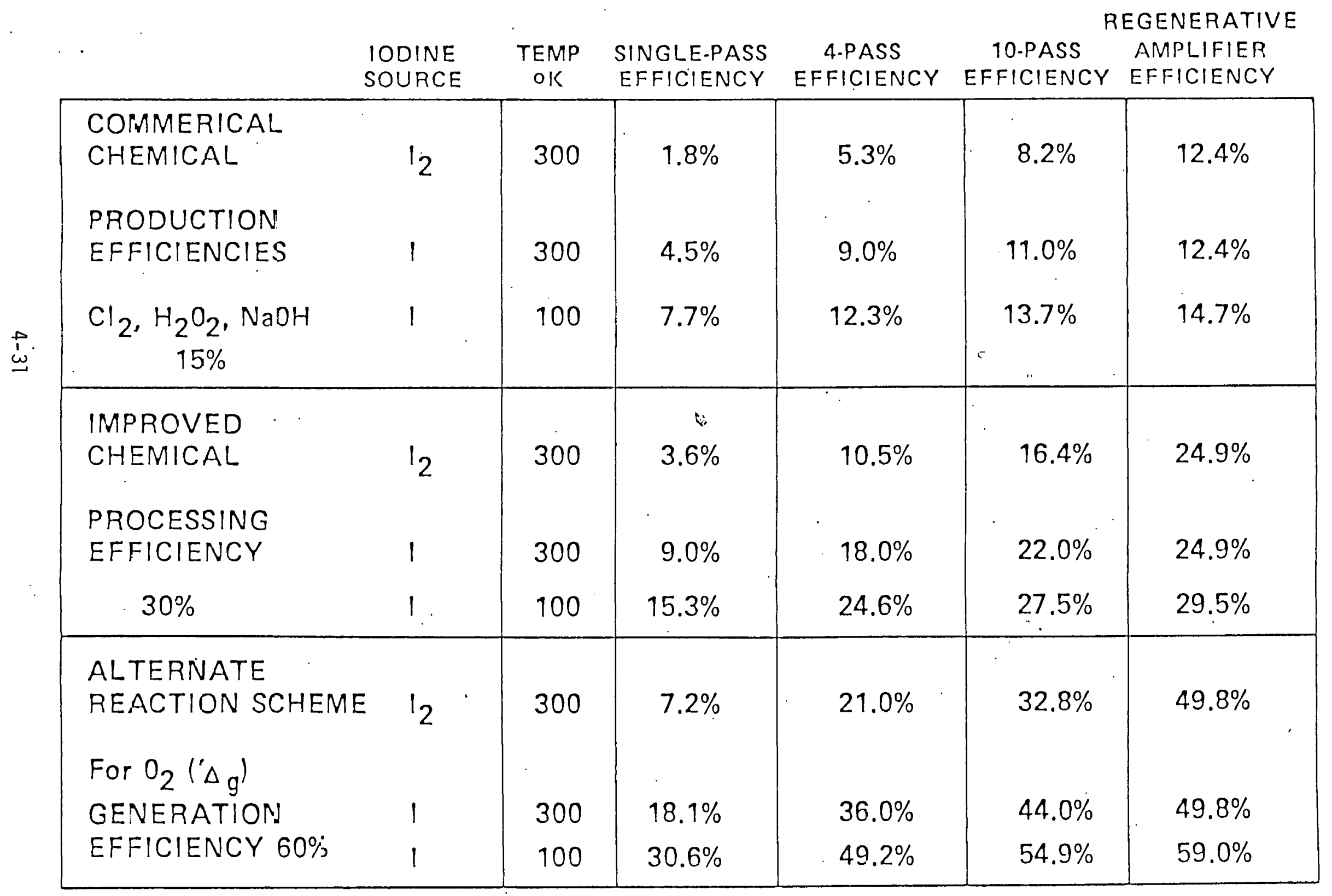




\subsubsection{The Free Electron Laser (FEL)}

S. B. Segall, L. D. Siebert, G. C. Catella, W. B. Colson*

We have also started analyzing the free electron laser (FEL) as a potential laser fusion driver. The FEL has been under investigation by Madey at Stanford University since 1970. (7) Recently, Stanford scientists demonstrated amplifier gain at $10.6 \mu \mathrm{m},{ }^{(8)}$ and operated the FEL as an oscillator at $3.4 \mu \mathrm{m} .{ }^{(9)}$ The free electron laser has several unique advantages which make it a very attractive ICF driver candidate.

\section{Vacuum Operation}

The FEL can operate totally in vacuum. This would eliminate the problem of self-focusing and damage in transparent optical elements which limits all other laser driver candidates. The only optical elements would be mirrors, and the laser beam diameter at the mirrors could be made large enough to prevent damage from high intensity laser light. Since there would be no thermal distortion or optical beam degradation in the amplifier, repetition rate would probably be limited by factors in the reactor system other than the laser and the laser beam could be passed many times through a laser amplifier.

\section{Tuneable}

The free electron laser is tuneable. The FEL could be tuned to whatever frequency is found to be optimum for the pellet implosion (and different frequencies may prove to be optimum for different pellet designs).

\section{Effirient.}

The FEL has the potential of being highly efficient. We are investigating FEL driver systems with potential overall efficiencies of at least $20 \%$. 


\section{Principle of FEL Operation}

The basic physical mechanism which produces light in a free electron laser is shown in Figure 4.3-11. Consider a beam of relativistic electrons interacting with a beam of electromagnetic radiation of wavelength $\lambda_{i}$. A small fraction of the incident photon flux will be backscattered and Doppler shifted to a higher frequency w given approximately by

$$
\omega \approx 4 \gamma^{2} \omega_{i}
$$

For a highly relativistic electron beam the upshift in photon energy can be very large. For example, for a $500 \mathrm{MeV}$ electron beam the photon energy would be increased by a factor of $3.8 \times 10^{6}$. This additional photon energy is obtained from the electrons which lose kinetic energy in the scattering process. Since the electrons are traveling at almost the speed of light, backscattered photons will continue to travel with the electron beam stimulating emission of additional photons at the same frequency. This is the mechanism that produces laser gain.

An incident electromagnetic pump field of wavelength $\lambda_{i}$ may be simulated by a stationary periodic magentic field of period $\lambda_{j} / 2$. The advantage of using a periodic magnetic field to produce radiation is that easily attained magnetic fields can simulate very intense electromagnetic fields. The density of a magnetic field is given in mks units by

$$
u_{B}=1 / 2 \frac{B^{2}}{\mu_{0}}
$$

Electruns passing through this field intercept an energy flux

$$
\phi_{B}=u_{B} c
$$

per unit area where $c$ is the speed of light. Electrons interacting with an incident el ectromagentic pump field of intensity $I_{i}$ intercept an energy 

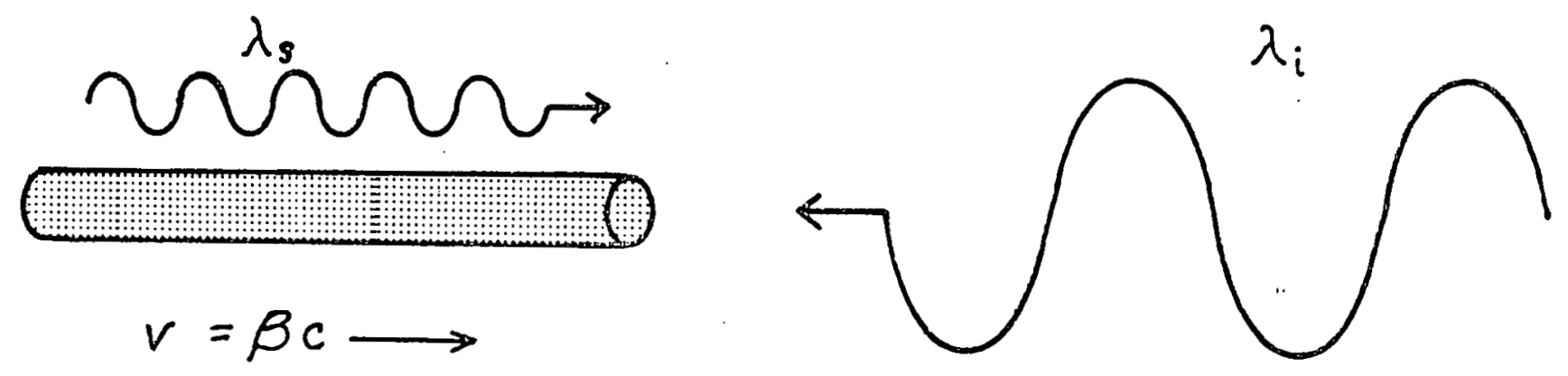

Relativistic Electrons

DOFPLER SHIFT OF BACKSCATTERED PHOTONS

$$
\lambda_{s}=\frac{\lambda_{i}}{(1+\beta)^{2} r^{2}} \approx \frac{\lambda_{i}}{4 r^{2}}
$$

FIGURE 4.3-11 Principle of FEL operation 
flux

$$
\phi_{i}=2 I_{i}
$$

An electromagnetic pump field of intensity $I_{i}$ can therefore be simulated by a magnetic field $B$ which satisfies

$$
I_{i}=\frac{c}{4 \mu o} B^{2}
$$

or

$$
I=59.5 B^{2}
$$

Where $I$ is in megawatts $/ \mathrm{cm}^{2}$ and $B$ is in kilogauss. The first working free electron laser $(8,9)$ was designed using a helical magnetic field of $2.3 \mathrm{kG}$ which is equivalent to a microwave field of $315 \mathrm{MW} / \mathrm{cm}^{2}$. 


\section{Multiple Pass FEL Systems}

While scientists at Stanford and el sewhere ${ }^{(10-13)}$ have studied the FEL in some detail, the extension to the ICF driver application is not trivial. Early analysis ${ }^{(14)}$ showed, for example, that the efficiency would not meet the 1 to $3 \%$ reactor driver minimum requirements. We have taken two approaches to improving FEL system efficiency. We are optimizing ampiifier designs to maximize single-pass energy extraction, and we are studying multiple-pass systems where the light beam is passed back and forth through the amplifier, while the e-beam is recirculated. By recirculating the electron beam it may be possible to recover and reuse energy that remains in the electrons after they leave the amplifier. A design suggested by Madey for an FEL oscillator using a recirculating e-beam is shown in Figure 4.3-12. (15)

The FEL oscillator of Figure 4.3-12 produces a continuous train of light pulses which pass through the partially transmitting end mirror. The power density inside the oscillator is always larger than the output power density. For ICF drivers, the output peak power would be too large to pass through these mirrors without damage. One would like to utilize the high power density which exists inside this oscillator cavity. One solution is to enlarge this "oscillator," and to fire the fusion pellet into the high power laser cavity after the laser beam is amplified to the required level as suggested by Segal1 ${ }^{(16)}$ (Figure 4.3-13). The pellet must be fired with a velocity and trajectory adequate to insure arrival at the laser focus simultaneously with the amplified laser beam, while avoiding the beam on the previous pass. For a round trip optical path length of a few hundred meters, the pellet velocity must be of the order of a few times $10^{5} \mathrm{~cm} / \mathrm{sec}$. 


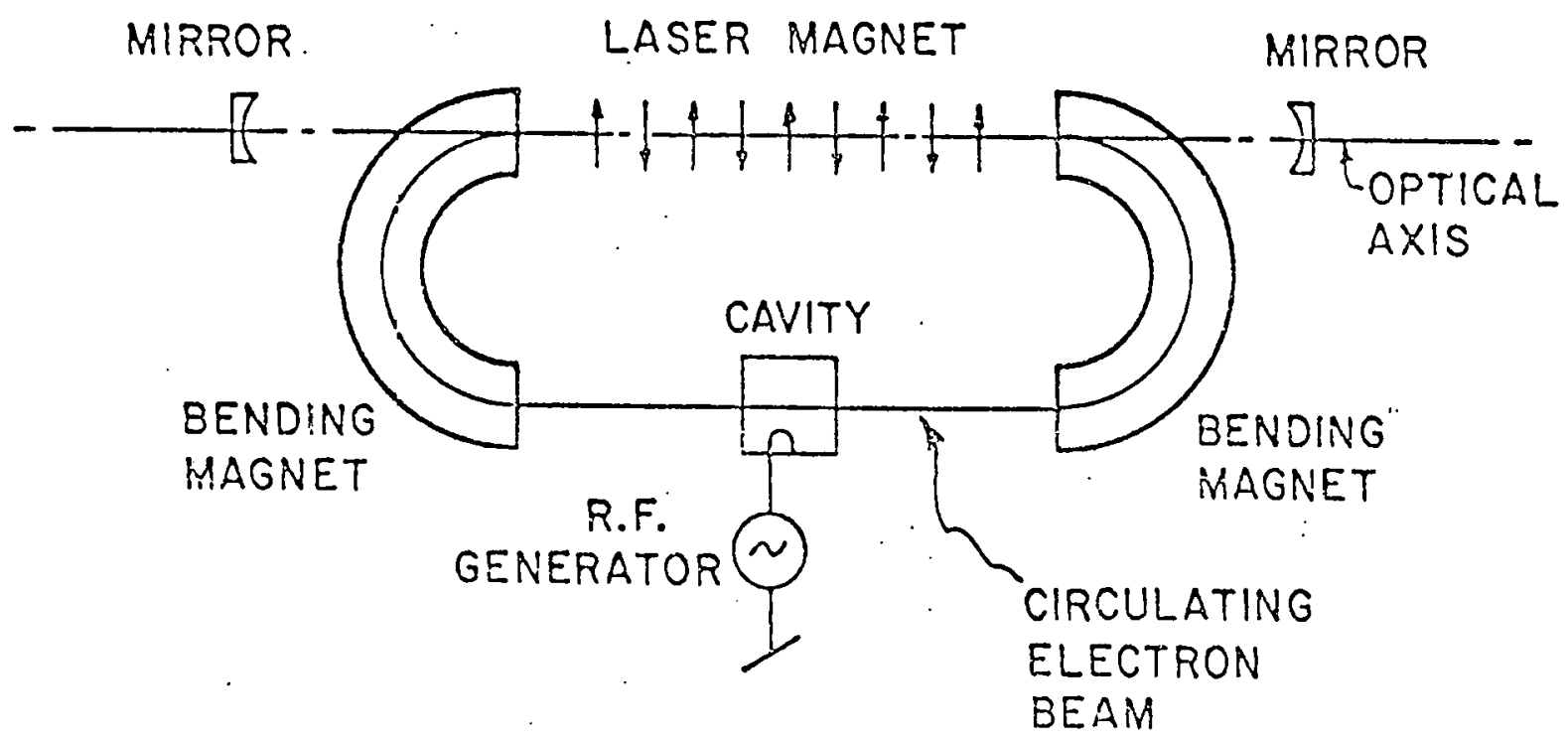

FIGURE 4.3-12 FEL oscililator with a recirculating electron beam 


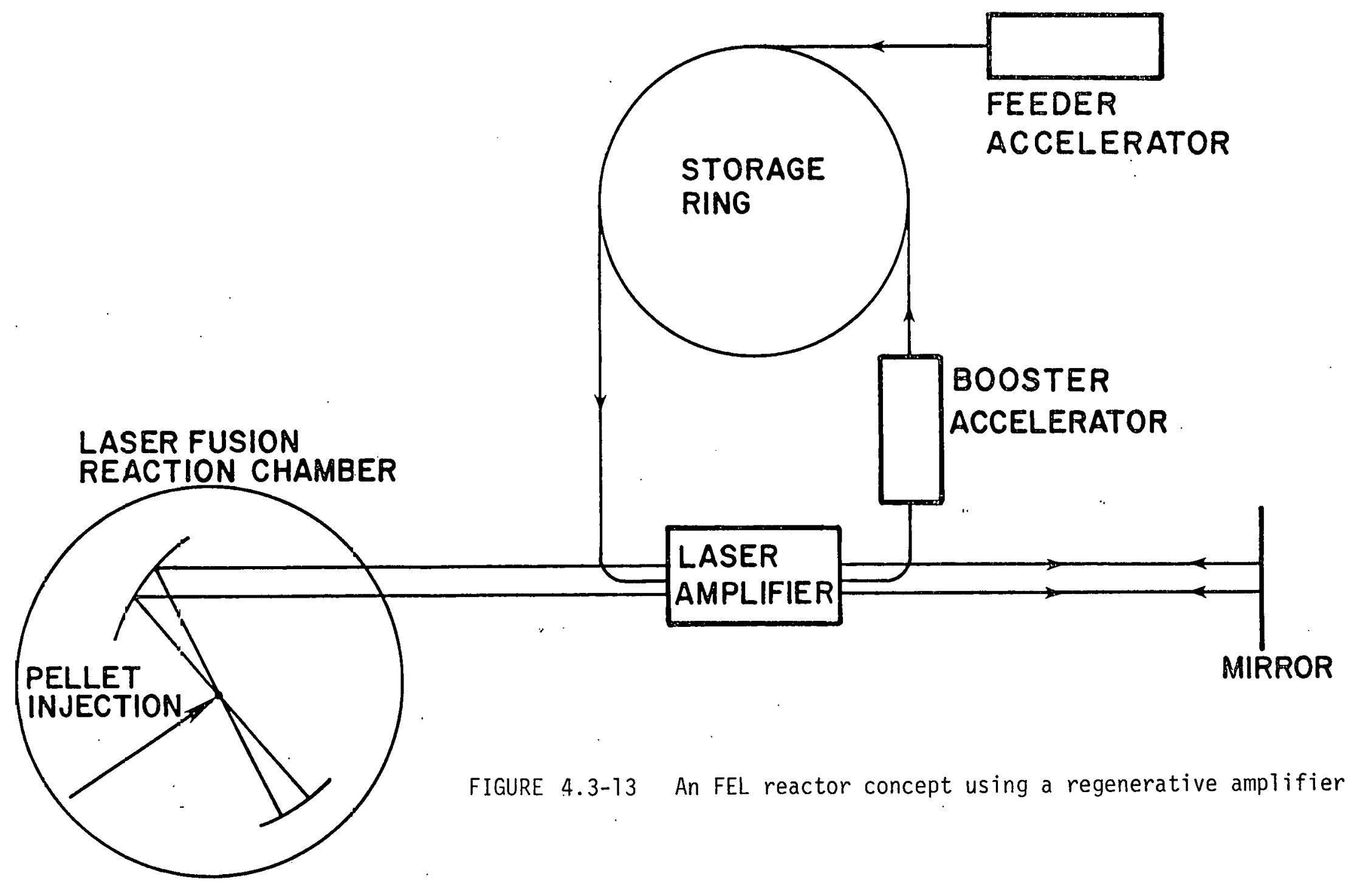


Two laser pulses which intersect at the focus on every pass can be produced in an optical cavity when the round trip transit time of the electrons is exactly half the round trip transit time for the optical pulses and the focal point is located at the center of the cavity (Figure 4.3-14). More than one pair of beams can be used to illuminate the target by increasing the number of optical cavities. A four beam configuration is illustrated in Figure 4.3-15. Figure 4.3-16 shows a possible storage ring configuration for the four beam laser of Figure 4.3-15. Four electron pulses are built up and stored in a large outer storage ring. The pulses are switched into the loops containing the FEL amplifiers when a laser pulse is required.

The current per electron beam required to achieve a given output power $P$ is a function of several variables, including the number of optical beams $M$, the number of amplifier passes per optical beam per target shot $N$, the fractional optical losses per round trip pass $\delta$, and the electron energy loss per amplifier $\eta E$, where $E$ is the electron energy and $\eta$ is the fractional energy extraction per pass. If we limit the number of passes to $1 / \delta$, then about $63 \%$ of -the total optical energy emitted will hit the target. In this case the current required for a given output power is given by.

$$
I(a m p)=1.6 \times 10^{4} \quad \frac{P}{M N n E}
$$

where $P$ is in TW and $E$ is in hundreds of MeV.

This relation is illustrated graphically in Figure 4.3-17 for three power levels. For the 4 beam system of Figure 4.3-15 operated with 500 round-trip optical passes per target shot $\left(\varepsilon=2 \times 10^{-3}\right)$, an output pulse of $100 \mathrm{TW}$ could be produced with an electron current of 1600 amp if the electron energy were $500 \mathrm{MeV}$ and an average of $5 \%$ of the electron energy were converted to laser 


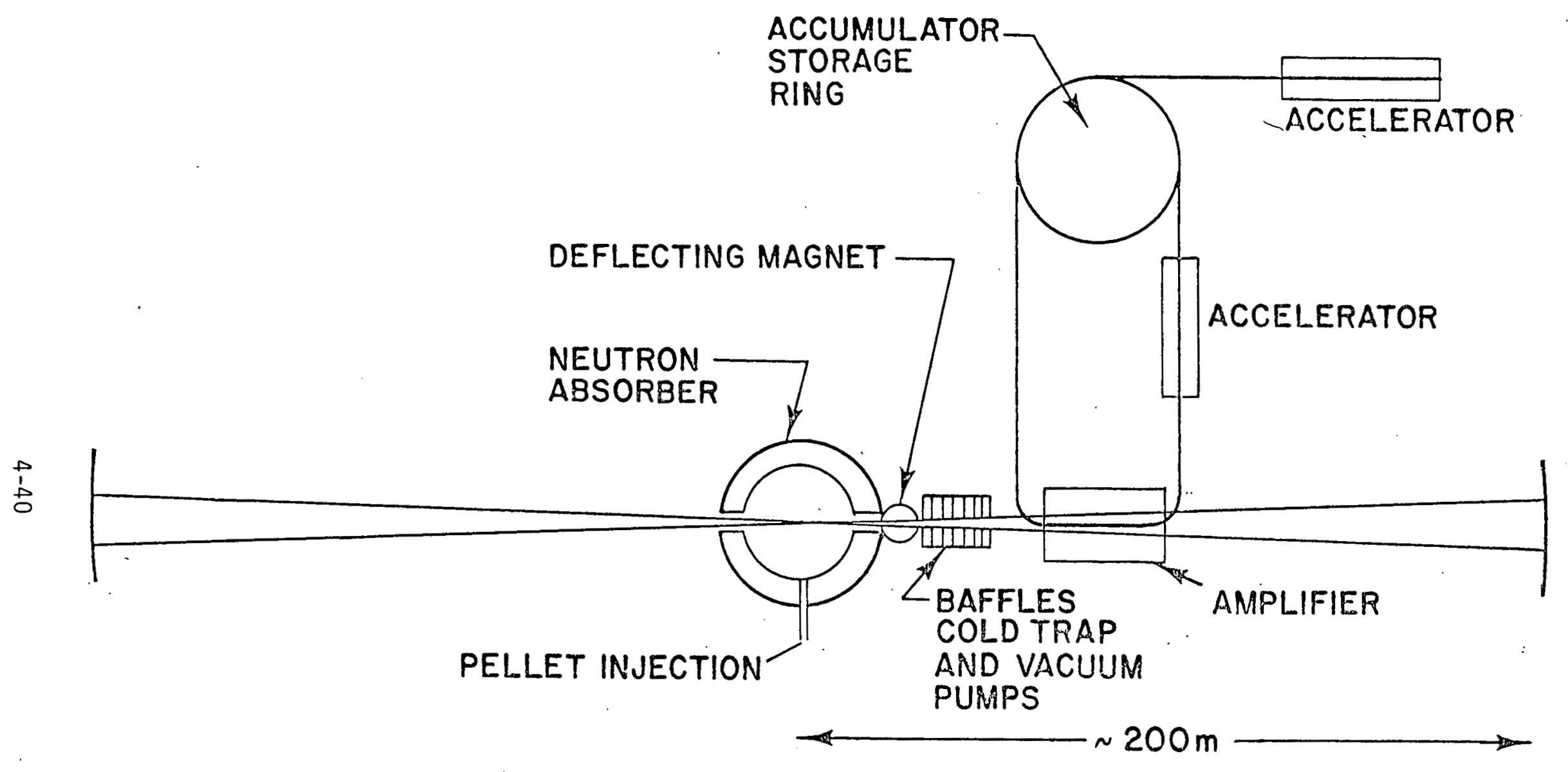

Kms

ivion 
1. INJECTOR

2. SYNCHROTRON OR MICROTRON 3. BAAIN-RING BOOSTER

4. AMPLIFIER-RING RE-ACCELERATOR $X$. SWITCH

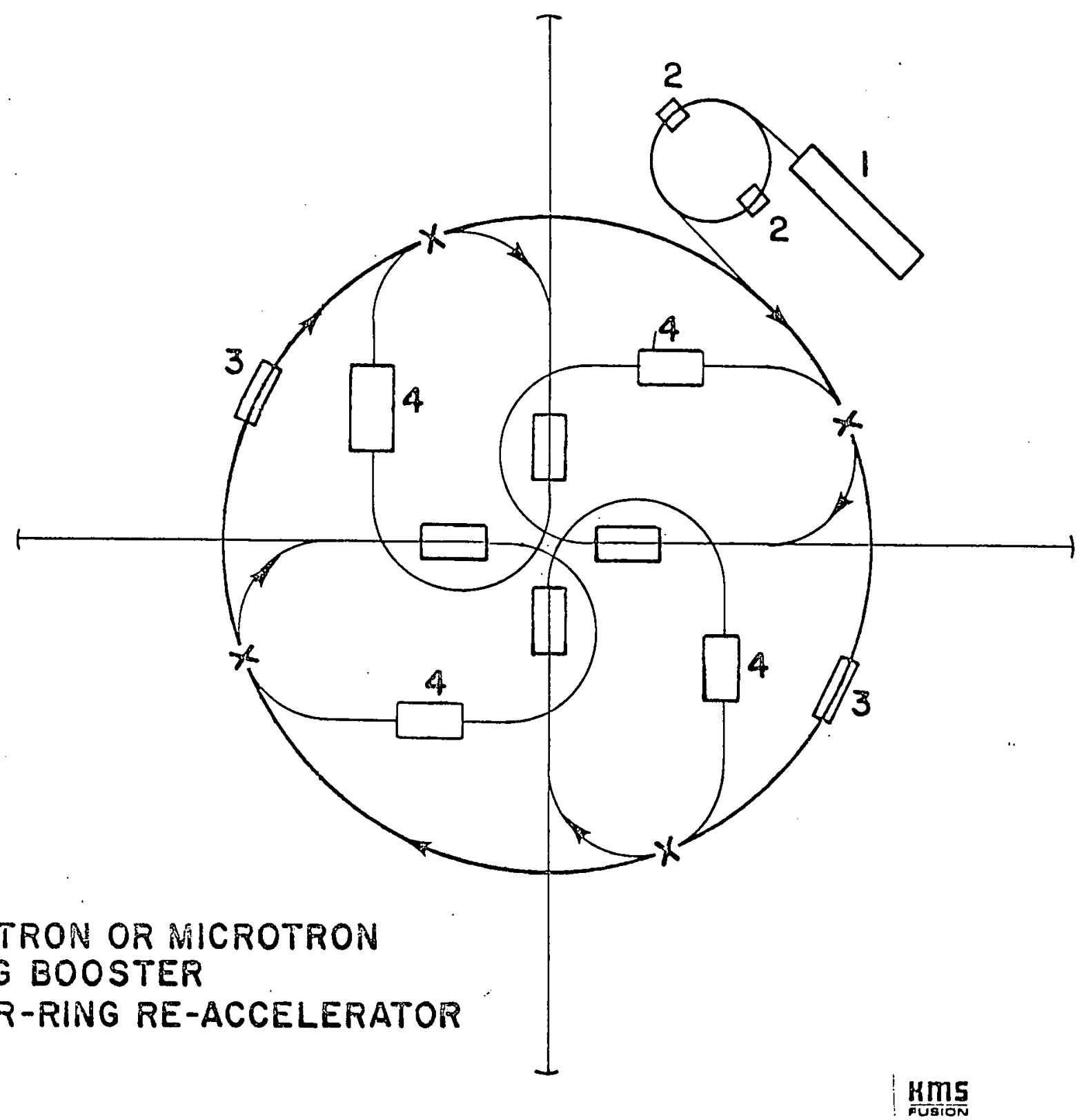

FIGUPE 4.3-16 Electron accelerators for the FEL 


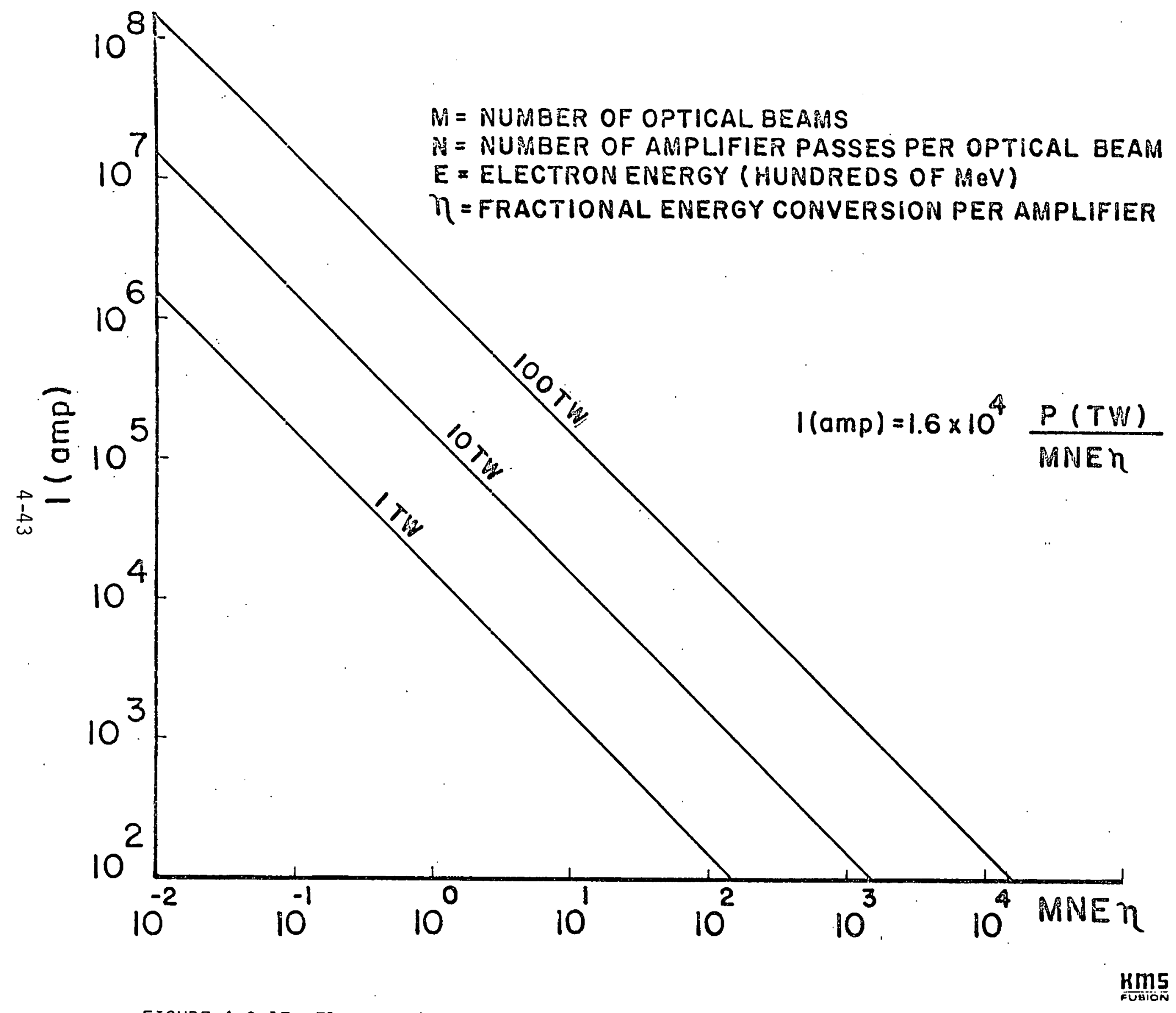

FIGURE 4.3-17 Electron beam current required for an FEL amplifier 
light per pass through an FEL amplifier.

The existing technologies best suited to producing high current electron beams at high energy are induction linacs and storage rings. Both of these devices can produce beams with very low energy spreads $\left(\frac{\Delta E}{E} \leq 10^{-3}\right)$ which are well suited for use in a free electron laser. The maximum peak current which can be obtained from these devices with existing technology is on the order of 10 kamp for an induction linac and a few kamps for a storage ring type device.

\section{Correction for Energy Spread}

Broadening of the electron energy distribution in the FEL amplifier is presently considered the most serious obstacle to recirculating the electron beam. Without compensation for energy spread, multi-pass laser gain would be drastically reduced. In conventional electron storage rings synchrotron radiation is used to damp oscillations and reduce energy spread. This is a very inefficient way to reduce spread, however, since only a small fraction of the energy lost by the electrons contributes to narrowing of the energy distribution. (14)

There are two possible approaches to efficiently compensating for energy spread - either the amplifier could be designed to be less sensitive to electron energy spread or a more efficient method of correcting for energy spread than synchrotron radiation damping must be found. A technique for reducing the sensitivity of the FEL amplifier to energy spread has been proposed by Smith et. al (17) at Stanford. Using this technique the electrons passing through a linear array of periodic magnets are dispersed transversely in energy. The magnitude of the magnetic field is then varied across the beam so that electrons with different energies all radiate at the same laser 
frequency.

It may also be possible to design the laser amplifier to maximize the ratio of mean energy loss to energy spread, thereby reducing the amount the distribution function must be corrected in order to compensate for energy spread. By varying the magnetic field amplitude or magnet period and shifting the phase of the periodic magnetic field at appropriate positions along the amplifier, it may be possible both to increase the fraction of the electron energy converted to photon energy and reduce the spread in the distribution of electron energies leaving the amplifier.

Any residual energy spread which is not corrected in the amplifier must be reduced in the remainder of the storage ring. A few methods of narrowing the electron energy distribution in the storage ring have been suggested in addition to synchrotron radiation. Particles with different energies have different equilibrium orbits in a storage ring. The orbits have slightly different path lengths causing particles of different energies to be accelerated in the booster accelerator at different phases of the RF cycle: As a result, electrons with higher energies are accelerated less than electrons with lower energies. Elias et. al ${ }^{(18)}$ have demonstrated the effects of this mechanism on narrowing of the electron energy distribution in Monte Carlo calculations of an FEL amplifier in a storage ring. Alternatively, electrons leaving the laser amplifier could be dispersed transversely in energy and a differential damping mechanism such as differential damping of image current as by means of electrical resistors embedded in the wall of the vacuum vessel through which the electron beam is transported, may be used to reduce the spread in the electron energy distribution more efficiently than synchrotron radiation. All of the concepts for correction of energy spread in a storage ring by mechanisms other than synchrotron radiation are 
prel iminary and require further study.

Correcting for residual energy spread may not be as severe for pulsed lasers as for continuously operating systems. Only a finite number of passes are made per target shot in a pulsed system, and some degradation of the laser gain may be tolerated. Even at repetition rates of 10 or 20 target shots per second the electron pulses are in the amplifier loops only a few percent of the time. In the intervals between target shots the electron beam could be restored to its initial distribution.

\section{Theoretical Studies}

In order to study FEL amplifier performance we have developed a computer code which simulates the passage of an electron beam through an FEL amplifier. At the present time this code is being used to analyze the behavior of constant period helical amplifiers. In the future we will. relax the constraints of constant period and field strength to permit optimization of the amplifier design. In the computer simulation we follow an electron beam, represented by a linear array of discrete particles as it passes through a lielical magnetic field together with an electromagnetic wave. Individiual electron dynamics are used to describe the beam-wave interaction, since collective effects can be neglected in the parameter range expected for high power, short-wavelength free-electron lasers.

\section{Basic Equations of the Single Marticle Model}

The basic equations used in the single particle model of the FEL

are the Lorentz force equations which govern the electron trajectories (19) These are given in Gaussian units by 


$$
\frac{d}{d t}(\gamma \vec{B})=\frac{e}{m c}\left(\vec{E}_{r}+\vec{\beta} \times\left(\vec{B}_{r}+\vec{B}_{m}\right)\right)
$$

and

$$
\frac{d \gamma}{d t}=\frac{e}{m c} \vec{B} \cdot \overrightarrow{E_{r}}
$$

where $\quad \gamma^{-2}=1-\vec{\beta} \cdot \vec{\beta}$.

Here $\beta$ is the ratio of the electron velocity to the speed of light $c, \gamma$ is the ratio of electron energy to electron rest mass $m, \vec{E}_{r}$ and $\vec{B}_{r}$ are the electric and magnetic fields of the electromagnetic wave, $\vec{B}_{m}$ is the magnetic field of the laser amplifier, and $e$ is the charge of an electron.

The static magnetic field is taken to be of the form

$$
\vec{B}_{\mathrm{m}}=\mathrm{B}_{\mathrm{m}} \cdot \quad\left(\cos \mathrm{k}_{\mathrm{m}} \mathrm{z}, \sin \mathrm{k}_{\mathrm{m}} \mathrm{z}, 0\right)
$$

where $k_{m}=2 \pi / \lambda_{m}$ and $\lambda_{m}$ is the magnet period. The electric and magnetic fields of the laser beam are given by

where

$$
\begin{aligned}
& \vec{E}_{r}=E_{r}(t)(\cos x,-\sin x, 0) \\
& \vec{B}_{r}=B_{r}(t)(\sin x, \cos x, 0)
\end{aligned}
$$

and in Gaussian units $E_{r}(t)=B_{r}(t), \lambda_{r}=2 \pi / k_{r}=2 \pi c / \omega{ }_{r}$ is the optical wavelength and $\phi$ is the optical phase.

Since all the fields are assumed to be transverse it is convenient to rewrite equations (11) - (13) in terms of axial and transverse components

$$
\begin{aligned}
& \frac{d}{d t}\left(r \vec{B}_{\perp}\right)=\frac{e}{m c}\left(\vec{E}_{r}+\vec{B}_{z} \times\left(\vec{B}_{r}+\vec{B}_{m}\right)\right) \\
& \frac{d}{d t}\left(\gamma \vec{B}_{z}\right)=\frac{e}{m c}\left(\vec{B}_{\perp} \times\left(\vec{B}_{r}+\vec{B}_{m}\right)\right)
\end{aligned}
$$




$$
\frac{d y}{d t}=\frac{e}{m c} \vec{E}_{r} \cdot \vec{\beta}_{\perp}
$$

and $\gamma^{-2}=1-\beta_{\perp}^{2}-\beta_{z}^{2}$

Because $\vec{\beta}_{z} \times \vec{B}_{r}=-\vec{\beta}_{z} \vec{E}_{r}$ equation (17) becomes

$$
\frac{d}{d t}\left(\gamma \vec{B}_{\perp}\right)=\frac{e}{m c}\left(\vec{E}_{r}\left(1-\beta_{z}\right)+\vec{B}_{z} \times \vec{B}_{m}\right) \text {. }
$$

Assuming $\beta_{z} B_{m} \gg E_{r}(t)\left(1-B_{z}\right)$,

which will always be true for the cases we are considering, we can neglect the first term on the right hand side in equation (21) giving

$$
\frac{\mathrm{d}}{\mathrm{dt}}\left(\gamma \vec{\beta}_{\perp}\right) \approx \frac{\mathrm{e}}{\mathrm{mc}} \vec{B}_{z} \times \vec{B}_{\mathrm{m}}
$$

Using the component form for $\vec{B}_{m}$ in equation (14) and the transformation

$$
\frac{d}{d t}\left(\gamma \vec{\beta}_{\perp}\right)=c \beta_{z} \frac{d}{d z} \quad\left(\gamma \vec{\beta}_{\perp}\right)
$$

equation (23) can be easily integrated. Assuming $B_{m}$ and $k_{m}$ are constants we obtain

Defining $\quad \alpha \equiv \frac{|\mathrm{e}| \mathrm{B}_{\mathrm{m}}}{\mathrm{mc}^{2} \mathrm{k}_{\mathrm{m}}}$

$$
\overrightarrow{B_{\perp}}=\frac{\overrightarrow{e B}}{\gamma m^{2} k_{m}} .
$$

the magnitude of $\vec{\beta}_{\perp}$ is given by

$$
B_{\perp}=\frac{\alpha}{\gamma}
$$

From equation (25) we see that $\vec{B}_{\perp}$ is parallel to $\vec{B}_{m}$. Using this in equation (18) we obtain

$$
\frac{d}{d t}\left(\ddot{\gamma} \vec{B}_{z}\right)=\frac{e}{m c}\left(\vec{B}_{\perp} \times \vec{B}_{r}\right) .
$$

Because the magnitude of $\vec{B}_{\perp} \times \vec{B}_{r}$ equals $\vec{B}_{\perp} \cdot \vec{E}_{r}$ we can write $(28)$ as

$$
\frac{d}{d t}\left(\vec{r}_{z}\right)=\frac{e}{m c}\left(\vec{E}_{r} \cdot \beta_{\perp}\right)
$$


The right hand side of equation (29) is identical to the right hand side of equation (19). We therefore see that the assumption $\beta_{z} \approx 1$ is required to make our system of equations self consistent in addition to the previous assumption that $k_{m}$ and $B_{m}$ are constant and relation (22) holds.

From equations (20) and (27) we obtain

$$
\frac{\left(1+\alpha^{2}\right)}{\gamma 2}=1-\beta_{z}^{2}
$$

Differentiating and then assuming $\beta_{z} \approx 1$ we obtain

where

$$
\begin{aligned}
\frac{d B_{z}}{d t} & =\frac{\left(1+\alpha^{2}\right)}{\gamma^{3}} \frac{d \gamma}{d t}=\frac{\left(1+\alpha^{2}\right)}{\gamma^{3}} \cdot \frac{e}{m c} \vec{E}_{r} \cdot \vec{B}_{\perp} \\
& =\frac{\left(1+\alpha^{2}\right) \alpha}{r^{4}} \frac{e}{m c} E_{r}(t) \cos \Psi \\
\Psi & =\left(k_{m}+k_{r}\right) z-\stackrel{\omega}{r}_{r} t+\phi
\end{aligned}
$$

An electron is said to be in resonance with the electromagnetic wave when the electron moves a distance of one magnet period during the time one wavelength of light passes over it.

When this is the case

$$
\frac{{ }^{\lambda} \mathrm{m}}{\mathrm{v}_{\mathrm{z}}}=\frac{{ }^{\lambda} \mathrm{m}+{ }^{\lambda} \mathrm{R}}{\mathrm{C}}
$$

where $v_{Z}={ }_{Z} c$ and $\lambda_{R}$ is the laser wavelength at resonance. This can be rewritten in the form

$$
\lambda_{R}=\lambda_{m} \frac{\left(1-\beta_{z}\right)}{\beta_{z}}
$$


Using equation (31) and assuming $\beta_{z} \approx 1$ except in the term $\left(1-\beta_{z}\right)$, we obtain

$$
\lambda_{R}=\frac{\lambda_{m}}{2 \gamma^{2}} \quad\left(1+\alpha^{2}\right)
$$

The position of an electron in the electron beam can be written in the form

$$
z(t)=\beta_{0 z} \quad c t+z^{\prime}(t)
$$

where $\beta_{0 z}$ is the velocity of a reference frame moving with the initial mean velocity of the electrons and $z^{\prime}(t)$ is the position of an electron in the moving frame as viewed from the laboratory frame. Using (36) we can write equation $\left(32^{\prime \prime}\right)$ as

$$
\psi=\left(\left(k_{m}+k_{r}\right) \beta_{0 z} c t+\left(k_{m}+k_{r}\right) z^{\prime}-\omega_{r} t+\phi\right.
$$

Defining

$$
\Delta \Omega \equiv\left(k_{m}+k_{r}\right) \beta_{o z} c-\omega_{r}
$$

equation (37) can be written

$$
\begin{aligned}
\Psi & =\left(k_{r}+k_{m}\right) z^{\prime}+\Delta \Omega t+\phi \\
& \approx k_{r} z^{\prime}+\Delta \Omega t+\phi
\end{aligned}
$$

From equation (38) we obtain

$$
\frac{\Delta \Omega}{1-\beta_{0 z}}=\frac{\beta_{O Z}}{1-\beta_{0 z}} \quad k_{m} c-\omega_{r}
$$

which from equation (34) becomes

$$
\frac{\Delta \Omega}{1-\beta_{0 z}}={ }^{\omega} R-\omega_{r}
$$

where $\omega_{R}$ is the resonance frequency for electrons moving with the initial electron velocity.

The relative angle $\Psi$ between $E_{r}$ and $\beta_{\perp}$ determines whether an electron loses or gains energy. From equation (39) we see that at any instant of time this angle varies by $2 \pi$ radians over a distance of one wavelength of light and, therefore, sections of an electron beam 
$\lambda_{r}$ in length will evolve identically in time provided the electron beam has an initially uniform spatial and velocity distribution and the laser intensity at the input end of the amplifier is uniform.

Electrons in a region where $\cos \Psi$ is positive will be decelerated and electrons in a region where $\cos \psi$ is negative will be accelerated. This effect will produce bunching of the electron beam on a scale of the wavelength of the laser light. If $\omega_{r}=\omega_{R}$ there will be no explicit time dependence in the phase factor $\Psi$, and the electrons will bunch symmetrically around the position $\psi=-\frac{\pi}{2}$ (Figure 4.3-18.) For convenience we define a new angle $\theta=\frac{\pi}{2}+\psi$ in Figure 4.3-18. so that $\sin \theta=\cos \psi$. Net transfer of energy to the laser field as a result of the bunching process will be zero at the resonance energy and there will be no laser gain.

If $\omega_{r} \neq \omega_{R}$ then $\theta$ will be explicitly dependent on time, the function $\sin \theta$ will shift relative to a reference frame moving with the electron beam, and the electron density distribution will no longer be symmetric about $\theta=\pi$. If $\Delta \Omega$ in equation (39) is positive the shift of the function $\sin \theta$ in the reference frame moving with the initial electron velocity will cause more electrons to lose energy than gain energy (Figure 4.3-19)

: resulting in amplification of the electromagnetic wave. If $\Delta \Omega$ were negative more electrons would be accelerated than decelerated, and the Taser beam would give up energy to the electrons.

It is assumed that energy lost or gained by the electrons is transferred to or from the laser beam so that energy is conserved. Under these conditions the changes in laser intensity, I, resulting.f.rom a change: in electron energy is given by:

$$
\begin{aligned}
\frac{d I}{d t} & =\frac{c}{\lambda_{r}} \int_{0}^{\lambda_{r}} \rho\left(z^{\prime}\right) m c^{2} \frac{d \gamma}{d t} d z^{\prime} \\
& =\frac{m c^{3}}{\lambda_{r}} \int_{0}^{\lambda_{r}} \rho\left(z^{\prime}\right) \frac{d_{\gamma}}{d t} d z^{\prime} \\
& 7-51
\end{aligned}
$$




\section{AT RESONANCE OBTAIN BUNCHING WITHOUT GAIN}

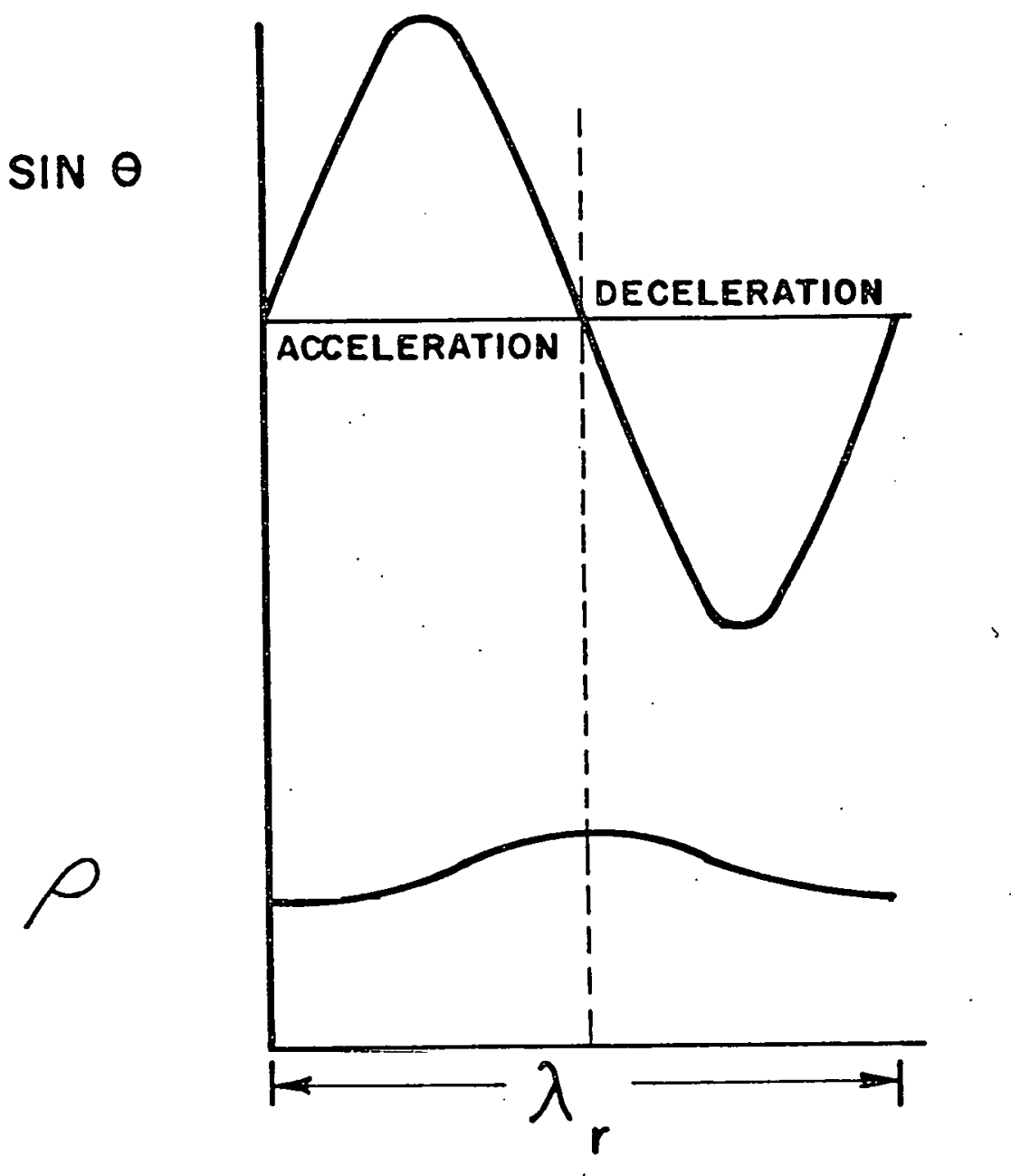

FIGURE 4.3-18 Illustrating electron bunching at resonance where $p=$ electron density

$$
4-52
$$




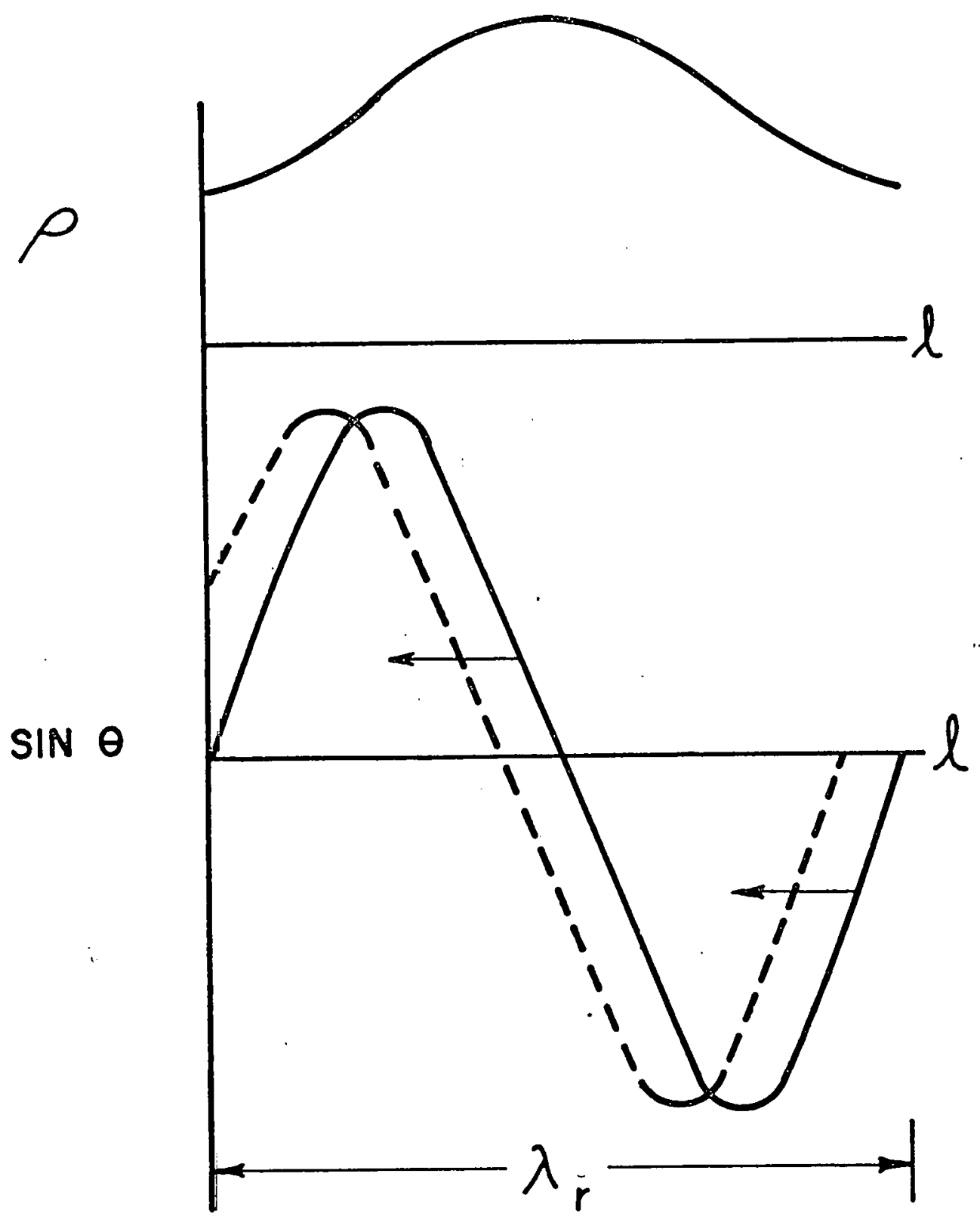

FIGURE 4.3-19 Electron condition for amplification. 
$\rho\left(z^{\prime}\right)$. is the electron density as a function of position in the moving frame and the integral is taken only over a segment one radiation wavelength long, since segments of the beam $\lambda_{r}$ in length evolve identically in time. The laser electric field $E_{r}(t)$ can be found from $I(t)$ using the formula

$$
\begin{aligned}
& \text { formula } \\
& I(t)=\frac{E_{r}(t)^{2}}{4 \pi}
\end{aligned}
$$

\section{Computer Simulations}

The basic equations derived in the last section have been used in the computer code which simulates the passage of an electron beam through an FEL amplifier.

In the code a segment of the electron beam one radiation wavelength long is followed through the amplifier. The beam is represented by a linear array of discrete charges initially positioned at equal intervals within a laser wavelength. At each time step the change in the electron energy, velocity, and position are calculated and the amplitude of the laser field is updated. Periodic boundary conditions are used. The electrons are assumed to be initially monoenergetic and the $^{\prime}$ laser beam traveling with the electrons is assumed to be monochromatic with frequency $\omega_{r}$.

To calculate maximum fractional energy conversion a laser frequency is chosen that differs from the resonance frequency $\omega_{R}$ by an amount $\Delta \omega$ ( $\omega_{R}$ is fixed by the amplitude of the helical magnetic field, $B_{m}$, the magnet period $\lambda_{m}$, and the initial electron energy $\left.E\right)$. For this frequency

$$
\omega_{r}=\omega_{R}-\Delta \omega
$$

electrons were permitted to progress down the amplifier until net transfer of energy to the laser field decreased to zero. This was done for a 
range of values of $\Delta \omega$ The laser frequency $\omega_{r}$ for which laser gain was a maximum was then assumed to be the frequency of the light propagating in the amplifier and the maximum fractional energy conversion was taken to be the maximum value for this frequency.

The evolution of the electron beam in the amplifier can be followed graphically in our computer code. At any time step we can obtain the electron density as a function of position, the phase of the power function $E_{r} \beta_{\perp}$ sin $\Theta$ relative to the density function, the position and velocity of each of the discrete particles we are tracking, and the electron energy distribution. An example of the computer graphics is shown in Figure 4.3-20. Using this computer code we expect to obtain a thorough understanding of the operation of constant period amplifiers in the regime of high laser power and high electron energies appropriate to the laser fusion application. Building on these results we will then begin design of optimized amplifier configurations.

\section{Analytic Model}

An analytic model has been developed which can be used together with the computer simulations to obtain scaling information. The analytic model uses the same basic equations as the computer simulations to obtain an upper limit on the fractional energy conversions of a constant period FEL amplifier as a function of magnet and electron beam parameters.

To derive this upper limit we begin by substituting equation (25) into équation (19)

$$
\begin{aligned}
\frac{d \gamma}{d t} & =\frac{e}{m c} \vec{E}_{r} \cdot \vec{B}_{\perp} \\
& =\left(\frac{e^{2} B_{m} \lambda_{m}}{2 \pi m^{2} c^{3} \gamma}\right) E_{r}(t) \cos \psi
\end{aligned}
$$


TIME $=9.23 E-08 \quad$ DWT $=-2.77$

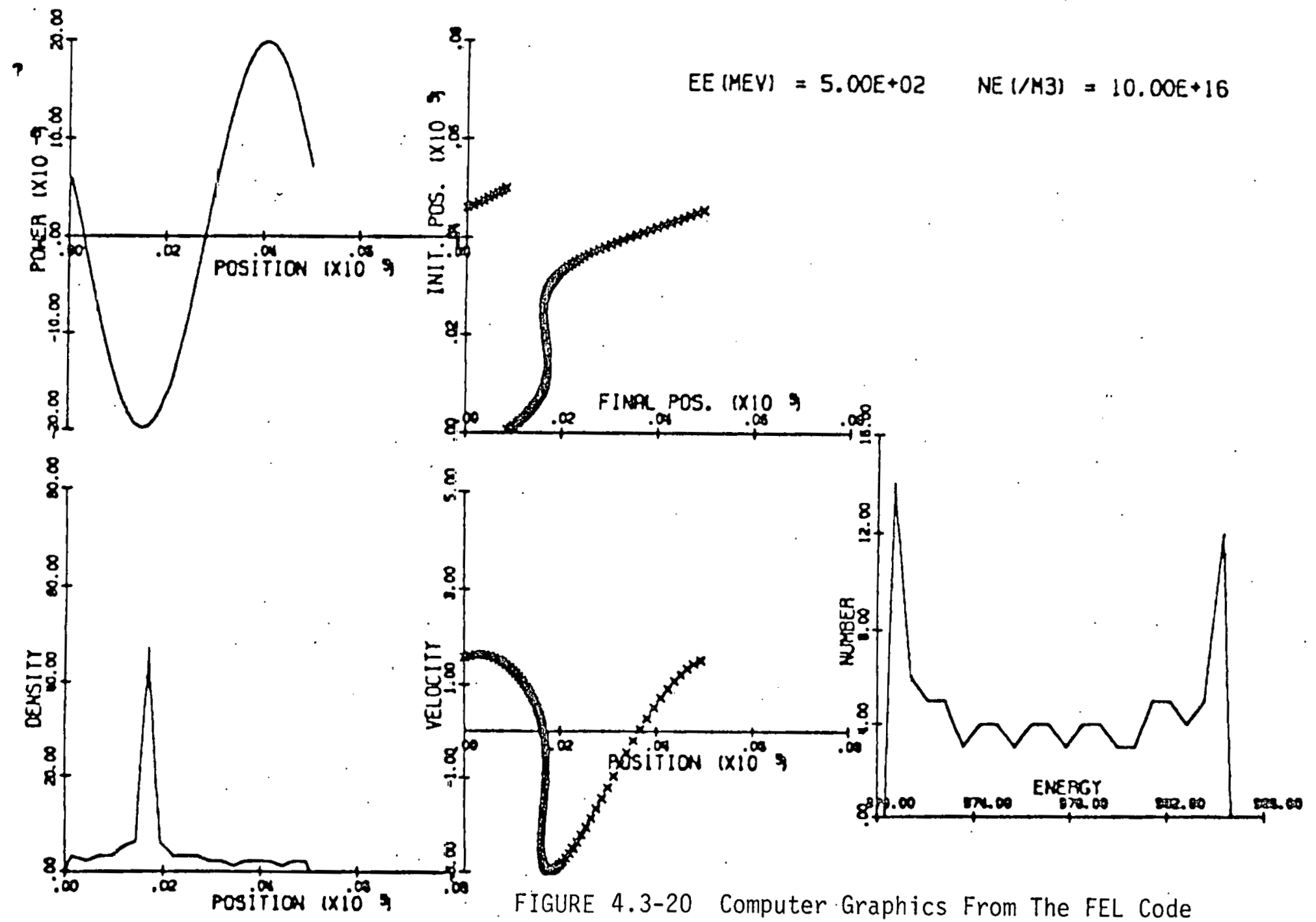


Using conservation of energy we can write

$$
\frac{E_{r}^{2}(t) d V}{4 \pi}+\sum_{i=1}^{N e} \gamma_{i}(t) m c^{2}=\text { constant, }
$$

where the sum is taken over all electrons in a volume element $d v$ in the electron beam. It is assumed here that the electron beam and optical beam occupy the same volume and that any energy lost by the electrons is transferred to the optical beam.

Equation (46) can be rewritten in terms of the initial values of electric field and electron energy in the form

$$
E_{r}^{2}(t)=E_{r}^{2}(0)+4 \pi \rho \gamma_{0} m c^{2}-\frac{4 \pi}{d V} \sum \gamma_{j}(t) m c^{2}
$$

where $\gamma_{0} m c^{2}$ is the initial electron energy and $\rho$ is the initial uniform electron density.

$$
\begin{aligned}
E_{r}^{2}(t) & =4 \pi \rho \gamma_{0} m c^{2}\left(\frac{E_{r}^{2}(0) / 4 \pi}{\rho \gamma_{0} m c^{2}}+\frac{N e \gamma_{0}-\Sigma \gamma_{j}(t)}{N e \gamma_{0}}\right) \\
& =4 \pi \rho \gamma_{0} m c^{2}(s+n)
\end{aligned}
$$

where

$$
s \equiv \frac{E^{2}(0) / 4 \pi}{\rho r_{0} m c^{2}}
$$

is the initial ratio of field energy density to electron energy density and

$$
n \equiv \frac{N_{e} \gamma_{0}-\Sigma_{\gamma_{j}}(t)}{N e \gamma_{0}}=\frac{1}{N e} \sum_{i=1}^{N e} \frac{\Delta \gamma_{j}}{\gamma_{0}}
$$


is the fraction of the initial electron energy that has been converted to laser energy at time $t$. Substituting equation (48) into equation (45) we obtain

$$
\gamma d \gamma=\left(\frac{e^{2} B_{m}}{m^{2} c^{3}} \frac{\lambda}{m}\right)\left(4 \pi \rho \gamma_{0} m c^{2}\right)^{1 / 2} \quad(s+n)^{1 / 2} \cos \psi d t
$$

Equation (51) holds for a single electron. We can sum both sides of equation (51) over all the electrons in a volume dV. Using $\gamma_{i}=\gamma_{0}+\Delta \gamma_{i}$ and assuming $\gamma_{0}>\Delta \gamma_{i}$, we obtain

$$
\gamma_{0} \sum_{i=1}^{N e} d r_{i}=\left(\frac{e^{2} B_{m}{ }^{\lambda} m}{m^{2} c^{3} 2 \pi}\right)^{\left(4 \pi \rho r_{0} m c^{2}\right)^{1 / 2}(s+n)^{1 / 2}} \sum_{i=1}^{N e} \cos \Psi_{i}(t) d t .
$$

Integrating over the length of the amplifier and utilizing the definition of $n$ in equation (50) we obtain

$$
\left.\int_{0}^{n_{f}} \frac{d \eta}{(s+n)}\right) / 2=\frac{-k c}{N \lambda_{m}}, \int^{N \lambda_{i} / c} \frac{1}{N e} \sum \cos \psi_{i}(t) d t
$$

where $n_{f}$ is the fractional energy conversion that has been attained at the output end of the amplifier, $N \lambda_{m} / \mathrm{c}$ is the time required for the electrons to transverse the amplifier assuming $\beta_{z} \approx 1$, and

$$
K \equiv \frac{e^{2} B_{m} \lambda^{2} m^{N o} !^{1 / 2}}{\pi^{1 / 2}\left(\gamma_{o} m c^{2}\right)^{3 / 2}}
$$

The evolution of the phase angle ${ }^{\psi}{ }_{i}$ as a function of time is a complicated function which we are able to determine by calculating electron trajectories in the computer simulation. To obtain an upper limit for the fractional energy conversion $n$ we can assume that the electrons are perfectly bunched 
throughout the amplifier so that $\cos { }^{\Psi}{ }_{i}$ always has the value -1 . With this assumption we can perform the integrals in equation (53): and obtain.

$$
\frac{\eta_{\max }}{s}=\frac{k}{\sqrt{s}}+\frac{k^{2}}{4 s}
$$

where $n_{\max }$ is the upper 1 imit on fractional energy extraction obtained by assuming the electrons were perfectly bunched. Equation (55) is a simple equation with only two variables, $n / s$ and $K / \sqrt{5}$, which provides an upper limit for the performance of all constant period free electron lasers. A graph of equation (55) is shown in Figure 4.3-21 together with points obtained from the computer simulation. The computer results, as expected are always lower than the limiting value $n_{\max }$. It is interesting to note that the particle simulation results all lie on a single curve in this parameter space. One of the benefits we obtain from the analytic model is, therefore, identification of a parameter space in which FEL data covering a wide range of electron energies, field strengths and input laser intensities can be plotted on a single curve. This will permit us to determine accurate scaling of FEL performance with less extensive computer mapping of the multidimensional FEL parameter space than would have been possible before identification of the scaling parameters $\mathrm{n} / \mathrm{s}$ and $\mathrm{K} / \sqrt{\mathrm{s}}$. 


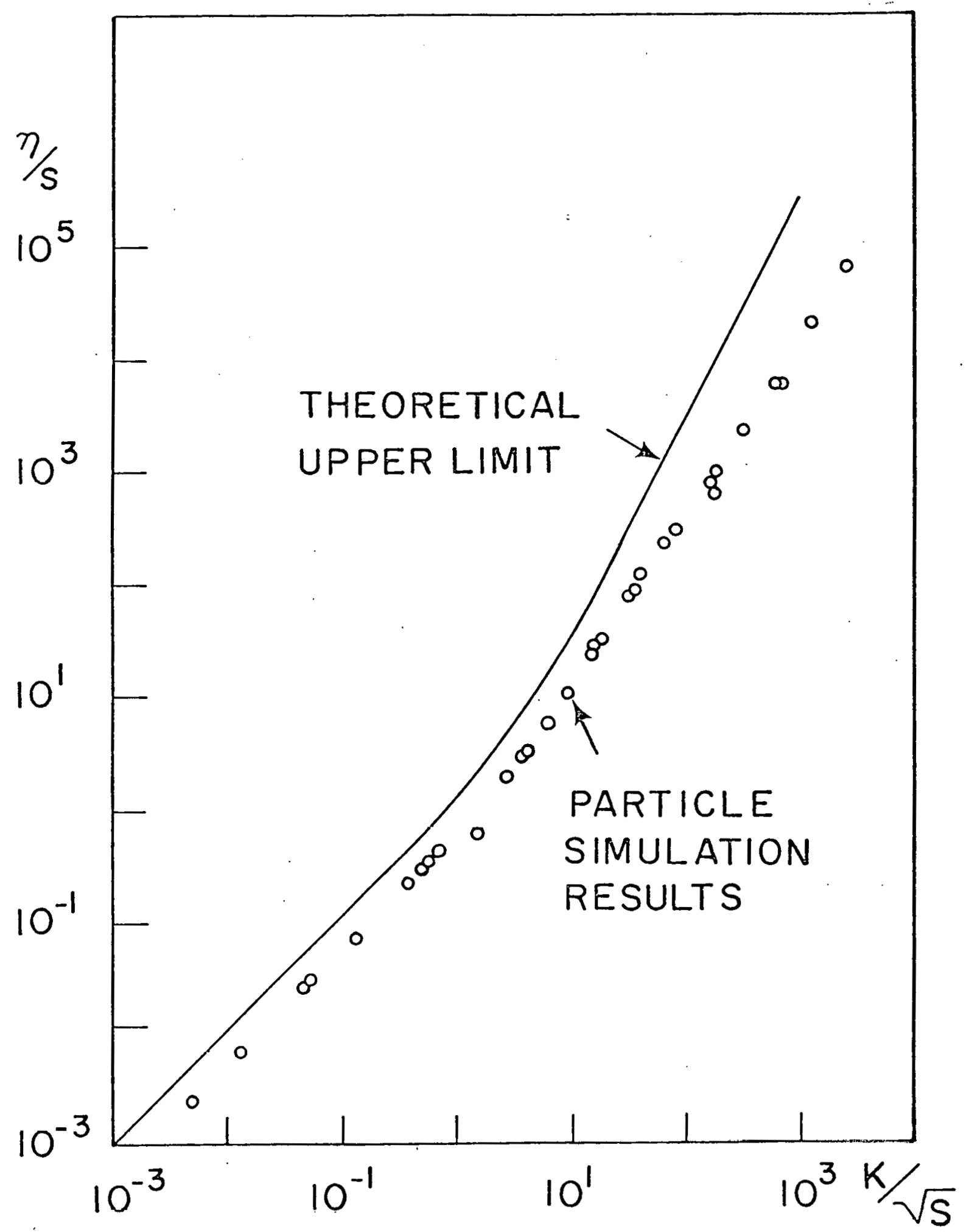

FIGURE 4.3-21 Maximum single pass fractional energy conversion for a constant period helix 


\subsection{Glass Laser Operation}

N. K. Moncur, M. A. Buoniconto, G. E. Busch, C. J. Edmonson,

C. M. Kinzer, W. W. Lawrence, F. J. Masters, R. G. Sanderson,

J. R. Vidolich and W. R. Wilson

The Nd:glass laser system as originally restaged in July of 1977 had internally damaged laser rods; funds were not available to replace these old rods. Early operation caused serious damage to the laser discs, and to the optical elements in the target bunker area. In September of 1977 the plasma spatial filter ${ }^{(1)}$ was re-installed in the system to retard further damage. In September of 1978, the $10 \mathrm{~cm}$ beam was expanded to $14 \mathrm{~cm}$ and two $14 \mathrm{~cm}$ amplifiers were added to each chain. In the following sections we will discuss the Nd:glass laser performance, some measurements of thermal heating effects, and our recent experience with disc cleaning and damage accumulation.

\subsubsection{Power On Target}

M. A. Buoniconto and J. G. Kubiak

The two-beam $10 \mathrm{~cm}$ laser system schematic is shown in Figure 4.4-1. Also shown are the amplifiers which were added in September of 1978 to increase power on target. The power on target for the $10 \mathrm{~cm}$ and for the $14 \mathrm{~cm}$ system is shown in Figure 4.4-2 as a function of the energy per 40 ps pulse into the first spatial filter. The power on target includes the optical losses for the target illumination system. Clearly the system is power saturated, as predicted by lumped-element computer codes which account for B-integral induced losses at spatial filter pinholes. The average power on target for 50 target shots during September and October, 1978 was 576 GW. 


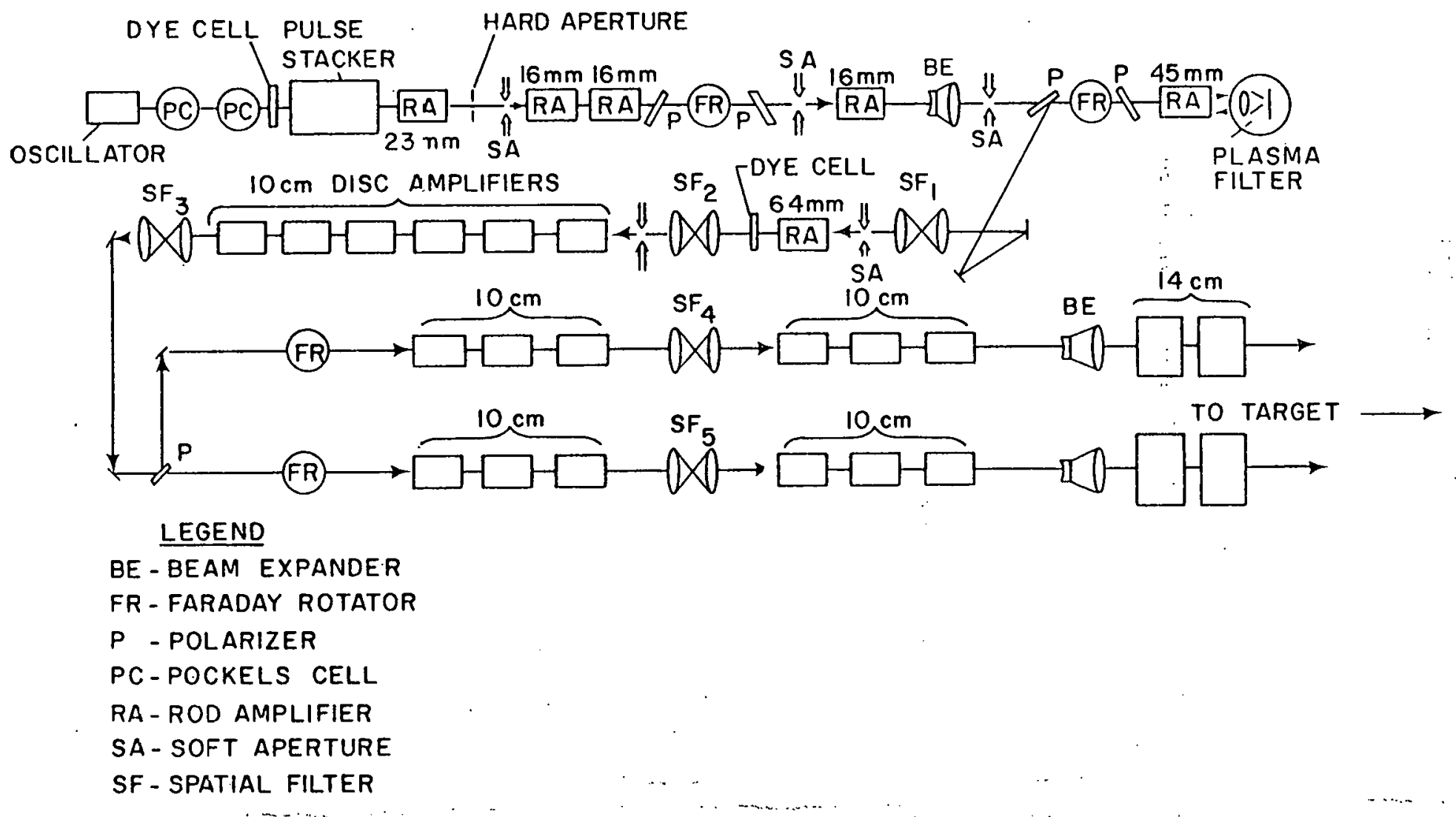

FIGURE 4.4-1 KMS laser configuration September 1978 with plasma filter 


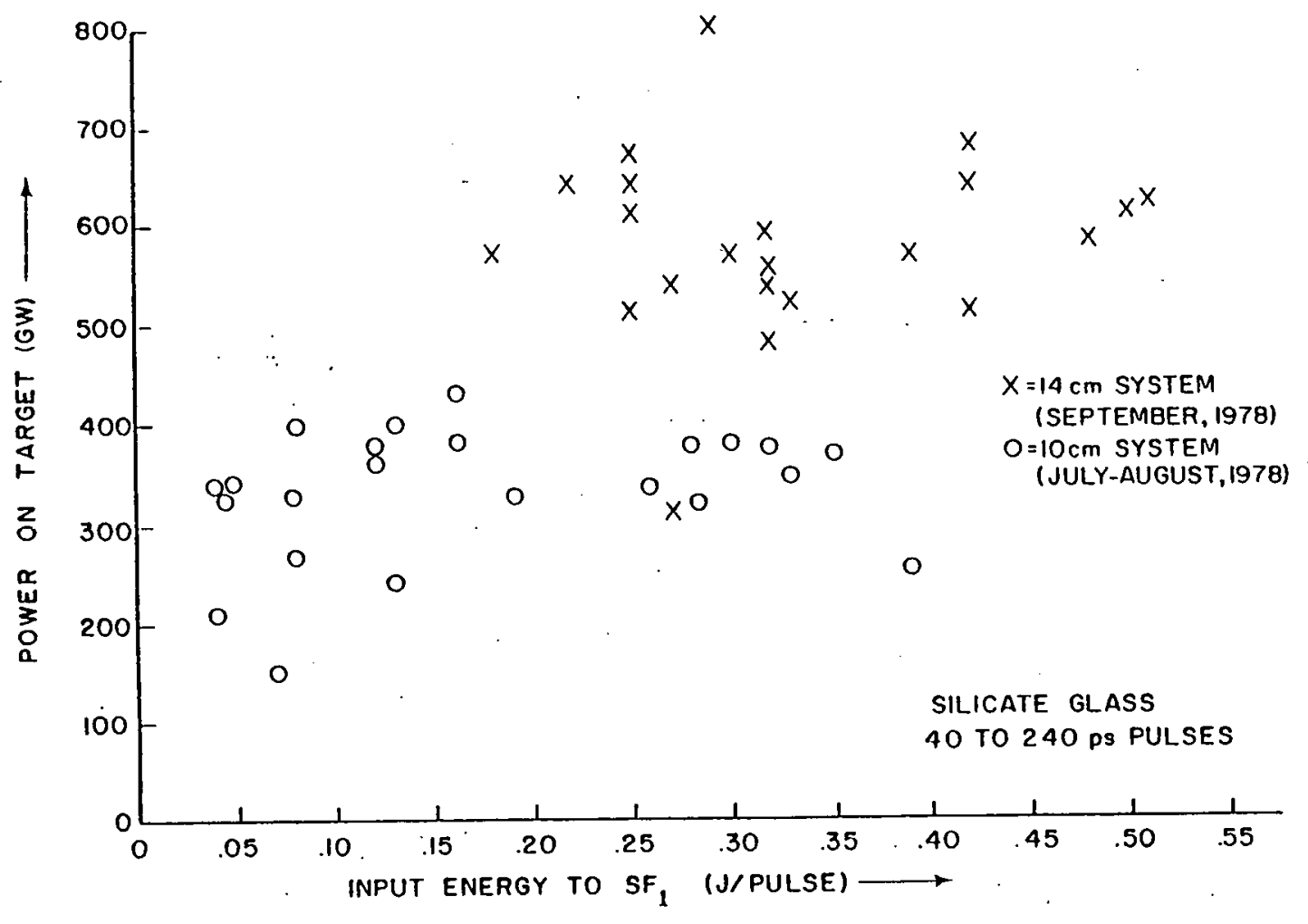

FIGURE 4..4-2 KMSF laser performance with the plasma spatial filter (input/output) 


\subsubsection{Far Field Data}

G. E. Busch, M. A. Buoniconto, G. C. Catella, and J. G: Kubiak The laser energy must be reasonably well-collimated to irradiate the $100 \mu \mathrm{m}$ type fusion pellets. The far-field camera records the incoming laser beam focal spot size on photographic film. This film is then developed, scanned, digitized, linearized, and integrated to yield estimates of the percentage of energy enclosed in spherical volumes at the target. The computer output for shot \#3981 is shown in Figure 4.4-3. The microdensitometer scan through the laser focal spot is shown in the lower left hand corner. After transformation through the film's $H$ and $D$ curve, the linearized focal spot is plotted in the lower right hand corner. The abscissa is in the microdensitometer distance units. This linearized function is then integrated assuming rotational symetry, with conversion to the target plane coordinates, as shown in the upper trace of Figure 4.4-3. The operator manually selects the $100 \%$ energy point by extrapolating this integrated curve prior to final plotting.

Representative far-field data are plotted in Figure 4.4-4 for recent target shots. All data represent the target diameters which would enclose $80 \%$ of the recorded energy on target. The horizontal lines indicate the cut-off diameter of the last pinhole in the laser chain. Energy ahnve these lines is therefore a direct result of nonlinear small-scale self-focussing. There are three discs amplifiers after this last pinhole spatial filter in the $10 \mathrm{~cm}$ system, and five amplifiers for the $14 \mathrm{~cm}$ system. 
)39BLFF

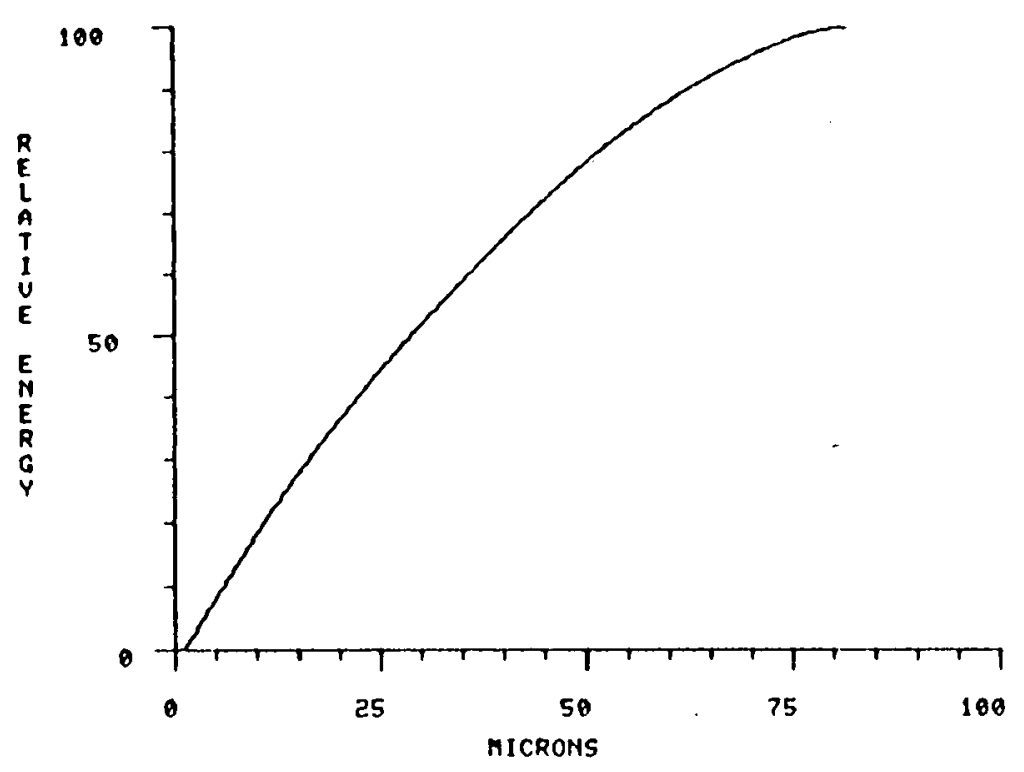

ì
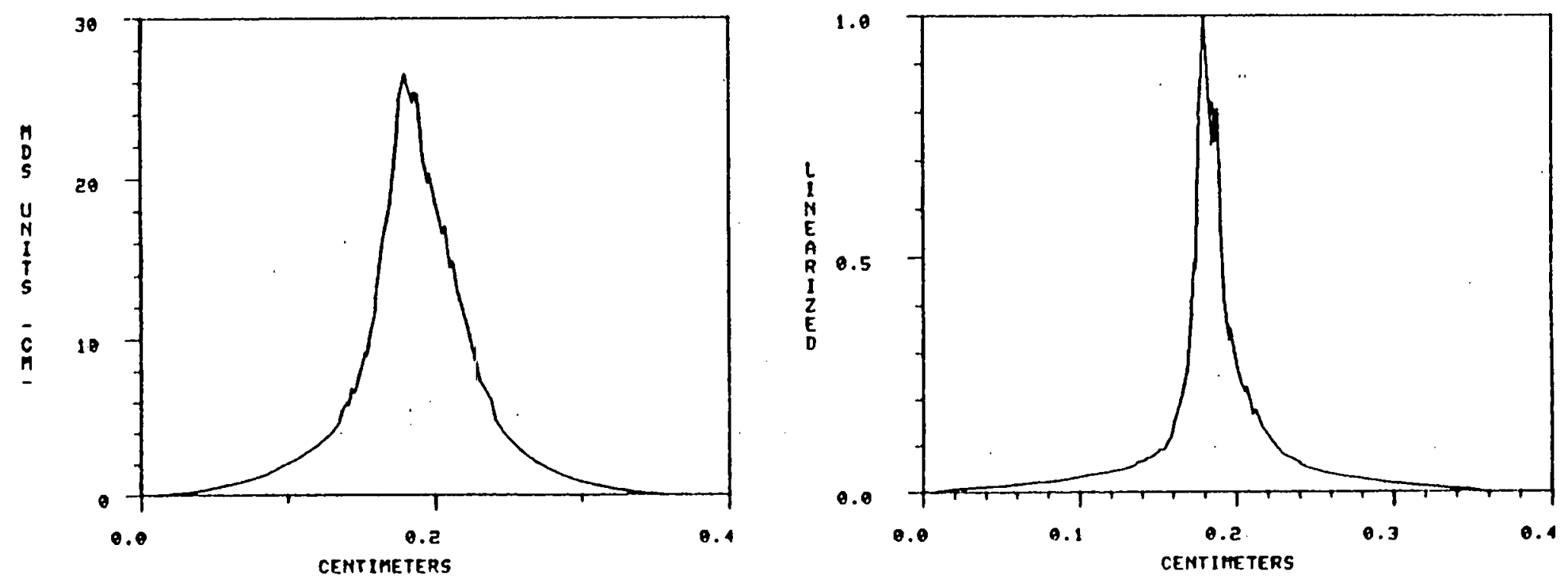

FIGURE 4.4-3 Computer output format for far-field image calculations 


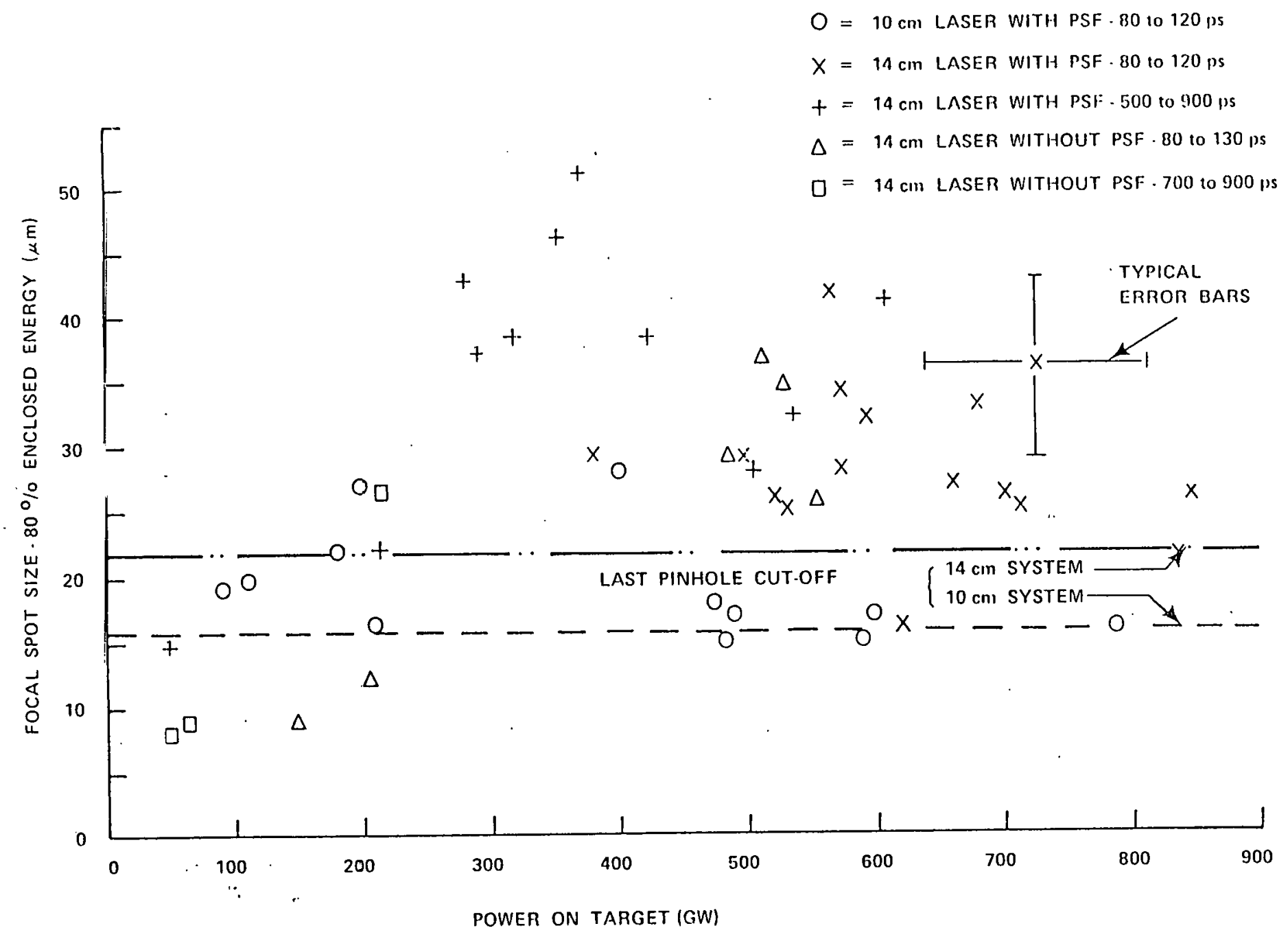

FIGURE 4.4-4 Laser focal spot size (80\% enclosed energy) at the target as a function of power on target 


\subsubsection{Near Field Data}

G. C. Catella, M. A. Buoniconto and J. G. Kubiak

Initial attempts to record near field images through a nonimaging Galilean 4:1 beam reducer were not successful, since the reduced images had significantly different spatial structure than the actual $14 \mathrm{~cm}$ laser beam. A relay lens system is necessary to reproduce the near field intensity distribution in the laser beam. But any image relay system necessarily produces a focal spot, so a vacuum cell was also required to prevent air breakdown.

The resulting 4:1 reduced images are recorded on Eastman Kodak High Speed Infrared film.

One dimensional microdensitometer scans of the near field films are scaled to full size and digitized. The Digital Equipment Corporation PDP $11 / 45$ then transforms the data through the appropriate $H$ and $D$ curve, corrects for any extraneous fog level, and then linearizes and normalizes the data. At this point a two dimensional circular approximation is made and an integration of energy versus radius performed.

A standard deviation is derived from the data across the high intensity portion of the beam to provide an objective measure of beam breakup. (see Figure 4.4-5) The "average" is obtained by averaging all of the data points (800/scan) and determining a standard deviation. The standard deviation is subtracted from the first average. Only the data between the two intersections of this line and the near-field curve is reaveraged and a new standard deviation calculated. These are the solid and dotted lines respectively in Figure 4.4-5. Since the "average" is an arbitrary value for this non-periodic function, a peakto valley variation is quoted instead of peak to average. Twice the standard deviation may be considered the average ripple or noise in 


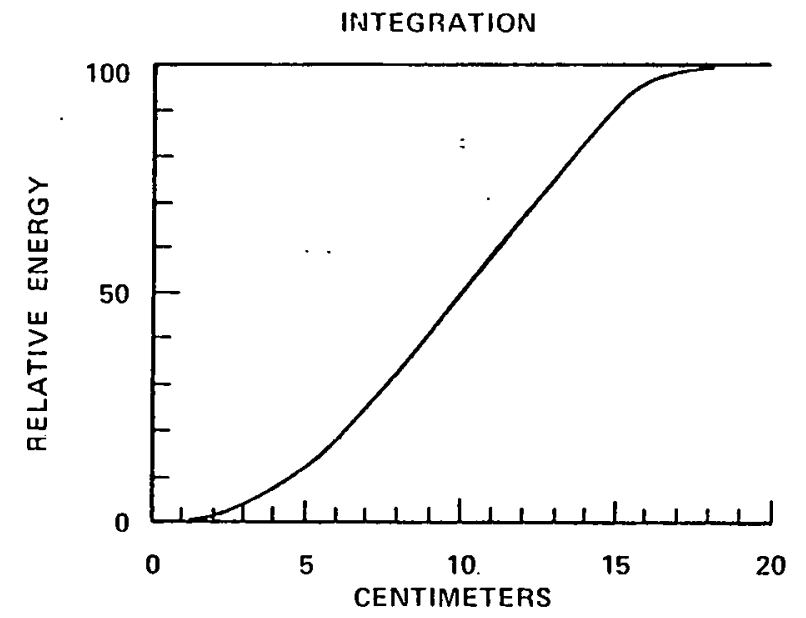

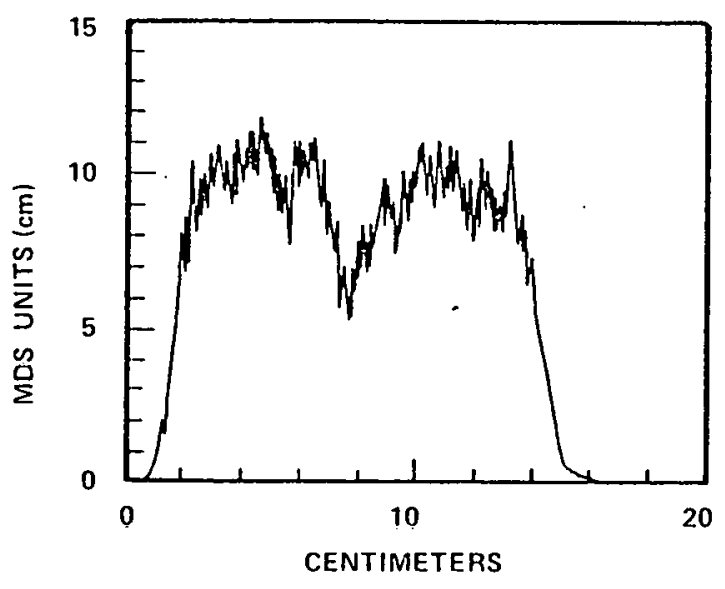

RAW DATA

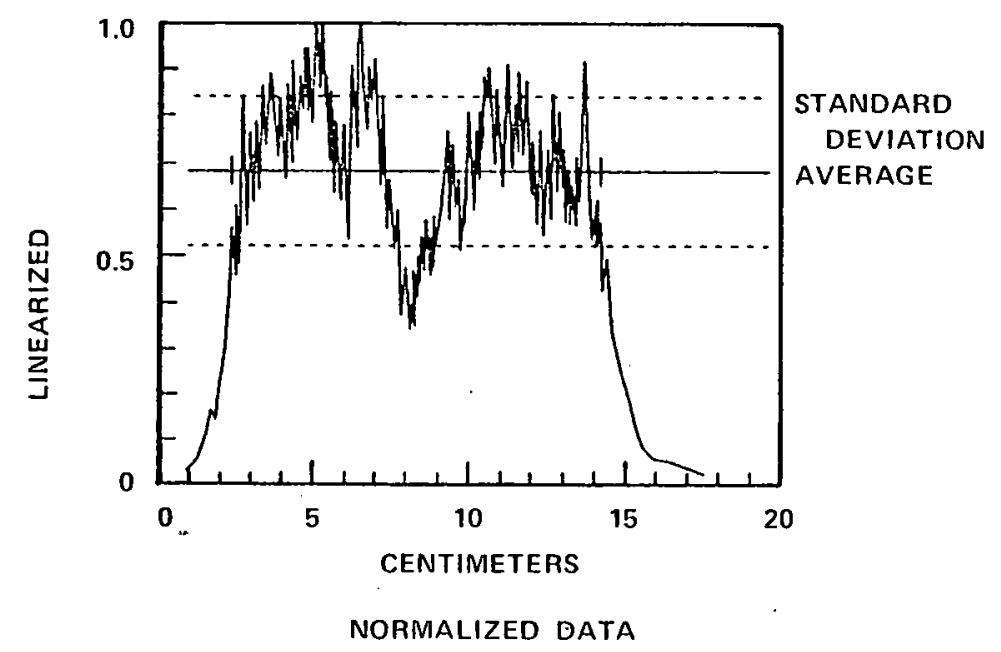

FIGURE 4.4-5 Computer output for near-field data analysis 
the beam. This peak to valley value, with the standard deviation or average ripple, is indicative of beam breakup.

When the plasma spatial filter was used to prevent pre-pulses, a small central area of the laser beam was blocked. An altered approach is required for reducing this annular field data. Data from the "hole" significantly increases the standard deviation compared to the actual standard deviation over the active annular area. To correct this distortion, the standard deviation is obtained separately for the two "halves" of the annulus (see figure 4.4-6). These are averaged to obtain a single value, which more accurately reflects the small-scale self-focusing present in the laser beam.

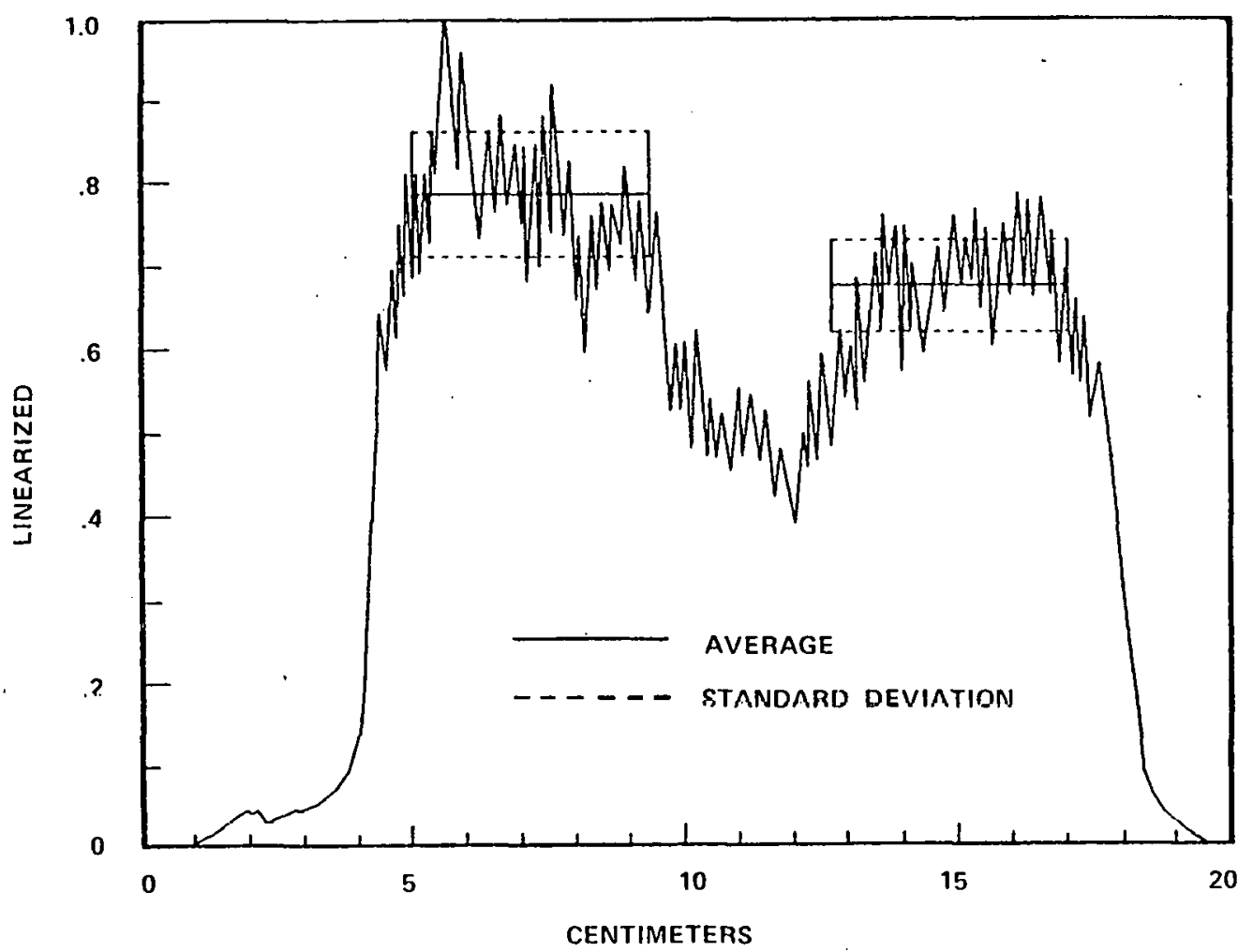

FIGURE 4.4-6 Near field data analysis for an annular laser beam 
The peak-to-valley beam quality is shown in Figure 4.4-7 for the $14 \mathrm{~cm}$ laser system with the plasma spatial filter. The peak to valley values average $1.25 \mathrm{~dB}$ over a wide range of peak power delivered on target.

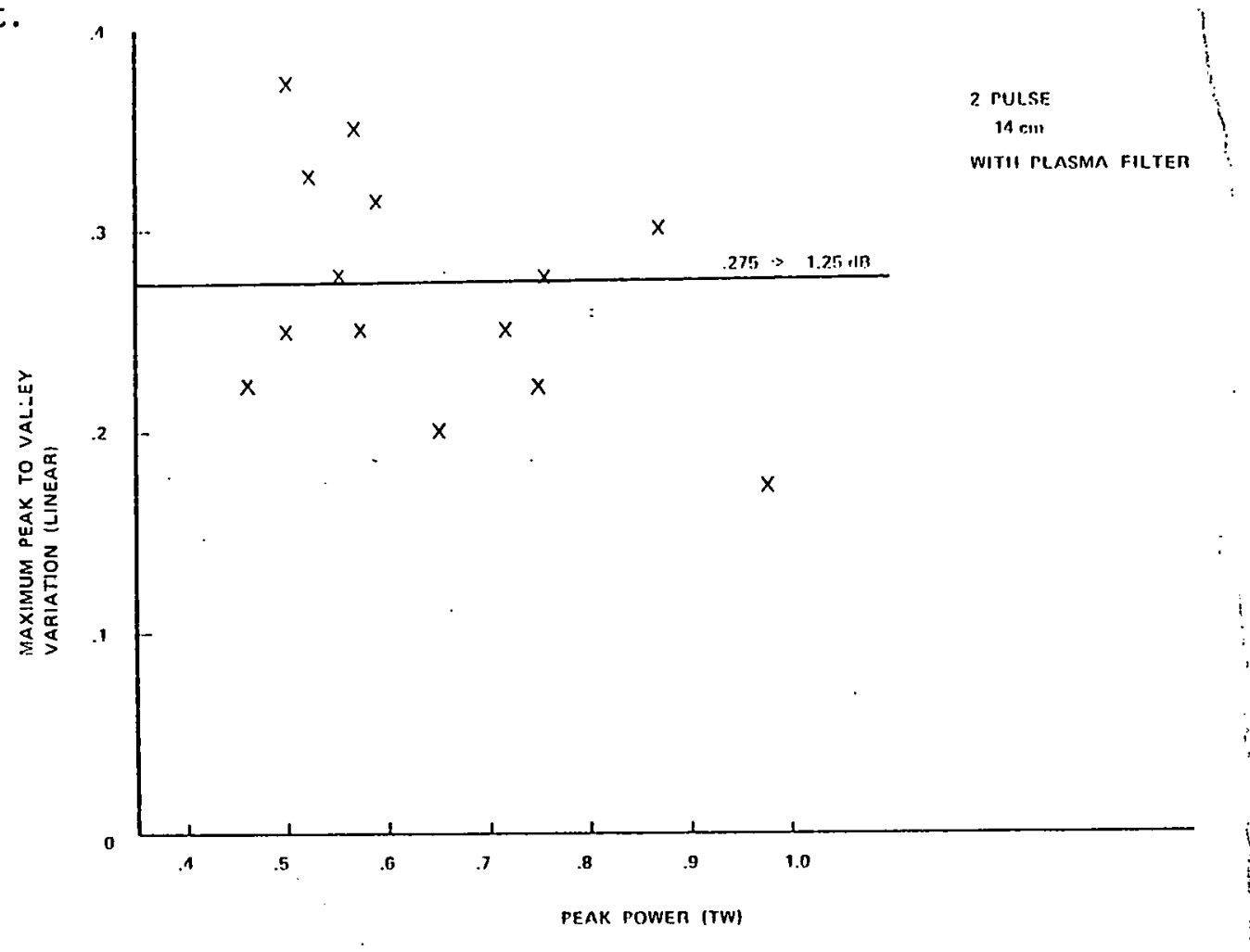

FIGURE 4.4-7 Near Field Peak-To-Valley Data With Plasma Spatial Filter.

The standard deviation figure-of-merit for near field beam quality is plotted in Figure 4.4-8 for the $14 \mathrm{~cm}$ laser system operating with long and short pulses and with and without the plasma spatial filter.

For short pulse experiments with the plasma spatial filter, the least mean square fit indicates that beam quality is improving with peak power. If the plasma spatial filter is turned off the beam quality degrades. Similar behavior is present in the long pulse data.

In all operating regimes the average beam break up or ripple is less than $30 \%$, with a value of $15 \%$ being representative for long pulse operation. 


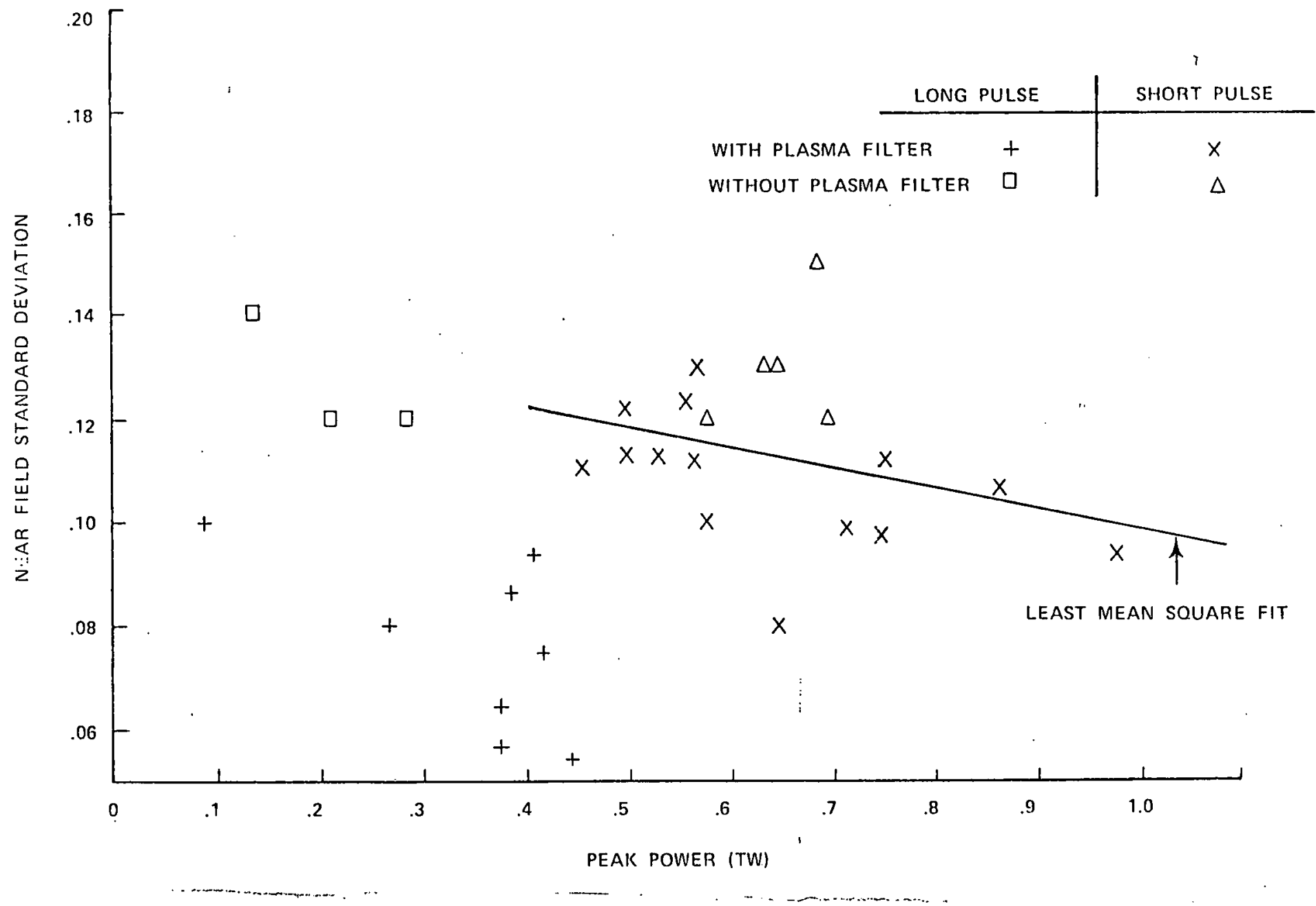

FIGURE L.4-8 Near field standard deviation 


\subsubsection{Disc Thermal Heating Effects}

R. D. Sigler

In the 1977 Annual Report, we showed that significant thermal distortion in the laser disc amplifiers persisted for up to four hours after a shot ${ }^{(20)}$. At that time we assumed that thermal distortions of the laser disc caused much of the optical wavefront perturbations. Subsequent experiments have shown, however, that even air gradients inside the disc amplifier enclosure can distort the beam for up to $1 / 2$ hour after the flashlamps are fired. We also found that water cooled flow tubes placed around the flashlamps reduced the thermal distortion time down to one hour.

To test the effects of air gradients, the laser discs were removed from a $10 \mathrm{~cm}$ amplifier, and the ends of the pyrex cylinder were enclosed with mylar. The air temperature inside the pyrex cylinder was then measured as a function of time after the flashlamps were fired. As shown in Figure 4.4-9, the air temperature remained $1^{\circ} \mathrm{C}$ above ambient for over two hours without air flow, and for 30 minutes with 4 cubic feet per minute (CFM) nitrogen flow through the flashlamp area surrounding the pyrex tube. Figure 4.4-10 shows the corresponding focal spot degradation of a Krypton laser beam (647 nm) which was passed. five times through this empty head. The spot growth ratio plotted in Figure 4.4-10 is the ratio of the square root of the sum of the squares of the maximum focal spot diameters taken in two orthogonal directions (to account for astigmatism), divided by the same quantity before the flashlamps were fired. 


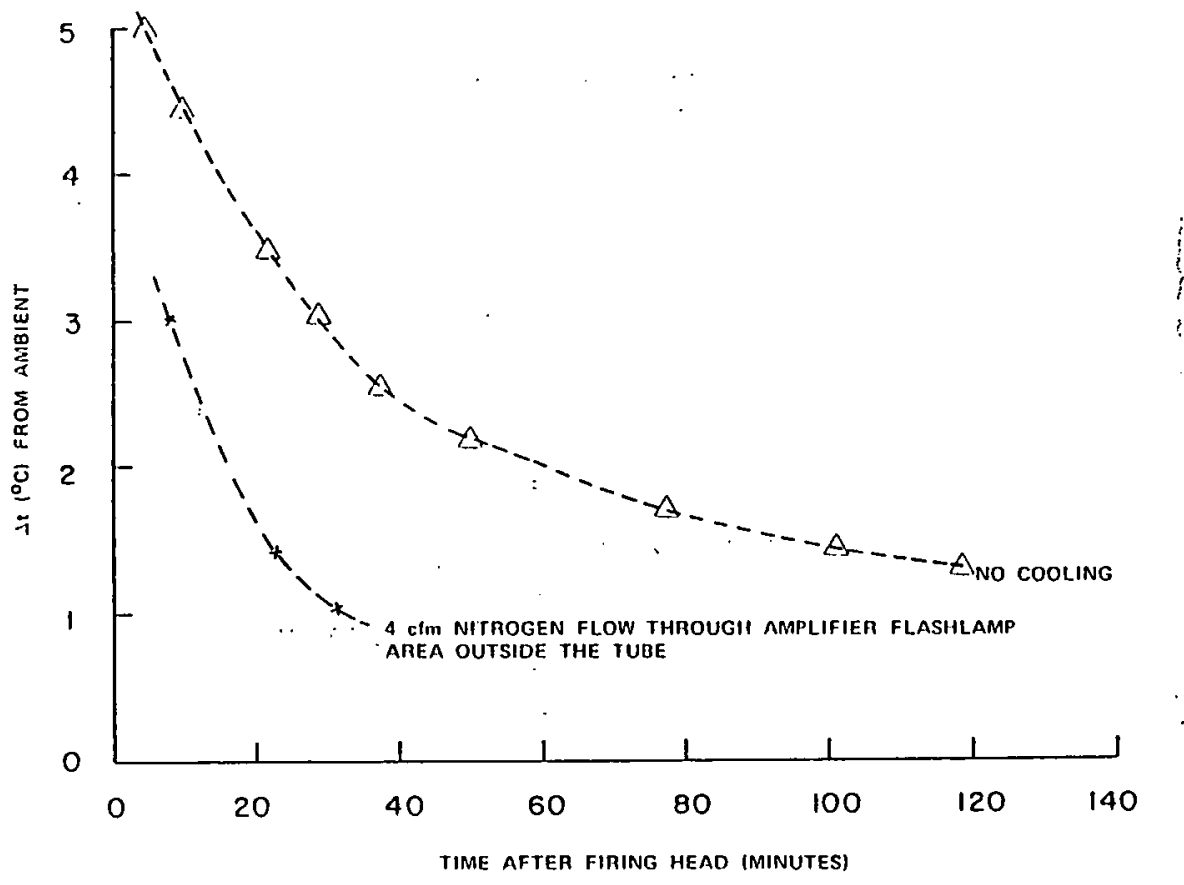

FIGURE 4.4-9 Temperature history inside amplifier: head without laser discs

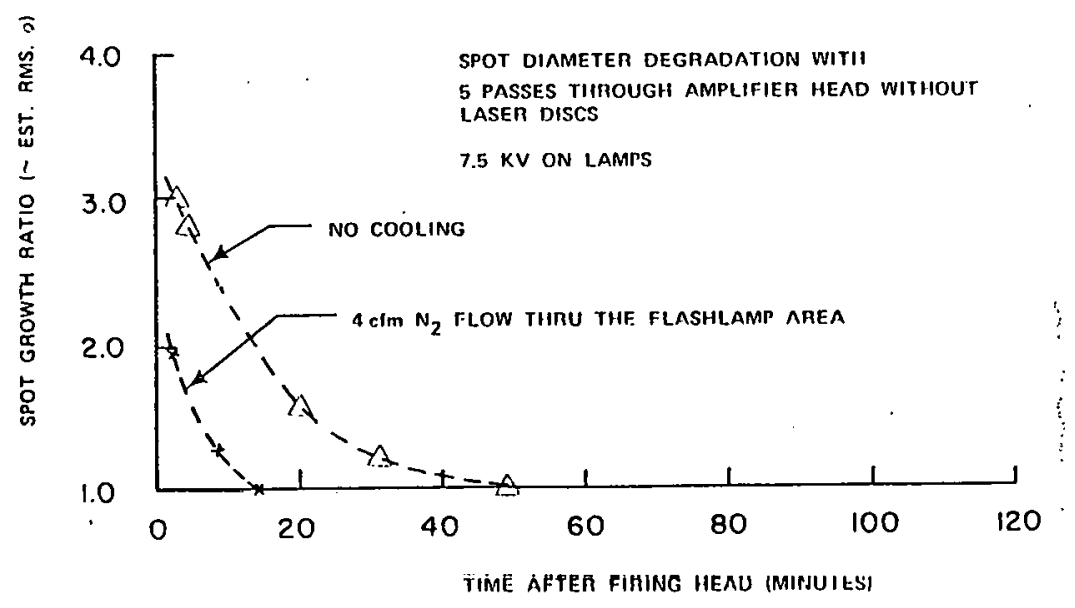

FIGURE 4.4-10 Ratio of laser spot size after flashlamp firing to spot size before firing 
Next the three $10-\mathrm{cm}$ laser discs were reinstalled in the amplifier, and the tests were repeated. Figure 4.4-11 shows the temperature history after flashlamp firing for the case of no cooling, cooling the flashlamp area with a 120 c.f.m. fan, and adding water-cooled flow tubes around the flashlamps. The corresponding beam spot size growth ratio is plotted in Figure 4.4-12, for these three cooling conditions. These data indicate that the water-cooled flow tubes are most effective in returning the beam to its original condition, and one hour cooling is sufficient. Tests with both flow tubes and cooling air showed no improvement over flow tubes alone. We therefore reinstalled the water cooled flow tubes on all disc amplifier flashlamps.

\subsubsection{Laser System Cleanliness}

B. W. Loughry, G. C. Catel11a, H. A. Laskowske, W. R. Wilson. All sources of spatial noise must be minimized for best performance of a high power laser system. Even though spatial filters remove high spatial frequency light ripples, there are always low frequency ripples which will pass through the spatial filter pinholes and which will limit system performance. Undesireable amplitude and phase modulation of the laser beam can originate from particulate contamination in the laser beam, and from damage in or on the glass. surfaces. Clean room techniques were instituted last year to improve system performance ${ }^{(21)}$. Our major conclusions after a years' operating experience under "clean conditions" are as follows:

1. The average noise level due to disc surface damage (as measured by per cent obscuration of the beam) increases at the rate of about $0.004 \% /$ shot. 


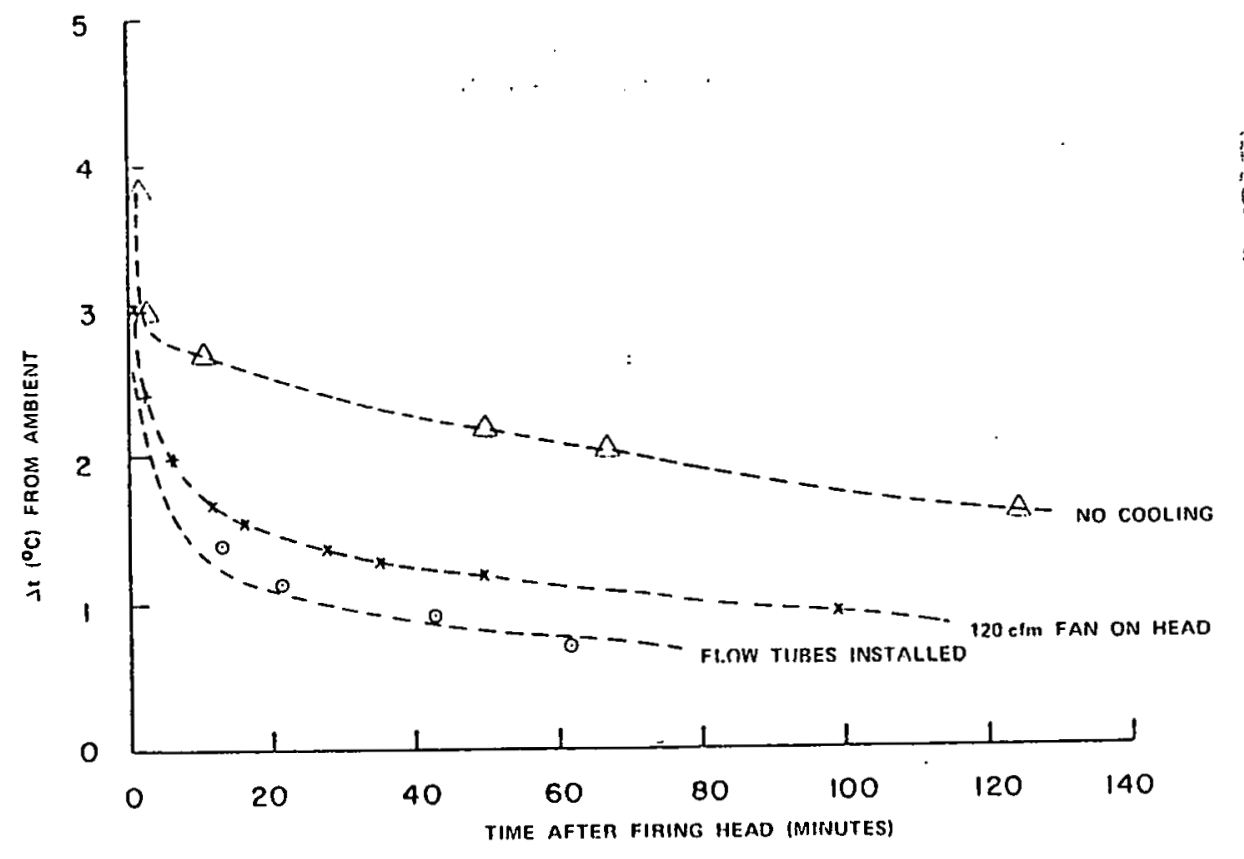

FIGURE 4.4-11 Temperature history at edge of laser discs after flashlamp firing

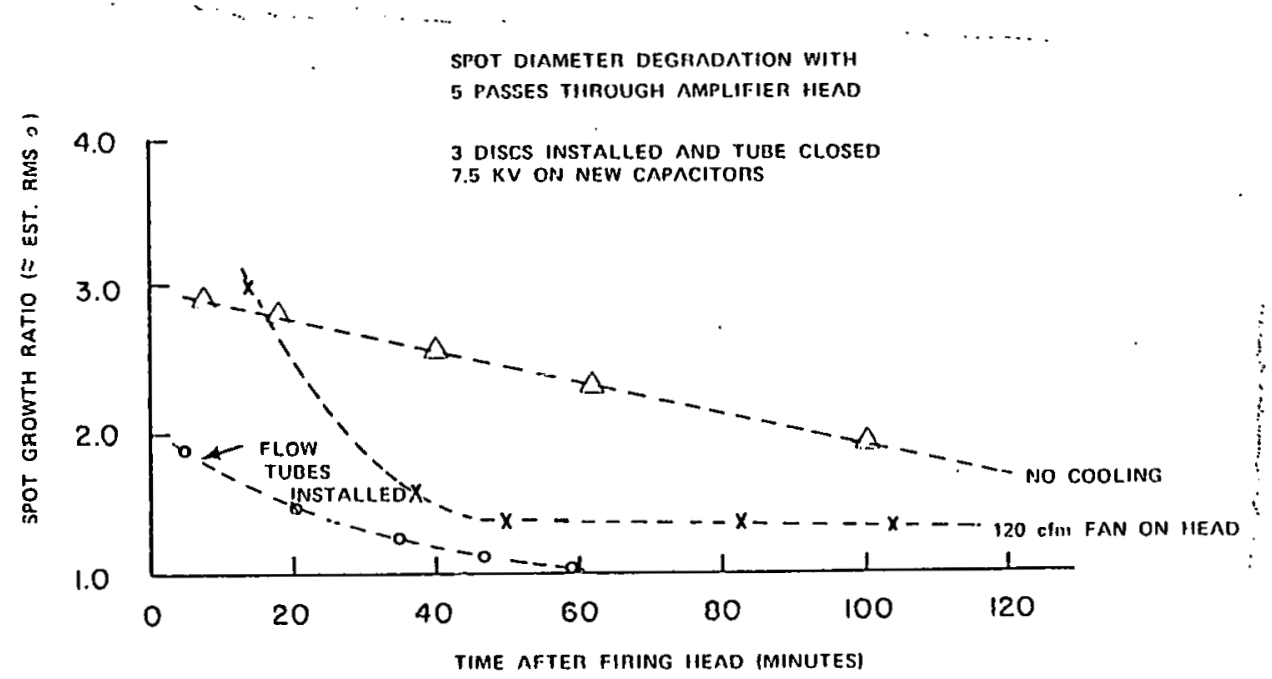

FISU? $4.5-12$ Laser beam focal spot degradation with laser discs after flashlamp firing 
2. The laser discs must be repolished periodically to maintain less than $1.5 \%$ obscuration due to surface damage.

3. The total beam obscuration (due to both damage and particulate contamination) is independent of the number of shots for the first 100 shots following cleaning, averaging about $1.25 \%$ of the beam area.

4. The airborne particulate contamination level inside the disc amplifier immediately after each shot increases dramaticaliy following the installation of a cleaned amplifier, and falls gradually over hundreds of shots.

5. Nitrogen purging of the amplifier beam path does not significantly reduce obscuration growth rate on the disc surfaces.

These conclusions are discussed in more detail in the following paragraphs:

The disc amplifiers are routinely removed from the laser and recleaned in a Class 100 clean room. The condition of each laser disc is estimated before and after cleaning by microscopically examining several areas on both sides of the discs. By counting the number of obscured areas before cleaning, we can estimate the percentage of the beam blocked by damage sites and contamination. After cleaning, another count establishes the percent area blocked by accumulated damage plus any residual particulate contamination after the cleaning operation.

The obscuration data presented here are extrapolated from one amplifier to the entire laser chain: We calculate the percentage of the beam which would be obscured if every amplifer had the same noise level as the one measured. To simplify the measurement procedure, we count the number of particles and damage sites, but we do not measure 
their sizes. The obscured area is then estimated from a previous detailed analysis of the average size of 513 damage sites measured on two representative laser discs. The obscuration data therefore assume that the obscuration sites are uncorrelated and also that the average particle size is equal to the average damage site size. The obscuration due only to surface damage on the laser discs is plotted in Figure 4.4-13 as a function of the number of flashlamp shots after cleaning. Each data point is based on damage site counts taken on three surfaces, with the upward facing surfaces (set at Brewster's angle with $45^{\circ}$ polarization) averaged together, and the downward facing surfaces averaged together. While there is wide scatter, these data strongly suggest that particulate matter settling on the upward facing ("top") surfaces leads to increased surface damage on these faces. Averaged over both top and bottom surfaces, the system damage obscuration accumulates at the rate of about .004\%/shot.

To remove this surface damage, we began repolishing laser discs in March, 1978. As shown in Figure 4.4-14, the system obscuration level due to surface damage fell from about $1.5 \%$ to 1 ess than $1.2 \%$ after repolishing.

If we include particulate contamination and surface damage, the percentage area obscured fluctuates dramatically from amplifierto-amplifier with an average system obscuration level of $1.25 \%$ as shown in Figure 4.4-15. For the first one hundred shots, then, particulate contamination dominates surface damage, and any increase due to accumulated surface: damage is masked by the particles prior to disc cleaning. 


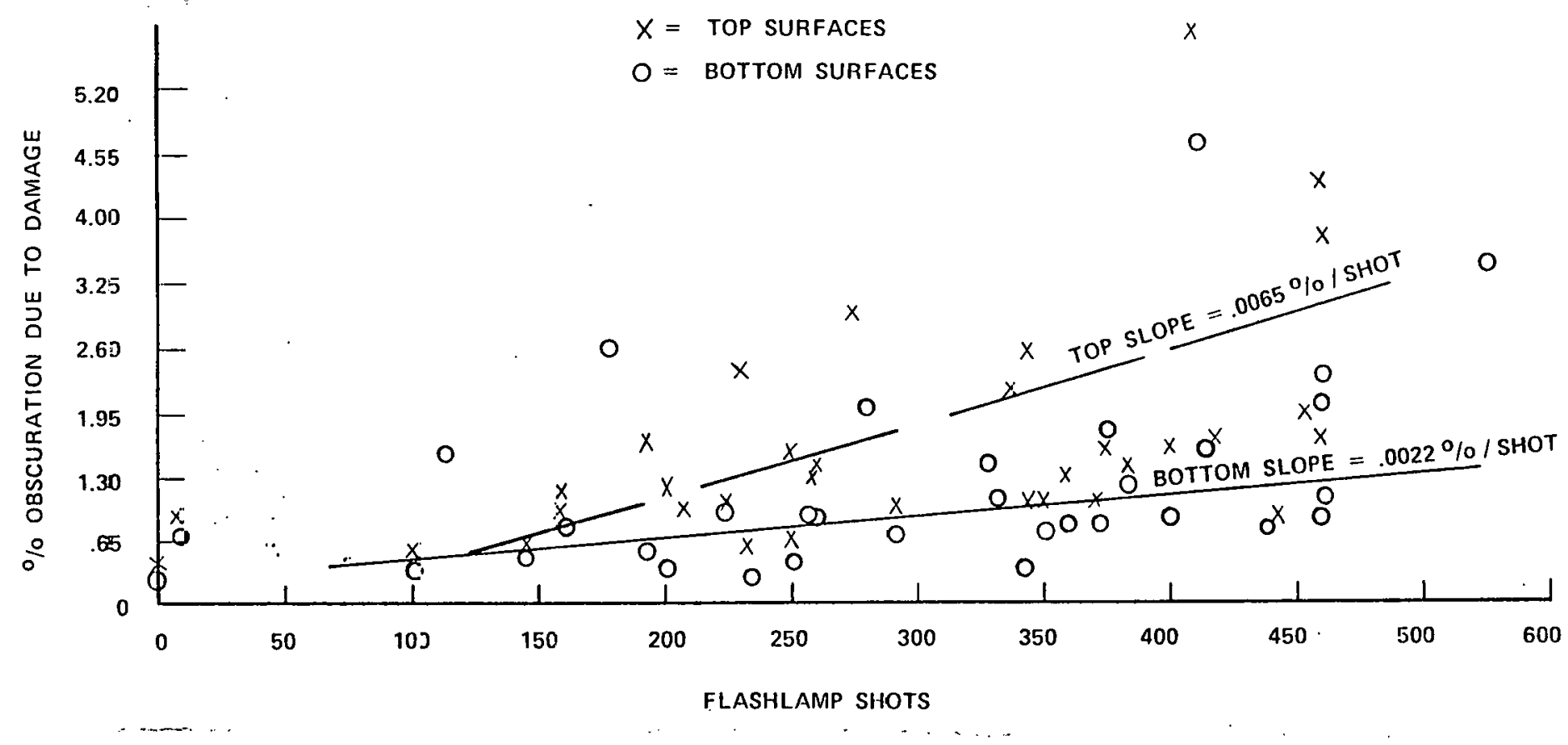

FIGURE 4.4-13 Beam obscuration due to disc surface damage as a function of the number of shots after cleaning 


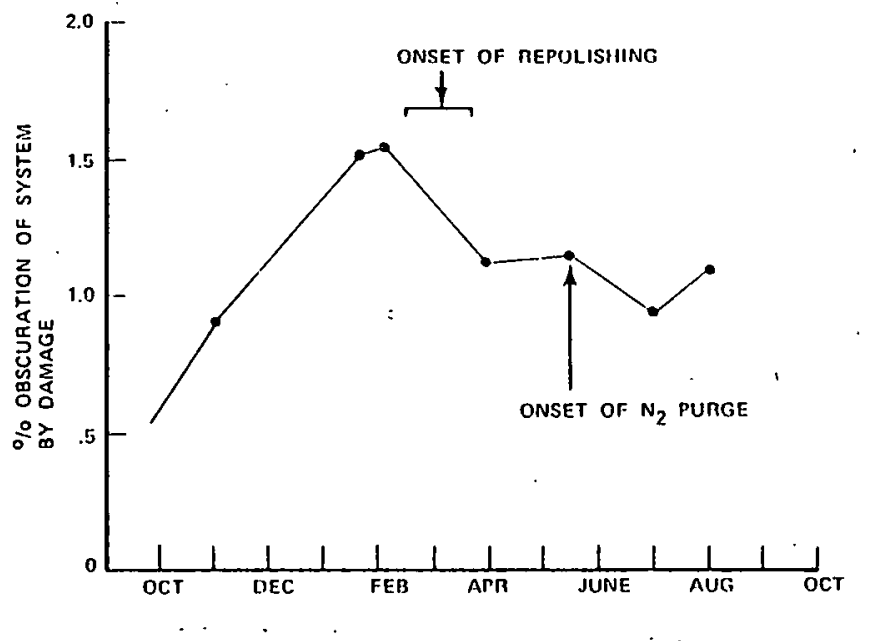

FIGURE 4.4-14 Laser system obscuration due to laser disc surfaca damage

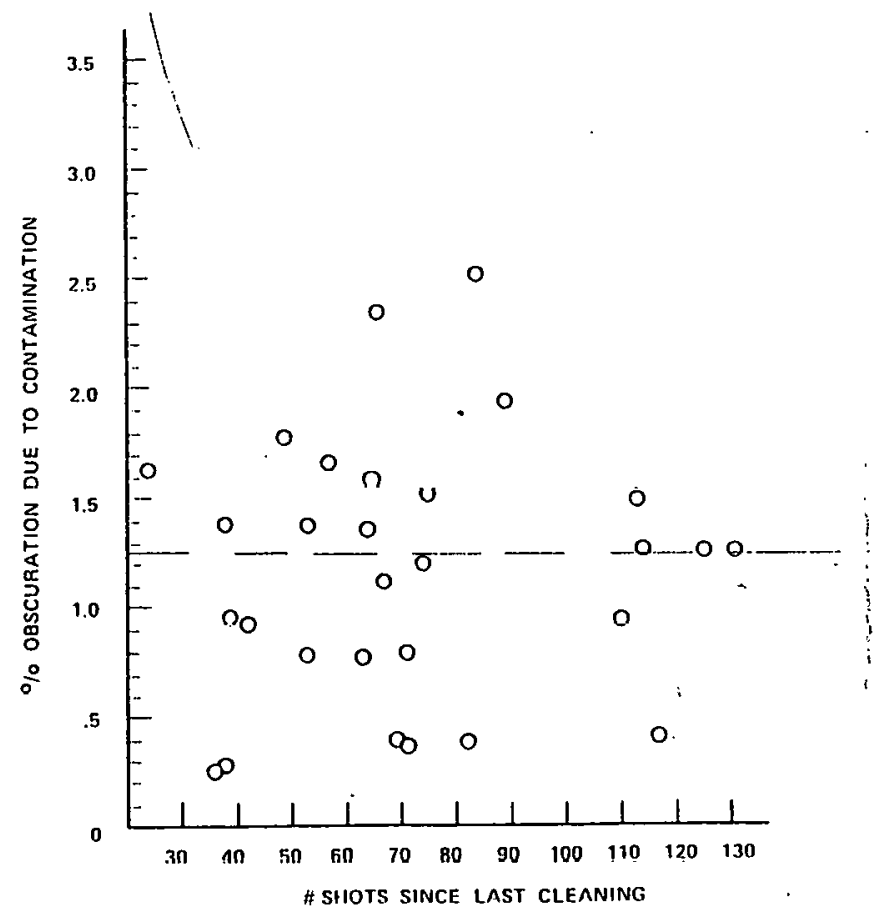

FIGURE 4.4-15 Total system obscuration due to disc surface damage and contamination without nitrogen purge 
To check the airborne particulate contamination inside the disc amplifiers, a particle counter probe was inserted into the beam enclosure area. A wide range of contamination from 600 particles per cubic foot (PPCF) to 13,000 PPCF was detected. (The laboratory air is typically about 1000 to 25000 PPCF; the laser amplifiers are assembled inside a clean room with less than 100 PP.CF). The unexpectedly high contanination levels suggested a series of beam enclosure particulate contamination tests extending over about 140 laser firings.

The test configuration is shown in Figure 4.4-16. Initially, a nitrogen purge was not used; the probe measured only contamination generated near the sampling point. Starting with shot \#3458.2, a steam of filtered nitrogen, at about $32 \mathrm{CFH}$ (cubic feet per hour) forced the particle laden air to pass the sampling probe. The flow rate within the enclosure was about 1.5 feet per minute. The nitrogen was left on continuously (except for some weekends) until shot \#3514, after which it was turned on immediately after a shot, while particulate data were being collected, and turned off about $1 / 2$ to 1 hour after the shot, to avoid disc thermal problems caused by the relatively cool nitrogen gas. Beginning with shot \#3550, the nitrogen purge was left on only during the data taking interval (12 or 18 minutes after each shot.)

A contamination meter which draws in and counts all airborne particles 0.5 micron and larger samples the air within the beam enclosure. After each laser shot, a 0.01 cubic foot sample was withdrawn and counted each 36 seconds. The data for each shot generally showed an increase in contamination immediately after the shot, with a subsequent slow (10 to 20 minutes) decrease to a relatively steady 


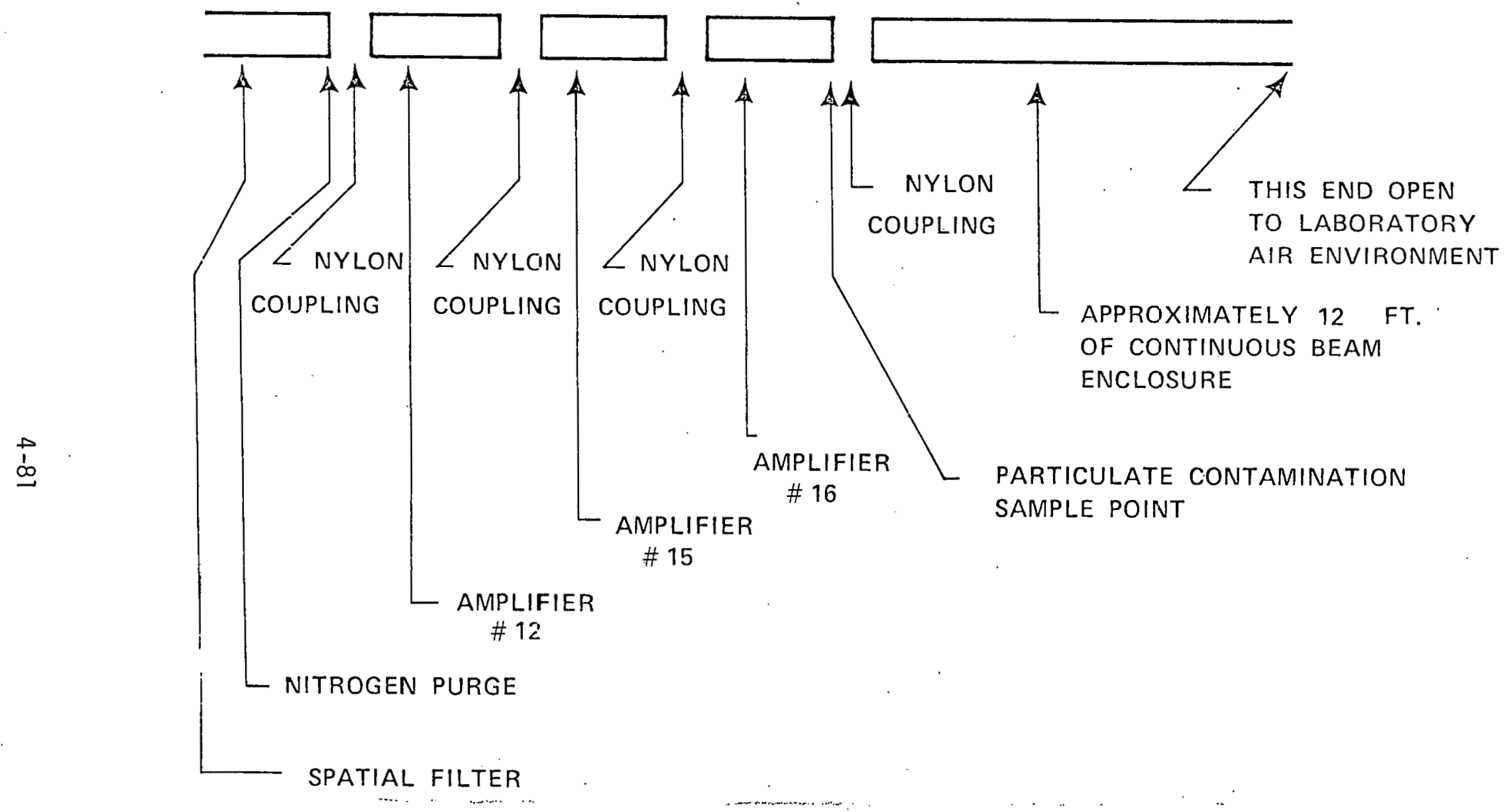

FIGURE 4.4-16 Beam enclcsure internal particulate contamination test configuration 


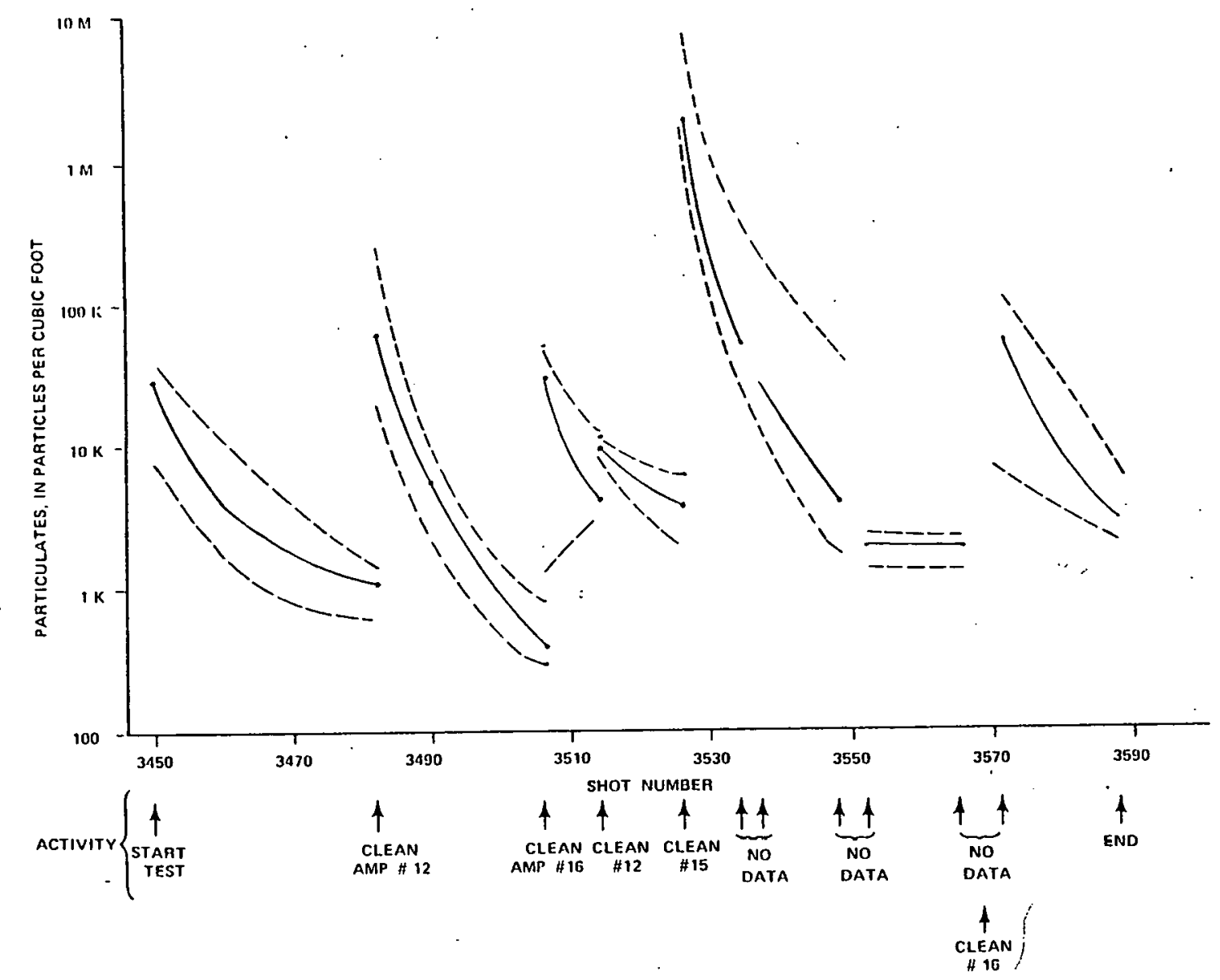

FIGURE 4.4-17 Airborne particulate contamination history inside

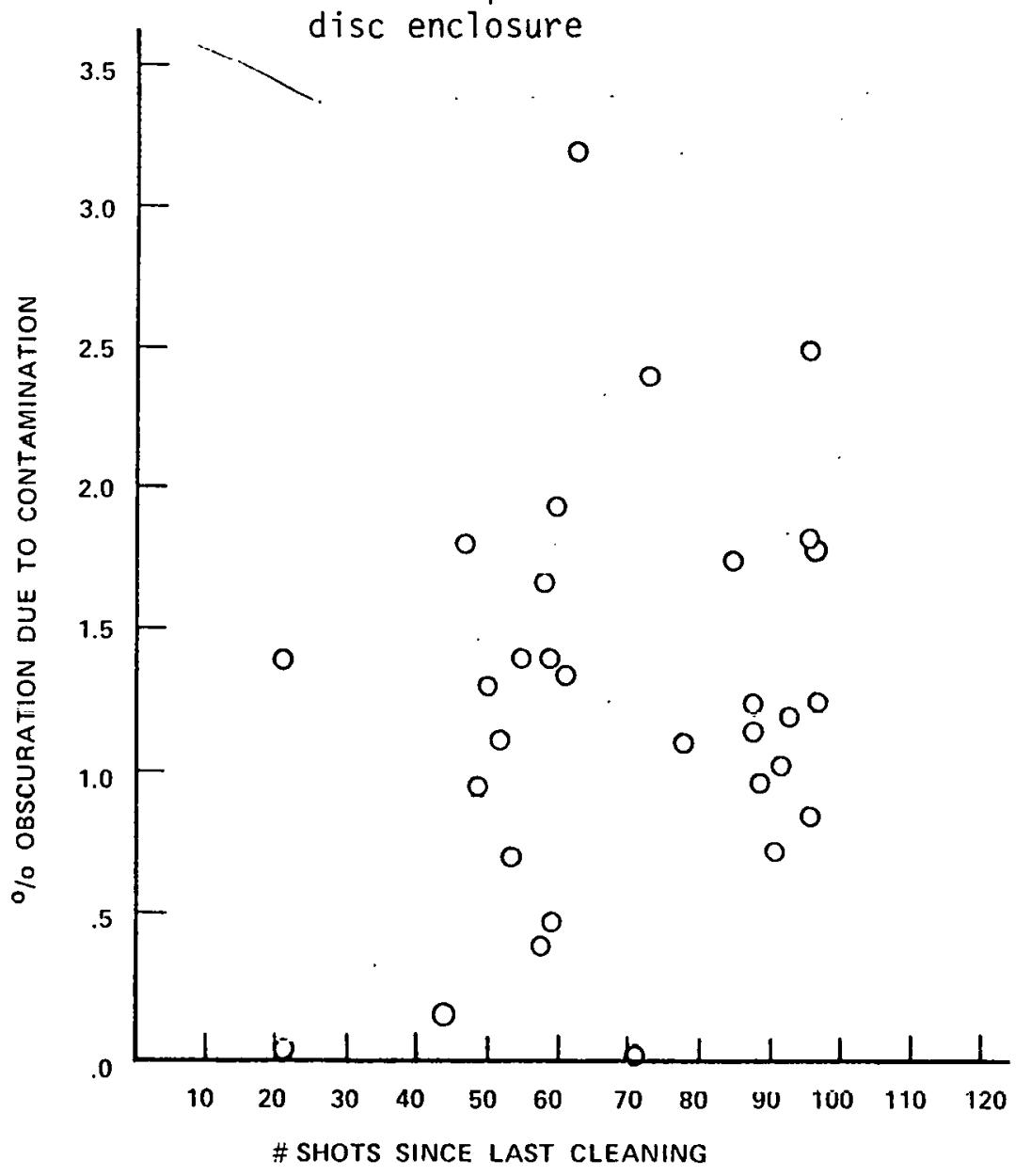

FIGURE 4.4-18 Total system obscuration due to disc surface damage and contamination with nitrogen purge 
level. The shot to shot data also showed a trend, indicated in Figure 4.4-17, characterized by very high contamination levels on the first shot after the installation of a cleaned amplifier, with a rapid decline on subsequent shots. In Figure 4.4-17, the average contamination level for the first six minutes after each shot was calculated and plotted as solid lines while the dashed lines indicate the data scatter.

Figure 4.4-17 shows that peak contamination levels rose several orders of magnitude on the first shot after a newly cleaned amplifier was installed. Presumably, the flashlamps blow the fine particlate matter off of the disc and amplifier enclosure surfaces. These very high peak levels rapidly (a few shots) diminished to much lower levels and continued a gradual decline.

As discussed above, several tests were run with and without nitrogen purging to determine if a 1.5 feet per minute flow would retard disc surface contamination. No improvement in surface contamination has been demonstrated. Figure 4.4-18 shows the total obscuration with nitrogen purging of the amplifiers; the average level is about $1.25 \%$, unchanged from the data without nitrogen purging. Similarly, Figure 4.4-19 shows limited data on damage obscuration with the nitrogen flow, averaged over both top and bottom surfaces (each data point represents the average of the six surfaces of the three discs per amplifier). The rate of increase of about $.004 \% /$ shot is similar to the increase without nitrogen flow. 


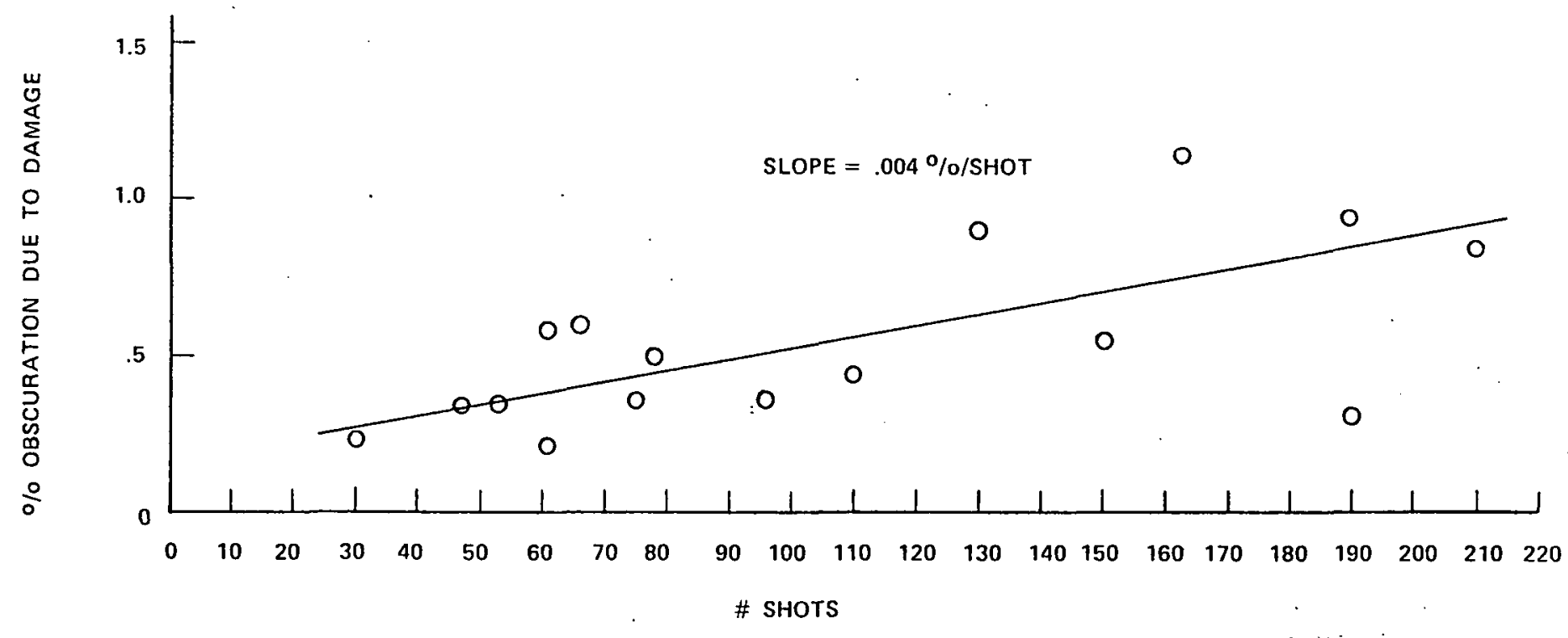

FIGURE 4.4-19 Surface damage on $10 \mathrm{~cm}$ laser discs with nitrogen purge

\subsection{TW Laser Upgrade}

C. E. Thomas, B. W. Loughry, D. L. Melmoth, N. K. Moncur, L. D. Siebert and R. D. Sigler

The project to upgrade our Nd:glass laser to 2 TW was started on August 1, 1978. The basic 2 TW system (Figure 4.5-1) was designed last year; (22) it includes increased disc amplifier pump power, conversion to phosphate laser glass, an actively mode-locked oscillator, and an all-reflective target illumination system. We plan to utilize the plasma spatial filter to retard damage, and two extra spatial filters have been added to relay the hard aperture image to the target chamber area. Early results on the disc amplifier modifications including conversion to phosphate laser glass are described below. 


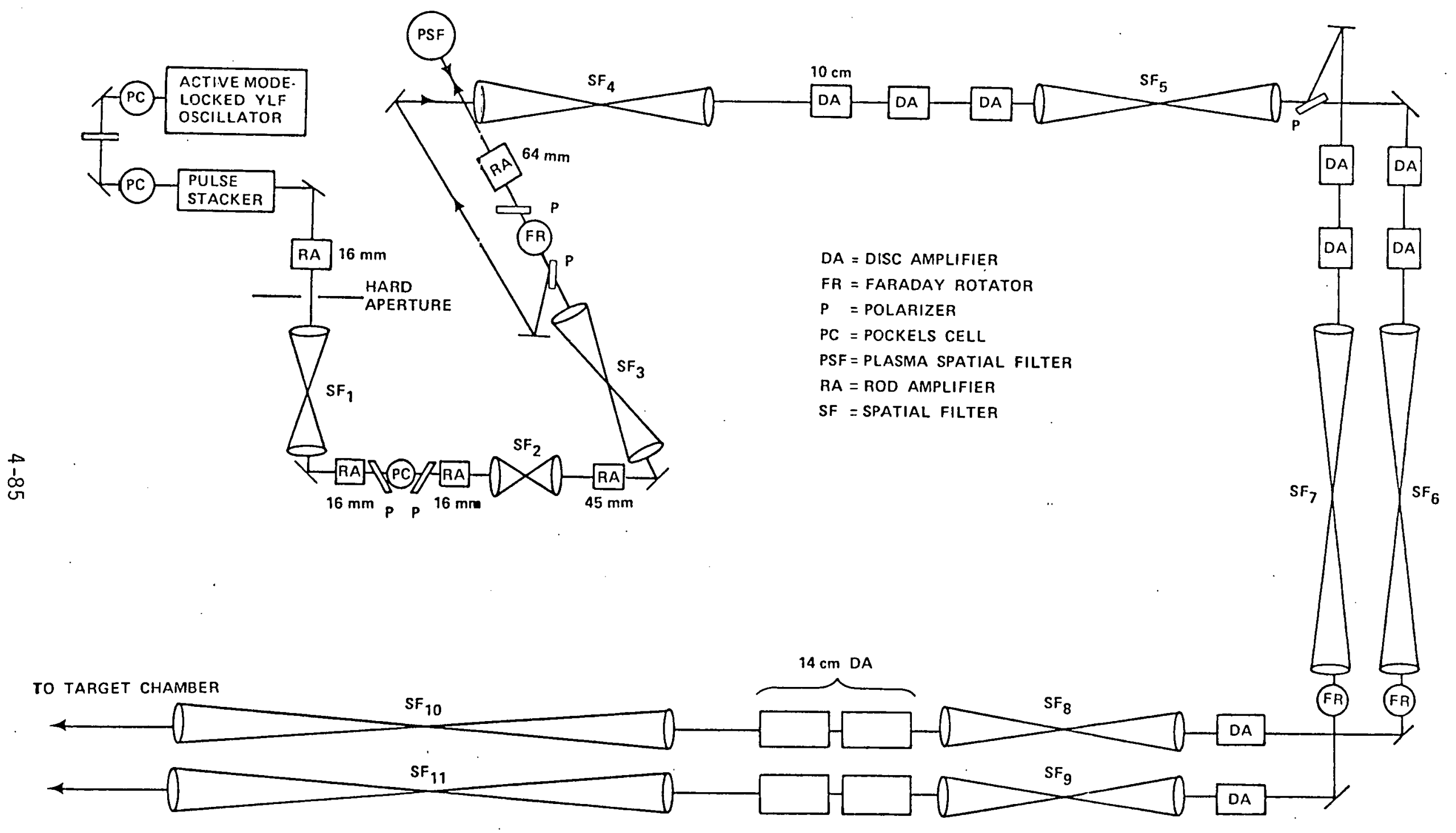

2 TW Nd: GLASS LASER SYSTEM

FIGURE 4.5-1. The Phase I Laser Upgrade To 2 TW 


\subsubsection{Phosphate Laser Disc Cladding}

G. C. Catella, J. G. Kubiak and N. K. Moncur

Special absorptive cladding is required on the edges of laser discs to attenuate parasitic oscillations within each disc; if allowed to grow, these oscillations would lower the stored energy before the desired main pulse arrived, thereby lowering system gain. These edge claddings reduce internal reflections by having the same index as the laser glass and by absorbing the Nd+ laser transitions.

Although the edge claddings are shielded from direct flashlamp exposure, they still must withstand the intense flashlamp radiation which is transmitted through the disc or leaks around the edge of the protective metal shield, without degrading or producing contaminating particles. The old edge cladding on our silicate laser discs do deteriorate under flashlamp exposure, and some of the debris from these claddings contaminates the laser disc surface. Very often particulate matter ejected from the cladding on one shot is burned into the active optical surface on subsequent shots, thereby producing permanent surface damage. We have had to periodically repolish the laser discs to minimize the system noise level (see section 4.4.5).

We have tested edge claddings on phosphate laser glass samples from three vendors by measuring the internal reflections and scattering, and by exposing the cladding directly to the flashlamp with a loading

of 25 to 30 joules $/ \mathrm{cm}^{2}$ for 10 shots. The reflection and scattering measurements at $1.064 \mu \mathrm{ill}$ are shown in Figure 4.5-2. for a variety of claddings from each vendor. The measurements for current silicate 
claddings on LHG-91H are shown for comparison. It is apparent that each vendor has improved the scattering and reflection of its claddings to an acceptable level.

Damage testing of these claddings showed that not all could be exposed to direct flashlamp radiation without damage. To improve the durability of its claddings, Kigre developed a thick monolithic cladding for its Q-88 glass. This monolithic cladding has a lower absorption coefficient, so that both the flashlamp light and the laser transitions are absorbed over a larger volume of glass than for the thin claddings. Kigre placed the monolithic cladding on $14 \mathrm{~cm}$ discs which we tested in a standard module. After 10 repeated firings with the edge cladding exposed directly to the flashlamps, we could find no damage on the surface of the cladding .

The other two vendors varied the compositions of their thin edge claddings; small samples also withstood direct exposure to the flashlamps with only minor ablation. Subsequently Owens-Illinois delivered a small sample with a monolithic edge cladding which was not damaged by flushlamp light.

In summary a11 three vendors have demonstrated durable edge claddings which reflect less than $4 \times 10^{-4}$ at $1.064 \mu \mathrm{m}$ (vs $10^{-3}$ for existing silicate claddings). Kigre and Owens-Illinois have demonstrated thick monolithic claddings which are not damaged by direct flashlamp exposure, while Hoya has demonstrated a durable thin edge cladding. Both Kigre and Owens-I17inois thin edge claddings show some small ablations after 10 flashlamp firings, but even these coatings are far 


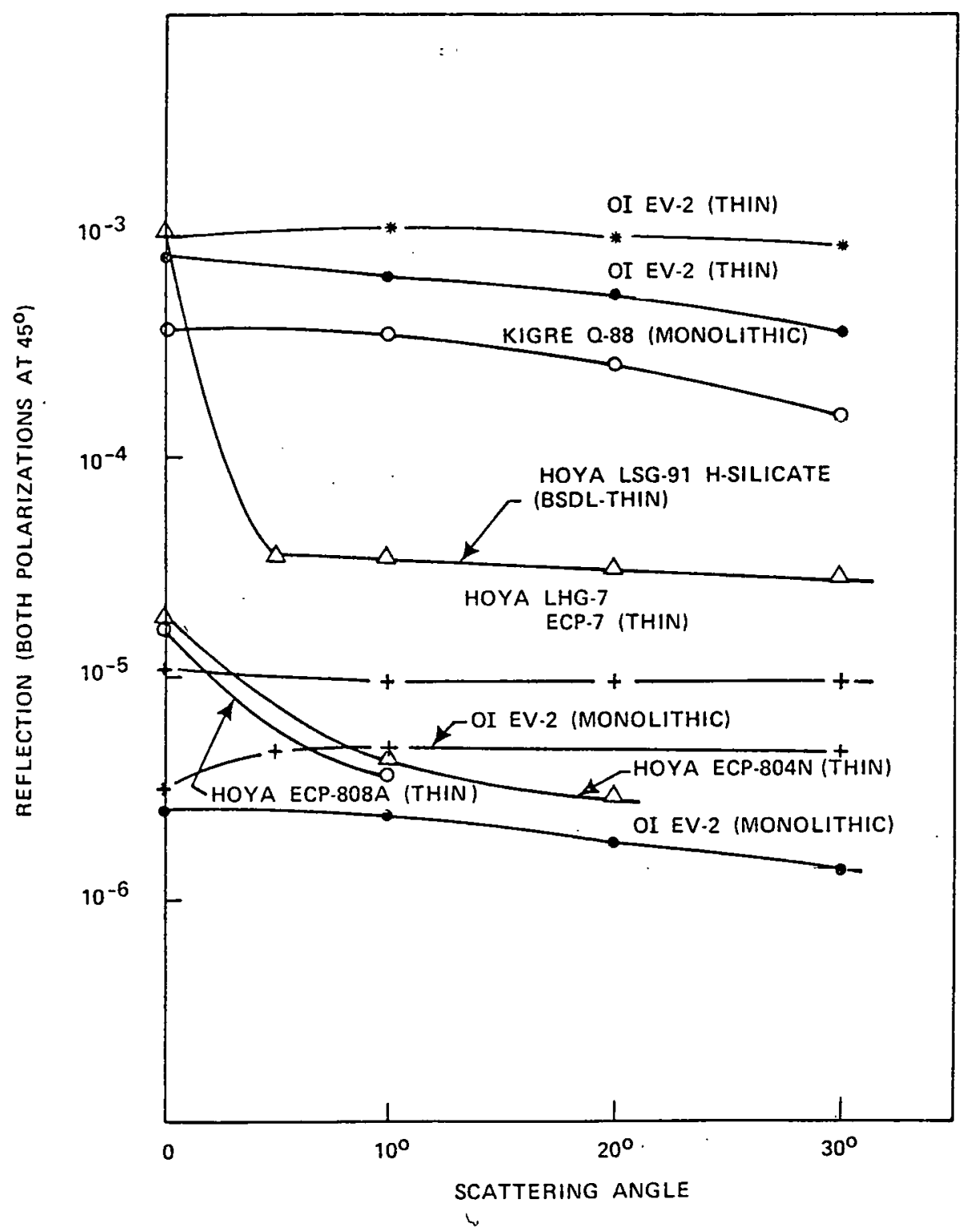

FIGURE 4.5-2 Internal reflection and scattering for various laser disc edge claddings 
superior to the old silicate frit-type thin claddings which are essentially destroyed by direct flashlamp exposure on the first shot.

\subsubsection{Disc Amplifier Modifications}

N. K. Moncur, P. D. Fairchild, H. A. Laskowski, W. W. Lawrence

D. L. Melmoth,

With proper staging; the focusable power from a B-limited, shortpulse laser system can generally be increased by increasing the smallsignal gain of the amplifiers. To improve the small-signal gain of the present KMSF 10 and $14 \mathrm{~cm}$ disc amplifiers, several modifications have been made which resulted in increased flashlamp pumping. These modifications included:

1. Changing the capacitance per lamp from $140 \mu \mathrm{f}$ to $210 \mu \mathrm{f}$.

2. Changing the lamp reflectors to close the open space behind the lamp.

3. Increasing the number of flashlamps per head from 8 to 10 in the 10-cm module. The number of lamps in the $14 \mathrm{~cm}$ module remains at 12 .

These modifications resulted in an improved small signal gain as shown in Figure 4.5-3. for silicate laser glass at the center of the modules. (The gain of the $10 \mathrm{~cm}$ amplifiers, which include three discs of $2.5 \mathrm{~cm}$ thick ED-2 silicate glass, was about 1.45 to 1.50 prior to these modifications). The gain was measured with a CW YAG laser. The design goal for the $10-\mathrm{cm}$ gain with these modifications was 1.72 $(6 \% / \mathrm{cm})$ with the silicate laser glass, which should translate to a gain of $2.2(9 \% / \mathrm{cm})$ with the phosphate laser glass at $1.054 \mu \mathrm{n}$. 'The goal for the 14-cm amplifiers was a gain of $1.48(6.5 \% / \mathrm{cm}$ for the two 2.5 $\mathrm{cm}$ thick silicate discs per amplifier) which should yield.a.gain of 1.80 $(9.8 \% / \mathrm{cm})$ with phosphate glass. ${ }^{(23)}$ The dashed lines of Figure $4.5-3$ indicate that we have exceeded the design goal for the 10 cin amplifier, 


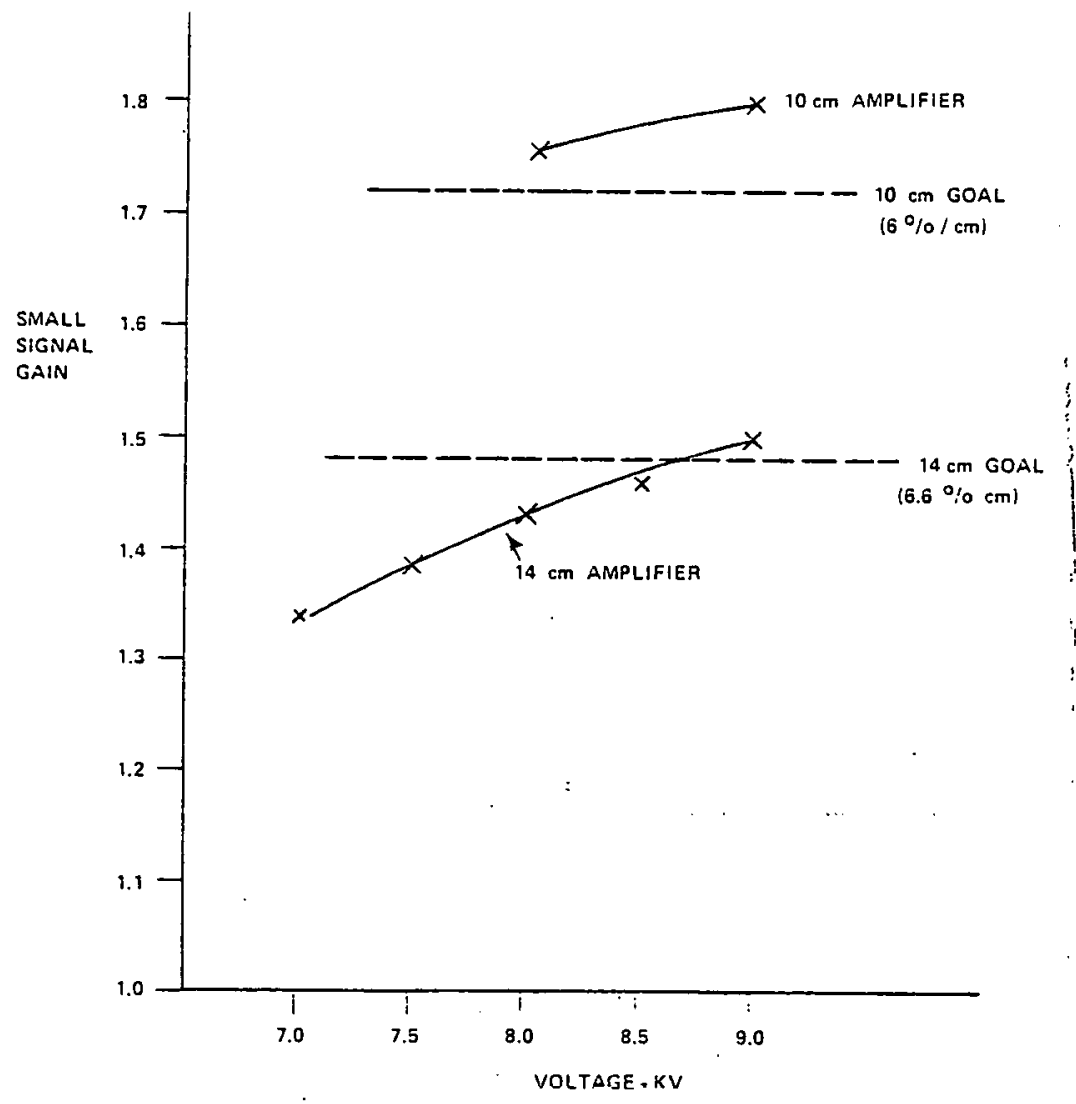

FIGURE 4.5-3 Small signal gain at center of modified 10 and $14 \mathrm{~cm}$ modules with silicate laser glass at $1.064 \mu \mathrm{m}$

and just met the goal for the $14 \mathrm{~cm}$ amplifier at $8.75 \mathrm{KV}$. Figure $4.5-4$ shows the radial gain profile of the modules.

A $14 \mathrm{~cm}$ module was assembled with two Q-88 phosphate discs. and the small signal gain was measured to be $1.49(6.6 \% / \mathrm{cm})$ at $1.064 \mu \mathrm{m}$. This should translate to a small signal gain of over $9 \% / \mathrm{cm}$ at $1.054 \mu \mathrm{m}$.

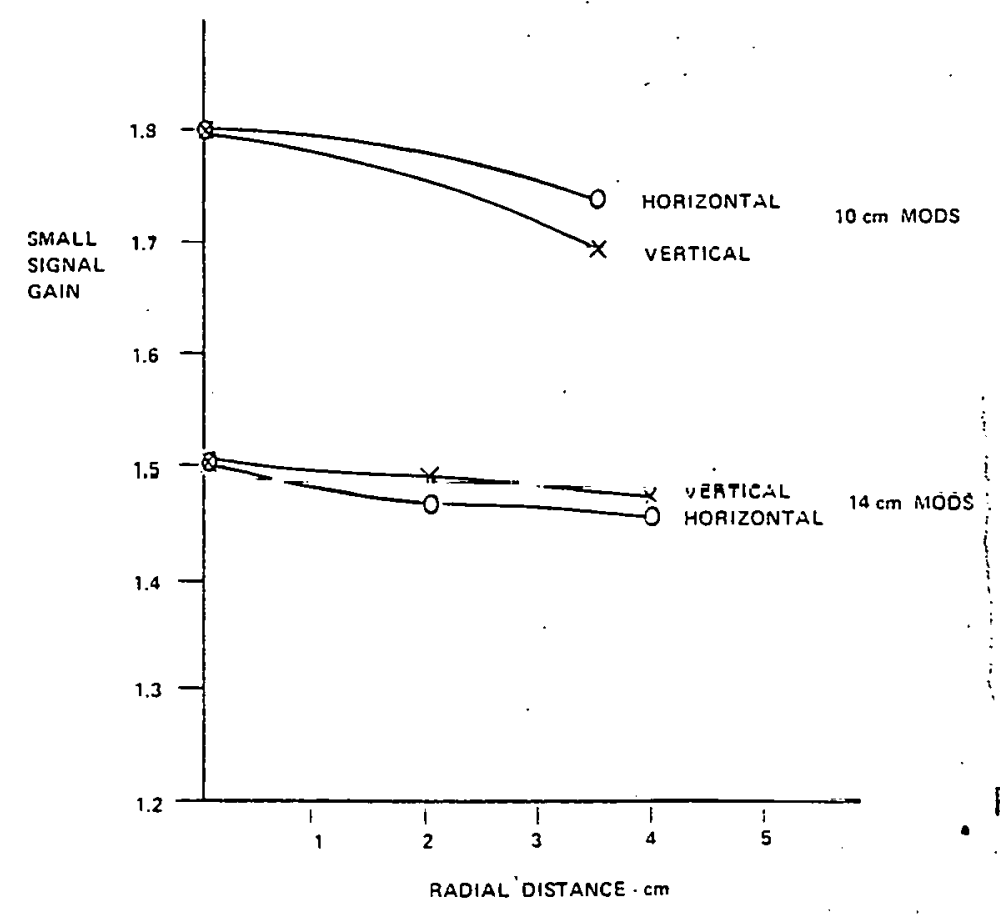

$4-90$
FIGURE 4.5-4 The radial gain profile for the modified disc arplifiers 


\section{REFERENCES}

1. 1977 Annual Report on Laser Fusion Research, KMSF \#U762, page 4-41.

2. Ibid, page 4-1

3. Ibid, page 4-22

4. Ibid, page $4-35$

5. W. E. McDermott, N. R. Pchelkin, D. J. Bernard, and R. R. Bousek, App 1. Phys. Lett, 32, 469 (1978).

6. A. W. Adamson, A Textbook of Physical Chemistry, Academic Press, New York, 1973, page 637.

7. J.M.J.Madey, J. App1. Phys, 42, 1906 (1971).

8. L. R. Elias, W. M. Fairbank, J. M. J. Madey, H. A. Schwettman, and T. I. Smith, Phys. Rev. Lett 36, 717 (1976).

9. D. A. G. Deacon, L. R. Elias, J. M. J. Madey, G. J. Ramian, H. A. Schwettman, and T. I. Smith, Phys. Rev. Lett. 38, 892, (1977):

10. W. B. Colson, Physics of Quantum Electronics, Vol 5, (1977).

11. A. Gover and A. Yariv, Applied Physics 16,121 (1978).

12. P. Sprangle, R. A. Smith, and V. L. Granatstein, NRL Memorandum Report 3888 (1978).

13. H. A. Kroll and W. A. McMultin, Phys. Rev. A 17, 300 (1978).

14. A. Renieri, "The Free Electron Laser: The Storage Ring Operation", report issued"by C. N. E. N., Frascati; Italy, December 1977.

15. J. M. J. Madey, U.S. Patent 3822410, July 2, 1974.

16. S. B. Sega11, KMSF Report No. KMSF,-U698 (T), February 1978.

17. T. I. Smith, J. M. J. Madey, L. R. Elias, and D. A. G. Deacon Stanford University Report No. HEPL-830 (August, 1978).

18. L. R. Elias, J. M. J. Madey and T. I. Smith, Stanford University Report HEPL-824 September 1978.

19. J. D. Jackson, Classical Electrodynamics, (Wiley, 1976)

20. 1977 Annual Report on Laser Fusion Research, KMS \#U762, page 4-19.

21. Ibid, page $4-8$.

22. Ibid, page $4-.23$

23. Ibid, page $4-23$ 